\title{
THE CRYOGENIC DARK MATTER SEARCH AND BACKGROUND REJECTION WITH EVENT POSITION INFORMATION
}

\author{
by \\ Gensheng Wang \\ Submitted in partial fulfillment of the requirements \\ for the degree Doctor of Philosophy
}

Thesis Adviser: Professor Daniel S. Akerib

Department of Physics

CASE WESTERN RESERVE UNIVERSITY

January 2005 


\section{CASE WESTERN RESERVE UNIVERSITY SCHOOL OF GRADUATE STUDIES}

We hereby approve the thesis/dissertation of

Gensheng Wang

candidate for the Ph.D degree.

(signed $) \frac{\text { Daniel S. Akerib }}{\text { (Chair of Committee })}$
$\frac{\text { Lawrence M. Krauss }}{\text { John E. Ruhl }}$

$\longrightarrow$

Daniel R. Stinebring

(Date) August 19, 2004 
To my family 


\section{Contents}

1 Modern Cosmology and Dark Matter 17

1.1 Introduction . . . . . . . . . . . . . . . . 17

1.2 Basics of Modern Cosmology . . . . . . . . . . . . . . 18

1.3 Observational Cosmology and Dark Matter . . . . . . . . . . . . 23

1.3.1 The Expansion of the Universe . . . . . . . . . . . . 23

1.3.2 The Cosmic Microwave Background . . . . . . . . . 26

1.3.3 Nucleosynthesis . . . . . . . . . . . . . . . . . . 30

1.3.4 The Structure of the Universe . . . . . . . . . . . . . . 33

1.3.5 Dark Matter in the Universe . . . . . . . . . . . . . 35

1.4 Dark Matter in Galaxies . . . . . . . . . . . . . . . . . . 42

1.4.1 Rotation of the Galaxy . . . . . . . . . . . . . . 42

1.4.2 Dark Matter in the Milky Way . . . . . . . . . . . . . 44

1.5 Candidates of Dark Matter . . . . . . . . . . . . . . . 47

1.5 .1 Neutrinos . . . . . . . . . . . . . . . . 48

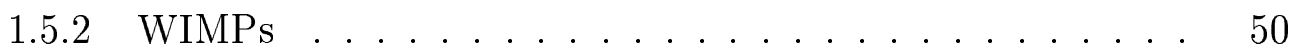

1.5.3 Axions . . . . . . . . . . . . . . 58

1.5.4 Other Particle Dark matter Candidates . . . . . . . . . 61

2 WIMP Detection $\quad 63$

2.1 Introduction . . . . . . . . . . . . . . . . 63

2.2 WIMP-Nucleon Scattering Cross Section . . . . . . . . 65

2.3 WIMP Direct Detection Rate . . . . . . . . . . . . 70

2.4 Signature of WIMP Direct Detection . . . . . . . . . . 75

2.4.1 Angular Distribution of Event Rate . . . . . . . . . 75

2.4.2 Annual Modulation . . . . . . . . . . . . . . . . 77

2.5 Indirect detection . . . . . . . . . . . . . . . . . 79

2.6 Dark Matter Search Experiments . . . . . . . . . . . 84

2.6 .1 DAMA . . . . . . . . . . . . . . . 84

2.6 .2 EDELWEISS . . . . . . . . . . . . . . 85

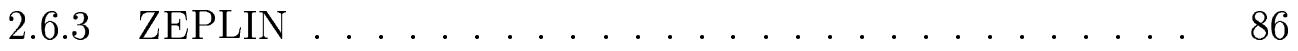

2.6 .4 Super Kamiokande . . . . . . . . . . . . . . . 86 
3 CDMS II in Soudan $\quad 88$

3.1 CDMS II Experiment Backgrounds . . . . . . . . . . . . . . 89

3.2 CDMS II Background in Soudan Mine . . . . . . . . . . 95

3.3 The Dilution Unit and Ice Box . . . . . . . . . . . . . . . 101

3.4 Readout Electronics and DAQ . . . . . . . . . . . . 107

3.4 .1 Front End Electronics _. . . . . . . . . . . . . 107

3.4 .2 DAQ . . . . . . . . . . . . . . . . . . . 109

3.5 Data Analysis . . . . . . . . . . . . . . . . 110

4 ZIP Detectors 112

4.1 Introduction . . . . . . . . . . . . . . . . . . 112

4.2 The Ionization Measurement . . . . . . . . . . . . . 116

4.2.1 The Dead Layer of Surface Events . . . . . . . . . . . . 119

4.2 .2 Charge Trapping . . . . . . . . . . . . . . 120

4.3 Phonon Measurement . . . . . . . . . . . . . . . . . 125

4.3.1 Phonons in ZIP Detectors . . . . . . . . . . . . 125

4.3.2 Quasi-particle Collection . . . . . . . . . . . . . 126

4.3.3 Electrothermal Feedback Circuit . . . . . . . . . . . . . 131

4.4 ZIP Detector Tests at CWRU . . . . . . . . . . . . 136

4.5 TES thermal parameters . . . . . . . . . . . . 142

5 Event Location Information in ZIP Detectors 151

5.1 Introduction . . . . . . . . . . . . . . 151

5.2 Primary Phonons . . . . . . . . . . . . . . . . . . 152

5.3 Neganov-Luke Phonons . . . . . . . . . . . . . . . . . . 155

5.4 Event Location Reconstruction . . . . . . . . . . . . . 162

5.5 Event Location Calculation . . . . . . . . . . . . . . 173

5.6 Understanding Event Position Information . . . . . . . . . . . 184

6 Detector Setup and Calibrations 194

6.1 Detector Neutralization . . . . . . . . . . . . . . . 195

6.2 Optimizing TES Bias . . . . . . . . . . . . . . . . 197

6.3 Position Dependence . . . . . . . . . . . . . . . 200

6.4 Energy Calibration . . . . . . . . . . . . . . . 203

6.5 The Detector Noise . . . . . . . . . . . . . . . . . 208

6.6 Inner Electrode Event Selection . . . . . . . . . . . . . 211

6.7 Data Quality Cuts . . . . . . . . . . . . . . . . . 214

6.8 Electron and Nuclear Recoil Bands . . . . . . . . . . . . . 219

6.9 Detector Stability . . . . . . . . . . . . . . . 226 
7 WIMP Search Data and Analysis 229

7.1 Tower I at Soudan . . . . . . . . . . . . . . . . 230

7.2 Background Discrimination with Ionization Yield . . . . . . . . 231

7.3 Rejecting Surface Events . . . . . . . . . . . . . . . 234

7.4 Phonon Partition for Events with Big Radius . . . . . . . . . 248

7.5 Position Based WIMP Search Data Analysis . . . . . . . . . . 259

7.6 Blind WIMP Search Data Analysis . . . . . . . . . . . 271

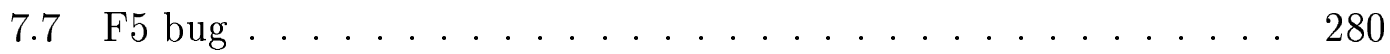

8 Conclusion 283

8.1 WIMP Exclusion Limit . . . . . . . . . . . . . . . . 283

8.2 Outlook . . . . . . . . . . . . . . . . 286

8.3 On My Contributions to A Broad Collaborative Experiment . . 287

A LED Study 293

A.1 Introduction . . . . . . . . . . . . . . . . . 293

A.2 LED I-V Measurement . . . . . . . . . . . . . . . . . 294

A.3 Charging Time Constant . . . . . . . . . . . . . . 297

A.4 Heating Power Estimation . . . . . . . . . . . . . . . 299

A.5 CDMS Detector Neutralization . . . . . . . . . . . 301

A.5.1 LED Photon Energy Spectrum . . . . . . . . . . . . 301

A.5.2 Intrinsic Excitation Threshold of Silicon . . . . . . . . 301

A.5.3 Detector's Conductance with LED Flashing . . . . . . . 303

A.5.4 Electron Diffusion in the Crystal . . . . . . . . . . 305

A.6 Summary . . . . . . . . . . . . . . 306

B Plots of Position Based Data Analysis 307

$\begin{array}{ll}\text { C The Efficiency } & 326\end{array}$ 


\section{List of Figures}

1.1 Hubble constant measurement . . . . . . . . . . . . . . . 24

1.2 SN Ia residual Hubble diagram $\ldots \ldots \ldots \ldots \ldots$

1.3 Anisotropy of the cosmic microwave background . . . . . . . . 27

1.4 The WMAP angular power spectrum . . . . . . . . . 28

1.5 Evolution of the light element abundances over time . . . . . . 31

1.6 The predicted abundance of the light elements vs.baryon density . 32

1.7 The large-scale structures in the galaxy distribution . . . . . . . 34

1.8 Power spectrum of density inhomogeneity today . . . . . . . . 34

1.9 The contours on $\Omega_{\mathrm{M}}$ and $\Omega_{\Lambda} \ldots \ldots \ldots \ldots \ldots \ldots \ldots \ldots$

1.10 Rotation curve of NGC $6503 \ldots \ldots \ldots$. . . . . . . . . . . 43

1.11 the density profile of dark matter halos as a function of radius . . 44

1.12 The rotation curve for the Milky Way . . . . . . . . . . . 45

1.13 The range of local dark matter densities . . . . . . . . . 46

1.14 Feynman diagrams contributing to early universe neutralino anni-

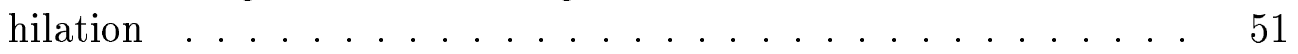

1.15 Evolution of a typical WIMP number density in the early universe 52

1.16 Relic density of the lightest neutralino as a function of its mass . 57

1.17 Laboratory, astrophysical, and cosmological constraints on the axion mass . . . . . . . . . . . . . . . . 59

1.18 Experimental constraints on the density of axions $\ldots \ldots \ldots 60$

2.1 Feynman diagrams contributing to neutralino-nucleon cross section 64

2.2 WIMP-nucleon elastic scattering cross section versus WIMP mass 69

2.3 The differential event rate of $\mathrm{Si}$ and of $\mathrm{Ge} \ldots \ldots . \ldots 74$

2.4 The integrated event rate of $\mathrm{Si}$ and of Ge . . . . . . . . . 74

2.5 The angular distribution of nuclear recoil events . . . . . . 76

2.6 Number of signal events required as a function of of threshold energy 77

2.7 Schematic view of the Earth motion around the Sun . . . . . 78

2.8 Simulation of a gamma-ray annihilation line from the annihilation of $\sim 48 \mathrm{GeV}$ neutralinos . . . . . . . . . . . . 82

2.9 Annual modulation of the total counting rate for seven years of data with the DAMA-NaI detectors . . . . . . . . 85 
2.10 Super-K 90 \% CL exclusion region in WIMP parameter space . .

3.1 Muon rate at Soudan . . . . . . . . . . . . . 96

3.2 Limiting sensitivity of WIMP-nucleon cross section as a function of raw exposure in kg-day . . . . . . . . . . . . . 101

3.3 The dilution refrigerator and ice box ........... 103

3.4 The tower that houses six CDMS ZIP detectors . . . . . . . 104

3.5 The FET card, SQUID card and the detectors . . . . . . . 105

4.1 A photograph of a ZIP detector in its housing . . . . . . . . 113

4.2 A schematic of ZIP detector surface structures . . . . . . . . 116

4.3 A schematic of the ionization readout. . . . . . . . . . . 117

4.4 The $60 \mathrm{keV}$ line from ${ }^{241} \mathrm{Am}$ source in CWRU run27 . . . . . . . . 121

4.5 The collected charge versus electron recoil energy of Z6 . . . . . 122

4.6 Phonon dispersion relation of silicon . . . . . . . . . . 125

4.7 Superconducting gap structure of the quasiparticle traps . . . . 127

4.8 A sketch of the ZIP detector phonon sensor . . . . . . . . . . 128

4.9 Simplified Circuit Diagram for the Phonon Sensor . . . . . . . . . 131

4.10 Tungsten Resistance Temperature Relation . . . . . . . . . . . . . 132

4.11 Critical Current Curves for Detector Z5 (G9) . . . . . . . . . . 139

4.12 IbIs curves for detector G9 (Z5), channel D . . . . . . . . . . 140

4.13 Americium source spots and Energy Spectrum in S11 . . . . . . 143

$4.14 R-I_{b}$ and $R-T$ relations, S11, sensor A . . . . . . . . . 144

$4.15 R-I_{b}$ and $R-T$ relations, S11, sensor B . . . . . . . . . 145

4.16 TES temperature and thermal coupling coefficient . . . . . . . 149

4.17 TES heat capacity . . . . . . . . . . . . 149

5.1 Average size of electrons and holes cloud of an electron recoil as a function of energy . . . . . . . . . . . . . 154

5.2 Diagram of electron and phonon wave vectors . . . . . . . . 156

5.3 The electron Neganov-Luke phonon emission rate at different frequencies for a germanium detector with -3V bias . . . . . . . 159

5.4 The hole Neganov-Luke phonon emission rate at different frequencies for a germanium detector with $-3 \mathrm{~V}$ bias . . . . . . . . 159

5.5 The electron Neganov-Luke phonon energy angle distribution for eermanium detector with $-3 \mathrm{~V}$ bias . . . . . . . . . 160

5.6 The hole Neganov-Luke phonon energy angle distribution for germanium detector with $-3 \mathrm{~V}$ bias . . . . . . . . . . 161

5.7 A particle interaction in quadrant A of a ZIP detector . . . . . . 162

5.8 Comparison of primary phonons and Neganov-Luke phonons . . . 163

5.9 The pre-given event locations in quadrant A of the ZIP detector . 165 
5.10 The reconstructed $\mathrm{X}$ and $\mathrm{Y}$ for the pre-given 185 locations . . . 170

5.11 The reconstructed $\mathrm{Z}$ for the pre-given 185 locations . . . . . . 171

5.12 Phonon propagation distance distributions . . . . . . . . . . 174

$5.13 \mathrm{Z2}$, phonon timing parameter distributions . . . . . . . . 175

$5.14 \mathrm{Z} 2$, normalized phonon front propagation distance distributions . 177

$5.15 \mathrm{Z} 2$, reconstructed event locations . . . . . . . . . . 177

5.16 Reconstructed event locations for G31, $1074 \ldots \ldots$. . . . . . . 178

5.17 Reconstructed event locations for G31, 2038 . . . . . . . . . . 179

5.18 Reconstructed event locations for G31, quadrant C . . . . . . . 184

5.19 Yield versus recoil energy plot for G31, quadrant C . . . . . . . 185

5.20 Z5: reconstructed event locations for high ionization yield events . 186

5.21 Z5: yield distribution as a function of radius for local quadrant events 187

$5.22 \mathrm{Z} 5$ : Phonon start time distribution as a function of radius for local quadrant events . . . . . . . . . . . . . 188

5.23 Z5: Rise time distribution as a function of radius for local quadrant

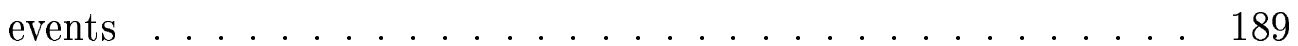

5.24 Z5: Neutron and surface events in the $\mathrm{x}$ and $\mathrm{y}$ plane . . . . . . 190

5.25 Z5: Neutron and surface events in the R and Z plane . . . . . . 191

$5.26 \mathrm{Z} 5$ : ZZ parameter versus ionization yield for neutron and surface events ......................... 191

5.27 Z5: ejectrons before and after ZZ parameter cut . . . . . . . 192

6.1 Noise Spectra of Z3 . . . . . . . . . . . . . . . . 197

6.2 Balancing phonon energy and timing parameters of Z3 . . . . 198

$6.3 \mathrm{Y}$ dependence of charge collection efficiency . . . . . . . . . . 201

6.4 Charge energy spectra after before and after position correction . 202

6.5 Charge energy spectra and MC simulation of ${ }^{133} \mathrm{Ba}$ calibration . . 204

6.6 Phonon energy spectra and MC simulation of ${ }^{133} \mathrm{Ba}$ calibration . . 205

6.7 Charge inner $10 \mathrm{keV}$ line in low background data . . . . . . . 206

6.8 Phonon $10 \mathrm{keV}$ line in low background data . . . . . . . . 207

6.9 The noise blobs of baseline fluctuation . . . . . . . . . . . 209

6.10 Inner electrode noise blob fit for threshold identification . . . . . 210

$6.113 \sigma$ band of inner electrode events . . . . . . . . . . . . 211

6.12 Inner electrode event selection . . . . . . . . . . . . 212

6.13 The qi cut efficiency .................. 213

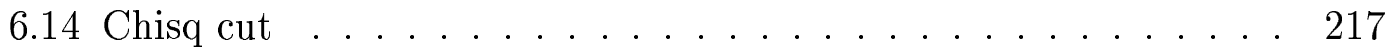

6.15 Event selection efficiency of Chisq cut . . . . . . . . . . . 218

6.16 Find the mean and standard deviation of gamma band . . . . . 220

6.17 electron recoil band and nuclear recoil band of Z3 . . . . . . . . . 221

6.18 The band width parameters of Z3 . . . . . . . . . . . 222 
6.19 Find the mean and standard deviation of nuclear recoil band with Gaussian fit . . . . . . . . . . . . . . . 223

$6.20 \mathrm{Z1}$-Z6 data and bands . . . . . . . . . . . . . . . . 224

6.21 Stability of electron recoil band . . . . . . . . . . . 227

6.22 Stability of inner electrode noise . . . . . . . . . . . . 228

7.1 Live time of CDMS run118 . . . . . . . . . . . . . 230

7.2 The ionization energy versus the recoil energy . . . . . . . . . 232

7.3 Ionization yield versus the recoil energy . . . . . . . . . . 233

7.4 A $60 \mathrm{keV}$ event raw traces in $\mathrm{Z} 2 \ldots \ldots \ldots \ldots \ldots . \ldots 235$

7.5 Ejectrons in $\mathrm{Z} 5 \ldots \ldots \ldots \ldots \ldots \ldots \ldots \ldots$

7.6 pdelc distribution of neutrons and ejectrons in Z5 . . . . . . 240

7.7 The optimized pdelc cut value search for Z5 . . . . . . . . . 241

7.8 The pdelc cut and efficiency of Z5. . . . . . . . . . . . . 241

7.9 ptrtc distribution of neutrons and ejectrons in Z5 . . . . . . 242

7.10 The optimized ptrtc cut value search for Z5. . . . . . . . . 243

7.11 The ptrtc cut and efficiency of Z5. . . . . . . . . . . 243

7.12 pminrtc distribution of neutrons and ejectrons in Z5 . . . . . 244

7.13 The optimized pminrtc cut value search for Z5. . . . . . . . 245

7.14 The pminrtc cut and efficiency of Z5. . . . . . . . . . 245

$7.15 \mathrm{ZZ}$ distribution of neutrons and ejectrons in Z5 . . . . . . 246

7.16 The optimized ZZ cut value search for Z5. . . . . . . . . 247

7.17 The ZZ cut and efficiency of Z5 . . . . . . . . . . . . 247

7.18 Separate events in Z5 into two regions. . . . . . . . . . . 253

7.19 High timing parameter events have big radius. . . . . . . . . 254

$7.20 \mathrm{Z} 5$, ptrtc distribution as a function of radius. . . . . . . . 254

$7.21 \mathrm{Z} 5$, pminrtc distribution as a function of radius. . . . . . . 255

$7.22 \mathrm{Z}$, pdelc distribution as a function of radius. . . . . . . 255

$7.23 \mathrm{Z}$, phonon partition distribution as a function of pdelc. $\ldots . .256$

$7.24 \mathrm{Z}$, phonon partition distribution as a function of pdelc, 3. . . . 257

7.25 Phonon partition as a function of radius $\mathrm{R} \ldots \ldots \ldots \ldots$

7.26 Phonon partition as a function of local quadrant phonon start time 258

$7.27 \mathrm{Z5}$, position based low background data analysis . . . . . . 266

$7.28 \mathrm{Z} 5$, phonon delay cut for small radius events in calibration data and low background data . . . . . . . . . . . 267

7.29 Z5, the efficiencies of neutron selection and surface event rejection for position information based data analysis . . . . . . . 267

$7.30 \mathrm{Z1}$, before and after phonon partition cut . . . . . . 268

$7.31 \mathrm{Z} 2$, before and after phonon partition cut . . . . . . . 268

7.32 Z3, before and after phonon partition cut . . . . . . . . 269

$7.33 \mathrm{Z} 4$, before and after phonon partition cut . . . . . . . . 269 
7.34 Z5, before and after phonon partition cut . . . . . . 270

$7.35 \mathrm{Z} 6$, before and after phonon partition cut . . . . . . . . 270

7.36 Setting of phonon timing parameter cuts for surface event rejection 272

7.37 Timing cut efficiencies on neutron selection and surface event rejection in blinding low background data analysis . . . . . . . 276

7.38 All low background data before timing cuts in blinding analysis . 277

7.39 Single scattering events after timing cuts in blinding analysis . . . 278

7.40 Muon anto-coincident multiple scattering events after timing cuts in blinding analysis . . . . . . . . . . . . . . . 279

7.41 Single scattering events in Z6 . . . . . . . . . . 280

7.42 A single scattering in Z $\quad \ldots \ldots \ldots \ldots \ldots \ldots \ldots$

8.1 Total nuclear recoil event selection efficiency of four germanium detectors . . . . . . . . . . . . . . . . . 284

8.2 WIMP exclusion limit . . . . . . . . . . . . . 285

A.1 LED I-V measurement circuit. . . . . . . . . . . . . . . . 294

A.2 LED I-V relation at different temperatures. Squares are LED at

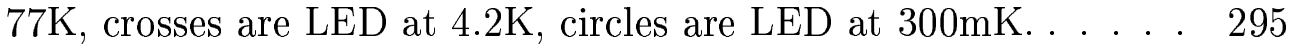

A.3 LED I-V relation at different temperatures. The Xes are for LED at $48.1 \mathrm{~K}$, squares are for LED at $26.1 \mathrm{~K}$, crosses are for LED at $13.5 \mathrm{~K}$, and circles are for LED at $7.4 \mathrm{~K} \ldots \ldots \ldots \ldots . \ldots 296$

A.4 LED turn-on time. . . . . . . . . . . . . . . . . . 298

A.5 LED turn-on time and heating power estimation. . . . . . . 300

A.6 Energy gap structure of silicon . . . . . . . . . . . . . . 302

A.7 Detector resistance with LED flashing current. . . . . . . . . . . 303

A.8 Photon absorption coefficient with photon energy . . . . . . . 304

B.1 Z1, phonon partition cut for big radius events . . . . . . 308

B.2 Z1, position based low background data analysis . . . . . . 309

B.3 Z1, phonon delay cut for small radius events in calibration data and low background data . . . . . . . . . . . . 310

B.4 Z1, the efficiencies of neutron selection and surface event rejection for position information based data analysis . . . . . . . 310

B.5 Z2, phonon partition cut for big radius events . . . . . . . . 311

B.6 Z2, position based low background data analysis . . . . . . . 312

B.7 Z2, phonon delay cut for small radius events in calibration data and low background data . . . . . . . . . . . . . 313

B.8 Z2, the efficiencies of neutron selection and surface event rejection for position information based data analysis . . . . . . . . 313

B.9 Z3, phonon partition cut for big radius events . . . . . . . . . 314 
B.10 Z3, position based low background data analysis . . . . . . 315

B.11 Z3, phonon delay cut for small radius events in calibration data and low background data . . . . . . . . . . . 316

B.12 Z3. the efficiencies of neutron selection and surface event rejection for position information based data analysis . . . . . . . 316

B.13 Z4, phonon partition cut for big radius events . . . . . . . 317

B.14 Z4, position based low background data analysis . . . . . . 318

B.15 Z4, phonon delay cut for small radius events in calibration data and low background data . . . . . . . . . . . . . . . 319

B.16 Z4, the efficiencies of neutron selection and surface event rejection for position information based data analysis . . . . . . . . 319

B.17 Z5, phonon partition cut for big radius events . . . . . . . . 320

B.18 Z5, position based low background data analysis . . . . . . . . 321

B.19 Z5, phonon delay cut for small radius events in calibration data and low background data . . . . . . . . . . . 322

B.20 Z5, the efficiencies of neutron selection and surface event rejection for position information based data analysis . . . . . . . 322

B.21 Z6, phonon partition cut for big radius events . . . . . . . . 323

B.22 Z6, position based low background data analysis . . . . . . . 324

B.23 Z6, phonon delay cut for small radius events in calibration data and low background data . . . . . . . . . . . . 325

B.24 Z6, the efficiencies of neutron selection and surface event rejection for position information based data analysis . . . . . . . 325

C.1 Efficiency of data quality cuts . . . . . . . . . . . 327

C.2 Efficiency of charge inner electrode cut . . . . . . . . . 328

C.3 Efficiency of charge energy threshold cut . . . . . . . . . . . 329

C.4 Efficiency of surface event rejection cuts . . . . . . . . 330

C.5 Preselection of neutrons . . . . . . . . . . . . . 331

C.6 Efficiency of nuclear recoil band cut . . . . . . . . . . . 332

C.7 Efficiency of nuclear recoil selection . . . . . . . . . . 333 


\section{List of Tables}

1.1 Standard Model particles and their superpartners in the MSSM . 54

3.1 Tower 1 stack configuration .............. 106

4.1 Silicon and germanium characteristic parameters . . . . . . . . 114

4.2 The trapping cross section of ionized donors at temperatures below $1 \mathrm{~K} \ldots \ldots \ldots \ldots \ldots \ldots . \ldots \ldots 124$

4.3 Basic phonon sensor properties for detector Z5 (G9). . . . . . 138

4.4 Thermal parameters of S11 sensor A . . . . . . . . . . 148

4.5 Thermal parameters of S11 sensor A . . . . . . . . . 148

5.1 Local quadrant phonon timing normalization coefficients of all six detectors . . . . . . . . . . . . . . . . 181

5.2 Neighbor quadrant phonon timing nromalization coefficients of germanium detectors . . . . . . . . . . . . . . 182

5.3 Neighbor quadrant phonon timing normalization coefficients of silicon detectors . . . . . . . . . . . . . . . . . 182

6.1 Charge inner energy threshold . . . . . . . . . . . . . 211

6.2 Charge outer $3 \sigma$ band fit coefficients . . . . . . . . . . 213

6.3 The Charge inner event selection .............. 214

6.4 Event selection efficiency with cPstdS cut for Tower I detectors. . 216

6.5 cChisq cut and event selection efficiency . . . . . . . . 219

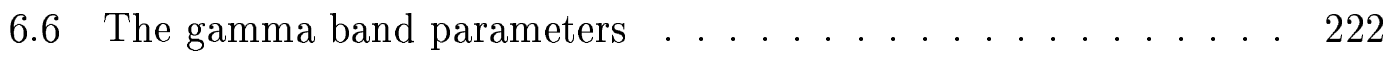

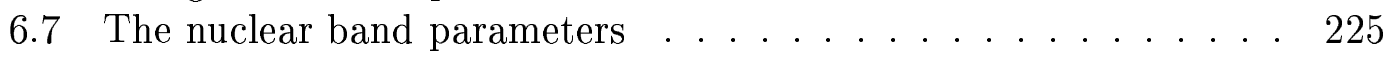

7.1 Surface event rejection parameters for events having small radius . 260

7.2 Surface event rejection parameters for events having big radius . . 261

7.3 Anti-coincident single scattering events in Z5 . . . . . . . . 263

7.4 Multiple scattering events in position information based low background data analysis . . . . . . . . . . . . . 265

7.5 Timing cut parameters in low background data blinding analysis . 273

7.6 Single scattering events in Z6 ............. 275 
7.7 Single scattering events in the blinding data analysis . . . . . 275 


\section{ACKNOWLEDGEMENTS}

I'd like to say thank you to the people at Case Western Reserve University and in CDMS collaboration, who have made my research and this thesis possible in the last five years.

First of all, I'd like to thank Dan Akerib, my thesis advisor. I have benefited tremendously from Dan's broad knowledge of physics, his ability to quickly grasp and explain new physics concepts, and his talent for separating the essentials from the details. Dan provided me not only with the opportunity to work on the challenging and interesting CDMS experiment, but with a large degree of academic freedom to pursue research in an independent way. Thank you, Dan, for your leadership, friendship and encouragement.

My association with Richard Schnee has been not only in data analysis and making fancy plots, but also in ping pong. Richard is the "Analysis Guru" in the CDMS collaboration. I turned to him for data analysis problems quite often. He was also very happy to explain what is the difference between cosmology and astrophysics. His patience and instruction always guided me to overcome the difficulties I encountered in the last few years. Thank you, Richard. I hope we can find a time to play bridge.

I'd like to thank the research associates I have worked with in the CWRU group: Alex Bolozdynya, Mike Dragowsky, and Darren Grant, for their guidance and friendship. I only worked with Alex Bolozdynya for a short period of time before he moved to Florida. I admire his broad knowledge in experimental physics. It has been a lot fun working with Mike Dragowsky. We had good times discussing topics like cryogenics, ionization charge collection, and phonon physics, rotating a plot $90^{\circ}$, and more. Darren Grant is such a nice person, he sometimes had his 
lunch two hours later for helping me make diagrams look better.

I'd like to thank my fellow graduate students in the CWRU group: Thushara Perera, Donald Driscoll, Sharmila Kamat, Raul Hennings-Yeomans, and Cathy Bailey. Thushara's ability to learn new knowledge is amazing. Our discussions included mechanical dynamics, quantum theory, cryogenics, SQUID, and Asian culture. Don's knowledge in computer and automatic control made a lot of our routine measurement easier; of course, phonon physics and quasi-particle collection were the most important parts of our discussions. I have had the pleasure of working with Sharmila Kamat. Her hard work in detector testing and Monte Carlo simulation of neutron backgrounds is impressive. Raul and Cathy are new graduate students in our group, I appreciate their kindness, and their questions in research. I would like to thank the technicians, Aaron Manalaysay and Adam Sirois, for their excellent work and for the discussions with them on cryogenics, labview, SQUID, etc. I'd also like to thank the undergraduates, Tim Peshek, Tim Janezic, Bryan Linkous, David Nielsen, and others, for their excellent lab work to make the lab a fun and lively workplace, and for managing our computer.

I'd like to thank my thesis committee members, Lawrence Krauss, John Ruhl, Dan Stinebring, and Dan Akerib, for their interest and patience.

I'd like to thank all the secretaries in the Physics Department for making my life much easier than it could have been in last five years.

I'd like to thank Rachel Goldberg for her helpful corrections of the draft of my thesis.

I owe many thanks to the CDMS collaboration. It has been my pleasure for having the great opportunity of working with Dan Bauer, Bruce Lambin, Rodney Choate, Bryan Johnson, Rich Schmitt, Jim Beaty, Paul Brink, Long Duong, Clarence Chang, Vuk Mandic, Joel Sanders, and others. We all shared the diffi- 
cult time during the Soudan experiment construction and the excitement of the eventual success in cryogenics in the Soudan mine. And special thanks to my fellow graduate students in the CDMS collaboration, Clarence Chang, Vuk Mandic, Joel Sanders, Walt Ogburn and others, for their hard work and excellent job in making the CDMS WIMP search data analysis so enjoyable, for their suggestions and support in the position based data analysis. I'd like to thank Bernard Sadoulet and Blas Cabrera for their guidance and instruction in semiconductor energy gap theory, electron dynamics in semiconductor, and phonon generation and propagation, etc. Paul Brink has been the person in the CDMS collaboration to whom I have most often communicated; most of my questions have been the details of the ZIP detector structure, phonon physics, charge transportation dynamics, superconductors, and semiconductor physics. Thank you, Paul.

Finally, let me thank my family and friends. The most important role has been played by my wife, Yitian. Without her love and support, I would not have survived the last five years. I missed many of the concerts, the music classes, and the holidays that I should have shared with my daughter, Xuanji. I'd like to say thank you to my daughter for your understanding. I thank my parents for the most important support and encouragement I have had during all my years of school. I also thank my brother, Yongsheng, and my sister, Xiaomei, for their support and understanding. I'd like to thank my friends, Yianpin Pan and others. 
The Cryogenic Dark Matter Search and Background

Rejection with Event Position Information

\author{
Abstract \\ by \\ Gensheng Wang
}

Evidence from observational cosmology and astrophysics indicates that about one third of the universe is matter, but that the known baryonic matter only contributes to the universe at 4\%. A large fraction of the universe is cold and nonbaryonic matter, which has important role in the universe structure formation and its evolution. The leading candidate for the non-baryonic dark matter is Weakly Interacting Massive Particles (WIMPs), which naturally occurs in the supersymmetry theory in particle physics.

The Cryogenic Dark Matter Search (CDMS) experiment is searching for evidence of a WIMP interaction off an atomic nucleus in crystals of Ge and Si by measuring simultaneously the phonon energy and ionization energy of the interaction in the CDMS detectors. The WIMP interaction energy is from a few $\mathrm{keV}$ to tens of $\mathrm{keV}$ with a rate less than 0.1 events $/ \mathrm{kg} /$ day. To reach the goal of WIMP detection, the CDMS experiment has been conducted in the Soudan mine with an active muon veto and multistage passive background shields.

The CDMS detectors have a low energy threshold and background rejection capabilities based on ionization yield. However, betas from contamination and other radioactive sources produce surface interactions, which have low ionization yield, comparable to that of bulk nuclear interactions. The low-ionization surface electron recoils must be removed in the WIMP search data analysis. An emphasis of this thesis is on developing the method of the surface-interaction rejection using 
location information of the interactions, phonon energy distributions and phonon timing parameters. The result of the CDMS Soudan run118 92.3 live day WIMP search data analysis is presented, and represents the most sensitive search yet performed. 


\section{Chapter 1}

\section{Modern Cosmology and Dark}

\section{Matter}

\section{$1.1 \quad$ Introduction}

Cosmology helps us to understand how the universe came into being, why it looks as it does now, and what its future holds. Cosmologists make astronomical observations that probe billions of years into the past, to the edge of the knowable universe. They seek the basis of scientific understanding, using the tools of modern physics, and fashion theories that provide unified and testable models of the evolution of the universe from its creation to the present and beyond. Modern cosmological efforts include: measuring the Hubble constant, the deceleration parameter, and the energy density parameters; understanding the formation of the large structure; and understanding the universe as a whole with the relic signatures, such as Cosmic Microwave Background (CMB), as the tools. As we will see when examining the observational evidence, the matter density can't be accounted for by ordinary baryonic matter. The work described in this thesis is a 
search for what the missing matter might be.

In this chapter, I review modern cosmology, discuss the evidence for the existence of cold dark matter, and describe the particle dark matter candidates. The main references for this chapter are [1], [2], and [3].

\subsection{Basics of Modern Cosmology}

Mathematical constructs or physical models are the basic tools in cosmology. Through these models we hope to explain the observed phenomena, and the current state of the universe, as well as its beginning and its end.

The first assumption that we make while constructing models for the universe is that it is homogenous and isotropic. Homogeneity is the property that makes every point indistinguishable from every other point and isotropy is the property that makes every direction indistinguishable from every other. Homogeneity is invariance under translations, while isotropy is invariance under rotations.

From cosmological and astrophysical observations, we know that the universe is homogeneous and isotropic on large scales. The universe expands as a function of time. The Robertson-Walker metric is used to describe the physical universe, and is described below.

In spatial coordinates analogous to two dimensional polar coordinates, the

four dimensional separation $d s$ between two events in space-time $(t, x, \theta, \phi)$ and $(t+d t, x+d x, \theta+d \theta, \phi+d \phi)$ satisfies:

$$
d s^{2}=d t^{2}-a(t)^{2}\left(d x^{2}+S_{k}(x)^{2}\left(d \theta^{2}+\sin ^{2} \theta d \phi^{2}\right)\right)
$$




$$
S_{k}(x)= \begin{cases}R \sin (x / R) & \text { if } k=+1 \\ x & \text { if } k=0 \\ R \sinh (x / R) & \text { if } k=-1\end{cases}
$$

where $R$ is the curvature radius of the comoving universe today; $a(t)$ is called the scale factor; $t$ is the cosmological proper time, or cosmic time, which is the time measured by an observer who sees the universe expanding uniformly around him; $x$ is the radial coordinate analogous to $\rho$ in two dimensional polar coordinates; $(t, x, \theta, \phi)$ are called comoving coordinates of a point in space; and $k$ is the curvature constant, where $k=0$ for a flat universe, $k=+1$ for a positively curved universe, and $k=-1$ for a negatively curved universe.

In spatial coordinates analogous to three dimensional spherical coordinates, $r=S_{k}(x)$, the four dimensional separation $d s$ between two events in the comoving space-time given by $(t, r, \theta, \phi)$ and $(t+d t, r+d r, \theta+d \theta, \phi+d \phi)$ satisfies:

$$
d s^{2}=d t^{2}-a(t)^{2}\left(\frac{d r^{2}}{1-k(r / R)^{2}}+r^{2}\left(d \theta^{2}+\sin ^{2} \theta d \phi^{2}\right)\right)
$$

The fundamental equations of cosmology include two equations from general relativity:

$$
\begin{gathered}
\frac{\ddot{a}}{a}=-\frac{4 \pi G}{3}(\rho+3 p)+\frac{\Lambda}{3} \\
\frac{\ddot{a}}{a}+2\left(\frac{\dot{a}}{a}\right)^{2}+2 \frac{k}{a^{2} R^{2}}=4 \pi G(\rho-p)
\end{gathered}
$$

where $G$ is the gravitational constant, $\rho$ is the density of matter, $p$ is pressure of matter (and photons), and $\Lambda$ is the vacuum energy (dark energy) of the universe.

Combining equations 1.4 and 1.5 together yields the Friedmann equation:

$$
\left(\frac{\dot{a}}{a}\right)^{2}=\frac{8}{3} \pi G \rho-\frac{k}{a^{2} R^{2}}+\frac{\Lambda}{3} .
$$

An isotropic perfect fluid obeys an equation of state

$$
p=w \rho
$$


where $w=\frac{1}{3}$ for photons, $0<w<\frac{1}{3}$ for matter, and $w=-1$ for dark energy.

The fluid equation in adiabatic processes is

$$
\frac{\partial \rho}{\partial t}=-3 \frac{\dot{a}}{a}(p+\rho)
$$

In principle, equations $1.6,1.7$, and 1.8 can tell us the whole story about the universe given initial conditions and boundary values. But we need to find out these initial conditions and boundary values from observational cosmology and experimental astrophysics to gain a better understanding of the universe. Hubble's law is the key to understanding the expanding universe, the redshift is a fundamental tool in observational cosmology, and the matter components are the topics that we will discuss in detail in this chapter. I summarize these concepts below.

\section{Hubble's law}

Along the spatial geodesic between the observer and the galaxy, the angle $(\theta, \phi)$ is constant,

$$
d s=a(t) d x
$$

The proper distance is

$$
d_{p}(t)=a(t) x
$$

To write it in spatial coordinates $(t, r, \theta, \phi)$, the proper distance is

$$
d_{p}(t)= \begin{cases}a(t) R_{0} \sin ^{-1}(r / R) & \text { if } k=+1 \\ a(t) r & \text { if } k=0 \\ a(t) R_{0} \sinh ^{-1}(r / R) & \text { if } k=-1\end{cases}
$$

In the comoving coordinates, $x$ and $r$ do not change, so there is a linear relation between the proper distance and the recession speed of the galaxy:

$$
v_{p}(t)=H d_{p}(t)
$$


where

$$
v_{p}(t)=\dot{d}_{p}(t)
$$

and

$$
H=\frac{\dot{a}}{a}
$$

The Hubble constant today is:

$$
H_{0}=\left(\frac{\dot{a}}{a}\right)_{t=t_{0}}
$$

\section{The redshift}

The scale factor $a(t)$ changes with time. The redshift of the observed lights from distant galaxies can tell us the scale factor for when the light was emitted. Suppose the galaxy emits light with wavelength $\lambda_{e}$ at time $t_{e}$, and the wavelength of the light we see today is $\lambda_{0}$. Then:

$$
\frac{\lambda_{e}}{a\left(t_{e}\right)}=\frac{\lambda_{0}}{a\left(t_{0}\right)}
$$

Using the definition of redshift, $z=\left(\lambda_{0}-\lambda_{e}\right) / \lambda_{e}$, the redshift of light from a distant object is related to the scale factor at the time it was emitted via the equation

$$
1+z=\frac{a\left(t_{0}\right)}{a\left(t_{e}\right)}=\frac{1}{a\left(t_{e}\right)} .
$$

Here we use the usual convention that $a\left(t_{0}\right)=1$.

Keeping the first three terms of the Taylor expansion, the scale factor in the recent past and near future can be approximated as

$$
a(t) \approx a\left(t_{0}\right)+\left.\frac{d a}{d t}\right|_{t=t_{0}}\left(t-t_{0}\right)+\left.\frac{1}{2} \frac{d^{2} a}{d t^{2}}\right|_{t=t_{0}}\left(t-t_{0}\right)^{2} .
$$

Using equation 1.15, we define the deceleration parameter as

$$
q_{0} \equiv-\left(\frac{\ddot{a} a}{\dot{a}^{2}}\right)_{t=t_{0}}=-\left(\frac{\ddot{a}}{a H_{0}{ }^{2}}\right)_{t=t_{0}} .
$$


The scale factor is customarily written in the form of

$$
a(t) \approx a\left(t_{0}\right)+H_{0}\left(t-t_{0}\right)+\frac{1}{2} q_{0} H_{0}^{2}\left(t-t_{0}\right)^{2} .
$$

In today's standard cosmology model, the deceleration parameter $q_{0}$ is directly related to the density parameters of the different components of the universe:

$$
q_{0}=\frac{1}{2} \sum_{w} \Omega_{w, 0}(1+w)
$$

The lookback time of the universe as a function of redshift is

$$
t_{0}-t_{e} \approx H_{0}^{-1}\left[z-\left(\frac{1+q_{0}}{2}\right) z^{2}\right]
$$

An approximate relation for the current proper distance to a galaxy with redshift $z$ can be expressed as

$$
d_{p}\left(t_{0}\right) \approx \frac{c}{H_{0}} z\left[1-\left(\frac{1+q_{0}}{2}\right) z\right]
$$

The linear Hubble relation $d_{p} \propto z$ holds true only in the limit $z<<2 /\left(1+q_{0}\right)$.

\section{Matter Densities in the Universe}

In terms of Hubble constant $H_{0}$ today, the critical density is defined as,

$$
\rho_{c} \equiv \frac{3 H_{0}^{2}}{8 \pi}
$$

We also define

$$
\begin{gathered}
\Omega_{M} \equiv \frac{\rho}{\rho_{c}} \\
\Omega_{k} \equiv \frac{-k}{R_{0}^{2} H_{0}^{2}} \\
\Omega_{\Lambda} \equiv \frac{\Lambda}{3 H_{0}^{2}}
\end{gathered}
$$


as the normalized densities at the present time of matter, curvature, and the cosmological constant, respectively. Equation 1.6 is reduced to:

$$
\Omega_{M}+\Omega_{k}+\Omega_{\Lambda}=1 .
$$

The following sections will explain the measurement of the matter densities in the universe within the framework described here.

\subsection{Observational Cosmology and Dark Matter}

The Big Bang model provides accurate and scientifically testable hypotheses in modern cosmology. The remarkable agreement with the observational data gives us considerable confidence in the model. These are the four observational cosmology pillars: the expansion of the universe, the origin of the cosmic background radiation, the nucleosynthesis of light elements, and the formation of galaxies and large-scale structures. These cosmology pillars and astrophysics observations give strong indications that dark matter exists in the universe.

\subsubsection{The Expansion of the Universe}

The expansion of the universe was discovered in 1929 by Edwin Hubble, who measured the distances to a sample of nearby galaxies, and established a correlation between distance and recession velocity. The slope of this relation is the Hubble constant. The latest results are from the Hubble Space Telescope (HST) Key

Project [4]. Cepheid variable stars were used to calibrate distance scales over the range of $25 \mathrm{Mpc}$. The luminosities and redshifts of galaxies were measured. Large systematic uncertainties in determining distance have made an accurate determination of the Hubble constant a challenge, and only recently have improvements 
in instrumentation, the launch of the Hubble Space Telescope (HST), and the development of several different measurement methods led to a convergence of its value. Accurate distances to nearby galaxies obtained as part of an HST Key Project have allowed calibration of 5 different methods for determining the distances to galaxies out to $500 \mathrm{Mpc}$. Fig. 1.1 shows that the velocity of galaxy increases with distance, where the error bars represent 1- $\sigma$ statistical and systematic uncertainties, respectively. All the techniques show good agreement within their respective uncertainties, and yield a value

$$
H_{0}=72 \pm 2 \pm 7 \mathrm{~km} \mathrm{sec}^{-1} \mathrm{Mpc}^{-1}
$$
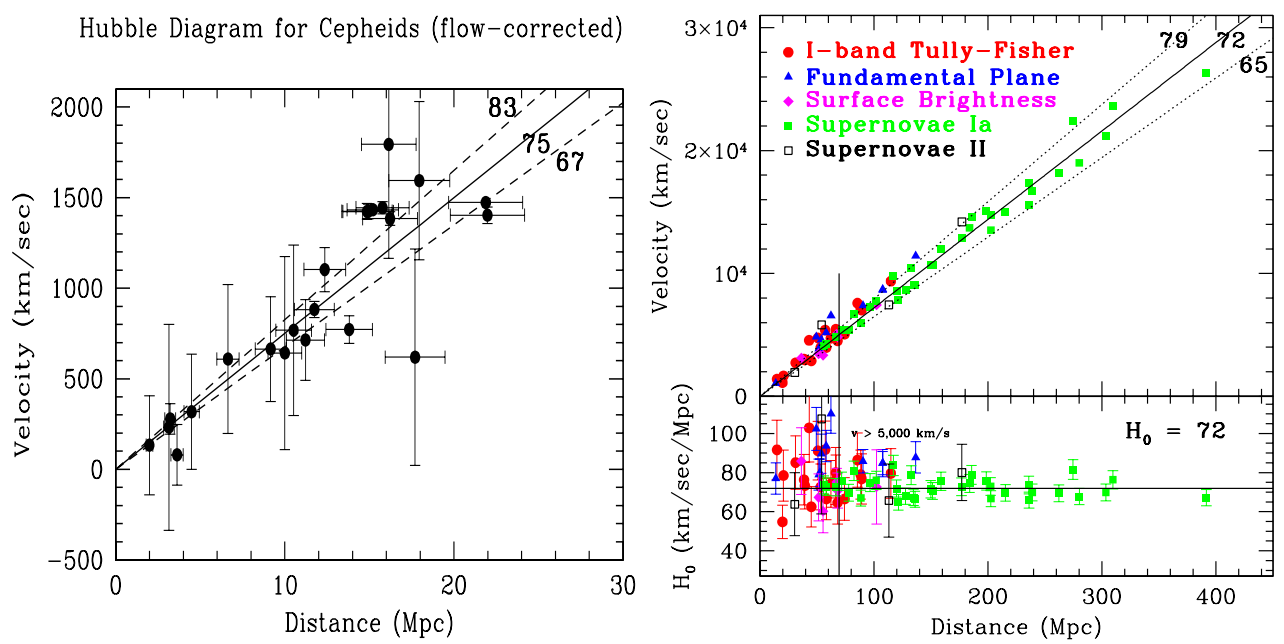

Figure 1.1: Left: Velocity versus distance for galaxies with Cepheid distance. Right: Low-redshift galaxies are used to establish the expansion of the universe and the Hubble constant; the consistency of the five different distance indicators is shown. The lower panel shows the value of the Hubble constant object by object and the convergence to $72 \mathrm{~km} / \mathrm{s} / \mathrm{Mpc}$. The scatter at distances less than $100 \mathrm{Mpc}$ arises due to gravitational induced "peculiar velocities" that arise from the inhomogeneous distribution of matter. Figure from [4].

Because light from very distant galaxies was emitted a long time ago, the Hubble diagram also provides a means of measuring the expansion at earlier times. 


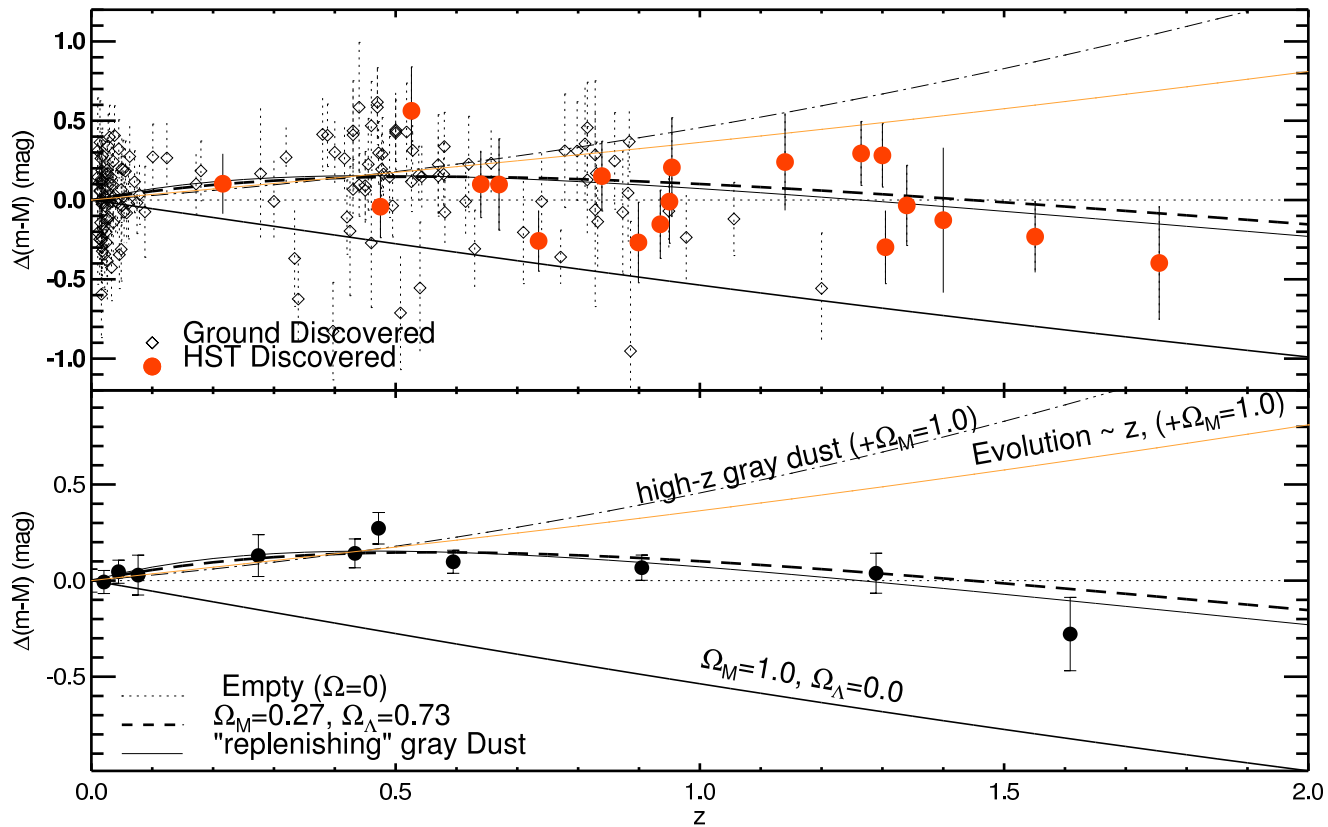

Figure 1.2: SN Ia residual Hubble diagram comparing cosmological models and models for astrophysical dimming. Upper panel: SNe Ia from ground-based discoveries in the gold sample are shown as diamonds, HST-discovered SNe Ia are shown as filled symbols. Bottom panel: weighted data points in the redshift bins are shown for illustrative purposes only. Data and models are shown relative to an empty universe model $(\Omega=0)$. Accelerated cosmic expansion is indicated by positive slope, decelerated by negative slope. Their transition (near $z=0.5$ ) from present acceleration to earlier deceleration is well fitted by the curve with cosmological parameters $\Omega_{M}=0.27$ and $\Omega_{\Lambda}=0.73$. Figure from [5].

For many decades, efforts have been directed toward measuring what was almost universally expected to be a slowing of the expansion over time due to the gravity of all the matter. However, observations by two independent groups have found that supernovae at high redshifts are fainter than predicted for a slowing expansion and indicate that the expansion is actually speeding up (see Fig. 1.2) [5, 6, 7]. Although systematic effects due to intervening dust or evolution of the supernovae themselves could explain such a dimming of high-redshift supernovae, several tests have failed to turn up any evidence for such effects. 
The fact that the expansion is speeding up, rather than slowing down, can be accounted for within Einstein's theory, as the source of gravity is proportional to $(\rho+3 p)$, where the pressure $p$ and energy density $\rho$ describe the bulk properties of the "substance". A substance that has negative pressure has repulsive gravity in Einstein's theory, and can be the cause of cosmic acceleration.

The deceleration parameter was introduced to quantify the slowing of the expansion; it is related to the mass-energy content of the universe:

$$
q_{0} \equiv \frac{-(\ddot{a} / a)_{0}}{H_{0}^{2}}=\frac{\Omega_{M}}{2}+\frac{3}{2} w_{\Lambda} \Omega_{\Lambda} \simeq-0.86 \pm 0.17
$$

where $w_{\Lambda} \equiv p_{\Lambda} / \rho_{\Lambda}$ characterizes the pressure of the dark-energy component. In the absence of dark energy, a flat universe would decelerate by its own self-gravity, whereas dark energy allows for acceleration. The supernova measurements are consistent with $w_{\Lambda}=-1$ and $\Omega_{\Lambda}=0.73$.

Strong indirect evidence for an additional energy component comes from a comparison of the density of matter with measurements of $\Omega_{M}$ from fluctuations in the CMB. As we will see, the concordance of various observations, along with spectacular confirmation by the CMB, yields a standard cosmology, in which nonbaryonic dark matter is one of the primary components, and where nature remains a mystery.

\subsubsection{The Cosmic Microwave Background}

Photons are the messengers in cosmology. At earlier times, the photons were the dominant part of the mass-energy budget, from which we ascertain that the infant universe was a hot thermal bath of elementary particles. CMB photons interacted closely with matter until the temperature of the universe had cooled enough for the ionized plasma to combine and form neutral atoms, allowing the photons to 
travel through the plasma. The Cosmic Microwave Background (CMB) photons are the photons from "last-scattering" with other particles, when the universe was about 400,000 years old and about 1100 times smaller than it is today.

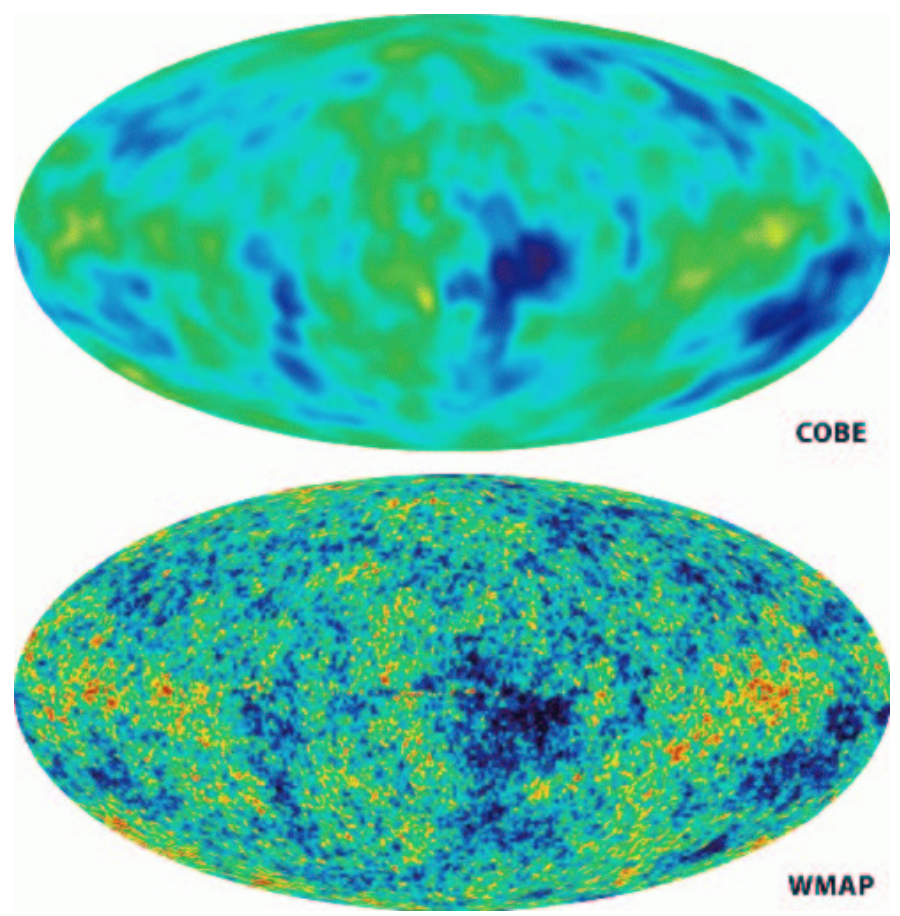

Figure 1.3: Anisotropy of the cosmic microwave background: All-sky maps, made by COBE and by WMAP (lower). Range of color scale is $(\Delta T) \pm 200 \mu K$. Figure from http://map.gsfc.nasa.gov/.

The existence of CMB photons was predicted by George Gamow and his collaborators in 1948 and inadvertently discovered by Arno Penzias and Robert Wilson in 1965. NASA's COBE satellite, a four-year mission launched in 1989, measured the temperature of the background radiation to better than one part in one thousand, $T_{0}=2.725 \pm 0.001 \mathrm{~K}$, and discovered tiny (tens of microKelvin) variations in the temperature of the CMB across the sky (see Figure 1.3). These tiny fluctuations arise from primeval lumpiness in the distribution of matter. In the early universe, outward pressure from the CMB photons, acting counter to the inward 


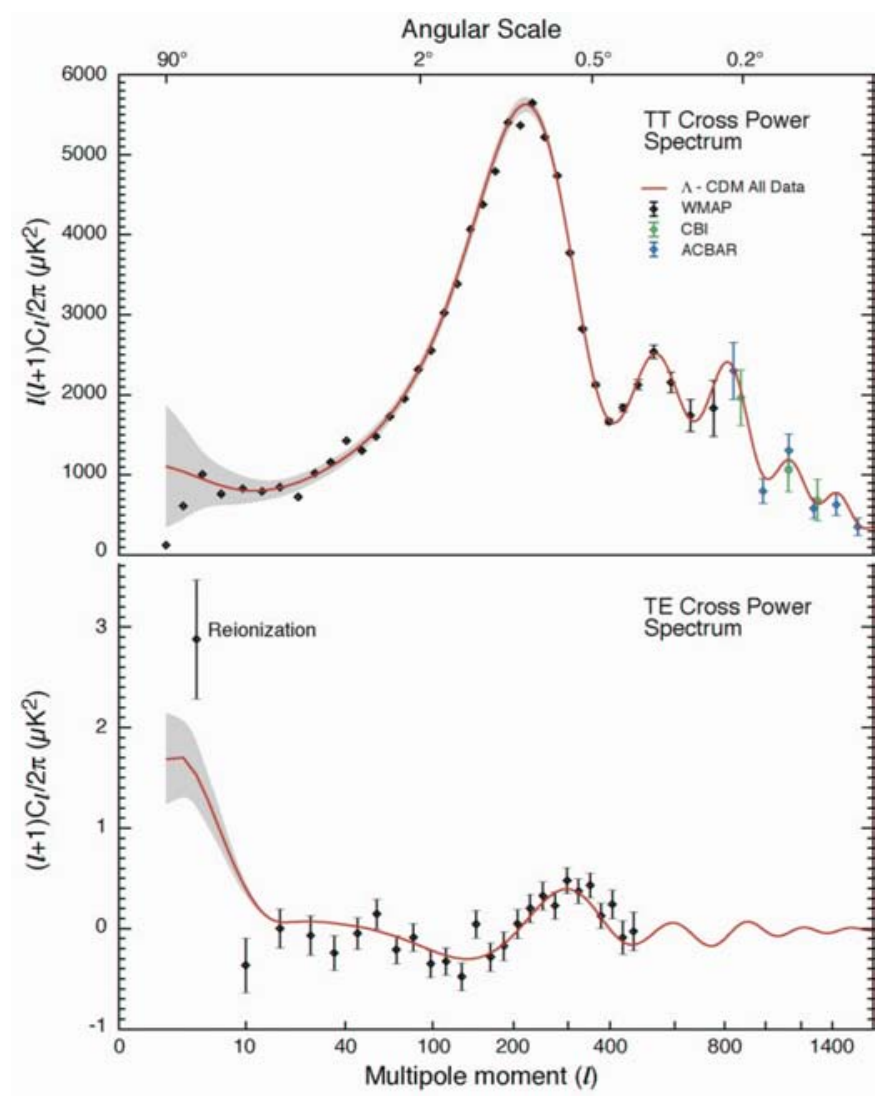

Figure 1.4: The WMAP angular power spectrum ( also includes data from CBI and ACBAR). The curve is the consensus cosmology model; the gray band includes cosmic variance. The WMAP measurements up to $l \sim 350$ are limited by cosmic variance. The lower panel shows the anisotropy across the polarization power spectrum; the high point marked "reionization" is the evidence for reionization of the universe at $z \sim 20$. Figure from [8].

force of gravity due to matter, set up oscillations whose frequencies are now seen imprinted in the CMB fluctuations. Evidence of these "acoustic oscillations" can be seen when the fluctuations are described by their spherical-harmonic power spectrum. In late 2002, the DASI collaboration detected the next important feature [9] predicted for the CMB: polarization. Because the CMB radiation is not isotropic (as evidenced by the anisotropy seen across the microwave sky) and Thomson scattering of electrons is not isotropic, CMB anisotropy should develop 
about a $5 \%$ polarization.

The analysis of CMB anisotropies enables accurate testing of cosmological models and puts stringent constraints on cosmological parameters.

The observed temperature anisotropies in the sky are usually expanded as

$$
\frac{\delta T}{T}(\theta, \phi)=\sum_{\ell=2}^{+\infty} \sum_{m=-\ell}^{+\ell} a_{\ell m} Y_{\ell m}(\theta, \phi)
$$

where $Y_{\ell m}(\theta, \phi)$ are spherical harmonics.

The variance $C_{\ell}$ of $a_{\ell m}$ is given by

$$
C_{\ell} \equiv<\left|a_{\ell m}\right|^{2}>\equiv \frac{1}{2 \ell+1} \sum_{m=-\ell}^{\ell}\left|a_{\ell m}\right|^{2}
$$

If the temperature fluctuations are assumed to be Gaussian, as appears to be the case, all of the information contained in CMB maps can be compressed into the power spectrum, essentially giving the behavior of $C_{\ell}$ as a function of $\ell$. Usually plotted is $\ell(\ell+1) C_{\ell} / 2 \pi$, as shown in Figure 1.4.

The methodology for extracting information from CMB anisotropy angular power spectrum is simple, at least in principle. Starting from a cosmological model with a fixed number of parameters, the best-fit parameters are determined from the peak of the N-dimensional likelihood surface.

From the analysis of the WMAP data alone, the following values are found for the abundance of baryons and matter in the universe [8]:

$$
\Omega_{b} h^{2}=0.024 \pm 0.001 \quad \Omega_{M} h^{2}=0.14 \pm 0.02
$$

Taking into account data from CMB experiments studying smaller scales, such as ACBAR and CBI, and astronomical measurements of the power spectrum from the large scale structure, such as 2dFGRS and the Lyman $\alpha$ forest, the constraints become [10]:

$$
\Omega_{b} h^{2}=0.0224 \pm 0.0009 \quad \text { and } \quad \Omega_{\mathrm{M}} \mathrm{h}^{2}=0.135_{-0.009}^{+0.008}
$$


The value of $\Omega_{b} h^{2}$ thus obtained is consistent with predictions from Big Bang nucleosynthesis [11]:

$$
0.018<\Omega_{b} h^{2}<0.023 \text {. }
$$

Besides those provided by CMB studies, the most reliable cosmological measurements are probably those obtained by the Sloan Digital Sky Survey (SDSS) team, which has recently measured the three-dimensional power spectrum, $P(k)$, using over 200,000 galaxies. An estimate of the cosmological parameters combining the SDSS and WMAP measurements can be found in reference [12], where they give a constraint on baryonic matter density in the universe of

$$
0.018<\Omega_{b} h^{2}<0.024
$$

at the $2 \sigma$ confidence level. Thus, the observational evidence for non-baryonic dark matter we see is from CMB and large scale structure. A key element of the story, the nature of dark matter comes from nucleosynthesis.

\subsubsection{Nucleosynthesis}

Thanks to the pioneering efforts of George Gamow and his collaborators, there now exists a satisfactory theory of the production of light elements in the early universe. In the very early universe the temperature was so great that all matter was fully ionized and nucleons were dissociated. Within minutes after the Big Bang itself, the temperature of the universe rapidly cooled from its phenomenal $10^{32}$ Kelvin to approximately $10^{9}$ Kelvin. At this temperature, nucleosynthesis, or the production of light elements, could take place. In a short time interval, protons and neutrons collided to produce deuterium. Most of the deuterium then collided with other protons and neutrons to produce helium and a small amount of 
tritium. Lithium-7 arises from the coalescence of one tritium and two deuterium nuclei. Figure 1.5 shows the evolution of the light element abundances over time.

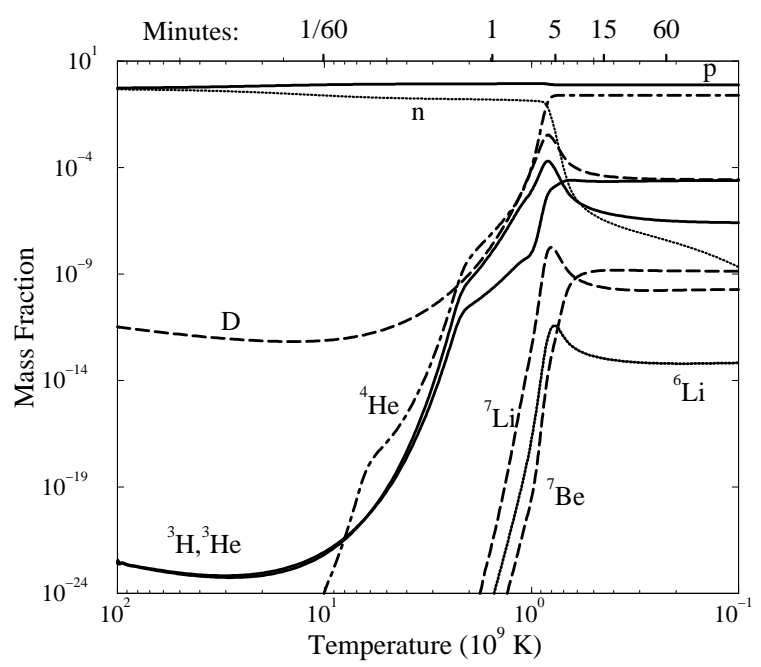

Figure 1.5: Evolution of the light element abundances over time(or temperature). When the universe has cooled sufficiently, the light elements are not dissociated by the energetic photons. Nucleosynthesis proceeds until the supply of free neutrons is exhausted. Figure from [13].

The yield of $\mathrm{D},{ }^{3} \mathrm{He},{ }^{4} \mathrm{He},{ }^{7} \mathrm{Li}$ elements depends on various physical parameters. Most importantly, they depend on the baryon-to-light ratio $\eta$. A high baryon-to-light ratio increases the temperature at which deuterium synthesis occurs. Getting an earlier start means that nucleosynthesis is more efficient at producing ${ }^{4} \mathrm{He}$, leaving less $\mathrm{D}$ and ${ }^{3} \mathrm{He}$ as leftovers. So the ratios between the light elements allow us to find out the baryon-to-light ratio, therefore calculating the baryon density of the universe [1].

Light element abundances place strong limits on the baryon density. Particularly, the production of deuterium is the most sensitive indicator of the baryon density. Measurements made with the HIRES spectrograph on the 10-meter W.M. Keck Telescopes of the amount of deuterium in high-redshift clouds of gas $[13,14]$ 


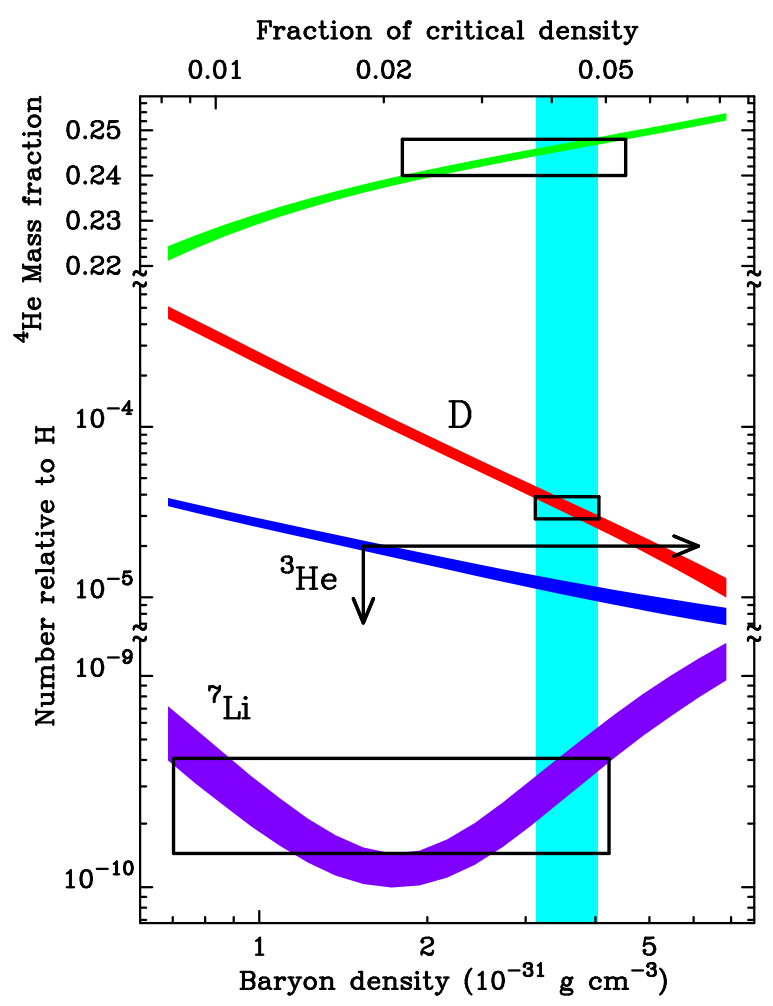

Figure 1.6: The predicted abundance of the light elements vs. baryon density. The vertical band indicates the narrow range of baryon densities consistent with the deuterium measurements; the boxes (the arrows for ${ }^{3} \mathrm{He}$ ) indicate the range in baryon density (horizontal extent of box) that is consistent with the measured light-element abundance (vertical extent of box). The overlap of the boxes with the deuterium band indicates the general consistency of the observed abundances of the other light elements with their predicted abundances for this baryon density. (Note, for the $\Omega_{B}$ scale at the top, $h^{2}=0.5$ is assumed.) Figure from [13].

yield a value of $\Omega_{b}$

$$
0.018<\Omega_{b} h^{2}<0.022
$$

accounting for less than $15 \%$ of the matter density $\Omega_{M}$. This result is comparable to the measured baryonic matter density from CMB in equation 1.35. This measurement implies that the majority of dark matter is non-baryonic.

The density of ordinary baryons within a narrow range is the predicted production consistent with what we actually measure (see Figure 1.6). BBN theory 
and baryonic matter density measurements, combined with CMB measurements, suggest that non-baryonic dark matter is an important component of matter in the universe.

\subsubsection{The Structure of the Universe}

Figure 1.7 shows a thin slice through the three-dimensional map of over 221,000 galaxies produced by the $2 \mathrm{dFGRS}[15]$. This $3^{\circ}$-thick slice passes through both the NGP strip (in the North Galatic Cap) on the left and the SGP strip (in the South Galatic Cap) on the right. The decrease in the number of galaxies toward higher redshifts is an effect of the survey selection by magnitude - only intrinsically more luminous galaxies are brighter than the survey magnitude limit at higher redshifts. The clusters, filaments, sheets and voids making up the largescale structures in the galaxy distribution are clearly resolved. The fact that there are many such structures visible in the figure is a qualitative demonstration that the survey volume comprises a representative sample of the universe; the small amplitude of the density fluctuations on large scales is quantified by the power spectrum, as shown in Figure 1.8.

The large-scale hierarchical structure of the universe in the distribution of galaxies arises from the gravitational instability of small fluctuations in the initial density field of the universe. A simple description of galaxy clustering is the twopoint correlation function $\xi$, which measures the excess probability of finding two galaxies separated by a given distance. It is found empirically to follow a simple power law [3],

$$
\xi=\left(r / 6 h^{-1} \mathrm{Mpc}\right)^{-1.8}
$$

which implies that finding another galaxy within $6 h^{-1} \mathrm{Mpc}$ from a given galaxy 


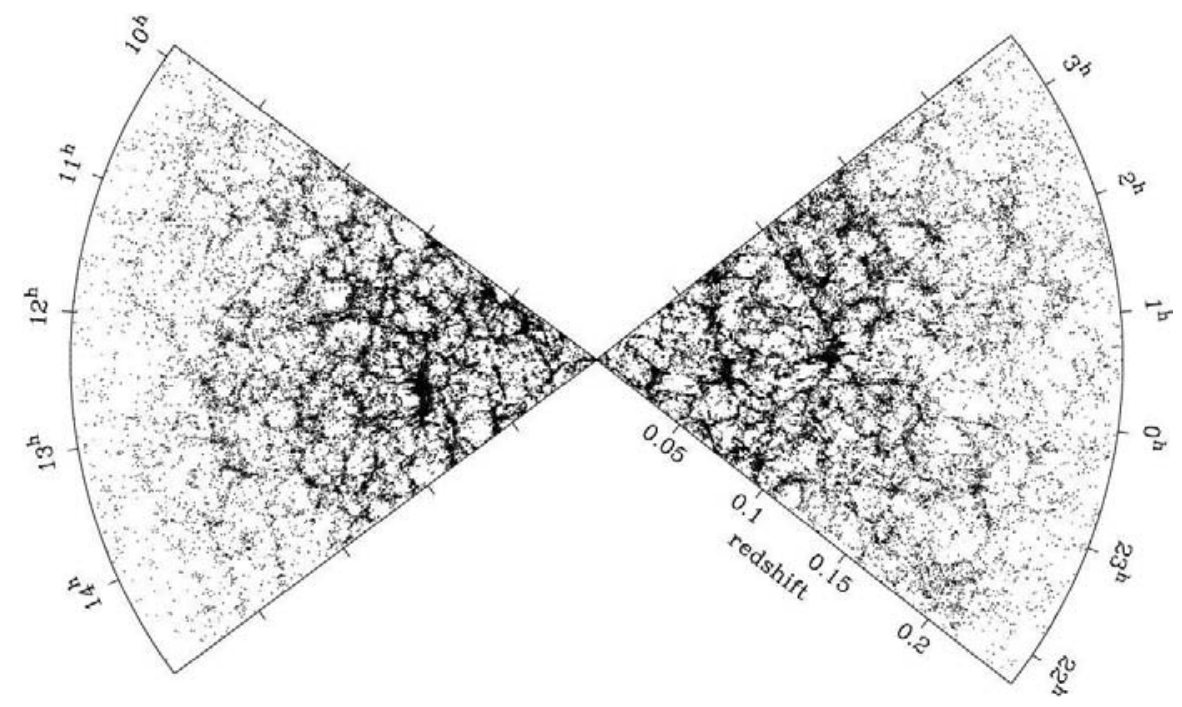

Figure 1.7: The large-scale structures in the galaxy distribution are shown in this $3^{\circ}$-thick slice through the 2dFGRS map. The slice cuts through the NGP strip (at left) and the SGP strip (at right), and contains 63, 000 galaxies. Figure from [16].

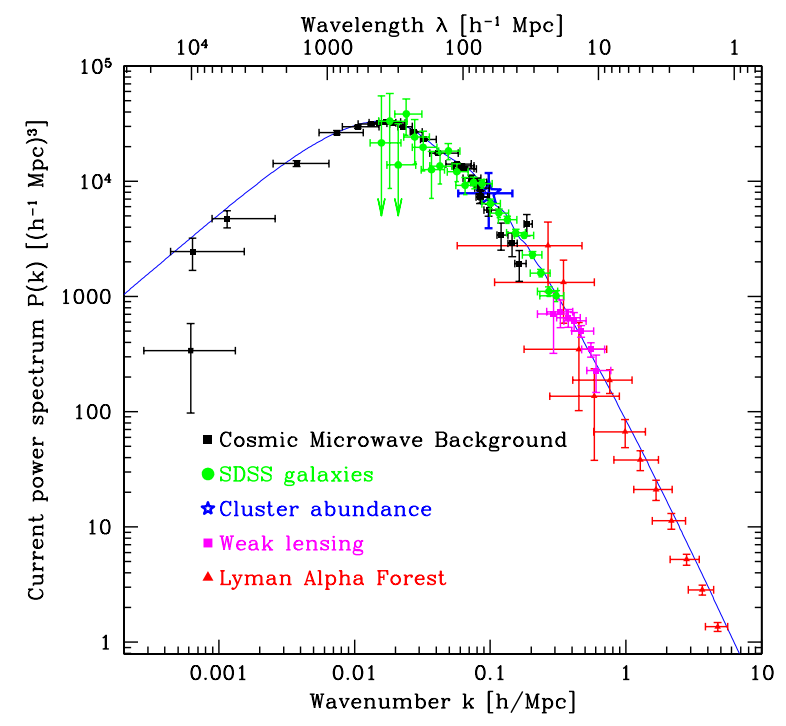

Figure 1.8: Power spectrum of density inhomogeneity today obtained from a variety of measurements, including large-scale structure, CMB, weak lensing, rich clusters, and the Lyman-alpha forest. The curve is the theoretical prediction for the consensus cosmology model. Figure from [17].

is twice as likely as finding a galaxy within a randomly placed circle of radius $6 \mathrm{Mpc}$ on the sky. The Fourier transform of the correlation function is the power 
spectrum of the distribution of galaxies. The power spectrum can be directly compared with theoretical predictions from inflation and the cold dark matter model. As shown in figure 1.8, the observed and predicted power spectra of matter density inhomogeneity compare well. Combined with the anisotropy of the cosmic microwave background, two large structure surveys (2df [15] and SDSS [17]) reached the same conclusion: the matter density $\Omega_{M} \sim 0.3$.

Furthermore, if most of the dark matter in the universe were hot dark matter, such as neutrinos, then we would expect the old structures in the universe to be superclusters, and that galaxies would be relatively young. In fact, the opposite seems to be true in our universe. Superclusters are just collapsing today, while the galaxies have been around since at least $z \sim 6$, when the universe was less than a gigayear old. Thus, most dark matter in the universe must be cold dark matter, for which the free streaming from hot dark matter has been negligible [1].

\subsubsection{Dark Matter in the Universe}

The matter density on large scales (Mpc) has been investigated extensively, using astrophysical probes beyond (and in some cases, prior to) the results of cosmological observations described earlier. For example, clusters of galaxies provide a laboratory for studying and measuring dark matter in a variety of ways. Measurements of the matter-density come from mass-to-light ratios, motions of cluster member galaxies, X-ray gas temperature profiles, gravitational lensing of background objects, and surveys using the Sunyaev-Zeldovich effect. Assuming that clusters provide a "representative sample" of matter in the universe, the total amount of matter can be inferred from the baryon density, and the results are in good agreement with that of cosmological profiles. That number is about one 
third of the critical density. The first part of this section follows closely from Barbara Ryden's book [1].

\section{Mass-to-Light Ratio}

In the B band, the total luminosity of the universe is

$$
J_{\star} \approx 1.2 \times 10^{8} L_{\odot} M p c^{-3}
$$

where $L_{\odot}$ is the luminosity of the Sun.

The mass-to-light ratio of stars in a galaxy depends on the mix of stars that it contains. Within 1 kiloparsec of the Sun, the mass-to-light ratio of stars is

$$
\frac{M}{L} \approx \frac{4 M_{\odot}}{L_{\odot}} \approx 1.7 \times 10^{5} \mathrm{~kg} \mathrm{watt}{ }^{-1}
$$

where $M_{\odot}$ is the mass of The Sun.

If the mass-to-light ratio of the stars within 1 kiloparsec is a typical in the universe, then the mass density of stars in the universe is

$$
\rho_{\star} \approx \frac{M}{L} \cdot J=5 \times 10^{8} M_{\odot} M p c^{-3}
$$

The current critical density of the universe is equivalent to a mass density of

$$
\rho_{c}=1.4 \times 10^{11} M_{\odot} M p c^{-3}
$$

The density parameter of stars in the universe today is

$$
\Omega_{\star}=\frac{\rho_{s t a r}}{\rho_{c}} \approx 0.004
$$

which is far below the critical density of the universe, and thus indicates that most of the matter in the universe is dark. 


\section{Virial Theorem}

In the 1930's Fritz Zwicky made a compelling case for the existence of a large amount of dark matter. In studying the Coma cluster of galaxies, he noticed that the dispersion in the radial velocity was very large, around $1000 \mathrm{~m} / \mathrm{s}$. The stars and gas visible in the Coma cluster did not provide enough gravitational attraction to hold the whole cluster together.

By applying the virial theorem to a self-gravitating system in a steady state like the Coma cluster, the kinetic energy equals half the potential energy [1]

$$
\frac{1}{2} M<v^{2}>=-\frac{\alpha}{2} \frac{G M^{2}}{r_{h}}
$$

where $M=\sum m_{i}$ is the total mass of all galaxies in the cluster, $\alpha$ is a numerical factor of order unity that depends on the density profile of the cluster, $r_{h}$ is the half-mass radius of the cluster, and $G$ is the gravitational constant. For the observed cluster of galaxies, it is found that $\alpha \approx 0.4$ is a good fit for the potential energy.

This means that we can use the virial theorem to estimate the mass of a cluster of galaxies:

$$
M=\frac{<v^{2}>r_{h}}{\alpha G}
$$

Assuming that the velocity dispersion is isotropic in the Coma cluster, the three dimensional mean square velocity $\left\langle v^{2}\right\rangle$ is found to be [1]:

$$
<v^{2}>=2.32 \times 10^{12} \mathrm{~m}^{2} \mathrm{~s}^{-2} .
$$

Assuming that the Coma cluster is spherical, the observed distribution of galaxies within the Coma cluster indicates a half-mass radius [1]

$$
r_{h} \approx 1.5 M p c \approx 4.6 \times 10^{22} \mathrm{~m} .
$$


The calculated total mass of the Coma cluster is therefore

$$
M=\frac{<v^{2}>r_{h}}{\alpha G} \approx 2 \times 10^{15} M_{\odot}
$$

The measured mass of stars and gas in the Coma cluster are $M_{\star} \approx 3 \times 10^{13} M_{\odot}$ and $M_{g} \approx 2 \times 10^{14} M_{\odot}$ [1], respectively, which indicates that about $85 \%$ of the mass in the Coma cluster is comprised of dark matter. As we will see, further studies of clusters using different techniques have confirmed this result.

\section{X-ray}

The measurements of X-ray emission from hot gravitationally bound gas in clusters of galaxies enable them to be used as good probes of the physics of these systems. The equation of hydrostatic equilibrium is:

$$
\frac{d P}{d r}=-\rho g,
$$

where $n$ is the particle density; $T$ is the temperatur; $k$ is the Boltzmann constant; $P=n k T$ is the pressure; $m$ is proton mass; $\mu \approx 0.6$ is the average molecular weight; $\rho=n \mu m$; and $g=G M(<r) / r^{2}$ is the local gravitational acceleration. Equation 1.47 can be rewritten as

$$
M_{\mathrm{T}}(<r)=-\frac{k T r^{2}}{G \mu m}\left(\frac{d \ln n}{d r}+\frac{d \ln T}{d r}\right) .
$$

The quantities on the right can be measured from X-ray spectral images yielding the gas mass profile $M_{\text {gas }}(r)$, the total mass profile $M_{\mathrm{T}}(r)$, and the gas fraction $f_{\text {gas }}$.

Allen et al [18] calculated the X-ray mass fraction with clusters PKS0745-191, Abell 2390, Abell 1835, MS2137-2535, RXJ1347-1145, and 3C295. In calculating the total baryonic mass in the clusters, the optically luminous baryonic mass in 
galaxies is $0.19 h^{0.5}$ times the X-ray gas mass [19]. Other sources of baryonic matter are expected to make only very small contributions to the total mass and are ignored.

Given the baryonic masses, and assuming that the regions of the clusters within $r_{2500}$ (the radius at 2500 times the critical density of the universe) provide a fair sample of the matter content of the universe, one can write

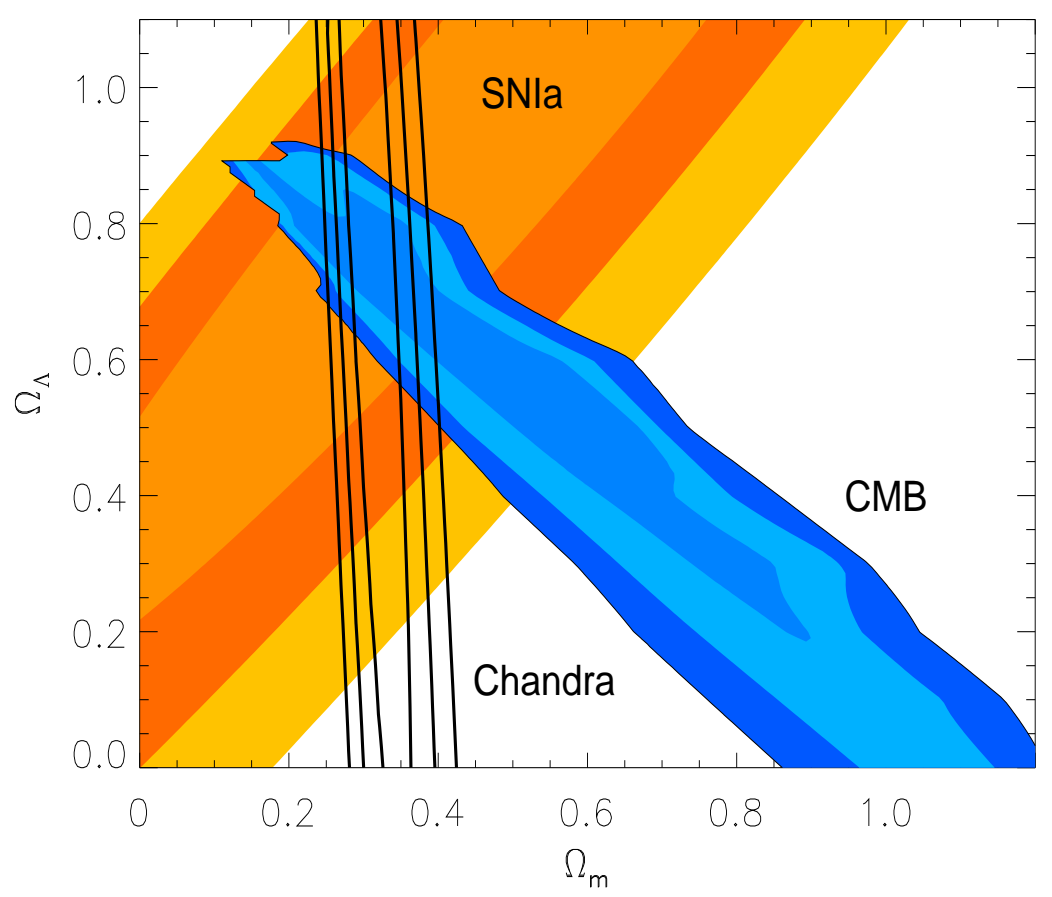

Figure 1.9: The joint 1,2 , and $3 \sigma$ confidence contours on $\Omega_{\mathrm{M}}$ and $\Omega_{\Lambda}$ determined from the Chandra $f_{\text {gas }}(\mathrm{z})$ data (bold contours), and independent analyses of cosmic microwave background (CMB) anisotropies and the properties of distant supernovae. Figure from [18]. (Note that the contours from SN and CMS have since tightened up.)

$$
\Omega_{\mathrm{m}}=\frac{\Omega_{\mathrm{b}}}{f_{\text {gas }}\left(1+0.19 h^{0.5}\right)} .
$$


For $\Omega_{\mathrm{b}} h^{2}=0.0205 \pm 0.0018$ and using the $\Lambda \mathrm{CDM}(h=0.7) f_{\text {gas }}$ values, Allen et al [18] obtained the result $\Omega_{\mathrm{m}}=0.319 \pm 0.032$ with a simple calculation with equation 1.49 .

In addition to the simple calculation of $\Omega_{\mathrm{m}}$ based on the weighted-mean $f_{\text {gas }}$ values, described above, the data for the present sample was used to obtain more rigorous constraints on cosmological parameters from the apparent variation of $f_{\text {gas }}$ with redshift $z$, as shown in Figure 1.9. Combined with SNIa and CMB, the $\Omega_{M}=0.270 \pm 0.035$.

\section{Gravitational Lensing}

Weak gravitational lensing can be used to measure the mass in a region by utilizing the fact that the path of a light bundle passing a gravitational potential will be bent by the potential. As a result, images of background galaxies that are near a massive structure, such as a cluster of galaxies, are deflected away from the structure, and distorted such that they are stretched tangentially to the center of the potential. This effect, known as gravitational shear, causes the background galaxies' ellipticities to deviate from an isotropic distribution, and the magnitude and direction of these deviations is used to measure the mass of the structures causing the lensing. This technique of measuring the mass does not make any assumptions about the dynamical state of the mass, and therefore is one of a few methods that can be used to measure the mass of a dynamically disturbed system.

Clowe D. et al found direct evidence for the existence of dark matter by using the interacting cluster 1E0657-558. With measured luminosity and reconstructed mass from weak lensing, they found the mass-to-light ratio to be between $200 M_{\odot} / L_{\odot}$ and $400 M_{\odot} / L_{\odot}[20]$, which indicates that dark matter exists. 


\section{Sunyaev-Zeldovich Effect}

The Sunyaev-Zeldovich Effect, an inverse electron Thomson scattering process of CMB photons passing through the hot gas of clusters, provides an independent measure of the mass of the intracluster medium, which is typically several times the mass responsible for the light from the galaxies. By combining the gas mass with a measure of the total mass determined either from gravitational lensing observations or from the virial theorem and the X-ray determined electron temperature, one can determine the fraction of mass of the galaxy cluster contained in baryons. An estimate of the baryonic to the total mass on the scale of massive galaxy clusters is important as it should represent the universal value. This fraction, together with a measure of $\Omega_{b}$, thus provide another measure of $\Omega_{M}$.

Using sensitive centimeter-wave receivers mounted on the Owens Valley Radio Observatory (OVRO) and Berkeley-Illinois-Maryland-Association (BIMA) millimeter arrays, the group led by Carlstrom has obtained interferometric measurements of the Sunyaev-Zel'dovich (SZ) effect toward massive galaxy clusters. They used the SZ data to determine the pressure distribution of the cluster gas, in combination with published X-ray temperatures, to infer the gas mass and total gravitational mass of 18 clusters. The gas mass fraction, $f_{g}$, is calculated for each cluster, and is extrapolated to the fiducial radius $r_{500}$ (the cluster radius at 500 times the critical density of the universe) using the results of numerical simulations. The mean of $f_{g}$ within $r_{500}$ is $0.081_{-0.011}^{+0.009} h$ (statistical uncertainty at $68 \%$ confidence level). They derived an upper limit for $\Omega_{M}$ from this sample under the assumption that the mass composition of clusters within $r_{500}$ reflects the universal mass composition: $\Omega_{M} h \leq \Omega_{B} f_{g}$. The gas mass fractions depend on cosmology through the angular diameter distance and the $r_{500}$ correction factors. For a flat 
universe $\left(\Omega_{\Lambda}=1-\Omega_{M}\right)$ and $\mathrm{h}=0.7$, they found $\Omega_{M} \sim 0.25[21]$.

In summary, there is a broad range of astrophysical and cosmological data that shows we live in an expanding universe, which is composed of about $73 \pm 4 \%$ dark energy, about $23 \pm 4 \%$ dark matter, and $4.4 \pm 0.4 \%$ baryons. The rest of the known composition of the universe are photons and neutrinos. The measurements from different methods, for example, CMB photons and large scale structure surveys, X-ray, and Sunyaev-Zeldovich survey, agree with each other well.

\subsection{Dark Matter in Galaxies}

The most convincing and direct evidence for dark matter on galactic scales comes from the observations of the rotation curves of galaxies, namely the graph of circular velocities of stars and gas as a function of their distance from the galactic center.

\subsubsection{Rotation of the Galaxy}

Rotation curves are usually obtained by combining observations of the redshifts of the $21 \mathrm{~cm}$ line, which originates from hydrogen, with optical surface photometry. Observed rotation curves usually exhibit a characteristic flat behavior at large distances. A typical example is shown in Figure 1.10.

In Newtonian dynamics, the circular velocity is expected to be

$$
v(r)=\sqrt{\frac{G M(r)}{r}}
$$

where $M(r) \equiv 4 \pi \int \rho(r) r^{2} \mathrm{~d} r$, and $\rho(r)$ is the mass density profile, which should be falling $\propto 1 / \sqrt{r}$ beyond the center of the optical disc. The fact that $v(r)$ 


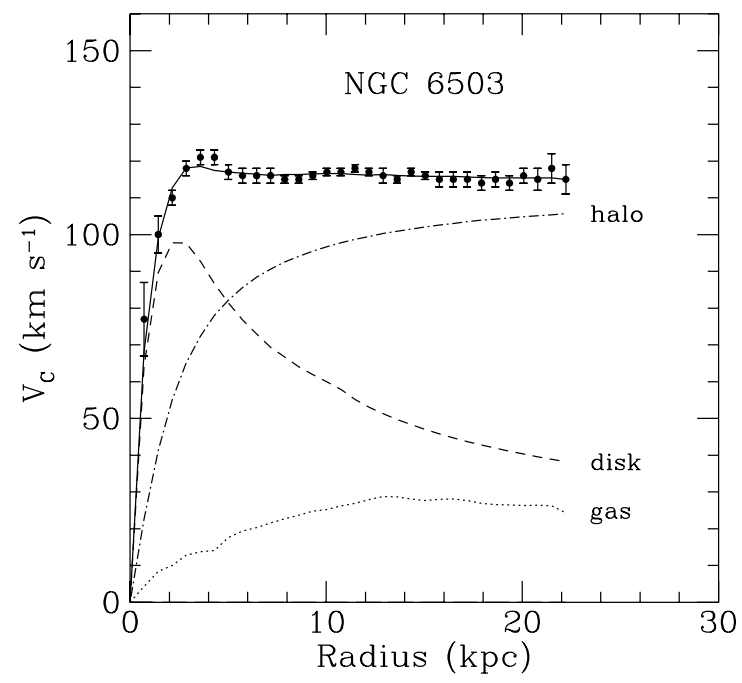

Figure 1.10: Rotation curve of NGC 6503. The dotted, dashed and dash-dotted lines are the contributions of gas, disk, and dark matter, respectively. Figure from [22].

is approximately constant implies the existence of a halo with $M(r) \propto r$ and $\rho \propto 1 / r^{2}$.

Cosmological N-body simulations based on observational data of galaxies have led to impressive strides in our understanding of the structure formation in the universe dominated by collisionless dark matter. Several models are shown in Figure 1.11. An example is the Navarro-Frank-White (NFW, 1996) cold dark matter simulation profile. It follows a simple formula:

$$
\frac{\rho(r)}{\rho_{\text {crit }}}=\frac{\delta_{c}}{\left(r / r_{s}\right)\left(1+r / r_{s}\right)^{2}}
$$

that describes the density profile of any halo with only two parameters, a characteristic density contrast $\delta_{c}$, and a scale radius, $r_{s}$. 

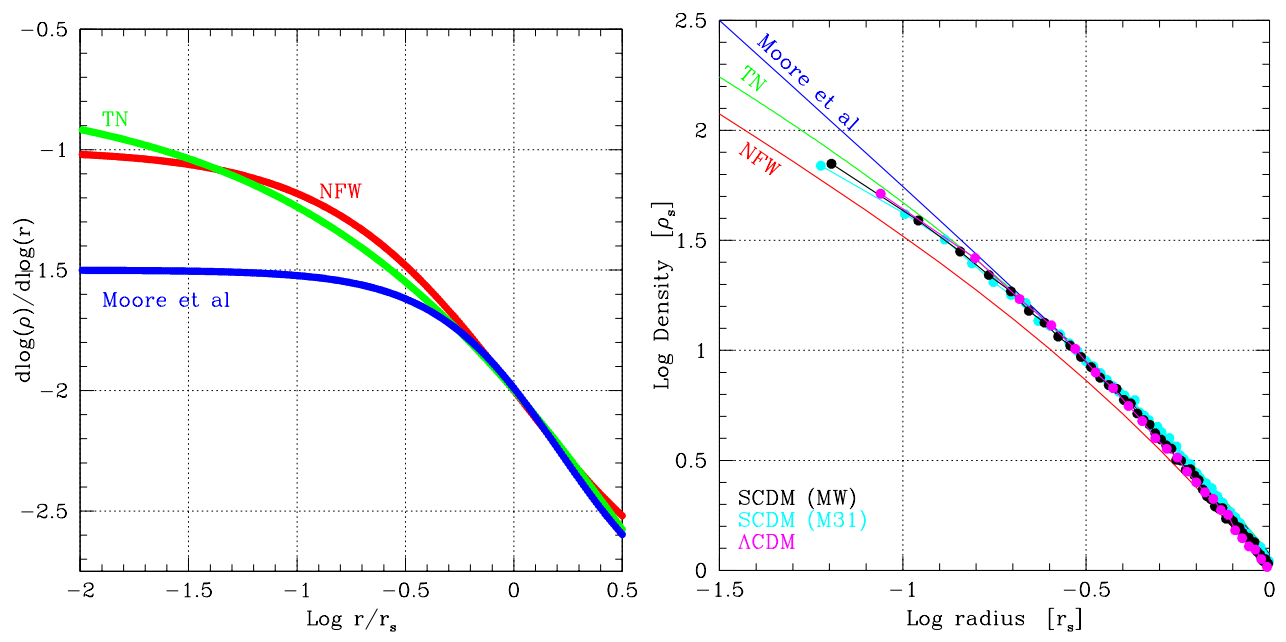

Figure 1.11: Left: Logarithmic slopes of three different models for the density profile of dark matter halos as a function of radius. Right: Density profiles of three simulated CDM halos (solid circles) compared, from bottom to top, to the models of NFW, TN, and Moore et al, respectively. Only radii within the NFW scale radius, $r_{s}$, are shown in order to emphasize details of the inner profiles. A significant excess over the NFW profile is seen for all three systems over the radial range shown, as expected from the 'critical solution' of TN. The Moore et al profile describes the inner profiles better than NFW in the range $0.15<r / r_{s}<0.5$, but deviates systematically at smaller radii reliably probed by the simulations. Figure from [23].

\subsubsection{Dark Matter in the Milky Way}

Our position within the Milky Way complicates the geometry when we study its structure and kinematics. It is therefore significantly harder to determine our own galaxy's rotation curve. In practice, the difficulty lies in knowing the Galactic radii of the objects we look at. One solution is to look at standard candles, whose distances can be estimated, and hence whose radii in the galaxy can be geometrically derived.

Similar to other galaxies, the Milky Way has a stellar bulge, a stellar disk, a gas disk and a dark matter halo. (See Figure 1.12.)

Very important to the prospects for direct and indirect detection is the density of dark matter in the region of our solar system. Although this quantity is 


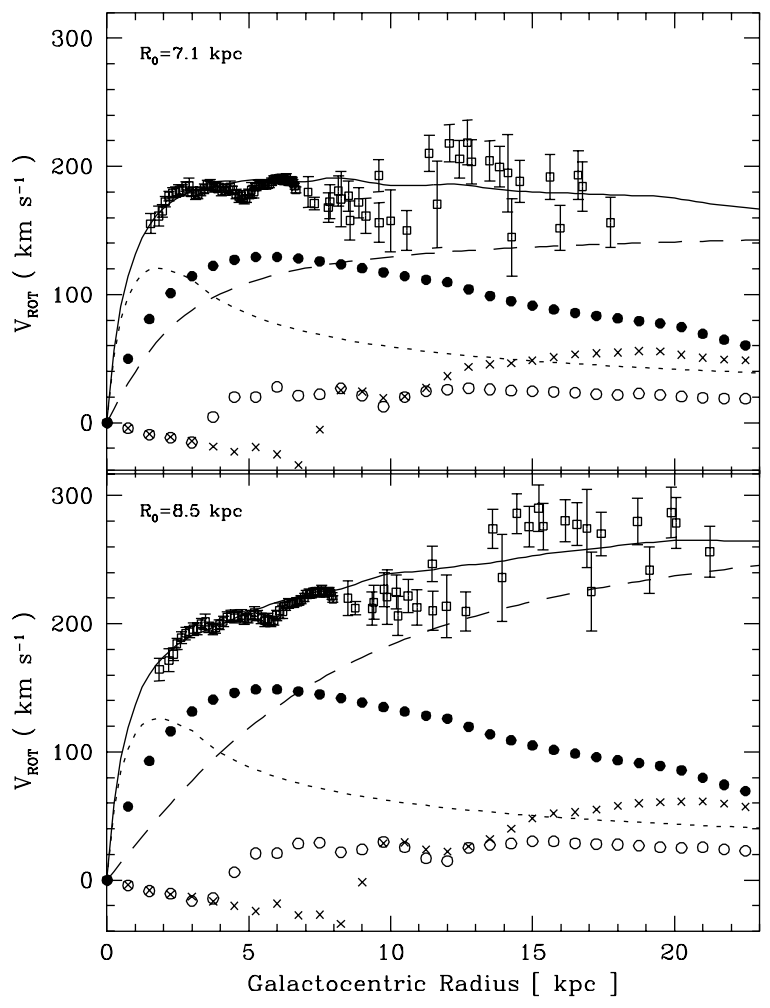

Figure 1.12: The rotation curve for the Milky Way for values of $R_{0}=7.1 \mathrm{kpc}$ ( $R_{0}$ is the distance from the center of the Milky Way to the solar system), $V_{0}=$ $185 \mathrm{~km} \mathrm{~s}^{-1}$, and $R_{0}=8.5 \mathrm{kpc}, V_{0}=220 \mathrm{~km} \mathrm{~s}^{-1}$. The figure also shows one of the ways in which the rotation curve can be decomposed into the contributions from different mass components: the bulge (dotted line); the stellar disk (filled circles); the gas (crosses refer to cold hydrogen gas, where negative values mean that the force is directed outwards. Circles refer to giant molecule cloud); and the dark halo (dashed line). The best fit model, which is obtained by summing the individual components in quadrature, is shown as a solid line. Figure from [24].

considerably more well-known than the density near the galactic center, there are still uncertainties associated with the local density, which we will discuss here.

The local density of dark matter is determined by observing the rotation curves of the Milky Way. As noted earlier, this is somewhat difficult to do from our location within the galaxy. Furthermore, rotation curves measure the total mass within an orbit. Thus the density distributions of the galactic bulge and disk are 


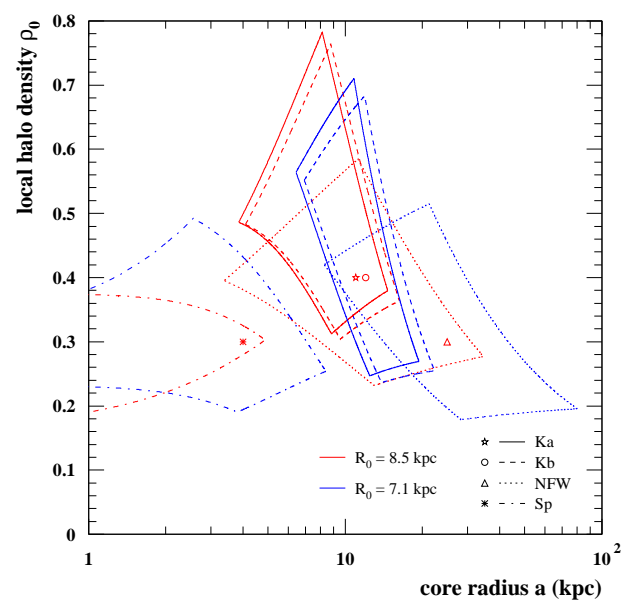

Figure 1.13: The range of local dark matter densities acceptable with observations of rotation curves for a variety of halo profiles and galactocentric distances. Densities in the range of $0.2-0.8 \mathrm{GeV} / \mathrm{cm}^{3}$ are shown to be acceptable. Figure from [25].

needed to accurately separate out the dark matter profile.

In addition to the local density, the velocity distribution of dark matter in the local region is needed to accurately calculate direct and indirect detection rates. This is also best inferred from observed rotation curves.

Different groups have come to somewhat different conclusions regarding the local density and velocity distribution of dark matter. For example, Bahcall et al found a best-fit value of $\rho_{0}=0.34 \mathrm{GeV} / \mathrm{cm}^{3}$ [26], Caldwell and Ostriker found $\rho_{0}=0.23 \mathrm{GeV} / \mathrm{cm}^{3}[27]$, while Turner calculates $\rho_{0}=0.3-0.6 \mathrm{GeV} / \mathrm{cm}^{3} \quad[28]$.

The velocity distribution of dark matter is typically assumed to be Maxwellain and thus described only by its RMS velocity, $v_{0}=<v^{2}>^{1 / 2} \cong 230 \mathrm{~km} / \mathrm{s}$. 


\subsection{Candidates of Dark Matter}

To define the search for dark matter, we need to gain insight into the specific candidates of dark matter and their particle properities within the astrophysics picture.

Since ordinary matter is baryonic, the most straightforward possibility is to assume this composition for the elusive dark matter. The contribution from stars and gas is not enough, so astrophysical bodies collectively known as MAssive Compact Halo Objects (MACHOs) are the main baryonic dark matter candidates [29]. These candidates include brown and white dwarfs, Jupiter-like objects, neutron stars, and stellar black hole remnants. However, the scenario of Big-Bang nucleosynthesis, which explains the origin of the elements after the Big Bang, taking into account measured abundances of helium, deuterium and lithium, sets a limit for the number of baryons that can exist in the universe, namely $\Omega_{b} \sim 0.04$. This density is clearly too small to account for all of the dark matter in the universe. Baryonic objects are likely components of some of the dark matter on galactic scales. But non-baryonic candidates are needed to address the dark matter problem on cosmological scales.

Particle physics provides candidates for dark matter. The three most promising are 'axions', 'neutrinos', and 'WIMPs' with masses of the order of $10^{-5} \mathrm{eV}$, $30 \mathrm{eV}$ and $100 \mathrm{GeV}$, respectively. Neutrinos are the only candidates that are known to exist, although they are disfavored as being the primary component of dark matter. The other particles are not present in the standard model, but they are crucial to solving important theoretical problems of this model. The existence of these particles is predicted in extensions of the standard model. 


\subsubsection{Neutrinos}

There are three known 'flavors' of neutrinos: the electron neutrino $\nu_{e}$, the muon neutrino $\nu_{\mu}$, and the tau neutrino $\nu_{\tau}$. Weak interactions produce the flavor eigenstates $\left|\nu_{e}\right\rangle,\left|\nu_{\mu}\right\rangle$, and $\left|\nu_{\tau}\right\rangle$ which are associated with their respective charged leptons. However, these flavor eigenstates are not energy eigenstates. Let $\left|\nu_{1}\right\rangle,\left|\nu_{2}\right\rangle$, and $\left|\nu_{3}\right\rangle$ denote the three energy eigenstates for the three-flavor system, with energies $E_{1}, E_{2}$ and $E_{3}$, respectively. Consider for simplicity two flavors of neutrinos instead of three, $\nu_{e}$ and $\nu_{\mu}$, for which we consider flavor transformations, since these provide evidence for non-zero neutrino mass.

Then the flavor and the energy eigenstates are connected by a unitary transformation,

$$
\left\{\begin{array}{l}
\left|\nu_{e}\right\rangle=\cos \theta\left|\nu_{1}\right\rangle+\sin \theta\left|\nu_{2}\right\rangle \\
\left|\nu_{\mu}\right\rangle=-\sin \theta\left|\nu_{1}\right\rangle+\cos \theta\left|\nu_{2}\right\rangle
\end{array}\right.
$$

So, there is a probability of conversion fron $\nu_{e}$ to $\nu_{\mu}$. Neutrino oscillations have been detected in two systems. Atmospheric muon neutrinos, which originate from the collision of cosmic rays with the Earth's atmosphere, have been observed to oscillate into tau neutrinos [30]:

$$
\nu_{\mu} \rightarrow \nu_{\tau}, \quad \Delta m_{23}^{2} \sim 3 \times 10^{-3} \mathrm{eV}^{2}
$$

Solar neutrinos, produced in the nuclear reactions that make the Sun shine, also show oscillations [31]:

$$
\nu_{e} \rightarrow \nu_{\mu} \text { or } \nu_{\tau}, \quad \Delta m_{12}^{2} \sim 7 \times 10^{-5} \mathrm{eV}^{2}
$$

These results can be used to set a lower limit on the mass of the heaviest neutrino. The mass of the heaviest neutrino must be greater than or equal to the square root of the largest mass-squared difference, assuming the mass of the other neutrino 
vanishes. This gives the lower limit

$$
\text { mass of heaviest neutrino } \gtrsim 0.05 \mathrm{eV}
$$

Upper limits on neutrino masses come from laboratory experiments, such as tritium decay and high-energy accelerator experiments [32]:

$$
m_{1}<2.8 \mathrm{eV}, \quad m_{2}<190 \mathrm{keV}, \quad m_{3}<18.2 \mathrm{MeV}
$$

However, the small mass differences implied by equations 1.53 and 1.54 show that the smallest of the three upper limits applies to all three active neutrino masses. Thus we have

$$
m_{i}<2.8 \mathrm{eV} \quad(i=1,2,3)
$$

It follows from this mass constraint that reactions such as $\nu_{e} \bar{\nu}_{e} \leftrightarrow e^{+} e^{-}$in the hot early universe were able to keep standard-model neutrinos in thermal equilibrium. The neutrino density then follows from a computation of the neutrino number density [33]. The result is

$$
\Omega_{\nu} h^{2}=\sum_{i=1}^{3} \frac{g_{i} m_{i}}{90 \mathrm{eV}}
$$

where $g_{i}=1$ for a neutrino that is its own antiparticle (Majorana neutrino), and $g_{i}=2$ for a neutrino that is not its own antiparticle (Dirac neutrino).

Cosmology provides an upper limit on the neutrino density $\Omega_{\nu} h^{2}$ from WMAP. A combined analysis of cosmic microwave background measurements, galaxy clustering measurements, and observations of the Lyman- $\alpha$ forest gives the upper limit [34]:

$$
\Omega_{\nu} h^{2}<0.0076 \quad \text { (95\% C.L.). }
$$

This translates into a cosmological upper limit on the neutrino mass using equation 1.58:

$$
g_{1} m_{1}+g_{2} m_{2}+g_{3} m_{3}<0.7 \mathrm{eV} .
$$


On the other hand, a lower bound on the cosmological density in neutrinos comes from equation 1.58 and inequality 1.55. Taking only one massive Majorana flavor,

$$
\Omega_{\nu} h^{2}>0.0006
$$

Thus neutrinos are a form of dark matter, although not abundant enough.

Suppose the neutrino is its own antiparticle by taking $g_{i}=1$ in equation 1.60. The results for showing the known neutrinos as elements of dark matter can be summarized by the constraints:

$$
\begin{gathered}
0.05 \mathrm{eV}<m_{1}+m_{2}+m_{3}<0.7 \mathrm{eV}, \\
0.0006<\Omega_{\nu} h^{2}<0.0076,
\end{gathered}
$$

The upper limit on $\Omega_{\nu} h^{2}$ means that currently known neutrinos cannot be the major constituents of dark matter.

Furthermore, being light and relativistic at the time of galaxy formation, neutrino free streaming erases fluctuations below a scale of $\sim 40 \mathrm{Mpc}$, called the freestreaming length [35]. This would imply that the big structures (superclusters) formed first in the universe, then the small structures (galaxies). The observations appear that the galaxies have been around since at least $\mathrm{z} \sim 6$, while superclusters are just collapsing today [1]. So neutrinos are not a viable dark matter candidate.

The neutrinos are hot dark matter, not the cold dark matter that we are looking for. Since the known non-baryonic neutrinos fail to be cold dark matter, we are led to consider hypothetical particles.

\subsubsection{WIMPs}

The Weakly Interactive Massive Particle (WIMP) could be the lightest neutralino in supersymmetry. The superpositions of the neutral gauginos and the neutral 
higgsinos in supersymmetric extensions of the Standard Model of particle physics are called neutralinos. It was realized by $[36,37,38]$ that a natural candidate for dark matter is the lightest neutralino. This particle is typically stable and massive. WIMPs could have a relic abundance in the universe that matches the dark matter density.

\section{The Relic Density of WIMPs}

WIMPs that were in thermal equilibrium in the early universe (thermal WIMPs) are particularly interesting. Their cosmological density is naturally of the right order of magnitude when their interaction cross section is of the order of the weak cross section. This also makes them detectable in the laboratory, as we will see later.

In the early universe, the temperature was higher than the mass of WIMPs; i.e., in units with $c=\hbar, T>m_{\chi}$, WIMPs could convert to standard model particles and standard model particles could convert to WIMPs, with the reaction mediated through Higgs or slepton exchange. See the Feynman diagrams in Figure 1.14.
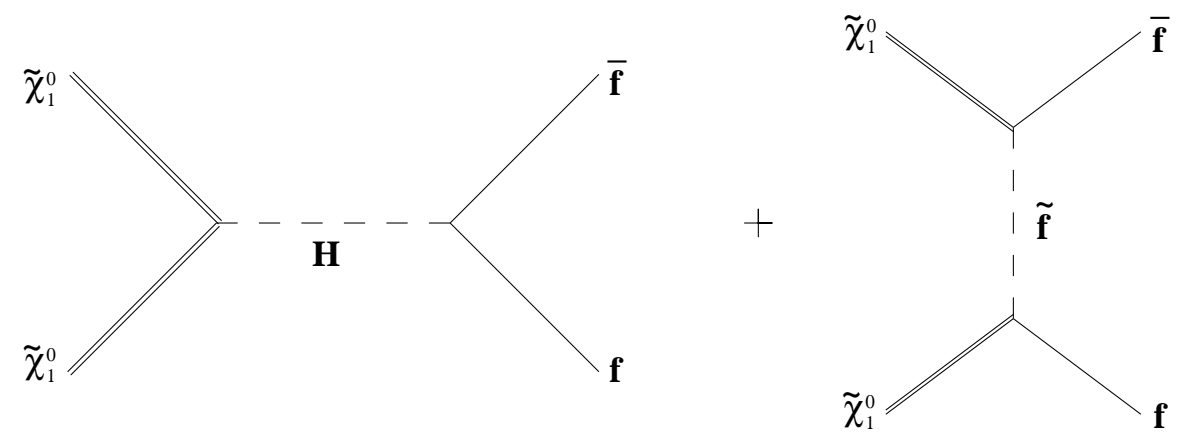

Figure 1.14: Feynman diagrams contributing to early universe neutralino $\left(\tilde{\chi}_{1}^{0}\right)$ annihilation into fermions through neutral Higgses $(H \equiv H, h, A)$ and through squarks and sleptons $(\tilde{f})$. 


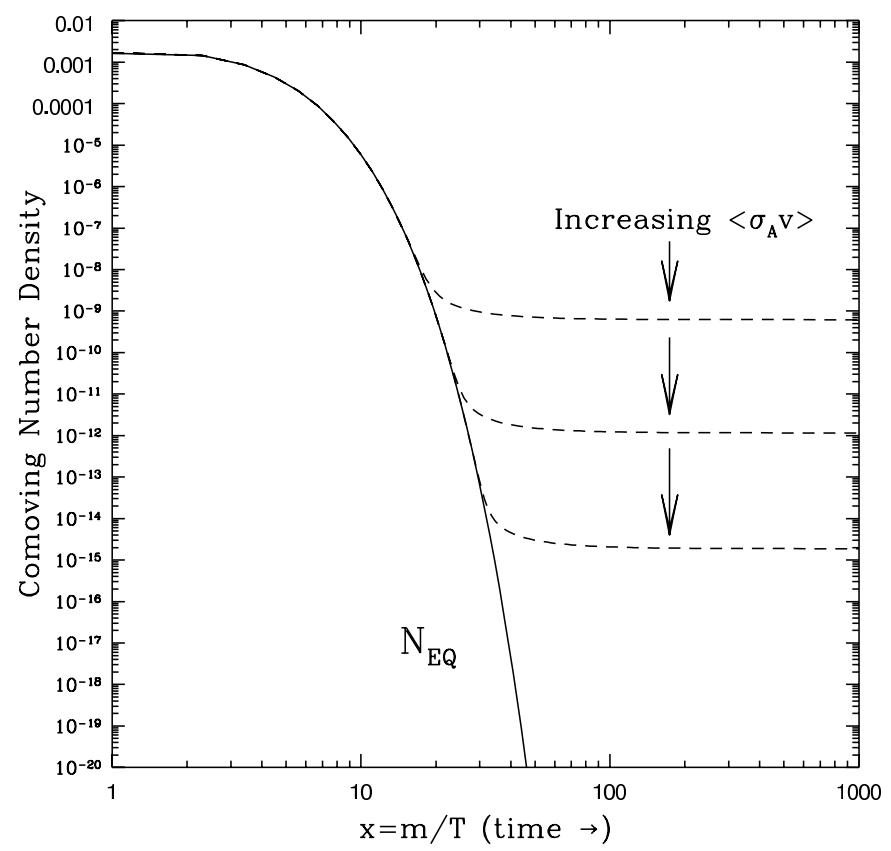

Figure 1.15: Evolution of a typical WIMP number density in the early universe. The number of WIMPs in a volume expanding with the universe (comoving density) first decreases exponentially due to the Boltzmann factor $e^{-m / T}$ and then 'freezes out' to a constant value when the WIMP annihilation reactions cannot maintain chemical equilibrium between WIMPs and standard model particles. In the figure, $\langle\sigma v\rangle$ is the thermally averaged annihilation cross section times relative velocity. WIMPs with a larger annihilation cross section end up with smaller densities. Figure from [39].

The annihilation reactions that convert WIMPs into standard model particles were initially in equilibrium with their opposite reactions. As the universe expanded, and the temperature became smaller than the WIMP mass, the gas of WIMPs, still in thermal equilibrium, diluted faster than the gas of standard model particles. The evolution of the number density $n$ of WIMPs is governed by the Boltzmman equation [40]

$$
\frac{d n}{d t}=-3 H n-<\sigma v>_{a n n}\left(n^{2}-n_{0}^{2}\right)
$$

where $H$ is the Hubble parameter, $n_{0}$ is the equillibrium number density of 
WIMPs, $v$ is the relative velocity of the annihilating WIMPs, and $\langle\sigma v\rangle_{\text {ann }}$ is the thermally averaged annihilation cross section.

The equilibrium number density of non-relativistic particles is suppressed by a Boltzmann factor $e^{-m / T}$ with respect to the number density of relativistic particles. As the universe cooled further, WIMPs became so rare that the WIMP annihilation reactions could no longer occur, and from then on the number density of WIMPs decreased inversely with volume (or in other words, the number of WIMPs per comoving volume remained constant). Chemical decoupling occured when the WIMP annihilation rate $\Gamma_{\mathrm{ann}}=\left\langle\sigma_{\mathrm{ann}} v\right\rangle n$ became smaller than the universe expansion rate $H$. Using Friedmann's equation to find the expansion rate $H$, then an order-of-magnitude estimation of relic density of WIMPs is $[39,41]$ :

$$
\Omega h^{2} \approx \frac{3 \times 10^{-27} \mathrm{~cm}^{3} / \mathrm{s}}{\left\langle\sigma_{\mathrm{ann}} v\right\rangle} .
$$

An important property of this equation is that smaller annihilation cross sections correspond to larger relic densities. This can be understood from the fact that WIMPs with stronger interactions remain in thermal equilibrium for a longer time, and hence decouple when the universe is colder, and their density could be further suppressed by a smaller Boltzmann factor. Figure 1.15 illustrates this relationship.

\section{Supersymmetry}

Supersymmetry is a proposed symmetry of space-time that was discovered in the process of unifying the fundamental forces of nature (electroweak, strong, and gravitational forces). It requires that particles exist in multiplets, related by a supersymmetric transformation. In supersymmetry, there is a fermionic degree of freedom for every bosonic degree of freedom, and vice versa. For every particle, 
there is a superpartner whose spin differs by $1 / 2$. In terms of its action on the components of the fields of the theory, a new discrete symmetry is defined by $\mathrm{R}$ parity:

$$
R=(-1)^{3(B-L)+2 S}
$$

where $B$ and $L$ are baryon and lepton number operators, and $S$ is the spin. This means that $R=1$ for ordinary particles and $R=-1$ for their superpartners. It is the conservation of R-parity that causes the neutralino to be stable-an essential feature for a dark matter candidate.

One of the primary motivations for supersymmetry is its role in stabilizing the masses of fundamental scalar particles, such as the Higgs boson.

\begin{tabular}{|c|c|c|c|c|c|}
\hline \multicolumn{2}{|c|}{ Standard Model particles and fields } & \multicolumn{4}{|c|}{ Supersymmetric partners } \\
\hline & & \multicolumn{2}{|c|}{ Interaction eigenstates } & \multicolumn{2}{|c|}{ Mass eigenstates } \\
\hline Symbol & Name & Symbol & Name & Symbol & Name \\
\hline$q=d, c, b, u, s, t$ & quark & $\tilde{q}_{L}, \tilde{q}_{R}$ & squark & $\tilde{q}_{1}, \tilde{q}_{2}$ & squark \\
\hline$l=e, \mu, \tau$ & lepton & $\tilde{l}_{L}, \tilde{l}_{R}$ & slepton & $\tilde{l}_{1}, \tilde{l}_{2}$ & slepton \\
\hline$\nu=\nu_{e}, \nu_{\mu}, \nu_{\tau}$ & neutrino & $\tilde{\nu}$ & sneutrino & $\tilde{\nu}$ & sneutrino \\
\hline$g$ & gluon & $\tilde{g}$ & gluino & $\tilde{g}$ & gluino \\
\hline$W^{ \pm}$ & $W$-boson & $\tilde{W}^{ \pm}$ & wino & & \\
\hline$H^{-}$ & Higgs boson & $\tilde{H}_{1}^{-}$ & higgsino & $\tilde{\chi}_{1,2}^{ \pm}$ & chargino \\
\hline$H^{+}$ & Higgs boson & $\tilde{H}_{2}^{+}$ & higgsino & & \\
\hline$B$ & $B$-field & $\tilde{B}$ & bino & & \\
\hline$W^{3}$ & $W^{3}$-field & $\tilde{W}^{3}$ & wino & & \\
\hline$H_{1}^{0}$ & Higgs boson & & & $\tilde{\chi}_{1,2,3,4}^{0}$ & neutralino \\
\hline$H_{2}^{0}$ & Higgs boson & $H_{1}^{0}$ & higgsino & & \\
\hline$H_{3}^{0}$ & Higgs boson & $\tilde{H}_{2}^{0}$ & higgsino & & \\
\hline
\end{tabular}

Table 1.1: Standard Model particles and their superpartners in the MSSM. Table from [42].

We are considering the minimal supersymmetric extension of the Standard Model (MSSM, for Minimal Supersymmetric Standard Model). The MSSM contains the minimal number of particles necessary to incorporate all of the standard Model(SM) particles and their supersymmetric partners. The MSSM contains all the known fields of the standard model and an extra higgs doublet, together 
with the partners required to form supersymmetric multiplets. No other fields are introduced in the MSSM. The interactions of the theory are all those allowed by the gauge symmetry $S U(3) \times S U(2) \times U(1)$ and by renormalizability. MSSM particles and interactions are listed in Table 1.1. More specifically:

- Fermionic superpartners are associated with all gauge fields. Gluons, $W^{ \pm}$, and $B$ bosons then get fermionic partners called gluinos $(\tilde{g})$, winos $\left(\tilde{W}^{i}\right)$ and binos $(\tilde{B})$, respectively. The common name for all partners of gauge fields is the gaugino.

- Scalar partners are associated with the fermions, i.e., quarks and leptons get scalar partners called squarks and sleptons.

- One additional Higgs field is introduced, so there are a total of two Higgs doublets, corresponding to five physical Higgs states. One spin 1/2 Higgsino is associated with each Higgs boson. This is done to give masses to both up and down-type quarks upon an electroweak symmetry breaking and also to preserve supersymmetry.

- Higgsinos and electroweak gauginos (binos and winos) have the same quantum number, so they can mix, and form charginos and neutralinos.

Minimal supersymmetric theories with R-parity conservation are attractive for the study of dark matter as they predict the existence of a new stable particle, which is the lightest stable particle dark matter candidate neutralino. Furthermore, for parameter values of interest to resolve the gauge hierarchy problem, the neutralio has an annihilation cross section that yields a relic density of cosmological interest, helping to form the structure of the early universe. 
In the MSSM, the superpartners of the $B, W_{3}$ gauge bosons (or the photon and $Z$, equivalently) and the neutral Higgs bosons, $H_{1}^{0}$ and $H_{2}^{0}$, are called binos $(\tilde{B})$, winos $\left(\tilde{W}_{3}\right)$, and higgsinos $\left(\tilde{H}_{1}^{0}\right.$ and $\left.\tilde{H}_{2}^{0}\right)$, respectively. These states mix into four Majorana fermionic mass eigenstates, called neutralinos. The four neutralino mass eigenstates are typically labelled $\tilde{\chi}_{1}^{0}, \tilde{\chi}_{2}^{0}, \tilde{\chi}_{3}^{0}$, and $\tilde{\chi}_{4}^{0}$, ordered by increasing mass. In the following sections we will refer to $\tilde{\chi}_{1}^{0}$, the lightest of the four neutralinos, as the neutralino, and denote it simply as $\chi \equiv \tilde{\chi}_{1}^{0}$.

In the basis $\left(\tilde{B}, \tilde{W}_{3}, \tilde{H}_{1}^{0}, \tilde{H}_{2}^{0}\right)$, the neutralino mass matrix can be expressed as

$$
M_{\chi}=\left(\begin{array}{cccc}
M_{1} & 0 & -M_{Z} \cos \beta \sin \theta_{W} & M_{Z} \sin \beta \sin \theta_{W} \\
0 & M_{2} & M_{Z} \cos \beta \cos \theta_{W} & -M_{Z} \sin \beta \cos \theta_{W} \\
-M_{Z} \cos \beta \sin \theta_{W} & M_{Z} \cos \beta \cos \theta_{W} & 0 & -\mu \\
M_{Z} \sin \beta \sin \theta_{W} & -M_{Z} \sin \beta \cos \theta_{W} & -\mu & 0
\end{array}\right)
$$

where $M_{1}$ and $M_{2}$ are the bino and wino mass parameters, respectively, $\theta_{W}$ is the Weinberg angle, and $\tan \beta$ is the ratio of the vacuum expectation values of the Higgs bosons. $\mu$ is the Higgsino mass parameter determined by the minimization of the Higgs effective potential

$$
\mu^{2}=\frac{m_{H_{1}}^{2}-m_{H_{2}}^{2} \tan ^{2} \beta}{\tan ^{2} \beta-1}-\frac{1}{2} M_{Z}^{2}
$$

As we have seen, the (lightest) neutralino is a linear combination of $\tilde{B}, \tilde{W}_{3}, \tilde{H}_{1}^{0}$, and $\tilde{H}_{2}^{0}$,

$$
\chi=N_{11} \tilde{B}+N_{12} \tilde{W}_{3}+N_{13} \tilde{H}_{1}^{0}+N_{14} \tilde{H}_{2}^{0} .
$$

It is commonly defined that $\chi$ is most gaugino-like if $P=N_{11}^{2}+N_{12}^{2}>0.9$, and Higgsino-like if $P<0.1$. Otherwise, $\chi$ is mixed.

The Weakly Interactive Massive Particle (WIMP) is most likely the electrically neutral lightest neutralino. It is the dark matter candidate that has been 
most widely investigated theoretically. The direct and indirect dark matter search experiments are sensitive to this particle, or its annihilation products.

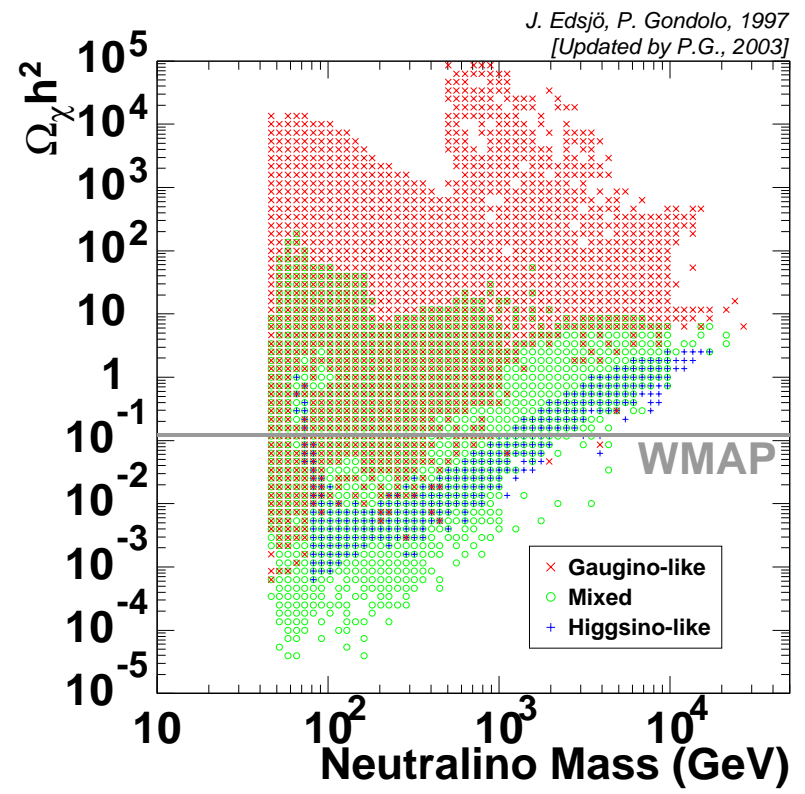

Figure 1.16: Relic density of the lightest neutralino as a function of its mass. For each mass, several density values are possible depending on the other supersymmetric parameters (seven in total in the scenario plotted). The color code shows the neutralino composition (gaugino, higgsino or mixed). The gray horizontal line is the current error band in the WMAP measurement for the cosmological cold dark matter density. Figure from [33]

The density of WIMPs was also calculated in supersymmetry theory. Even the minimal supersymmetric standard model (MSSM) has 106 parameters beyond those in the Standard Model: 102 supersymmetry-breaking parameters, 1 complex supersymmetric parameter $\mu$, and 1 complex electroweak symmetry-breaking parameter $\tan \beta[32]$. Since it has little predictive power given with many parameters, simplified scenarios with a reduced number of parameters are used in phenomenological studies. One of the most studied cases is minimal supergravity, which reduces the number of parameters to five: three real mass parameters at 
the Grand Unification scale (the scalar mass $m_{0}$, the scalar trilinear coupling $A_{0}$, and the gaugino mass $m_{1 / 2}$ ) and two real parameters at the weak scale (the ratio of Higgs expectation values $\tan \beta$ and the sign of the $\mu$ parameter). Another scenario relevant to dark matter studies is a class of models with seven parameters specified at the weak scale [39]: $\mu, \tan \beta$, the gaugino mass parameter $M_{2}$, the mass $m_{A}$ of the CP-odd Higgs boson, the sfermion mass parameter $\widetilde{m}$, and the bottom and top quark trilinear couplings $A_{b}$ and $A_{t}$.

An example of calculations for the density of the lightest neutralino is given in Figure 1.16. This figure was obtained in a scenario with seven supersymmetric parameters at the weak scale. The relic density is not fixed once the neutralino mass is given, because the neutralino annihilation cross section depends on the masses and composition of many other supersymmetric particles. Therefore the density in Figure 1.16 is not a single-valued function of the neutralino mass, and the plot was obtained through an extended computer scan in seven-dimensional parameter space [33].

\subsubsection{Axions}

Axions were suggested in [43] to solve the so-called "strong CP problem". Out of the vacuum structure of Quantum Chromodynamics there arises a large CPviolating phase, which is at variance with stringent measurements of the electric dipole moment of the neutron. A possible solution to this problem is that the CP-violating phase is the vacuum expectation value of a new field, called the axion, which relaxes dynamically to a very small value. The original axion model of Peccei and Quinn is experimentally ruled out, but other axion models based on the same idea have been proposed. Among them are the invisible axions [44], 
the KSVZ axion [45] and the DFSZ axion [46]. They differ in their strength of couplings to matter and radiation.

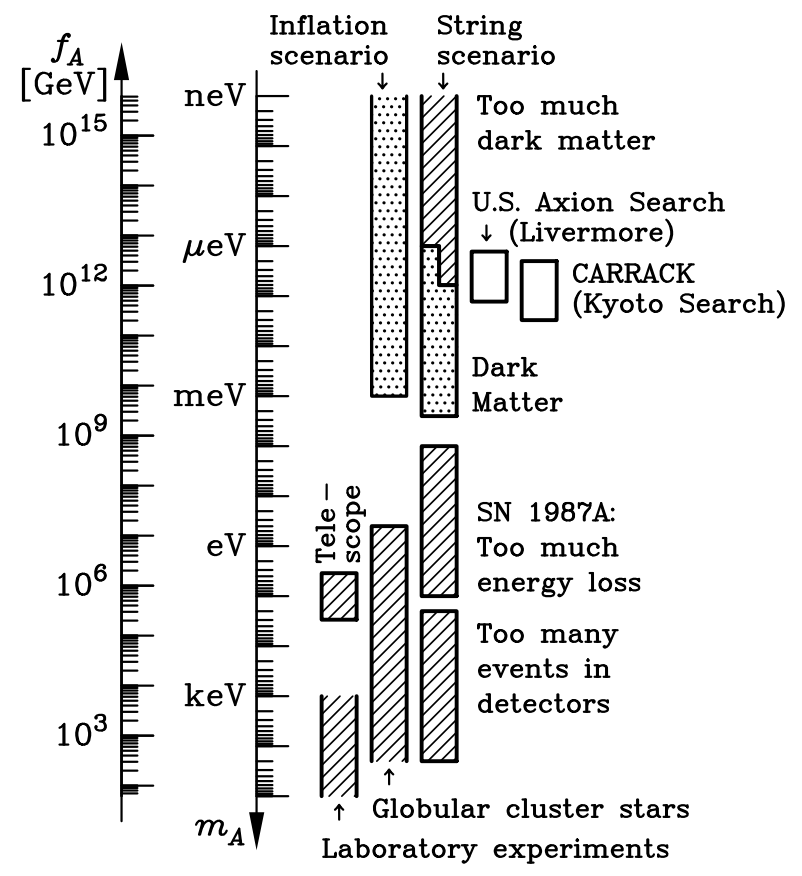

Figure 1.17: Laboratory, astrophysical, and cosmological constraints on the axion mass $m_{A}$. The inflation scenario and the string scenario are referred to in the text as the vacuum alignment scenario and the string emission scenario, respectively. $f_{A}$ is the axion decay constant, which is inversely related to $m_{A}$. The axion is a good dark matter candidate for $1 \mu \mathrm{eV} \lesssim m_{A} \lesssim 1 \mathrm{meV}$. Figure from [32].

In a cosmological context, axions, contrary to neutrinos and neutralinos, are generally produced non-thermally. The two main mechanisms for non-thermal axion production are vacuum alignment and emission from cosmic strings. In the vacuum alignment mechanism, a potential is generated for the axion field by chiral symmetry breaking. The axion field, which can in principle be at any point in this potential, starts moving toward the minimum of the potential, then oscillates around it. In Quantum mechanics, the field oscillations correspond to the generation of axion particles. In the other main non-thermal mechanism for 


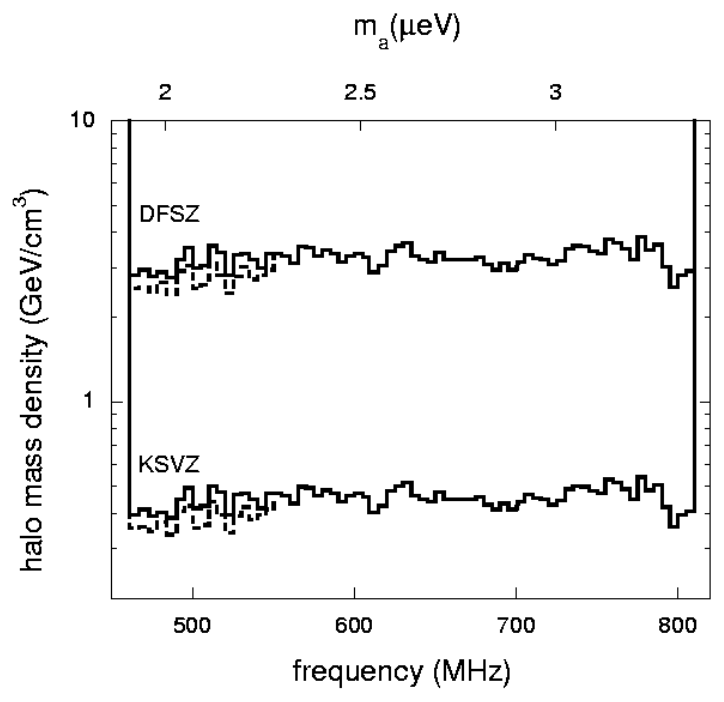

Figure 1.18: Experimental constraints on the density of axions in the galactic halo near the Sun as a function of the axion mass (upper scale) and cavity frequency (lower scale). The regions above the curves marked 'DFSZ' and 'KSVZ' are excluded from the respective axion models. The currently accepted value for the local dark halo density is $0.45 \mathrm{GeV} / \mathrm{cm}^{3}$, which is approximately the extension of the excluded region for the KSVZ axion. Figure from [47].

axion production, axions are emitted in the wiggling or decay of cosmic strings. In both cases, axions are produced with small momentum, $\ll \mathrm{keV}$, and thus they are cold dark matter despite having tiny masses, between $1 \mu \mathrm{eV}$ and $1 \mathrm{meV}$. This is in fact the range of masses in which axions are good dark matter candidates. Figure 1.17 shows the current constraints on the axion mass from laboratory, astrophysical, and cosmological data.

Searches for axions as galactic dark matter rely on the coupling of axions to two photons. An incoming galactic axion can become a photon in the magnetic field in a resonant cavity. For this to happen, the characteristic frequency of the cavity has to match the axion mass. Since the latter is unknown, searches for galactic axions use tunable cavities, and scan over the cavity frequency, a time-consuming 
process. The U.S. axion search at Lawrence Livermore National Laboratory is currently exploring a wide range of interesting axion masses, and has put some constraint on the KSVZ axion as a dominant component of the galactic halo [47]. Figure 1.18 shows the constraints on the local galactic density in axions as a function of the axion mass. KSVZ axions with mass in the range 1.91-3.34 $\mu \mathrm{eV}$ cannot be the main component of galactic dark matter. The Lawrence Livermore National Laboratory search is still continuing to a larger range of axion masses.

\subsubsection{Other Particle Dark matter Candidates}

The list of dark matter candidates is by no means complete. In addition to the neutralino there are other potential SUSY candidates for dark matter. In principle one of them might be the sneutrino [48] of the MSSM. One finds that the sneutrino relic density is in the region $0.1 \lesssim \Omega_{\tilde{\nu}} h^{2} \lesssim 1.0$ for $550 \mathrm{GeV} \lesssim m_{\tilde{\nu}} \lesssim$ $2300 \mathrm{GeV}$ [49]. This is consistent with the LEP limits on $Z^{0} \rightarrow$ invisible neutral particles, suggesting $m_{\tilde{\nu}} \gtrsim m_{Z^{0}} / 2$, as in the case of the heavy neutrino above. However, sneutrino-nucleus interaction is similar to the heavy neutrino-nucleus interaction, and therefore direct detection experiments [50] impose limits similar to those discussed above on the sneutrino mass. Such a heavy sneutrino cannot be the lightest stable particle in SUSY models.

The supersymmetric partner of the graviton, the gravitino, has also been proposed as a candidate for dark matter [51]. In the absence of inflation, the gravitino could give rise to the correct relic abundance if its mass is of order $\mathrm{keV}$. This is unlikely in specific theoretical models. For example, in gravity mediated SUSY, breaking the masses of the superpartners is of the order the gravitino mass, and therefore this should be of order $1 \mathrm{TeV}$. Gravitinos as dark matter would be un- 
detectable since their interactions with ordinary matter are extremely weak.

Instead of using WIMPs with typical masses of order a hundred $\mathrm{GeV}$, the authors of [52] studied scenarios with nonthermal WIMPs in the range $10^{12}$ to $10^{16} \mathrm{GeV}$. They called these objects WIMPZILLAS.

The lightest Kaluza-Klein particle, LKP, in models with TeV extra dimensions, has been studied as a viable dark matter candidate [53]. It is actually a typical WIMP (the most studied possible particle is a Kaluza-Klein 'photon'), with a mass in the range $400-1200 \mathrm{GeV}$. 


\section{Chapter 2}

\section{WIMP Detection}

\section{$2.1 \quad$ Introduction}

WIMPs are regarded as one of the primary dark matter candidates. WIMPs not only can form a background density in the Universe, but they will also cluster gravitationally with ordinary stars in the galactic halos. In particular they will be present in our own galaxy, the Milky Way. This raises the hope of detecting relic WIMPs directly, by performing experiments on the Earth through scattering off the atomic nucleus of a detector material. Since the detection will be on the Earth, we need to know the properties of our galaxy in order to be sure that such a detection is feasible. Analyses have been carried out with the conclusion that the Milky Way disc contains large amounts of dark matter as discussed in Chapter 1. Some observational evidence seems to point at a roughly spherical distribution of dark matter with a dispersion velocity $v_{0}=230 \mathrm{~km} / \mathrm{s}$ in the Milk Way. At the position of the Sun, around $8.5 \mathrm{kpc}$ away from the galactic center, the mean density of elementary particles trapped in the gravitational potential well of the galaxy is expected to be from $0.2 \mathrm{GeV} / \mathrm{cm}^{-3}$ to $0.8 \mathrm{GeV} / \mathrm{cm}^{-3}[26,27,28]$. 
The experimental approach to WIMP detection in the laboratory is to measure the recoil energy $E_{r}$ of a nucleus off of which a WIMP has scattered elastically. The WIMP detection rate is determined by the WIMP distribution in the Milk Way and the WIMP-nucleus elastic-scattering cross section. WIMP elastic scattering is generic depending on the spin independent or spin-dependent coupling between WIMPs and nucleons, and can be calculated in general terms with particle physics and dark matter halo assumptions.
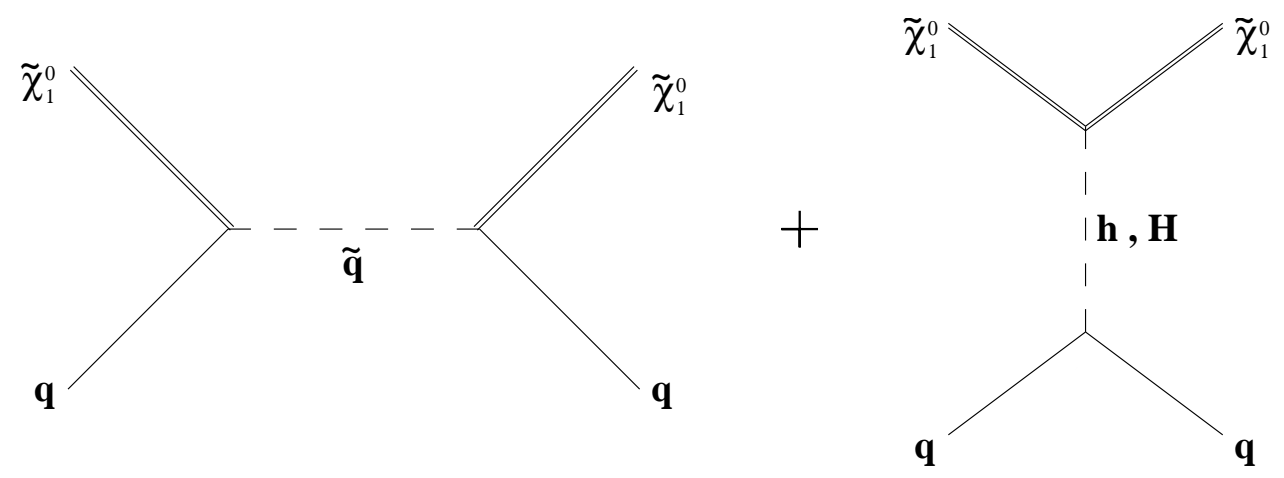

Figure 2.1: Feynman diagrams contributing to the neutralino-nucleon cross section through squark $(\tilde{q})$ exchange and CP-even light $(h)$ and heavy $(H)$ neutral Higgs exchange.

Figure 2.1 shows Feynman diagrams contributing to the scalar elastic-scattering of a neutralino from quarks through the exchange Higgs bosons or squarks. The elastic scattering of a WIMP off a nucleus in a detector depends fundamentally on the interactions of WIMPs with quarks and gluons. The distribution of quarks in the nucleon and the distribution of nucleons in the nucleus play crucial roles. The interaction of the WIMP with a whole nucleus causes it to recoil kinematically. The WIMP scattering rate of the detector nucleus is determined by the WIMP density and WIMP-nucleus scattering cross section. With a Boltzman velocity distribution centered at $230 \mathrm{~km} / \mathrm{s}$, the energy spectrum of recoils is a falling exponential with a typical energy in the $10-\mathrm{keV}$ range. Current experi- 
ments can detect recoils at considerably low energy, as low as a few keV, and are thus sensitive to these recoils.

Indirect WIMP detection is also possible. Gravitationally trapped WIMPs will annihilate once the density of WIMPs is above the thermal equilibrium density in the gravitational potential. They will emit neutrinos and gamma rays.

I will start with a discussion of WIMP-nucleon scattering cross section calculation in section 2.2. Section 2.3 discusses the spin-independent WIMP scattering event rate off ordinary matter in the isothermal halo model, and section 2.4 describes the signature of the WIMP signal. Section 2.5 is a brief summary of the indirect detection of WIMPs. Section 2.6 is a general overview of past and present dark matter search experiments.

\subsection{WIMP-Nucleon Scattering Cross Section}

WIMPs can be detected directly via the recoiling of a nucleus (A, Z) in the elastic scattering process.

$$
\chi+(A, Z) \rightarrow \chi+(A, Z)^{\star}
$$

The neutralino-nucleus elastic scattering cross section depends on the interaction of neutralinos with quarks and gluons. It was thought before that the axial-vector coupling provided the only interaction of neutralinos with a laboratory detector target (ordinary matter). However, it was then realized from a heavy quark expansion that there may be significant scalar coupling of neutralinos to nuclei if the neutralino is a mixed gaugino/higgsino state [54]. For a mixed neutralino state, the scalar coupling would be enhanced additionally by the exchange of the lightest Higgs boson [55], and since the lightest Higgs boson is relatively light, this contribution could be significant. Moreover, the contribution to the 
scalar coupling from squark mixing has been shown to be important $[56,57]$. This contribution is proportional to the quark/squark mass ratio. Since the top quark is known to be quite heavy, squark mixing may have a significant effect on the scalar-nucleus coupling, even if the neutralino is in a pure state [39]. In the theoretical calculations of the neutralino-nucleon elastic scattering cross section, the contraints of the Higgs mass, measured masses of the top and bottom quarks, and the tau lepton, and the bounds on SUSY masses from LEP and Tevatron are important [58].

For non-relativistic Majorana particles, the elastic cross section $\sigma_{\chi}$ of relic neutralinos can be divided into two separate types. The coherent part described by an effective scalar coupling between the WIMP and the nucleus is proportional to the number of nucleons in the nucleus. It receives a tree-level contribution from scattering off quarks, $\chi q \rightarrow \chi q$, as described by a Lagrangian $\mathcal{L} \sim(\chi \chi)(\bar{q} q)$, through a Higgs exchange or a squark exchange. The incoherent component of the WIMP-nucleus cross section results from an axial-current interaction of a WIMP with constituent quarks, given by $\mathcal{L} \sim\left(\chi \gamma^{\mu} \gamma_{5} \chi\right)\left(\bar{q} \gamma_{\mu} \gamma_{5} q\right)$, through a $\mathrm{Z}$ boson exchange or a squark exchange, and couples the spin of the WIMP with the total spin of the nucleus.

The differential cross section for a WIMP (with mass $m_{\chi}$ ) scattering off a nucleus $X_{Z}^{A}$ with mass $m_{A}$ is therefore given by

$$
\frac{d \sigma}{d|\vec{q}|^{2}}=\frac{d \sigma^{\text {scalar }}}{d|\vec{q}|^{2}}+\frac{d \sigma^{\text {axial }}}{d|\vec{q}|^{2}}
$$

where the transferred momentum $\vec{q}=\frac{m_{A} m_{\chi}}{m_{A}+m_{\chi}} \vec{v}$ depends on the velocity $\vec{v}$ of the incident WIMP. The effective WIMP-nucleon cross sections $\sigma^{\text {scalar }}$ and $\sigma^{\text {axial }}$ are computed by evaluating nucleonic matrix elements of corresponding WIMP-quark and WIMP-gluon interaction operators [39]. 
In the scalar part, contributions from individual nucleons in the nucleus add coherently and the finite size effects are accounted for by including the scalar nuclear form factor $F(q)$. The effective interaction in general also includes tensor components, but the relevant nucleonic matrix elements can be expanded in the low momentum-transfer limit in terms of the nucleon four-momentum and the quark (gluon) parton distribution function. The differential cross section for the scalar part then takes the form [39]

$$
\frac{d \sigma^{\text {scalar }}}{d|\vec{q}|^{2}}=\frac{1}{\pi v^{2}}\left[Z f_{p}+(A-Z) f_{n}\right]^{2} F^{2}(q)
$$

where $f_{p}$ and $f_{n}$ are the effective WIMP couplings to protons and neutrons, respectively, and typically $f_{n} \approx f_{p}$. Explicit expressions for the case of the supersymmetric neutralino can be found in the Appendix of reference [39].

Usually the Saxon-Woods scalar form factor is adopted [59]

$$
F\left(Q_{r}\right)=\frac{3 j_{1}\left(q R_{0}\right)}{q R_{1}} e^{-\frac{1}{2}(q s)^{2}}
$$

where $R_{1}=\sqrt{R^{2}-5 s^{2}}, R=A^{\frac{1}{3}} \times 1.2 \mathrm{fm}, j_{1}$ is a spherical Bessel function and $s=1 \mathrm{fm}$.

At the zero momentum transfer limit,

$$
\sigma_{0}^{\text {scalar }}=\int_{0}^{4 m_{r}^{2} v^{2}} \frac{d \sigma^{\text {scalar }}(q=0)}{d|\vec{q}|^{2}} d|\vec{q}|^{2}=\frac{4 m_{r}^{2}}{\pi}\left[Z f_{p}+(A-Z) f_{n}\right]^{2}
$$

where $v$ is the velocity of incident WIMP, and $m_{r}=\frac{m_{A} m_{\chi}}{m_{A}+m_{\chi}}$ is the reduced mass.

A convenient quantity customarily used in comparing theory and experimental results for spin independent interaction is the cross section $\sigma_{p}^{S I}$ for WIMP elastic scattering off a free proton in the limit of zero momentum transfer:

$$
\sigma_{p}^{S I}=\frac{4}{\pi} m_{r p}^{2} f_{p}^{2}
$$


where $m_{r p}=\frac{m_{p} m_{\chi}}{m_{p}+m_{\chi}}$. The analogous quantity for a target with nuclei with mass number $A$ can then be expressed in terms of $\sigma_{p}^{S I}$ as

$$
\sigma_{A}^{S I}=\left(\frac{m_{r}}{m_{r p}}\right)^{2} A^{2} \sigma_{p}^{S I}
$$

However, note that $\sigma_{0}^{\text {scalar }}$ is not really the total cross section. The actual total cross section is obtained by integrating equation 2.3 with the Woods-Saxon form factor.

Effective axial WIMP coupling to the nucleus depends on the spin content of the nucleon $\Delta q_{p, n}$ and the overall expectation value of the nucleon group spin in the nucleus $<S_{p}>$ and $<S_{n}>$. For a nucleus with a total angular momentum $J$ we have [39]:

$$
\frac{d \sigma^{\text {axial }}}{d|\vec{q}|^{2}}=\frac{8}{\pi v^{2}} \Lambda^{2} J(J+1) S(q)
$$

with $\Lambda=\frac{1}{J}\left[a_{p}\left\langle S_{p}\right\rangle+a_{n}\left\langle S_{n}\right\rangle\right]$. The axial couplings

$$
a_{p}=\frac{1}{\sqrt{2}} \sum_{u, d, s} d_{q} \Delta q^{(p)}, \quad a_{n}=\frac{1}{\sqrt{2}} \sum_{u, d, s} d_{q} \Delta q^{(n)}
$$

are determined by the experimental values of the spin constants $\Delta u^{(p)}=\Delta d^{(n)}=$ $0.78, \Delta d^{(p)}=\Delta u^{(n)}=-0.5$, and $\Delta s^{(p)}=\Delta s^{(n)}=-0.16$. The effective couplings $d_{q}$ depend on the WIMP properties, which can be found in the Appendix of reference [39] for the neutralino.

At the zero momentum transfer limit and for axial (or incoherent) interactions, one finds

$$
\sigma_{0}^{a x i a l} \sim(32 / \pi) G_{F}^{2} m_{r}^{2} \Lambda^{2} J(J+1)
$$

where $G_{F}$ is the Fermi constant.

In the minimal supersymmetry frame work, Bednyakov [60] showed that the scalar WIMP-nucleon scattering cross section is between $10^{-5} \rightarrow 10^{-12} \mathrm{pb}$, and that the axial WIMP-nucleon scattering cross section is between $10^{-2} \rightarrow 10^{-7} \mathrm{pb}$. 


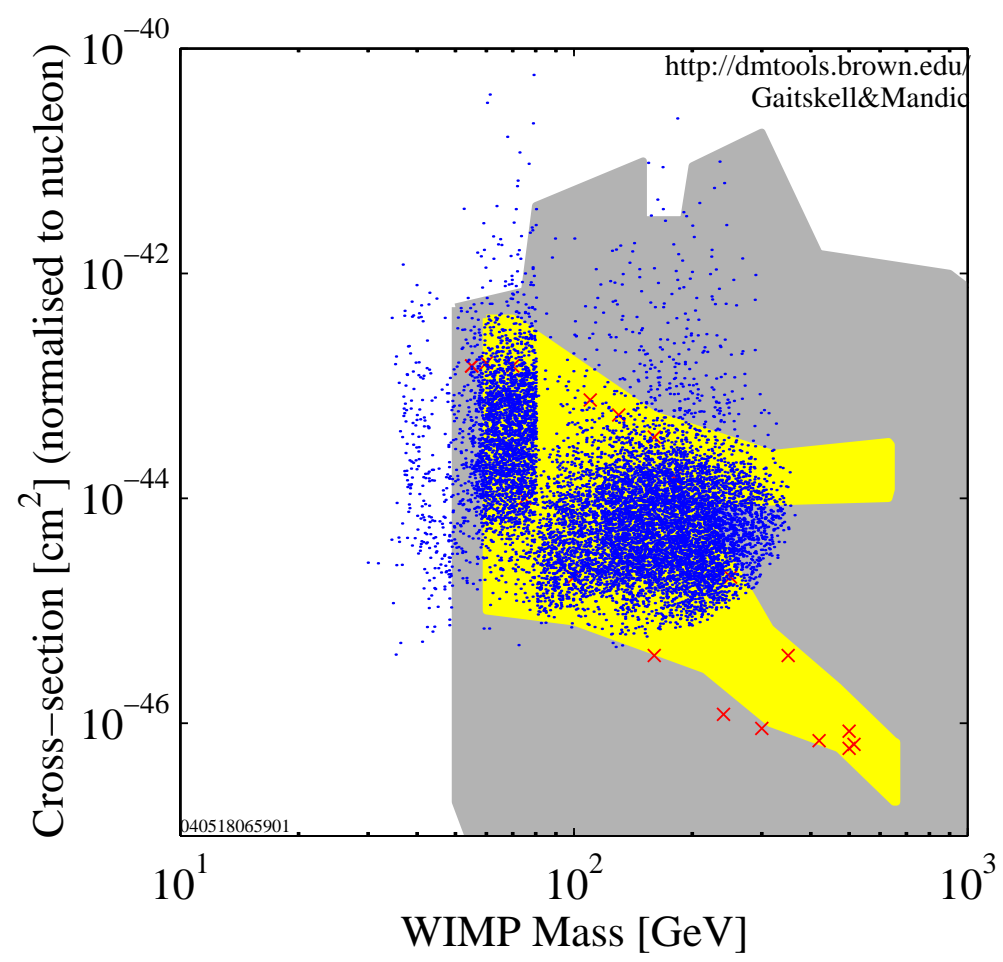

DATA listed top to bottom on plot

V. Bednyakov et al., Z.Phys.A 357 (1997) 339 SUSY MSSM

Chattopadhyay et. al Theory results - post WMAP

Baer et. al 2003

Baltz and Gondolo 2003

040518065901

Figure 2.2: WIMP-nucleon elastic scattering cross section versus WIMP mass allowed in MSSM.

The minimal supergravity (mSUGRA) model [61] assumes the minimal supersymmetric standard model, or MSSM, is valid at all energy scales from $M_{\text {weak }}$ up to $M_{G U T} \simeq 2 \times 10^{16} \mathrm{GeV}$. The mSUGRA model could arise as the low energy limit of a supergravity theory, where supersymmetry is broken in the hidden sector of the model at energy scale $M \sim 10^{10} \mathrm{GeV}$. Supersymmetry breaking is communicated to the observable sector via gravitational interactions, leading to soft SUSY breaking mass terms of the order of the electroweak scale, $\tilde{m} \sim 100-1000$ GeV. At the GUT scale (with the added assumption of an approximate global $U(n)$ symmetry for the mSUGRA Lagrangian), this leads to a common mass for 
all scalars $m_{0}$ and a common trilinear coupling $A_{0}$. Motivated by the apparent unification of gauge coupling constants, it is also assumed that all gaugino masses are unified to $m_{1 / 2}$ at $M_{G U T}$. The weak scale sparticle spectrum is derived from the renormalization group (RG) running of the SUSY soft breaking parameters. Requiring radiative electroweak symmetry breaking allows the determination of the superpotential Higgsino mass squared $\mu^{2}$, and allows the expression of the soft SUSY breaking bilinear term $B$ in terms of $\tan \beta$, which is the ratio of vacuum expected values (vev's) of the two Higgs fields. Thus, all sparticle masses and couplings are derived in terms of the parameter set

$$
m_{0}, m_{1 / 2}, A_{0}, \tan \beta, \text { and } \operatorname{sign}(\mu)
$$

With laboratory constraints of $b \rightarrow s+\gamma$, of $g-2$, and of $B_{s}^{0} \rightarrow \mu^{+} \mu^{-}$, and with the new result of dark matter density from WMAP, the mSUGRA model gives a scalar WIMP-nucleon scattering cross section between $10^{-6}$ and $10^{-11} \mathrm{pb}$, and an axial WIMP-nucleon scattering cross section between $10^{-3}$ and $10^{-8} \mathrm{pb}$ [62]. Figure 2.2 shows the allowed parameter region in the WIMP-nucleon cross section versus WIMP mass space in several MSSM calculations.

\subsection{WIMP Direct Detection Rate}

The differential rate of interactions per unit mass of target material of a particle with interaction cross section $\sigma$ is given by

$$
d R=\frac{N_{0}}{A} \sigma v d n
$$

where $n$ is the number density of incoming particles having speed $v$ relative to the target with atomic mass $A$. $N_{0}$ is Avogadro's number. The total WIMP interaction rate is given by integration over the WIMP velocity distribution. 
The WIMP velocity distribution is given by the WIMP phase space distribution. For simplicity, it is standard to assume the WIMPs occupy an isothermal, isotropic phase space distribution, appropriate for a fully gravitationally relaxed population of WIMPs. The formulism below follows Lewin and Smith's review paper [63] closely. The differential particle density is given by

$$
d n=\frac{n_{0}}{k} f\left(\vec{v}, \overrightarrow{v_{E}}\right) d^{3} v
$$

where $k$ is the normalization constant

$$
k=\int_{0}^{2 \pi} d \phi \int_{-1}^{+1} d(\cos \theta) \int_{0}^{v_{e s c}} f\left(\vec{v}, \overrightarrow{v_{E}}\right) v^{2} d v
$$

with

$$
n_{0}=\int_{0}^{v_{e s c}} d n
$$

Assume a Maxwellian velocity distribution

$$
f\left(\vec{v}, \overrightarrow{v_{E}}\right)=\exp \left(-\frac{\left(\vec{v}+\overrightarrow{v_{E}}\right)^{2}}{v_{0}^{2}}\right)
$$

where $\vec{v}$ is the WIMP velocity relative to the earth, and $\overrightarrow{v_{E}}$ is the Earth's velocity relative to the nonrotating halo of the galaxy. The quantity $v_{0}^{2}$ is characteristic of the WIMP kinetic energy, and has value $v_{0} \simeq 230 \mathrm{kms}^{-1}$. $v_{\text {esc }}$ is the halo escape velocity. $n_{0}=\frac{\rho_{\chi}}{M_{\chi}}$ is the mean dark matter particle number density. For convenience, we define

$$
k=k_{0}=\left(\pi v_{0}^{2}\right)^{3 / 2}
$$

for the non-physical case of the escape velocity $v_{e s c}=\infty$. Then for the same Maxwellian distribution truncated at $\left|\vec{v}+\overrightarrow{v_{E}}\right|=v_{e s c}$, we can write

$$
k=k_{1}=k_{0}\left[\operatorname{erf}\left(\frac{v_{e s c}}{v_{0}}\right)-\frac{2}{\pi^{1 / 2}} \frac{v_{e s c}}{v_{0}} e^{-v_{e s c}^{2} / v_{0}^{2}}\right]
$$


where $\operatorname{erf}$ is the error function. By using the low momentum transfer approximation, $\sigma=$ constant $=\sigma_{0}$,

$$
R=\frac{N_{0}}{A} \sigma_{0} \int v d n=\frac{N_{0}}{A} \sigma_{0} n_{0}<v>
$$

We define $R_{0}$ as the event rate per unit mass for $v_{E}=0, v_{e s c}=\infty$

$$
R_{0}=\frac{2}{\sqrt{\pi}} \frac{N_{0}}{A} \frac{\rho_{\chi}}{M_{\chi}} \sigma_{0} v_{0}
$$

so that

$$
R=R_{0} \frac{\sqrt{\pi}}{2} \frac{<v>}{v_{0}}=R_{0} \frac{k_{0}}{k} \frac{1}{2 \pi v_{0}^{4}} \int v f\left(\vec{v}, \overrightarrow{v_{E}}\right) d^{3} v .
$$

The differential form of this equation is

$$
d R=R_{0} \frac{k_{0}}{k} \frac{1}{2 \pi v_{0}^{4}} v f\left(\vec{v}, \overrightarrow{v_{E}}\right) d^{3} v
$$

The recoil energy of a nucleus struck by a dark matter particle of kinetic energy $E=\frac{1}{2} M_{\chi} v^{2}$ and scattered at angle $\theta$, is

$$
E_{r}=E \alpha \frac{(1-\cos \theta)}{2}
$$

where $E_{r}$ is the recoil energy, and $\alpha=\frac{4 m_{r}^{2}}{M_{\chi} M_{N}}=\frac{4 M_{\chi} M_{N}}{\left(M_{\chi}+M_{N}\right)^{2}}$. We assume the scattering is isotropic; i.e. uniform in $\cos \theta$, so that the recoils are distributed in $E_{r}$ over the range $0 \leq E_{r} \geq E \alpha$. Hence

$$
\begin{gathered}
\frac{d R}{d E_{r}}=\int_{E_{\min }}^{E_{\max }} \frac{1}{E \alpha} d R(E)=\frac{1}{E \alpha} \int_{v_{\min }}^{v_{\max }} \frac{v_{0}^{2}}{v^{2}} d R(v) \\
\frac{d R}{d E_{r}}=\frac{R_{0}}{E_{0} \alpha} \frac{k_{0}}{k} \frac{1}{2 \pi v_{0}^{2}} \int_{v_{\min }}^{v_{\max }} \frac{1}{v} f\left(\vec{v}, \overrightarrow{v_{E}}\right) d^{3} v
\end{gathered}
$$

where $E_{\text {min }}=E_{r} / \alpha$, the smallest particle energy that can give a recoil energy of $E_{r} ; E_{0}=\frac{M_{\chi} v_{0}^{2}}{2}$; and $v_{\min }$ is the dark matter particle velocity corresponding to $E_{\text {min }} ;$ i.e.,

$$
v_{\min }=\left(\frac{2 E_{\min }}{M_{\delta}}\right)^{1 / 2}=\left(\frac{E_{r}}{E_{0} \alpha}\right)^{1 / 2} v_{0}
$$


For this we obtain

$$
\frac{d R(0, \infty)}{d E_{r}}=\frac{R_{0}}{E_{0} \alpha} e^{-\frac{E_{r}}{E_{0} \alpha}}
$$

This is a good approximation with the velocity of WIMPs from 0 to $v_{e s c}$, and $v_{e s c}>>v_{0}$. Combined with the nuclear form factor $F\left(q^{2}\right)$, where $q^{2}=2 M_{N} E_{r}$, the expected WIMP nucleus recoil energy spectrum is

$$
\begin{gathered}
\frac{d R}{d E_{r}}=\frac{R_{0}}{E_{0} \alpha} e^{-\frac{E_{r}}{E_{0} \alpha}} F^{2}\left(q^{2}\right) \\
R_{0}=\frac{2}{\sqrt{\pi}} \frac{N_{0}}{A} n_{0} \rho_{0} v_{0} \\
E_{0} \alpha=\frac{1}{2}\left(\frac{M_{\chi}}{G e V c^{-2}}\right)\left(\frac{v_{0}}{c}\right)^{2} \frac{4 M_{\chi} M_{N}}{\left(M_{\chi}+M_{N}\right)^{2}} G e V \\
=\left(\frac{M_{\chi}}{100 G e V c^{-2}}\right)\left(\frac{v_{0}}{230 k m s^{-1}}\right)^{2} \frac{4 M_{\chi} M_{N}}{\left(M_{\chi}+M_{N}\right)^{2}} \times 26.7 k e V \\
R_{0}=\frac{540}{A M_{\chi}}\left(\frac{\sigma_{0}}{1 p b}\right)\left(\frac{\rho_{\chi}}{0.4 G e V c^{-2} c m^{-3}}\right)\left(\frac{v_{0}}{230 k m s^{-1}}\right) k g^{-1} d^{-1}
\end{gathered}
$$

Suppose the WIMP has a mass $M_{\chi}=100 \mathrm{GeV}$, and $\rho_{\chi}=0.3 \mathrm{GeVcm}^{-3}$, and the WIMP-nucleon cross section is $\sigma_{\chi p}=10^{-42} \mathrm{~cm}^{2}$. Thus, for silicon, $R_{0}=$ $4.4 \times 10^{-2} \mathrm{~kg}^{-1} d^{-1}, E_{0} \alpha=17.6 \mathrm{kev}$, and for germanium, $R_{0}=5.4 \times 10^{-1} \mathrm{~kg}^{-1} d^{-1}$, $E_{0} \alpha=26.7 \mathrm{kev}$. Figure 2.3 shows the elastic differential rate as a function of recoil energy in silicon and in germanium, and Figure 2.4 is the integrated event rate under the WIMP mass and cross section assumptions stated above.

For completeness, the differential scattering rate of silicon nuclei and of germanium nuclei can be calculated. At zero $v_{E}$ and finite $v_{e s c}$, the differential event rate is

$$
\frac{d R\left(0, v_{e s c}\right)}{d E_{r}}=\frac{k_{0}}{k_{1}}\left[\frac{d R(0, \infty)}{d E_{r}}-\frac{R_{0}}{E_{0} \alpha} e^{-v_{e s c}^{2} / v_{0}^{2}}\right]
$$

At non-zero $v_{E}$ and infinite $v_{e s c}$, the differential event rate [63] is

$$
\frac{d R\left(v_{E}, \infty\right)}{d E_{r}}=\frac{R_{0}}{E_{0} \alpha} \frac{\pi^{1 / 2}}{4} \frac{v_{0}}{v_{E}}\left[\operatorname{erf}\left(\frac{v_{\min }+v_{E}}{v_{0}}\right)-\operatorname{erf}\left(\frac{v_{\min }-v_{E}}{v_{0}}\right)\right]
$$




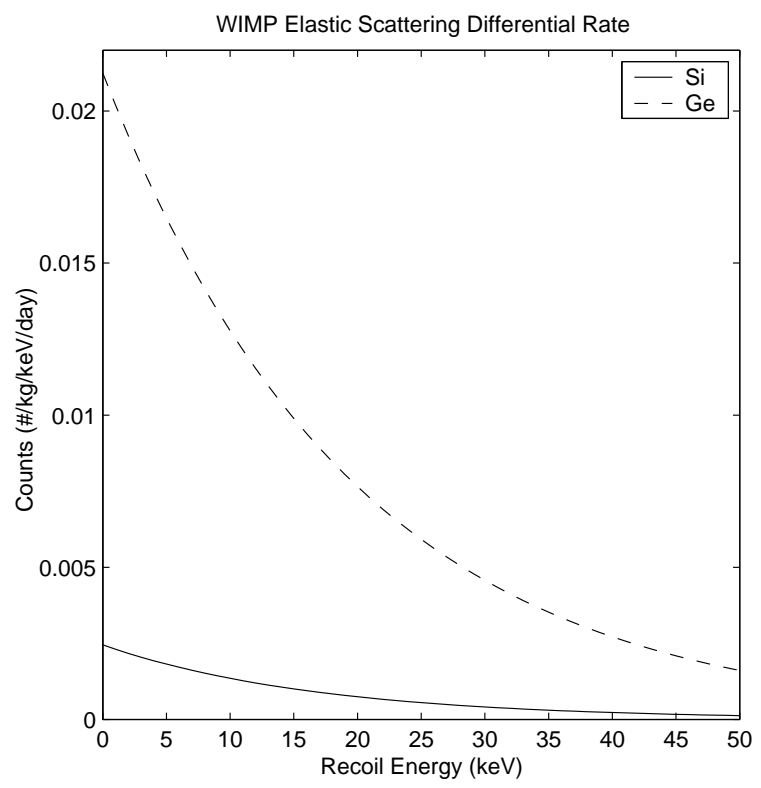

Figure 2.3: The differential event rate of $\mathrm{Si}$ and of Ge for a WIMP-nucleon cross section at $10^{-42} \mathrm{~cm}^{2}$. Suppose $m_{\chi}=100 \mathrm{GeV}$.

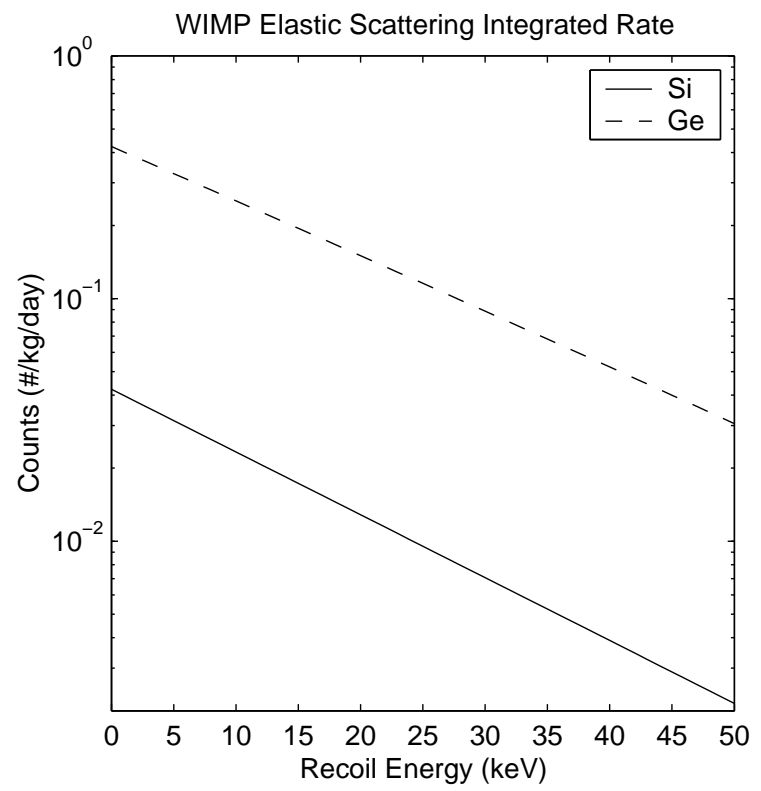

Figure 2.4: The integrated event rate of $\mathrm{Si}$ and of Ge for a WIMP-nucleon cross section at $10^{-42} \mathrm{~cm}^{2}$. Suppose $m_{\chi}=100 \mathrm{GeV}$. X-axis is the recoil energy threshold of the event rate integration. 
At non-zero $v_{E}$ and finite $v_{e s c}$, the differential event rate is

$$
\frac{d R\left(v_{E}, v_{e s c}\right)}{d E_{r}}=\frac{k_{0}}{k_{1}}\left[\frac{d R\left(v_{E}, \infty\right)}{d E_{r}}-\frac{R_{0}}{E_{0} \alpha} e^{-v_{e} s c^{2} / v_{0}^{2}}\right]
$$

\subsection{Signature of WIMP Direct Detection}

The sensitivity of WIMP direct detection experiments is limited by background, mainly gammas, betas and neutrons (muon-induced or from radioactivity), even though the experiments are sited underground. The best strategy is to shield the experiment volume carefully, to use low radioactivity materials, and to use detector technology that rejects the residual background. Also, the signatures of a incoming WIMPs can help experimenters identify them.

\subsubsection{Angular Distribution of Event Rate}

A powerful signature of WIMP direct detection comes from measuring nuclear recoil directions. The Earth's rotation and its motion around the Sun, and the Sun's motion around the galactic disk, lead to energy and direction information. For example, the event rate changes with the detector nucleus recoil angle. Angular sensitivity can provide a key additional tool that might allow unambiguous separation of a signal due to galactic halo WIMPs from other possible backgrounds.

Copi and Krauss [64] provided a formalism that allows a calculation of the expected angular distribution of events in terrestrial detectors with angular sensitivity for a distribution of galactic halo dark matter. They utilized this formalism to examine the expected signature for WIMP dark matter and found that as few as 40 events might be required to disentangle the signal from backgrounds if the 


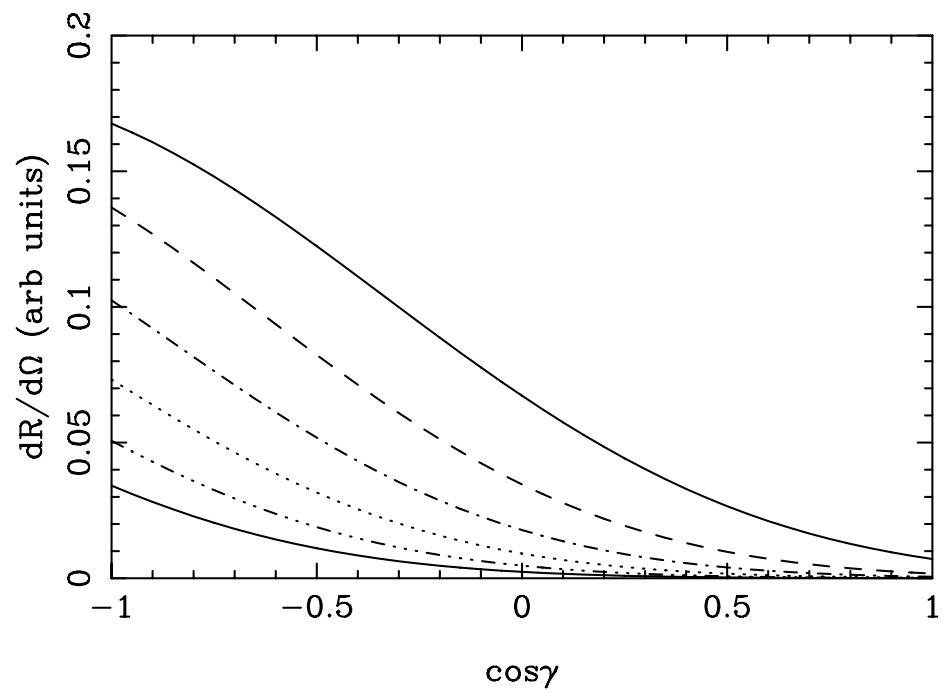

Figure 2.5: The angular distribution of nuclear recoil events $d R / d \Omega$ for an isothermal model with $\phi=0$ and $v_{0}=220 \mathrm{~km} / \mathrm{s}$ as a function $\cos \gamma$ (Z axis points to the Sun) and for various threshold energies. Here the thresholds are (from upper to lower) $E_{t h}=0 \mathrm{keV}$ (the upper solid curve), $2 \mathrm{keV}, 4 \mathrm{keV}, 6 \mathrm{keV}, 8 \mathrm{keV}$, and 10 $\mathrm{keV}$ (lower solid curve). Figure from [64].

WIMP distribution resembles an isothermal sphere.

Copi and Krauss assumed the statistical signal to noise ratio to be 1 . In their study, a ${ }^{73}$ Ge detector target was used, and both spin-independent and spindependent cross sections were included. They chose a $\mathrm{Z}$ axis pointing to the Sun. Figure 2.5 shows the WIMP event rate angular distribution, and Figure 2.6 gives the event number required as a function of the energy threshold for an unambiguous WIMP identification by using the directional information in their formalism.

The DRIFT dark matter search experiment seeks to exploit this directional information. The detector is a low pressure time projection chamber (TPC) with a mixture of target gas (argon or xenon) and an electronegative gas (Carbon Disulfide is used as the electronegative component). The recoil energy and direction 


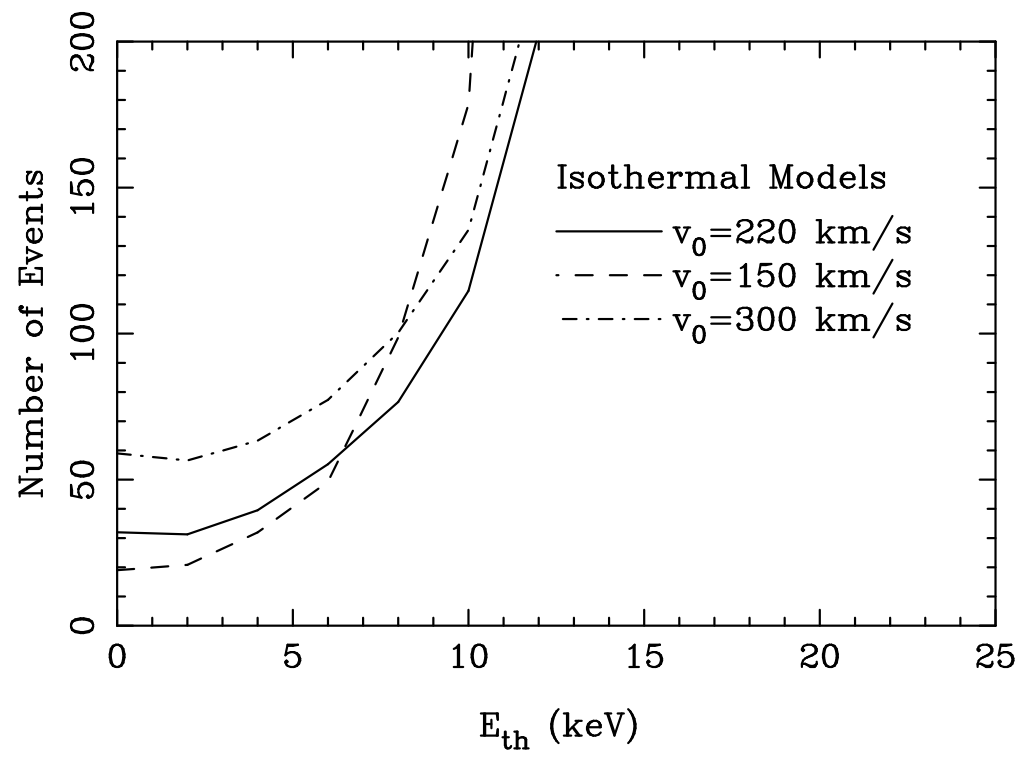

Figure 2.6: Number of signal events required as a function of of threshold energy to distinguish an isothermal distribution of WIMPs in the halo from a flat background. Three values for the dispersion in the WIMP halo, $v_{0}=220 \mathrm{~km} / \mathrm{s}$ (solid line), $v_{0}=150 \mathrm{~km} / \mathrm{s}$ (dashed line), and $v_{0}=300 \mathrm{~km} / \mathrm{s}$ (dahsed dot line) are considered. Figure from [64].

are measured by drifting the ionization created along the recoil track to a suitable readout plane. The recoil track length is a few $\mathrm{mm}$ from the WIMP-nucleus interaction with tens of $\mathrm{keV}$ recoil energy [65], and carries the direction information of the recoiling nucleus.

\subsubsection{Annual Modulation}

Another signature of WIMPs is the annual modulation of the scattering rate off the detector target material nuclei. The annual modulation of the signal rate is induced by the Earth's revolution around the Sun; as a consequence, the Earth is crossed by a larger WIMP flux in June (when its rotational velocity is added to that of the solar system with respect to the galaxy) and by a smaller one in 
December (when the two velocities are subtracted, see Figure 2.7).

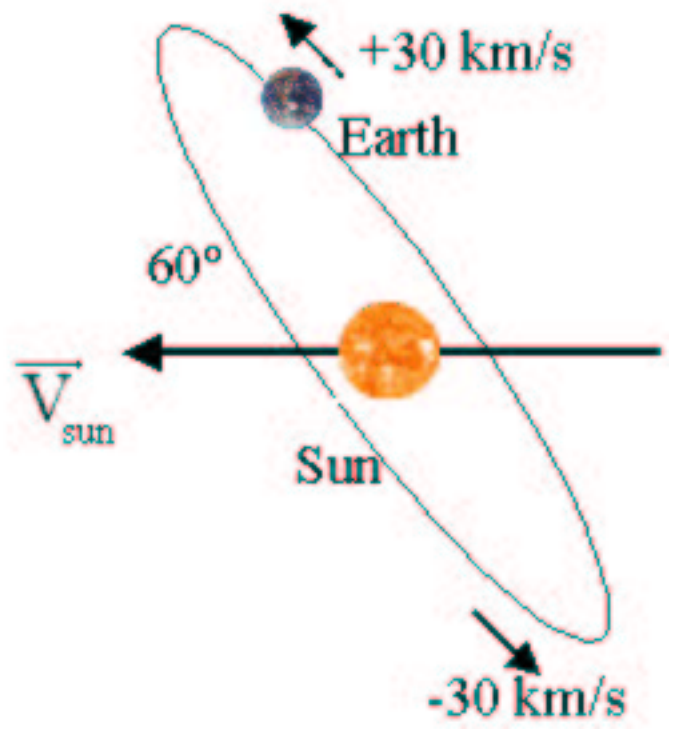

Figure 2.7: Schematic view of the Earth's motion around the Sun. Figure from [66].

In particular, the expected differential rate as a function of the recoil energy $d R / d E_{r}$ depends on the WIMP velocity distribution and on the Earth's velocity in the galactic frame, $\vec{v}_{E}(t)$. Projecting $\vec{v}_{E}(t)$ on the galactic plane, one can write:

$$
v_{e}(t)=v_{\odot}+v_{\oplus} \cos \gamma \cos \omega\left(t-t_{0}\right)
$$

Here $v_{\odot}$ is the Sun's velocity with respect to the galactic halo $\left(v_{\odot} \simeq v_{0}+12 \mathrm{~km} / \mathrm{s}\right.$ and $v_{0}$ is the local velocity whose value is about $\left.230 \mathrm{~km} / \mathrm{s}\right) ; v_{\oplus}=30 \mathrm{~km} / \mathrm{s}$ is the Earth's orbital velocity around the Sun on a plane with inclination $\gamma=60^{\circ}$ with respect to the galactic plane; furthermore, $\omega=2 \pi / \mathrm{T}$ with $\mathrm{T}=1$ year, and roughly $\mathrm{t}_{0} \simeq 2^{\text {nd }}$ June (when the Earth's speed is at maximum). The Earth's velocity can be conveniently expressed in units of $v_{0}: \eta(t)=v_{E}(t) / v_{0}=\eta_{0}+\Delta \eta \cos \omega\left(t-t_{0}\right)$, which depends on the assumed value of the local velocity. $\eta_{0} \approx 1.052$ is the yearly average of $\eta$ and $\Delta \eta \approx 0.065$. Since $\Delta \eta \ll \eta_{0}$, the expected counting rate can be 
expressed by the first order Taylor approximation [66]:

$$
\frac{d R}{d E_{r}}[\eta(t)]=\frac{d R}{d E_{r}}\left[\eta_{0}\right]+\frac{\partial}{\partial \eta}\left(\frac{d R}{d E_{r}}\right)_{\eta=\eta_{0}} \Delta \eta \cos \omega\left(t-t_{0}\right) .
$$

In reference to equations 2.27 and $2.29, \frac{\partial}{\partial \eta}\left(\frac{d R}{d E_{r}}\right)_{\eta=\eta_{0}}$ is close to $\frac{d R}{d E_{r}}$, so there is about $6.5 \%$ annual modulation depth.

When the annual modulation signature is used to test for a WIMP signal, radioactive background modulation over the course of a year should be carefully excluded and variation in the experimental environment should be minimized.

Another signature can be the diurnal modulation of the WIMP detector scattering rate due to the rotation of the Earth. It has the advantage of placing less stringent requirements on the stability of the detector and its associated electronics. But this modulation effect is less than 1\%. Hasenbalg has shown that at least a 20-kg-year data is required to reach the $0.5 \%$ diurnal modulation sensitivity [67], with typical background levels of $2-3$ counts $/ \mathrm{keV} / \mathrm{kg} / \mathrm{day}$.

With limited experimental exposure, the best method for WIMP direct detection is to discriminate the backgrounds. An event-by-event background discrimination method allows us to identify nuclear recoils at high confidence levels. I will describe the CDMS experiment background shield and active veto in chapter 3, and background rejection methods of CDMS ZIP detectors in chapter 7.

\subsection{Indirect detection}

Beyond the direct detection of galactic neutralino dark matter in the laboratory, the search of dark matter neutralinos can be carried out by looking for the products of their annihilation. There are three types of searches, categorized according to the place where neutralino annihilations occur. The first is the case of neutralino 
annihilation in the Sun or the Earth, which gives rise to a signal in high-energy neutrinos. The second is the case of neutralino annihilation in the galactic halo, or in the halo of external galaxies, which generates gamma-rays and other cosmic rays such as positrons and antiprotons; the third is the case of neutralino annihilations around black holes, in particular around the black hole at our galactic center.

All these annihilation signals share the property of being proportional to the square of the neutralino density. This follows from the fact that the neutralino is a Majorana fermion, i.e. is identical to its antiparticle. Two neutralinos can annihilate to produce standard model particles. The annihilation rate is

$$
\Gamma_{\mathrm{ann}}=\frac{\sigma_{\mathrm{ann}} v \rho^{2}}{m^{2}}
$$

where $\Gamma_{\text {ann }}$ is the neutralino annihilation rate per unit volume (i.e. the number of neutralinos that are annihilated per unit volume and unit time), $\sigma_{\mathrm{ann}}$ is the neutralino-neutralino annihilation cross section, $v$ is the relative speed of the two annihilating neutralinos, $\rho$ is the neutralino mass density, and $m$ is the neutralino mass. The annihilation cross section $\sigma_{\text {ann }}$ goes at $1 / v$ at small speeds, as required by kinematical arguments, and thus the product $\sigma_{\mathrm{ann}} v$ does not vanish linearly with $v$. Galaxies and clusters of galaxies are overdense in the dark matter field, as is any possible substructure in galactic halos. Furthermore, dark matter may be concentrated gravitationally around massive objects, and may even get trapped inside planets and stars. The neutralino annihilation rate, proportional to the square of the neutralino density, increases substantially in these dark matter concentrations, possibly to the point of giving observable signals. 


\section{High Energy Neutrinos}

Neutralinos orbiting around the solar system can occasionally collide with nuclei in the Sun and in the Earth. In these collisions, they may lose enough kinetic energy to end up with a speed smaller than the escape speed, thus becoming gravitationally trapped. After some time, the trapped neutralinos will sink to the core of the celestial body in which they are captured, and will possibly reach a condition of thermal equilibrium.

Once concentrated in the center, neutralinos annihilate frequently. The annihilation rate is maximal when all incoming neutralinos annihilate, i.e. an equilibrium between capture and annihilation. Whether this condition is satisfied depends on the relative strength of the annihilation and scattering cross sections, and ultimately on the parameters of the particle and halo models [39].

Of the annihilation products produced in the center of the Earth and the Sun, only the neutrinos make it to the surface; all the other products are absorbed or decay within a short distance of production. All three flavors of neutrinos are produced for neutralino masses that are currently allowed. Direct production of a neutrino pair is, however, strongly suppressed in neutralino annihilation, due to the Majorana nature of the neutralino. Annihilation neutrinos are instead produced as secondaries in the decay chains of the primary particles produced in the neutralino-neutralino annihilation. As a result, the neutrino energy spectrum is a continuum, and the typical energy of neutrinos from neutralino annihilations is about a tenth of the neutralino mass. Given the current constraints, this means a neutrino energy between a few GeVs and a few TeVs.

Neutrinos of this energy can be detected in Cherenkov neutrino telescopes. A charged-current interaction in the material surrounding the detector (typically 
rock, ice, or water) converts the neutrino into its corresponding charged lepton, which then radiates Cherenkov light in the detector medium (ice or water).

Several experiments are potentially able to detect the flux of high energy neutrinos from dark matter annihilations in the solar core. The AMANDA experiment [68] is currently the largest operating neutrino telescope. ANTARES [69] with low energy threshold $(10 \mathrm{GeV})$, and IceCube [70], with a much greater effective area, will each function as dark matter experiments. The Super-Kamiokande detector [71] in Japan gave a WIMP exclusion limit already (see Figure 2.10), and will continue to contribute to the search for dark matter.

\section{Gamma-rays and Cosmic Rays}

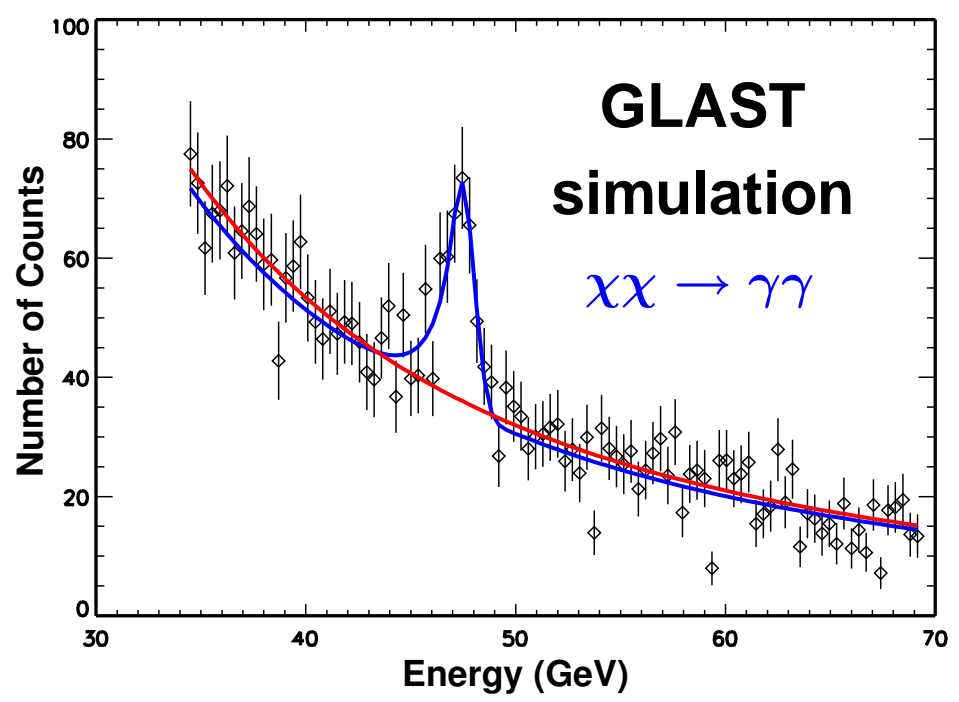

Figure 2.8: Simulation of a gamma-ray annihilation line from the annihilation of $\sim 48 \mathrm{GeV}$ neutralinos, superimposed on a gamma-ray background of astrophysical origin. The simulation includes the finite energy resolution of the upcoming GLAST detector. Figure from [33].

Neutralino annihilations produce signals in the halo of our galaxy or in the 
halo of external galaxies. The annihilation products of importance are those that are either rarely produced in astrophysical environments or otherwise have a peculiar characteristic that make them easily recognizable. In the first category are rare cosmic rays such as positrons, antiprotons, and antideuterons. In the second category are gamma-rays, whose spectrum is expected to contain a gammaray line at an energy corresponding to the neutralino mass. The gamma-ray line is produced directly by the primary neutralino annihilation into $\gamma \gamma$ or $Z \gamma$. Positrons, antiprotons, deuterons, and the gamma continuum are generated in the particle cascades that follow the decay of the primary annihilation products. Their energy spectra are therefore broad, with a typical energy that is only a fraction of the neutralino mass, and a shape whose details depend on the dominant annihilation channels. Two neutralinos can in fact annihilate into a variety of primary products, depending on their masses and compositions: fermion pairs $f \bar{f}$, Higgs boson pairs $H_{i} H_{j}$, gauge boson pairs $W^{+} W^{-}, Z Z$, etc. Good energy resolution is crucial for detecting the neutralino gamma-ray line. The simulation in Figure 2.8 shows that the upcoming GLAST detector should have an adequate energy resolution.

The EGRET (the Energetic Gamma Ray Experiment Telescope) experiment has reported an excess of gamma-rays in the region of the galactic center, where the radiation is well above the gamma ray emission expected from interactions with primary cosmic rays with the interstellar medium [72]. It is intriguing to imagine that such excess emission could be the product of dark matter annihilations near the galactic center. However, it should be noted that some difficulties exist, related to this interpretation. For example, as shown in reference [73], the EGRET source is not exactly coincident with the galactic center. This makes the interpretation of the EGRET signal as dark matter annihilation in a density spike problematic. 


\subsection{Dark Matter Search Experiments}

Besides CDMS experiments, which I will describe in detail later, there are more than 20 experiments aimed at the detection of dark matter that are currently running or in preparation around the world. Most of them use the elastic-scattering technique. For example, germanium is a very pure material and has been used for many years for trying to detect dark matter. In this type of experiment, in order to detect the nuclear recoil energy, physicists measure the ionization produced by collisions with nuclei. In fact, ${ }^{76} \mathrm{Ge}$ ionization detectors have been applied to WIMP searches since 1987 [50]. With the data from these experiments, together with the data from the Heidelberg-Moscow [74] and IGEX experiments [75] located at the Gran Sasso (L'Aquila, Italy) and Canfranc (Huesca, Spain) Underground Laboratories, respectively, physicists were able to exclude a WIMPnucleon cross section larger than about $10^{-5} \mathrm{pb}$ for masses of WIMPs $\sim 100 \mathrm{GeV}$, due to the negative search result. Below are several well known dark matter search experiments.

\subsubsection{DAMA}

The DAMA (DArk MAtter) experiment is one of a number of experiments using $\mathrm{NaI}(\mathrm{Tl})$ crystals as dark matter detectors. With a detector mass of $\sim 100 \mathrm{~kg}$ (consisting of 9 individual crystals) and a total exposure of $\sim 107731$ kg-days, the DAMA experiment has the highest sensitivity of the various $\mathrm{NaI}$ experiments. In addition to background rejection with pulse shape discrimination, the DAMA exposure is sufficient to be sensitive to the WIMP annual modulation signature. Figure 2.9 shows the annual modulation signal that has been observed over a period of seven years. If this signal were interpreted as being caused by galactic halo WIMPs, it 
would correspond to a WIMP mass of from a few tens to several hundred $\mathrm{GeV} / \mathrm{c}^{2}$ with a cross section around $10^{-5} \mathrm{pb}$. As we will see later, under the standard astrophysical, nuclear, and particle physics assumptions, the CDMS experiment excludes the same range of parameter space.

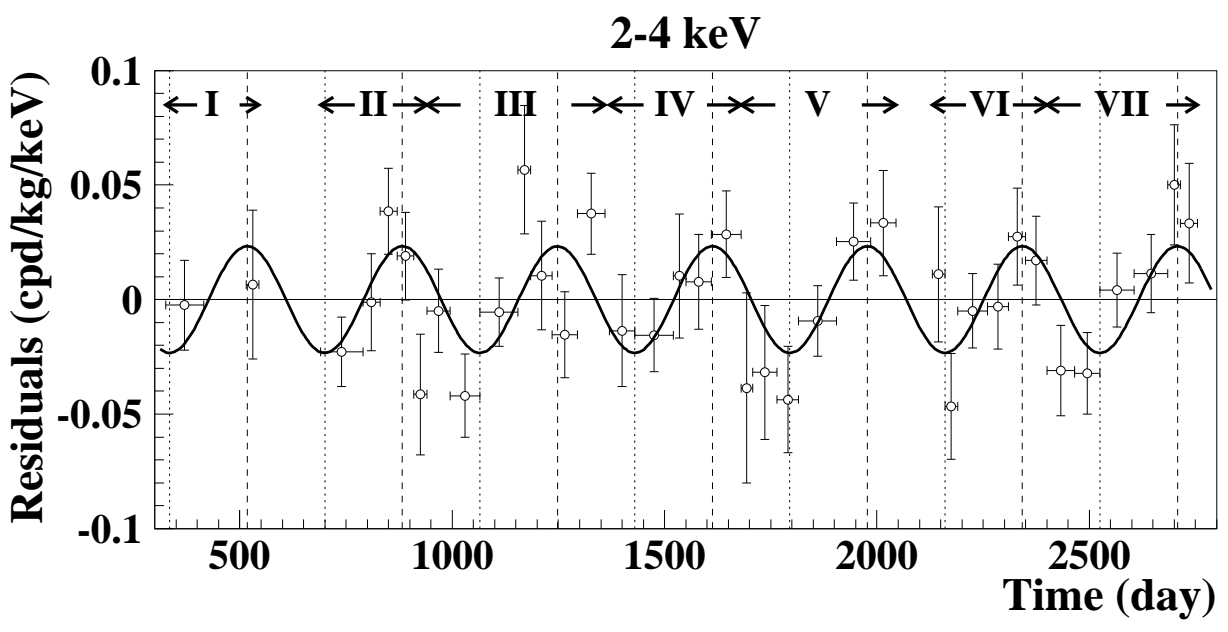

Figure 2.9: Annual modulation of the total counting rate (background plus possible dark matter signal) for seven years of data with the DAMA-NaI detectors. A constant counting rate has been subtracted to give the 'residuals'. The significance of the modulation is $6 \sigma$ and its period is one year. The interpretation of the yearly modulation as being a WIMP signal is controversial. (Figure from [66].)

\subsubsection{EDELWEISS}

Of the various dark datter direct detection experiments, the EDELWEISS experiment most resembles CDMS. The EDELWEISS experiment is based on the measurement of a germanium crystal's ionization and bolometric responses to a recoiling particle. The detectors are cylindrical in shape, $70 \mathrm{~mm}$ in diameter, 20 $\mathrm{mm}$ thick, and weigh $\sim 320 \mathrm{~g}$ each. Electrodes on the top and bottom surfaces are used to measure the number of excited charge carriers created by a recoiling par- 
ticle (ionization response), while NTD thermistors measure the temperature rise ( $\sim$ tens of $\mu K)$ of the entire crystal (bolometric or heat response). The combination of the two channels provides information on the energy as well as the nature of the recoil, allowing the detectors to discriminate between a WIMP signal and the majority of background sources. Operating at a depth of 4800 m.w.e. in the Laboratoire Souterrain de Moudane beneath the French-Italian Alps, the EDELWEISS experiment is well-shielded from undiscriminated background sources that result from the flux of cosmic ray muons at the surface. An $11.7 \mathrm{~kg}$-day exposure with one of the detectors, based on a combination of data taken in the years 2000 and 2002, resulted in no observed WIMP candidate events.

\subsubsection{ZEPLIN}

The ZEPLIN II - IV experiments, currently under construction, implement event by event discrimination by applying an electric field across a liquid Xe target. The electric field suppresses the recombination of the ionized electrons resulting in one prompt and one delayed signal. The relative sizes of the two signals could be a powerful discrimination parameter between electron and nuclear recoils. The ZEPLIN IV detector proposes to instrument up to 1 ton of Xe, allowing for sensitivities to cross-sections down to $10^{-45} \mathrm{~cm}^{2}$.

\subsubsection{Super Kamiokande}

As discussed in section 2.5, other experimental searches for WIMPs are based on the detection of WIMP annihilation products such as high energy cosmic rays, photons, or neutrinos. Such experiments exploit the fact that WIMPs, after having elastically scattered within the cores of the Earth, Sun, or Galactic bulge, 


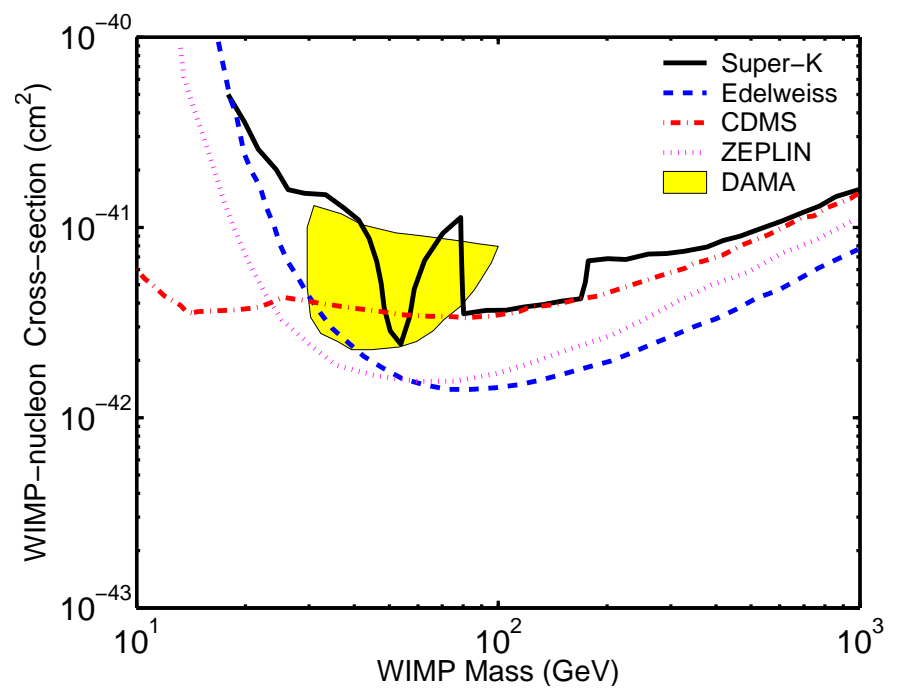

Figure 2.10: Super-K 90 \% CL exclusion region in WIMP parameter space (solid line) for a WIMP with scalar coupling obtained using limits on WIMP-induced muons from the Sun and the Earth. Also shown are the DAMA $3 \sigma$ allowed region (filled) and the $90 \%$ CL exclusion region from CDMS (dot-dashed), EDELWEISS (dashed), and ZEPLIN (dashed). Figure from [71].

may become trapped in their gravitational wells. Such an effect will lead to the accumulation of WIMPs until equilibrium is achieved. This occurs when the rate of infalling WIMPs is balanced by the rate of WIMP annihalation. The SuperKamiokande experiment searched for an excess of energetic, upward going muons resulting from $\nu_{\mu}$ arising from WIMP annihilation. The lack of a muon flux in excess of atmospheric neutrinos allowed the Super-Kamiokande experiment to set the WIMP exclusion limit shown in Figure 2.10, where part of the DAMA region is excluded. This result demonstrates that indirect detection techniques can be competitive and complementary to direct detection experiments in the search for dark matter candidates, WIMPs. 


\section{Chapter 3}

\section{CDMS II in Soudan}

We know from observational cosmology and supersymmetry that WIMPs interact with ordinary matter rarely. The spin-independent WIMP-nucleon cross section can be somewhere in the range from $10^{-5}$ to $10^{-11} \mathrm{pb}$. This means that a WIMP search experiment needs a low background environment for the effective WIMP event count. The surface or shallow site experiment is limited by the muoninduced neutron background, (see section 3.1), so a low natural radioactivity deep underground site has been chosen. The low background in the Soudan deep mine (2090 meters of water equivalent) allows CDMS experiments to explore WIMPnucleon cross section at the $2 \times 10^{-8} \mathrm{pb}$ level [76].

The commissioning of the CDMS II experiment in Soudan started in 2001 with Fermi lab engineers and technicians. Since the beginning of 2002, physicists have taken over the responsibility of the experiment setup, including dilution fridge debugging and tests. The dilution refrigerator reached its base temperature, which was below $30 \mathrm{mK}$, and the ice box was cooled down to a temperature lower than $40 \mathrm{mK}$, for the first time at the end of 2002 in the Soudan mine. Two towers, six germanium detectors, and six silicon detectors were installed in the summer of 
2003. The detector cooling down was in August 2003. After 40 days of electronic noise debugging, detector neutralization, and TES bias tuning, 92.8 live day raw WIMP-search data with Tower I was collected from Oct. 112003 to Jan. 11, 2004 .

This chapter will describe the background of CDMS experiment, the Soudan underground lab, the dilution fridge, the ice box, the active and passive shieldings, signal readout electronics and the data acquisition (DAQ).

\subsection{CDMS II Experiment Backgrounds}

In low background experiments of rare events, such as the CDMS experiment, several factors in the experimental backgrounds can obscure the signal counts of interest. These experiment backgrounds are environmental radioactivity, intrinsic contamination of the detector and shielding material, airborne radioactivity (radon), and cosmic rays induced particles.

CDMS II will detect the WIMP-nucleus interaction at a sensitivity better than 0.01 events $/ \mathrm{kg} /$ day. To reach this goal, background reduction and discrimination are necessary. The CDMS experiment background includes gammas, betas and neutrons from cosmic rays and natural radioactivities. The CDMS detector can discriminate gammas and betas from WIMPs at high efficiency, because WIMPs interact with nuclei; gammas and betas interact with electrons. But neutrons and WIMPs are indistinguishable.

In general, background particle sources can be divided into two categories: muon induced and non-muon induced. Muon-induced particles are produced promptly by a muon interaction in the experimental apparatus or surrounding material. Muons are produced in the Earth's atmosphere mainly from the decay 
of pions and kaons produced by primary cosmic rays [77]. The flux of muons at the earth's surface is about 170 muons $/ \mathrm{m}^{2} / \mathrm{sec}$ [78], with an average energy of about $4 \mathrm{GeV}$ [77]. While muons are propagating through the earth, they lose their energy by radiative processes and ionization.

Above several hundred-GeV, muon interactions are dominated by radiative processes. These radiative processes produce energetic electromagnetic and hadronic particles and showers [79]:

- Bremsstrahlung photons due to the muon's acceleration by the Coulomb fields of nuclei as it passes.

- Electron-positron pairs due to the high energy virtual photon from muons.

- Neutrons or hadrons in the processes of photonuclear reactions of the high energy real bremsstrahlung photon from muons.

- Energetic electrons - $\delta$-ray from the Coulomb scattering interaction of muons with electrons.

Below several hundred $\mathrm{GeV}$, the dominant energy loss mechanism of muons is by ionization of atoms. Once a muon has slowed down sufficiently, it may be captured by an atom, taking up residence in a Bohr orbital and quickly decaying down to the 1s level. The muon may be captured by the nucleus with neutron emission:

$$
\mu^{-}+p \rightarrow n+\nu_{\mu}
$$

Muon induced neutron production processes take place in the surrounding rock and the shielding material. Neutrons produced by these mechanisms in the shielding material can be rejected using the muon veto. Since no vetoeing is 
available for neutrons produced by muons in the surrounding rock, it is important to shield the detectors from these neutrons. Generally, muon induced neutrons have relative high energy (above several $\mathrm{MeV}$ ). Polyethylene's moderation is not as efficient for high energy neutrons as for low energy neutrons from the radioactivity in the surrounding rock.

Non-muon induced particles are from radioactive decay of unstable isotopes. For example, ${ }^{232} \mathrm{Th},{ }^{238} \mathrm{U}$, and ${ }^{40} \mathrm{~K}$ are radioactive isotopes with large naturally occurring abundance and long-lived decay products. Uranium and thorium possess complex decay chains that include alpha, beta, and gamma emissions. Potassium is a naturally occurring radioisotope, decaying via electron emission or electron capture, the latter accompanied by a high-energy photon. Radioisotopes of these three elements are present in metallic construction materials and rock. Other radioisotopes include ${ }^{222} \mathrm{Rn},{ }^{210} \mathrm{~Pb},{ }^{14} \mathrm{C},{ }^{7} \mathrm{Be},{ }^{22} \mathrm{Na},{ }^{60} \mathrm{Co},{ }^{68} \mathrm{Ge},{ }^{3} \mathrm{H}$, and ${ }^{63} \mathrm{Ni}$ from radioactive decay or from cosmogenic activation.

Of these radioisotopes, radon, which is a daughter of ${ }^{238} \mathrm{U}$, has been recognized as a potentially significant background to the CDMS detectors. About $6 \times 10^{8}$ ${ }^{222} \mathrm{Rn}$ atoms are released from the earth's surface per square-meter per day [80], and these radon atoms become air-borne. The radon level is higher in deep mines than on the surface. ${ }^{222} \mathrm{Rn}$ has a half-life of 3.8 days. This period of time is long enough to permit the radon to permeate many of the materials surrounding the CDMS detectors and to migrate to sensitive regions. The daughters of radon can produce alpha, beta and gamma particles with energies directly applicable to the range of interest for the CDMS experiment. Exposure of radon to the detectors may occur in three possible ways:

- production via $\mathrm{U} / \mathrm{Th}$ contamination 
- migration/permeation

- outgassing

Primordial radionuclides in ores and other raw materials results in a wide range of contamination in the final product. Material selection in experiment construction is important. There are relatively radio-pure materials available, such as copper and plastics. Copper is routinely purified through repeated electrolytic dissolution and redeposition; redeposition of uranium, thorium, and potassium is chemically disfavored. Plastics tend to be quite low in such radioisotopes because they are produced from natural gas. Possible contaminants would be ${ }^{3} \mathrm{H}$ and ${ }^{14} \mathrm{C}$ produced through nuclear spallation by cosmic rays. However, natural gas is mined from very old petroleum deposits, so such isotopes are expected to have decayed away. Germanium and silicon also tend to be quite clean because of the extensive purification performed during the production of detector grade crystals.

Other radioactive isotopes are created by cosmic-ray spallation. Of particular interest are $\mathrm{Mn}, \mathrm{Fe}$, and Co isotopes created in copper and Ge isotopes created in the detectors. Stock material for the cryostat and detectors is stored underground to minimize creation of such isotopes, but it is impossible to prevent radioisotope production completely because of the need for some surface exposure during processing or machining. There are no fission or $\alpha$-decay modes in these lower-Z radioisotopes, so they only give rise to the electromagnetic background.

Photon backgrounds can be attenuated effectively by high-Z materials such as lead. The CDMS detectors are surrounded by both Pb (two layers of lead, $4 \mathrm{~cm}$ ancient lead with low ${ }^{210} \mathrm{~Pb}$ after $22.5 \mathrm{~cm}$ lead) and $\mathrm{Cu}$, which serve as gamma attenuators. The ${ }^{238} \mathrm{U},{ }^{232} \mathrm{Th}$, and ${ }^{40} \mathrm{~K}$ activities in these materials are low because these isotopes and their daughters are removed efficiently during the 
smelting and refining processes.

A small amount of neutrons is produced by fission and $(\alpha, \mathrm{n})$ reactions, with the $\alpha$ 's coming from radioactive decays. Neutrons produced by fission and $(\alpha, \mathrm{n})$ reactions are easily moderated by the polyethylene shielding between the outer shield and the cryostat. Cryostat construction materials and the inner lead shield, which lie inside the polyethylene shielding, are not significant neutron sources because of their intrinsically low uranium and thorium (and daughter) levels. Neutrons from natural radioactivity have a very soft spectrum, with a steep falloff above $2 \mathrm{MeV}$, so they can easily be moderated by polyethylene shielding [80].

With external backgrounds well understood, the detectors themselves and the materials immediately surrounding them are the primary concerns. These internal backgrounds are due to the accumulation of radionucleides on the surface of the detectors, in the bulk contamination of the detectors, and in the inner volume of the experiment. The method by which the detector crystals are grown precludes the majority of backgrounds that do not arise from isotopes of Ge or Si. However, cosmogenically created radioisotopes will begin to accumulate within the bulk of the crystals during periods when the detectors are exposed to the high cosmic ray flux above ground. Some such background may even be continuously produced by periodic neutron calibrations [81].

${ }^{222} \mathrm{Rn}$ can contaminate the experiment inner volume and the surfaces of the detectors. The short lived ${ }^{222} \mathrm{Rn}$ leads to the long lived ${ }^{210} \mathrm{~Pb}$ via intermediate daughters within about 4 days. Several of these decays involve a high energy particle, which could result in the recoiling nucleus being implanted in the detector with depths of up to several $\mu \mathrm{m} .{ }^{210} \mathrm{~Pb}$ has a half-life of 22 years, long enough to provide a nearly constant background throughout the experiment. ${ }^{210} \mathrm{~Pb}$ also undergoes beta decay, with an energy mean of $6.2 \mathrm{keV}$, and an end-point of $63.5 \mathrm{keV}$. 
To prevent radon contamination, CDMS detectors are purged with nitrogen gas from liquid nitrogen when the detectors are in storage; an air-tight can filled with pure nitrogen gas is used when the detectors are in the process of transportation.

${ }^{14} C$ contamination comes from detector processing or handling. ${ }^{14} C$ undergoes beta decay, resulting in a spectrum with an energy mean of $50.1 \mathrm{keV}$, and an end-point at $156 \mathrm{keV}$. Because ${ }^{14} \mathrm{C}$ is long lived (a half-life of 5730 years), its contamination provides a constant background source throughout the experiment.

Another long-lived contamination isotope is ${ }^{40} \mathrm{~K}$ from detector procession or handling. Its dominant decay mode is beta decay with an end-point energy of $1.46 \mathrm{MeV}$. While energy deposition of this magnitude is outside the interesting WIMP search range, the betas have enough energy to produce an electron positron pair and could lead to secondary particles, such as betas and gammas, with sufficiently low energy to fall within the region of interest. Potassium is present in human beings in large amounts. Potassium is transferred for example through fingerprints and perspiration. The use of gloves and other cleanroom wear during cleaning steps and the handling of cleaned materials are necessary to prevent the deposition of potassium.

The common feature of these three radioisotopes $\left({ }^{210} \mathrm{~Pb},{ }^{14} \mathrm{C}\right.$ and $\left.{ }^{40} \mathrm{~K}\right)$ is the production of low energy betas, a background that results in "surface" electron recoils; that is, electron recoils within the first few microns of the detector surface. All of these backgrounds are sufficiently long lived that they will not decay on the time scale of the experiment.

Interactions between the hadronic component of cosmic-rays and Ge results in the creation of ${ }^{68} \mathrm{Ge}$ and ${ }^{65} \mathrm{Zn}$ isotopes. The decay chains of these isotopes result in low energy gammas ( about $10 \mathrm{keV}$ ). Electron recoil discrimination remains sufficiently high at that energy to reduce the possibility of such backgrounds being 
mistaken for nuclear recoils.

Interaction of neutrons (both thermal and high energy) with the Ge detectors result in the creation of ${ }^{71} \mathrm{Ge}$ and ${ }^{73 m} \mathrm{Ge}$ isotopes. The sources of the neutrons are muon interactions during the storage and transportation of the detectors, and the ${ }^{252} \mathrm{Cf}$ neutron calibrations to be discussed later. The muon induced neutrons, however, will be suppressed by several orders of magnitude at the Soudan deep site. These Ge isotopes result in $10 \mathrm{keV}$ x-rays and $67 \mathrm{keV}$ gammas.

The CDMS II experiment at the Stanford Underground Facility (SUF) was done in 2001 and 2002. SUF is located at a depth of 10.6 meter. Using cosmic ray intensities, this location was determined to be at 16 meters of water equivalent. At $3 \mathrm{~V}$ charge bias, 93 days of low background data were taken from December 2001 to April 2002, resulting in 65.8 live day and $4.6 \times 10^{6}$ events. After cuts, this became 28.3 kg-day data, with 20 single scattering nuclear recoil candidates in germanium detectors and 2 in silicon detectors. Also, there are 2 triple scattering nuclear recoil events and 1 non neighbor double nuclear recoil event. With Monte Carlo simulation, it was confirmed that most or all of the 20 nuclear recoils in germanium detectors are neutrons. The conclusion was that the shallow site CDMS experiment was limited by the muon induced neutron background [82]. The CDMS experiment needed to move to a deep site.

\subsection{CDMS II Background in Soudan Mine}

The Soudan mine is $780 \mathrm{~m}$ underground, which is a depth of 2090 meters of water equivalent. The muon flux reduced by 10000 times compared to the Stanford Underground Facility (16 meters of water equivalent). (See figure 3.1.) This reduces the muon flux to $1.8 \times 10^{-3}$ muons $/ \mathrm{m}^{2} / \mathrm{s}$. The muon induced neutron 
rate is below $1 \times 10^{-4}$ events $/ \mathrm{keV} / \mathrm{kg} /$ day [80].

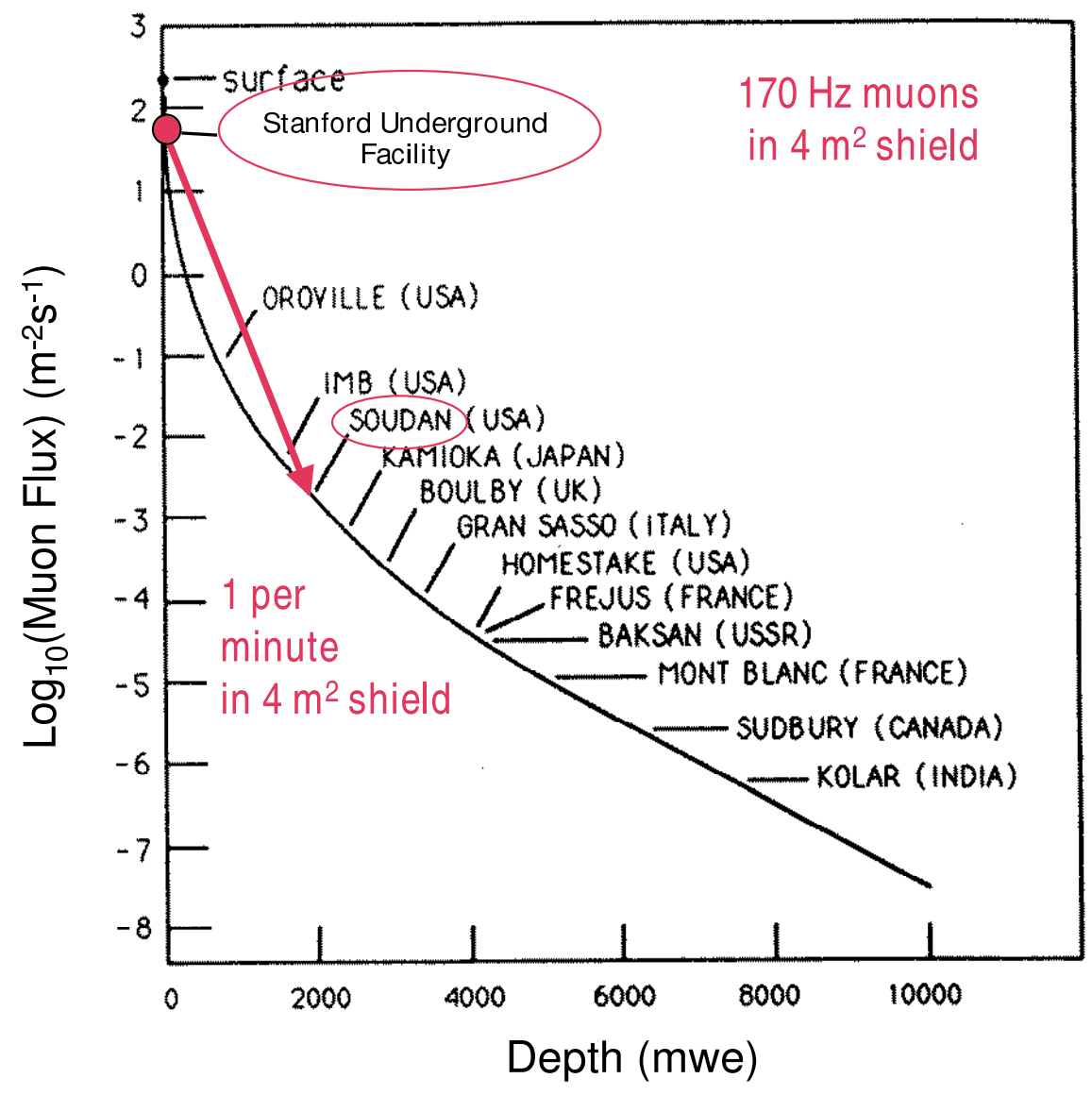

Figure 3.1: Muon rate at Soudan.

The strategy for achieving an extremely low background is insulating the detectors from the natural environmental radioactivity to a level that allows the CDMS dark matter search experiment to be highly sensitive to WIMP recoils. Active muon veto paddles and passive shielding have been installed. See figure 3.3 in section 3.3. Any veto coincident event in the detectors within the $50 \mu$ s scale will be excluded from the data. The muon veto efficiency is better than $99.9 \%$. 
$50 \mathrm{~cm}$ of Polyethylene moderates neutrons to lower energies; $10 \mathrm{~cm}$ of additional polyethylene inside the lead further moderates contained neutrons. $22.5 \mathrm{~cm}$ lead reduces $\gamma$ rays from radioactivity, $4 \mathrm{~cm}$ inner "old" lead (low in ${ }^{210} \mathrm{~Pb}$ ) attenuates gamma rays from ${ }^{210} \mathrm{~Pb}$ in the outer lead, and $3 \mathrm{~cm}$ radioactively clean copper is next to the towers, providing additional gamma attenuation.

With the mechanism for shielding external backgrounds well established, minimizing the amount of radiation originating within the shield is important. The dilution refrigerator and the ice box are in a class 10000 clean room. The scientists come to an ante room before going to the experiment space. They suit up with clean room clothes and booties, and wear double-pair gloves, face masks and caps. These protocols ensure that there is a low background in the CDMS experimental space.

Throughout the construction of the Soudan low background experiment, we have been extremely careful in our choice of materials. One serious limitation is that we cannot use any stainless stell near our detector package because of the presence of ${ }^{60} \mathrm{Co}$ used as a thickness monitor in iron smelting furnaces. We use a dilution refrigerator to cool our detectors to $\sim 40 \mathrm{mK}$, and we cannot avoid some use of stainless steel. As can be seen in Figure 3.3 in section 3.3, we isolate our commerically-purchased dilution refrigerator from a custom made experimental chamber, called the "ice box". The ice box is made primarily of high-purity copper, which can be made electrochemically to contain few radioactive isotopes.

The ice box is purged with old air throughout the experiment, to prevent radioactive radon accumulation in the area close to the ice box.

At the Soudan deep site, one expects a lower background event rate (as compared to that at the surface). These events fall into several classes: 


\section{Gammas}

- Gammas originate within the rock of the Soudan mine from radionucleides of the ${ }^{238} U$ and ${ }^{232} T h$ decay chains as well as from ${ }^{40} K$. Lead and copper shieldings attenuate these gammas. And CDMS detectors reject these gammas that come from natural radioactivity.

- $10.36 \mathrm{keV}$ gammas from ${ }^{68} \mathrm{Ga}$ and $11.4 \mathrm{keV}$ gammas from ${ }^{71} \mathrm{Ge}$. They come from cosmogenic activity, and are rejected as background by CDMS detectors.

2. Betas

- Energetic gammas can knock off electrons from copper or neighbor detectors. These electrons are called "ejectrons". Ejectrons are rejected as surface events by CDMS detectors. The ejectrons from neighbor detectors are tagged as multiple scattering events. And ejectrons from copper are excluded in the low background data after the charge outer electrode cut.

- Radionucleides such as ${ }^{222} \mathrm{Rn},{ }^{210} \mathrm{~Pb},{ }^{40} \mathrm{~K},{ }^{63} \mathrm{Ni}$, and ${ }^{14} \mathrm{C}$ are beta emitters. Air-borne radon is the main beta source in the Soudan mine, though the old air purge suppresses the radon level quite a bit. Electrons from radioactivity are rejected as surface events by CDMS detectors.

\section{Neutrons}

- Neutron production from natural radioactivity in the rock are expected to produce 0.005 neutrons $/ \mathrm{kg} /$ day. These neutrons have an energy 
spectrum that is predominantly below $2 \mathrm{MeV}$. They have a large cross section on protons. The flux of such neutrons is attenuated by approximately $1 \times 10^{6}$ by the 50 -cm thick polyethylene shield surrounding the detectors. This makes the contribution from this background negligible for the entire CDMS II exposure. The materials chosen for the detector volume and the inner shielding have all been screened and carefully handled to minimize the levels of residual radioactivity that might yield neutrons, mainly from $\mathrm{U} / \mathrm{Th}$ fissions or (alpha,n) reactions.

- Muon induced neutrons inside the shield and in the detectors have an energy spectrum between a few Mev and tens of MeV. An active plastic scintillator is used to identify and veto muons or muon related events. Such a veto system is crucial in eliminating the flux of gammas and neutrons due to muon interactions with the shield material. And the shield of the CDMS experiment includes passive layers of lead and copper to shield the flux in addition to polyethylene used to moderate the neutrons as shown in various $\mathrm{Pb}$ and polyethylene layers in figure 3.3. Such a shielding configuration is expected to reduce the neutron rates to 0.003 events $/ \mathrm{keV} / \mathrm{kg} /$ day within the recoil energy range $10-100 \mathrm{keV}$, which is typical of WIMPs.

- Muon induced neutrons outside the shield can be divided into three categories: slow (thermal) neutrons with energies below $1 \mathrm{MeV}$, fast neutrons $(E \sim f e w M e V)$, and high neutrons $(E>$ tens of $\mathrm{MeV})$. High neutrons have a small cross section on protons, so the polyethylene is less effective at stopping them. These neutrons could eventually set a limit on the sensitivity of the CDMS experiment at Soudan. Extensive 
studies have been done for the energy spectrum and event rate of this class of neutrons. The precision Monte Carlo simulations will help us to get this neutron background energy spectrum. And also, there are two powerful tools that can be used for this neutron background rejection. WIMPs can only scatter off nuclei in one detector, but neutrons can scatter off nuclei in several detectors. Secondly, WIMPs have a higher event rate in germanium detectors than in silicon detectors, while neutrons have higher event rate in silicon detectors than in germanium detectors. This neutron background can be excluded by using a multiple nuclear recoil event rate and nuclear recoil event ratio between germanium detectors and silicon detectors

The expected muon-induced external neutron backgrounds have been estimated from Monte Carlo simulations with uncertainty of a factor of a few. The muon-induced neutron single scattering rate is $1 \times 10^{-4}$ events $/ \mathrm{keV} / \mathrm{kg} /$ day at $20 \mathrm{keV}$ in the germanium detector, and $2 \times 10^{-4}$ events $/ \mathrm{keV} / \mathrm{kg} /$ day at $20 \mathrm{keV}$ in the silicon detector [83]. The coadded neutron event rate in the energy from $5 \mathrm{keV}$ to $100 \mathrm{keV}$ is 0.0053 events $/ \mathrm{kg} /$ day in germanium detectors, and 0.0112 events $/ \mathrm{kg} /$ day in silicon detectors [83].

With the ZIP detector's active rejection capability applies gammas and betas, the limiting external background is neutrons. The low energy neutrons from natural radioactivity are moderated with the polyethylene shield. The muon-induced internal neutrons are tagged as muon-coincident events. The muon-induced high energy neutrons will eventually set a limit for the CDMS experiment. But this limit can be lowered signicantly with the signature of neutrons, as described above: the multiple nuclear recoil event rate, the nuclear recoil event ratio between ger- 


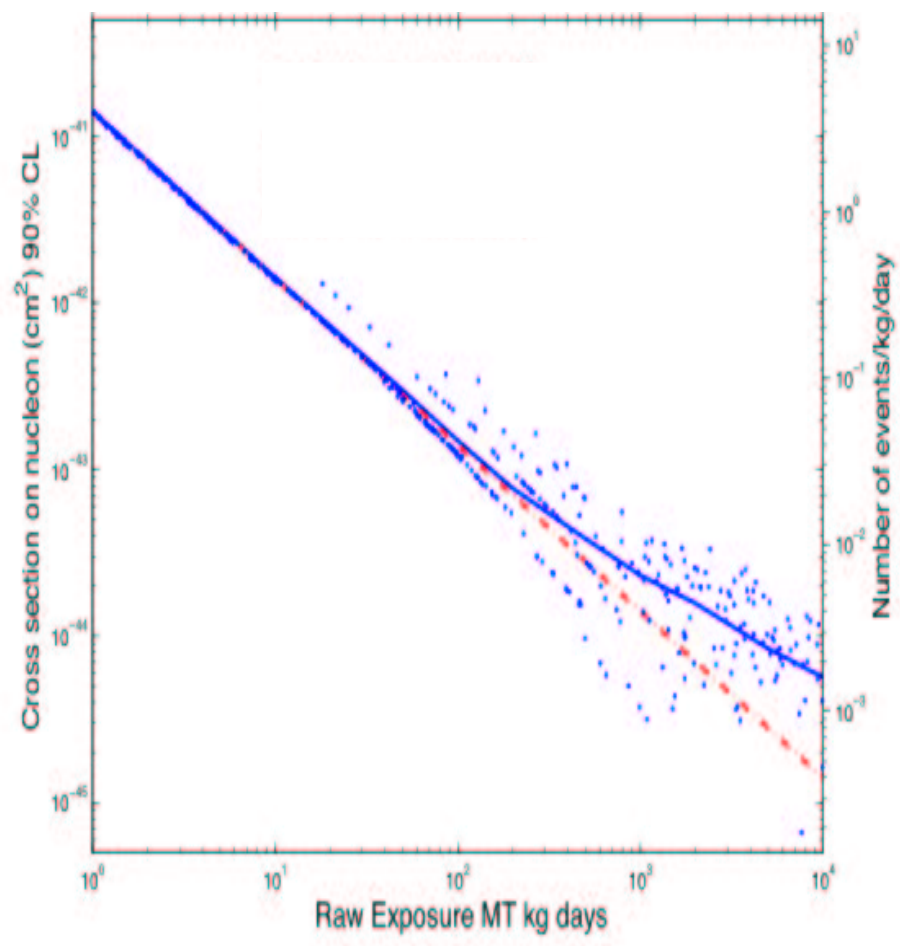

Figure 3.2: Limiting sensitivity of WIMP-nucleon cross section as a function of raw exposure in kg-day. The dashed red line is the case of background free, the blue solid line is after background subtraction. Blue points illustrate random fluctuation from experiment to experiment. Figure from [76].

manium detectors and silicon detector, as well as the energy spectrum of neutrons from Monte Carlo simulation. Figure 3.2 shows the CDMS experiment expected sensitivity as a function of raw exposure.

\subsection{The Dilution Unit and Ice Box}

The dilution refrigerator, ice box, shielding and veto are shown in figure 3.3. An Oxford Kelvinox 400 dilution refrigerator provides the cooling necessary to bring the detectors and their surrounding experimental volumes down to a temperature 
of $40 \mathrm{mK}$. The cooling power of the refrigerator, nominally $0.4 \mathrm{~mW}$ at $100 \mathrm{mK}$, decreases quadratically with temperature. The power budget is dominated primarily by black body radiation from the $600 \mathrm{mK}$ thermal shields surrounding the detector volume and the infrared radiation leakage at warmer stages. The main power dissipation on the fridge come from heat sinking the FETs at $4 K$. The FET power load of $48 \mathrm{~mW}$, combined with thermal conduction along the electronic readout wiring (from room temperature to $4 K$ ) and thermal radiation, results in the consumption of 150 liters of liquid ${ }^{4} \mathrm{He}$ per day. The CDMS refrigerator is modified from the standard design. The thermal and mechanical connection between the mixing chamber and the experimental volume is accomplished by a horizontal cold finger (at 90 degrees to the mixing chamber) about $2 \mathrm{~m}$ long. The advantage of this configuration is apparent: the material from which dilution refrigerator is constructed cannot conform to the strict radioactivity cleanliness limits required by the experiment will be kept away from the ice box. The disadvantage is that the temperature of the experiment volume will be slightly higher than the base temperature of the dilution refrigerator. There are even multistage thermal shieldings to minimize the thermal load to the ice box.

The detectors are housed in the ice box. The ice box consists of 6 cans made of OFHC copper, and heat sunk to the $40 m K, 50 m K, 600 m K, 4 K$ (LHe), $77 K$ (LN), and room temperature stages, respectively. This provides the mechanical support (designed to account for the differential contraction at all temperatures) and the necessary heat sinking to the refrigerator. The innermost can of the ice box consists of a volume of about one cubic ft and is designed to hold a payload of 42 ZIP detectors in 7 towers.

The tower in Figure 3.4 is the mechanical structure holding the detectors together and anchoring them to the various thermal levels $(40 \mathrm{mK}, 50 \mathrm{mK}, 600 \mathrm{mK}$, 


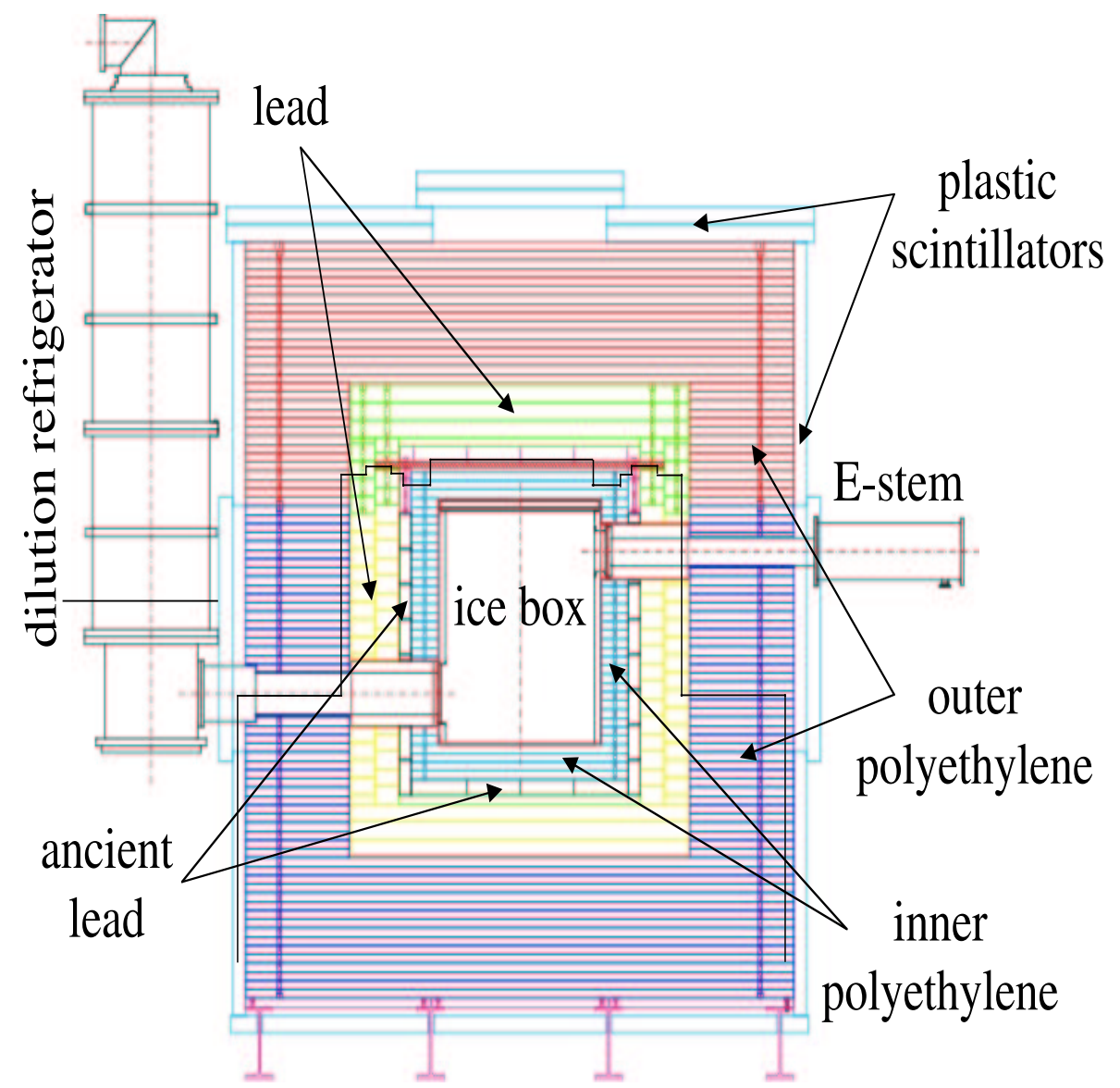

Figure 3.3: The dilution refrigerator and ice box alignment.

$4 K)$ within the ice box. The detectors are at the lowest temperature layer $(40 \mathrm{mK})$, which is thermally isolated by graphite tubes. The electrical connections are made via superconducting $\mathrm{NbTi}$ wires, which were soldered with a custommade ancient-lead (low-radioactivity) solder. These wires are packaged in a series of vacuum coaxes that run down one of the six hexagonal faces to each of the six detectors in the stack, heat sunk at each thermal layer.

Though the primary function of the tower is to provide mechanical support 


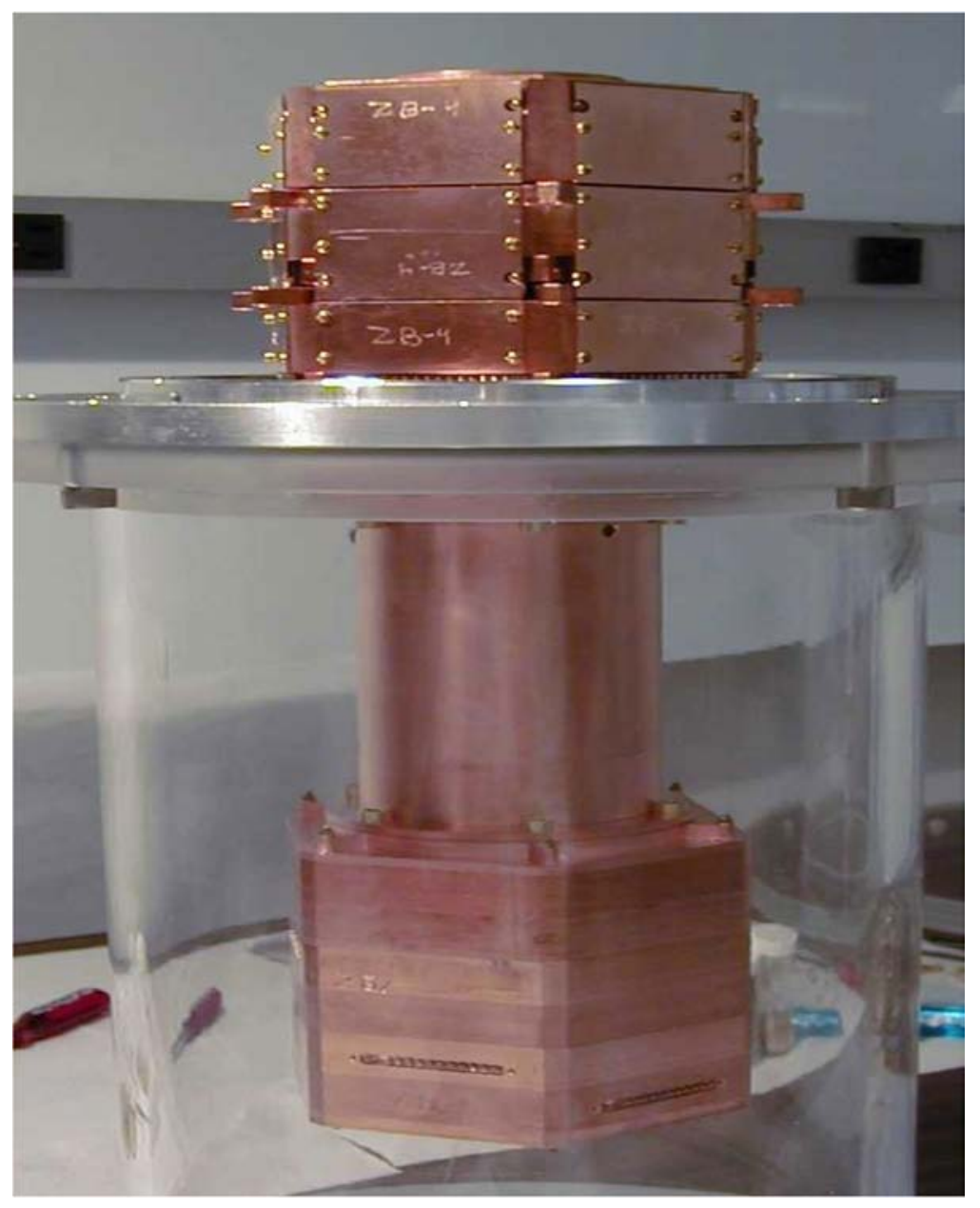

Figure 3.4: The tower that houses six CDMS ZIP detectors.

for the detectors, cryogenic electronics, and wiring, it also serves the important function of thermally isolating the various layers of the ice box while being mechanically connected to them. This is necessary because the heat dissipation and thermal noise requirements of the various cryogenic electronic components can not be satisfied by heat sinking them to a single thermal stage. The optimal noise performance of the FETs is obtained by operating them at a temperature of about $130 \mathrm{~K}$. This was accomplished by isolating them on a membrane with the appro- 
priate thermal conductance to allow the FETs to self heat to that temperature. The $4 \mathrm{~K}$ thermal stage is the only one capable of sinking the tens of $\mathrm{mW}$ of power dissipated by the FETs. SQUIDs, on the other hand, dissipate only a few hundred $\mathrm{nW}$ and can thus be heat sunk to the $600 \mathrm{mK}$ stage, resulting in lower SQUID noise.

In the CDMS experiment, the term "tower" refers to the functional unit of six ZIP detectors described above, and arranged vertically as shown in figure 3.5. Detectors within the tower are numbered from one to six in descending vertical order, and will be referred to by their number, such as Z\#, throughout this thesis, when a particular detector needs to be identified. The detailed configuration information of Tower I is in Table 3.1.

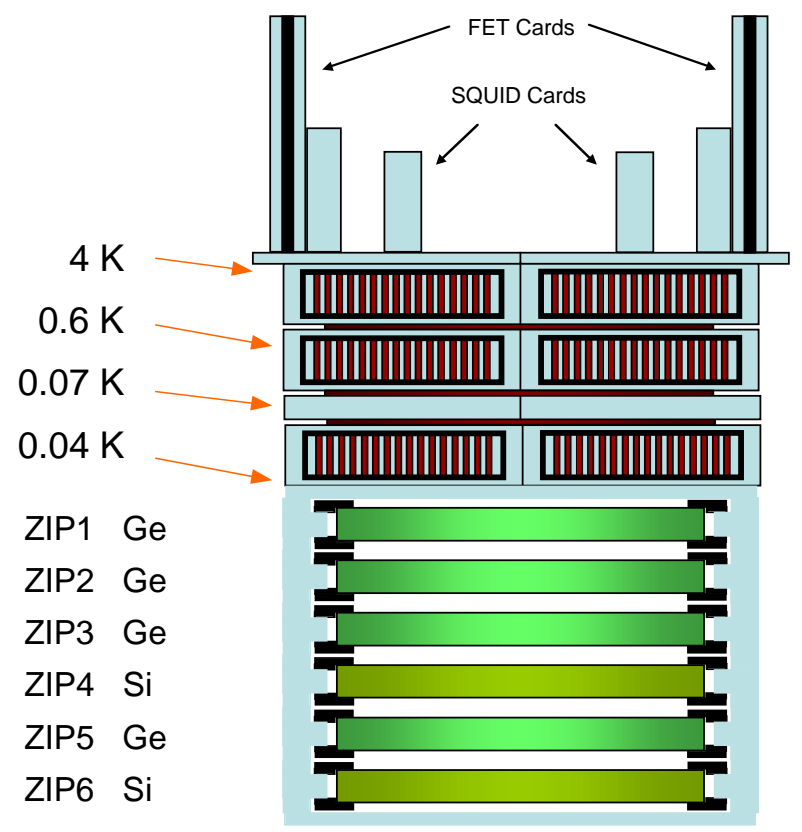

Figure 3.5: The FET card, SQUID card and the detectors. 
The electric connections to outside stages are realized through the FET card thermally sunk at the $4 \mathrm{~K}$ stage. The SQUID card at $600 \mathrm{mK}$ is connected to the FET card with superconducting wire flyovers for the purpose of thermal isolations. The electric connection wires to the detectors are anchored in the side coax, and each detector is likewise connected to the FET card through the side coax.

\begin{tabular}{|c|c|c|c|}
\hline Stack Position & Detector Name & Side Coax & SQUET \\
Z1 & G6 & ZB1-6 & ZB11 \\
Z2 & G11 & ZB2-4 & ZB8 \\
Z3 & G8 & ZB3-1 & ZB9 \\
Z4 & S3 & ZB4-3 & ZB4 \\
Z5 & G9 & ZB5-1 & ZB5 \\
Z6 & S1 & ZB6-3 & ZB6 \\
\hline
\end{tabular}

Table 3.1: Tower 1 stack configuration.

As in all other precision experiments, a large amount of time and man power have been put into noise debugging in electronics and leakage debugging in the cryogenic system. Dielectric breaks on all cryo lines going through the RF wall were installed to reduce the environmental electromagnetic noise coupled to the detector signal readout electronics. In 2002, the LHe bath to the IVC leak and mixing chamber to IVC leak gave us a lot trouble. During the summer of 2003, we battled ebox (housing the room temperature end of the electrical stripelines) air leaks. We needed to deal with the LHe bath to OVC leak and watch out for the berp throughout Soudan Run118. We are very thankful for the cooperation of the dilution refrigerator, allowing us to keep collecting WIMP-search data in the Soudan mine. 


\subsection{Readout Electronics and DAQ}

The data acquisition system (DAQ) and readout electronics are an important part of the CDMS experiment. The readout electronics fall into 2 major categories: room temperature (warm) and cryogenic (cold). Cold electronics refer to the SQUID and FET circuits that are located inside the fridge and are operated at cryogenic temperatures. The cold electronics will be described in the detector chapter. The warm electronics are described in the following sections.

\subsubsection{Front End Electronics}

The electronics chain can best be understood by following the path of the analog signals from the detectors to the digital information stored on hard drives. Signals from the cold electronics are transmitted outside the refrigerator via strip lines. These are about $3 \mathrm{~m}$ long, $2.5 \mathrm{~cm}$ wide strips of kapton on which a thin copper layer (0.018 mm thick) has been etched to form 50 distinct conductors. This design was adopted to minimize the heat load on the $4 \mathrm{~K}$ stage since the strip lines, by necessity, connect the $4 \mathrm{~K}$ stage to the room temperature area. The room temperature end of a strip line is connected to an electronics board known as the Front End Board (FEB). The FEBs are the room temperature portion of the detector readout circuits. They contain the feedback amplifiers, which dissipate too much power to be installed inside the fridge. The FEBs are robust enough to be well separated from the detectors and cold portions of the circuit, in terms of noise

and impedance considerations. An FEB contains all the electronics required to read out and operate a single detector, namely four phonon channels and two ionization channels. The FEB controls the LED flashing for detector neutralization. It also contains various control electronics, such as digital to analog converters 
(DACs) that supply the bias currents and voltages, amplifiers for providing further gain stages, and digital logic circuits to allow remote communication with and control of the boards and detectors. The detector signals, which are amplified upon reaching the $\mathrm{FEB}$, are large enough $(0.5 \mathrm{~V})$ that they're unlikely to suffer degradation while being transmitted over cables for a distance of about $15 \mathrm{~m}$. In other words, any further noise that might be acquired will be smaller that the amplified, resolution limited noise determined by the cryogenic components of the circuit. Leaving the FEBs, the signals are transmitted to Receiver-Trigger-Filter (RTF) boards.

RTF boards receive and filter signals for the digitizers. RTF boards determine whether there exists a pulse of sufficient amplitude to issue a trigger. The RTFs are programmable, allowing a selection of trigger levels on the scale of $\mathrm{mV}$, corresponding to fractions of a $\mathrm{keV}$ phonon energy. It is important to have a low trigger level in order to identify and record any recoils with energies down to the detector noise limit. The trigger level, however, cannot be set arbitrarily low so that the trigger rate is dominated by random noise. Leaving the RTF boards, the detector signals are fed into high-speed, high-resolution digitizers. The signals are continuously digitized at $1.25 \mathrm{MHz}$ with 12 bit resolution and a dynamic range of $4 \mathrm{~V}$. When a trigger is issued, a 2048 bin long trace (corresponding to $1.6 \mathrm{~ms}$ ) is recorded by the DAQ.

The muon veto counters cover a surface area of $20 \mathrm{~m}^{2}$ with panels of $4.5 \mathrm{~cm}$ thick NE-110 plastic scintillators. The panels are grouped into functional units representing the six sides (or faces) of the cube formed by the $\mathrm{Pb}$ shield. Each panel is read out by photomultiplier tubes, connected to the panels via wavelength shifter bars and light guides. The photomultiplier tube signals from each panel are then summed and digitized in 0.8 mus time bins. A continuous record of the 
digitized muon veto information is then sent into a memory buffer to be accessed whenever an event related trigger is issued.

\subsubsection{DAQ}

The DAQ consists of a combination of custom built electronic hardware and software modules written in java programming language. The DAQ is an umbrella name for a system that performs various tasks, only one of which is recording the actual detector signals. Signals from the RTF boards and muon-veto logic, along with their timing information, are continuously collected and stored in a memory buffer. When an event occurs that satisfies the triggering criteria, a command is issued to download the contents of the digitizers as well as the contents of the memory buffer. This provides information about the activity in the detectors and the muon veto that immediately preceeds, follows and corresponds to the event in question; allowing us to determine whether the event was associated with particles originating within or outside the shield. In addition to recording the data, the DAQ handles the task of controlling the detectors and coordinating several activities that occur during data taking. Additionally, quantities such as trigger and veto rates, as well as various detector diagnostics are continuously monitored (approximately once a minute) to ensure that the experiment is operating within acceptable parameters. In case any of the monitored parameters, whether detector or refrigerator related, are outside of the acceptable range, the DAQ is capable of issuing phone calls to alert the operators.

The main CDMS run control is through the home-made software package, control GUI. FEB settings are accessible through FEB control in the GUI. The GUI also allows for fridge and ice box historical data, as well as the necessary 
detector noise plots.

Commercial VME waveform digitizers sample each signal, and history buffers record times of veto and triggers. Veto and trigger electronics were designed and built at UCSB, with contributions from Fermilab.

The features of the CDMS DAQ include:

- Java/Web based server/client data acquisition

- Remote control/monitoring - Expert can be 'on call' a 1000 miles away

- Can control from the surface (evening and weekend running)

- High data rate capability

- Integrated error reporting and monitoring

- Historical data collection and plots

- Diagnostic noise plots

\subsection{Data Analysis}

A first pass data analysis is called DarkPipe, which is run offline on a cluster of PCs running the Linux operating system. DarkPipe transforms the digitized pulse information from a digitizer bin vs. time format into rudimentary physical quantities such as pulse integrals, start times, rise times, etc. The analysis is automated such that as soon as a data file (usually 500 events) becomes available, it is promptly sent to the first available CPU. In low background running mode, the event trigger rate is less than $0.1 \mathrm{~Hz}$ in the Soudan mine, and a single CPU is sufficient to keep up with the data rate. When calibration data is taken, trigger 
rates can be as high as $15 \mathrm{~Hz}$, which can be handled in real time by the $10 \mathrm{CPU}$ cluster. A typical day of background data consists of 10000 events in the Soudan mine. The data collection in low background running is divided into two near half-day long periods. This is because the DAQ is turned off twice a day for a period of 1 hour during cryogenic transfers.

A second pass data analysis is called PipeCleaner, which is run on the outputs of DarkPipe. This consists primarily of applying calibration constants to produce quantities with meaningful units (such as $\mathrm{keV}$ for energy, or $\mu s$ for time) and of calculating derived quantities, such as the x-y coordinates of an event. The second pass analysis is fast, it only takes a few minutes for 10000 events, which are collected in a one day time scale in low background operation mode. Usually the final processed version of the data (known as RRQs - Relational Reduced Quantities) is available almost immediately at the end of the acquisition of a data series.

Higher level analysis of the data (i.e. searching for Dark Matter candidates) is called CAP (CDMS Analysis Package), which is run with home made software built upon the Matlab programming language. 


\section{Chapter 4}

\section{ZIP Detectors}

\subsection{Introduction}

The CDMS ZIP detector, shown in Figure 4.1, is a high-purity, single-crystal cylinder of $\mathrm{Ge}(\mathrm{Si}), 250 \mathrm{~g}$ (100 g) in mass, $7.6 \mathrm{~cm}$ in diameter, and 1-cm thick. The detector has two concentric charge-collection electrodes, $Q_{\text {inner }}$ and $Q_{\text {outer }}$. One side of the detector is patterned with active aluminum/tungsten films that define four independent phonon sensors. The other side is patterned with gold grids, which are used as the inner electrode and outer electrode.

The energy deposited in the detector by an interacting particle is called "recoil energy" $E_{r}$. There are two kind of interactions in the detector: electron recoils and nuclear recoils. The electron recoil sources include electrons and $\gamma$ rays, which interact with the shell electrons in the detector. Nuclear recoil sources include neutrons and WIMPs, which interact with nuclei in the detector.

When a particle with kinetic energy traverses a semiconductor, it scatters by ionization and phonon emission. Particle semiconductor interaction can be summarized as the following cascade process: 


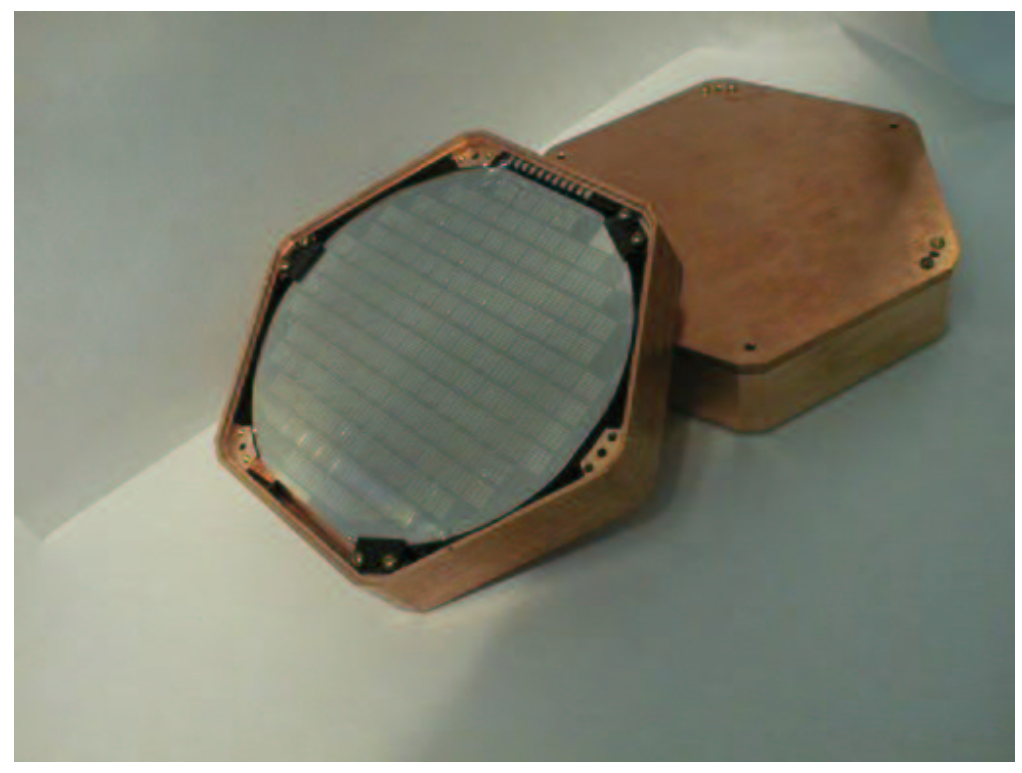

Figure 4.1: A photograph of a ZIP detector in its housing. The exposed surface shows the phonon sensor pattern.

- There is an energy gap $E_{g}$ for a semiconductor. To create an electron hole pair in an intrinsic semiconductor, an average energy $\epsilon\left(>E_{g}\right)$ is needed.

- The deposited kinetic energy from the interaction of particles with a semiconductor generates electrons, holes, and phonons in a cascade process in semiconductors.

- Semiconductor materials exhibit a characteristic plasmon (a cloud of energetic electrons and holes) energy $\hbar \Omega_{p}$ (with typical energy $10-25 \mathrm{eV}$ ). The formation of plasmons is the dominant initial particle energy loss mechanism in a semiconductor.

- Optical phonon (typical energy is $\hbar \omega_{0}$ ) emission is another energy loss mechanism for direct nucleus interaction.

- Further energy loss mechanism includes the creation of more electron hole 


\begin{tabular}{|l|l|l|l|l|}
\hline type & $E_{g}$ & $\hbar \Omega_{p}$ & $\hbar \omega_{0}$ & $\epsilon$ \\
\hline $\mathrm{Ge}$ & 0.74 & 15.6 & 0.037 & 2.96 \\
\hline $\mathrm{Si}$ & 1.12 & 16.6 & 0.063 & 3.82 \\
\hline
\end{tabular}

Table 4.1: Silicon and germanium characteristic parameters (all quantities are in $\mathrm{eV})$.

pairs and optical phonon emissions.

- Most of the electron and hole energy are lost in the optical phonon cascade. The residual energy is given off to acoustic phonons when the charge carriers have cooled to within a single optical phonon energy of the band edge.

The cascade particle detector interaction process takes less than 10 ns [84, 85]. For electron recoils, the particle knocks off a shell electron initially, with few optical phonons. Then this energetic electron creates phonons, more electrons and holes during its thermalization in the semiconductor crystal. This process keeps going until the energy of electrons is so low that ionization and phonon emission can no longer occur.

For nuclear recoils, the particle hits on the nucleus initially, producing high energy phonons, and energetic electrons. Then these energetic electrons follow the same processes as electron recoils. There are phonons and ionization charges in both kind of recoils, but there are more phonons in nuclear recoils than in electron recoils.

For electron recoils, one electron-hole pair is produced for every $\epsilon \approx 2.96 \mathrm{eV}$ $(3.82 \mathrm{eV})$ of energy in $\mathrm{Ge}$ (in $\mathrm{Si}$ ). The "ionization energy" $E_{\mathrm{Q}}$ is defined for convenience as the recoil energy inferred from the detected number of charge pairs $N_{\mathrm{Q}}$ by assuming that the event is an electron recoil with $100 \%$ charge-collection efficiency:

$$
E_{\mathrm{Q}} \equiv N_{\mathrm{Q}} \times \epsilon
$$


This is also refered to as the "electron equivalent" energy and conventionally written in units of keVee. The ionization yield is $Y \equiv E_{\mathrm{Q}} / E_{\mathrm{r}}$, so $Y \approx 1$ for electron recoils with "complete" charge collection.

Nuclear recoils produce fewer charge pairs, and hence less ionization energy $E_{\mathrm{Q}}$ than electron recoils of the same recoil energy do. The ratio between the ionization yield $Y_{e r}$ of electron recoils and the ionization yield $Y_{n r}$ of nuclear recoils depends on both the material and the recoil energy, with $Y_{e r} / Y_{n r}$ in the range of $2.5 \sim$ 5 [86] for the CDMS germanium ZIP detetcor.

The drifting of charges dissipates energy in the external electric field, increasing phonon production by an amount equal to the work done by the external electric field. These "Neganov-Luke" phonons contribute to the total observed phonon signal $E_{P}$, yielding

$$
E_{\mathrm{P}}=E_{\mathrm{r}}+e V_{\mathrm{b}} N_{\mathrm{Q}}=E_{\mathrm{r}}+\frac{e V_{\mathrm{b}}}{\epsilon} E_{\mathrm{Q}}
$$

where $V_{\mathrm{b}}$ is the bias voltage across the detector $[87,88]$. Since $E_{\mathrm{Q}}=E_{\mathrm{r}}$ for electron recoils with full charge collection, $E_{\mathrm{P}}=\left(1+\frac{e V_{\mathrm{b}}}{\epsilon}\right) E_{\mathrm{r}}$ for these events. In practice, the recoil energy $E_{\mathrm{r}}$ of an event is calculated from measurements of the phonon and ionization energies:

$$
E_{\mathrm{r}}=E_{\mathrm{P}}-\frac{e V_{\mathrm{b}}}{\epsilon} E_{\mathrm{Q}}
$$

When charge trapping happens in the semiconductor, equation 4.3 no longer holds. The trapped charge will lose its energy by photon emission or by phonon emission, so the measured charge energy is a reduced ionization energy. Moreover, the measured phonon energy is more complicated than primary phonon energy and Neganov-Luke phonon energy together. 


\subsection{The Ionization Measurement}

A ZIP detector is instrumented with two charge collection channels as shown in

Figure 4.2. $Q_{\text {outer }}$ is about $2 \mathrm{~mm}$ wide. Because of poor charge collection (due to non-uniform electric field near the edge of the ZIP detector) and poor phonon collection (due to the limited TES coverage near the edge of the ZIP detector), the $Q_{\text {outer }}$ electrode is used to reject background only. The events that fall in $Q_{\text {inner }}$ are the low background events for WIMP hunting in the CDMS experiment. The entire phonon sensor side is used as the ground plane. Because the bias voltage over phonon sensors is only at the $100 \mu V$ scale, it has no effect on charge channel biases. See figure 4.2 for details.
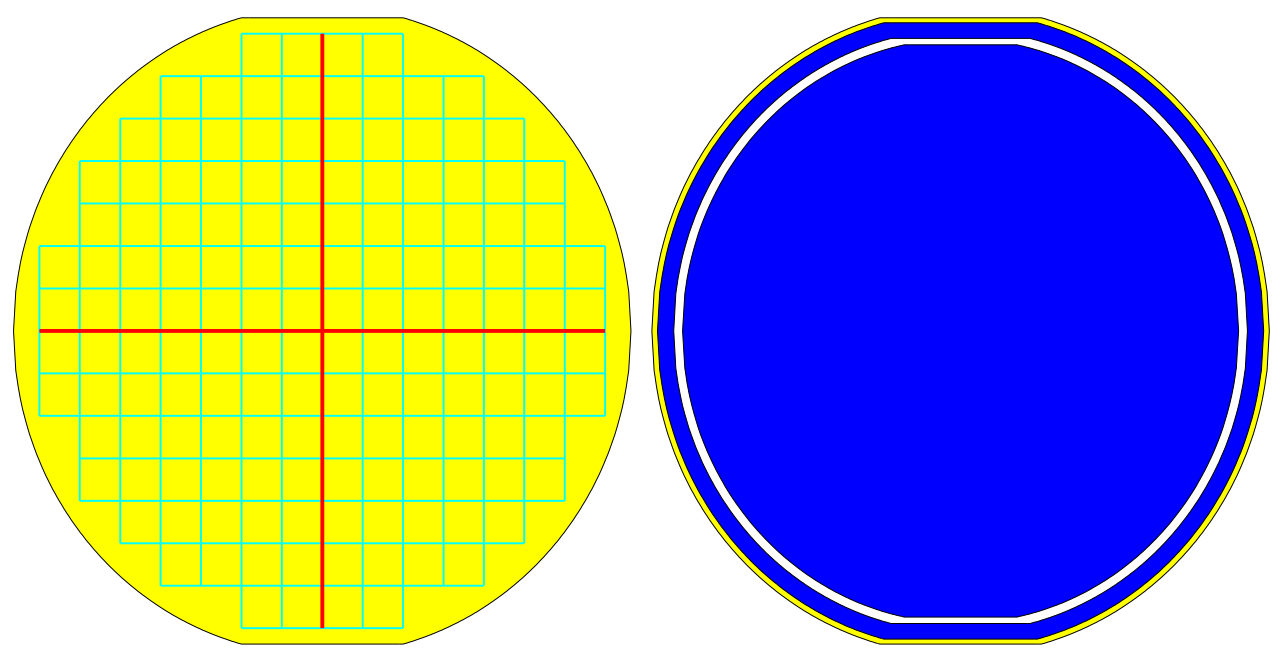

Figure 4.2: A schematic of ZIP detector surface structures. The phonon side is instrumented with four phonon sensors. There are 37 QET dies in each phonon sensor. The charge side is instrumented with two charge collection channels, $Q_{\text {inner }}$ and $Q_{\text {outer }}$.

The ionization charges are read out with a silicon JFET charge integrator [89] as shown schematically in figure 4.3. An electric field of typically 3-6 Volts $/ \mathrm{cm}$ is applied across the detector via the $V_{b}$ line, causing the electrons and holes to 


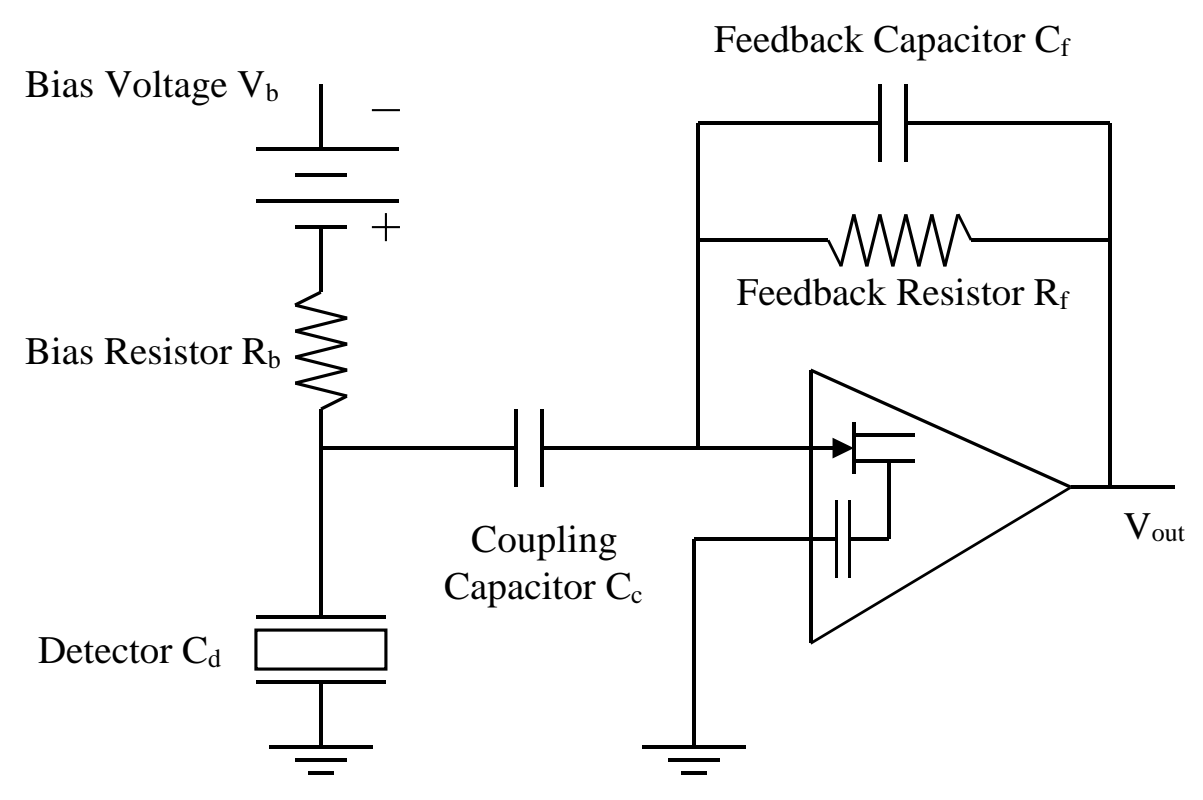

Figure 4.3: A schematic of the ionization readout. The detector is represented by the detector capacitor $C_{d}$. The charge coupling capacitor $C_{c}$ sends the signal to the charge integrator, which has a typical time constant $R_{f} C_{f}$.

drift to their respective electrodes. As the drifting electrons begin to develop a voltage across $C_{d}$, the amplifier responds by lowering its output voltage in order to attract the charge carriers onto the feedback capacitor, maintaining the JFET gate voltage at virtual ground. The size of the signal that appears at the output of the amplifier is given by $V_{\text {out }}=Q / C_{f} \sim E_{Q}$. The charge amplifier circuit produces voltage pulses whose pulse height depends linearly on the ionization energy. For a complete ionization charge collection of an electron recoil, the ionization energy equals the recoil energy, $E_{Q}=E_{r}$. The feedback capacitor is allowed to discharge through the feedback resistor $R_{f}$ with a time constant of $\tau=R_{f} / C_{f} \sim 40 \mu s$. The coupling capacitor $C_{c}$ serves to isolate the JFET gate from the ionization DC bias voltage, allowing it to remain at virtual ground. The presence of the 
coupling capacitor stabilizes the charge channel base line. Empirically, a bigger $C_{c}(300 \mathrm{pF})$, relative to $C_{d}(50 \mathrm{pF})$, can increase the measurement sensitivity of the charge readout.

The performance of the ionization readout circuit is mainly determined by thermal noise in the readout circuit loop and by JFET noise. The thermal noise of the voltage across the various capacitors in the circuit can be written as

$$
V_{r m s}=\sqrt{4 k_{B} T / C}
$$

where $T$ is temperature and $C$ is capacitance. The equivalent charge fluctuation is

$$
Q_{r m s}=\sqrt{4 k_{B} T C}
$$

The capacitance of the detector is $C_{d}=50 p F$. Given that the detectors are operated at a temperature of $50 \mathrm{mK}$, a noise contribution of 74 electron-hole pairs arises from the thermal fluctuations. This is equivalent to a $220 \mathrm{eV}$ energy for electron recoils in $\mathrm{Ge}$ detectors ( $282 \mathrm{eV}$ in $\mathrm{Si}$ ). For a more accurate calculation, one must consider, in addition to the detector capacitance, the various other elements that can contribute to thermal fluctuations. For example, there is the coupling capacitor $C_{c}$, which adds in series to the detector capacitance, and the parasitic capacitance $C_{p}=10 p F$, which adds in parallel to the detector/coupling capacitance combination.

The charge amplifier noise is quantified by the voltage fluctuations at the gate of the JFET, which is $\sim 1 n V / \sqrt{H z}$. The optimal noise performance of the JFET is obtained when the JFET is operated at a temperature of $130 \mathrm{~K}$. This is accomplished by isolating the JFET on a membrane that is heat-sinked in the $4 \mathrm{~K}$ bath, allowing it to self heat to $130 \mathrm{~K}$. 


\subsubsection{The Dead Layer of Surface Events}

The reduced charge collection of the event near the surface of the detector was a problem in the early stage of CDMS detector development. This problem was solved with the introduction of amorphous silicon.

The ionization charge collection is realized by separating the electrons and holes into a positive side and negative side, respectively, with the applied external electric field. Two factors need to be considered in the ionization charge collection. First, electrons and holes generated by an interaction have high kinetic energy before their thermalization by emitting phonons. They can diffuse away from the original interaction location before they get enough kinetic energy from the external electric field along the direction that they are supposed to go. This diffusion process is in a random direction; some of them may diffuse back into the nearby electrode, in opposition to the direction that they are supposed to go in the drift field. Second, the ionization charge cloud has a self-shielding effect. Electrons and holes need time to pick up kinetic energy from the external electric field to overcome the initial diffusion. Hence, some of the charges produced near the surface of the detector can diffuse against the applied electric field into the nearby electrode. A fraction of the event ionization charges cannot be collected, so the surface of the detector appears to be the detector's "dead layer" [90].

In order to reduce the loss of ionization near the detector surfaces, the ZIP detectors are made with hydrogenated, amorphous-silicon contacts. Amorphous Si possesses a bandgap $E_{\mathrm{g}}=1.2 \mathrm{eV}$, almost twice as large as that of bulk Ge $(0.74$ $\mathrm{eV}$ ). As long as the bands of the bulk Ge and the deposited layer of amorphous silicon are nearly centered on each other, the amorphous Si can block the back diffusion of charges of both polarities. This amorphous silicon layer is $40 \mathrm{~nm}$ thick. 
The dead layer is a problem particularly for electrons that interact with the detector on the surfaces, since electrons at the energy comparable to WIMP recoils have a very small penetration depth. The $90 \%$ stopping length in Ge (in $\mathrm{Si})$ is $0.5 \mu \mathrm{m}(0.7 \mu \mathrm{m})$ at $10 \mathrm{keV}$, and $10 \mu \mathrm{m}(23 \mu \mathrm{m})$ at $60 \mathrm{keV}$ [86]. Although most low-energy electrons suffer incomplete ionization collection even with our amorphous electrodes, only a small fraction electrons produce an ionization yield indistinguishable from that characteristic of nuclear recoils.

\subsubsection{Charge Trapping}

Charges can be trapped within the bulk of the detector at shallow impurity sites. Impurities (acceptors/donors) in the detector bulk create shallow energy levels just above/below the valence/conduction bands. The depth of these impurity levels is $\sim 10 \mathrm{meV}$. At $50 \mathrm{mK}$, the acceptors/donors $\left(\sim 10^{11} / \mathrm{cm}^{3}\right)$ are actually in ground states. But these impurities could be left in ionization states due to imperfect neutralization. (Neutralization refers to removing the charge states of ionized impurities or ionized defects in a germanium or silicon ZIP detector. Because the long lasting charge states can trap the electrons and holes from intrinsic excitation in a particle interaction in the ZIP detector temporarily or permanently, they result in a charge measurement defficiency. The ZIP detector neutralization is important for background rejection in CDMS experiment.) The ionized impurities therefore trap electrons or holes from event interaction. Polarized neutral impurities (dipoles) could also act as temporary charge trapping centers in germanium and silicon.

The charge trapping physics of ionized charge trapping centers can be understood with the Thomson Model. The Thomson Model is especially useful in 
helping us to understand the charge trapping as it depends on the allied external bias voltage and the detector netraliztion processes. When the drifting charge approaches the trapping center, the drifting charge could lose its kinetic energy by emitting a photon, or by emitting a phonon. If this occurs, the charge will be trapped in the trapping center's Coulomb potential, and it will get to the ground state (neutralized state) by interacting with a third body ( for example, a phonon emission). The following is a charge trapping analysis from first principles. The experimental study of charge trapping can be found in reference [91]; two examples of two silicon detectors are in Figures 4.4 and 4.5.

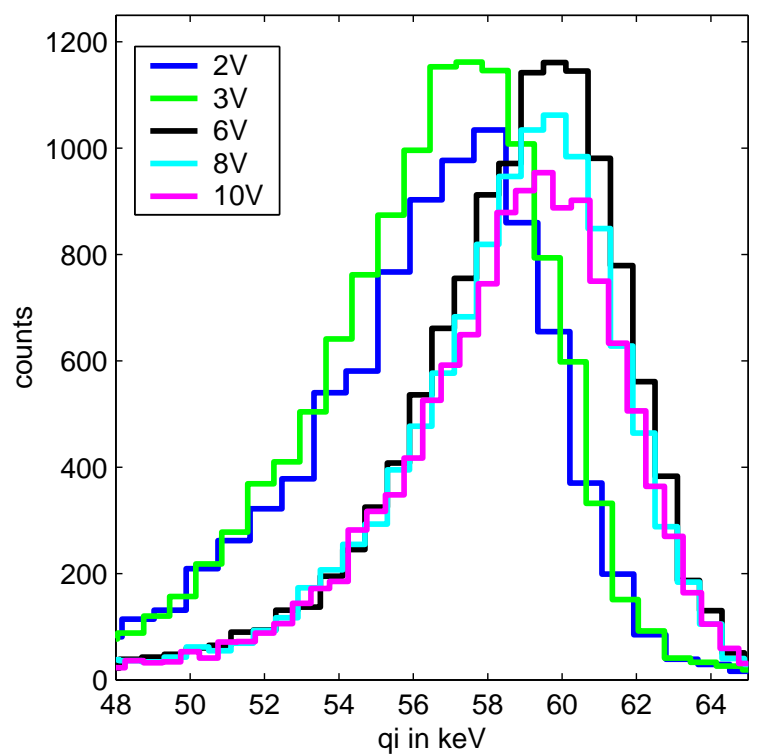

Figure 4.4: The $60 \mathrm{keV}$ line from ${ }^{241} \mathrm{Am}$ source in CWRU run27 at different bias voltages. At $2 \mathrm{~V}$ and $3 \mathrm{~V}$ biases, the $60 \mathrm{keV}$ line shifts down to $57.5 \mathrm{keV}$. The $60 \mathrm{keV}$ line peaks at the same energy at $6 \mathrm{~V}, 8 \mathrm{~V}$ and $10 \mathrm{~V}$ biases. This is an experimental support that there is a Rydberg series $(n=3)$ between $3 \mathrm{~V}$ bias and $6 \mathrm{~V}$ bias. (See the theoretical explanation in this section.)

Near a charge trapping center, the electron total energy is

$$
E=-\frac{1}{4 \pi \epsilon \epsilon_{0}} \frac{e^{2}}{r}+E_{k}
$$




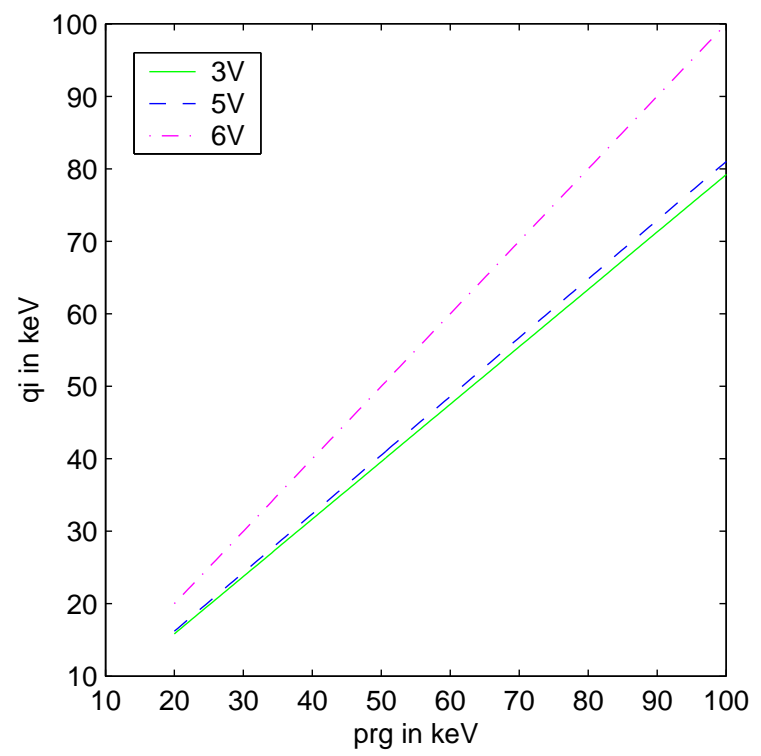

Figure 4.5: The collected charge versus electron recoil energy (prg is electron equivalent recoil energy) of $Z 6$. The straight lines are fitted to the data [91] in Soudan run118. There is a charge collection efficiency jump between $5 \mathrm{~V}$ bias and $6 \mathrm{~V}$ bias. This is an indication that there is a Rydberg series $(n=3)$ between $5 \mathrm{~V}$ bias and $6 \mathrm{~V}$ bias. (See the theoretical explanation in this section).

$E_{k}$ is electron kinetic energy, $\epsilon_{0}$ is vacuum permittivity, and $\epsilon$ is silicon relative permittivity. When $E>0$, electrons cannot be trapped. When $E \leq 0$, there is probability that the electron is trapped. So the possible bound state formation radius for an electron with kinetic energy $E_{k}$ is

$$
r=\frac{1}{4 \pi \epsilon \epsilon_{0}} \frac{e^{2}}{E_{k}}
$$

The charge trapping cross section in the Thomson Model is [92, 93]

$$
\sigma=\pi r^{2} \frac{4}{3} \frac{r}{l}
$$

where $l$ is the mean free path of electrons for an energy losing collision. $l=$ $7.5 \times 10^{-4} \mathrm{~cm}$ for silicon at low temperatures [94].

Analogous to hydrogen atoms, there is a Rydberg series for ionized shallow 
donors in silicon,

$$
\begin{gathered}
E=-\frac{R}{n^{2}} \\
R=\frac{m}{m_{0}} \frac{1}{\epsilon^{2}} R_{0}
\end{gathered}
$$

with $R_{0}=13.6 \mathrm{eV}, m$ is electron effective mass in silicon, and $m_{0}$ is electron bare mass. $R=31.2 \mathrm{meV}$ for silicon, close to $44 \mathrm{meV}$ for ionized phosphorous impurities and $54 \mathrm{meV}$ for ionized arsenic impurities in silicon. The actual excitation energy of phosphorous in silicon is used in the following calculations.

In silicon, the electron average drift velocity [94] at low temperature (high electric field approximation) is

$$
v_{d}=3.3 \times 10^{4}(V / D)^{0.2}
$$

where $V$ is the voltage across the silicon detector, and $D$ is the detector thickness. The average electron thermal velocity is

$$
v_{r m s}=1.085 v_{d}
$$

The average electron kinetic energy is

$$
E_{k}=\frac{1}{2} m v_{r m s}^{2} .
$$

The electron trapping condition is

$$
E_{k}<\frac{R}{n^{2}}
$$

Corresponding to Rydberg energy level $n$, the external bias voltage should be

$$
V=\left(\frac{R}{n^{2}}\left(\frac{1}{2} m\left(1.085 \cdot 3.3 \times 10^{4}\right)^{2}\right)^{-1}\right)^{2.5}
$$

For a given Rydberg energy level n, the minimum kinetic energy for an electron to escape the ionized charge trapping center can be calculated with equation 4.14. 


\begin{tabular}{|c|c|c|c|c|c|c|}
\hline $\mathrm{n}$ & 1 & 2 & 3 & 4 & 5 & 6 \\
\hline $\mathrm{V}($ volts $)$ & 1500 & 46.9 & 6.17 & 1.46 & 0.48 & 0.19 \\
\hline $\mathrm{r}\left(10^{-8} \mathrm{~cm}\right)$ & 43.6 & 1774.4 & 392.4 & 697.6 & 1090 & 1569 \\
\hline$\sigma\left(10^{-16} \mathrm{~cm}^{2}\right)$ & 4.64 & 296 & 3376 & 18960 & 72320 & 216000 \\
\hline
\end{tabular}

Table 4.2: The trapping cross section of ionized donors at temperatures below $1 \mathrm{~K}$ at different bias voltages for the CDMS silicon detector. $\mathrm{n}$ is Rydberg series number, $\mathrm{V}$ is the external bias voltage required for preventing an electron falling in that Rydberg energy level.

The electron trapping radius and trapping cross section can be calculated with equations 4.7 and 4.8 .

Table 4.2 summarizes the corresponding bias voltage, charge trapping radius, and charge trapping cross section at different Rydberg energy levels for silicon. The voltage at $n=1$ is normalized to 1500 volts, which is the typical breakdown voltage of high purity silicon at the same thickness.

Generally, Coulomb attractive centers have a large trapping cross section $2 \times 10^{-12} \mathrm{~cm}^{2}$ below $1 \mathrm{~K}$ under 4 volts external bias. The neutral trapping centers' cross section is small, $\sim 10^{-20} \mathrm{~cm}^{2}$ below $1 \mathrm{~K}$, with no significant effect at the centimeter length scale. See reference [93]

Suppose the Coulomb center density is $10^{+11} / \mathrm{cm}^{3}$. Then the mean transportation length of charge in silicon should be $1 /\left(2 \times 10^{-12} \times 10^{+11}\right)=5 \mathrm{~cm}$, much bigger than the thickness of the detector. This result is consistant with Penn's experimental investigation [95]. Also, we know that $10^{+11} / \mathrm{cm}^{3}$ charge trapping center density is the upper limit; the actual value is much smaller for a well-neutralized ZIP detector. Because of the high mobility of electrons and holes in germanium, their drifting velocity is much bigger. The trapping cross section is smaller in germanium than in silicon, therefore the mean transport length of electrons is longer in germanium than in silicon. 


\subsection{Phonon Measurement}

The CDMS ZIP detector measures phonons created from particle interaction and from drifting electrons and holes across the detector. In this section, I will describe the phonon components in the ZIP detector, the phonon measurement physics and technology, and the phonon energy readout electronic circuit.

\subsubsection{Phonons in ZIP Detectors}

As explained in section 4.1, phonons in ZIP detectors are in two categories: primary phonons and Neganov-Luke phonons. The primary phonons are mainly optical phonons. These optical phonons have small group velocity, and they decay quickly into acoustic (ballistic) phonons. See Figure 4.6 for the dispersion relation.

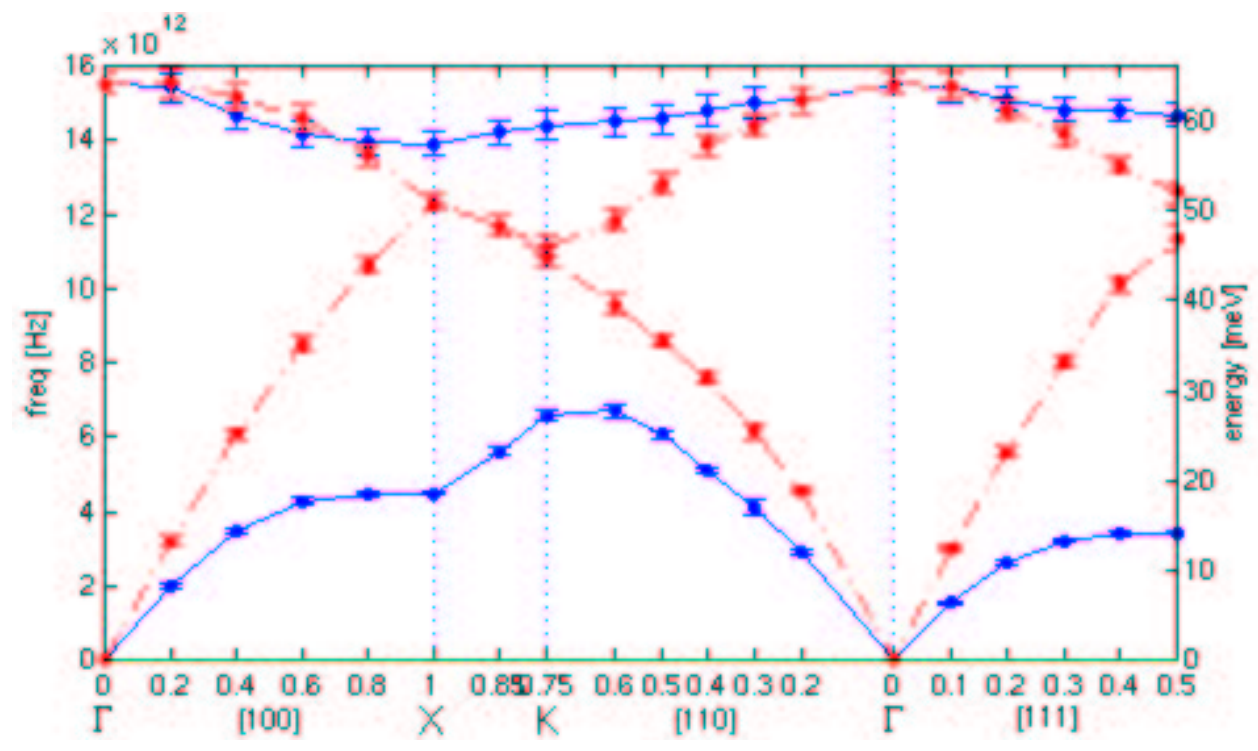

Figure 4.6: Phonon dispersion relation of silicon generated from Dolling neutron scattering data. The longitudinal phonons are in red, the transverse phonons are blue. Figure from [96]. Phonon group velocity is proportional to the first derivative of the dispersion curves. Lower energy acoustic phonons are faster. 
The high energy optical phonons decay in two main processes: phonon anharmonic decay and elastic scattering. These are also called phonon down conversion propagation.

For anharmonic decay [97], the rate is

$$
\tau_{B}{ }^{-1}=\frac{B}{\mu s}\left(\frac{\nu}{T H z}\right)^{5}
$$

with $B=0.074$, and $\nu$ is the phonon frequency in THz. $\tau_{B}$ is in $\mu s$.

For elastic scattering (isotopes, impurities, and defects), the scattering time is given by the well-known formula [97],

$$
\tau_{A}^{-1}=A\left(\frac{\nu}{T H z}\right)^{4}
$$

with $A=2.43$, and $\tau_{A}$ given in $\mu s$.

On the $\mu s$ time scale, all optical phonons decay into ballistic phonons. Typical phonon propagation distance is about $1 \mathrm{~mm}$ during optical phonon decay.

The Neganov-Luke Phonons are instant phonons. When charges drift across the silicon or germanium crystal, the charges interact with the crystal's deformation potential, and the kinetic energy of these charges is lost as the emission of phonons. Basically, Neganov-Luke phonons are sub-THz phonons, and most of them are pointed to the charge drifting direction. The next chapter will explain Neganov-Luke phonons in detail, because the Neganov-Luke phonon energy distribution as a function of location is used for a phonon propagation-time calculation in event position reconstruction.

\subsubsection{Quasi-particle Collection}

Phonon energy is measured with Quasi-particle trap assisted Electrothermal feedback Transition edge sensors (QET). QETs are structures that have aluminum 
phonon capture fins and tungsten superconducting Transition Edge Sensors (TES). The QET collects the phonon energy coming from the interaction in the detector crystal. The QET consists of $300 \mathrm{~nm}$ thick aluminum films, covering macroscopic areas, connected to $35 \mathrm{~nm}$-thick tungsten films at discrete locations throughout the surface of the detector. The tungsten films are the energy sensing elements.

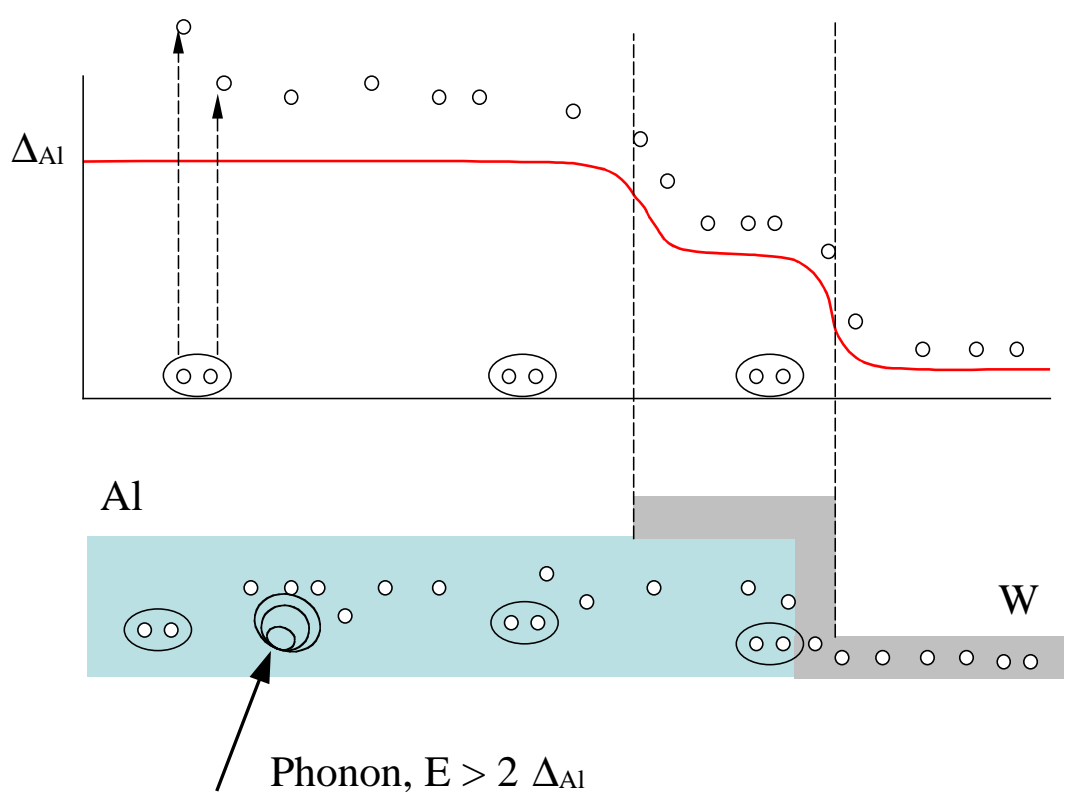

Figure 4.7: Superconducting gap structure of the quasiparticle traps. At the bottom is a sketch of the sensor, with a representation of a scattering event. A phonon incident on the aluminum fins breaks cooper pairs and creates quasiparticles. As shown in the sketch of the band structure at the top, the tungsten has a lower superconducting gap than the aluminum and the quasiparticles diffuse towards the tungsten meander. Once inside the tungsten, they scatter into lowerenergy quasiparticles and are trapped in the tungsten. Finally, the quasiparticles thermalize and increase the temperature of the tungsten meander.

The deposition and patterning of the QET structure are done with photolithographic processes similar to those used in CMOS technology. One surface of 


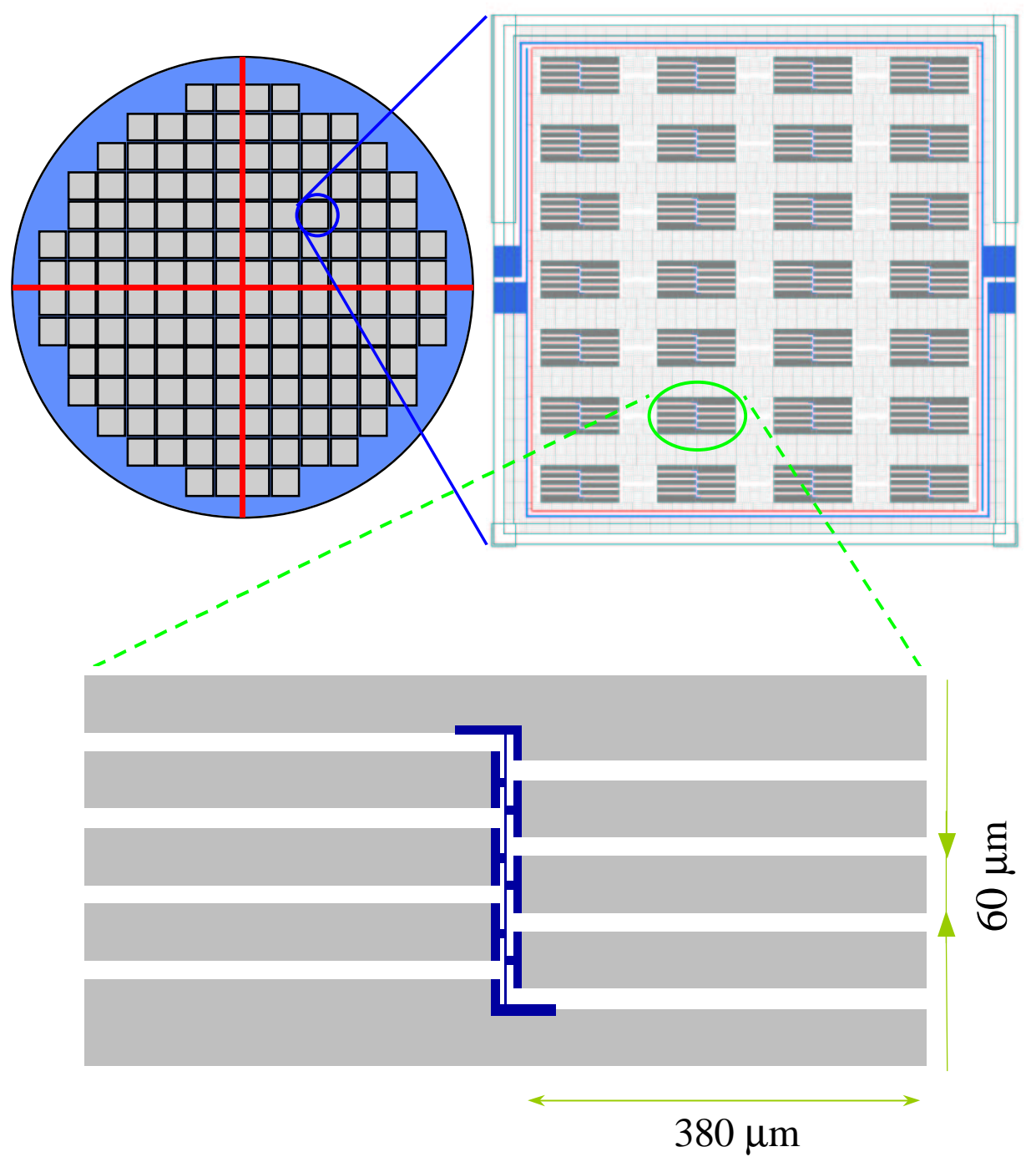

Figure 4.8: A sketch of the ZIP detector phonon sensor. The top left graph shows a schematic of one detector phonon side, which contains four 37-5 mm $\times 5 \mathrm{~mm}$ square dies. The top right graph shows a magnified view of one of those dies, each of which contains $4 \times 7=28$ sensors. The lower drawing is of an isolated sensor, with the tungsten meander shown in dark blue and the aluminum quasiparticle traps shown in light grey. 
the detector is divided into four phonon channels. Each channel consists of 37 repeated, electrically connected dies. Each die contains identical $4 \times 7$ QET cells.

The connection between the $\mathrm{Al}$ and $\mathrm{W}$ films is accomplished via a $4 \mu \mathrm{m}$ wide overlap region. Figure 4.7 shows a schematic of the resulting electronic band structure. The size of the superconducting energy gap in $\mathrm{Al}$ is $2 \Delta_{A l}=340 \mu \mathrm{eV}$. This energy corresponds to a phonon frequency of $84 \mathrm{GHz}$. Phonons with higher frequencies are able to break Cooper pairs in the $\mathrm{Al}$ film, creating two quasiparticles. Since phonons become ballistic at frequencies of $0.6-1 \mathrm{THz}$ the average energy that they'll impart to the quasiparticles will be about $2 \sim 4 \mathrm{meV}$. These initial quasiparticles then decay down to the gap edge by shedding phonons that in turn are able to create more quasiparticles. This process, known as a cascade, transforms a single quasiparticle with energy $10 \times 2 \Delta_{A l}$ into a collection of quasiparticles with energy $\Delta_{A l}$. During this process, however, approximately half the initial energy is lost to phonons with energies less than the Al energy gap. As these phonons are unable to create further quasiparticles, they are undetectable, and thus pose an unavoidable energy collection loss. Similarly, a small fraction of the initial phonon spectrum will be sub-gap, contributing an additional $10 \%$ inefficiency.

The quasi-particle dynamics is determined by the following equation [98]

$$
D \nabla^{2} n-\Gamma n^{2}-\frac{n}{\tau_{d}}=\frac{\partial n}{\partial t}
$$

where $n$ is the number density of quasi-particles, $D$ is the diffusion coefficient of quasi-particles, $\Gamma$ is the annihilation rate of quasi-particles in the alumium strip, and $\tau_{d}$ is the diffusion time of quasi-particle due to scattering of impurities and defects in aluminum. 
The quasi-particle effective lifetime time is defined by

$$
\frac{1}{\tau}=\Gamma n+\frac{1}{\tau_{d}}
$$

The quasi-particle diffusion length is described by the equation

$$
l_{d}=\sqrt{D \tau}
$$

which is determined by two parameters, the diffusivity D and the quasi-particle lifetime $\tau$.

The diffusivity and lifetime are determined by the quasiparticle mean free path and film thickness respectively. The diffusion length increases with aluminum thickness, but because of the step connection between aluminum and tungsten, ZIP detectors use $300 \mathrm{~nm}$ thick aluminum films.

Measurement of the quasiparticle diffusion length in $150 \mathrm{~nm}$ thick Al films has yielded a result of $l_{d} \sim 180 \mu m$ [99]. Consequently, quasiparticles created more than a few diffusion lengths from a TES will unlikely be able to reach and deposit their energy in the tungsten TES.

The current quasi-particle trapping aluminum fins are designed by simulations [81]. There are 28 TES cells in each die. In each cell, a tungsten TES is connected to ten $380 \mu m \times 60 \mu m$ aluminum fins, as shown in figure 4.8. This configuration is expected to have a quasi-particle collection efficiency of $24 \%$.

When first fabricated, the TES transition temperatures range from 120 to $150 \mathrm{mK}$. The desired TES transition temperature of $80 \mathrm{mK}$ is realized by ionimplantation of ${ }^{56} \mathrm{Fe}$ into tungsten films, based on the measured initial transition temperature distribution pattern on the surface of the ZIP detector. This information is obtained from diagnostic tests. The transition temperature of TESs are generally tuned in the range of 70 to $90 \mathrm{mK}$. 


\subsubsection{Electrothermal Feedback Circuit}

In this section, the TES signal readout is described, taking into account of the thermal equation of the TES and the electrothermal feedback effect of the voltage biased TES circuit.

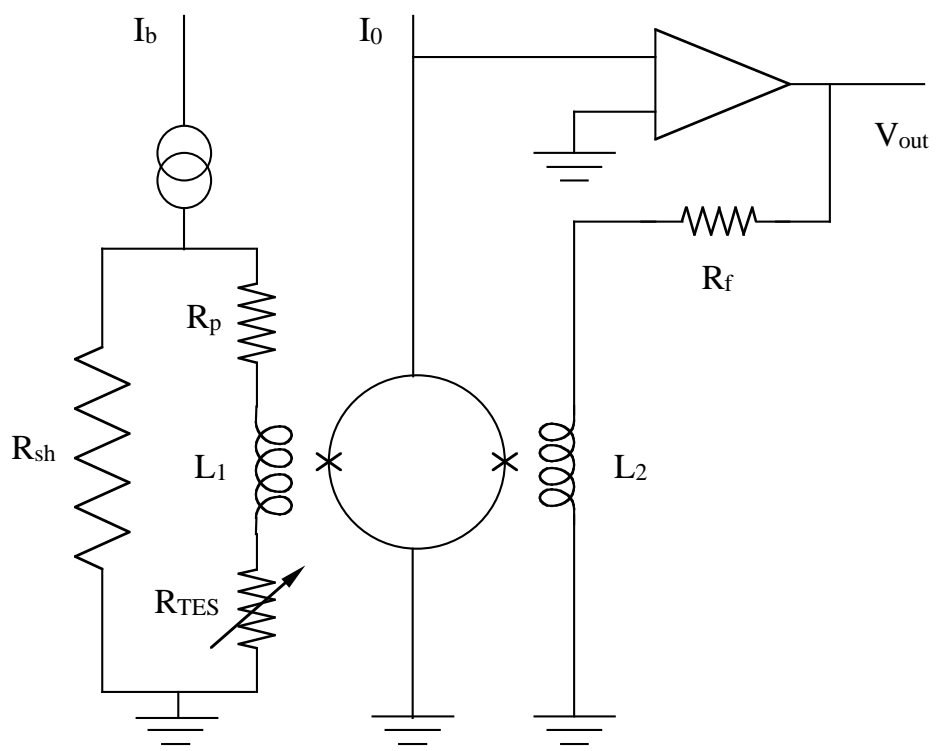

Figure 4.9: Simplified Circuit Diagram for the Phonon Sensor. The sensor $\left(R_{T E S}\right)$ is voltage biased by applying a DC current in parallel with a shunt resisitor $\left(R_{s h}=20 \mathrm{~m} \Omega\right)$. We measure the current in the sensor via inductive coupling to a DC-biased SQUID array, which is amplified via a feedback loop (shown here schematically as a single amplifier) and read out at $V_{\text {out }}$.

For a collected phonon energy Q, in the case of quasi-particle collection efficiency 1 , the TES thermal energy change rate is TES heat capacity times TEStemperature derivative

$$
C \frac{d T}{d t}=\frac{V^{2}}{R(T)}-\kappa\left(T^{n}-T_{s}^{n}\right)=\delta(t) Q
$$




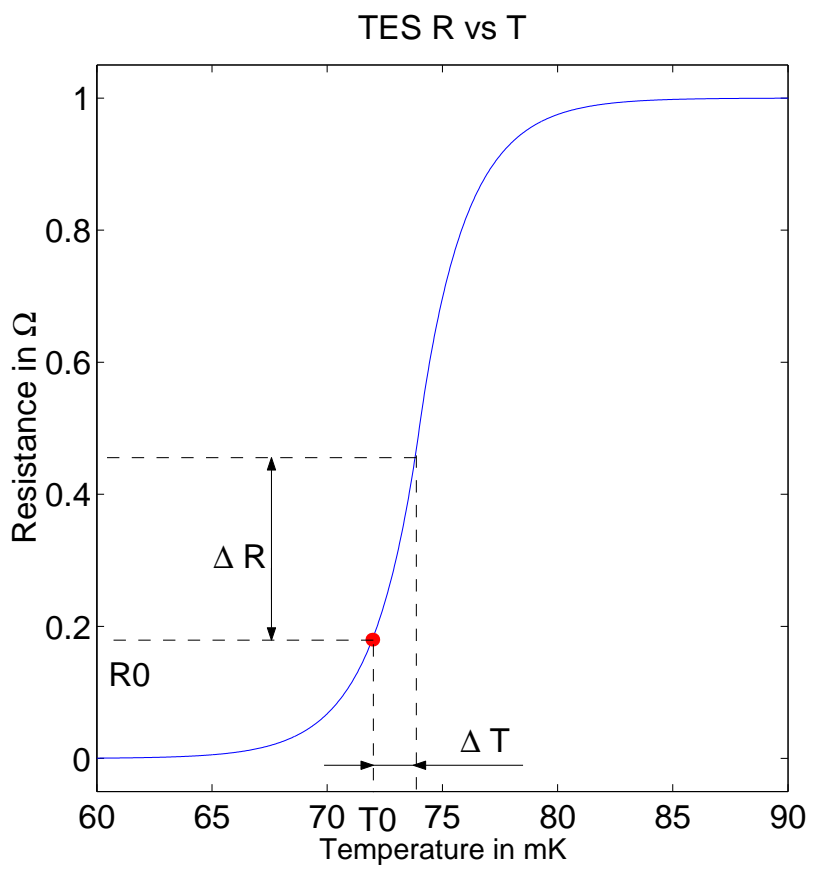

Figure 4.10: TES sensor resistance and temperature relation. Collected phonon energy raises the TES temperature. There is a corresponding resistance change due to the temperature rising.

where $C$ is heat capacity of the TES; $T$ is the electron temperature in the TES; $T_{s}$ is the substrate temperature (semiconductor crystal); $V \approx I_{b} R_{s h}$ is the bias voltage; $R$ is the TES resistance; and $\kappa$ is the thermal coupling coefficient between the electron system in tungsten and the substrate, a geometry- and materialdependent quantity; the index $n$ depends on the dominant thermal impedance between the sensor and the substrate. For metal thin films at low temperatures, the electronic and phonon systems are decoupled and $n=5[100,101]$.

For a given bias current $I_{b}$, the TES temperature is $T_{0}$, and its resistance is $R_{0}$. At equilibrium

$$
\frac{V^{2}}{R_{0}}=\kappa\left(T_{0}^{n}-T_{s}^{n}\right)
$$


We define the resistance temperature slope as

$$
\beta=\frac{d R}{d T}
$$

and at the bias point

$$
\beta_{0}=\left(\frac{d R}{d T}\right)_{0}
$$

We define the quality factor of the TES as

$$
\alpha=\frac{T_{0}}{R_{0}} \beta_{0}
$$

The temperature change of the TES is

$$
\Delta T=T-T_{0}
$$

where $\Delta T$ and $T$ are functions of time. Then the thermal equation is rewritten as

$$
\frac{d \Delta T}{t}=-\frac{\Delta T}{\tau}+\frac{Q}{C} \delta(t)
$$

and the time constant of the TES thermal relaxation in the electro-thermal feedback circuit is

$$
\tau=\frac{C}{\kappa T_{0}^{n-1}(n+\alpha)} .
$$

Usually the parasitic resistance $R_{p}$ is small ( a few $m \Omega$ ), and can be ignored. Near the TES bias point (see Figure 4.10), approximately,

$$
R=R_{0}+\beta \Delta T
$$

The current through TES is $I_{s}$. In reference to Figure 4.9, applying Ohm's law gives

$$
I_{s} R=\left(I_{b}-I_{s}\right) R_{s h} .
$$


When the resistance of the TES changes from $R_{0}$ to $R$, the current change in TES is

$$
\Delta I_{s}=-\frac{I_{b} R_{s h} \cdot\left(R-R_{0}\right)}{\left(R_{0}+R_{s h}\right)\left(R+R_{s h}\right.} .
$$

The TES current $\left(I_{s}\right)$ change in terms of the temperature change is

$$
\Delta I_{s}=-\frac{I_{b} R_{s h} \cdot \beta \Delta T}{\left(R_{0}+R_{s h}\right)\left(R_{0}+R_{s h}+\beta \Delta T\right)} .
$$

We write $\Delta T$ in term of $\Delta I_{s}$

$$
\Delta T=-\frac{\Delta I_{s}\left(R_{0}+R_{s h}\right)^{2}}{\beta\left(I_{b} R_{s h}+\Delta I_{s}\left(R_{0}+R_{s h}\right)\right)} .
$$

Solving equation 4.27 and equation 4.33 under the boundary condition $\Delta T=\frac{Q}{C}$ at $t=0$, the phonon energy readout becomes

$$
\Delta I_{s}=-\frac{I_{s 0}}{1+\frac{\left(R_{0}+R_{s h}\right) C}{\beta Q} \exp (t / \tau)}
$$

with

$$
I_{s 0}=\frac{I_{b} R_{s h}}{R_{0}+R_{s h}} .
$$

Under the small energy approximation defined by $\left(R_{0}+R_{s h}\right) C>>\beta Q$

$$
\Delta I_{s}=-\frac{\beta Q I_{s 0}}{C\left(R_{0}+R_{s h}\right)} \exp (-t / \tau)
$$

Equation 4.36 means that the phonon readout amplitude is linearly proportional to collected phonon energy $\mathrm{Q}$ at a small perturbation approximation. Also, the phonon relaxation is determined by the parameter $\tau$, which is a function of the TES sensor thermal and dynamic parameters, such as heat capacity, thermal coupling constant $\kappa$, and quality factor $\alpha$.

Equation 4.36 is the physics basis by which we interpret the phonon pulse data in the CDMS experiment. The arriving phonons are functions of time and 
interaction location, phonon energy collection is a continuous process until all phonons above the energy threshold are collected or thermalized. So, phonons originating from different interactions (electron recoils or nuclear recoils) or from different locations (on the surface of the detector or in the bulk of the detector) have different propagation velocities, and different distributions in space and time. Therefore, phonon pulses have start times and rise times that correspond to their interaction types and interaction locations. We will make extensive use of these quantities in the data analysis and position reconstruction.

Superconducting Quantum Interference Devices(SQUIDs) are used to read out the current change originating from phonon energy pickup in the phonon sensors. The CDMS collaboration uses SQUID arrays made by Martin Huber at NIST facilities in Boulder, Co. There are 100 SQUID cells in series. This SQUID has $2 \mathrm{MHz}$ bandwidth, low input impedance and low noise, and is ideally suited for use with the low impedance TES sensors.

The TESs are inductively coupled to the SQUIDs as shown in figure 4.9. Current flowing through the TES induces magnetic flux in the SQUID, which results in a voltage change across its terminals. This change in voltage drives an amplifier that feeds a current back into the feedback coil in order to cancel the change in magnetic flux through the SQUID. An input to the feedback coil ratio of 10:1, and a feedback resistor of $R_{f}=1 \mathrm{k} \Omega$ convert the TES current into an output voltage given by $V_{\text {out }}=10 \times 1000 \times \Delta I_{s}$. The CDMS SQUIDs are characterized by a modulation depth of $5 \mathrm{mV}$, one flux quantum per $25 \mu \mathrm{A}$, and a nominal noise performance of $2 p A / \sqrt{H z}$ (both in reference to the input coil). A more complete description of the SQUID characteristics and details can be found in reference [100]. The bias resistor $R_{s h}$ and bias current $I_{b}$ provide the TES bias voltage. $R_{s h}=20 \mathrm{mV}$ provides a stiff voltage source to keep TES resistances 
above $200 m \Omega$.

The main noise source in the SQUID phonon energy readout circuit is the thermal noise of resistors. They are $R_{T E S}$ at $80 \mathrm{mk}, R_{p}$ at $4 \mathrm{~K}, R_{s h}$ at $0.6 \mathrm{~K}$, and the feedback Resistor $R_{f}$ at $4 \mathrm{~K}$. The overall thermal noise level is around $10 p A / \sqrt{H z}$.

\subsection{ZIP Detector Tests at CWRU}

ZIP detectors are fabricated at the Center for Integrated Systems (CIS) at Stanford University. The microfabration includes amorphous silicon layer deposition, electrode formation, and aluminum quasi-particle capturing fin and W TES meander processing. Once the packaging is complete and the appropriate wire bonds are made, these detectors are sent to the test facilities at CWRU or collaborators in the Sadoulet group at UC Berkeley. At the test facilities, the basic performance of the detector and the distribution of tungsten Tc's are measured. If a detector passes the necessary checks, it is sent back to the CIS for ion implantation,

which lowers Tc's to the required range. After ion implantation, the detector is tested again to confirm that the transition temperatures of each phonon sensor are indeed in the designed range, and to check for electrical connectivity and basic performance before deployment in a physics run of CDMS experiments.

To minimize radioactive contamination of detectors, they are kept in a radon and dust free environment during storage and transportation. Ground transport is preferred due to the higher rate of cosmic-ray-induced spallation at high altitudes. When not in use, the detectors are stored in clean, polyethylene shielded containers to minimize exposure to cosmic rays and thermal neutrons. Detector mounting and installation are performed under clean-room conditions. 
The CWRU CDMS detector testing facility includes a Kelvinox 400 dilution refrigerator made by Oxford Instruments housed inside a "Faraday Cage" for RF shielding; a clean room for detector and test sample setup and storage; an LR $700 \mathrm{AC}$ resistance bridge for thermometry and four-wire resistance measurements of individual tungsten pieces cut from test wafers; Joerger cards installed in a VXI crate for data collection; two FLUKE PM339413 oscilloscopes; and standard CDMS equipment, for example, 3U power supply, 9U crate and front end boards, RTF crate and boards, GPIB control interfaces, etc.

There is a calibrated $\mathrm{RuO}_{2}$ thermometer on the mixing chamber of the dilution refrigerator. The base temperature of this dilution refrigerator is $12 \mathrm{mK}$, with an actual cooling power of $160 \mu \mathrm{W}$ at $100 \mathrm{mK}$. Three ten foot long CDMS striplines run from the vacuum bulk head at the top of the dilution refrigerator to below the experiment chamber, so we can test three CDMS detectors in a single refrigerator cooling down. Infrared light absorbers are distributed at the lower flanges. CDMS detectors are maintained in a neutralized through the test runs. Also, with the individual test sample holder sitting on the top of the CDMS tower, we can test 40 TES samples during the same run as the detector diagnostic test.

Both the charge measurement and phonon measurement are tested at CWRU, but most of the diagnostic tests involve the four phonon sensors, labeled as A, $\mathrm{B}, \mathrm{C}$, and $\mathrm{D}$ according to their location relative to the detector interface board (DIB). The first test of a phonon sensor's quality is to measure its normal and parasitic resistances. When the meander is superconducting, a typical $10 \mathrm{~m} \Omega$ parasitic resistance in the circuit, which comes from wiring connections at the $4 \mathrm{~K}$ stage, remains. A high value for parasitic resistance indicates an obvious problem with the circuit, whether in the sensor itself or somewhere else in the wiring from the $4 \mathrm{~K}$ stage on the FET card, through the $600 \mathrm{mK}$ stage on the SQUID card, 
and down to the sensor wire binding pads. The normal resistance is measured by heating the dilution refrigerator mixing chamber to a temperature of $\sim 250 \mathrm{mK}$, well above our tungsten transition temperature, but below the transition of the aluminum leads.

\begin{tabular}{|c|c|c|c|c|c|c|}
\hline \hline \multirow{2}{*}{ Channel } & \multicolumn{3}{|l|}{ Pre-implantation } & \multicolumn{3}{|l|}{ Post-implantation } \\
\cline { 2 - 7 } & $R_{\text {para }}$ & $R_{\text {norm }}$ & $T_{\text {trans }}$ & $R_{\text {para }}$ & $R_{\text {norm }}$ & $T_{\text {trans }}$ \\
\hline $\mathrm{A}$ & $9.1 \mathrm{~m} \Omega$ & $1.55 \Omega$ & $123-127 \mathrm{mK}$ & $12.1 \mathrm{~m} \Omega$ & $1.48 \Omega$ & $79-85 \mathrm{mK}$ \\
\hline $\mathrm{B}$ & $9.5 \mathrm{~m} \Omega$ & $1.66 \Omega$ & $129-139 \mathrm{mK}$ & $10.9 \mathrm{~m} \Omega$ & $2.17 \Omega$ & $83-87 \mathrm{mK}$ \\
\hline $\mathrm{C}$ & $11.2 \mathrm{~m} \Omega$ & $1.85 \Omega$ & $112-116 \mathrm{mK}$ & $16.5 \mathrm{~m} \Omega$ & $2.17 \Omega$ & $81-85 \mathrm{mK}$ \\
\hline $\mathrm{D}$ & $13.0 \mathrm{~m} \Omega$ & $2.00 \Omega$ & $121-126 \mathrm{mK}$ & $21.1 \mathrm{~m} \Omega$ & $1.53 \Omega$ & $82-99 \mathrm{mK}$ \\
\hline
\end{tabular}

Table 4.3: Basic phonon sensor properties for detector Z5 (G9). Data are given for each of the four phonon channels. Shown are the normal and parasitic resistances, along with the $T_{c}$ range derived by a resistance-vs.-temperature sweep. Values are shown both prior to and after the implantation. Summarized by [102].

In reference to figure 4.9, application of Ohm's law gives $\left(I_{b}-I s\right) \times R_{s h}=I_{s} \times R$; $I_{b}$ is the TES circuit input current, and is known; $R_{s h}=20 \mathrm{~m} \Omega$ is the value of the shunt resistor; $I_{s}$ is the current flowing through the TES sensor; $R$ is the TES resistance, including the parasitic resistance; $R=R_{p}$ when the TES is in a superconducting state. The measured resistance is simply expressed by

$$
R=\left(\frac{I_{b}}{I_{s}}-1\right) \times R_{s h}
$$

The transition temperature is measured by using external triangle waves. Figure 4.9 shows a simplified circuit diagram of a ZIP phonon sensor. We start at a temperature at which the quadrant is in a superconducting state, while applying a small triangle wave $\left(I_{b} \sim 3 \mu A\right.$ peak-to-peak at $\left.100 \mathrm{~Hz}\right)$. Then the dilution refrigerator is heated up slowly from the base temperature, and the output triangle wave of a quadrant is monitored. Table 4.3 lists two numbers for the transition 

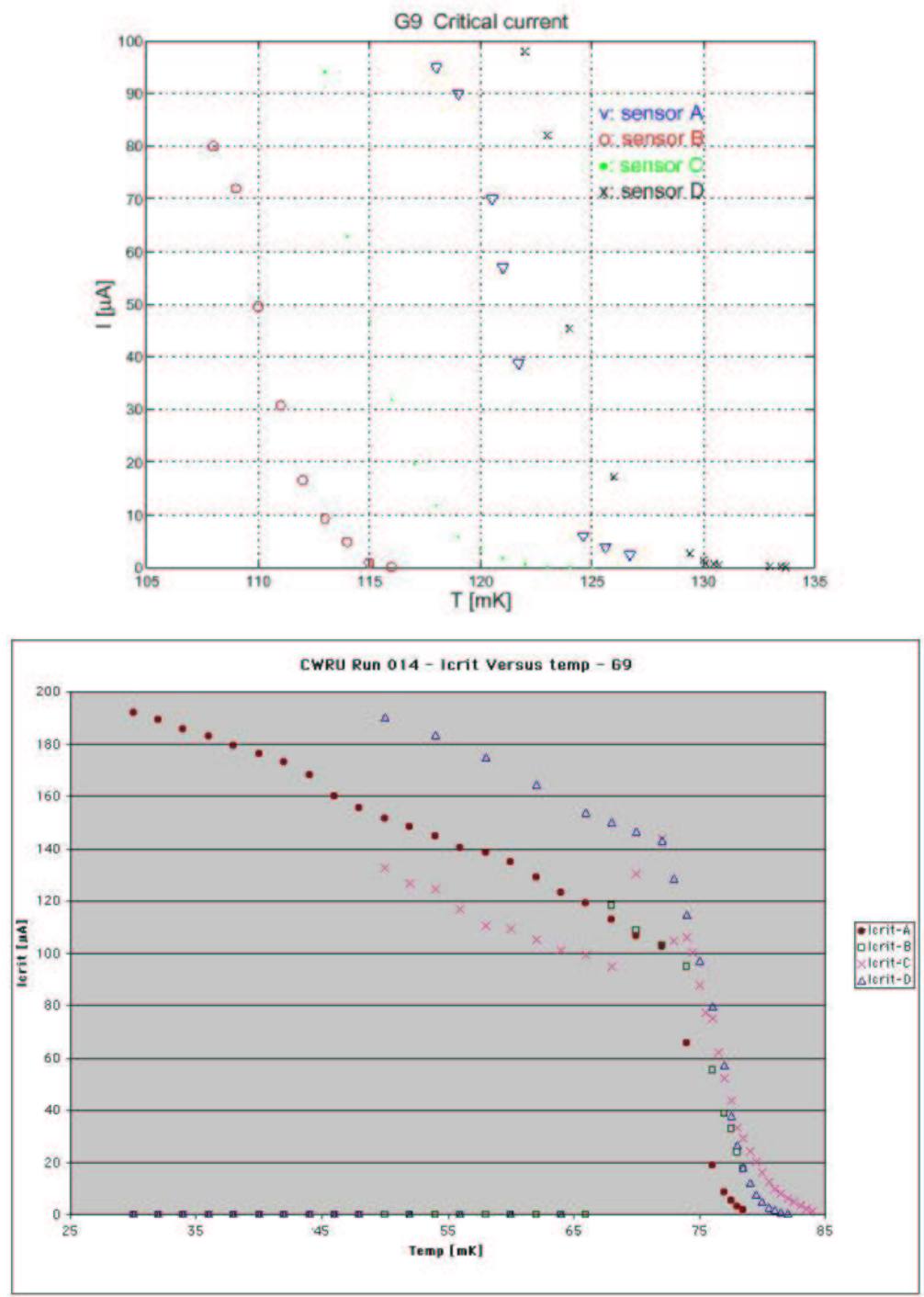

Figure 4.11: Critical Current Curves for Detector Z5 (G9). The top graph shows the critical current curves for G9 before implantation(UCB Run270). A $T_{c}$ gradient is indicated as the four quadrants (Sensors A-D) have curves that are separated by $\sim 15 \mathrm{mK}$. The bottom graph is for the critical currents after implantation(CWRU R14). These curves are much more uniform and have a gradient of less than $10 \mathrm{mK}$.

temperature. The first number represents the temperature at which the triangle wave output starts to differ from the superconducting value. This does not 

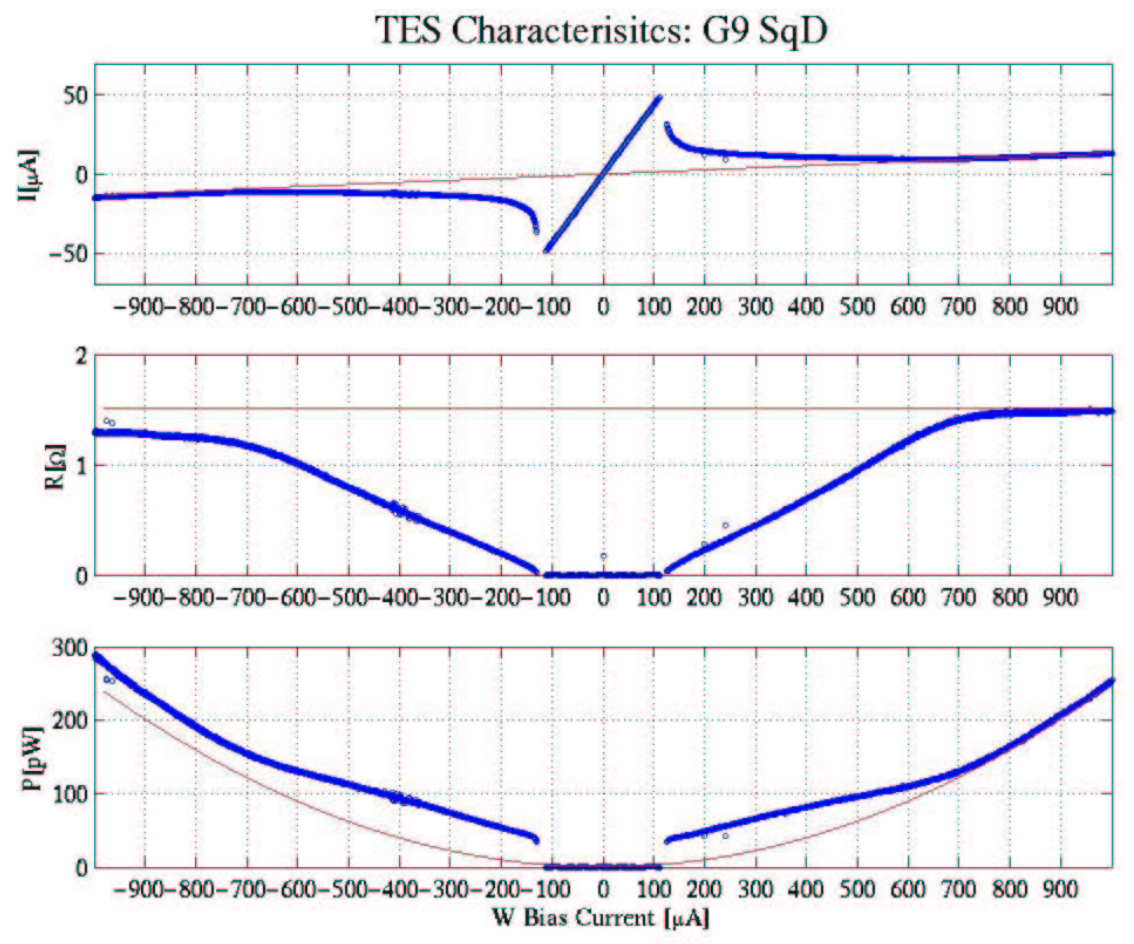

Figure 4.12: IbIs curves for detector G9 (Z5 in tower I), channel D (post-implant). The dark blue circles represent the data. The $\mathrm{x}$-axis in all three curves is the bias current $I_{b}$. The top graph is of the current measured in the sensor. The red line is a linear fit to the part of the curve where the large bias current has driven the sensor normal, as seen in the calculated graph of resistance-vs-bias current (middle). Near zero bias current, the slope of the sensor current represents a small parasitic resistance $(\sim 10 \mathrm{~m} \Omega)$. The bottom graph is of the calculated power dissipation in the sensor, which is parabolic in the normal region $\left(P=I^{2} R\right)$. For sufficiently narrow transitions, the power should be constant in the transition region. The fact that the power is curved within the transition region reflects two things: 1. There is a $T_{c}$ gradient in that quadrant; 2 . The TES transition range has TES dynamic information, which needs to be better understood.

happen at the temperature of the lowest $T_{c}$ meanders, but rather when the input triangle wave starts to drive normal the few remaining sensors at the top of the R-T curve. The second number is the point at which the triangle wave output reaches its normal value. 
Another useful piece of TES information in the evaluation of the ZIP detecttor is critical current versus temperature. It's the current required to drive each sensor to normal (critical current) at the base temperature of the dilution refrigerator, which was about $20 \mathrm{mK}$ during these measurements. Figure 4.11 shows how the critical currents changed with temperature for ZIP detector G9. Measurements of critical current as a function of refrigerator temperature can be used to estimate the variation of transition temperatures within a sensor.

There are 888 TES meanders in each phonon sensor (channel). The four phonon sensors distribute on the detector surface in the area of $40 \mathrm{~cm}^{2}$. It is challenging to make uniform phonon sensors. The compensation method is to adjust TES transition temperature according to the diagnostic test results by ion implantation. These diagnostic results are mainly transition temperatures and critical currents. These allow Paul Brink and Betty Young at CIS to map out the transition temperature distribution pattern, and decide the ion implantation pattern and implantation doses for different areas. Detailed information of TES transition temperature gradient mapping and TES implantation can be found in references $[102,81]$.

Measurements of sensor current (Is) versus bias current (Ib) at base temperature provide another powerful sensor diagnostic. These $I_{b}-I_{s}$ data for the G9, channel $\mathrm{D}$ are shown in figure 4.12. Data was obtained by input current $I_{b}$ sweeping through the $5 \mathrm{~V}$ DAC in a very slow mode in order to keep the TES close to the thermal equilibrium state. This data acquisition and analysis package was developed by Tarek Saab [81].

There are three regions in the $I_{b}-I_{s}$ curve. When TES is in its normal state, 
the relation between $I_{s}$ and $I_{b}$ is linear:

$$
I_{s}=\frac{R_{s h}}{R_{T E S}} I_{b}
$$

The second state is biased. If the TES transition temperature is narrow and there is no superconducting normal phase separation occurring, an approximation relation under constant TES power dissipation $P$ is

$$
P=\frac{R_{s h}^{2} I_{b}^{2}}{R_{T E S}} .
$$

When the bias current is sufficiently low, and TES is in a superconducting state, then

$$
I_{s}=\frac{R_{s h}}{R_{\text {para }}+R_{s h}} I_{b}
$$

\subsection{TES thermal parameters}

The phonon pulse shape is determined by the thermal parameters and the arriving phonon energy flux as a function of time. Section 4.3.3 tells us that the start time and rise time of phonon pulse depend on the particle interaction type and location in the ZIP detector. The information in TES thermal parameters will directly lead to the full understanding of phonon pulse as a whole, for example, the relation between phonon pulse height and TES heat capacity, the phonon pulse relaxation time, etc. These will help us to increase phonon energy measurement resolution, and have uniform phonon timing parameter distribution. The direct results of these are better background rejection capability of the CDMS ZIP detector.

To study the TES thermal properties, we need to look into the TES dynamic range, i.e., the TES superconducting transition range. The whole TES dynamic is in the biased region, as mentioned in the last section. TES thermal parameters, 
such as the thermal coupling coefficient $\kappa$ between the TES sensor the substrate crystal, TES heat capacity $C$, etc, can be extracted with the $I_{b}-I_{s}$ relation plus the $R-T$ relation of phonon sensors in the superconducting transition range.
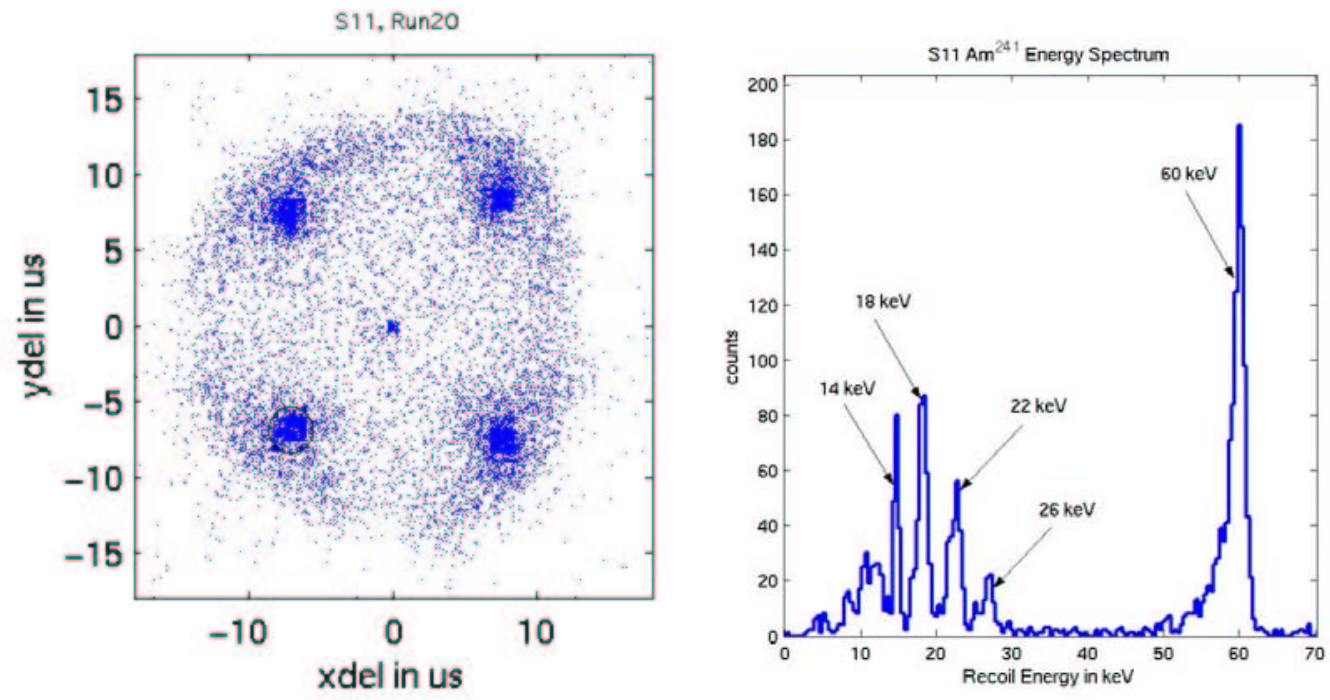

Figure 4.13: Americium source spots (the left graph)and energy spectrum from S11 (the right graph)in CWRU run20.

At CWRU, we tested the thermal properties of silicon detector S11 with and without elevated external bias voltage. The investigation of the thermal properties and possible application as a neutrino magnetic moment measurement detector using a silicon ZIP detector with elevated bias voltage (10-200 volts) has been summarized by Dan Akerib [103]. The summary here is the research work on basic TES properties measurement with no elevated external bias voltage in this section.

Four ${ }^{241} A m$ sources were used in the CWRU test facility with S11. The source spots and energy spectrum are shown in Figure 4.13.

$I_{b}-I_{s}$ data were taken at $26 \mathrm{mK}$ in the slow mode. Each step equals a $4.9 \mu \mathrm{A}$ input current. The wait time for each step is $1 \mathrm{~ms}$, which is long enough compared 
to the TES thermal time constant (from $100 \mu s$ to $200 \mu s$, estimated from the fall time of phonon pulses) so that the TES is in equilibrium with the substate. $R-I_{b}$ is calculated by using $R=\left(\frac{I_{b}}{I_{s}}-1\right) \times R_{s h}$. See Figure 4.14 for sensor A, and Figure 4.15 for sensor B.
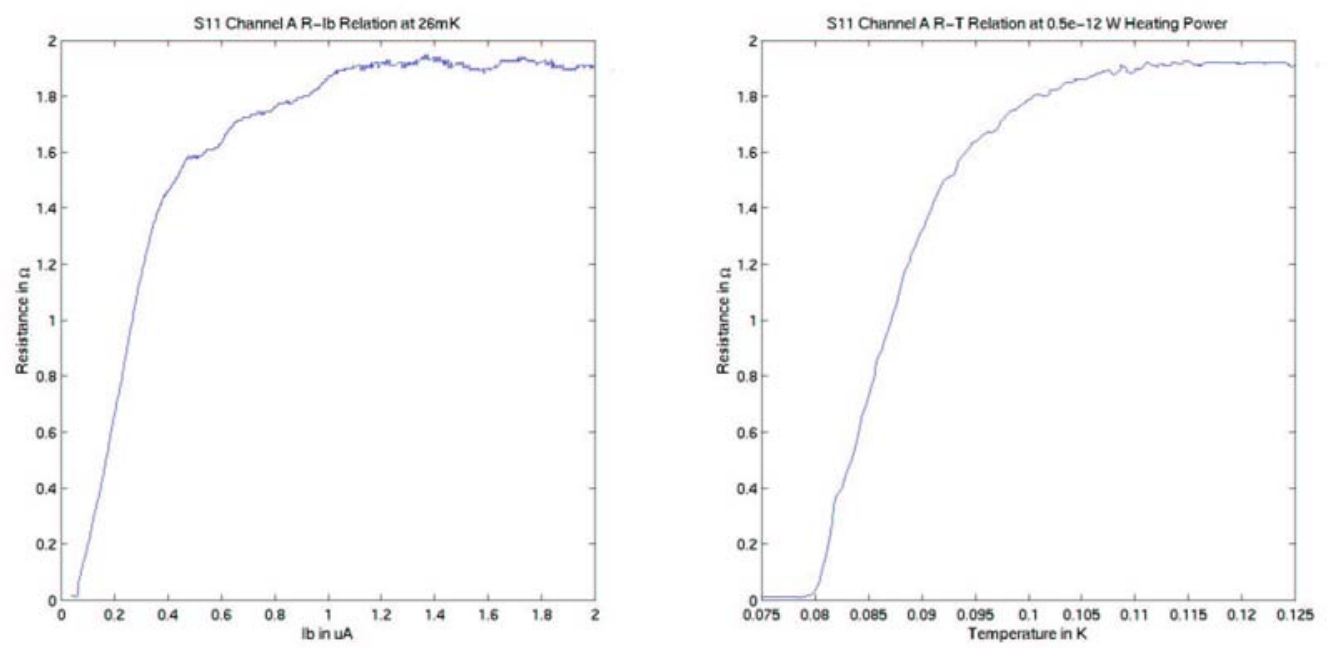

Figure 4.14: The measured $R-I_{b}$ (the left graph) and the measured $R-T$ relations (the right graph) of S11, sensor A.

The second piece of information we need to determine the thermal parameters is the $R-T$ relation of the TES. At low constant excitation power, $P_{0}=0.5 p W$, we measured the $R-T_{0}$ relation. $T_{0}$ is the TES temperature corresponding to $R$ at the excitation power. This curve was obtained in a very slow mode. I started the VI before I left for the day, and let it finish overnight. $T_{0}$ is close to the detector crystal temperature.

There is a $1 \mathrm{~K}$ resistor in series with the electro-thermal feedback circuit(Figure 4.9) in the current input line. A $12 \mathrm{bit} \pm 5 \mathrm{~V}$ DAC is the power source, so the input current step is $2.44 \mu \mathrm{A}$. The $0.5 \mathrm{pW}$ controlled heating power error is $3.3 \%$, when TES resistance is $100 \mathrm{~m} \Omega$. When TES resistance is high, the heating power error 

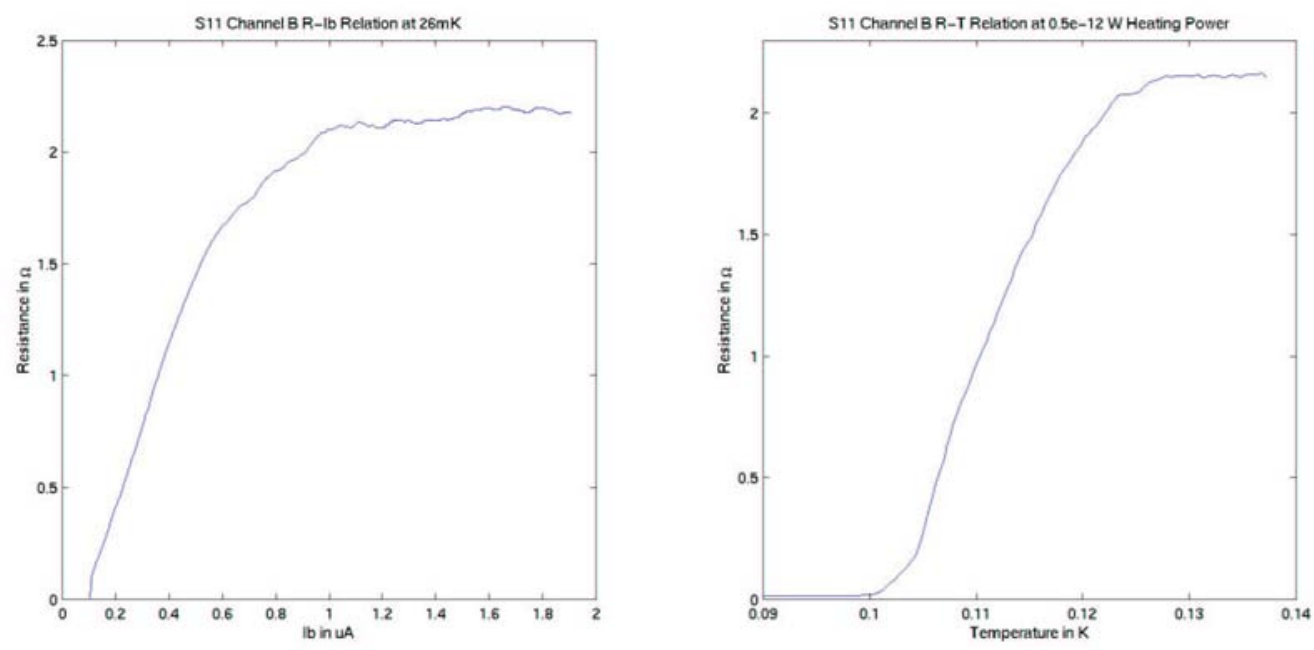

Figure 4.15: The measured $R-I_{b}$ (the left graph) and the measured $R-T$ relations (the right graph) of S11, sensor B.

comes from the output line. A 12bit Joerger collected the data. The SQUID output feedback resistor has a value of $1000 \Omega$. We count the SQUID input and output inductance ratio 10 , so the output current step is $0.09765 \mu \mathrm{A}$. To have a precision control, a 2.85 gain DC amplifier is used before the Joerger. The actual current measurement step is $0.0343 \mu A$. The $0.5 \mathrm{pW}$ controlled heating power error is $5.0 \%$ when TES resistance is $1.0 \Omega$. The measured $R-T_{0}$ is in Figure 4.14 for sensor A, and Figure 4.15 for sensor B.

With the two pieces of information, $R-T_{0}$ and $R-I_{b}$, we can calculate the thermal coupling constant $\kappa$. For a TES resistance $R$ at $T_{0}$,

$$
P_{0}=\kappa\left(T^{n}-T_{0}^{n}\right) .
$$

Corresponding to $I_{b}$ at base temperature $T_{s}$

$$
P=\kappa\left(T^{n}-T_{s}^{n}\right) .
$$


The Joule heating power is

$$
P=\frac{I_{b}^{2} R_{s h}^{2}}{\left(R+R_{s h}\right)^{2}} R .
$$

Putting equations $4.41,4.42$, and 4.43 together, the thermal coupling constant becomes

$$
\kappa=\left(\frac{I_{b}^{2} R_{s h}^{2}}{\left(R+R_{s h}\right)^{2}} R-P_{0}\right) \frac{1}{T_{0}^{n}-T_{s}^{n}} .
$$

The TES temperature is

$$
T=\left(T_{0}^{n}+\frac{P_{0}}{\kappa}\right)^{1 / n}
$$

Let

$$
\beta_{0}=\frac{d R}{d T_{0}}
$$

The resistance temperature slope is

$$
\beta=\frac{1}{T_{0}^{n-1}}\left(T_{0}^{n}+\frac{P_{0}}{\kappa}\right)^{\frac{n-1}{n}} \beta_{0} .
$$

We define the TES quality factor

$$
\alpha=\frac{T}{R} \frac{d R}{d T}
$$

Then

$$
\alpha=\frac{1}{R T_{0}^{n-1}}\left(T_{0}^{n}+\frac{P_{0}}{\kappa}\right) \beta_{0} .
$$

In reference to equation 4.31 , for small delta pulses,

$$
\Delta I_{s}=\frac{I_{s 0}}{1+\frac{\left(R+R_{s h}\right) C}{\beta Q}}
$$

We define

$$
c q=\frac{C}{Q}
$$

Then

$$
c q=\left(\frac{I_{s 0}}{\Delta I_{s}}-1\right) \frac{\beta}{R} .
$$


There is a ${ }^{241} \mathrm{Am}$ source in the middle of each phonon sensor. The amount of collected phonon energy in the location quadrant for a typical energy line (say a $60 \mathrm{keV}$ line) at a constant external bias voltage is the same. This is why we need quantity $c q . c q$ is a measure of TES heat capacity. It tells us how the heat capacity changes with bias current $I_{b}$ in ${ }^{241} \mathrm{Am}$ data.

From equation 4.36 , the actual phonon pulse fall time is

$$
\tau_{2}=\tau \ln \left(e+(e-1) \frac{\beta}{R} \frac{1}{c q} .\right.
$$

Then we have

$$
\tau=\frac{\tau_{2}}{\ln \left(e+(e-1) \frac{\beta}{R} \frac{1}{c q}\right.} .
$$

Here $\tau$ is defined in equation 4.28. The TES heat capacity is

$$
C=\tau \kappa T^{n-1}(n+\alpha) .
$$

$I_{s 0}$ is calculated with equation 4.35. $\Delta I_{s}$ is extracted from the phonon pulse height. At phonon channel gain $20, \Delta I_{s}=0.09765 \times \frac{V_{\text {out }}}{20} . V_{\text {out }}$ is in Joerger bins.

$\tau_{2}$ is the phonon pulse fall time in data. So far, all the TES thermal parameters can be calculated with $R-T_{0}, R-T_{b}$, and the external source data at different TES bias currents.

The thermal parameters, thermal coupling constant $\kappa$, resistance-temperature slope $\beta$, TES quality factor $\alpha$, TES heat capacity-phonon energy ratio $c q$, and TES heat capacity $C$ are calculated with measured detector temperature $T_{0}$, measured TES resistance $R$, measured phonon pulse height $\Delta I_{s}$, and measured phonon pulse fall time $\tau_{2}$ at a given TES bias current with ${ }^{241} \mathrm{Am}$ data at the $60 \mathrm{keV}$ line under 0 volts bias. The measured quantities and calculated thermal parameters for detector S11 sensor A are summarized in Table 4.4; for S11 sensor B they are summarized in table 4.5 . 


\begin{tabular}{|c|c|c|c|c|c|c|c|}
\hline \hline parameter & \multicolumn{7}{|c|}{ Values } \\
\hline$I_{b}(\mu A)$ & 66.0 & 88.0 & 110.0 & 132.0 & 154.0 & 231.0 & 319.0 \\
\hline$T_{0}(\mathrm{mK})$ & 80.2 & 80.6 & 81.1 & 81.8 & 82.5 & 85.6 & 89.0 \\
\hline$\kappa\left(10^{-6} W / K^{5}\right)$ & 4.45 & 4.72 & 4.83 & 4.83 & 4.97 & 5.07 & 5.53 \\
\hline $\mathrm{T}(\mathrm{mK})$ & 80.8 & 81.1 & 81.6 & 82.3 & 83.0 & 86.0 & 89.3 \\
\hline $\mathrm{R}(\Omega)$ & 0.068 & 0.144 & 0.235 & 0.341 & 0.445 & 0.855 & 1.255 \\
\hline$\beta_{0}(\Omega / \mathrm{K})$ & 189.5 & 177.4 & 164.5 & 152.1 & 144.3 & 128.4 & 101.5 \\
\hline$\beta(\Omega / \mathrm{K})$ & 193.8 & 181.6 & 168.2 & 155.4 & 147.2 & 130.5 & 102.8 \\
\hline$\alpha$ & 230.2 & 102.3 & 58.4 & 37.5 & 27.4 & 13.2 & 7.3 \\
\hline$I_{s 0}(\mu A)$ & 15.34 & 10.73 & 8.63 & 7.31 & 6.62 & 5.82 & 5.00 \\
\hline$\Delta I_{s}(\mu A)$ & 1.479 & 0.996 & 0.863 & 0.767 & 0.725 & 0.710 & 0.511 \\
\hline $\mathrm{cq}\left(10^{4} / K\right)$ & 2.671 & 1.233 & 0.644 & 0.389 & 0.269 & 0.098 & 0.072 \\
\hline$\tau_{2}\left(10^{-6} s\right)$ & 165 & 156 & 122 & 131 & 144 & 220 & 238 \\
\hline$\tau\left(10^{-6} s\right)$ & 155 & 147 & 114 & 122 & 134 & 201 & 222 \\
\hline $\mathrm{C}\left(10^{-12} J / K\right)$ & 6.901 & 3.220 & 1.553 & 1.152 & 1.023 & 1.011 & 0.964 \\
\hline
\end{tabular}

Table 4.4: S11 sensor A, thermal parameters. Index $\mathrm{n}=5 .{ }^{241} A m 60 \mathrm{keV}$ is used for phonon pulse fall time and phonon pulse amplitude $\Delta I_{s}$ under $0 \mathrm{~V}$ external bias voltage. $I_{b}$ is the independent parameter.

\begin{tabular}{|c|c|c|c|c|c|c|c|}
\hline \hline parameter & \multicolumn{7}{|c|}{ Values } \\
\hline$I_{b}(\mu A)$ & 120.0 & 144.0 & 168.0 & 192.0 & 216.0 & 300.0 & 432.0 \\
\hline$T_{0}(\mathrm{mK})$ & 103.3 & 104.1 & 104.8 & 105.5 & 106.1 & 108.3 & 112.5 \\
\hline$\kappa\left(10^{-6} W / K^{5}\right)$ & 2.65 & 2.61 & 2.63 & 2.62 & 2.74 & 2.93 & 3.18 \\
\hline $\mathrm{T}(\mathrm{mK})$ & 103.6 & 104.4 & 105.1 & 105.8 & 106.4 & 108.5 & 112.7 \\
\hline $\mathrm{R}(\Omega)$ & 0.139 & 0.214 & 0.294 & 0.385 & 0.460 & 0.775 & 1.253 \\
\hline$\beta_{0}(\Omega / \mathrm{K})$ & 78.0 & 106.7 & 124.8 & 136.1 & 140.8 & 132.1 & 99.9 \\
\hline$\beta(\Omega / \mathrm{K})$ & 79.0 & 108.0 & 126.0 & 137.7 & 142.3 & 133.3 & 100.6 \\
\hline$\alpha$ & 58.9 & 52.7 & 45.2 & 37.8 & 32.9 & 18.7 & 9.1 \\
\hline$I_{s 0}(\mu A)$ & 15.09 & 12.31 & 10.70 & 9.48 & 9.00 & 7.55 & 6.79 \\
\hline$\Delta I_{s}(\mu A)$ & 0.932 & 0.775 & 0.677 & 0.619 & 0.595 & 0.595 & 0.546 \\
\hline $\mathrm{cq}\left(10^{4} / K\right)$ & 0.8634 & 0.7512 & 0.6360 & 0.5120 & 0.4370 & 0.2011 & 0.0918 \\
\hline$\tau_{2}\left(10^{-6} s\right)$ & 97 & 122 & 17 & 147 & 155 & 171 & 205 \\
\hline$\tau\left(10^{-6} s\right)$ & 93.2 & 117.1 & 1121.9 & 140.9 & 148.5 & 162.4 & 194.5 \\
\hline $\mathrm{C}\left(10^{-12} J / K\right)$ & 1.850 & 2.098 & 1.958 & 1.975 & 1.972 & 1.564 & 1.400 \\
\hline
\end{tabular}

Table 4.5: S11 sensor B, thermal parameters. Index $\mathrm{n}=5 .{ }^{241} A m 60 \mathrm{keV}$ is used for phonon pulse fall time and phonon pulse amplitude $\Delta I_{s}$ under $0 \mathrm{~V}$ external bias voltage. $I_{b}$ is the independent parameter. 

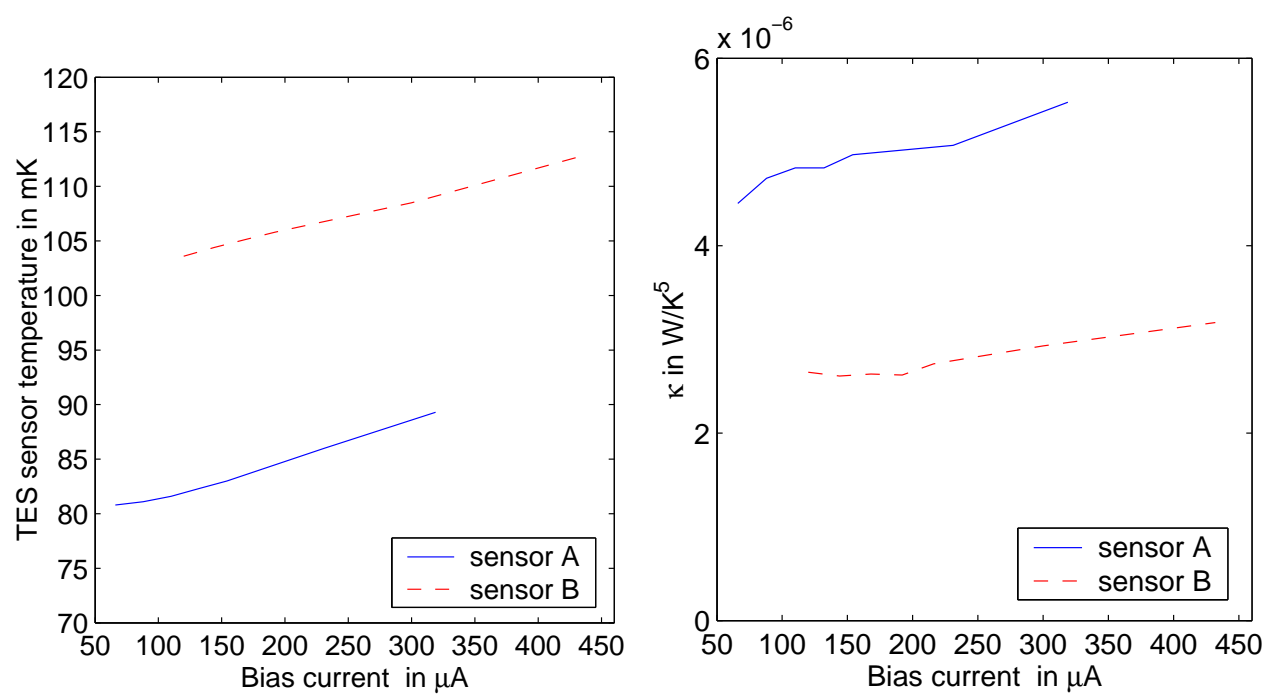

Figure 4.16: S11, TES temperature (the left graph) and thermal coupling coefficients (the right graph) at different bias currents.
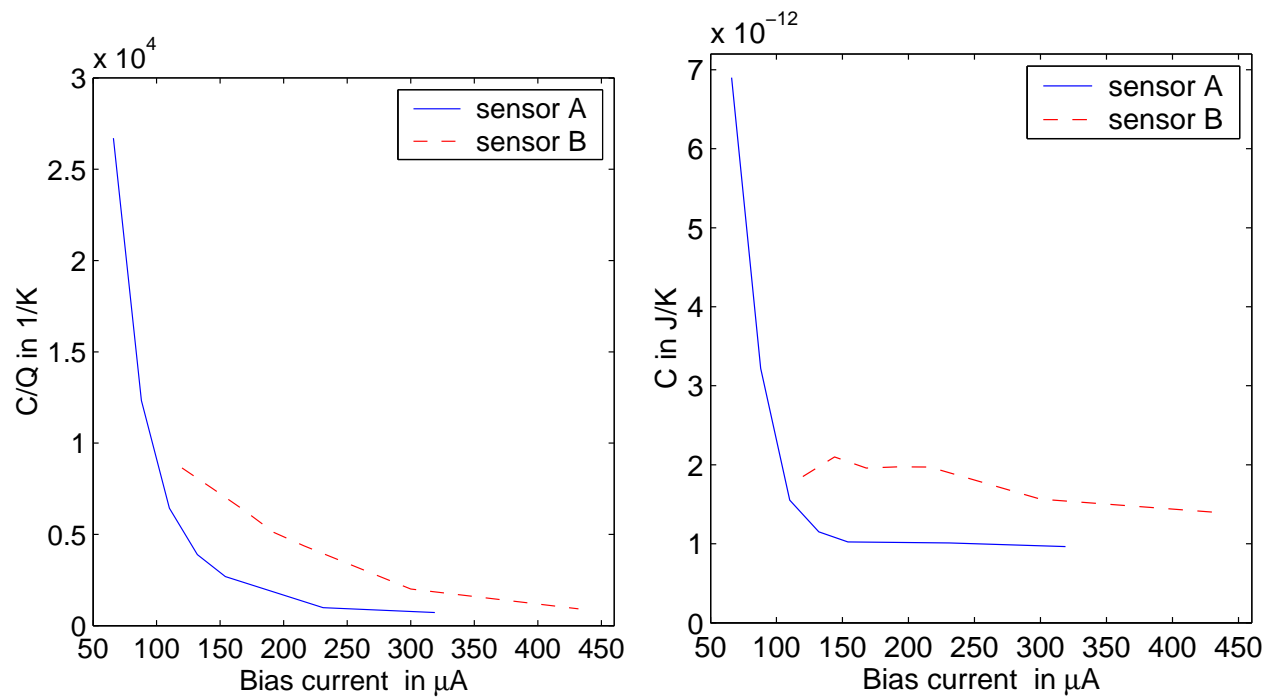

Figure 4.17: S11, TES heat capacity parameters at different bias currents.

$T-I_{b}$ and $\kappa-I_{b}$ plots are in Figure 4.16. There is a very weak bias current dependence of thermal coupling constant $\kappa$. But the differences in the thermal coupling constant $\kappa$ between phonon sensors are clear. There are two possible reasons for the thermal coupling coefficient difference between sensor A and sensor 
B: one is the amorphous silicon layer gradient; another is the index $n$ in equations 4.41 and 4.42. When $n=2$, the values of $\kappa$ are close to each other in different channels [104].

The $c q-I_{b}$ and $C-I_{b}$ plots are in Figure 4.17. Since the ${ }^{241} A m$ source is right in the middle of each sensor, the local quadrant phonon pulse height of the $60 \mathrm{keV}$ line is a good measure of the TES heat capacity at a given TES bias. $c q$ tells us how TES heat capacity changes with TES bias current $I_{b}$. The plot on the right has the absolute value of the TES heat capacity, but because the phonon pulse fall time parameter is used, the calculation error is bigger. For example, there is an uncertainty of $C$ at $I_{b}=120 \mu A$ for sensor B.

For the first time, we have the values of TES thermal parameters. These parameters are very useful in helping us to understand the CDMS detector TES biases and phonon timing parameters. The first application of TES thermal parameters is in understanding of how to choose the TES bias current to have uniformly distributed phonon timing parameters, and uniformly distributed collected phonon energy in the four phonon sensors on the surface of the ZIP detector. 


\section{Chapter 5}

\section{Event Location Information in ZIP Detectors}

\subsection{Introduction}

Event location information helps us to understand the collected phonon energy distribution and phonon timing parameter distributions in the ZIP detector. In the conventional delay plot of a CDMS ZIP detector (see Section 6.2), a radial degeneracy exists for charge inner electrode events that are near the charge outer electrode. The reconstructed $\mathrm{x}$ and $\mathrm{y}$ parameters described in this chapter break this degeneracy. With event $\mathrm{x}$ and $\mathrm{y}$ known, we can use the phonon energy distribution in the four phonon sensors on the surface of the ZIP detector and the phonon timing parameters together to reject surface events more effectively.

The reconstructed $\mathrm{z}$ parameter described in this chapter is based on the local phonon sensor and two neighbor phonon sensors' timing information. It can be used as a surface rejection parameter together with a reconstructed radial parameter $R=\sqrt{x^{2}+y^{2}}$. The reconstructed $\mathrm{z}$ parameter is not a true measure of 
the $\mathrm{z}$ coordinate of the event in that surface events from both sides of the ZIP detector have reconstructed $\mathrm{z}$ close to 0 . However, events from the bulk of the ZIP detector have bigger values of the reconstructed $\mathrm{z}$, and so a cut can be placed on $\mathrm{z}$ to discriminate bulk from surface event.

The ZIP detector event position reconstruction needs two classes of inputs. The first class of inputs come from phonon physics modelling, and include the phonon energy spectrum and phonon front propagation time, both as a function of event location. The phonon propagation time to each phonon sensor can be calculated with the specific phonon physics model for a given event at a particlular location in the ZIP detector. The second class of inputs are the measured phonon timing parameters in the local quadrant phonon sensor and in the two neighbor quadrant phonon sensors.

The ZIP detector event position reconstruction algebra is described in this chapter. I will start with general phonon properties in the ZIP detector, such as the optical phonon decay time and ballistic phonon propagation in Section 2. Neganov-Luke phonon distributions are in Section 3. With the phonon energy spectrum known, Sections 4 and 5 have the details of event reconstruction. Features of event location information are summarized in Section 6.

\subsection{Primary Phonons}

As discussed in the last chapter, the primary phonons refer to phonons from a particle interaction in a ZIP detector, as well as the phonons from hot charge carriers' thermalization in the interaction. The primary phonon propagation is a phonon down conversion process, i.e., high frequency phonons decay into lower frequency phonons, until the phonons are ballistic. The time that the optical 
phonons takes to decay into one $\mathrm{THz}$ ballistic phonons is in the $\mu s$ range, while the corresponding phonon propagation distance is from $1 \mathrm{~mm}$ to $2 \mathrm{~mm}$ [105].

Phonon decay is a phonon-impurity or phonon-anharmonic-potential scattering process. Two kinds of impurities must be distinguished in a ZIP detector when considering long wave optical phonon scattering. The first kind of impurities have a rigid electronic structure with excitation energies of the order of the atomic energy. Such impurities are closed shell atoms with all the valence electrons participating in chemical bonding with neighbors (ionized donors and acceptors, isotopes). The second kind of impurities have a soft electronic structure with low lying excited levels. Such impurities are unfilled shell ions, neutral donors and acceptors, and surface defects. This means the phonon scattering rate changes significantly for the shallow impurities. The scattering rate can be 4 orders higher for the shallow impurities based on Rayleigh scattering calculations [106]. This is an important characteristics that has been used in the CDMS surface event rejection algorithm. Because there are hanging chemical covalent bond on the surface of a ZIP detector, the optical phonons can decay into ballistic phonons immediately for surface events.

Perera did Monte Carlo simulation [80] for the the size of electron and hole cloud originated from a particle interaction in CDMS semiconductor detectors. The electrons and holes' spatial distribution from the original particle interaction is called a clump. It is initiated by either an interaction of any particle other than an electron or by the interaction of an electron incident on a detector. Figure 5.1 shows that the clump size is about $7 \mu m(27 \mu m)$ in $\mathrm{Ge}(\mathrm{Si})$ for $100 \mathrm{keV}$ recoil energy deposition. So, the initial interaction size of a particle in Ge or Si is small, and has no significant effect on phonon propogation.

For a particular particle interaction in a ZIP detector, there is no direction 


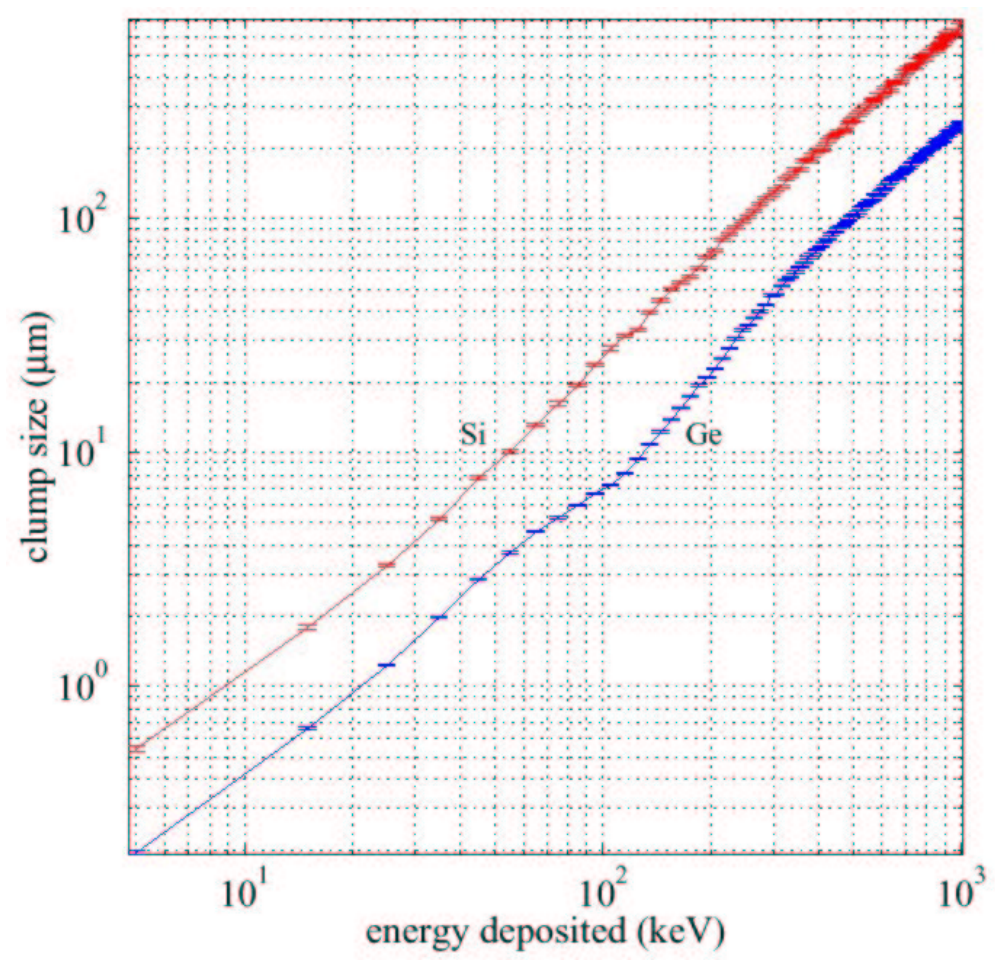

Figure 5.1: Average size of electrons and holes cloud of an electron recoil as a function of energy. Figure from [80].

information left, because of Umklapp processes [107]. In general, the primary phonons are treated as isotropic.

When a germanium ZIP detector is biased with 3 volts, the total phonons should be $E_{r}+\frac{e V}{\epsilon} E_{r}=2.0135 E_{r}$ for electron recoils, but the Neganov-Luke phonon is dominant. It is $\frac{e V}{\epsilon} E_{r}=1.0135 E_{r}$. The electron hole pair recombination phonon energy is $\frac{E_{R}}{\epsilon} E_{r}=0.2432 E_{r}$, so the primary phonon's contribution is only $0.7568 E_{r}$, about $37.6 \%$ of the phonon total. Here $E_{r}$ is recoil energy, and $\epsilon=2.96 \mathrm{eV}$.

In summary, the primary phonons in a ZIP detector can be treated as isotropic ballistic phonons at one $\mathrm{cm}$, the length scale in which we are interested. The possible error is less than $2 \mathrm{~mm}$ in length. Before we start event location reconstruction, 
we need to examine the Neganov-Luke phonon distribution in section 5.3.

\subsection{Neganov-Luke Phonons}

Blas Cabrera summarized the dynamics of electrons and holes, as well as NeganovLuke phonons, in his "Electron and phonon scattering" [94], which contained two important conclusions for the Neganov-Luke phonons in semiconductors. First, for phonon emission, the electron velocity must be greater than the sound speed in the crystal; otherwise, there is no phonon emission. This is analogous to Cherenkov radiation. Second, Neganov-Luke phonons go forward in reference to the electron traveling direction. The angle between the electron and the emitted phonon must satisfy the energy and momentum conservation equations.

For a two body interaction, energy conservation and momentum conservation are reflected in the electron wave vector and phonon wave vector relation,

$$
\begin{gathered}
q=2\left(k \cos \theta-k_{s}\right) \\
k_{s}=\frac{m s}{\hbar}
\end{gathered}
$$

where $\mathrm{q}$ is the phonon wave vector, $\mathrm{k}$ is the electron wave vector, $\theta$ is the angle between the electron and the phonon, $m$ is the electron effective mass, and $s$ is the sound speed.

For later use, let's define $\alpha$ as the angle between the electron wave vector and

$\mathrm{Z}, \beta$ as the polar angle of the electron wave vector, $\chi$ as the angle between the phonon wave vector and $\mathrm{Z}$, and $\delta$ as the polar angle of the phonon wave vector. $\mathrm{Z}$ points to phonon sensors on the surface of the ZIP detector. See figure 5.2.

For the low frequency Luke phonons (below $1 \mathrm{THz}$ ), the dispersion relation satisfies the long acoustic wave approximation, i.e., $\omega$, the phonon angular frequency, 


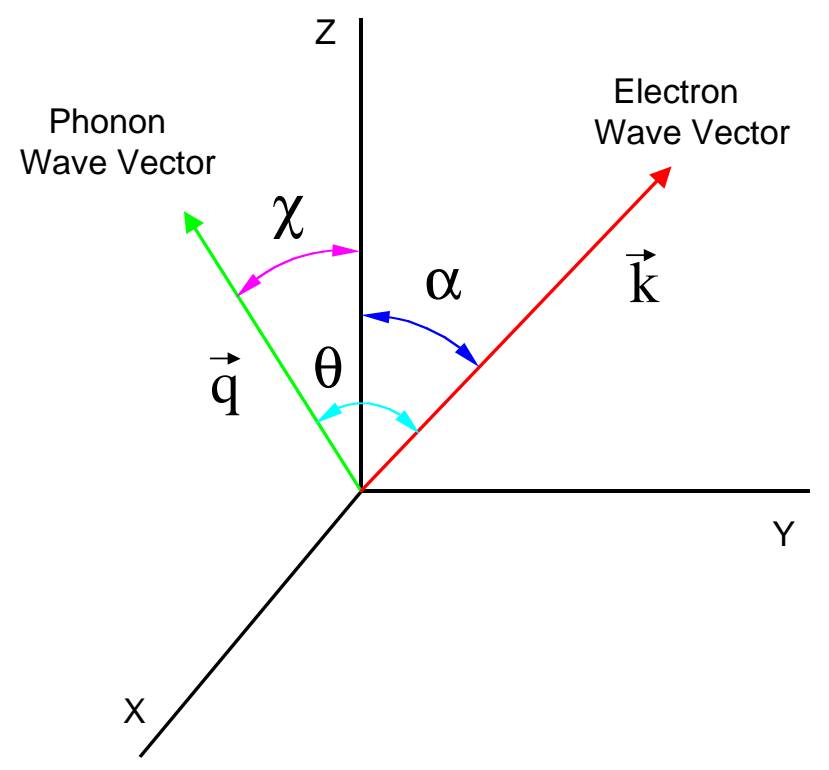

Figure 5.2: Diagram of electron and phonon wave vectors.

is a linear function of the phonon wave vector:

$$
\omega=s q
$$

The drifting electrons emit phonons by interacting with the deformation potential of the germanium crystal. The probability that an electron $\vec{k}$ emits a phonon $\vec{q}$ is given by the Fermi Golden rule

$$
P=\frac{2 \pi}{\hbar}|<\vec{k}-\vec{q}| H|\vec{k}>|^{2} \delta\left(E-\left(E^{\prime}+\hbar \omega\right)\right)
$$

where $H$ is the deformation potential, $E=\frac{\hbar^{2} k^{2}}{2 m}$ is electron kinetic energy before scattering, and $E^{\prime}=\frac{\hbar^{2}(\vec{k}-\vec{q})^{2}}{2 m}$ is the electron kinetic energy after scattering. The probability can be rewritten as [94]:

$$
P=\frac{2 \pi}{\hbar} \frac{E_{1}^{2} \hbar \omega}{2 V \rho s^{2}}\left(n_{q}+1\right) \frac{m}{\hbar^{2} k q} \delta\left(\cos \theta-\cos \theta_{0}\right)
$$


where the deformation potential $E_{1}=13.3 \mathrm{eV}$ for electrons in germanium, and $n_{q}$ is the thermal equilibrium phonon distribution, which is a Planck distribution. We suppose the probability to have phonons above the sensor energy threshold is zero at $40 \mathrm{mK}$ because of the extremely low excitations. $V$ is the semiconductor crystal volume, $\cos \theta_{0}=\frac{q^{\prime}}{k}$, and $q^{\prime}=\frac{q}{2}\left(1+\frac{2 m s}{\hbar q}\right)$.

The drifting electrons or holes are not in thermal equilibrium with the semiconductor crystal, but statistically speaking, they are in a temporary steady state with an average drifting velocity in the external electric field. The displaced Boltzmann distribution is used for the electron distribution in the external electric field [108]:

$$
f(\vec{k})=\left(\frac{h^{2}}{2 \pi m k_{B} T_{e}}\right) \exp \left(-\frac{\hbar^{2}\left(\vec{k}-\overrightarrow{k_{d}}\right)^{2}}{2 m k_{B} T_{e}}\right)
$$

where $\overrightarrow{k_{d}}$ is the characteristic electron drifting velocity in the external electric field, $T_{e}$ is electron effective temperature, and $k_{B}$ is the Boltzmann constant. It can be confirmed by integration that

$$
\hbar k_{d}=\int_{0}^{\infty} \int_{0}^{\pi} f(\vec{k}) \hbar k \cos \alpha \sin \alpha d \alpha 2 \pi k^{2} d k
$$

where $\hbar k_{d}$ is the average momentum of an electron in a semiconductor. It becomes apparent that

$$
\hbar k_{d}=m V_{d}
$$

where $V_{d}$ is the electron average drifting velocity in the external electric field.

The phonon rate of emission of phonons with wavevector $q$ into a particular direction $\chi$ and $\delta$ is

$$
P_{q}=\frac{V}{(2 \pi)^{3}} \int P f(\vec{k}) \sin \alpha d \alpha d \beta k^{2} d k .
$$

In expanded form, this is

$$
P_{q}=2 \pi\left(\frac{h^{2}}{2 \pi m k_{B} T_{e}}\right)^{3 / 2}
$$




$$
\begin{aligned}
& \int_{k_{0}}^{\infty} \int_{0}^{2 \pi} P \exp \left(-\frac{\hbar^{2} k_{d}^{2}}{2 m k_{B} T_{e}}\right) \exp \left(-\frac{\hbar^{2} k^{2}}{2 m k_{B} T_{e}}\right) \\
& \exp \left(\frac{\hbar^{2} k k_{d} \cos \alpha_{0}}{2 m k_{B} T_{e}}\right) d \eta k^{2} d k
\end{aligned}
$$

where $\cos \alpha_{0}=\cos \chi \cos \theta-\sin \chi \sin \theta \cos \eta$. Where $\eta$ is an integration parameter, it ranges from 0 to $2 \pi$. Note that $\cos \theta=\frac{q-k_{s}}{2 k}$ is known for a given electron wave vector $\vec{k}$ and the emitted phonon wave vector $\vec{q}$, and $k_{0}=\frac{q+k_{s}}{2}$.

There is one more parameter not determined yet: the effective electron temperature $T_{e}$. Again we use momentum conservation: in unit time, the total momentum of the emitted phonons equals the momentum that electron can get from the external electrical field

$$
e E=\int_{0}^{\infty} \int_{0}^{\pi} P_{q} \hbar q \cos \chi \sin \chi d \chi 2 \pi q^{2} d q
$$

and energy conservation: in unit time, the total phonon energy of the emitted phonons equals the energy that the electron can get from external electrical field

$$
e E V_{d}=\int_{0}^{\infty} \int_{0}^{\pi} P_{q} \hbar \omega \cos \chi \sin \chi d \chi 2 \pi q^{2} d q
$$

where $E$ is the external electric field. $E=\frac{V}{D}, V$ in volts is the bias voltage, and $D$ in meters is the ZIP detector thickness.

For a given electron drift velocity, there is an electron effective temperature and electric field relation, obtained by using momentum conservation equation 5.11. There is another electron effective temperature and external electrical field relation that comes from energy conservation equation 5.12. These two curves cross each other; the cross point gives the effective electron temperature under the external electric field.

On the other hand, the average electron velocity can be calculated with known $T_{e}$ by using equations 5.6, 5.7, and 5.8. Solving equations $5.7,5.8,5.11$, and 5.12 


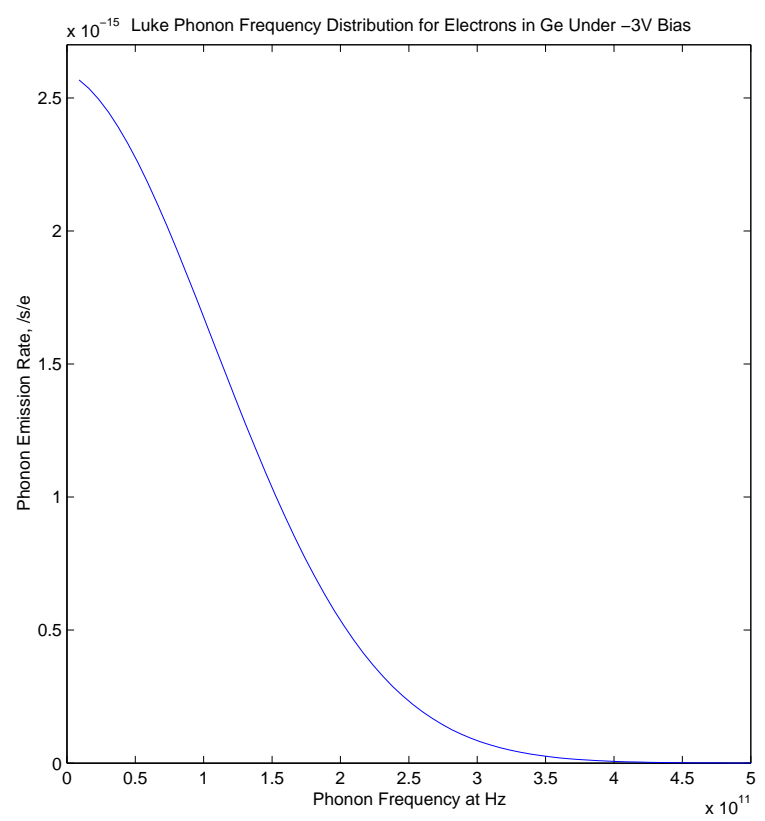

Figure 5.3: The electron Neganov-Luke phonon emission rate at different frequencies for a germanium detector with $-3 \mathrm{~V}$ bias. Low frequency is cut off at $84 \mathrm{GHz}$.

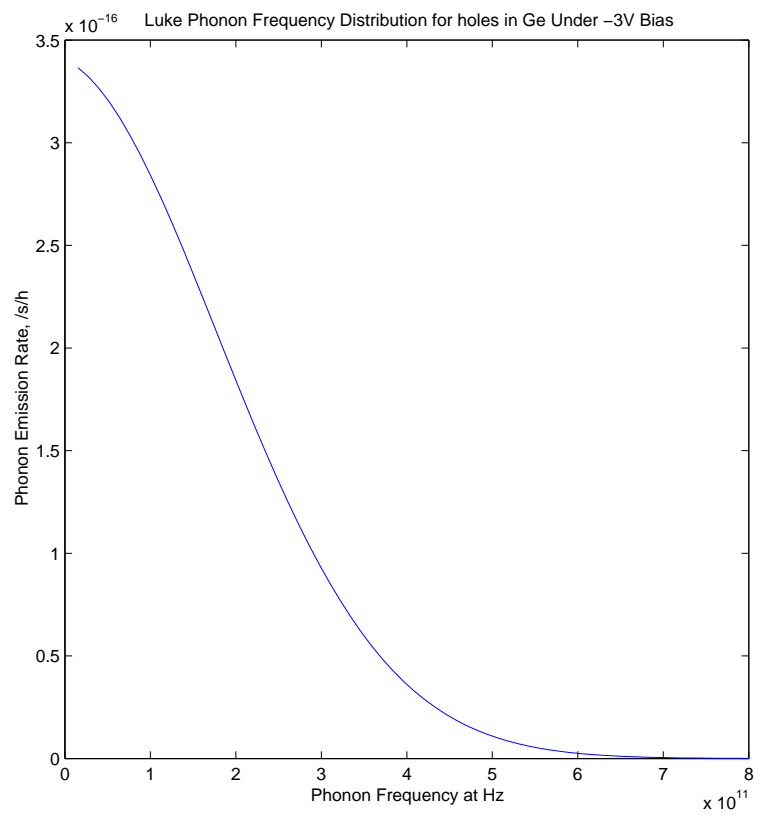

Figure 5.4: The hole Neganov-Luke phonon emission rate at different frequencies for a germanium detector with $-3 \mathrm{~V}$ bias. Low frequency is cut off at $84 \mathrm{GHz}$. 
iteratively with numerical methods, the electron average drifting velocity and its effective temperature can be found. The electron's average drift velocity is $1.756 \times 10^{4} \mathrm{~m} / \mathrm{s}$, and the electron's effective temperature is $17.87 \mathrm{~K}$. The hole's average drift velocity is $1.738 \times 10^{4} \mathrm{~m} / \mathrm{s}$, and the hole's effective temperature is $28.53 \mathrm{~K}$ at $-3 \mathrm{~V}$ bias for Germanium ZIP detector. The deformation potential is $E_{1}=13.3 \mathrm{eV}$ for an electron in germanium, and $E_{1}=4.73 \mathrm{eV}$ for a hole in germanium.

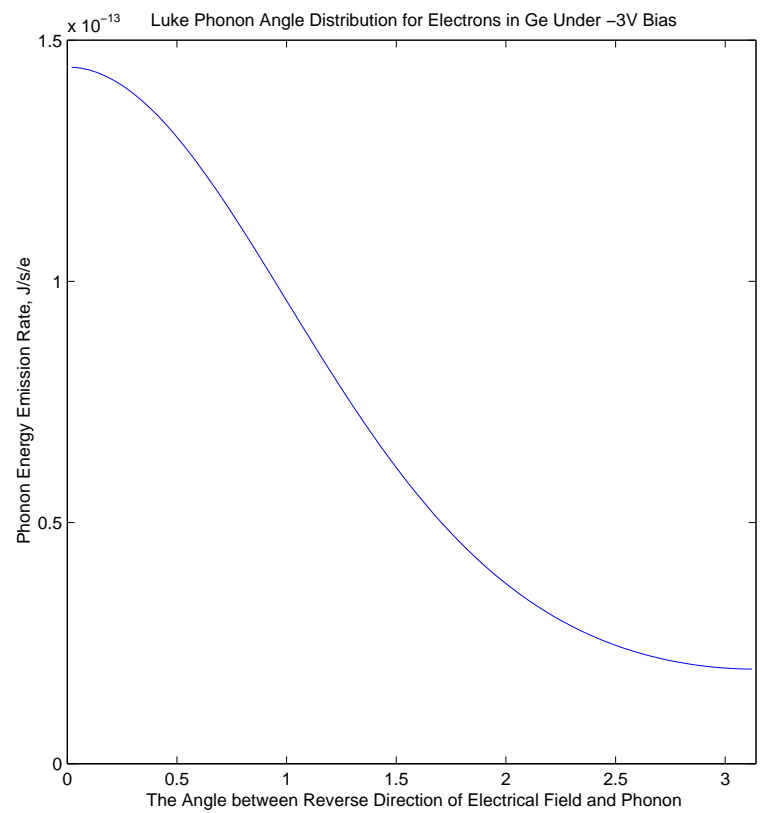

Figure 5.5: The electron Neganov-Luke phonon energy angle distribution for germanium detector with $-3 \mathrm{~V}$ bias.

Once we have the probability distribution $P_{q}$ of the Neganov-Luke phonons, we can calculate the Luke phonon emission rate-frequency distribution (see Figure 5.3 for electron and Figure 5.4 for hole), and the angular energy distribution (see Figure 5.5 fo relectron and Figure 5.6 for hole). For example, the Luke phonon emission rate at different frequencies for one electron is $P_{q}$ integrated over the 


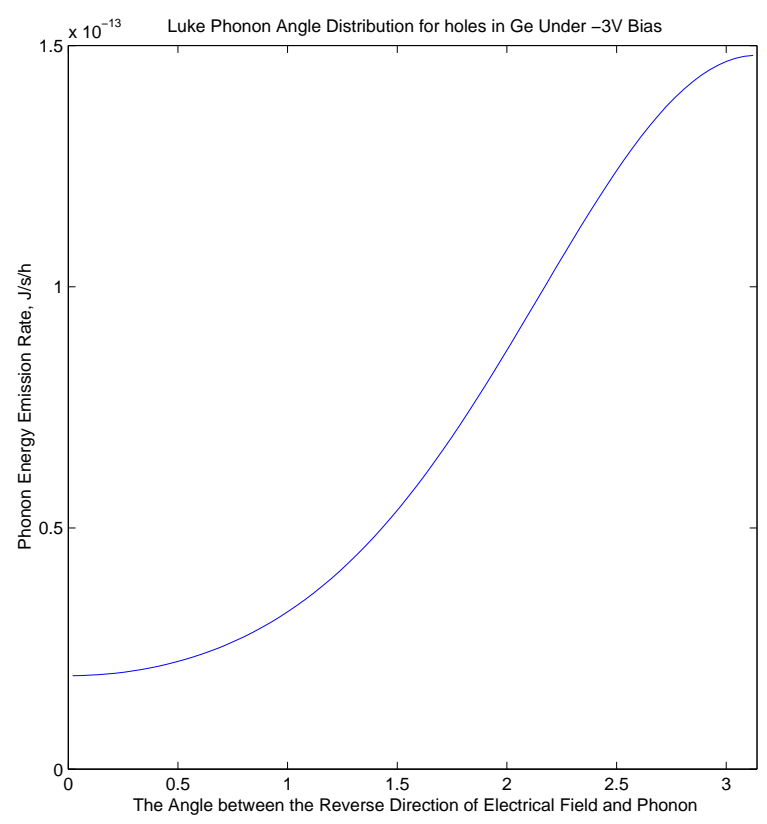

Figure 5.6: The hole Neganov-Luke phonon energy angle distribution for germanium detector with $-3 \mathrm{~V}$ bias.

solid angle

$$
P_{E}(f)=\frac{1}{(2 \pi)^{3}} \int_{0}^{\pi} P_{q} 2 \pi \sin \chi d \chi
$$

The Luke phonon energy emission rate as a function of the azimutal angle (with respect to $\mathrm{Z}$ in Figure 5.2) from one electron is

$$
P_{E}(\chi)=\frac{1}{(\pi)^{3}} \int_{0}^{\infty} P_{q} \hbar \omega 2 \pi q^{2} d q
$$

The total Luke phonon energy emission rate from one electron is

$$
P_{E}=\frac{1}{(2 \pi)^{3}} \int_{0}^{\infty} \int_{0}^{\pi} P_{q} \hbar \omega \sin \chi d \chi 2 \pi q^{2} d q
$$

98.23\% of the Luke phonons produced by electrons have frequencies above $84 \mathrm{GHz}$. (This frequency corresponds to an energy of $0.34 \mathrm{meV}$, equalling the energy gap of superconducting aluminum, $2 \cdot \Delta_{A l}$. Phonons that have frequency below $84 \mathrm{GHz}$ cannot break Cooper pairs in the aluminum phonon capture fins). 
99.86\% of the Luke phonons produced by holes have frequencies above $84 \mathrm{GHz}$. We conclude that almost all Neganov-Luke phonons are collectable, because their energy is above $2 \cdot \Delta_{A l}$.

With the distributions of primary phonons and Neganov-Luke phonons known, we are ready to consider the event location reconstruction in the ZIP detector.

\subsection{Event Location Reconstruction}

We need three parameters to identify an event interaction location in a ZIP detector. What we have in a ZIP detector are the timing parameters in two charge channels and four phonon sensors. Since $Q_{\text {outer }}$ is used as a background rejection electrode, we only select events within the $Q_{\text {inner }}$ electrode. The phonon timing parameters relative to $Q_{\text {inner }}$ are the parameters that we need for event location reconstruction. First, for an event in a ZIP detector in quadrant A, as shown in figure 5.7, we will take the time of the phonon pulse in a local phonon sensor as the time it reaches $20 \%$ of its peak amplitude relative to the time of the $Q_{i n n e r}$ pulse as phonon propagation time to local phonon sensor A, and similarly for the phonon propagation times to two neighbor the phonon sensors B and D.

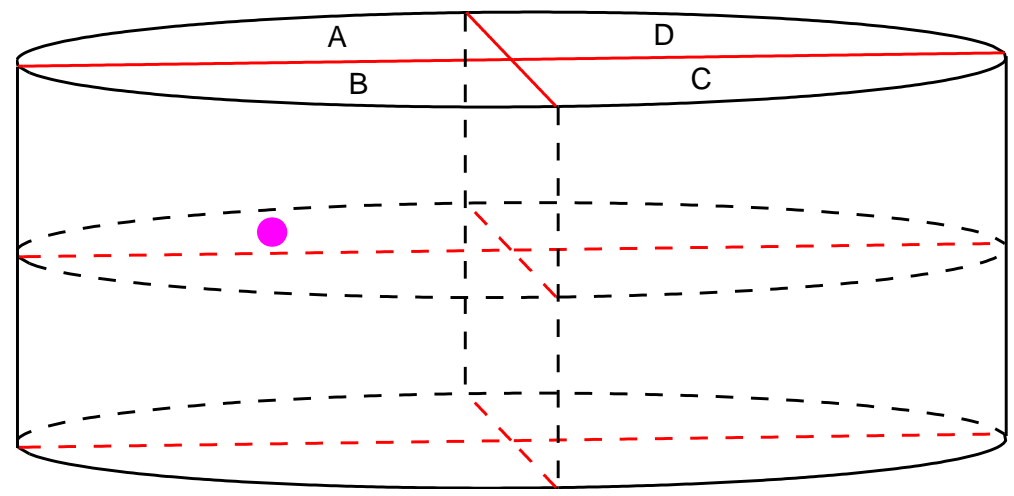

Figure 5.7: A particle interaction in quadrant A of a ZIP detector. 


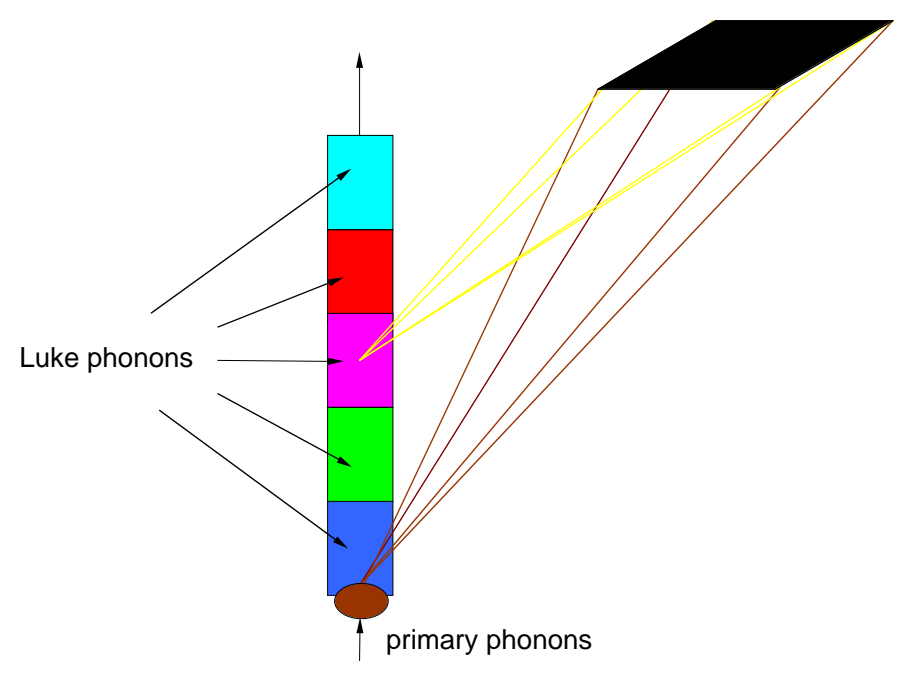

Figure 5.8: Comparison of primary phonons and Neganov-Luke phonons. The black patch is one die of a phonon sensor. Neganov-Luke phonon emission center is the moving charge. Primary phonons are from a unique source point.

Second, we need to know how to calculate the event location $\mathrm{x}, \mathrm{y}$ and $\mathrm{z}$ with phonon timing parameters, which relies on phonon modeling in a ZIP detector and is described in this section.

All phonons in the ZIP detector are treated as ballistic phonons, i.e., phonon propagation distance is linearly proportional to phonon propagation time, $L \propto t$. This is a good approximation at the cm length scale of a ZIP detector, because the optical phonon decay is completed in less than $2 \mathrm{~mm}$.

Ballistic phonon reflections in the ZIP detector could exist. Because we only use phonon pulse start time, the effect of ballistic phonon reflection is not considered in this work.

We further simplify the phonon evolution and propagation processes for event location reconstruction. Suppose all phonon components have the same absorption coefficient on the surface of the ZIP detector. 
For modeling purposes, the effective direct hit phonon propagation distance to each phonon channel can be calculated by weighting the distance from the particle interaction point to each die in the phonon sensor with the direct hit phonon flux. We notice that the primary phonons spread out from a single point, but the Neganov-Luke phonon emission center follows the drifting charge. (See figure 5.8.)

The third phonon component is the electron and hole recombination phonons on the surfaces of the ZIP detector. The Fermi energy in n-type germanium is about $10 \mathrm{meV}$ below the edge of the electron conduction band [109]. Germanium has an energy gap $E_{g}=743 \mathrm{meV}$. The released phonon energy from the hole recombination at the phonon sensor side is the Fermi energy difference at the two sides of the aluminum-amorphous-silicon-germanium structure. The released phonon energy from the electron recombination at the charge electrode side is the Fermi energy difference at the two sides of the gold-amorphous-silicon-germanium structure. Data for these structures is not available, but the total recombination phonon energy for an electron at the phonon sensor side and for a hole at the charge electrode side must sum to the gap energy $E_{g}$. I equally split this energy for the two sides in this model.

We define the primary phonon energy flux to each die in phonon sensor A as $E_{P i}$, the propagation distance to the die as $D_{P i}$, the direct Luke phonon energy flux to each die in phonon sensor $\mathrm{A}$ as $E_{L i}$, the propagation distance from the moving emission center as $D_{L i}$, the recombination phonon energy flux to each die in phonon sensor $\mathrm{A}$ as $E_{R i}$, and the propagation distance as $D_{R i}$. Then the average phonon propagation distance to phonon sensor A is

$$
D_{A}=\frac{\sum E_{P i} D_{P i}+\sum E_{L i} D_{L i}+\sum E_{R i} D_{R i}}{\sum E_{P i}+\sum E_{L i}+\sum E_{R i}}
$$


where $i$ is the index of each die in phonon sensor A.

Similarly, the average phonon propagation distances to two neighboring phonon sensors, $D_{B}$ to sensor B and $D_{D}$ to sensor D, can be calculated.

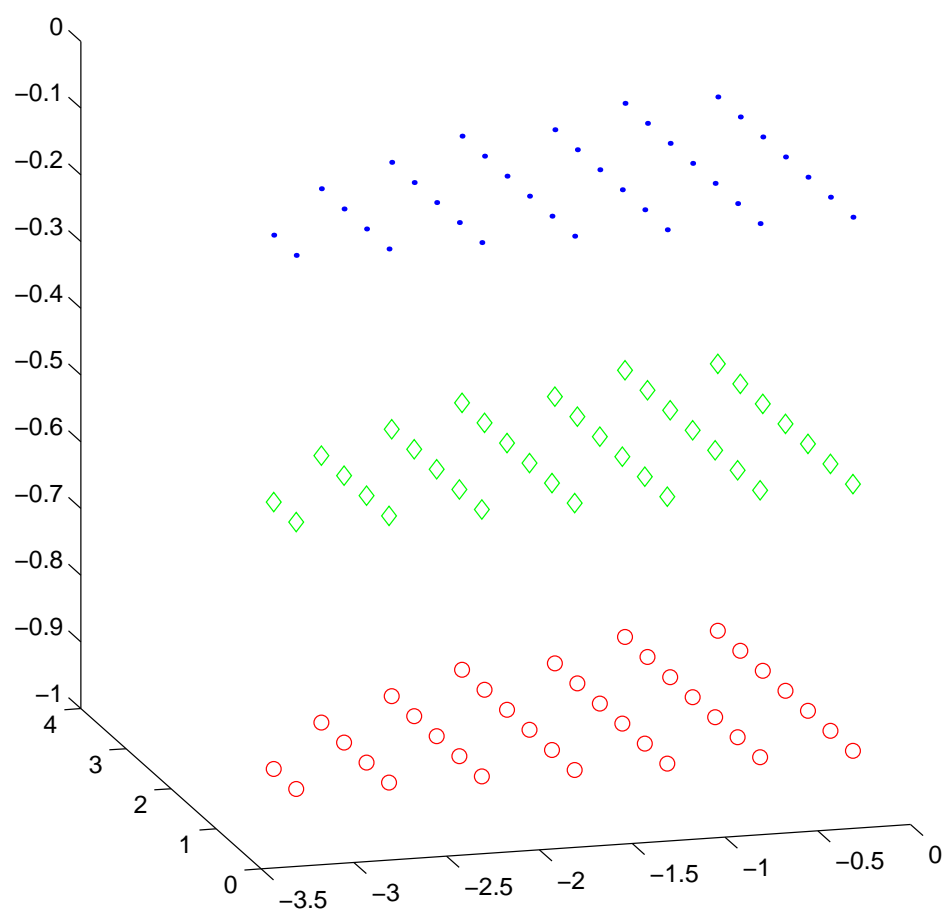

Figure 5.9: The pre-given event locations in quadrant $\mathrm{A}$ of the ZIP detector. The axis unit is $\mathrm{cm}$. Three layers of the five are shown.

We define the phonon sensor surface as the $Z=0$ plane, and the electrode side surface as $Z=-1$ plane. We consider 185 event locations in quadrant A. There are 37 event locations just under the center of each die of phonon sensor A at $Z=-0.1$; similarly, 37 event locations at $Z=-0.3 ; 37$ event locations at $Z=-0.5 ; 37$ event locations at $Z=-0.7$; and 37 event locations at $Z=-0.9$. These locations are labeled as $\left(x_{0 j}, y_{0 j}, z_{0 j}\right)$. (See Figure 5.9.)

For a given event located at $\left(x_{0 j}, y_{0 j}, z_{0 j}\right)$ in quadrant $\mathrm{A}$, the phonon propagation distance to local phonon sensor $\mathrm{A}$ is $D_{A j}$, to neighbor phonon sensor $\mathrm{B}$ 
is $D_{B j}$, and to neighbor phonon sensor $\mathrm{D}$ is $D_{D j}$. Note that $\mathrm{x}$ and $\mathrm{y}$ symmetry exists. If $\mathrm{x}$ and $\mathrm{y}$ coordinates are switched, the given event is relocated at $\left(x_{0 l}=y_{0 j}, y_{0 l}=x_{0 j}, z_{o l}=z_{0 j}\right)$ in quadrant $\mathrm{A}$, the phonon propagation distance to local phonon sensor $\mathrm{A}$ is the same, i.e., $D_{A l}=D_{A j}$. The propagation distance to neighbor phonon sensor $\mathrm{B}$ is $D_{B l}=D_{D j}$, and to neighbor phonon sensor $\mathrm{D}$ is $D_{D l}=D_{B j}$.

Now we need to build three functions for the calculations of $\mathrm{x}, \mathrm{y}$ and $\mathrm{z}$. The method is to recalculate the event location $\left(x_{j}, y_{j}, z_{j}\right)$ with the established functions and phonon transportation parameters $\left(D_{A j}, D_{B j}, D_{D j}\right)$ of the given locations $\left(x_{0 j}, y_{0 j}, z_{0 j}\right)$.

Suppose that $x_{j}=f_{1}\left(D_{A j}, D_{B j}, D_{D j}\right), y_{j}=f_{2}\left(D_{A j}, D_{B j}, D_{D j}\right)$, and $z_{j}=$ $f_{3}\left(D_{A j}, D_{B j}, D_{D j}\right)$ are cubic polynomials of $\left(D_{A j}, D_{B j}, D_{D j}\right)$.

Using the symmetry between $x$ and $y$, we write $x_{j}, y_{j}, z_{j}$ in the following explicit forms

$$
\begin{aligned}
x_{j}= & \operatorname{vect} 1(1) \cdot D_{D j}^{3}+\operatorname{vect} 1(2) \cdot D_{D j}^{2}+\operatorname{vect} 1(3) \cdot D_{D j} \\
& +\operatorname{vect} 1(4) \cdot D_{B j}^{3}+\operatorname{vect} 1(5) \cdot D_{B j}^{2}+\operatorname{vect} 1(6) \cdot D_{B j} \\
& +\operatorname{vect} 1(7) \cdot D_{A j}^{3}+\operatorname{vect} 1(8) \cdot D_{A j}{ }^{2}+\operatorname{vect} 1(9) \cdot D_{A j} \\
& +\operatorname{vect} 1(10) \cdot\left(D_{D j} D_{B j} D_{A j}\right)+\operatorname{vect} 1(11) \cdot\left(D_{D j}^{2}+D_{B j}^{2}\right) D_{A j} \\
& +\operatorname{vect} 1(12) \cdot\left(D_{D j}+D_{B j}\right) D_{A j}^{2}+\operatorname{vect} 1(13) \cdot\left(D_{D j}^{2} D_{B j}\right) \\
& +\operatorname{vect} 1(14) \cdot\left(D_{D j} D_{B j}^{2}\right)+\operatorname{vect} 1(15) \cdot\left(D_{D j}+D_{A j}\right) D_{B j}^{2} \\
& +\operatorname{vect} 1(16) \cdot\left(D_{B j}+D_{A j}\right) D_{D j}^{2}+\operatorname{vect} 1(17) \cdot\left(D_{D j} D_{B j}\right) \\
& +\operatorname{vect} 1(18) \cdot\left(D_{D j}+D_{B j}\right) D_{A j}+\operatorname{vect} 1(19) \\
y_{j}= & \operatorname{vect} 1(1) \cdot D_{B j}^{3}+\operatorname{vect} 1(2) \cdot D_{B j}^{2}+\operatorname{vect} 1(3) \cdot D_{B j} \\
& +\operatorname{vect} 1(4) \cdot D_{D j}^{3}+\operatorname{vect} 1(5) \cdot D_{D j}^{2}+\operatorname{vect} 1(6) \cdot D_{D j}
\end{aligned}
$$




$$
\begin{aligned}
& +\operatorname{vect} 1(7) \cdot D_{A j}^{3}+\operatorname{vect} 1(8) \cdot D_{A j} \cdot{ }^{2}+\operatorname{vect} 1(9) \cdot D_{A j} \\
& +\operatorname{vect} 1(10) \cdot\left(D_{D j} D_{B j} D_{A j}\right)+\operatorname{vect} 1(11) \cdot\left(D_{B j}^{2}+D_{D j}^{2}\right) D_{A j} \\
& +\operatorname{vect1}(12) \cdot\left(D_{B j}+D_{D j}\right) D_{A j}^{2}+\operatorname{vect} 1(13) \cdot\left(D_{B j}^{2} D_{D j}\right) \\
& +\operatorname{vect} 1(14) \cdot\left(D_{B j} D_{D j}^{2}\right)+\operatorname{vect} 1(15) \cdot\left(D_{B j}+D_{A j}\right) D_{D j}^{2} \\
& +\operatorname{vect} 1(16) \cdot\left(D_{D j}+D_{A j}\right) D_{B j}^{2}+\operatorname{vect} 1(17) \cdot\left(D_{B j} D_{D j}\right) \\
& +\operatorname{vect} 1(18) \cdot\left(D_{B j}+D_{D j}\right) D_{A j}+\operatorname{vect} 1(19) \\
& z_{j}=\operatorname{vect} 2(1) \cdot D_{D j}^{3}+\operatorname{vect} 2(2) \cdot D_{D j}^{2}+\operatorname{vect} 2(3) \cdot D_{D j} \\
& +\operatorname{vect} 2(4) \cdot D_{B j}^{3}+\operatorname{vect} 2(5) \cdot D_{B j}^{2}+\operatorname{vect} 2(6) \cdot D_{B j} \\
& +\operatorname{vect} 2(7) \cdot D_{A j}^{3}+\operatorname{vect} 2(8) \cdot D_{A j} \cdot{ }^{2}+\operatorname{vect} 2(9) \cdot D_{A j} \\
& +\operatorname{vect} 2(10) \cdot\left(D_{D j} D_{B j} D_{A j}\right)+\operatorname{vect} 2(11) \cdot\left(D_{D j}^{2}+D_{B j}^{2}\right) D_{A j} \\
& +\operatorname{vect} 2(12) \cdot\left(D_{D j}+D_{B j}\right) D_{A j}^{2}+\operatorname{vect} 2(13) \cdot\left(D_{D j}^{2} D_{B j}\right) \\
& +\operatorname{vect} 2(14) \cdot\left(D_{D j} D_{B j}^{2}\right)+\operatorname{vect} 2(15) \cdot\left(D_{D j}+D_{A j}\right) D_{B j}^{2} \\
& +\operatorname{vect} 2(16) \cdot\left(D_{B j}+D_{A j}\right) D_{D j}^{2}+\operatorname{vect} 2(17) \cdot\left(D_{D j} D_{B j}\right) \\
& +\operatorname{vect} 2(18) \cdot\left(D_{D j}+D_{B j}\right) D_{A j}+\operatorname{vect} 2(19)
\end{aligned}
$$

where vect1(1:19) and vect2(1:19) represent 38 over-determined parameters to be calculated.

For each location $\left(x_{0 j}, y_{0 j}, z_{0 j}\right)$, we calculate location $\left(x_{j}, y_{j}, z_{j}\right)$ using equations 5.17- 5.19 to arrive at a one to one mapping. We do least square fit by minimizing

$$
\text { sum }=\sum\left(x_{j}-x_{0 j}\right)^{2}+\sum\left(y_{j}-y_{0 j}\right)^{2}+\sum\left(z_{j}-z_{0 j}\right)^{2}
$$


so vect1 and vect2 are found.

$$
\begin{array}{r}
\text { vect } 1=[-0.01463714812983 \\
0.22606497969797 \\
-0.10441568013323 \\
-0.00267469710467 \\
0.06894581409225 \\
-0.24949327191634 \\
-1.56856755800621 \\
-0.42106246670328 \\
0.09201339802508 \\
0.02395379755088 \\
-0.79117977908479 \\
0.36739821084906 \\
-0.73226521417559 \\
-0.72826440684161 \\
0.69435414144089 \\
0.71631003557788 \\
0.22774781227942 \\
0.14251401056308 \\
-0.00001921258570] \\
\end{array}
$$




$$
\begin{array}{r}
\text { vect }=[0.00316675816152 \\
-0.02686621398716 \\
0.03761137355237 \\
0.00316675816107 \\
-0.02686621398264 \\
0.03761137354088 \\
-0.06775231098052 \\
-1.08224384461143 \\
0.35630924561140 \\
0.07597734726841 \\
0.21356332309292 \\
-0.13935473730309 \\
0.15827219670593 \\
0.15827219670586 \\
-0.16224101972826 \\
-0.16224101972808 \\
0.02020673277740 \\
-0.22578871953728 \\
0.00000877157406] \\
0
\end{array}
$$

As a cross check of the constructed functions with the 38 above coefficients, we compare the 185 pre-given event locations $\left(x_{0 j}, y_{0 j}, z_{0 j}\right)$ in quadrant $\mathrm{A}$ with the result of equations $5.17,5.18$ and 5.19 to calculate the reconstructed locations $\left(x_{j}, y_{j}, z_{j}\right)$. The predetermined and calculated positions are compared in Figure 5.10 for $\mathrm{x}$ and $\mathrm{y}$, and in Figure 5.11 for $\mathrm{z}$.

The reconstructed $\mathrm{x}$ and $\mathrm{y}$ are correct except that the error for events at 


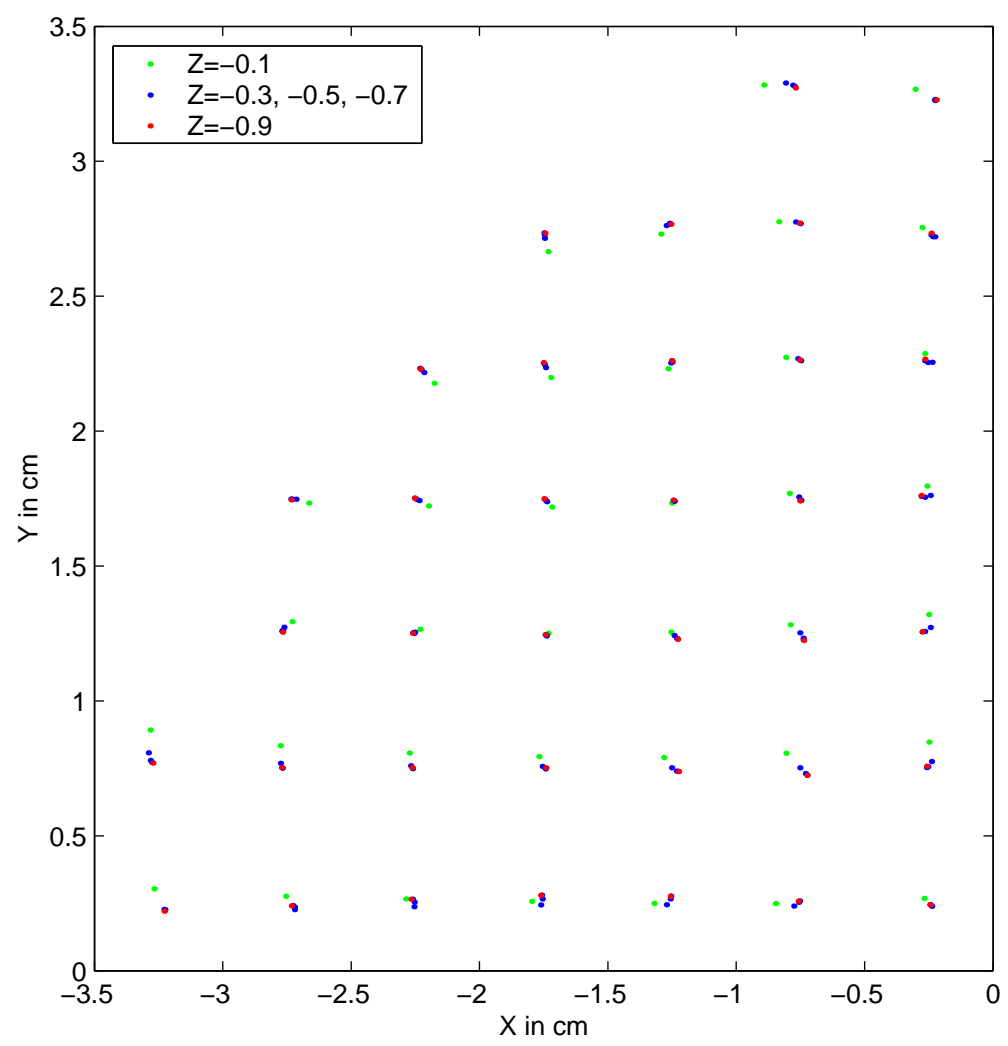

Figure 5.10: The reconstructed $\mathrm{X}$ and $\mathrm{Y}$ for the pre-given 185 locations. The pregiven locations have $X_{0}=-0.25,-0.75,-1.25,-1.75,-2.25,-2.75,-3.25$, and $Y_{0}=-0.25$, $-0.75,-1.25,-1.75,-2.25,-2.75,-3.25$, respectively.

$z=-0.1$ is slightly bigger. The effective phonon propagation distance calculation error increases when the event location approaches the phonon sensors, the solid angle to the local quadrant phonon sensor is large. The reconstruction z parameter is sharp for events inside the detector crystal, and the error of reconstructed $\mathrm{z}$ is slightly bigger when the event is close to the surfaces of the ZIP detector.

For any event in quadrant $\mathrm{A}$, once the phonon transportation distances $D_{A}$, $D_{B}$ and $D_{D}$ to phonon sensors $\mathrm{A}, \mathrm{B}$ and $\mathrm{D}$, are determined from the data using the prescription described earlier (more details in section 5.5) respectively, the 


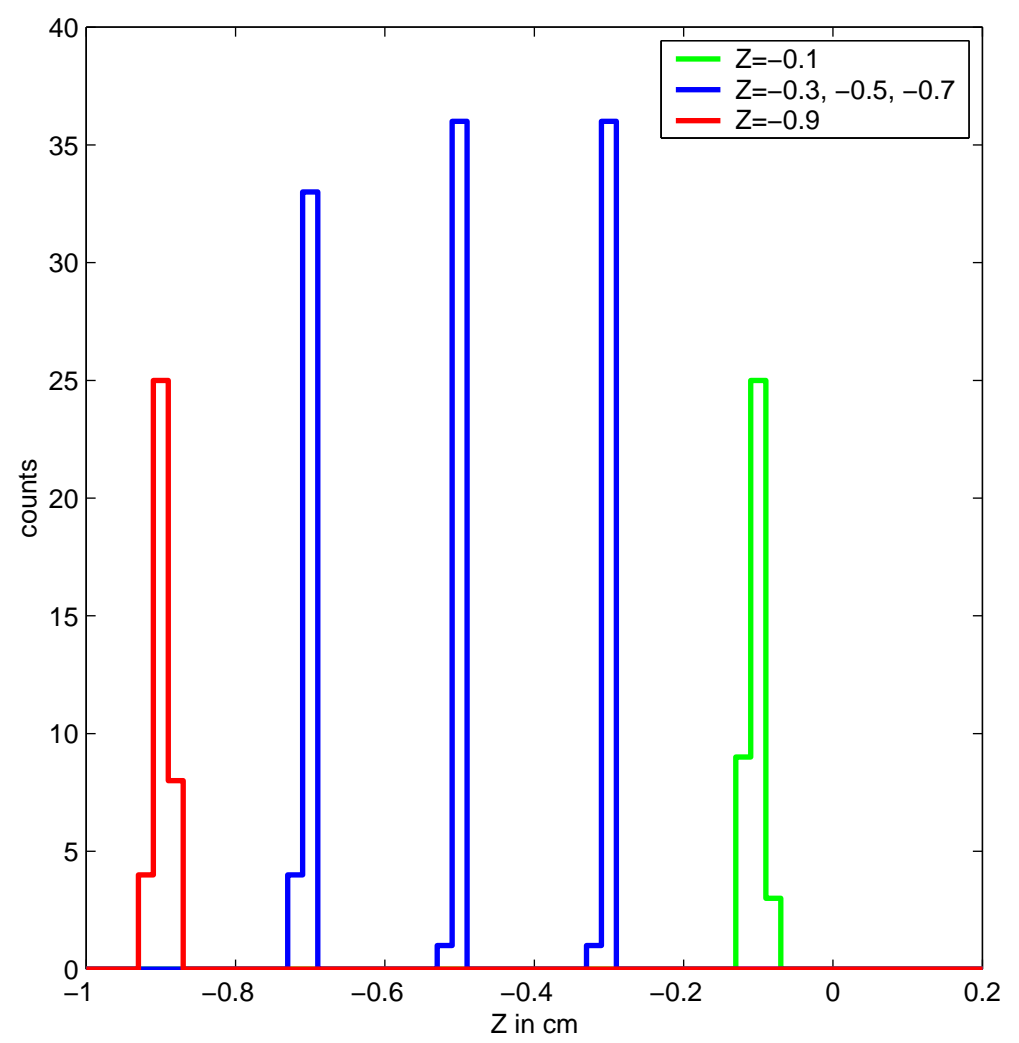

Figure 5.11: The reconstructed $\mathrm{Z}$ for the pre-given 185 locations.

event location can be calculated with the following equations:

$$
\begin{aligned}
x= & \operatorname{vect} 1(1) \cdot D_{D}^{3}+\operatorname{vect} 1(2) \cdot D_{D}^{2}+\operatorname{vect} 1(3) \cdot D_{D} \\
& +\operatorname{vect} 1(4) \cdot D_{B}^{3}+\operatorname{vect} 1(5) \cdot D_{B}^{2}+\operatorname{vect} 1(6) \cdot D_{B} \\
& +\operatorname{vect} 1(7) \cdot D_{A}^{3}+\operatorname{vect} 1(8) \cdot D_{A} \cdot^{2}+\operatorname{vect} 1(9) \cdot D_{A} \\
& +\operatorname{vect} 1(10) \cdot\left(D_{D} D_{B} D_{A}\right)+\operatorname{vect} 1(11) \cdot\left(D_{D}^{2}+D_{B}^{2}\right) D_{A} \\
& +\operatorname{vect} 1(12) \cdot\left(D_{D}+D_{B}\right) D_{A}^{2}+\operatorname{vect} 1(13) \cdot\left(D_{D}^{2} D_{B}\right) \\
& +\operatorname{vect} 1(14) \cdot\left(D_{D} D_{B}^{2}\right)+\operatorname{vect} 1(15) \cdot\left(D_{D}+D_{A}\right) D_{B}^{2} \\
& +\operatorname{vect} 1(16) \cdot\left(D_{B}+D_{A}\right) D_{D}^{2}+\operatorname{vect} 1(17) \cdot\left(D_{D} D_{B}\right) \\
& +\operatorname{vect} 1(18) \cdot\left(D_{D}+D_{B}\right) D_{A}+\operatorname{vect} 1(19)
\end{aligned}
$$




$$
\begin{aligned}
y= & \operatorname{vect} 1(1) \cdot D_{B}^{3}+\operatorname{vect} 1(2) \cdot D_{B}^{2}+\operatorname{vect} 1(3) \cdot D_{B} \\
& +\operatorname{vect} 1(4) \cdot D_{D}^{3} \operatorname{vect} 1(5) \cdot D_{D}^{2}+\operatorname{vect} 1(6) \cdot D_{D} \\
& +\operatorname{vect} 1(7) \cdot D_{A}^{3}+\operatorname{vect} 1(8) \cdot D_{A} \cdot^{2}+\operatorname{vect} 1(9) \cdot D_{A} \\
& +\operatorname{vect} 1(10) \cdot\left(D_{D} D_{B} D_{A}\right)+\operatorname{vect} 1(11) \cdot\left(D_{D}^{2}+D_{B}^{2}\right) D_{A} \\
& +\operatorname{vect} 1(12) \cdot\left(D_{D}+D_{B}\right) D_{A}^{2}+\operatorname{vect} 1(13) \cdot\left(D_{B}^{2} D_{D}\right) \\
& +\operatorname{vect} 1(14) \cdot\left(D_{B} D_{D}^{2}\right)+\operatorname{vect} 1(15) \cdot\left(D_{B}+D_{A}\right) D_{D}^{2} \\
& +\operatorname{vect} 1(16) \cdot\left(D_{D}+D_{A}\right) D_{B}^{2}+\operatorname{vect} 1(17) \cdot\left(D_{D} D_{B}\right) \\
& +\operatorname{vect} 1(18) \cdot\left(D_{B}+D_{D}\right) D_{A}+\operatorname{vect} 1(19) \\
z= & \operatorname{vect} 2(1) \cdot D_{D}^{3}+\operatorname{vect} 2(2) \cdot D_{D}^{2}+\operatorname{vect} 2(3) \cdot D_{D} \\
& +\operatorname{vect} 2(4) \cdot D_{B}^{3}+\operatorname{vect} 2(5) \cdot D_{B}^{2}+\operatorname{vect} 2(6) \cdot D_{B} \\
& +\operatorname{vect} 2(7) \cdot D_{A}^{3}+\operatorname{vect} 2(8) \cdot D_{A}{ }^{2}+\operatorname{vect} 2(9) \cdot D_{A} \\
& +\operatorname{vect} 2(10) \cdot\left(D_{D} D_{B} D_{A}\right)+\operatorname{vect} 2(11) \cdot\left(D_{D}^{2}+D_{B}^{2}\right) D_{A} \\
& +\operatorname{vect} 2(12) \cdot\left(D_{D}+D_{B}\right) D_{A}^{2}+\operatorname{vect} 2(13) \cdot\left(D_{D}^{2} D_{B}\right) \\
& +\operatorname{vect} 2(14) \cdot\left(D_{D} D_{B}^{2}\right)+\operatorname{vect} 2(15) \cdot\left(D_{D}+D_{A}\right) D_{B}^{2} \\
& +\operatorname{vect} 2(16) \cdot\left(D_{B}+D_{A}\right) D_{D}^{2}+\operatorname{vect} 2(17) \cdot\left(D_{D} D_{B}\right) \\
& +(18) \cdot\left(D_{D}+D_{B}\right) D_{A}+\operatorname{vect} 2(19) \\
& \\
& \\
& \\
&
\end{aligned}
$$

The event location in quadrant B is calculated in the same way, using phonon propagation distance to phonon sensor $\mathrm{B}$, to phonon sensor $\mathrm{C}$, and to phonon sensor A, as well as equations 5.21, 5.22 and 5.23. Event locations in quadrant $\mathrm{C}$ and quadrant D are calculated similarly. 


\subsection{Event Location Calculation}

In the actual data, we have only phonon timing parameters. Charge collection is fast, so the inner electrode charge time QIst is used as the time reference. For an event in quadrant A, local phonon sensor A's start time is its $20 \%$ amplitude time, so the phonon propagation time to sensor $\mathrm{A}$ is $t a=P A r 20-Q I s t$, where these variable names refer to standard quantities in the CDMS event data set; neighbor phonon sensor B's start time is its $20 \%$ amplitude time, so the phonon propagation time to sensor $\mathrm{B}$ is $t b=P B r 20-Q I s t$; and neighbor phonon sensor D's start time is its $20 \%$ amplitude time, so the phonon propagation time to sensor D is $t d=P D r 20-Q I s t$. These phonon timing parameters are transformed into the phonon front propagation distance under the assumption that they are ballistic. This means that we can use a uniform velocity to calculate the phonon average propagation distance to a particular phonon sensor. For the event in quadrant A, the phonon front propagation distance to phonon sensor $\mathrm{A}$ is $D_{A}=t a \cdot v_{p}$, to phonon sensor $\mathrm{B}$ is $D_{B}=t b \cdot v_{p}$, and to phonon sensor $\mathrm{D}$ is $D_{D}=t d \cdot v_{p} \cdot v_{p}$ is average phonon propagation velocity.

We discuss here the appropriate phonon velocity to use in this reconstruction. For uniformly distributed events in quadrant A, we calculated the phonon average propagation velocity with the timing paramters of the local quadrant phonon sensor and two neighbor quadrant phonon sensors, because we know the exact geometry of the ZIP detector. It turned out that the phonon propagation velocity in the ZIP detector is about one third of the sound speed of the crystal. The exact phonon velocity value is not important here; we only use the relative phonon timing parameters with a uniform phonon velocity for event location reconstruction.

The key step of the reconstruction is to normalize the phonon timing parame- 
ters. For events uniformly distributed in quadrant A, the local quadrant phonon sensor time is normalized in the range from $0.28 \mathrm{~cm}$ to $0.84 \mathrm{~cm}$. The neighbor quadrant phonon sensor time is normalized in the range from $0.7 \mathrm{~cm}$ to $4.8 \mathrm{~cm}$. These numbers come from the modeling discussed in equation 5.16 of Section 5.4. (See figure 5.12.)
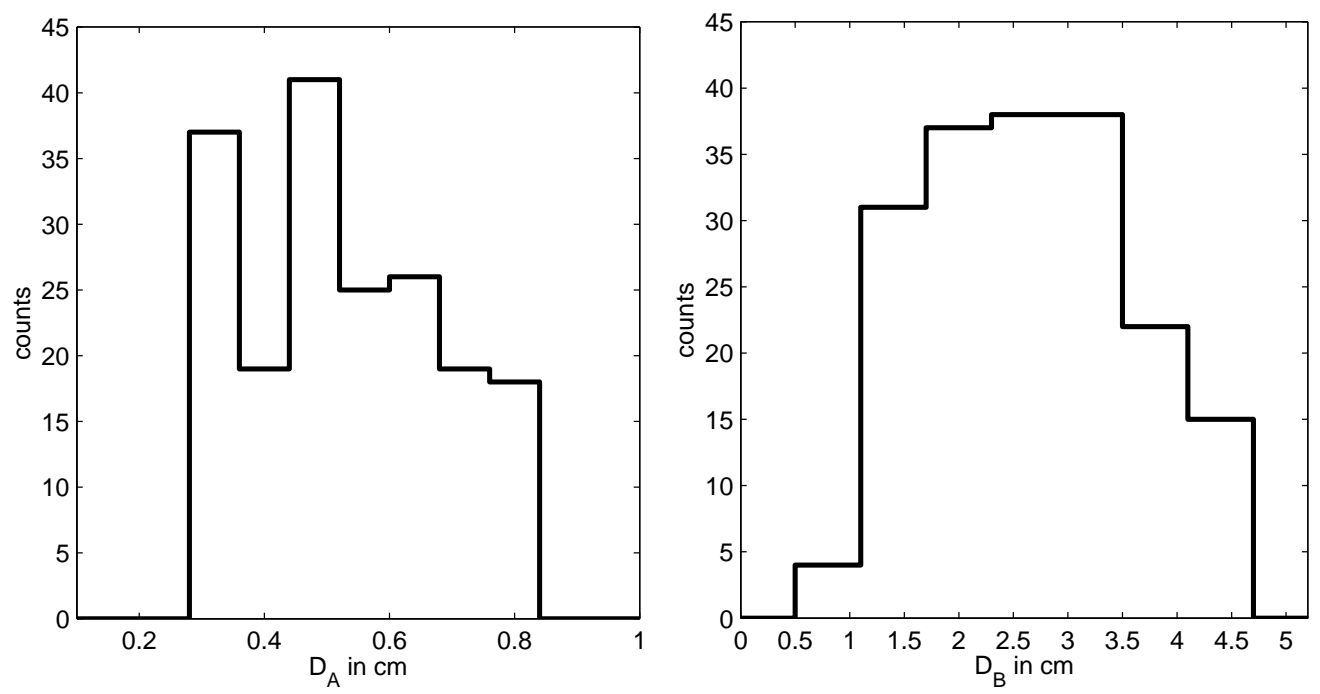

Figure 5.12: These graphs use the 185 uniformly distributed event locations in quadrant $\mathrm{A}$ given in the last section to calculate phonon front propagation distance distributions in the local quadrant phonon sensor (left) and in the neighbor quadrant phonon sensor B(right). The calculated phonon front propagation distance to neighbor quadrant $\mathrm{D}$ has the same distribution as for events in quadrant A to sensor B.

Let's use Z2, quadrant A as our first example. ${ }^{133}$ Ba calibration data in Soudan Run118 is used here as a source events for timing parameter analysis. The important point is that the ${ }^{133} \mathrm{Ba}$ calibration provides broad illumination across the detector, and so serves to define the full range of phonon propagation times. The calibration data and Soudan run118 timing normalization will be summarized at the end of this section. 

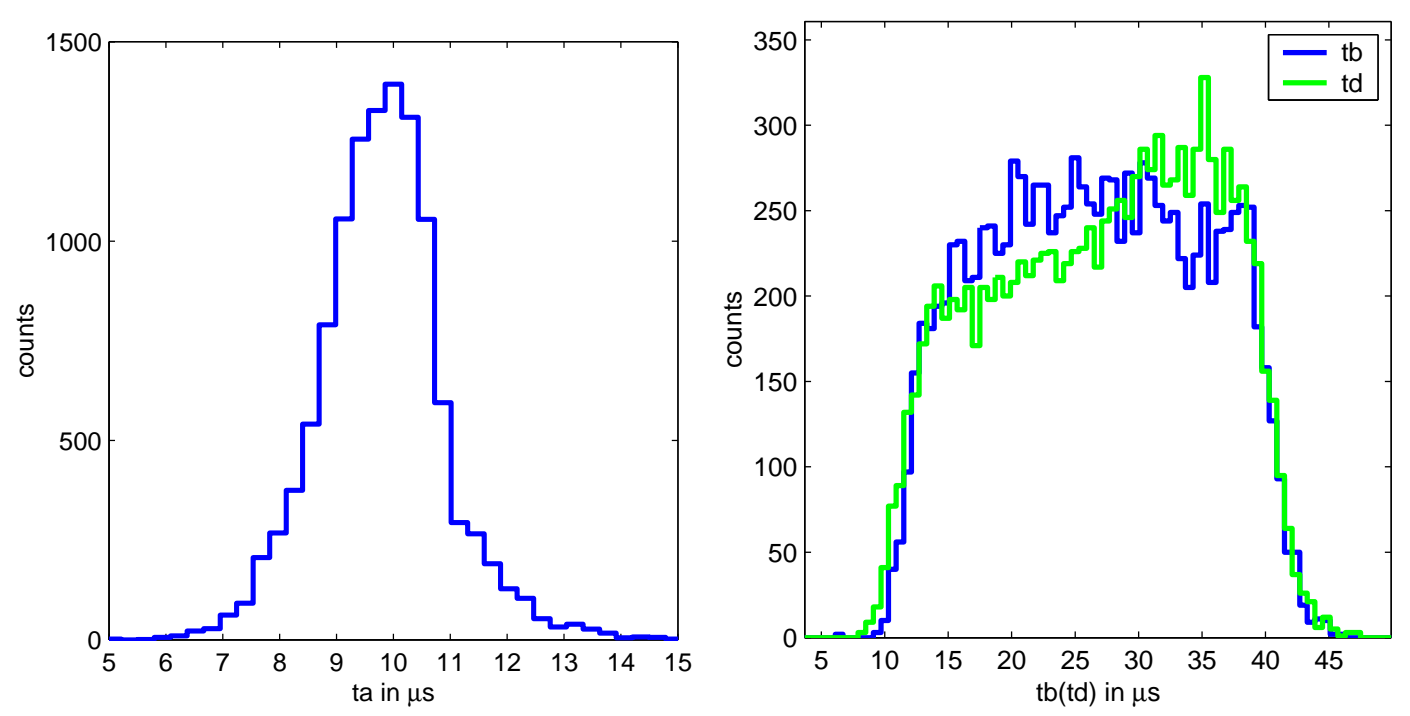

Figure 5.13: Soudan run118, Z2, ${ }^{133} \mathrm{Ba}$ calibration data. The graphs show, for events in quadrant $\mathrm{A}$, the phonon front propagation time to the local quadrant phonon sensor (left) and to the neighbor quadrant phonon sensors (right), prior to phonon timing normalization.

In the local phonon sensor $\mathrm{A}$, we take

$$
D_{A}=a \cdot t a+b
$$

Set $D_{A}=0.28 \mathrm{~cm}$ at $t a=7.8 \mu \mathrm{s}$, which is the time at $15 \% \mathrm{ta}$ distribution amplitude at the fast timing side (the left edge of the ta distribution in the left graph of Figure 5.13); and set $D_{A}=0.84 \mathrm{~cm}$ at $t a=12.4 \mu \mathrm{s}$, which is the time at $5 \% \mathrm{ta}$ distribution amplitude at the slow timing side (the right edge of the $t a$ distribution in the left graph of Figure 5.13). Then $a$ and $b$ can be calculated. After this normalization, for any event in quadrant A with phonon propagation time $t a$, equation 5.24 is used to calculate the phonon front propagation distance to the local quadrant phonon sensor.

Similarly, in the neighbor phonon sensor B for events in quadrant A, we take

$$
D_{B}=c_{1} \cdot t b+d_{1}
$$


Set $D_{B}=0.7 \mathrm{~cm}$ at $t b=10.6 \mu \mathrm{s}$, which is the time at $20 \% \mathrm{tb}$ ditribution shoulder at the fast timing side (the left edge of the $t b$ distribution in the right graph of Figure 5.13); and set $D_{B}=4.8 \mathrm{~cm}$ at $t b=43.1 \mu \mathrm{s}$, which is the time at $10 \% \mathrm{tb}$ distribution shoulder at the slow timing side (the right edge of the $t b$ distribution in the right graph of Figure 5.13). Then $c_{1}$ and $d_{1}$ can be calculated. After this normalization, for any event in quadrant A with phonon front propagation time $t b$ to phonon sensor channel $\mathrm{B}$, equation 5.25 is used to calculate the phonon front propagation distance from the interaction point to phonon sensor B.

Finally, in the neighbor phonon sensor channel D for events in quadrant A, we take

$$
D_{D}=c_{2} \cdot t d+d_{2}
$$

Set $D_{D}=0.7 \mathrm{~cm}$ at $t d=9.8 \mu \mathrm{s}$, which is the time at $20 \% \mathrm{td}$ distribution shoulder at the fast timing side (the left edge of the $t d$ distribution in the right graph of Figure 5.13); and set $D_{D}=4.8 \mathrm{~cm}$ at $t d=43.4 \mu \mathrm{s}$, which is the time at $10 \% \mathrm{td}$ distribution shoulder at the slow timing side (the right edge of the $t d$ distribution in the right graph of Figure 5.13). Then $c_{2}$ and $d_{2}$ can be calculated. After this normalization, for any event in quadrant A with phonon front propagation time $t d$ to phonon sensor channel D, equation 5.26 is used to calculate the phonon front propagation distance from the interaction point to phonon sensor D.

Figure 5.13 shows the phonon start time distributions in the local quadrant phonon sensor and in two neighbor quadrant phonon sensors for events in Z2 quadrant $\mathrm{A}$ and in the energy range from $10 \mathrm{keV}$ to $100 \mathrm{keV}$. Figure 5.14 shows the normalized phonon front propagation distance distributions in the local phonon sensor and in two neighbor phonon sensors for the same data. The phonon time normalization is important in the reconstruction, it ensures that we have correct 

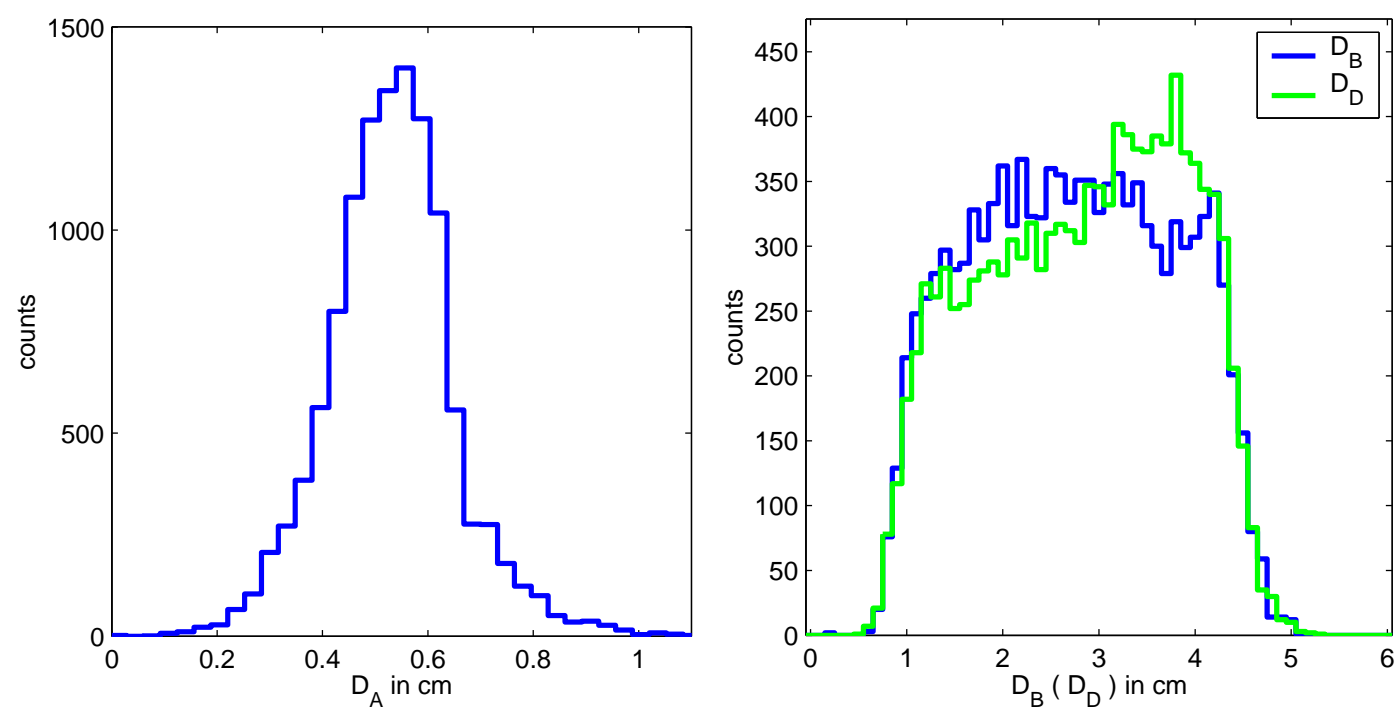

Figure 5.14: Soudan run118, Z2, ${ }^{133} \mathrm{Ba}$ calibration data. The graphs show, for events in quadrant $\mathrm{A}$, the phonon front propagation distance to the local quadrant phonon sensor (left) and to the neighbor quadrant phonon sensors (right), after phonon timing normalization.
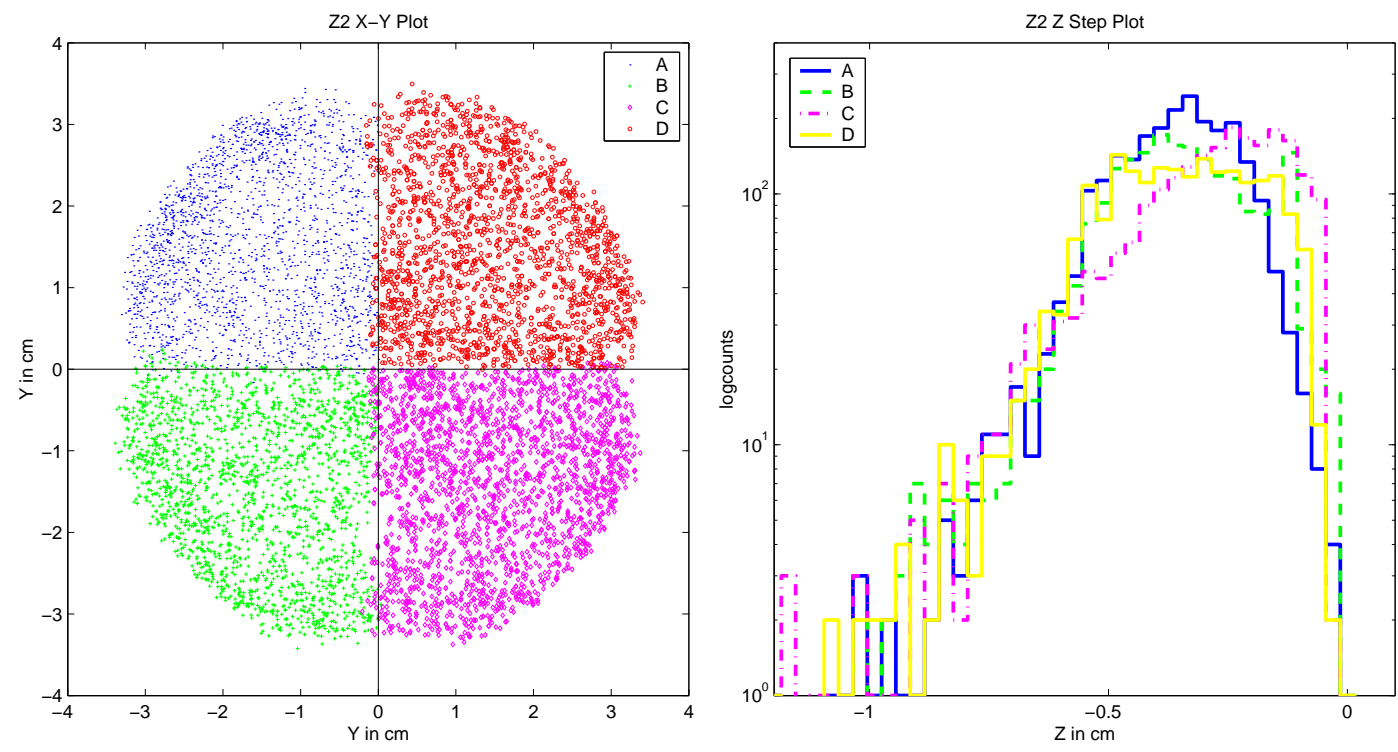

Figure 5.15: The reconstructed event locations for Z2 charge inner electrode events from ${ }^{133} \mathrm{Ba}$ calibration data in Soudan run118. Event $\mathrm{x}-\mathrm{y}$ distribution (left) and the $\mathrm{Z}$ parameter distribution (right) are shown. 
reconstructed event location. Figure 5.15 shows the reconstructed x, y (the graph on the left) and $\mathrm{z}$ (the graph on the right) parameters for $\mathrm{Z} 2$ with ${ }^{133} \mathrm{Ba}$ calibration data. The events distribute in charge inner electrode area, which are chosen by applying charge inner cut. The reconstructed $\mathrm{z}$ paremeter is in the range of -1 to 0 in $\mathrm{cm}$, which is the thickness of the ZIP detector. The calculation is done with equations 5.21, 5.22, and 5.23, as well as the normalized phonon timing parameters $D_{A}, D_{B}$, and $D_{D}$, using equation $5.24,5.25$, and 5.26 .
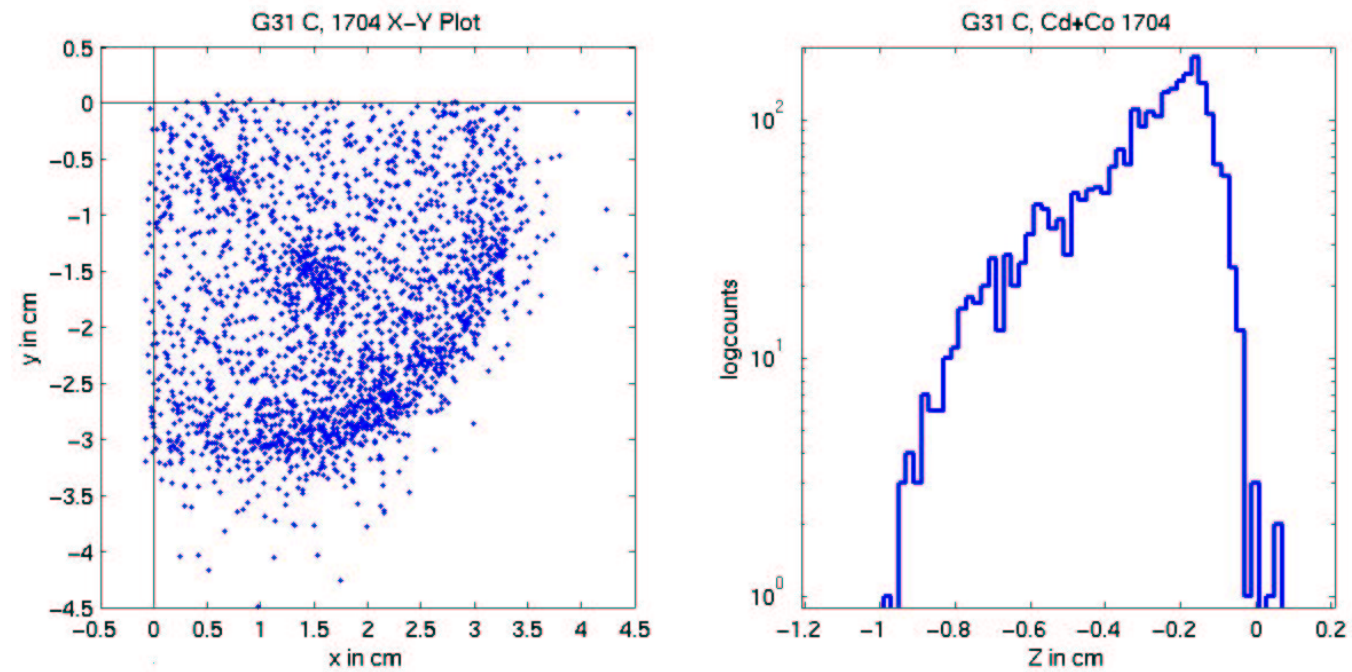

Figure 5.16: Reconstructed event locations for G31, quadrant C. Cadmium source spots are at the phonon sensor side, they are barely visible in the left graph because aluminum foil was used for blocking the sources.

Figure 5.16 shows the reconstructed event locations for G31 with data set 221122_1074, a special calibration run performed in a test setup by collaborators at UCB. In this run, there were two ${ }^{109} \mathrm{Cd}$ spot sources on the top of quadrant $\mathrm{C}$ ( phonon sensor side). A ${ }^{252} \mathrm{Cf}$ fission source was outside the dilution refrigerator providing uniform gamma and neutron illumination. Figure 5.17 shows the reconstructed event locations for G31 with data set 221107_2038. There were two ${ }^{109} \mathrm{Cd}$ spot sources on the bottom of quadrant $\mathrm{C}$ (electrode side) this time. The 

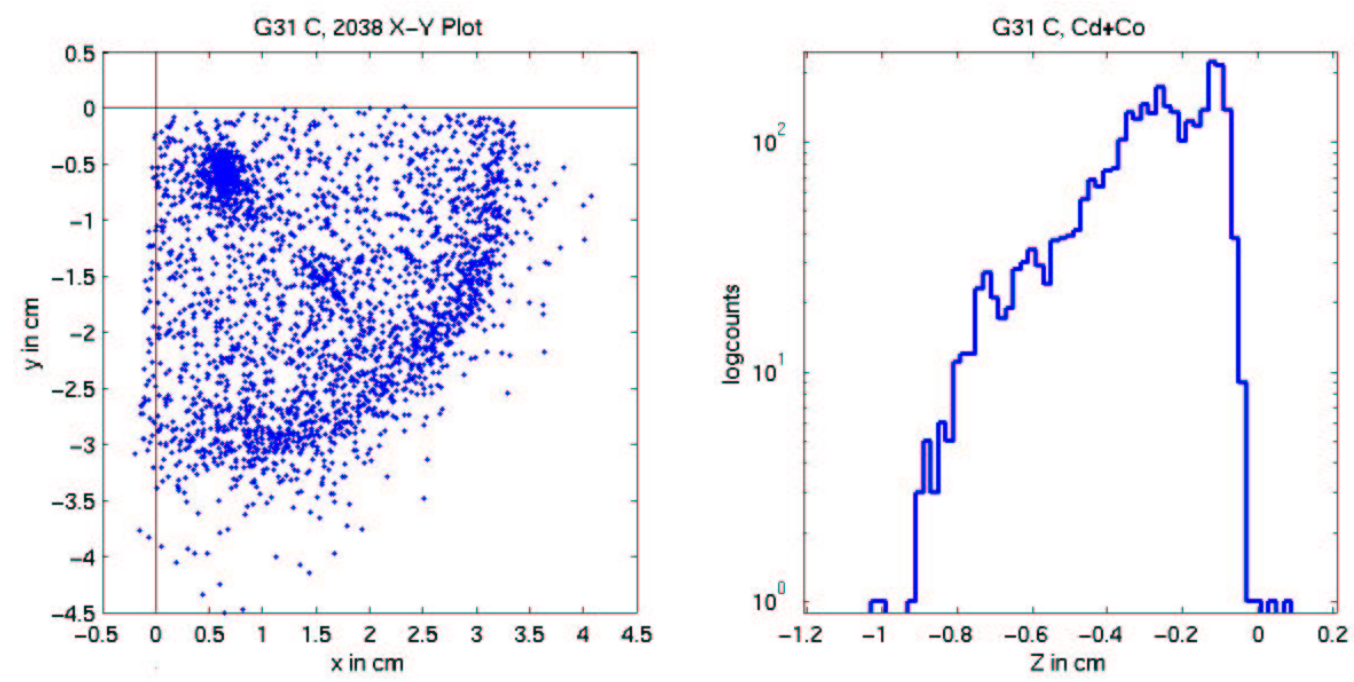

Figure 5.17: Reconstructed event locations for G31, quadrant C. Cadmium source spots at the charge electrode side are visible in the left graph. There are much more events for the source spot that is close to the center of the detector than for the source spot in the center of quadrant $\mathrm{C}$. The reason is that the event trigering energy was set on the phonon total of the four phonon sensors.

${ }^{252} \mathrm{Cf}$ source was again outside the dilution refrigerator. The ${ }^{109} \mathrm{Cd}$ source spots are visible in both data sets. And the reconstructed $\mathrm{z}$ parameter in $\mathrm{cm}$ is between -1 and 0.

All events are in the bulk of the ZIP detector in the modeling in section 5.4. This requires that we should use bulk events only to normalize the phonon timing parameters. But this is difficult, because there is a large fraction of events near the surfaces in the energy range from $10 \mathrm{keV}$ to $100 \mathrm{keV}$ in the Barium calibration data. Alternatively, we use high ionization yield events between 10 $\mathrm{keV}$ and $100 \mathrm{keV}$, most of which have a few $\mathrm{mm}$ or less penetration depth in the ZIP detector, then carefully balance the normalized phonon timing parameters for electron recoils (high yield single scattering events in ${ }^{133} \mathrm{Ba}$ calibration) and nuclear recoils (events in nuclear recoil band in the ${ }^{252} \mathrm{Cf}$ calibration). Ultimately, 
the reconstructed positions will be used empirically, so the choice will not bias the outcome.

There are other factors in the phonon timing parameter normalization for event location reconstruction. For example, phonon $20 \%$ amplitude time is energy dependent, so a corresponding correction should be made. Also, the reconstructed parameter $\mathrm{x}, \mathrm{y}$ and $\mathrm{z}$ needs to be considered as a whole both for electron recoils and for nuclear recoils. Here I summarize the phonon timing normalizations for Z1 through Z6 detectors of the Tower I in Soudan run118.

For a germanium detector, we write the local quadrant phonon start time parameter as

$$
t t 1=P \star r 20-Q I s t-c c 11 \cdot P \star O F e V+10 \times 10^{-6}
$$

where $P \star r 20$ stands for the phonon pulse time at $20 \%$ amplitude for phonon sensors $\mathrm{A}, \mathrm{B}, \mathrm{C}$, and $\mathrm{D}, Q I$ st is charge inner pulse start time, $\mathrm{P} \star \mathrm{OFeV}$ is collected phonon energy in $\mathrm{eV}, c c 11$ is for the start time energy dependence correction, and $t t 1$ is in seconds.

We write the normalized phonon front propagation distance as

$$
D 10=c c 12 \cdot t t 1^{2}+c c 13 \cdot t t 1+c c 14
$$

where $D 10$ is in $\mathrm{cm} . c c 12, c c 13$, and $c c 14$ are start time normalization coefficients. They are obtained with high yield single scattering events between $10 \mathrm{keV}$ and $100 \mathrm{keV}$ from ${ }^{133} \mathrm{Ba}$ calibration data and the following settings:

- $D 10=0.28 \mathrm{~cm}$ at the time of $15 \%$ of tt1 distribution amplitude at the fast timing side.

- $D 10=0.42 \mathrm{~cm}$ at the time of tt1 distribution peak. 
- $D 10=0.84 \mathrm{~cm}$ at the time of $\mathrm{X} \%$ of tt1 distribution amplitude at the slow timing side. $\mathrm{X}$ is between 2 and 5; both Barium calibration data and neutron calibration data are needed for determining X. The goal is to make sure that there are the same $\mathrm{Z}$ parameter distributions for events in all four quadrants.

$c c 11, c c 12, c c 13$, and $c c 14$ are given in Table 5.1 for germanium detectors.

\begin{tabular}{|c|c|c|c|c|c|}
\hline \hline Detector & Parameter & $\mathrm{A}$ & $\mathrm{B}$ & $\mathrm{C}$ & $\mathrm{D}$ \\
\hline $\mathrm{Z1}$ & $\mathrm{cc11}$ & 0.0900 & 0.1000 & 0.0600 & 0.0500 \\
& $\mathrm{cc12}\left(10^{8}\right)$ & -2.2083 & -0.2474 & -3.4791 & -2.3988 \\
& $\mathrm{cc13}\left(10^{4}\right)$ & 3.4339 & 2.6267 & 4.4827 & 4.7868 \\
& $\mathrm{cc14}$ & 0.1318 & -0.0245 & -0.2382 & -0.3600 \\
\hline $\mathrm{Z} 2$ & $\mathrm{cc11}$ & 0.0300 & 0.0400 & 0.0300 & 0.0300 \\
& $\mathrm{cc12}\left(10^{9}\right)$ & 3.4286 & 8.2483 & 0.2972 & 4.5585 \\
& $\mathrm{cc13}\left(10^{5}\right)$ & -0.6480 & -2.3987 & 0.5374 & -1.0350 \\
& $\mathrm{cc14}$ & 0.4003 & 2.0212 & -0.5666 & 0.8091 \\
\hline $\mathrm{Z3}$ & $\mathrm{cc11}$ & 0.0300 & 0.0300 & 0.0300 & 0.0300 \\
& $\mathrm{cc12}\left(10^{9}\right)$ & 6.9672 & 6.2525 & 0.7164 & 10.1480 \\
& $\mathrm{cc13}\left(10^{5}\right)$ & -1.6547 & -1.1366 & 0.5313 & -2.6781 \\
& $\mathrm{cc14}$ & 1.2165 & 0.6657 & -0.5011 & 1.9828 \\
\hline $\mathrm{Z5}$ & $\mathrm{cc11}$ & 0.0500 & 0.0650 & 0.0700 & 0.0550 \\
& $\mathrm{cc12}\left(10^{9}\right)$ & 0.8597 & -2.7260 & -1.9695 & -1.8519 \\
& $\mathrm{cc13}\left(10^{5}\right)$ & 0.9712 & 1.4746 & 1.2379 & 1.5315 \\
& $\mathrm{cc14}$ & -0.9840 & -0.9687 & -0.7710 & -1.2463 \\
\hline $\mathrm{Z4}$ & $\mathrm{cc11}$ & 0.0600 & 0.0800 & 0.0300 & 0.0400 \\
& $\mathrm{cc12}\left(10^{10}\right)$ & 1.5861 & 2.5954 & 1.0109 & 0.9245 \\
& $\mathrm{cc13}\left(10^{5}\right)$ & -3.2741 & -5.9950 & -1.5976 & -1.3322 \\
& $\mathrm{cc14}$ & 1.8432 & 3.6911 & 0.6529 & 0.3779 \\
\hline $\mathrm{Z6} 6$ & $\mathrm{cc11}$ & 0.0300 & 0.0400 & 0.0300 & 0.0300 \\
& $\mathrm{cc12}\left(10^{10}\right)$ & 0.5260 & 1.4900 & 2.2490 & 0.3265 \\
& $\mathrm{cc13}\left(10^{5}\right)$ & -0.1620 & -2.6012 & -5.1130 & 2.3184 \\
& $\mathrm{cc14}$ & -0.4373 & 1.0520 & 3.0326 & -2.2291 \\
\hline
\end{tabular}

Table 5.1: Local quadrant phonon timing normalization coefficients of all six detectors in Soudan Tower I.

The neighbor quadrant phonon timing normalization is done in a similar way. 


\begin{tabular}{|c|c|c|c|c|c|c|c|c|c|}
\hline \hline Detector & Parameter & A to B & A to D & B to C & B to A & C to D & C to B & D to A & D to C \\
\hline Z1 & cc21 & 0.0500 & 0.0500 & 0.0500 & 0.0300 & 0.0300 & 0.0500 & 0.0300 & 0.0500 \\
& cc22 & 0.8942 & 0.8567 & 0.8751 & 0.7111 & 0.8681 & 0.9246 & 0.7865 & 0.9169 \\
& $\mathrm{cc} 23\left(10^{-6}\right)$ & -0.3209 & -0.4082 & 0.6846 & 1.3511 & 0.0031 & 0.3971 & -0.2961 & -0.2912 \\
\hline Z2 & $\mathrm{cc} 21$ & 0.0500 & 0.0500 & 0.0500 & 0.0300 & 0.0300 & 0.0500 & 0.0500 & 0.0300 \\
& $\mathrm{cc} 22$ & 0.9801 & 0.9402 & 0.9776 & 1.0443 & 1.0145 & 0.9516 & 0.9760 & 1.0034 \\
& $\mathrm{cc} 23\left(10^{-6}\right)$ & -4.0392 & -2.7339 & -2.2183 & -4.9922 & -2.2817 & -2.8988 & -4.0545 & -1.5355 \\
\hline $\mathrm{Z3}$ & $\mathrm{cc} 21$ & 0.0500 & 0.0500 & 0.0500 & 0.0300 & 0.0300 & 0.0500 & 0.0300 & 0.0500 \\
& $\mathrm{cc} 22$ & 1.0039 & 0.9241 & 0.9331 & 0.9264 & 0.9439 & 0.9253 & 0.9035 & 1.0324 \\
& $\mathrm{cc} 23\left(10^{-6}\right)$ & -0.7517 & -1.2454 & 1.1684 & -0.8812 & -1.2427 & 1.0170 & -1.2640 & -1.2454 \\
\hline $\mathrm{Z5}$ & $\mathrm{cc} 21$ & 0.0900 & 0.1200 & 0.0900 & 0.0900 & 0.0600 & 0.0800 & 0.1000 & 0.1200 \\
& $\mathrm{cc} 22$ & 1.0807 & 1.0886 & 1.0336 & 1.0665 & 1.0653 & 0.9880 & 1.0391 & 1.1118 \\
& $\mathrm{cc} 23\left(10^{-6}\right)$ & 2.7922 & 2.5852 & 3.5661 & 2.7204 & 2.6066 & 3.3245 & 2.6565 & 3.0462 \\
\hline
\end{tabular}

Table 5.2: Neighbor quadrant phonon timing nromalization coefficients of germanium detectors Z1, Z2, Z3 and Z5. There are two neighbor phonon sensors for events in a particular quadrant.

\begin{tabular}{|c|c|c|c|c|c|c|c|c|c|}
\hline \hline Detector & Parameter & A to B & A to D & B to C & B to A & C to D & C to B & D to A & D to C \\
\hline Z4 & $\mathrm{cc} 21\left(10^{-6}\right)$ & 80 & 90 & 80 & 80 & 100 & 76 & 88 & 74 \\
& $\mathrm{cc} 22$ & 3.1551 & 2.5422 & 2.9614 & 2.9459 & 2.4588 & 3.0089 & 2.9255 & 3.4274 \\
& $\mathrm{cc} 23\left(10^{-6}\right)$ & -8.2916 & -6.4035 & -6.8856 & -7.5306 & -5.4163 & -7.0155 & -8.3802 & -9.9492 \\
\hline $\mathrm{Z} 6$ & $\mathrm{cc} 21\left(10^{-6}\right)$ & 80 & 80 & 74 & 78 & 74 & 74 & 84 & 80 \\
& $\mathrm{cc} 22$ & 2.9617 & 2.8122 & 3.2075 & 3.0222 & 3.2692 & 3.1336 & 2.6357 & 2.8814 \\
& $\mathrm{cc} 23\left(10^{-6}\right)$ & -7.6983 & -5.9146 & -8.9925 & -8.0444 & -9.5846 & -8.8313 & -5.2155 & -7.3017 \\
\hline
\end{tabular}

Table 5.3: Neighbor quadrant phonon timing normalization coefficients of silicon detectors Z4 and Z6. There are two neighbor phonon sensors for events in a particular quadrant.

We write the phonon start time parameter as

$$
t t 2=P \star r 20-Q I s t-c c 21 \cdot P \star O F e V
$$

where $\star$ stands for phonon sensors A, B, C, and D, $c c 21$ is for the start time energy dependence correction, and $t t 2$ is in seconds.

We write the normalized phonon front propagation distance as

$$
D 20=(c c 22 \cdot t t 2+c c 23) \cdot 1.25 \times 10^{5}
$$

where $D 20$ is in cm. $c c 22$ and $c c 23$ are start time normalization coefficients. They are calculated with high yield single scattering events between $10 \mathrm{keV}$ and 100 $\mathrm{keV}$ from ${ }^{133} \mathrm{Ba}$ calibration data and the following settings: 
- $D 20=0.70 \mathrm{~cm}$ at the time of $20 \%$ of $\mathrm{tt} 2$ distribution shoulder at the fast timing side.

- $D 20=4.80 \mathrm{~cm}$ at the time of $10 \%$ of tt2 distribution shoulder at the slow timing side.

We need to keep in mind that there are two neighbor phonon sensors for each detector quadrant. $c c 21, c c 22, c c 23$ are given in Table 5.2 for germanium detectors.

For silicon detectors, the local quadrant phonon timing normalization is done just as they are for the germanium detectors. The timing normalization coefficients for silicon detectors $\mathrm{Z} 4$ and $\mathrm{Z} 6$ are in Table 5.1.

The neighbor quadrant phonon timing normalization is done in a different way for silicon detectors: We write the phonon start time parameter as

$$
t t 21=P \star r 20-Q I s t
$$

and

$$
t t 22=t t 21 \cdot(1-(t t 21 / c c 21))
$$

where $\star$ stands for phonon sensors A, B, C, and D. We write the normalized phonon front propagation distance as

$$
D 20=(c c 22 \cdot t t 22+c c 23) \cdot 1.25 \times 10^{5}
$$

$c c 21$ is special: the purpose is to have uniform event location distribution inside a $3.5 \mathrm{~cm}$ radius of the ZIP detector in the energy range of $10 \mathrm{keV}$ to $100 \mathrm{keV}$. There are several possible reasons for this. The old QET structure has low quasi-particle collection efficiency because of the long aluminum phonon trap stripes. Phonon energy collection defficiency can lead to long phonon start times for events far 
away from the phonon sensor. Another possible reason is the phonon focusing effect [110]. $c c 22$ and $c c 23$ are start time normalization coefficients.

The neighbor quadrant timing parameter normalization coefficients for silicon detectors are in Table 5.3.

\subsection{Understanding Event Position Information}

In this section, we describe how the collected phonon energy distribution and the phonon timing parameter distributions in the ZIP detector change with the reconstructed $\mathrm{x}, \mathrm{y}$, and $\mathrm{z}$ parameters.
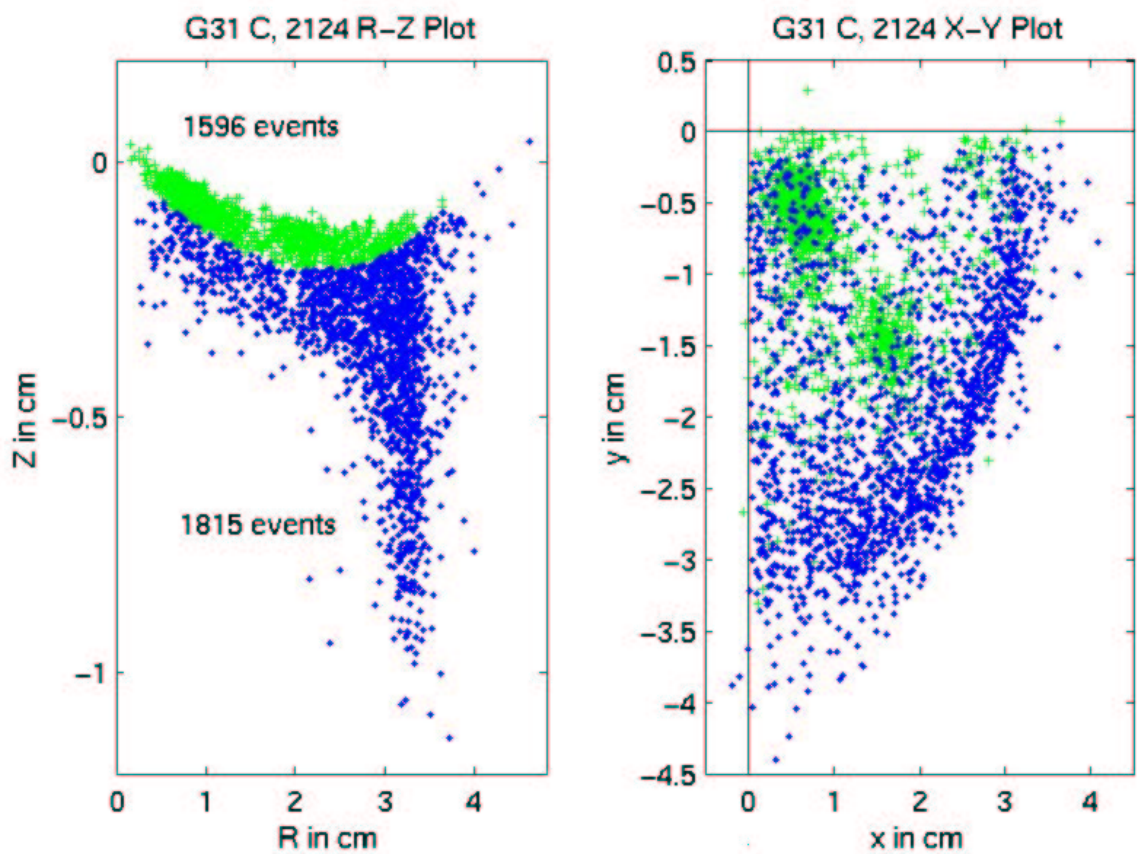

Figure 5.18: Reconstructed event locations for G31, quadrant C. The two ${ }^{109} \mathrm{Cd}$ source spots are visible. External sources: ${ }^{60} \mathrm{Co}+{ }^{252} \mathrm{Cf}$, data set 221110_2124.

Two important facts become clear from figure 5.18. The events (green crosses) selected in the R-Z plane correspond to Cadmium source positions. The recon- 


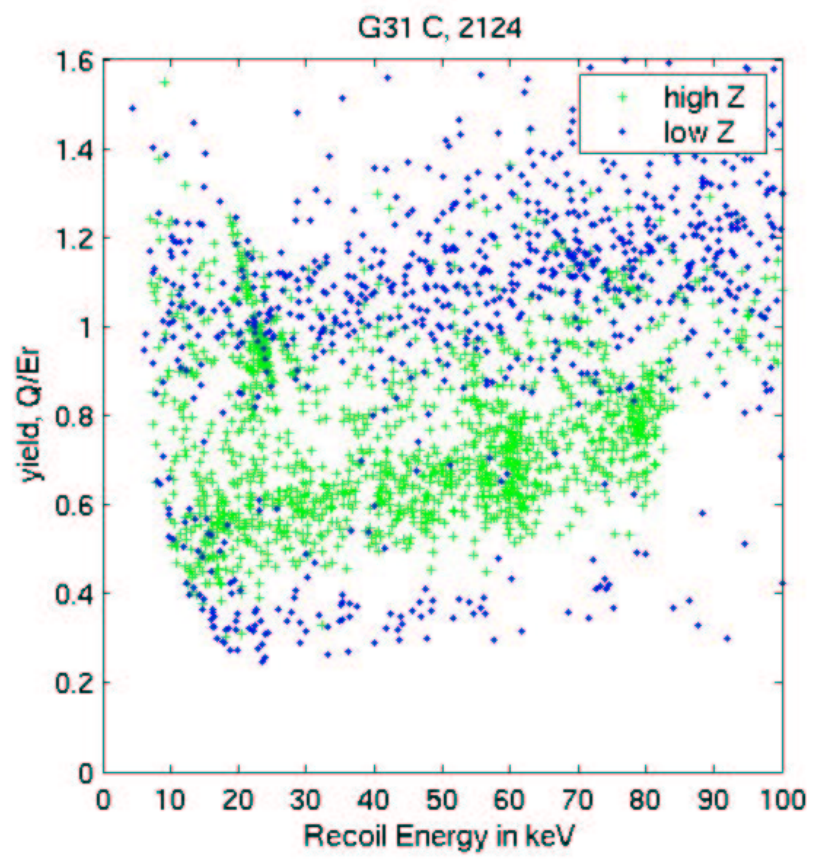

Figure 5.19: Yield versus recoil energy plot for G31, quadrant C. It confirms that the events with $\mathrm{Z}$ parameter close to 0 are from ${ }^{109} \mathrm{Cd}$ sources. External sources: ${ }^{60} \mathrm{Co}+{ }^{252} \mathrm{Cf}$.

structed $\mathrm{x}$ and $\mathrm{y}$ parameters do give the correct event $\mathrm{x}$ and $\mathrm{y}$ position in the ZIP detector. The large number of events at a big radius comes from the Compton scattering in copper of the DIB (see Chapter 3). The reconstructed z parameter carries the surface event discrimination message. Most surface events (from the Cadmium electron source) have a $\mathrm{z}$ parameter close to zero. This is because surface events have fast timing. They are the $22 \mathrm{keV} \gamma$ line, $63 \mathrm{keV}$ and $84 \mathrm{keV} \beta$ lines from the Cadmium source. The yield versus recoil energy plot in figure 5.19 confirms that this is the case. Most of the events that have a $\mathrm{Z}$ parameter close to zero are in the electron-electron recoil band (the green cross band), or are the $22 \mathrm{keV}$ gammas.

We notice that there are events with a low value $\mathrm{z}$ parameter beyond the Cad- 
mium source spots in Figure 5.18 and in the gamma band (yield=1) in Figure 5.19. They are gamma events close to the surfaces of the ZIP detector.
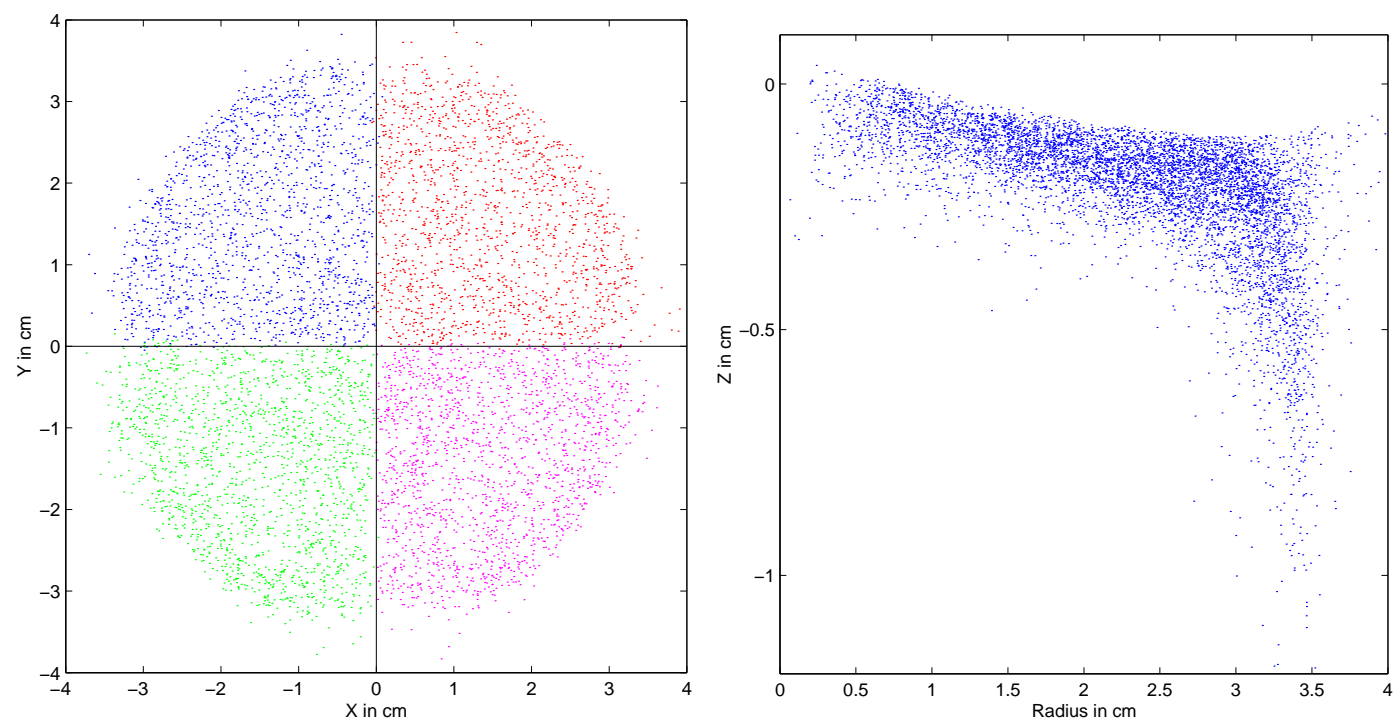

Figure 5.20: Z5: reconstructed event locations for high ionization yield events in ${ }^{133} \mathrm{Ba}$ calibration data of Soudan run118.

The reconstructed event location parameters reflect the fundamental physics processes in the ZIP detector. We explore the possible application of the reconstructed parameters by using Z5 as an example. The reconstructed $\mathrm{x}, \mathrm{y}$, and $\mathrm{z}$ parameters from Soudan run $118{ }^{133} \mathrm{Ba}$ calibration data are in Figure 5.20. The left plot shows two dimensional event distributions, while the right plot shows the event depth distribution.

Ionization yield, phonon start time and phonon rise time are used as background discrimination parameters in the CDMS experiment. These parameters' distributions as a function of radius are shown in Figures 5.21, 5.22, and 5.23. The limited phonon capture aluminum fin coverage for events at a big radius lead to phonon collection efficiency decreasing and phonon timing increasing. Although both phonon energy and phonon timing position corrections have been made for 

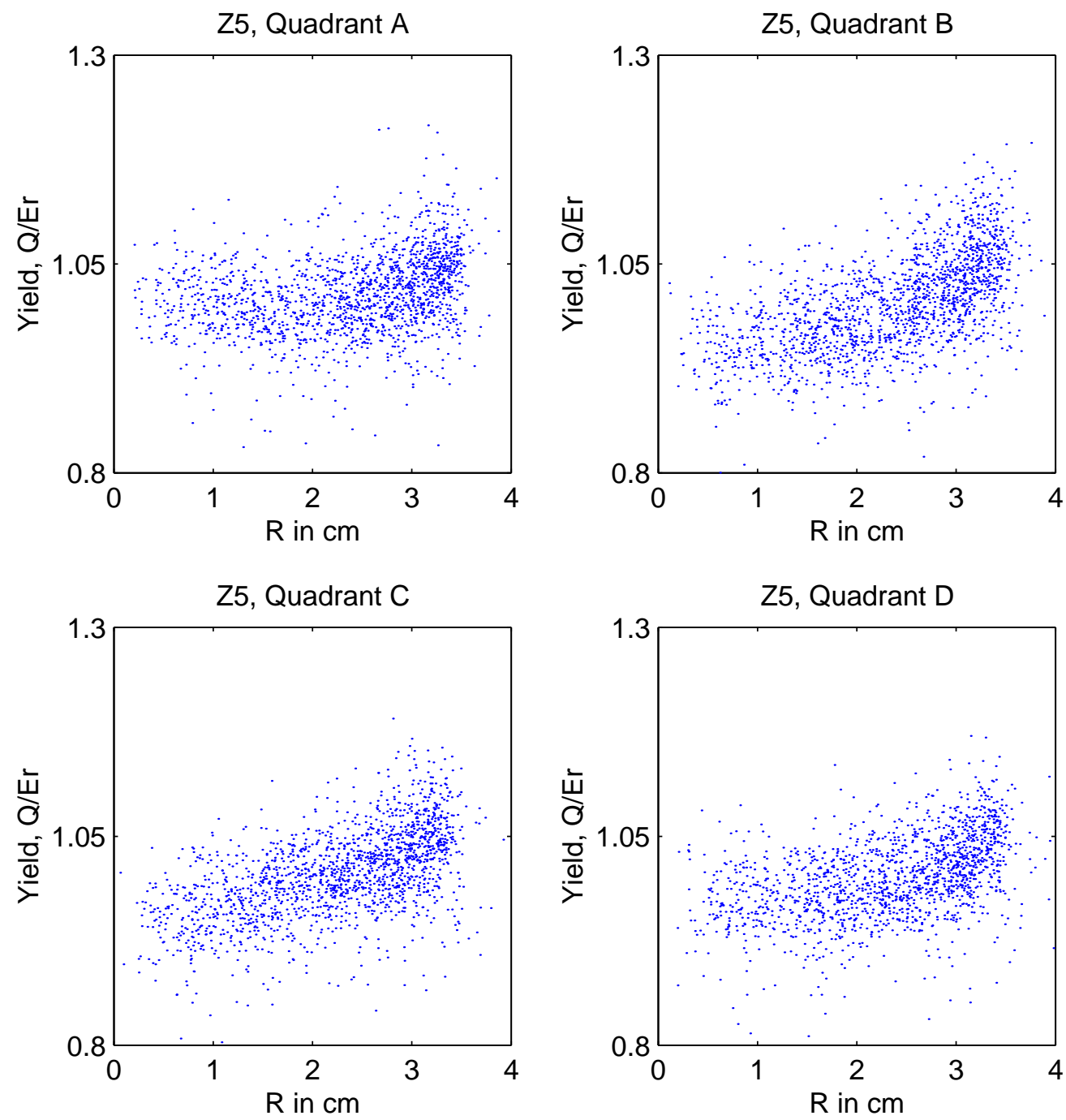

Figure 5.21: Z5: yield distribution as a function of radius for local quadrant events for high ionization yield events in ${ }^{133} \mathrm{Ba}$ calibration data of Soudan run118.

dealing with these problems, we still need to pay attention to low background events at a big radius. As we will see in Chapter 7, surface electron recoils that fail rise time cut most likely have big radius parameter.

Comparing the neutrons and surface events (which are defined as low yield nearest neighbor double scattering event as shown in the left plot in figure 5.27) is the method by which we define surface background rejection cuts. Figure 5.24 

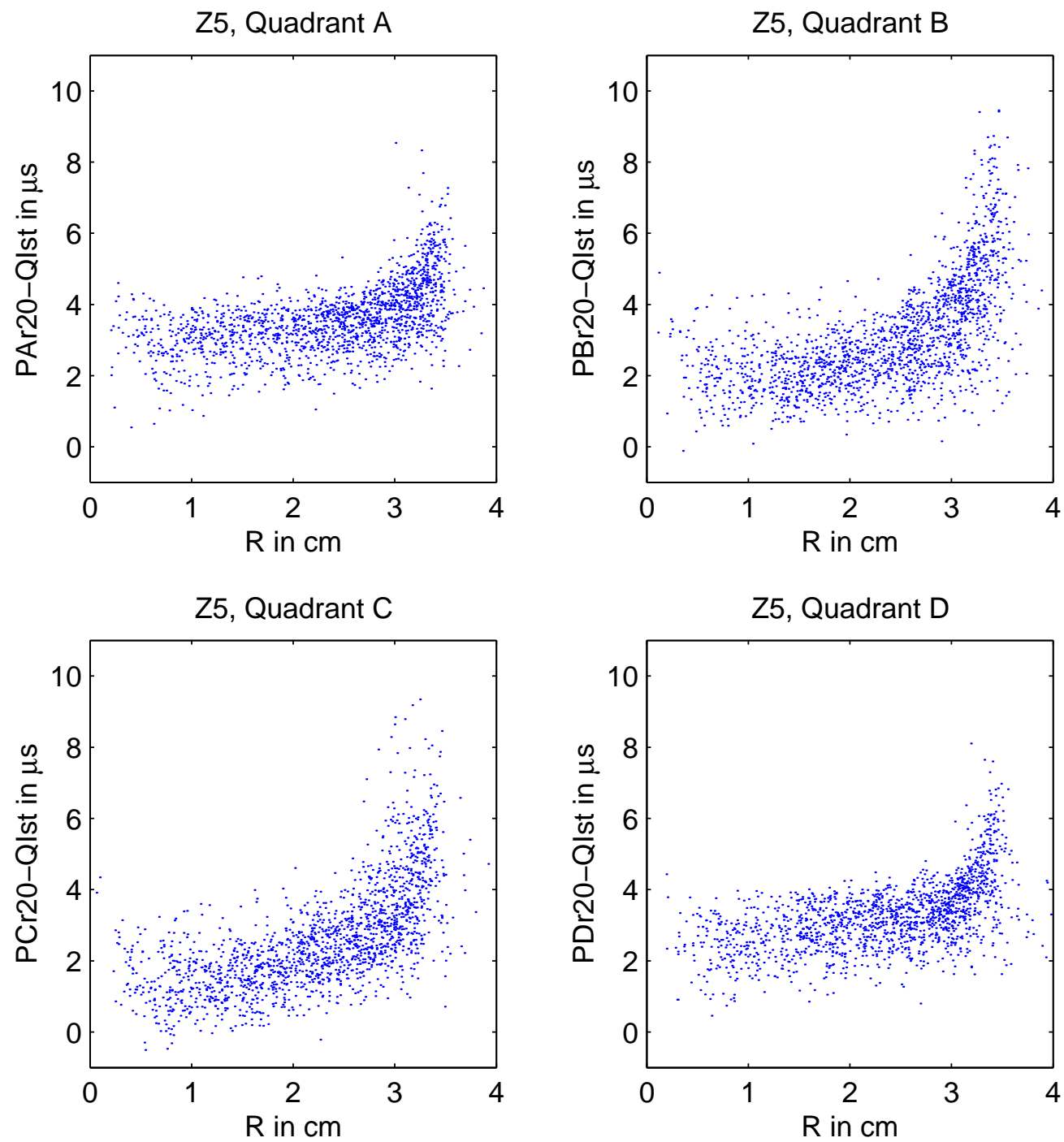

Figure 5.22: Z5: Phonon start time distribution as a function of radius for local quadrant events for high ionization yield events in ${ }^{133} \mathrm{Ba}$ calibration data of Soudan run118.

shows neutrons and surface events in $\mathrm{X}$ and $\mathrm{Y}$ plane. Neutrons generate more optical phonons in the bulk of the ZIP detector. Both the big radius and the diamond structure in the $\mathrm{X}$ and $\mathrm{Y}$ plot are the reflection of slow optical phonon decay. In contrast, surface events have a smaller radius parameter distribution.

The reason may be because nuclear recoils have suppressed ionization yield, 

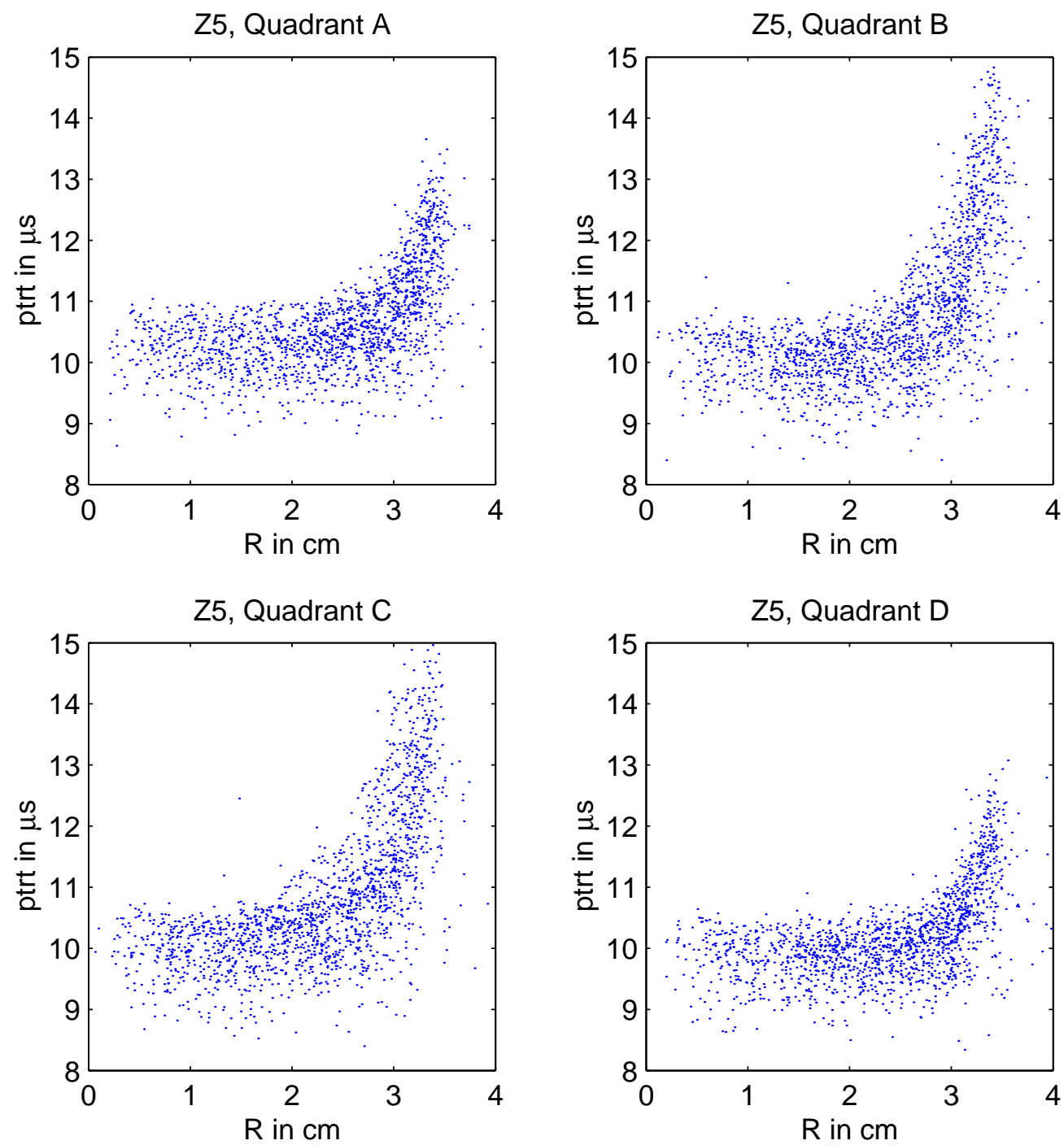

Figure 5.23: Z5: Rise time distribution as a function of radius for local quadrant events for high ionization yield events in ${ }^{133} \mathrm{Ba}$ calibration data of Soudan run118.

so the slow propagating, high energy primary phonons dominate. This is especially true for the low recoil energy nuclear recoil events, because the suppressed ionization yield means fewer fast Neganov-Luke phonons.

In Figure 5.25, the $\mathrm{Z}$ parameter is flattened by making a radial correction defined by the red line (for $f(R)$ as shown by the red line in the left plot), a new 


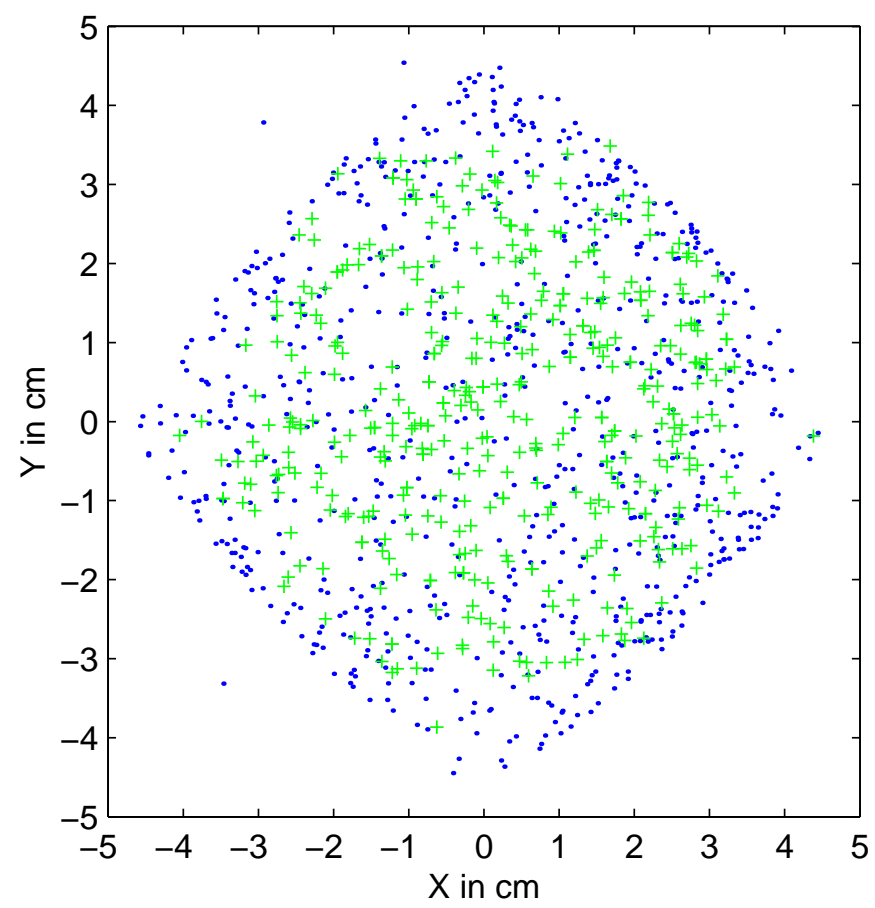

Figure 5.24: Z5: Neutron (blue dots) and surface events (green crosses) in the $\mathrm{x}$ and y plane. From ${ }^{252} C_{f}$ calibration data and ${ }^{133} \mathrm{Ba}$ calibration data in Soudan run118.

parameter is defined as $Z Z=Z-f(R)$. A plot of $Z Z$ versus $\mathrm{R}$ plot is on the right side of Figure 5.25. The left plot in Figure 5.26 shows the ZZ parameter versus ionization yield. The right plot in Figure 5.26 shows surface event discrimination capability of the ZZ parameter for the events with ionization yield less than 0.5. The blue line $(\alpha)$ is neutron selection efficiency as a function of ZZ; the dashed green line $(\beta)$ is surface event selection fraction as a function of the ZZ; and the magenta dashed dot line is quality factor $Q[111]$

$$
Q=\frac{\beta(1-\beta)}{(\alpha-\beta)^{2}}
$$

If we set the bulk event selection line at the minimum value of $Q$ and keep only events with $Z Z<-0.28 \mathrm{~cm}$ in the right plot in Figure 5.26 , then $72 \%$ of neutrons are selected, $97 \%$ surface events are rejected. The overall surface event rejec- 

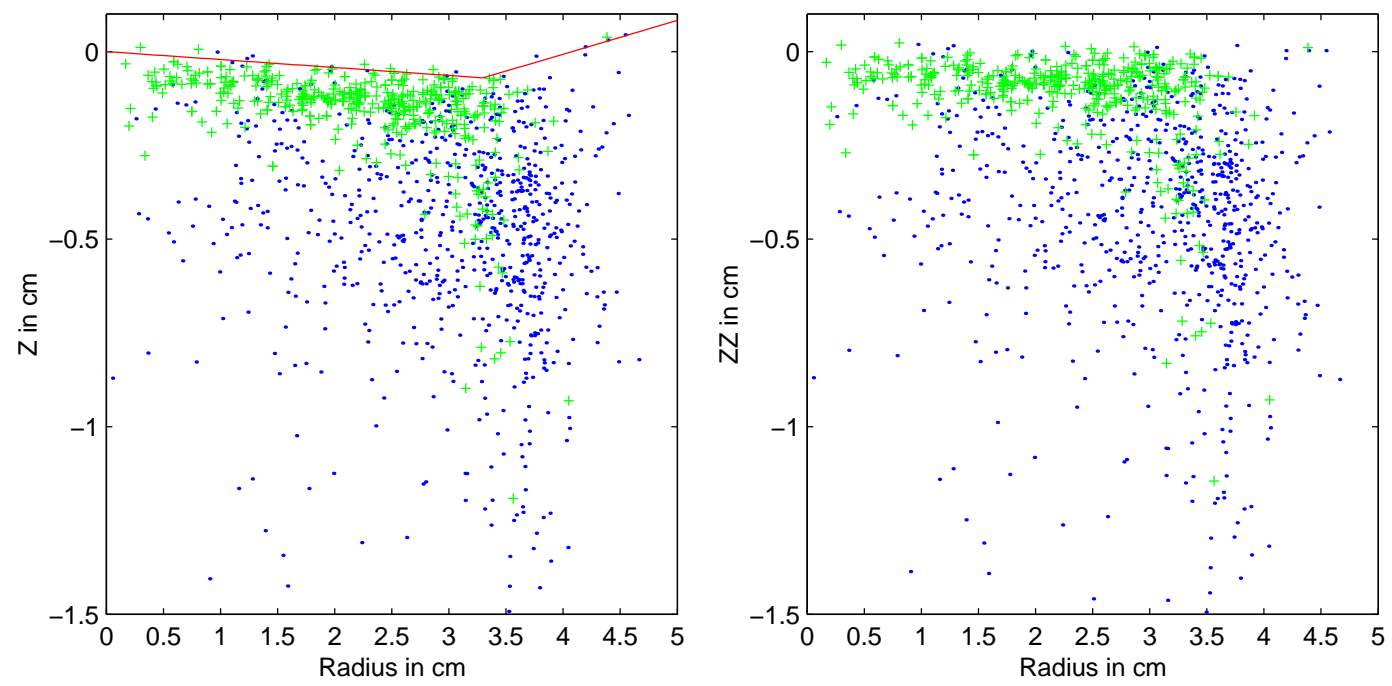

Figure 5.25: Z5: Neutron (blue dots) and surface events (green crosses) in the R and $\mathrm{Z}$ plane. From ${ }^{252} C_{f}$ calibration data and ${ }^{133} \mathrm{Ba}$ calibration data in Soudan run118. The right plot shows the flattened $\mathrm{Z}$ parameter along the red line in the upper part of the left plot.
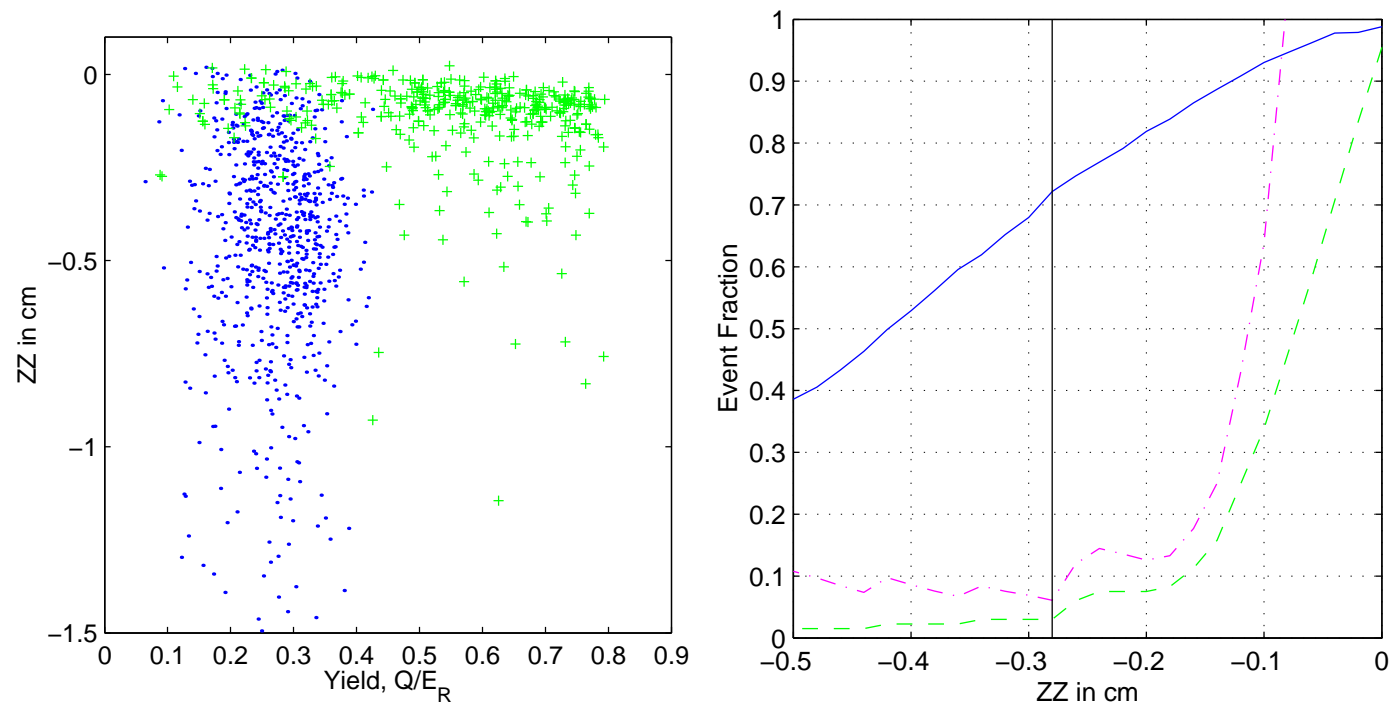

Figure 5.26: Left: Z5, ZZ versus ionization yield for neutron (blue dots) and surface events (green crosses). Right: Z5, The discrimination capability of ZZ for neutron (blue solid) and surface events (green dashed line). From ${ }^{252} C_{f}$ calibration data and ${ }^{133} \mathrm{Ba}$ calibration data in Soudan run118. 

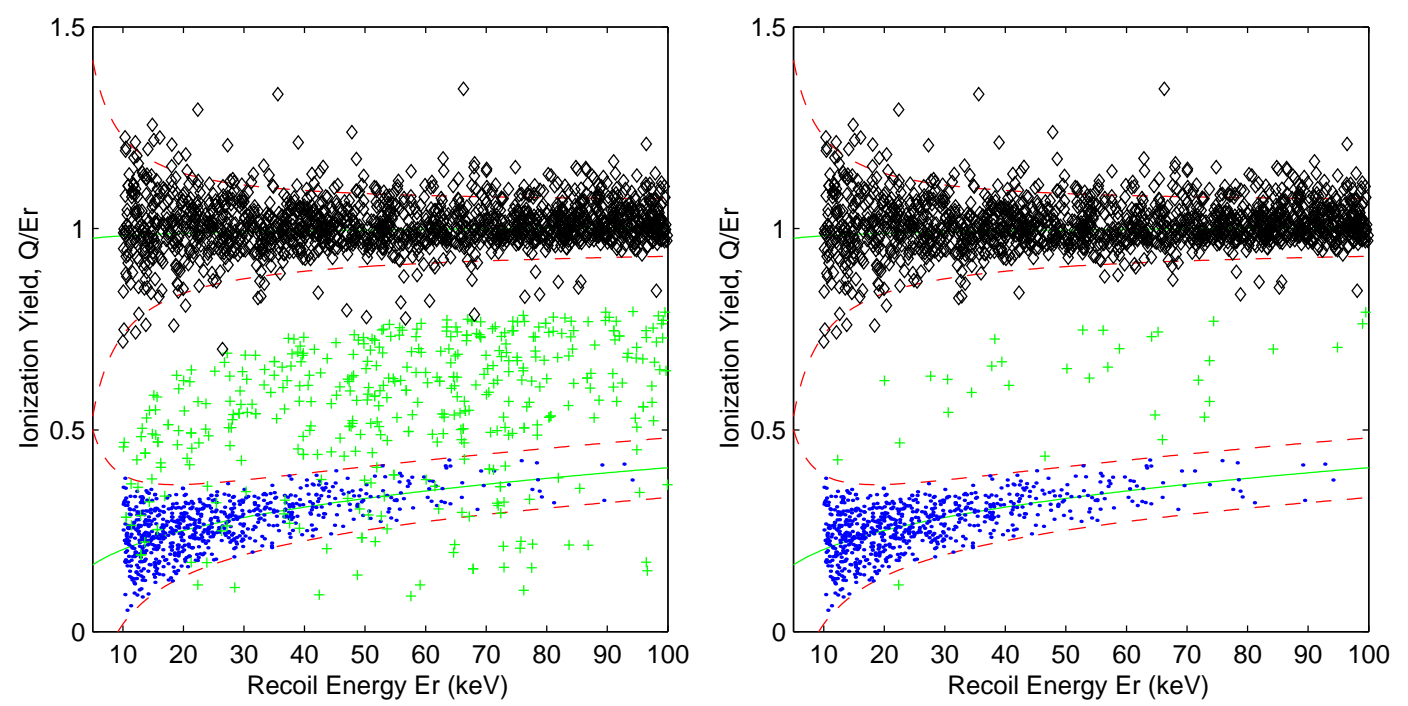

Figure 5.27: Left: $\mathrm{Z} 5$, before the $\mathrm{ZZ}=-0.28 \mathrm{~cm}$ cut applied to ejectrons (green crosses). The ejectrons are from data set 131211_0920(85k) and data set 131211_1159(56k). Right: Z5, after the $\mathrm{ZZ}=-0.28 \mathrm{~cm}$ cut applied to the ejectrons (green crosses). From ${ }^{252} C_{f}$ calibration data and ${ }^{133} \mathrm{Ba}$ calibration data in Soudan run118. The number of high ionization yield events is reduced by a factor of 20 .

tion efficiency is much higher than $97 \%$ once we include ionization yield, phonon timing, and event location information. We will fully discuss the surface event rejection in Chapter 7 when the full analysis of WIMP search data is described.

Figure 5.27 shows the ejectrons before the $\mathrm{ZZ}=-0.28 \mathrm{~cm}$ cut (left) and after the $\mathrm{ZZ}=-0.28 \mathrm{~cm}$ cut (right) in ionization yield versus recoil energy plot.

The good surface rejection parameters should include not only z information (and other timing parameters), but also $\mathrm{x}$ and $\mathrm{y}$. This is true for events that have a radius bigger than $2.7 \mathrm{~cm}$. This will be the main topic of Chapter 7 .

There is still a lot of room for improvement of event location reconstruction in a ZIP detector. For example, several phonon and detector physics processes need to be understood better. Knowledge of phonon reflection coefficients on the surface of a ZIP detector will allow us to find a more precise phonon energy flux as 
a function of time. TES thermal parameters, such as TES heat capacity and the thermal coupling coefficient $\kappa$, will allow us to have a more precise TES thermal response time. The quasi-particle diffusion process corrects the phonon timing parameter with quasi-particle diffusion time. The phonon energy flux $E_{p}(t)$ as a function of time can be identified with a better understaning of phonon physics processes in the ZIP detector, allowing for more precise event timing and location information.

The collected phonon energy distribution and phonon timing parameter distributions in the ZIP detector will be used for surface event rejection in the Soudan run118 low background data analysis in chapter 7 . 


\section{Chapter 6}

\section{Detector Setup and Calibrations}

The science results reported in this thesis are based on operating 6 ZIP detetcors, collectively refered as "Tower I" in the low-background cryostat at Soudan. "Tower I" detectors underwent many procedures before they were installed in the ice box in the Soudan mine. They were fabricated in CIS at Stanford University, and underwent numerous tests and calibrations at Case Western Reserve University and at UC Berkeley. They were run for nearly a year in the CDMS-1 ice box at the Stanford Underground Facility, and are well understood by the CDMS collaboration.

${ }^{133} \mathrm{Ba}$ gamma calibrations and ${ }^{252} \mathrm{Cf}$ neutron calibrations were done from the beginning to the end of Soudan run118. The purpose of these calibrations was to check the state of detector neutralization for good charge collection efficiency, to determine the best TES biases of these detectors compatible with the base temperature of the dilution refrigerator at Soudan, and to get the correct energy scale for charge and phonon channels. Another important application of the calibrations is to define the electron recoil band and nuclear recoil band. These bands are the first step to low background data analysis. This chapter will explain, in 
some detail, the ZIP detector setup and calibrations, quality data selection, and the gamma and nuclear recoil bands of the detectors in CDMS Soudan run118.

\subsection{Detector Neutralization}

The ionization yield, collected charge energy divided by recoil energy, is the most important background rejection parameter in the CDMS experiment, because low ionization yield nuclear recoil events are candidates for WIMPs. But the yield strongly depends on the neutralization state of the detectors. The trapped charges (ionization states of impurities and defects) in silicon and germanium must be removed before data collection. To achieve this goal, we shined infrared light onto the detectors. The light penetrated the surface of the silicon and germanium, creating free electrons and holes, so the trapped charge can be neutralized with the combination of these injected free charges.

During the first two weeks of the detectors' cooling down to base temperature, the main task was flashing the infrared LEDs installed in the DIB of the tower, neutralizing the detectors. The details of the LED baking schemes and their physics are in Appendix A.

To check the neutralization state, we look at the Barium calibration data energy spectra, which have several typical energy lines. The energy scales for both the ionization channels and phonon channels are also established this way. The pulse height to energy conversion for the ionization channel has no free parameters. This is only true, however, once the detectors have been fully neutralized. The ionization energy calibration therefore becomes, in essence, a determination of whether the detectors are properly neutralized.

An ideal way to check a detector neutralization state is to expose it to an 
external monochromatic radiation source and determine whether the shape and position of the spectral peak are as expected. Sources that produce a gamma line in the energy range of interest, $10<E<100 \mathrm{keV}$ would be ideal. However, gamma rays of this energy are quite efficiently absorbed by the $\mathrm{Cu}$ cans of the ice box and the tower, with the resulting flux reaching the detectors becoming extremely attenuated and dominated by continuum Compton gamma rays.

In Soudan run118, a barium source is used for the initial calibrations. Barium produces several typical energy lines with an energy spectrum with features. The two apparent lines are the $356 \mathrm{keV}$ line and the $384 \mathrm{keV}$ line. Relative high energy gammas can pass through the Si and Ge detectors easily. A sufficient number of gammas are absorbed to help neutralize the detectors when the detectors are grounded.

Z1 through Z5 were neutralized during the first two weeks of cooling down, but Z6 was troublesome. With additional LED baking schemes, it was eventually neutralized. We believed that this behavior was the consequence of two effects: (1) the detector, at the bottom of the stack, has been only exposed to its own LEDs above the silicon crystal, and (2) the LEDs themselves may have been mounted incorrectly in the detector housing, resulting in a diminished photon flux at the detector. Once the detectors have been neutralized, they remain so as long as their temperature doesn't rise above $1 \mathrm{~K}$. No evidence of deterioration was seen throughout the runs in the test facilities, in run21 at the Stanford Underground Facility, and in Soudan run118.

As will be discussed in section 6.4 about detector calibration, the agreement between data and the Monte Carlo simulation demonstrated adequate detector neutralization. 


\subsection{Optimizing TES Bias}
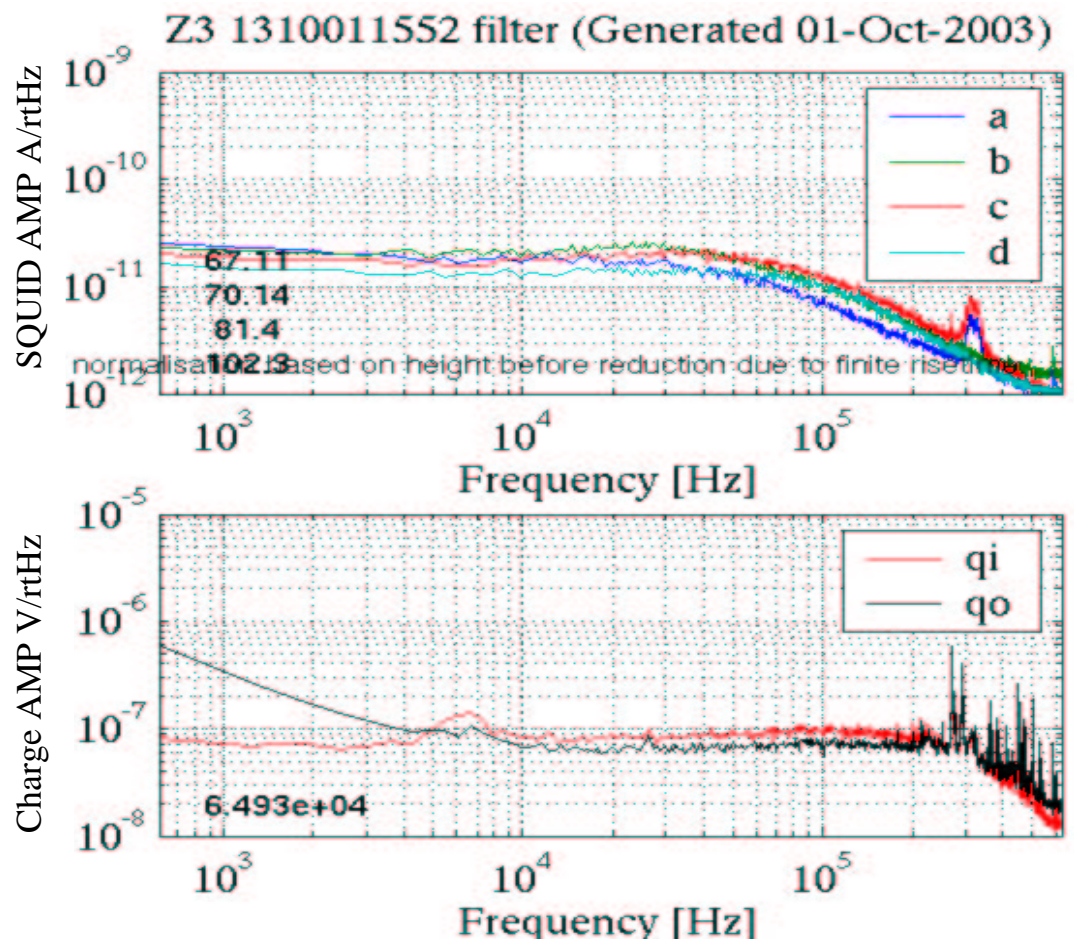

Figure 6.1: Noise Spectra of Z3.

We need to know the two most frequent used plots in the CDMS experiment before we discuss TES bias tuning. Roughly speaking, these two plots are quasi $\mathrm{x}-\mathrm{y}$ position plots based on phonon timing or energy sharing between the four phonon sensors.

- phonon delay plot: neighbor quadrant phonon start time minus local quadrant phonon start time. For events in quadrant $\mathrm{A}$, the $\mathrm{x}$ axis is $x d e l=$ $-(P D r 20-P A r 20)$, and the y axis is $y d e l=(\operatorname{PCr} 20-P A r 20)$; for events in quadrant $\mathrm{B}, x d e l=-(\operatorname{PCr} 20-P B r 20)$ and $y d e l=-(\operatorname{PAr} 20-P B r 20)$, and so on. $P A r 20, P B r 20, P C r 20$, and $P D r 20$ are phonon pulse $20 \%$ amplitude time in phonon sensors A, B, C, and D. The upper left graph in 

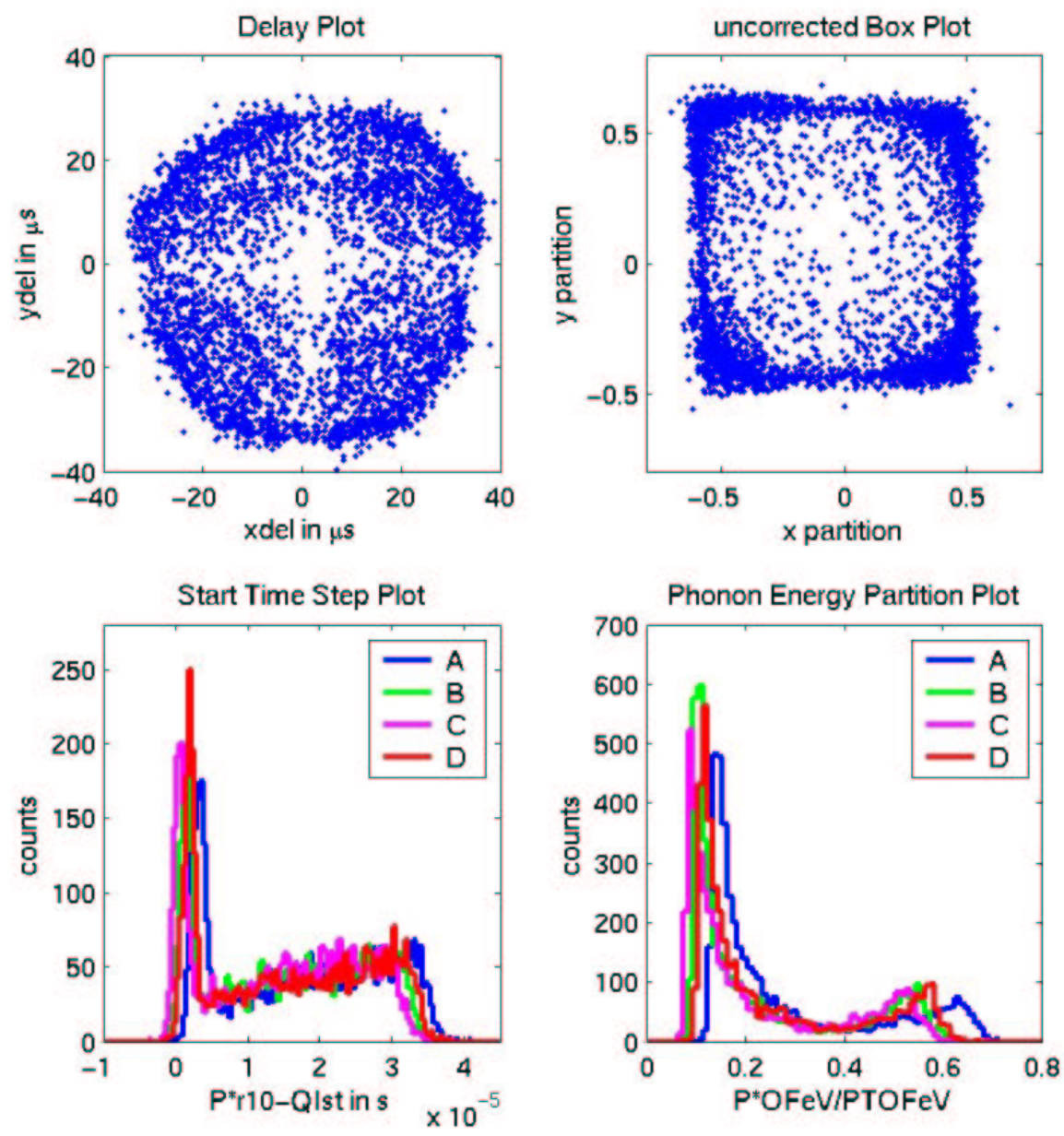

Figure 6.2: Balancing phonon energy and timing parameters of Z3.

figure 6.2 is the delay plot.

- Phonon box plot: also referred to as phonon energy partition plot. For events in quadrant $\mathrm{A}, \mathrm{x}$ axis is $-(p a+p b-p c-p d) /(p a+p b+p c+p d), \mathrm{y}$ axis is $(p a+p d-p b-p c) /(p a+p b+p c+p d)$; for events in quadrant $\mathrm{B}, \mathrm{x}$ axis is $-(p a+p b-p c-p d) /(p a+p b+p c+p d), \mathrm{y}$ axis is $-(p b+p c-p a-$ $p d) /(p a+p b+p c+p d)$, and so on. $p a, p b, p c$, and $p d$ are collected phonon energy in phonon sensors A, B, C, and D. The upper right plot in figure 6.2 
is the phonon box plot.

Optimization of the TES bias includes maximizing signal to noise ratio, and balancing the phonon delay and phonon box plots. The phonon delay plot carries four phonon sensors' timing parameters. It strongly depends on the TES bias of each phonon sensor. The phonon box plot carries four phonon sensors' thermal dynamic information and TES bias information.

When the TES bias is low, the electric thermal feedback circuit and the SQUID readout system give high noise output, but when TES bias is high, the signal output amplitude is small. A balanced TES bias range should be identified [112]. We did this by looking at the noise spectra of the phonon sensors. Low TES bias gives a low frequency noise spectrum above $10^{-10} A / \sqrt{H z}$ range; this is because of the TES sensors' low impedance. TES sensors are almost in a superconducting state at low bias. High TES bias gives an extremely low noise spectrum at high frequency; it is an indication that we are losing the signal to noise ratio. The balanced TES bias gives a flat noise spectrum around $10^{-11} A / \sqrt{H z}$ from a low frequency up to $5 \times 10^{5} \mathrm{~Hz}$. See Figure 6.1 for the noise spectra of the well biased detector Z3.

The delay plot and box plot together are used to make position corrections of phonon energies and timing parameters. The corrected ionization yield and timing are used to reject background, and to identify nuclear recoils. So, the second reason to tune TES biases is to balance the delay plot and box plot.

THe TES L/R time is sensitive to the TES bias; especially when the TES bias is low. We can use the L/R time for TES tuning, i.e., the four phonon channels' start times relative to charge start time allow us to find out whether the TES bias should be increased or should be decreased to balance delay plot and box plot. 
The optimized TES biases of the four quadrants generate similar phonon pulse shapes and phonon timing values. This will allow us to reject background in a near perfect way, and select nuclear recoils at high confidence levels.

In Figure 6.2, the four phonon channels' start times relative to charge are balanced, and the delay plot and box plot are close to the ideal case. The high phonon energy partition value of channel A could come from two facts: slightly low TES bias current and small TES heat capacity. To fine tune the phonon delay plot and phonon partition plot, two parameters, L/R (the TES time constant) and Q/C (the phonon pulse height for a collected phonon energy Q; C is TES heat capacity at bias point) should be ideally balanced. The thermal parameter study in Section 4.5 describes the underlying basis tuning TES bias.

The phonon box plot and phonon timing parameters (such as phonon pulse start time and rise time) can be corrected in the off-line data analysis. Because phonon energy measurement resolution depends on TES bias, and the off-line correction needs to use the phonon delay plot, proper the TES bias settings of the ZIP detector in the first place ensure good phonon energy resolution and the effectiveness of background rejection with phonon timing parameters.

\subsection{Position Dependence}

The position dependence of charge collection was pointed out by Blas Cabrera. The charge collection efficiency varies with event location along the y axis. This effect could come from the tunneling effects of electrons and holes in the amorphous silicon layer. The reason that the amorphous silicon layer was introduced was to overcome the dead layer problem by preventing the back diffusion of electrons and holes by using the energy gap difference between amorphous silicon and crystalline 
germanium $[90,79]$. But the potential energy difference of charges changes with the thickness of amorphous silicon. This could result in a slight change in charge collection efficiency. The charge collection efficiency position dependence mainly happens in germanium detectors. The energy gap difference between crystal silicon and amorphous silicon is small, so there is no charge collection efficiency change with event location.

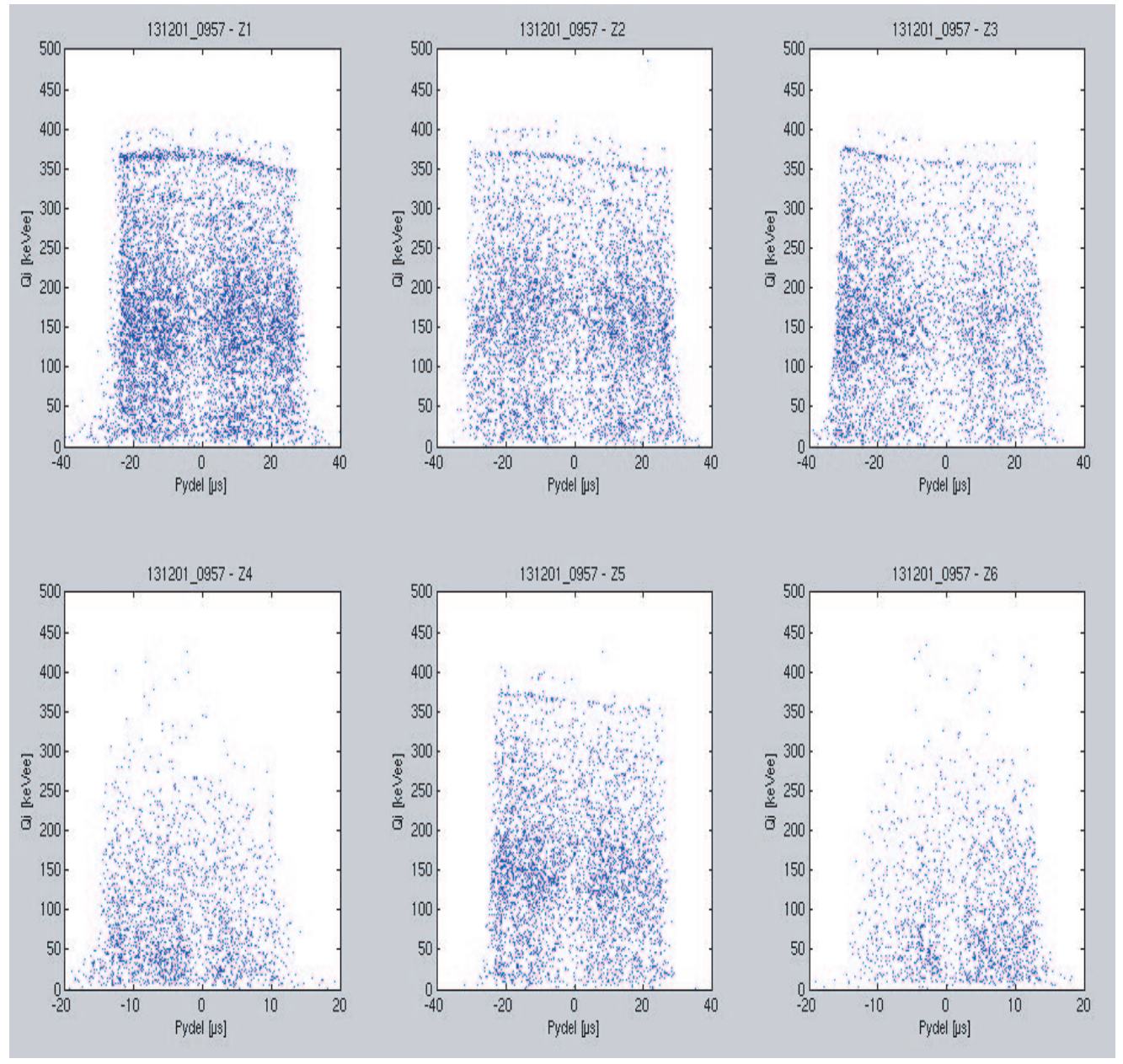

Figure 6.3: Y dependence of charge collection efficiency. Charge inner electrode events are selected. Qi is charge inner electron equivalent evergy, Pydel is a time parameter that gives event y position in the ZIP detector. The $356 \mathrm{keV}$ line in germanium detectors changes with Pydel. Figure from Blas Cabrera. 


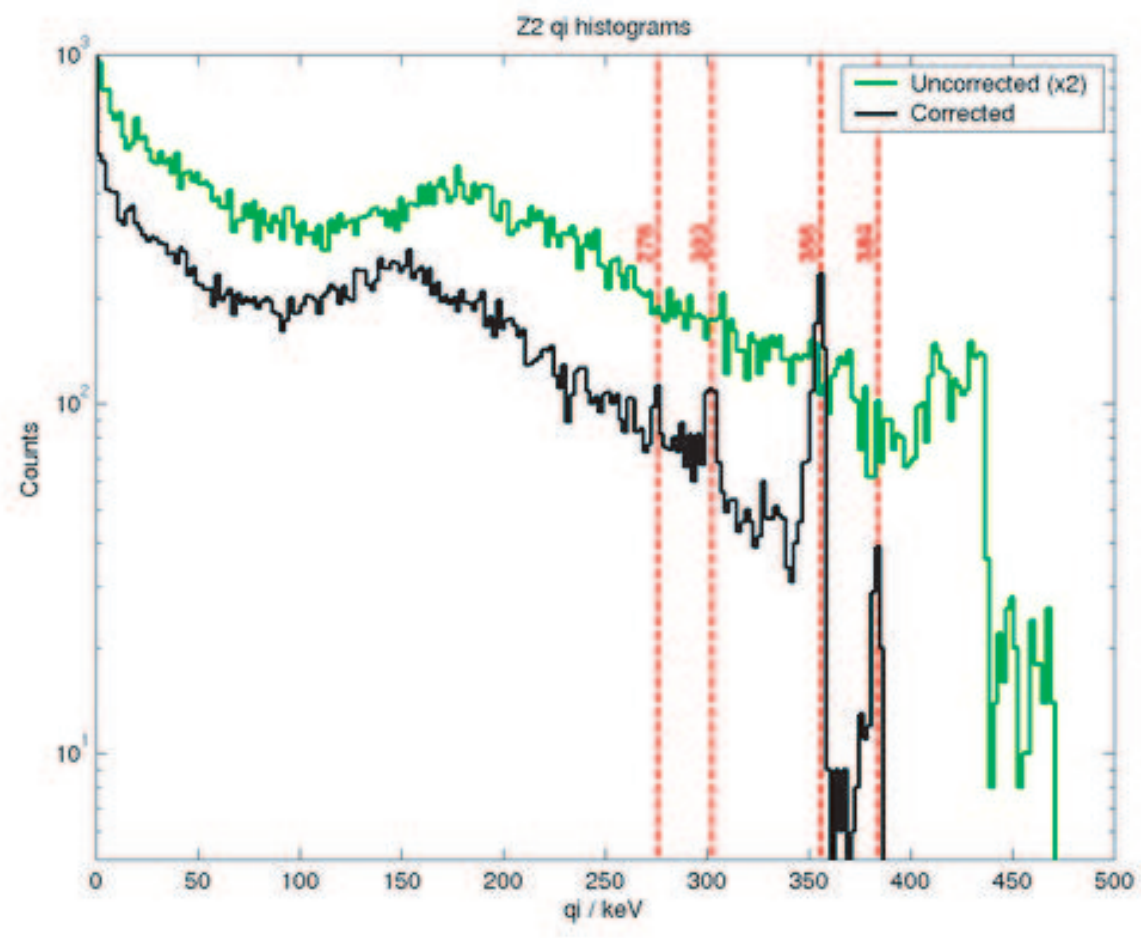

Figure 6.4: Charge energy spectra before and after position correction. The typical lines $(276 \mathrm{keV}, 303 \mathrm{keV}, 356 \mathrm{keV}$ and $384 \mathrm{keV})$ from ${ }^{133} \mathrm{Ba}$ can be seen. Figure from [113].

There are several lines visible around $300 \mathrm{keV}$ in the ${ }^{133} \mathrm{Ba}$ source. This makes it much easier to see this effect. In each of the four germanium detectors of Tower I, there is a strong dependence on ydel (see figure 6.3) and a weaker dependence on xdel.

These charge collection efficiency changes can be corrected with event location information from phonon channels, and the particular lines in the Barium calibration. To correct the ydel dependence, we fit a sine wave to the $356 \mathrm{keV}$ peak as it varies with ydel, then make the corresponding corrections. Similarly, to correct the xdel dependence, we fit a sine wave to the $356 \mathrm{keV}$ peak as it varies with xdel, then again make the corresponding corrections. The corrections were 
done by Walt Ogburn at Stanford University.

Figure 6.4 compares the charge inner electrode before and after correction. The barium source lines, $276 \mathrm{keV}, 303 \mathrm{keV}, 356 \mathrm{keV}$, and $384 \mathrm{keV}$ match well with the data after correction.

The corrections of position dependence of ionization yield and timing parameters were also done at Stanford University by using the delay parameters and phonon partition parameters.

\subsection{Energy Calibration}

The energy calibration was done by comparing energy spectra between our data and the Monte Carlo simulations carried out by Laura Baudis. The data match the Monte Carlo very well in the germanium detectors for the $303 \mathrm{keV}$, the $356 \mathrm{keV}$, and the $384 \mathrm{keV}$ lines. The Compton energy spectra are also excellent matches in all six detectors. Figure 6.5 is for the charge comparison between data and MC. Figure 6.6 is for the recoil energy comparison between data and MC.

The internal $10 \mathrm{keV}$ line, which comes from cosmogenic activation or from the activated nuclei during neutron calibration in germanium detectors, is also a very

good reference for detector charge energy scale, phonon energy scale, recoil energy resolution, and detector stability. This $10 \mathrm{keV}$ line of the Soudan low background data in 52.6 live days is shown in figures 6.7 for the charge, and in 6.8 for the recoil energy. The worse resolutioin in $\mathrm{Z} 1$ phonons is due to a known gradient in the tungsten critical temperature that was not effectively removed. 

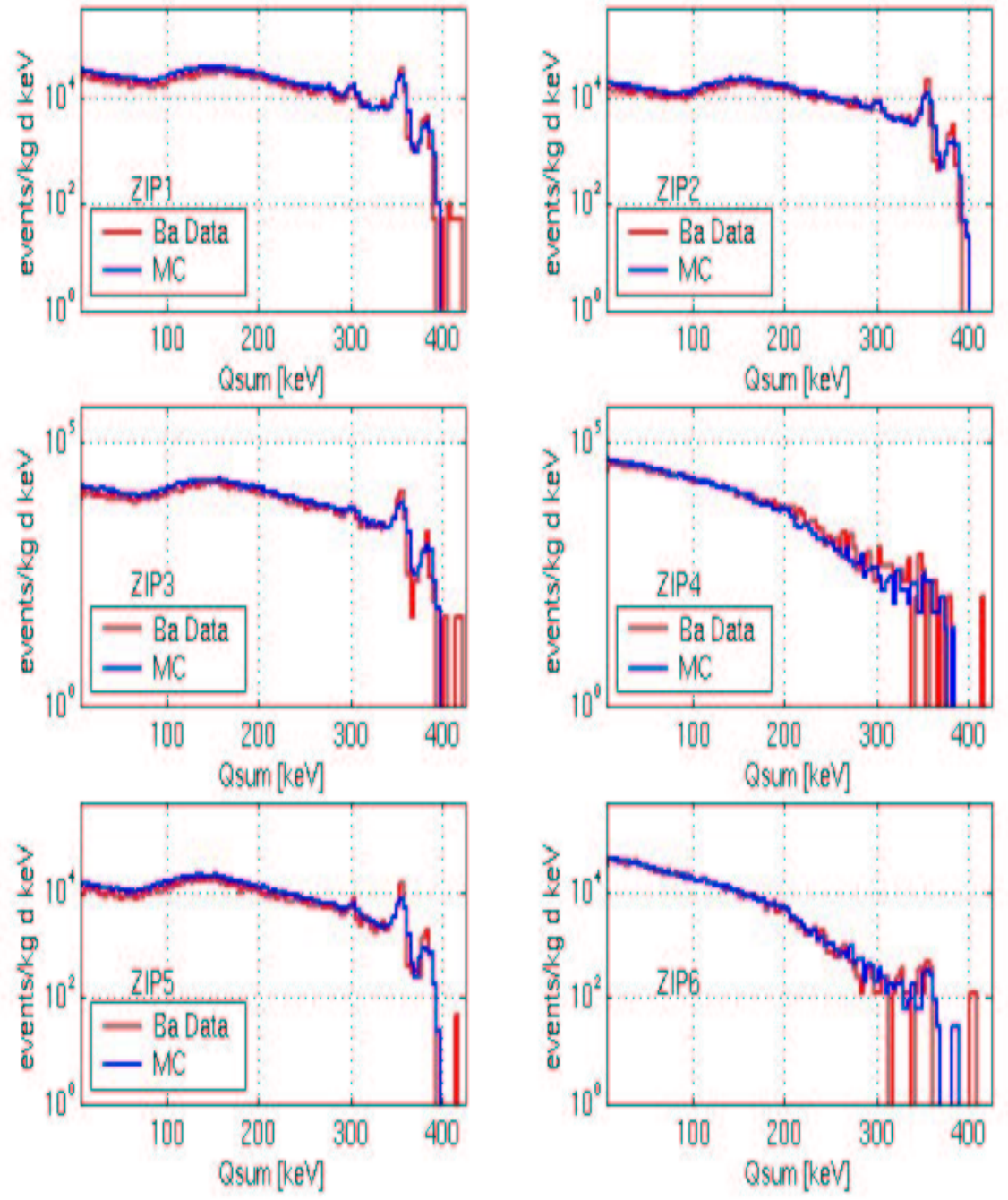

Figure 6.5: Comparison of charge energy spectra and Monte Carlo simulation of the ${ }^{133} \mathrm{Ba}$ calibration. Excellent match between data and Monte Carlo simulation for the ${ }^{133} \mathrm{Ba}$ typical lines $(356 \mathrm{keV}$ and $384 \mathrm{keV})$ and the low energy Compton spectra. Figure from [114]. 

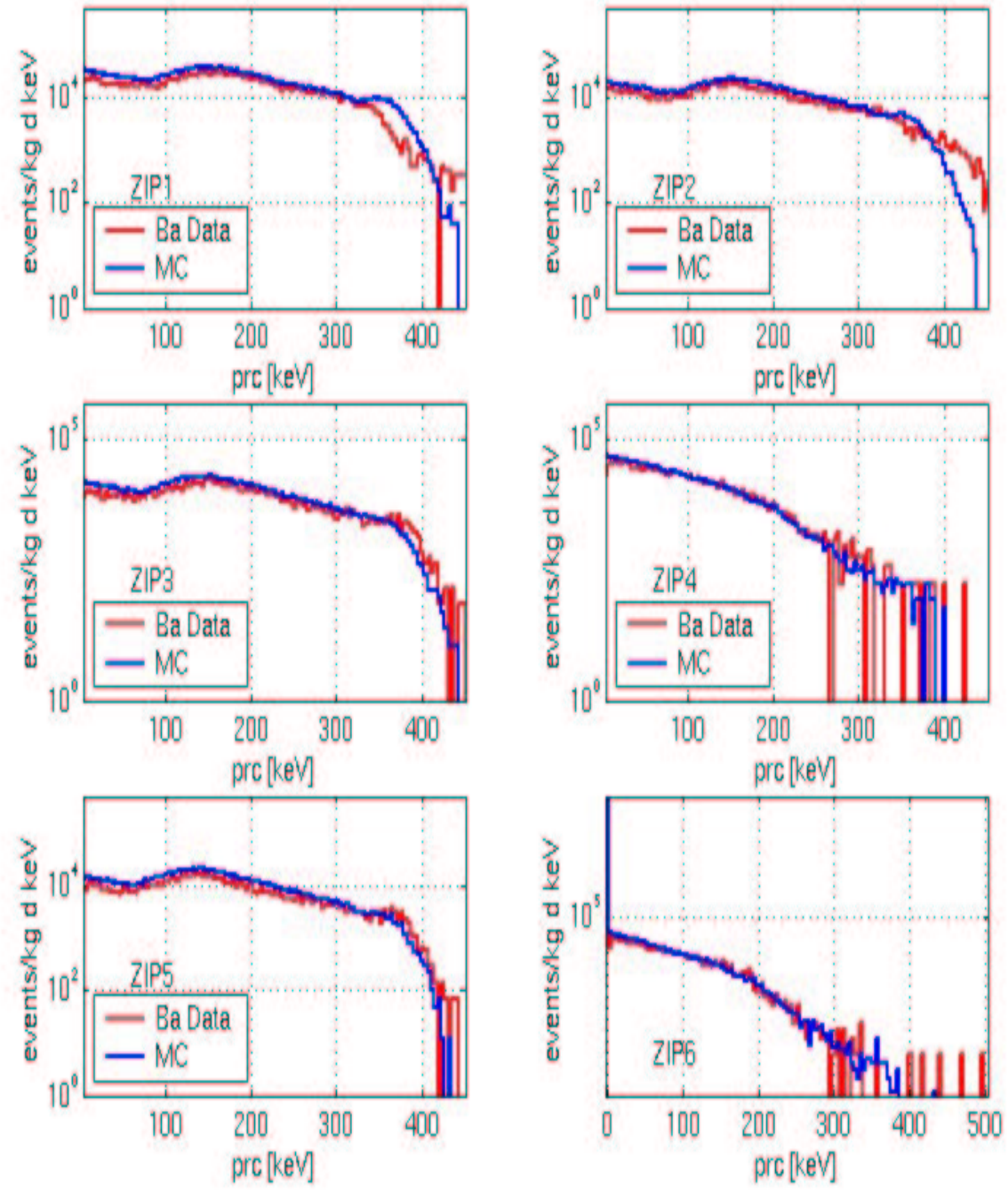

Figure 6.6: Comparison of phonon energy spectra (prc for position corrected recoil energy) and Monte Carlo simulation of the ${ }^{133} \mathrm{Ba}$ calibration. Good energy end edge and energy spectrum matches. The smearing of typical lines of ${ }^{133} \mathrm{Ba}$ comes from energy resolution dependence of phonon channels [81]. Figure from [114]. 

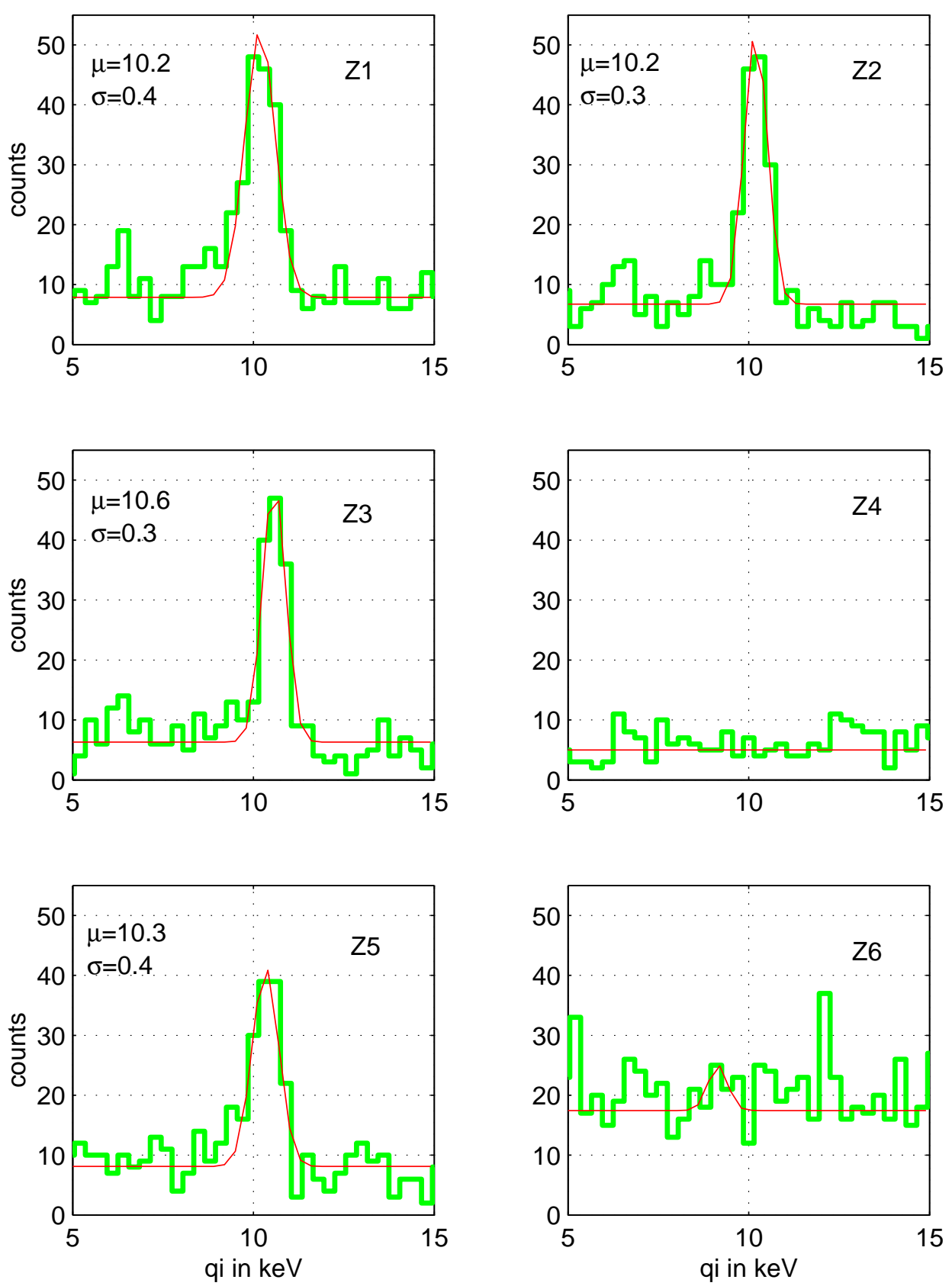

Figure 6.7: Charge inner $10 \mathrm{keV}$ line in germanium detectors for the 52.6 live day low background data in the Soudan run118. The mean and standard deviation are shown, as expected, the Z4 and Z6 detectors do not exhibit this feature. 

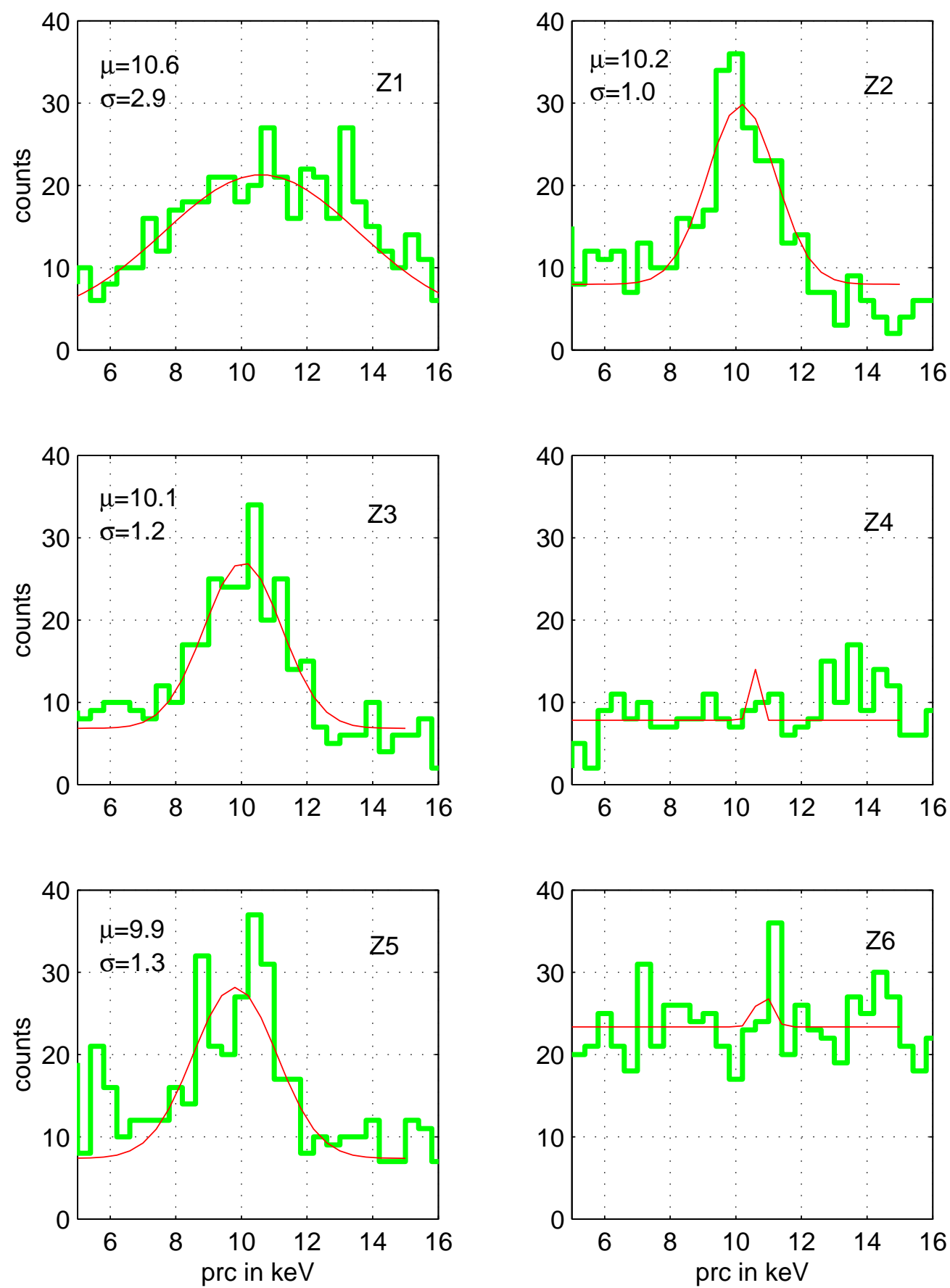

Figure 6.8: Phonon $10 \mathrm{keV}$ line in germanium detectors for the 52.6 live day low background data in the Soudan run118. The mean and standard deviation are shown, as expected, the Z4 and Z6 detectors do not exhibit this feature. 


\subsection{The Detector Noise}

The noise sources in the experiment determine the energy threshold, energy resolution, and the effectiveness of background discrimination parameters (such as the ionization yield). There are three noise sources in a ZIP detector. First, the baseline noise contribution arises from a combination of detector thermal fluctuations and readout electronics circuit responses. Second, the Poisson counting fluctuation scales as the square root of the relevant quantity, such as the number of charge carriers. Third, there is a systematic error in estimating a quantity such as a position dependent signal height and scale linearity with signal size. The contribution of this last term mainly comes from phonon channels. This is because there are $888 \mathrm{~W}$ meanders in parallel in each quadrant, but in the superconducting transition edge sensors, W meanders have transition temperature gradients, resulting in position dependence. Also, the limited phonon capture aluminium fin at the outer edge phonon channels could also be a source of energy non-linearity. The Poisson noise and the systematic noise are proportional to the size of the signal. The baseline noise is determined by the detector and readout electronics, directly effecting the detector energy threshold and sensitivity. Figure 6.9 shows the baseline noise resolution of the six detectors in Soudan Tower I. The baseline resolution is within $0.60 \mathrm{keV}$ for the phonon sensors, and within $0.90 \mathrm{keV}$ for the ionization charge.

For the reduced ionization energy collection of nuclear recoils, the energy threshold is determined by charge. For the WIMP search data analysis, it is important to set a charge energy threshold. Because only events falling in the inner charge electrode are chosen in the WIMP search data analysis, this charge energy threshold is set as charge inner energy threshold. This was done by look- 

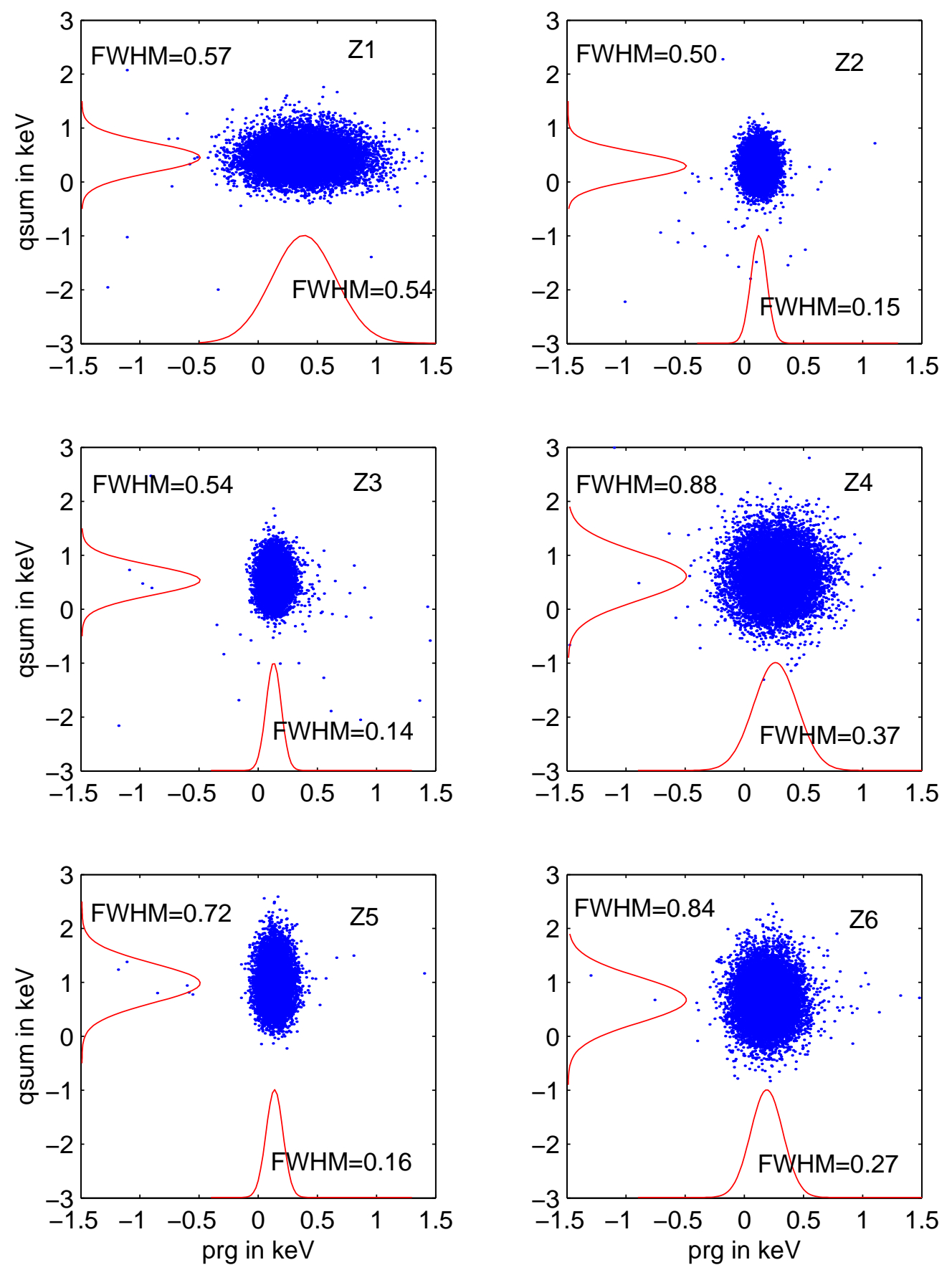

Figure 6.9: The noise blobs of baseline fluctuation. Full width at half maxima of charge and phonon are shown. prg is electron equivalent recoil energy. 

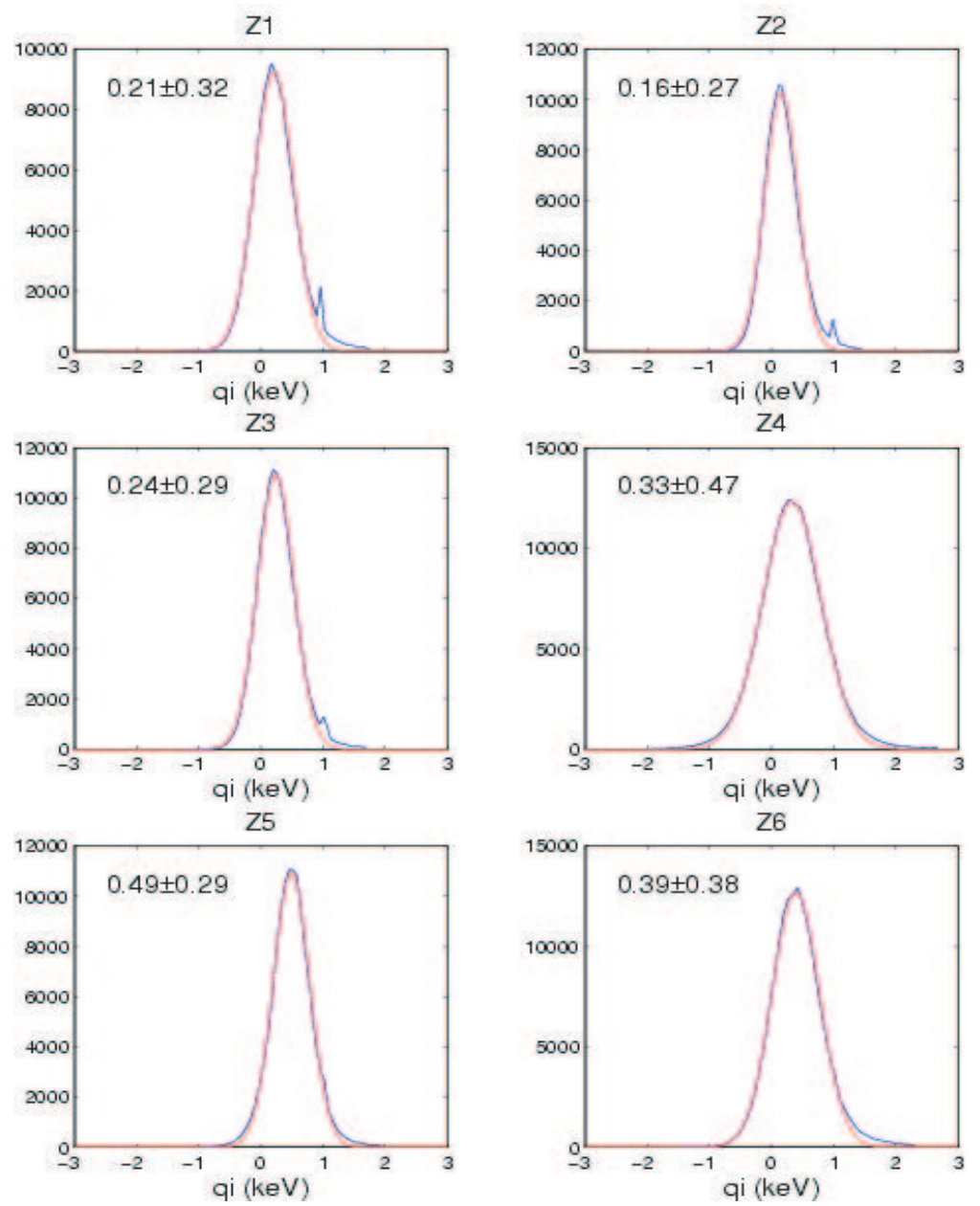

Figure 6.10: Inner electrode noise blob fit for threshold identification. The mean and standard deviation of charge inner baseline energy are shown. Figure from [115].

ing at low energy events both in ${ }^{133} \mathrm{Ba}$ calibration data and in ${ }^{252} \mathrm{Cf}$ calibration data. The low charge $($ qsum $<3 \mathrm{keV})$ and low phonon energy events were selected as noise events. A Gaussian fit was performed as in figure 6.10. The charge inner electrode baseline threshold is the mean of charge inner baseline plus its $5 \sigma$ standard deviation. They are listed in Table 6.1.

The nuclear recoils (neutrons) selection efficiency with the charge energy threshold cut is in Figure C.3 of Appendix C. The efficiency is close to ideal above $10 \mathrm{keV}$ 


\begin{tabular}{|c|c|c|c|c|c|c|}
\hline Detector & Z1 & Z2 & Z3 & Z4 & Z5 & Z6 \\
\hline cQThresS (keV) & 1.8 & 1.5 & 1.7 & 2.7 & 2.0 & 2.3 \\
\hline
\end{tabular}

Table 6.1: Charge inner energy threshold of Tower I detectors.

for Z2, Z3, Z5, and Z6, but it is low for Z1 and Z4 between $10 \mathrm{keV}$ and $15 \mathrm{keV}$.

\subsection{Inner Electrode Event Selection}

Defining a fiducial volume of a ZIP detector away from the edges of the detector crystal can ensure more uniform ionization response and phonon collection. And this can help reduce a large number of background electron recoils, because the outer circle is exposed directly to the DIB copper, a potential source of ejectrons (electrons knocked off by gammas).

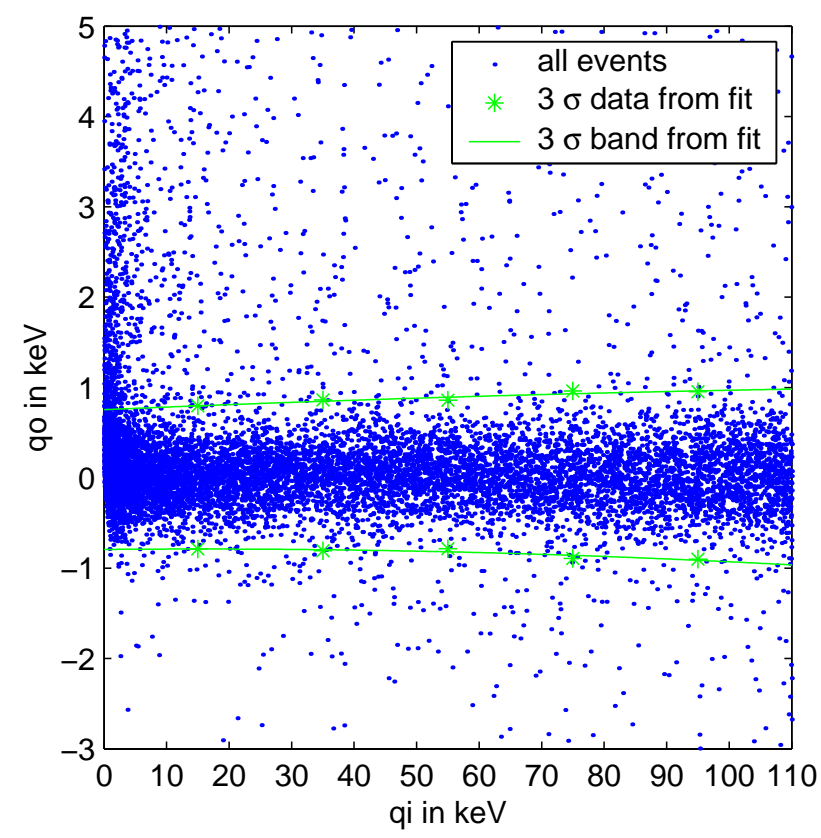

Figure 6.11: $3 \sigma$ band of inner electrode events in Z3. The blue dots are low charge outer electrode energy events from ${ }^{133} \mathrm{Ba}$ calibration data, the green lines are the fittings by using equations 6.1 and 6.2 with the $3 \sigma$ up bounds and low bounds (the green stars) at each energy bin. 
The charge inner electrode band can be defined as the $3 \sigma$ band of qo (qo and qi refer to the charge pulse height in the outer and inner electrodes, respectively) fit for all qo low events [116], as shown in Figure 6.11. The upper bound of the $3 \sigma$ band is a second order polynomial

$$
q o u=a \cdot q i^{2}+b \cdot q i+c .
$$

The lower bound of the $3 \sigma$ band is also a second order polynomial

$$
q o l=d \cdot q i^{2}+e \cdot q i+f
$$

The fit coefficients are given in Table 6.2.

But the tolerance of the measurement error increases with energy. The upper

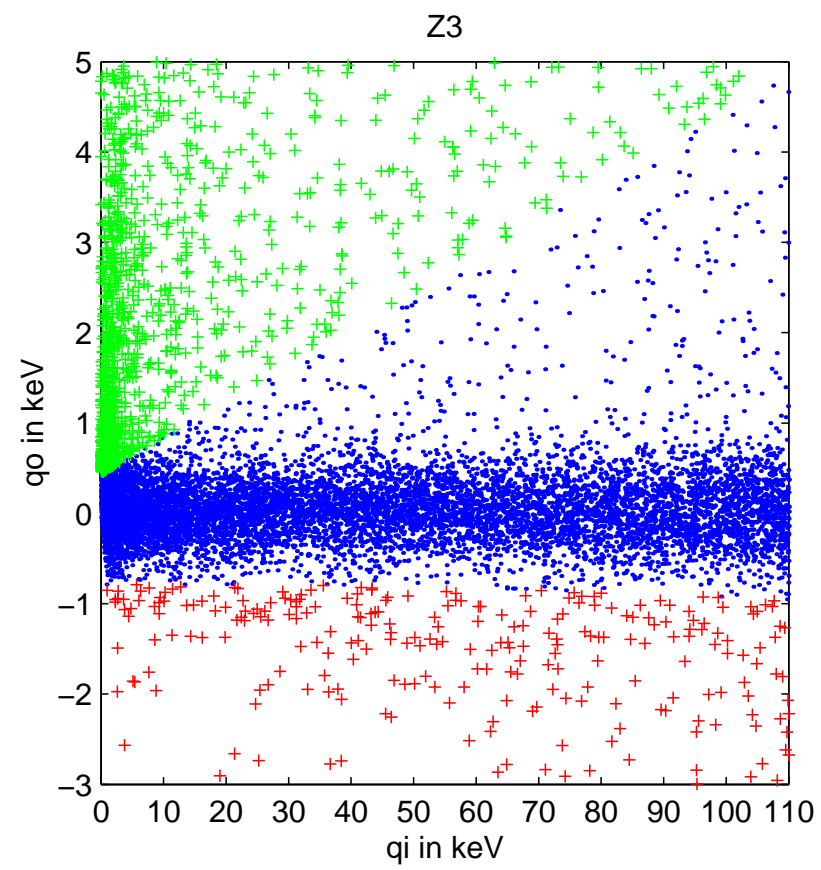

Figure 6.12: Inner electrode event selection in Z3. Events above the $3 \sigma$ lower bound in equation 6.2 and below the line in equation 6.3 are selectd. 


\begin{tabular}{|c|c|c|c|c|c|c|}
\hline Detector & Z1 & Z2 & Z3 & Z4 & Z5 & Z6 \\
\hline $\mathrm{a}\left(10^{-5}\right)$ & -4.303 & -2.267 & -0.964 & -4.696 & 1.464 & -5.464 \\
$\mathrm{~b}\left(10^{-3}\right)$ & 7.408 & 4.839 & 4.740 & 9.941 & 3.419 & 10.910 \\
$\mathrm{c}$ & 0.7337 & 0.7539 & 0.9483 & 1.0768 & 1.3687 & 0.6709 \\
$\mathrm{~d}\left(10^{-5}\right)$ & -3.125 & -6.232 & -2.250 & 0.125 & -2.928 & -2.964 \\
$\mathrm{e}\left(10^{-3}\right)$ & 2.242 & 7.430 & 1.475 & -1.272 & 5.521 & 3.060 \\
$\mathrm{f}$ & -0.8008 & -1.0080 & -0.9936 & -1.4415 & -1.3040 & -0.9426 \\
\hline
\end{tabular}

Table 6.2: Charge outer $3 \sigma$ upper bound and low bound fit parameters in equations 6.1 and 6.2 for Tower I detectors.
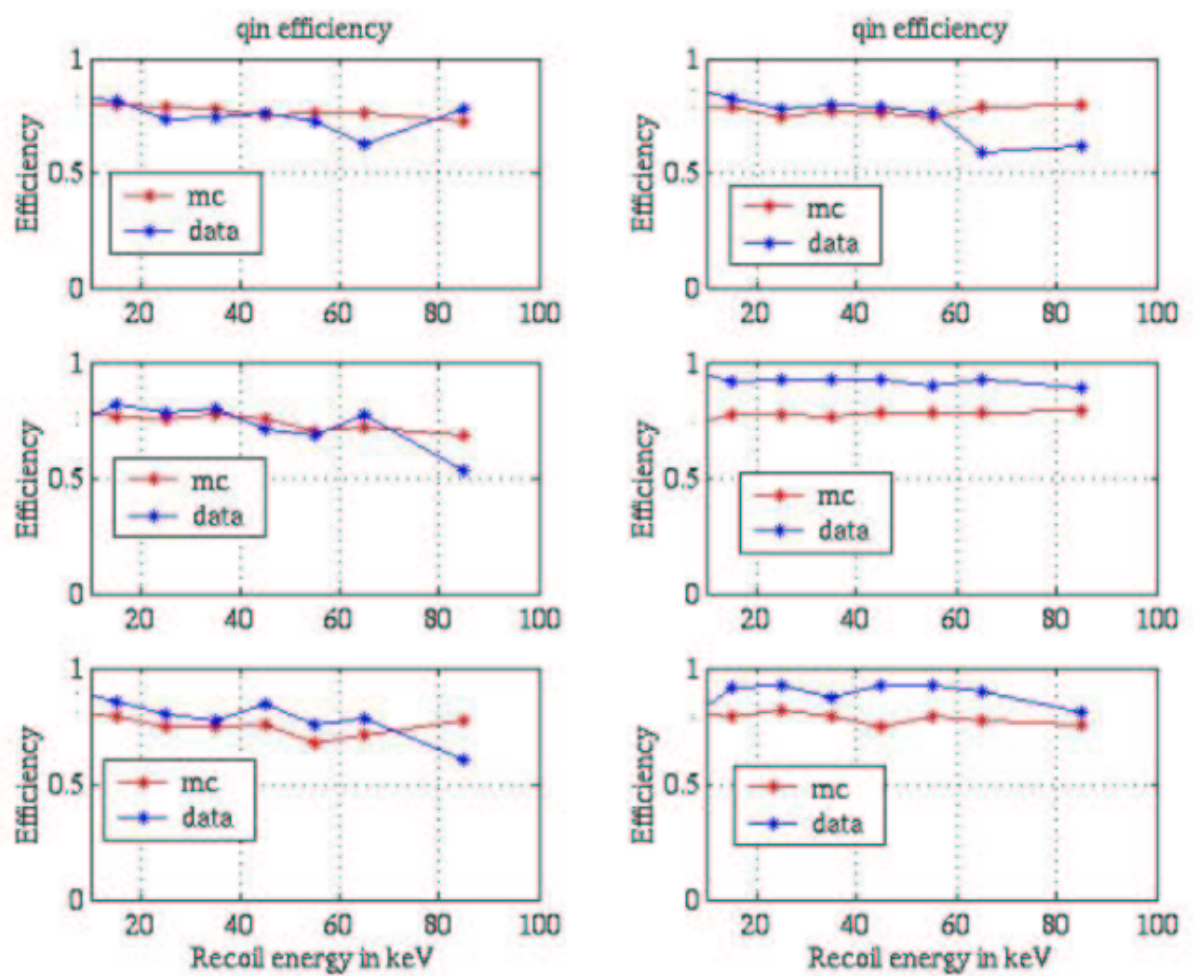

Figure 6.13: The qi cut efficiency as a function of recoil energy. Blue is from ${ }^{252} \mathrm{Cf}$ calibration data, red is from Monte Carlo simulation. Figure from [117].

bound of the charge inner events can be higher than the $3 \sigma$ limit. Also, the negative values of $q o$ is generally from qi-qo cross talk and random fluctuations in $q o$, the $3 \sigma$ lower bound selection line is appropriate. The charge inner cut is set such that any event below the $3 \sigma$ lower bound is removed, and any event above 


\begin{tabular}{|c|c|c|c|c|c|c|}
\hline Detector & Z1 & Z2 & Z3 & Z4 & Z5 & Z6 \\
\hline a & 0.38 & 0.34 & 0.45 & 0.46 & 0.70 & 0.57 \\
b & 0.04 & 0.04 & 0.04 & 0.04 & 0.04 & 0.04 \\
\hline
\end{tabular}

Table 6.3: The charge inner event upper bound selection parameters in equation 6.3 for Tower I detectors.

the line

$$
q o=a+b * q i,
$$

is removed. $a$ equals the mean of qo plus its $1 \sigma$ standard deviation in the 10-20 $\mathrm{keV}$ energy bin. $a$ and $b$ are given in table 6.3 .

Figure 6.13 is the cQinS cut efficiency for neutrons of the six detectors of Tower I in Soudan run118. The left three from top to bottom are Z1, Z3, and Z5. The right three from top to bottom are Z2, Z4, and Z6. The efficiency of decreasing at high energy for germanium (Z1, Z2, Z3 and Z5) detectors is that there are only a few nuclear recoil events above $60 \mathrm{keV}$ in the detectors. It is limited by statistics. We have only 3 hours of ${ }^{252} \mathrm{Cf}$ calibration data, because of the need to avoid activating radioiostopes in the germanium.

\subsection{Data Quality Cuts}

The data quality cuts operate on the properties of raw data traces and low background data collection experimental conditions. These cuts do not depend on the nature of the recoil. The following data quality cuts are applied to calibration data and WIMP search data.

- cBadData: removes a few bad datasets. These data sets have no operational phonon channels, or have bad phonon baseline chi-square, or have bad charge chi-square, or failed KS test significancies on the phonon partition and delay plots [118]. 
- cGlobTrig: removes events without a global trigger. There are less than $1 \%$ of low background data having no timing stamp in the history buffer, because of DAQ bug. There are no time dependence and no recoil type dependence in these data.

- cQbias: removes events with "bad" Qbias RQ. This happened rarely. The run control intended to stop the run, setting the charge bias to ground through GPIB, but the event builder kept collecting data.

- cZ2badph: removes datasets with bad phonons on Z2 based on 2D KS tests on the phonon partition and delay plots, as well as on the yield vs recoil energy [118].

- cZ4burst: removes datasets with trigger bursts on Z4, presumably due to instrumental baseline drifts.

- cZ5Bacalib: removes events in Z5 Ba calibrations when noise was bad.

- cCfbadfile: removes one file in the ${ }^{252} \mathrm{Cf}$ calibration data, which has spurous events.

- cGlitch: cuts out the multiple-trigger glitch events.

- cTransfer: cuts out parts of two datasets that inadvertently ran through cryogen transfers.

- cBad: combines all of the above.

- cPstd: removes events with bad pre-trigger baseline noise on phonons.

- cChisqS: removes events with big chi-square in the charge pulse fit. 
- cGoodEvS: cBad \& cPstd \& cChiSqS-for convenience, a combined cut.

Of all the data quality cuts, $c$ Pstd and cChisqS are directly related to the raw data of individual events.

The phonon sensor baseline quality cut, cPstd, was defined by using $61 \mathrm{k}$ randoms from the background data [119]. It is desirable to only use the random events that have clearly good Pstd. cPstd cuts events whose Pstd is more than 5 standard deviations of the phonon baseline standard deviation away from the mean of the phonon baseline standard deviation. The overall background data selection efficiency with the cPstd cut is in table 6.4.

\begin{tabular}{|c|c|c|c|c|c|c|}
\hline Detector & Z1 & Z2 & Z3 & Z4 & Z5 & Z6 \\
\hline Efficiency(\%) & 99.93 & 98.78 & 99.97 & 99.92 & 99.94 & 98.13 \\
\hline
\end{tabular}

Table 6.4: Event selection efficiency with cPstdS cut for Tower I detectors.

The purpose of the cChisqS cut is to remove the high $\chi^{2}$ events from the raw data. The value of $\chi^{2}$ is calculated from the charge template fitting. These unusual pulse shapes with high $\chi^{2}$ can be the result of:

- Pileup of two real events in the $\mu s$ or tens of $\mu s$ time scale.

- Pileup of a real event with a noise glitch.

- A noise event caused by an electronic or thermal transient.

- A noise when the sliding seal heater at the top of the dilution refrigerator turned on or off.

- Cross talk between detectors.

The charge cChisqS cut is defined as QSOFchisq $<c 1 * q s u m^{2}+c 2$ in reference [120]. The values of $\mathrm{c} 1$ and $c 2$, as well as the average event selection efficiency 

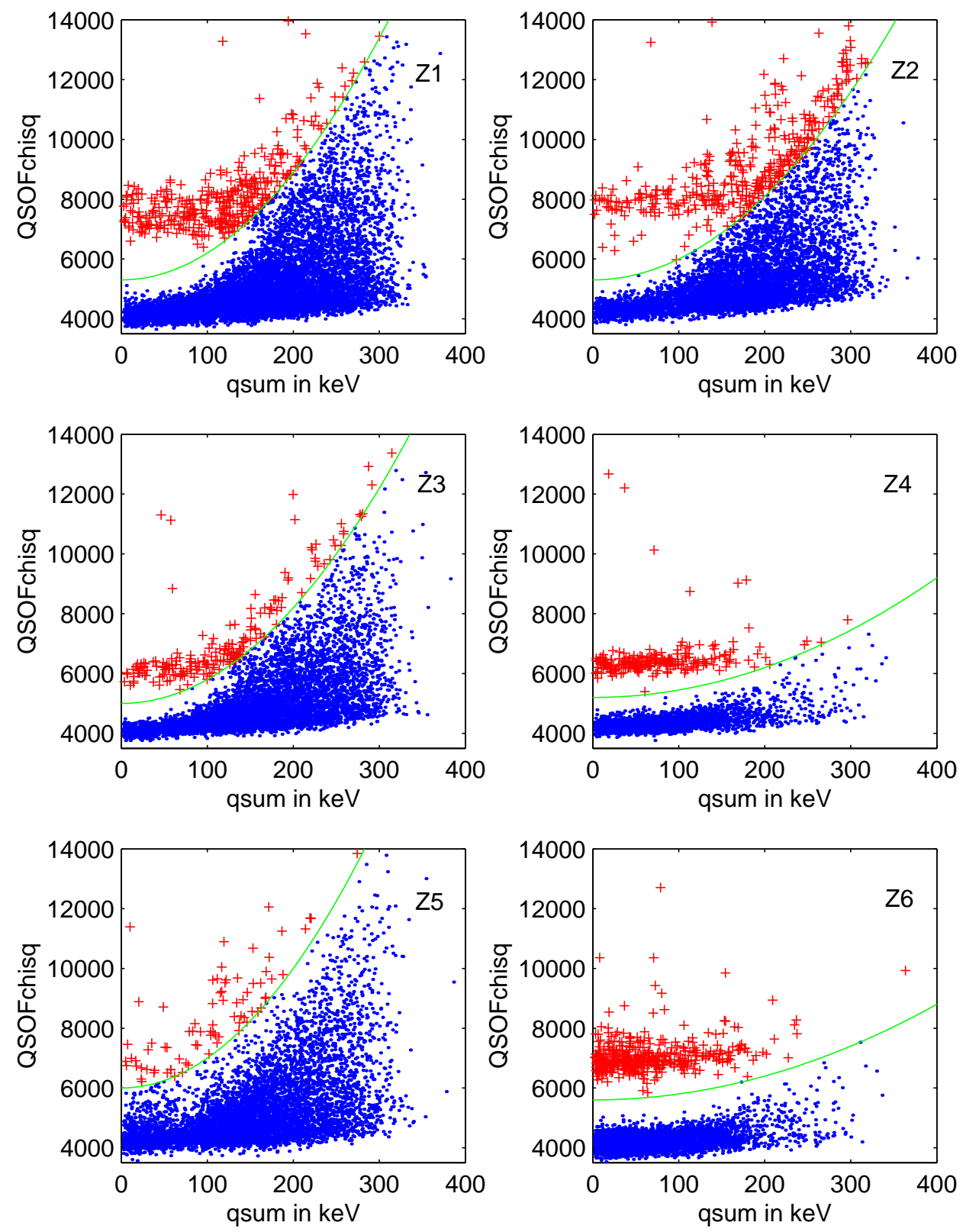

Figure 6.14: The cChisqS cuts for Tower I detectors. The $\chi^{2}$ is not normalized; we rely on the bimodel property of the variable to define the cut. The red crosses above the green line are cut off, the blue dots are selected.

between $5 \mathrm{keV}$ and $100 \mathrm{keV}$, are in Table 6.5 for all six detectors. The application of the cChisqS to the ${ }^{133} \mathrm{Ba}$ calibration data is shown in Figure 6.14. The event 

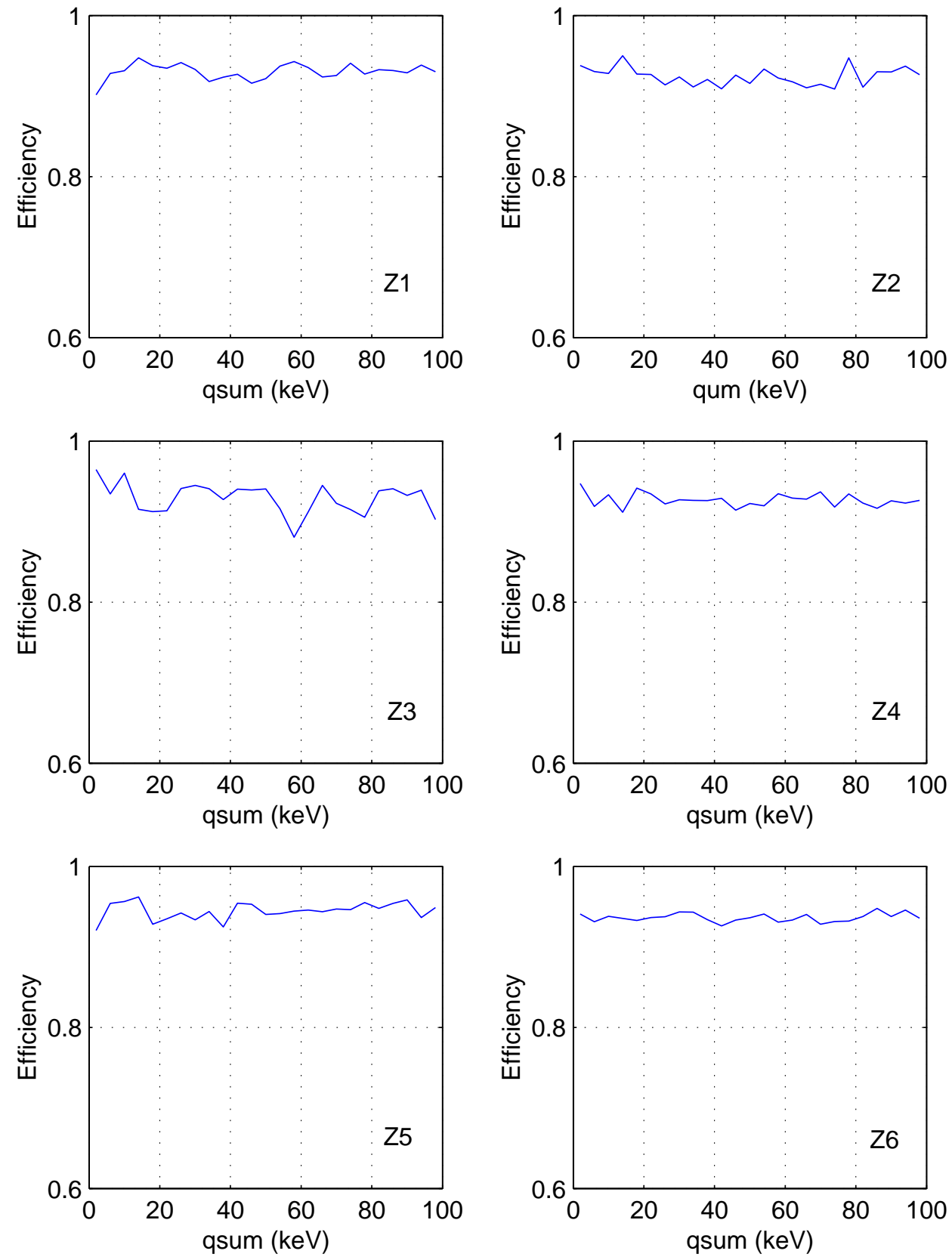

Figure 6.15: Event selection efficiency of the cChisqS cut for Tower I detectors.

selection efficiency distribution with the cChisqS cut is shown in Figure 6.15. 


\begin{tabular}{|c|c|c|c|c|c|c|}
\hline Detector & $\mathrm{Z} 1$ & $\mathrm{Z} 2$ & $\mathrm{Z} 3$ & $\mathrm{Z} 4$ & $\mathrm{Z} 5$ & $\mathrm{Z} 6$ \\
\hline $\mathrm{c} 1$ & 0.09 & 0.07 & 0.08 & 0.025 & 0.10 & 0.02 \\
$\mathrm{c} 2$ & 5300 & 5300 & 5000 & 5200 & 6000 & 5600 \\
\hline Efficiency(\%) & 93.04 & 92.45 & 92.88 & 92.67 & 94.48 & 93.65 \\
\hline
\end{tabular}

Table 6.5: The cChisqS cut and event selection efficiency for Tower I detectors.

\subsection{Electron and Nuclear Recoil Bands}

The ${ }^{133} \mathrm{Ba}$ calibration data and ${ }^{252} \mathrm{Cf}$ calibration data allow us to study the detectors in detail and to define the key parameters in low background data analysis; for example, the data quality cuts, the gamma and nuclear recoil bands, the surface electron recoil discrimination parameters, etc.

The ${ }^{133} \mathrm{Ba}$ source produces 356 and $384 \mathrm{keV}$ lines that result, after Compton scattering in the shielding and material surrounding the detectors, in a continuum spectrum with a significant number of low energy (i.e. $E<100 \mathrm{keV}$ ) events. Since we are primarily concerned with the energy region below $100 \mathrm{keV}$, these low recoil energy calibration data are the templates for WIMP search data analysis.

The ${ }^{252} \mathrm{Cf}$ calibration is for nuclear recoil band definition and for characterizing the nuclear recoils in ZIP detectors. ${ }^{252} \mathrm{Cf}$ produces neutrons by spontaneous fission. The neutrons leaving the source have a mean energy of $2.14 \mathrm{MeV}$, with 95\% below $5 \mathrm{MeV}$. Neutrons reach the detector by penetrating the inner lead, the inner polyethylene, and the copper can. The scattering is isotropic, and the mean energy loss is $3 \%$ of the energy of the incoming neutron. The neutron scattering produces a featureless, exponentially falling recoil energy spectrum, with a mean energy of $20 \mathrm{keV}[121]$.

Determination of the electron recoil band is made by slicing up the ${ }^{133} \mathrm{Ba}$ calibration data into recoil energy bins. For each such slice the yield parameter is then histogrammed and fitted to a Gaussian as shown in Figure 6.16. In order 
to obtain an accurate representation of the behavior of the yield parameter, it is necessary to apply data quality cuts to remove spurious events and misleading event populations. The cuts that were used are summarized below.

\section{Z3, Gamma Band Fits}
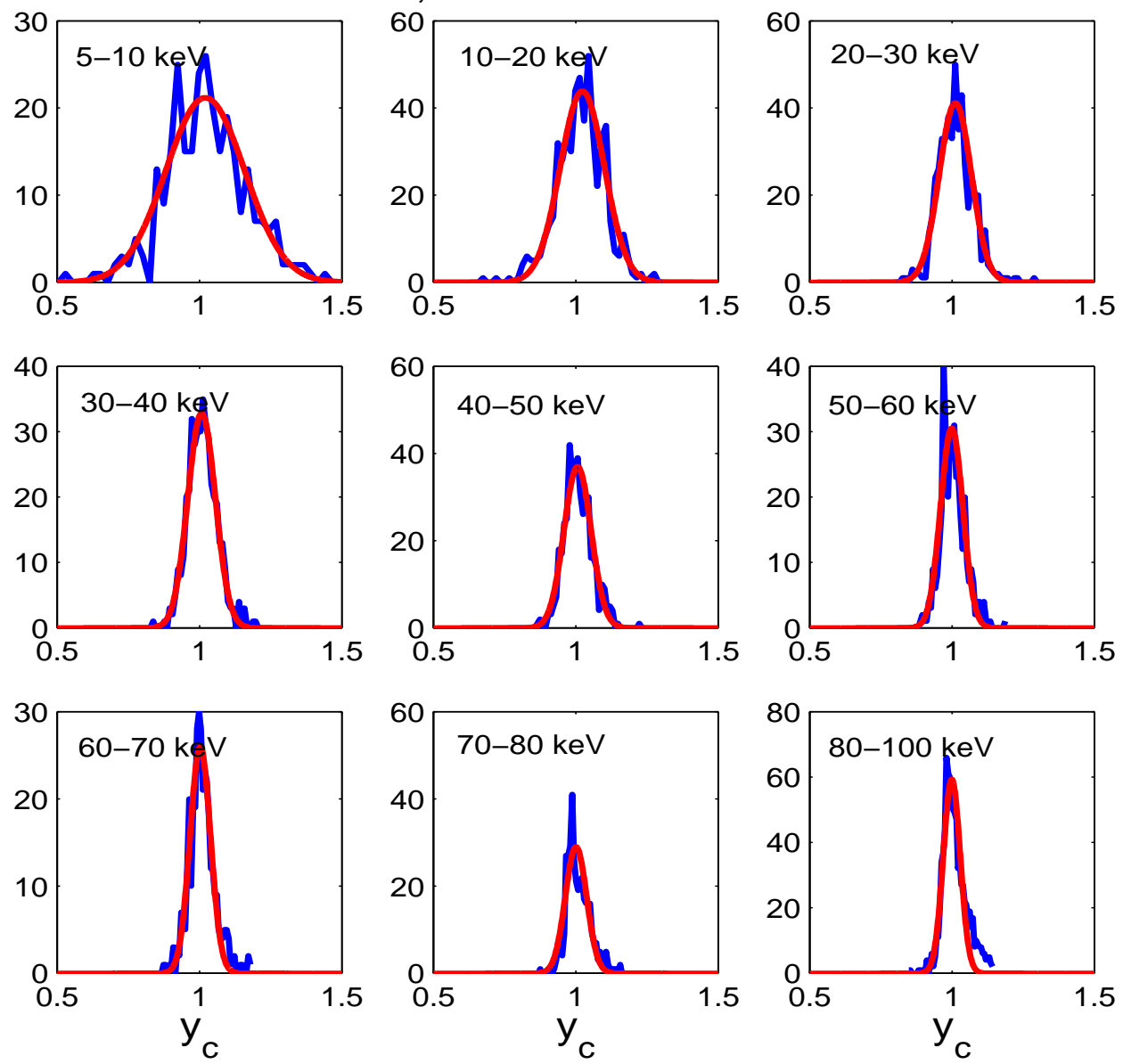

Figure 6.16: Method for finding the mean and standard deviation of the gamma band. Figure from [122].

1. Good Event: A generic cut on variables such as chisq and baseline standard deviation, intended to remove pile-up events as well as those associated with periods of unstable electronics.

2. Inner Electrode: Defines a fiducial volume away from the edges of the crystal 
to ensure uniform ionization response and sufficient phonon collection.

For the electron recoil band, we used events in the Ba calibration with $0.5<\mathrm{yc}<1.7$. At different energy bins, 5- 10, 10-20, 20-30, 30-40, 40-50, 50-60, 60-70, 70-80, and 80-100, we found the mean and standard deviation yield distribution.

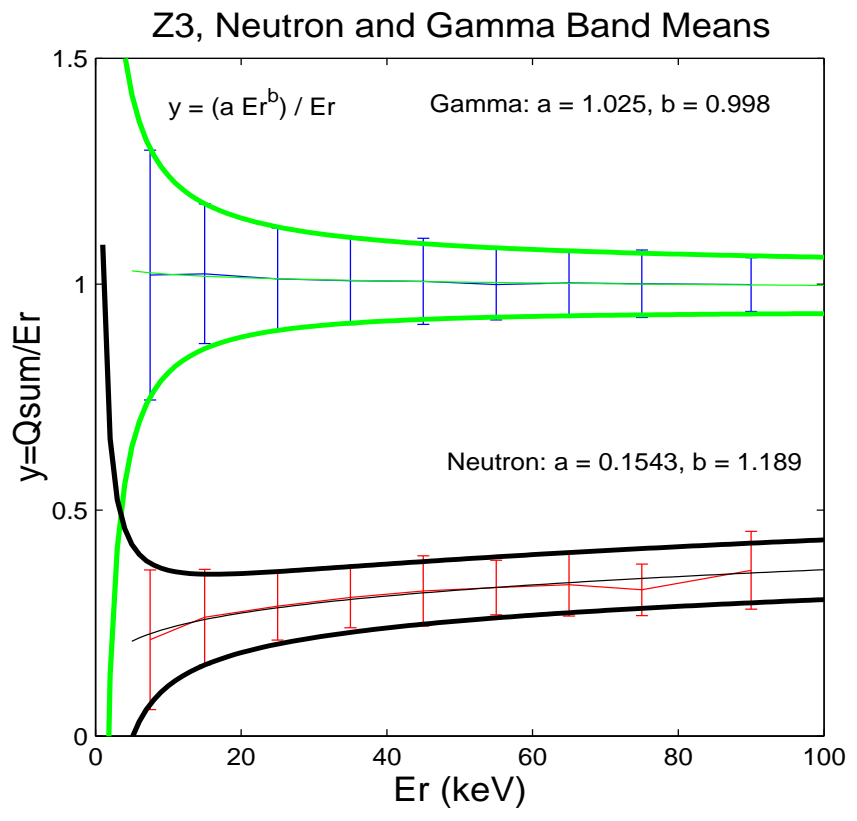

Figure 6.17: The defined electron recoil band and nuclear recoil band of Z3.

The position of the electron recoil band mean is parameterized as an exponential fit of recoil energy:

$$
y_{E R}=\frac{a E r^{b}}{E r}
$$

with parameters a and b determined by a fit are shown in Figure 6.17 for Z3.

The width of the $2 \sigma$ gamma band is also parametrized as a function of recoil energy

$$
\sigma_{E R}=c E r+d
$$

The sigma and recoil energy to a first order polynomial are shown in Figure 6.18 for Z3. 
The fitting parameters of the electron recoil band are in Table 6.6 for all six detectors.

\begin{tabular}{|c|c|c|c|c|c|c|}
\hline Detector & Z1 & Z2 & Z3 & Z4 & Z5 & Z6 \\
\hline a & 0.8427 & 0.9591 & 1.0248 & 0.9953 & 0.9616 & 1.0026 \\
b & 1.0352 & 1.0099 & 0.9960 & 1.0009 & 1.0090 & 1.0001 \\
c & 0.0609 & 0.0422 & 0.0297 & 0.0421 & 0.0258 & 0.0368 \\
d & 1.4024 & 0.7097 & 0.6606 & 1.1739 & 0.9782 & 1.0836 \\
\hline
\end{tabular}

Table 6.6: The gamma band parameters defined in equations 6.4 and 6.5 for the Soudan Tower I detectors.

For the nuclear recoil band, we use events in the ${ }^{252} \mathrm{Cf}$ calibration with $0<\mathrm{yc}<0.5$. At different energy bins, 5-10, 10-20, 20-30, 30-40, 40-50, 50-60, 60-70 70-80, and 80-100, we found the mean and standard deviation yield distribution.

The position of the nuclear recoil band mean is parameterized as an exponential
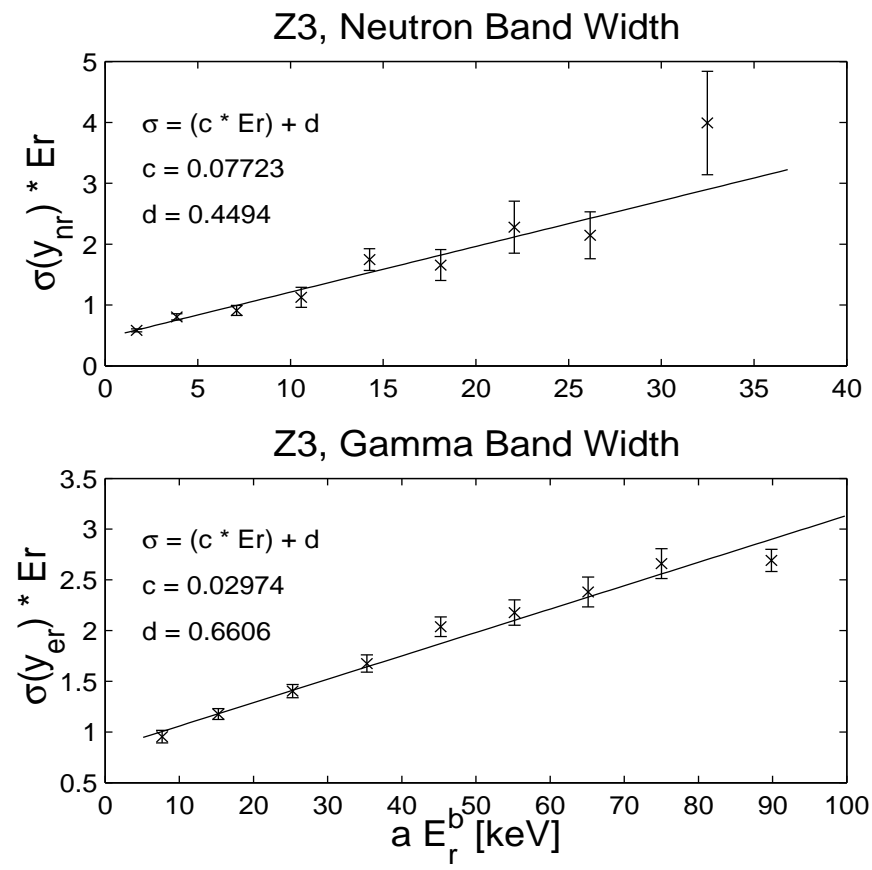

Figure 6.18: The band width parameters of Z3 


\section{Z3, Neutron Band Fits}
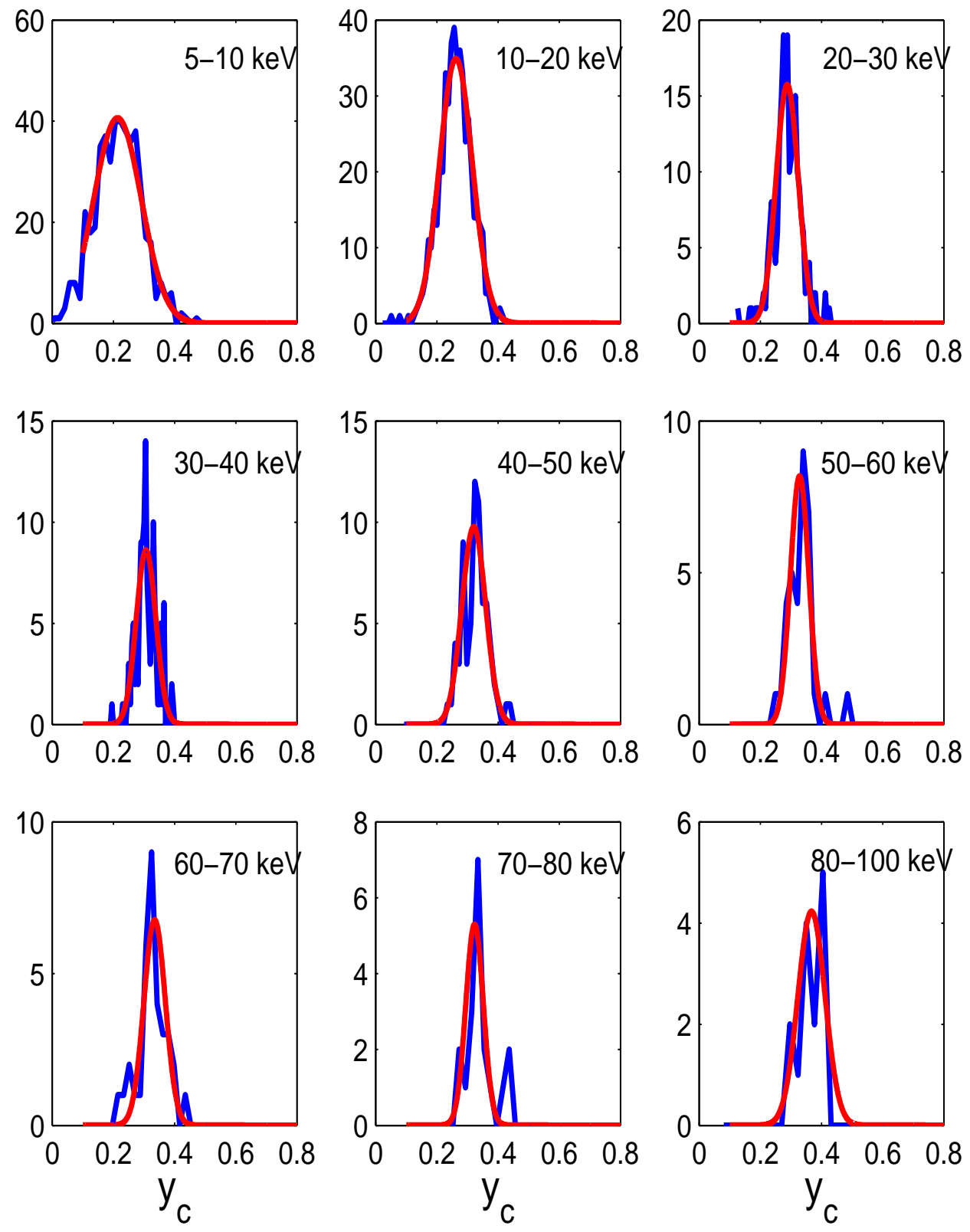

Figure 6.19: Method for finding the mean and standard deviation of nuclear recoil band with Gaussian fit. Blue is data, red is Gaussian fit. Figure from [122].

fit of recoils:

$$
y_{N R}=\frac{a E r^{b}}{E r}
$$



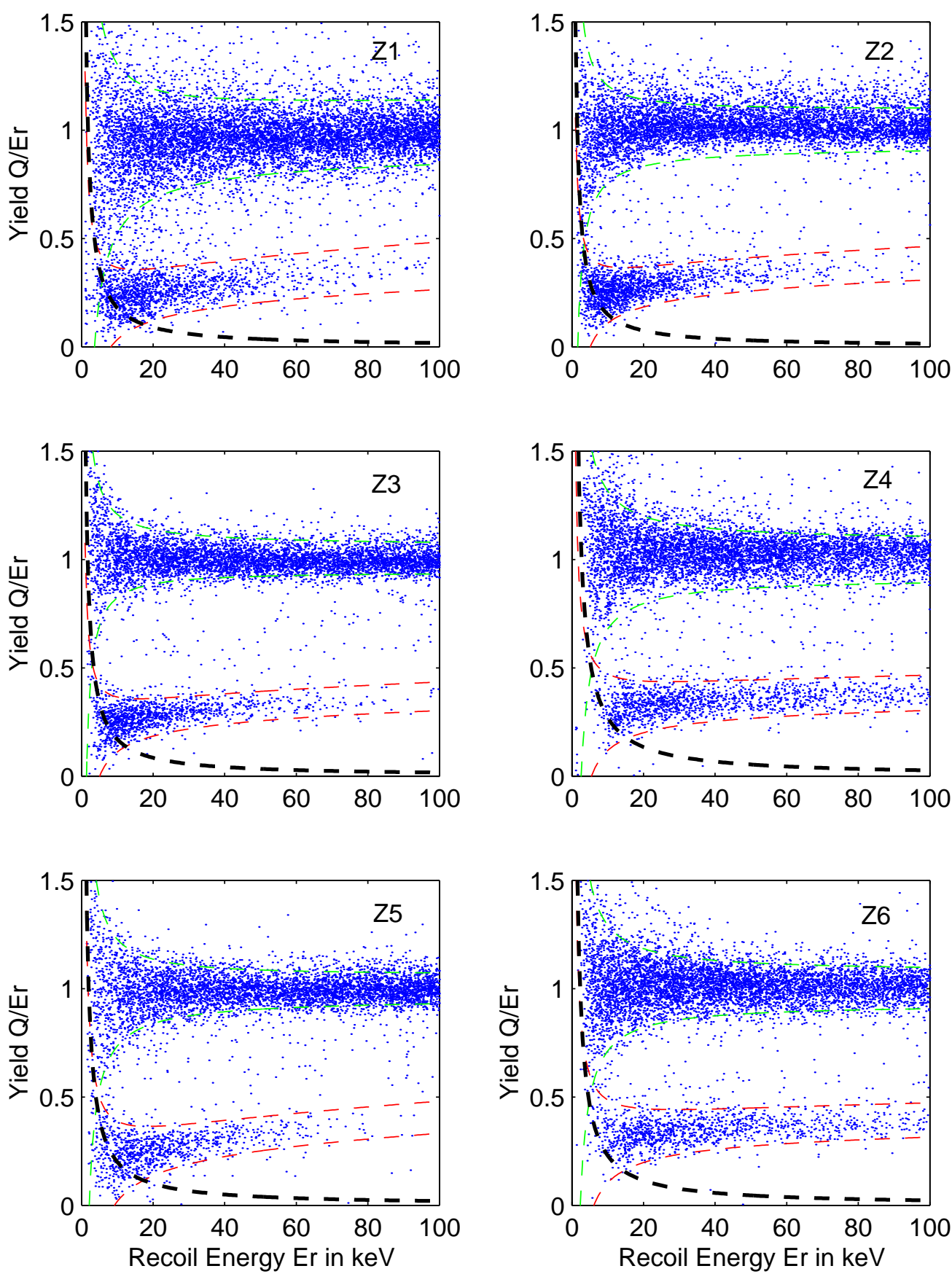

Figure 6.20: Z1-Z6 data and bands. The blue dots are data from ${ }^{252} \mathrm{Cf}$ neutron calibration and from ${ }^{133} \mathrm{Ba}$ calibration. The red dashed lines define the nuclear recoil band. The green dashed lines define the electron recoil band. The black dashed line shows the ionization yield for given charge threshold energy. 
with parameters a and b determined by a fit as shown in Figure 6.17 for Z3.

The width of the $2 \sigma$ nuclear recoil band is also parametrized as a function of recoil energy

$$
\sigma_{E R}=c E r+d
$$

with parameters c and d determined by a fit as shown in Figure 6.18 for Z3.

The fitting parameters of the nuclear recoil band are listed in Table 6.7 for all six detectors.

\begin{tabular}{|c|c|c|c|c|c|c|}
\hline Detector & $\mathrm{Z} 1$ & $\mathrm{Z} 2$ & $\mathrm{Z} 3$ & $\mathrm{Z} 4$ & $\mathrm{Z} 5$ & $\mathrm{Z} 6$ \\
$\mathrm{a}$ & 0.1064 & 0.1493 & 0.1543 & 0.2263 & 0.1019 & 0.2216 \\
$\mathrm{~b}$ & 1.2731 & 1.2067 & 1.1891 & 1.1154 & 1.3005 & 1.1250 \\
$\mathrm{c}$ & 0.1314 & 0.0885 & 0.0772 & 0.0896 & 0.0720 & 0.0823 \\
$\mathrm{~d}$ & 0.5692 & 0.4420 & 0.4494 & 0.6194 & 0.7803 & 0.7134 \\
\hline
\end{tabular}

Table 6.7: The nuclear band parameters defined in equations 6.6 and 6.7 for the Soudan Tower I detectors.

Figure 6.20 shows the defined electron recoil bands and the defined nuclear recoil bands for the Tower I detectors. The dashed curves are the energy threshold for each detector. The data in the plots are from the ${ }^{133} \mathrm{Ba}$ calibration and the ${ }^{252} \mathrm{Cf}$ calibration. 


\subsection{Detector Stability}

The $10 \mathrm{keV}$ line in the germanium detectors (see Figure 6.7 and Figure 6.8 in section 6.4) is the evidence of stable low background data collection in Soudan run118. The mean and width of the gamma bands, and the baseline noise of the charge tell us how stable the detectors were. Figure 6.21 shows the mean and width of the gamma band in the recoil energy range from $40 \mathrm{keV}$ to $60 \mathrm{keV}$ divided into 10 day slices during the 93 day low background data collection period. The standard deviations of the gamma band mean are 0.022, 0.019, 0.008, 0.009, 0.006 and 0.016 for detectors $\mathrm{Z} 1$ through Z6. The standard deviations of gamma band $2 \sigma$ width are $0.052,0.048,0.028,0.028,0.027$, and 0.033 for detectors Z1 through Z6.

Figure 6.22 shows the charge inner electrode baseline noise levels for the events where phonon total energy $p t$ is less than $3 \mathrm{keV}$ and for those that have not been trigged on. The baseline noise level is getting lower with time for Z2, which is good. The high base noise levels and the fluctuations in Z5 and Z6 are understood. There was noise coupling to the detector readout electronics at the vacuum bulkhead for Z5 and Z6 during Soudan run118. The average value of the charge inner ionization energy for Z1 through Z6 was $0.25 \mathrm{keV}, 0.21 \mathrm{keV}, 0.28 \mathrm{keV}, 0.41 \mathrm{keV}, 0.42 \mathrm{keV}$, and $0.36 \mathrm{keV}$. The FWHM of the charge inner for Z1 through Z6 was $0.63 \mathrm{keV}$, $0.47 \mathrm{keV}, 0.45 \mathrm{keV}, 0.88 \mathrm{keV}, 0.64 \mathrm{keV}$, and $0.74 \mathrm{keV}$. These values are consistent with the definition of the charge inner energy threshold in section 6.5.

The ZIP detector operations, energy calibration, energy threshold, data quality cuts, stability, electron recoil band and nuclear recoil band in Soudan run118 are now well defined. The next chapter discusses the WIMP search analysis with Soudan run118, which relies on these definations. 

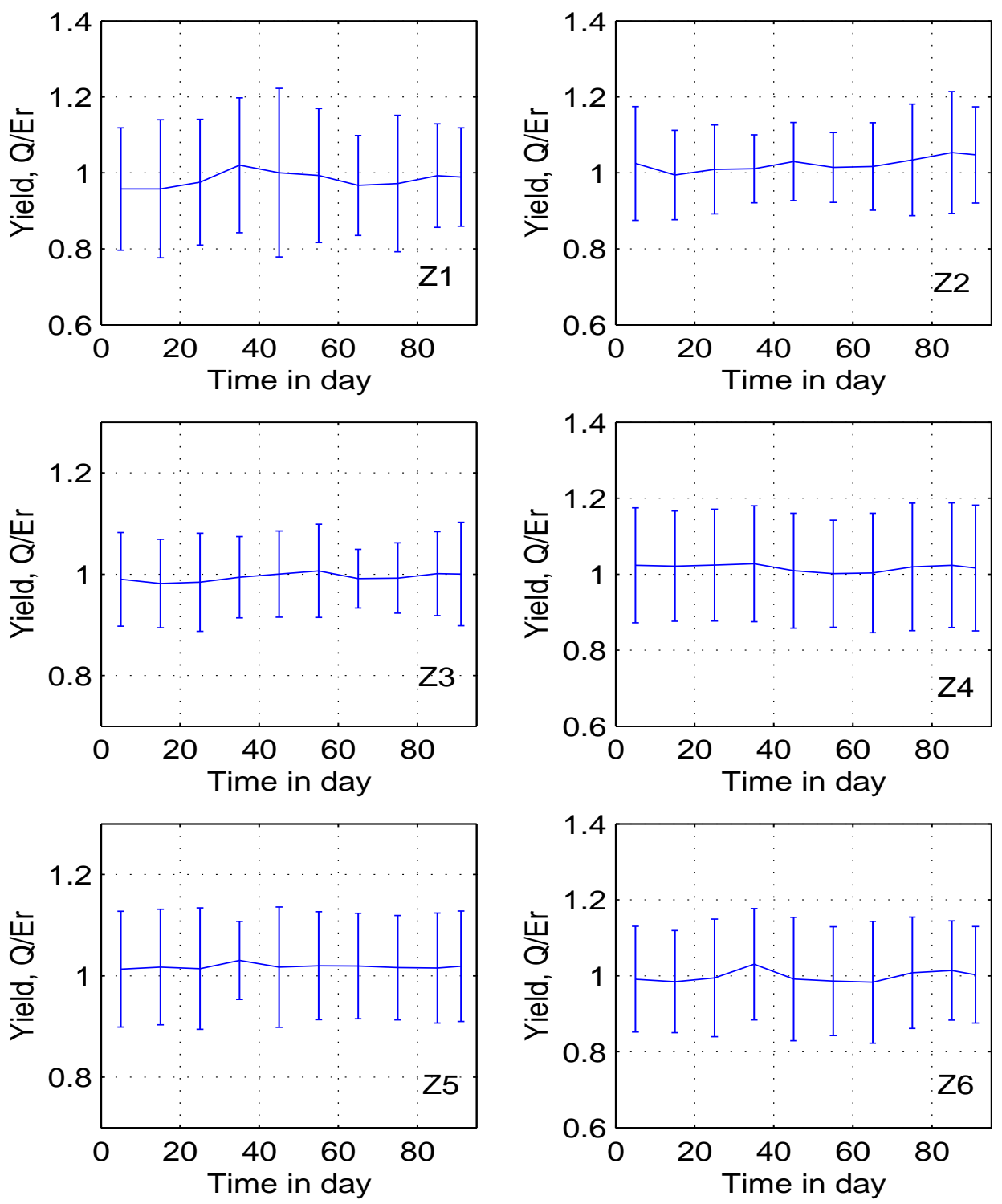

Figure 6.21: Stability of the electron recoil band. The bars show the $2 \sigma$ gamma band width in the 40-60 keV bin. The standard deviation of the gamma band width is $0.052,0.048,0.028,0.028,0.027$, and 0.033 for $\mathrm{Z} 1$ through $\mathrm{Z} 6$, respectively. 

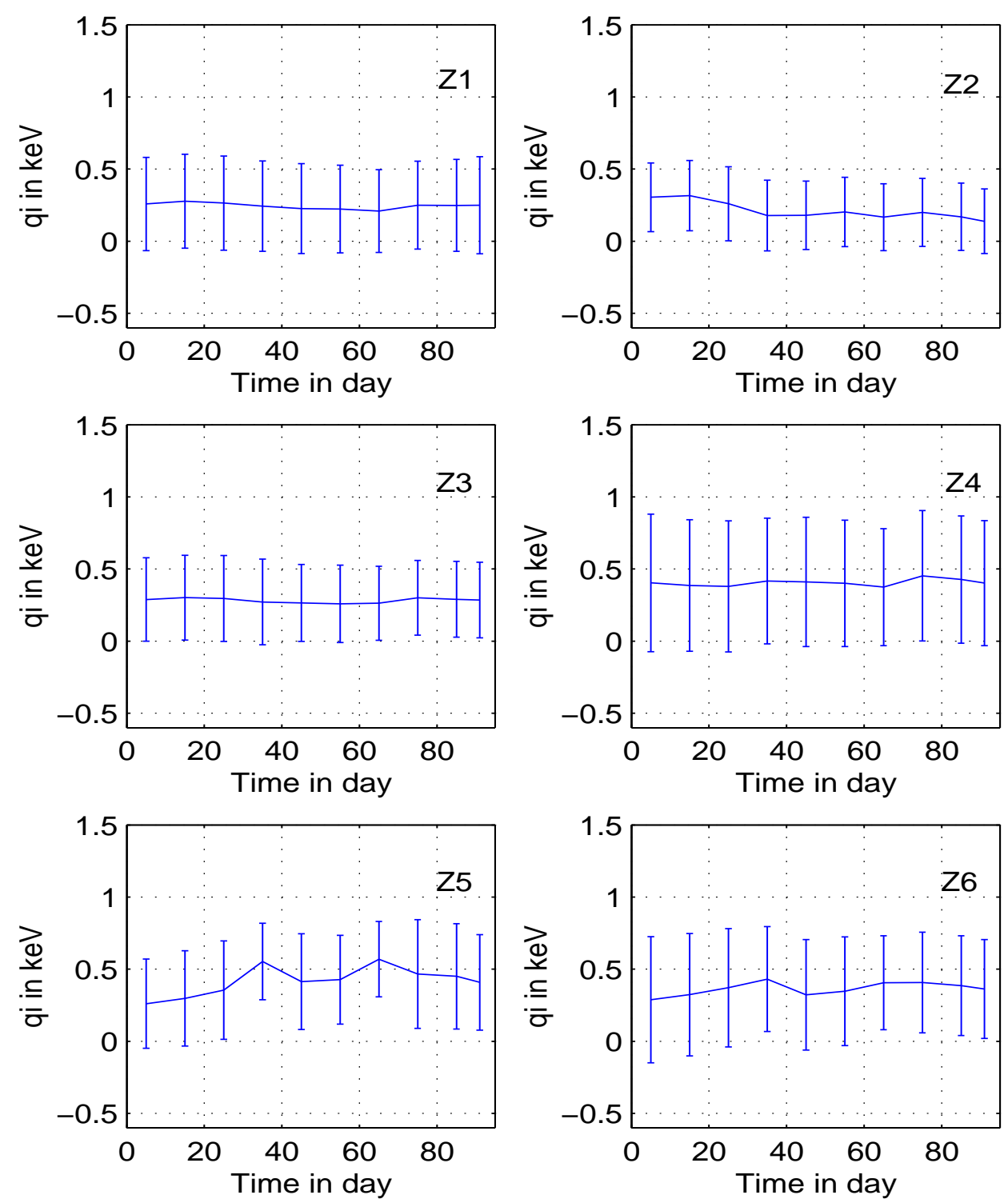

Figure 6.22: Stability of the inner electrode noise mean and full width at half maximum. The standard deviation of the charge inner mean at different time bins is $0.0037 \mathrm{keV}, 0.0333 \mathrm{keV}, 0.0026 \mathrm{keV}, 0.0051 \mathrm{keV}, 0.0862 \mathrm{keV}$ and 0.0129 $\mathrm{keV}$ for $\mathrm{Z1}$ through $\mathrm{Z6}$, respectively. 


\section{Chapter 7}

\section{WIMP Search Data and Analysis}

One important step in WIMP direct detection in the CDMS experiment is to identify nuclear recoils in the WIMP search data, which we collected with the CDMS ZIP detectors in Tower I during CDMS Soudan run118. The methods of nuclear recoil identification involve removing the electron interactions that are from background particles. CDMS ZIP detectors have event by event electron interaction background discrimination capabilities based on ionization yield, phonon timing parameters, and event interaction location parameters. In this chapter, I will describe the parameters that have been used for electron interaction rejection in the CDMS experiment, as well as two distinct treatments of the WIMP search data: a position information based analysis, and a blind analysis primarily using ionization yield and phonon timing parameters.

The distinguishing feature of these two analyses is that the blind anlaysis was designed and carried out as an unbiased straightforward set cuts on historically well-understood analysis parameters. The motivation was to rapidly evaluate our world's-best data set. In parallel, though on a somewhat longer time scale, we sought to investigate more sophisticated and optimal mehtods to improve background rejection and nuclear recoil selection efficiency. My sophiticated analysis, 
using the tools and insight from the work in Chapter 5, is one such result that will hopefully inform blind analysis of future large data sets.

\subsection{Tower I at Soudan}

CDMS II Soudan run118 started in August 2003. Two towers, with total 12 CDMS ZIP detectors, were installed. We only turned on the detectors in Tower I for WIMP search data (also referred to as low background data) collection in Soudan run118, because these detectors were well-characterized from Stanford Underground Facility (SUF) running. Noise debugging, detector neutralization and TES bias tuning took about 40 days for the first CDMS experiment in the Soudan mine. Stable low background data collection started on October 11, 2003,

and ended on January 11, 2004. 52.6 days of live time were accumulated during 93 calendar days. Other time was for cryogenic system maintetnnce, such as cryogen

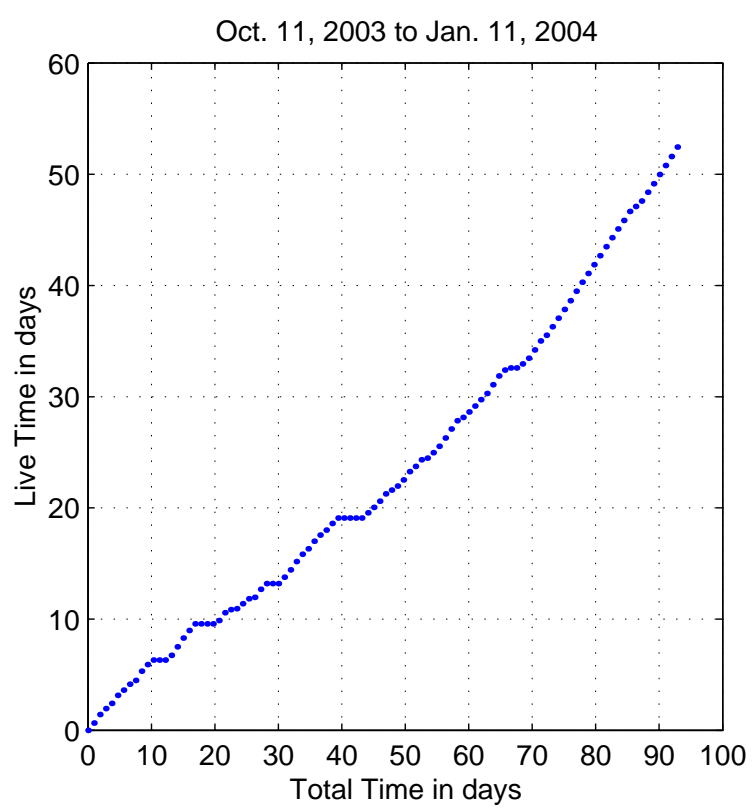

Figure 7.1: Accumulated exposure in live days for Tower I run118 at Soudan from October 11, 2003 to January 11, 2004. 
transfer, gamma calibrations with the Barium source and neutron calibrations with the Californium source, periods of bad noise, etc. Figure 7.1 shows the accumulation of live time.

\subsection{Background Discrimination with Ionization Yield}

The principle of the CDMS detectors is that photons and electrons interact with the electrons in the detectors, but WIMPs only interact with nuclei. The electron interaction has a high ionization yield (ionization yield is defined as collected charge energy divided by recoil energy in section 4.1 of chapter 4), which is usually normalized to 1 . Nuclear recoils have a lower ionization yield [123]. The ionization yield suppression factor for nuclear interactions is between 2.5 and 5 [86], depending on the detector (Ge or $\mathrm{Si}$ ) and recoil energy, and is understood according to Lindhard's theory [109].

Ionization energy versus phonon (recoil energy) is shown in Figure 7.2 for Z3 (germanium detector) and for Z4 (silicon detector). The events in the upper band are normalized so that ionization energy approximately equals phonon energy (recoil energy) for electron recoils; the events in the lower band with ionization energy much less than the phonon energy (recoil energy) are nuclear recoils [124]. In the formal data analysis, ionization yield, which is ionization energy divided by recoil energy, is used as the primary background discrimination parameter [86]. Nuclear recoils have a low ionization yield, while electron interactions have a high ionization yield, as shown in figure 7.3. Events in the $2 \sigma$ nuclear recoil band (the lower band in Figure 7.3) in the WIMP search data are considered as possible WIMP candidates, but surface event rejection cuts must be used with ionization 

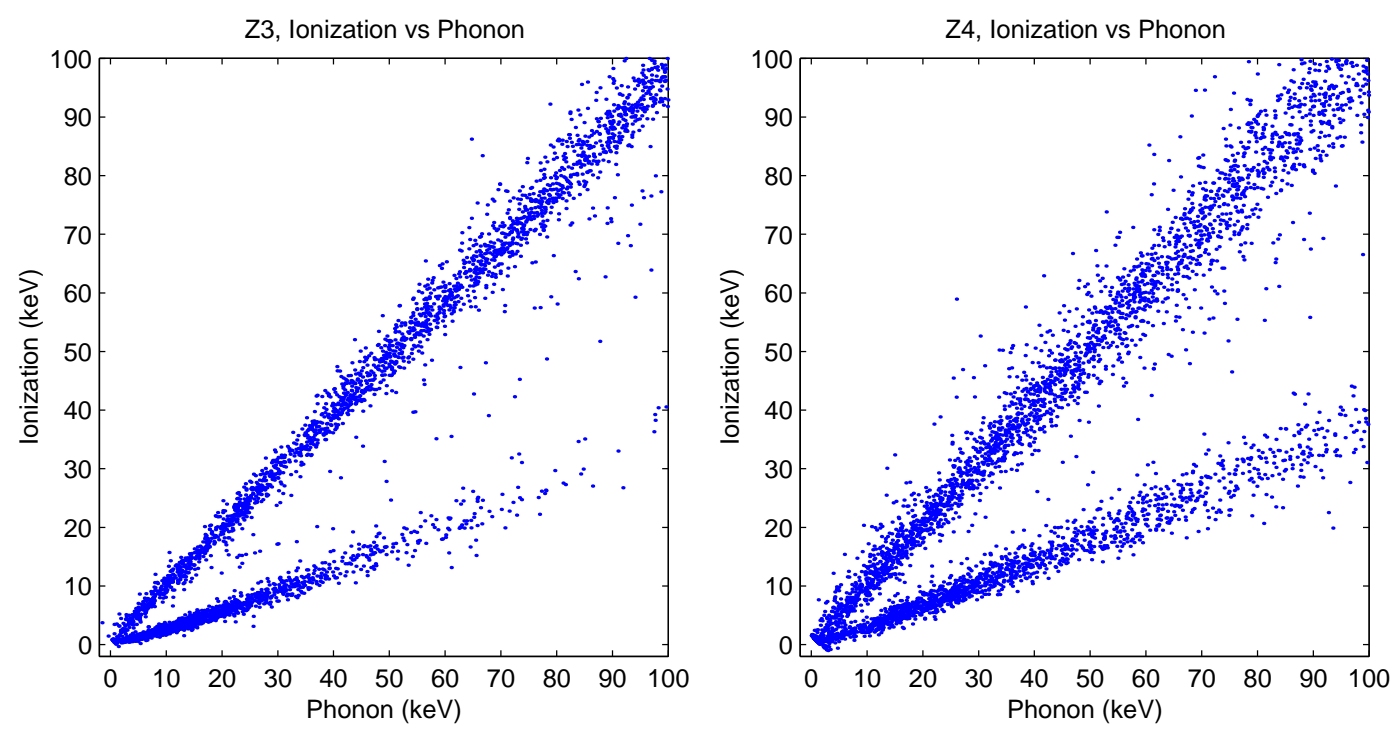

Figure 7.2: The ionization energy versus phonon (the recoil energy) for electron interactions and nuclear interactions. For the electron interactions, the slope is normalized to 1. For the nuclear interactions, there are more phonons than charges, so the ionization vs phonon slope is small. The plot on the left is for germanium detector $\mathrm{Z} 3$, the plot on the right is for silicon detector $\mathrm{Z} 4$.

yield together for nuclear recoil identification.

The electron interactions, which are from photons and electrons, can happen on the surface of the detectors. Because the interaction location is close to the electrode, and the electrons and holes created from the interaction are energetic, as noted earlier, they can back diffuse into the electrodes before charge collection. For example, under $-3 \mathrm{~V}$ external bias voltage for a germanium detector, the electrons and holes are supposed to separate. Electrons go to the positive electrode; holes go to the negative electrode. But for the interactions near the surface of the detector, say at the negative electrode side, the electrons with kinetic energy can back diffuse into the negative electrode and become trapped in, causing the loss of ionization collection. Therefore the surface interactions have reduced ionization yield, and appear to be a nuclear interaction in terms of ionization yield. 

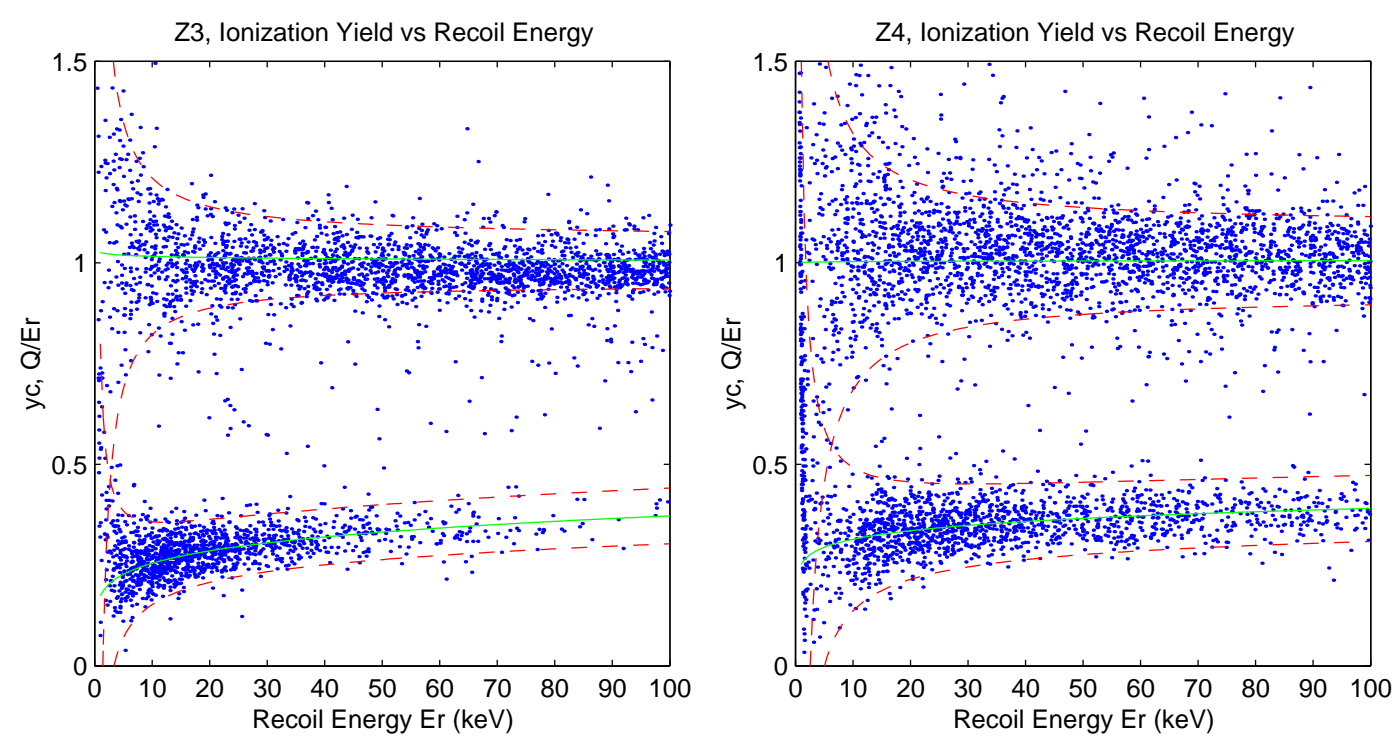

Figure 7.3: Ionization yield (yc is position corrected ionization yield) versus the recoil energy. The two dashed red lines that surround the lower ionization yield events define $2 \sigma$ nuclear recoil band. The two dashed red lines that surround the higher ionization yield events define $2 \sigma$ electron recoil band. The plot on the left is for germanium detetcor Z3, the plot on the right is for silicon detector Z4.

The surface interactions are rejected with phonon pulse timing parameters and interaction location parameters. The high impurity densities on the surface result in interactions having fast phonon pulse timing parameters, due to the high scattering rate of the primary optical phonon of the interaction [125]. The interaction location parameters are calculated with the local phonon sensor pulse start time and two neighbor phonon sensor pulse start times, as described in chapter 5 . Since surface interactions of low-energy electrons are the limited internal background in the experiment (neutrons are the dominant external background), the importance of surface event rejection cannot be over emphasized. 


\subsection{Rejecting Surface Events}

For an event in a detector, the CDMS data analysis package, DarkPipe, extracts the pulse start time, rise time, and fall time of each channel, and of the total of the four phonon sensors. (In one detector, there are four phonon sensors A, B, $\mathrm{C}$, and $\mathrm{D}$, and two charge collections, $\mathrm{QI}$ and $\mathrm{QO}$, as described in section 4.2 of chapter 4.) Figure 7.4 shows the raw traces of a $60 \mathrm{keV}$ event in the $\mathrm{Z} 2$ charge inner electrode. The timing parameters of events in ZIP detectors are used as surface event rejection, and for location reconstruction parameters, as discussed in Chapter 5.

The phonon timing parameters that are used in Soudan run118 data analysis include:

- The time at which the charge pulse reaches $20 \%$ of its peak amplitude. They are charge start time, QIst and QOst.

- The time at which the phonon pulse reaches $20 \%$ of its peak amplitude. They are phonon start time PAr20, PBr20, PCr20, PDr20, and PTr20.

- The time at which the phonon pulse reaches $10 \%$ of its peak amplitude. They are $\operatorname{PAr} 10, \operatorname{PBr} 10, \operatorname{PCr} 10, \operatorname{PDr} 10$, and $\operatorname{PTr} 10$.

- The time at which the phonon pulse reaches $40 \%$ of its peak amplitude. They are PAr40, PBr40, PCr40, PDr40, and PTr40.

- The difference between $40 \%$ pulse amplitude time and $10 \%$ pulse amplitude time is called phonon rise time. It is ptrt for the phonon total.

- The phonon rise time in the channel in which the maximum phonon pulse occurs is called minimum phonon rise time. It is pminrt. 

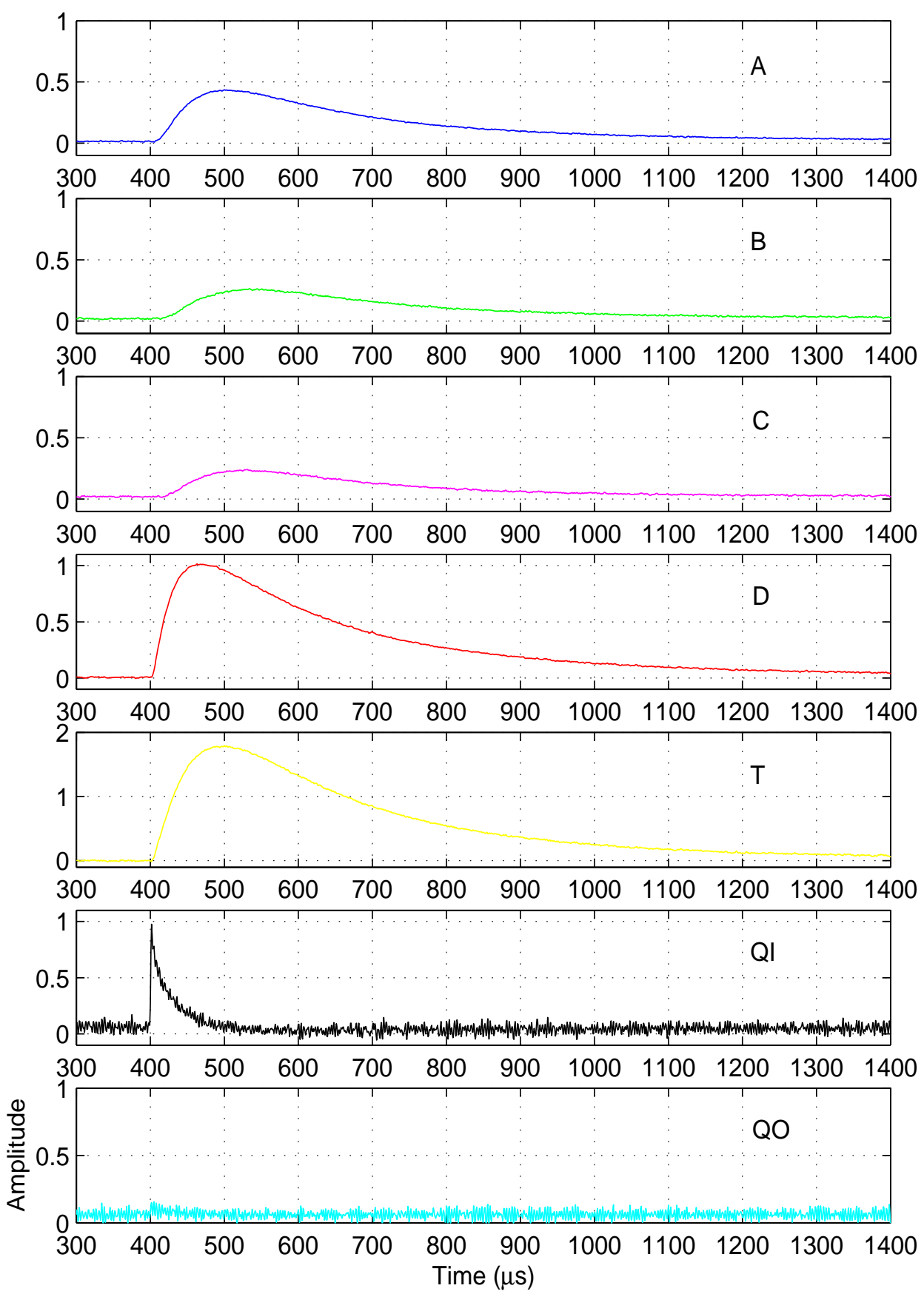

Figure 7.4: The $60 \mathrm{keV}$ event raw traces in the $\mathrm{Z} 2$ charge inner electrode. The traces are phonon sensor A, B, C, D, phonon total, charge inner and charge outer from top to bottom. The event is in quadrant $\mathrm{D}$. 
- The time difference between phonon start time and charge start time is a measure of phonon propagation time from event location to phonon sensor. It is phonon delay.

- The phonon delay in the channel at which the maximum phonon pulse occurs is called minimum phonon delay. It is pdel.

The timing parameters are renamed with a suffix of letter 'c' after their position corrections. For example, the phonon total rise time ptrt is renamed as ptrtc after this correction.

With the ionization yield cut, most of the electron recoil background is removed. But some surface electron recoils can leak into the nuclear recoil band. The surface event rejection remains as the central part of the CDMS WIMP search data analysis. Phonon rise time ptrtc was used as the surface event rejection parameter in ZIP detectors before CDMS Soudan run118 [86, 125]. The application of phonon delay for event location information and surface event discrimination was investigated after SUF rum21 in 2002. (internal references [126], [127], [128], [129], [130]). Phonon delay carries information from two physics processes. One is well understood with phonon rise time ptrtc for surface events. The high primary optical phonon scattering rate due to high impurity density on the detector surface results in fast phonon timing. The second one comes from the NeganovLuke phonon effect. The ionization charges are separated and collected completely for bulk electron interactions, but surface events have reduced ionization charge collection. Because Neganov-Luke phonons are emitted instantaneously, phonon delay will reject surface events as well as bulk electron events.

Another surface rejection parameter is the $\mathrm{z}$ coordinate parameter $Z Z$, which is described in chapter 5. $Z Z$ comes from event location transformation. It carries 
phonon timing information in the local quadrant phonon sensor and two neighbor phonon sensors.

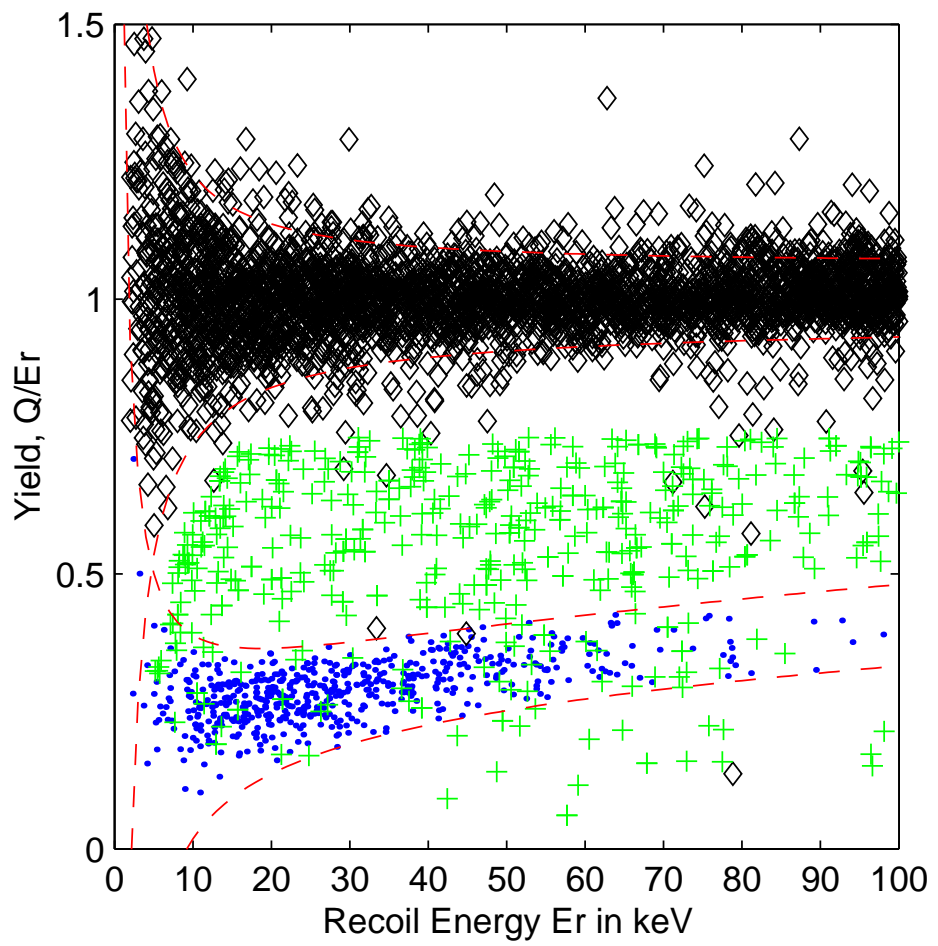

Figure 7.5: Ejectron selection in Z5. Ejectrons(green crosses) are low yield nearest double scattering events below the $3 \sigma$ lower bound of the gamma band and yield yc $<0.75$ in ${ }^{133} \mathrm{Ba}$ calibration data. The blue dots are neutrons in the nuclear recoil band. The black diamonds are single scatters in ${ }^{133} \mathrm{Ba}$ calibration data.

We use Z5 as an example to understand the surface event rejection capability of timing parameters ptrtc, pminrtc, and pdelc, and location parameter $Z Z .{ }^{252} \mathrm{Cf}$ calibration data in the nuclear recoil band and ejectrons (low yield nearest double scattering events below the $3 \sigma$ lower bound of the gamma band and ionization yield yc $<0.75)$ in ${ }^{133} \mathrm{Ba}$ calibration data are used as nuclear recoils and surface events, respectively, as shown in Figure 7.5.

In this thesis, neutrons in ${ }^{252} \mathrm{Cf}$ calibration data are used as 'WIMP-like' test particles, because both neutrons and WIMPs primarily interact with nuclei. 
The distributions of pdelc in 10-20 keV, 20-30 keV, 30-40 keV, and 40-100 keV energy bins for neutrons and ejectrons are in Figure 7.6. pdelc has good ejectron rejection capability, whereas most of the nuclear recoils are kept.

For a given cut line of the surface event rejection parameter, for example, pdelc, the neutron selection fraction is $\alpha$, and the ejectron selection fraction is $\beta$. As defined earlier, the minimum of the quality factor [111]:

$$
Q=\frac{\beta(1-\beta)}{(\alpha-\beta)^{2}}
$$

determines optimized cut value.

For pdelc, the quality factor Q decreases monotonically both in the $10-30 \mathrm{keV}$ energy bin and the 30-100 keV energy bin, as shown in Figure 7.7. We can set the surface event rejection cut at pdelc $=7.8 \mu \mathrm{s}$ in the $10-30 \mathrm{keV}$ energy bin, at pdelc $=6.8 \mu \mathrm{s}$ in the $30-100 \mathrm{keV}$ energy bin, because Q starts to decrease slowly at these values. These lead to the surface event cut line shown in the left graph in Figure 7.8. With this surface event cut line, one surface event between $10 \mathrm{keV}$ and $20 \mathrm{keV}$ is selected; $75 \%$ to $85 \%$ neutrons are selected. The nuclear recoil selection efficiency and the surface event rejection efficiency are shown in the right graph in figure 7.8 .

Similarly, The distributions of ptrtc, pminrtc, and ZZ in 10-20 keV, 20-30 keV, 30-40 keV, and 40-100 keV energy bins for neutrons and ejectrons are in figures 7.9, 7.12, and 7.15. The optimizations of the surface event cuts in $10-30 \mathrm{keV}$ energy bin and 30-100 keV energy bin for ptrtc, pminrtc, and ZZ are in figures 7.10, 7.13, and 7.16. The applicable surface event cuts and the efficiencies for ptrtc, pminrtc, and $\mathrm{ZZ}$ are in figures $7.11,7.14$, and 7.17 .

The phonon timing parameters pdelc, ptrtc and pminrtc, and the ZZ parameter all have good surface event rejection efficiency with most of the nuclear recoils 
selected. Timing parameters ptrtc, pminrtc, and pdelc are position corrected, i.e., the TES gradient dependence and the radius dependence of the TES response of these quantities are removed. But $\mathrm{ZZ}$ has not been position corrected yet. It is expected that $\mathrm{ZZ}$ would have better neutron selection efficiency after the position correction.

Because of the relatively high neutron selection efficiency of pdelc (phonon delay), pdelc has been used as the main surface rejection parameter in CDMS background data analysis. But other timing parameters are needed to further improve surface event rejection. There are two potential problems with data reduction cuts using phonon timing parameters only. One is the risk of surface event leakage by using only one phonon timing parameter cut, for example, pdelc at low recoil energy. Another is the low nuclear recoil event selection efficiency by using two or more timing parameters. 

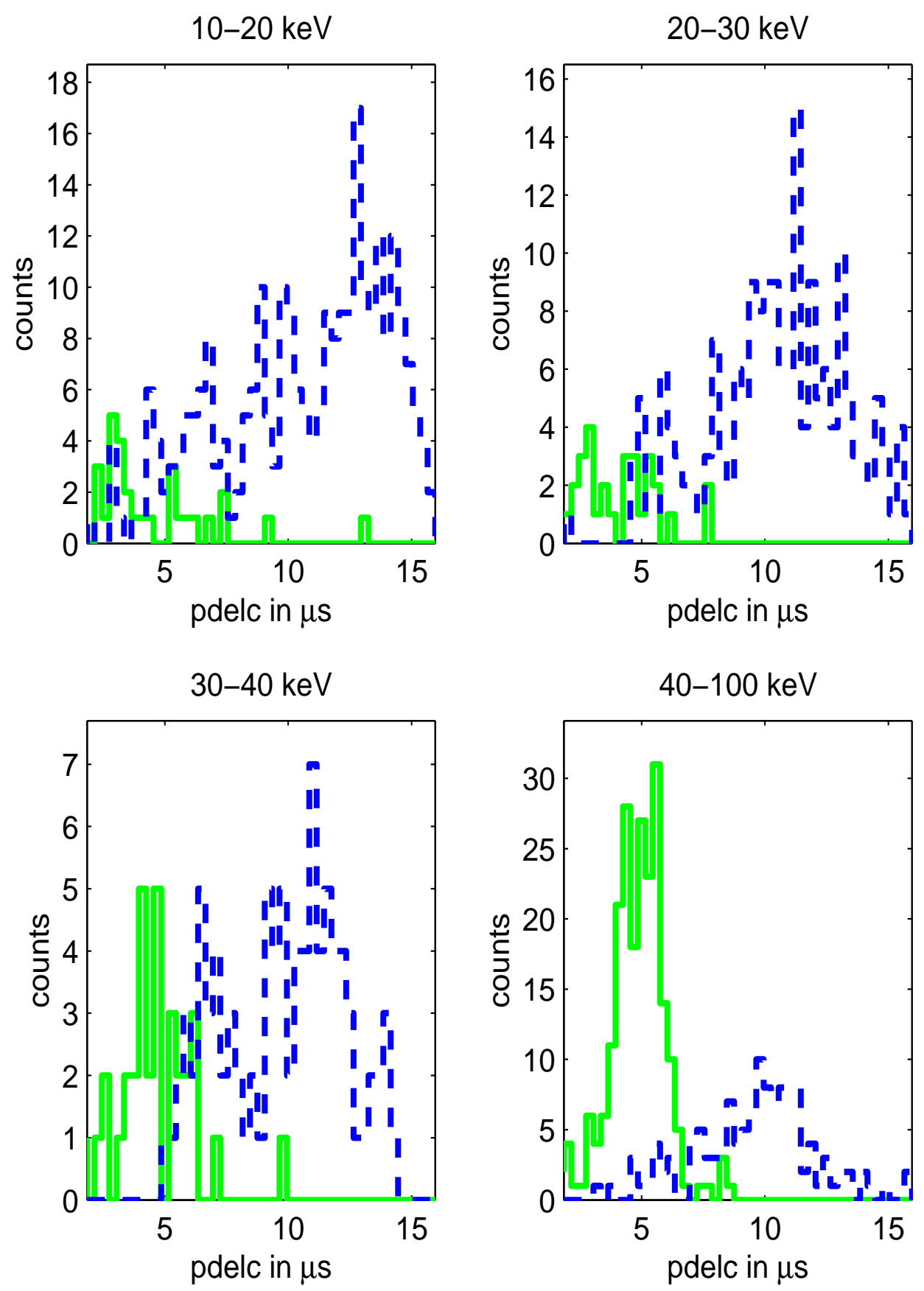

Figure 7.6: pdelc distribution of neutrons (blue dashed lines) and ejectrons (green solid lines) of Z5 in different energy bins. 

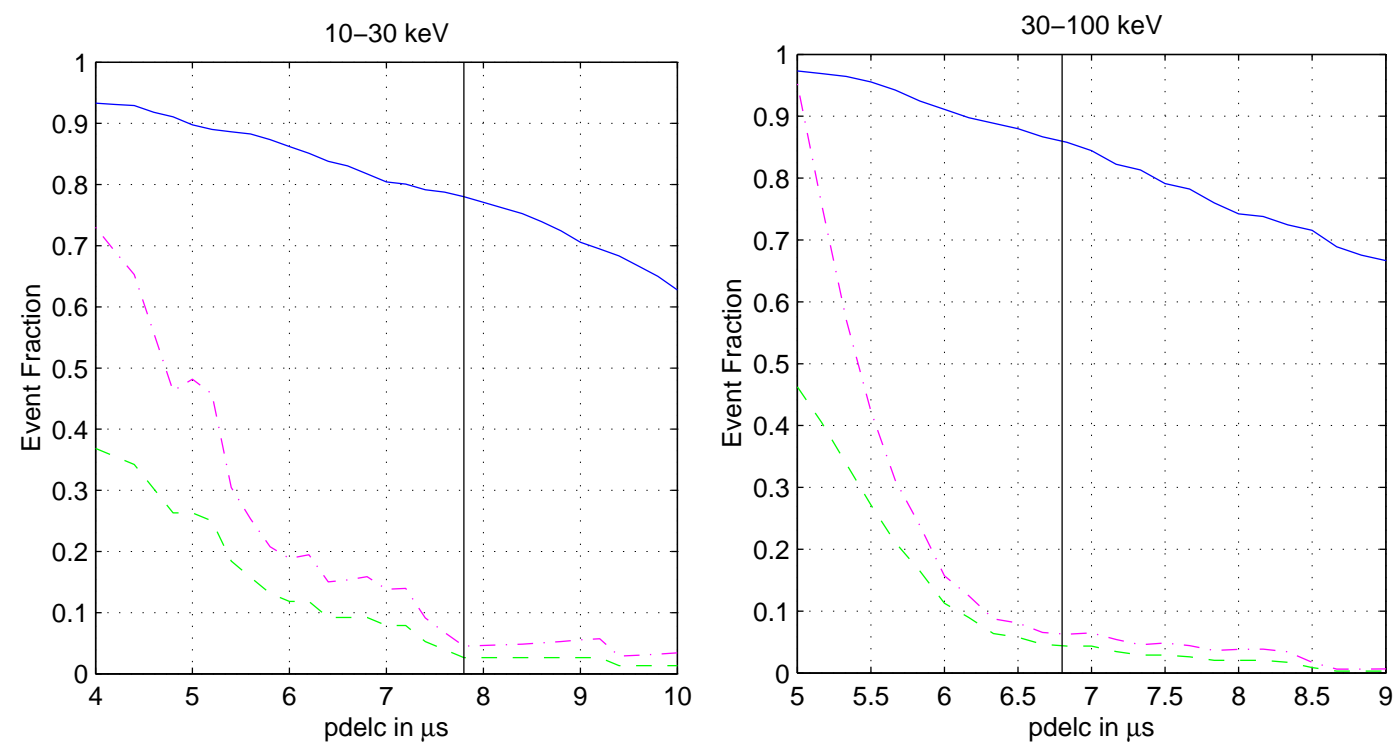

Figure 7.7: The optimized pdelc cut value search for Z5 in energy bins 10-30 $\mathrm{keV}$ (the left graph) and $30-100 \mathrm{keV}$ (the right graph). The blue solid line is the selected neutron fraction, the green dashed line is the selected ejectron fraction, the dashed dot line is the quality factor $Q$.
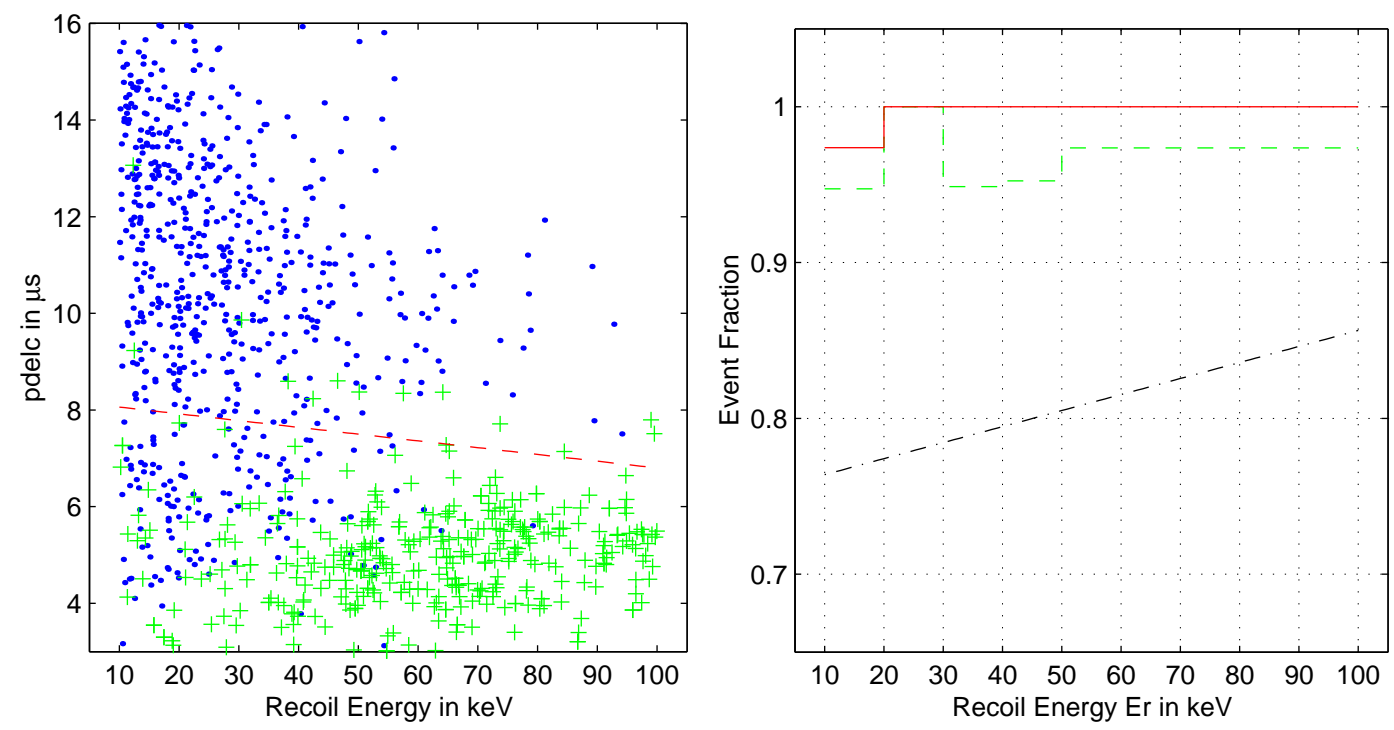

Figure 7.8: The pdelc cut (the left graph) and efficiencies (the right graph) of Z5. The black dashed dot line is the neutron selection efficiency, the green dashed line is ejectron rejection efficiency without ionization yield cut, the solid red line on the top is the ejectron rejection efficiency with ionization yield cut ( $2 \sigma \mathrm{NR}$ band) and pdelc cut. 

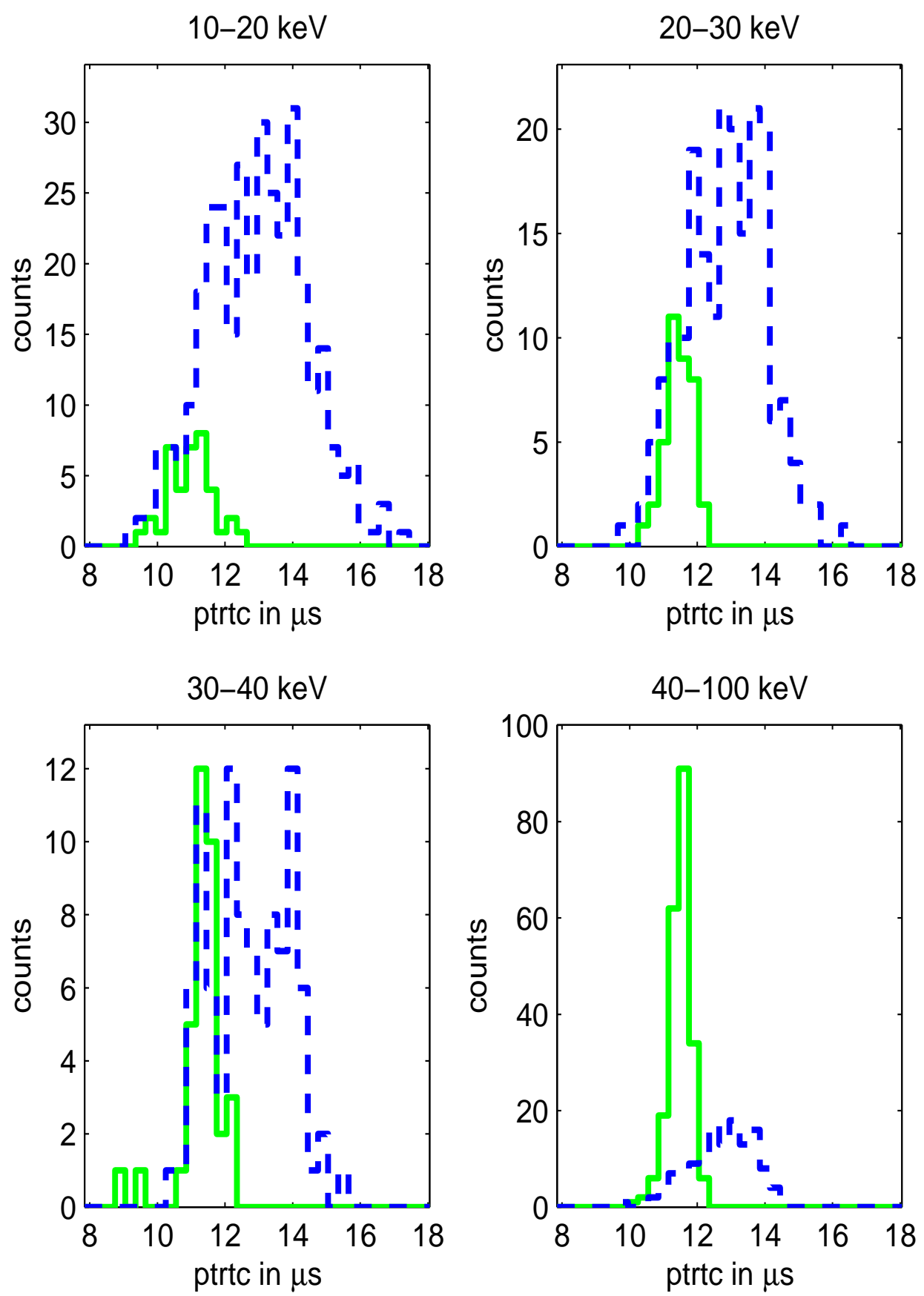

Figure 7.9: ptrtc distribution of neutrons (blue dashed lines) and ejectrons (green solid lines) of Z5 in different energy bins. 

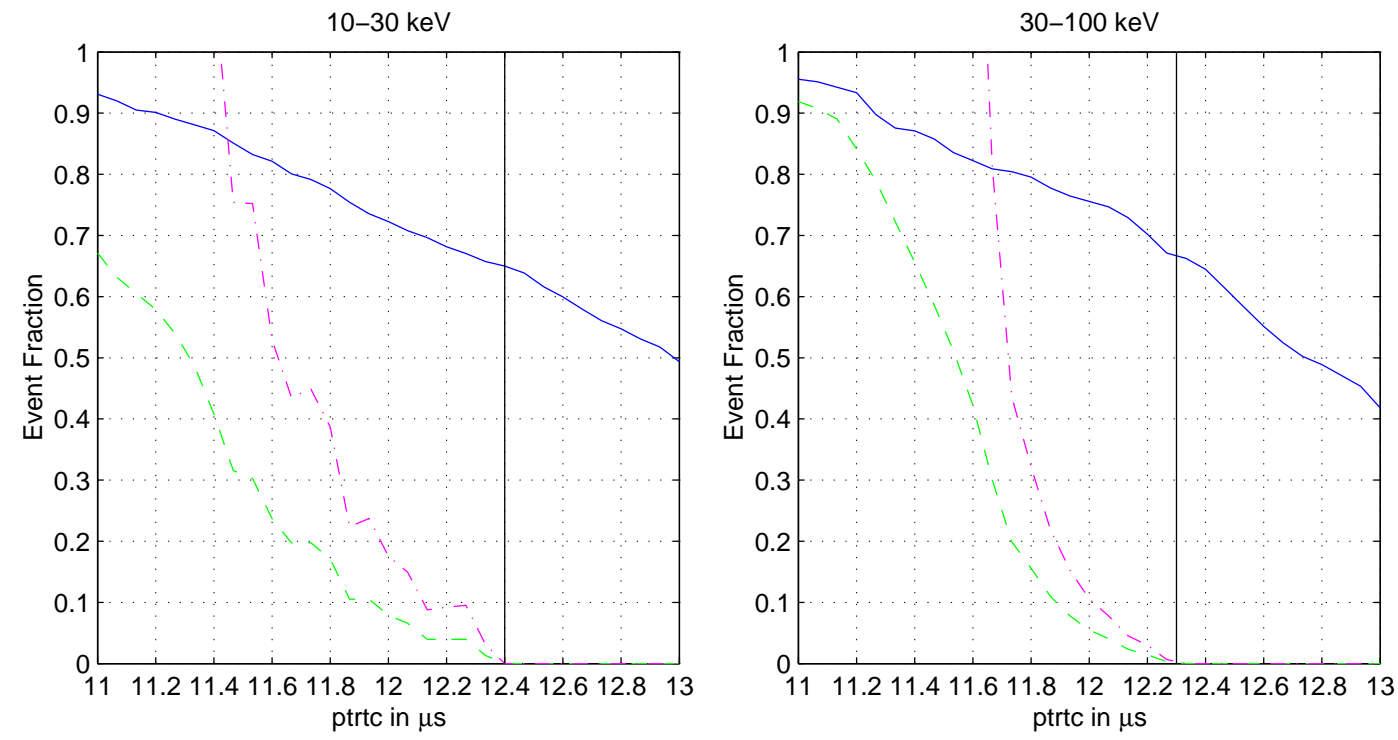

Figure 7.10: The optimized ptrtc cut value search for Z5 in energy bins $10-30 \mathrm{keV}$ (the left graph) and 30-100 keV (the right graph). The blue solid line is selected neutron fraction, the green dashed line is the selected ejectron fraction, the dashed dot line is the quality factor $Q$.
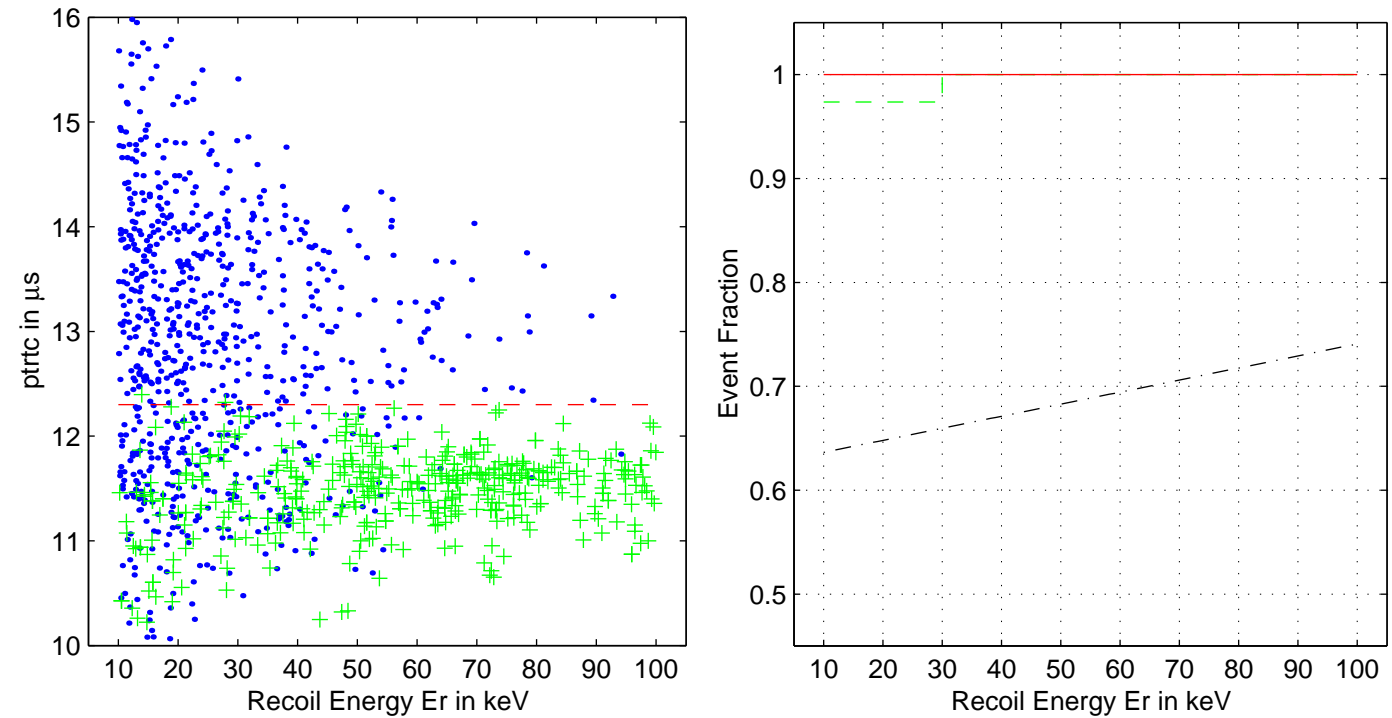

Figure 7.11: The ptrtc cut (the left graph) and efficiencies (the right graph) of Z5. The black dashed dot line is the neutron selection efficiency, the green dashed line is ejectron rejection efficiency without ionization yield cut, the solid red line on the top is the ejectron rejection efficiency with ionization yield cut ( $2 \sigma \mathrm{NR}$ band) and ptrtc cut. 

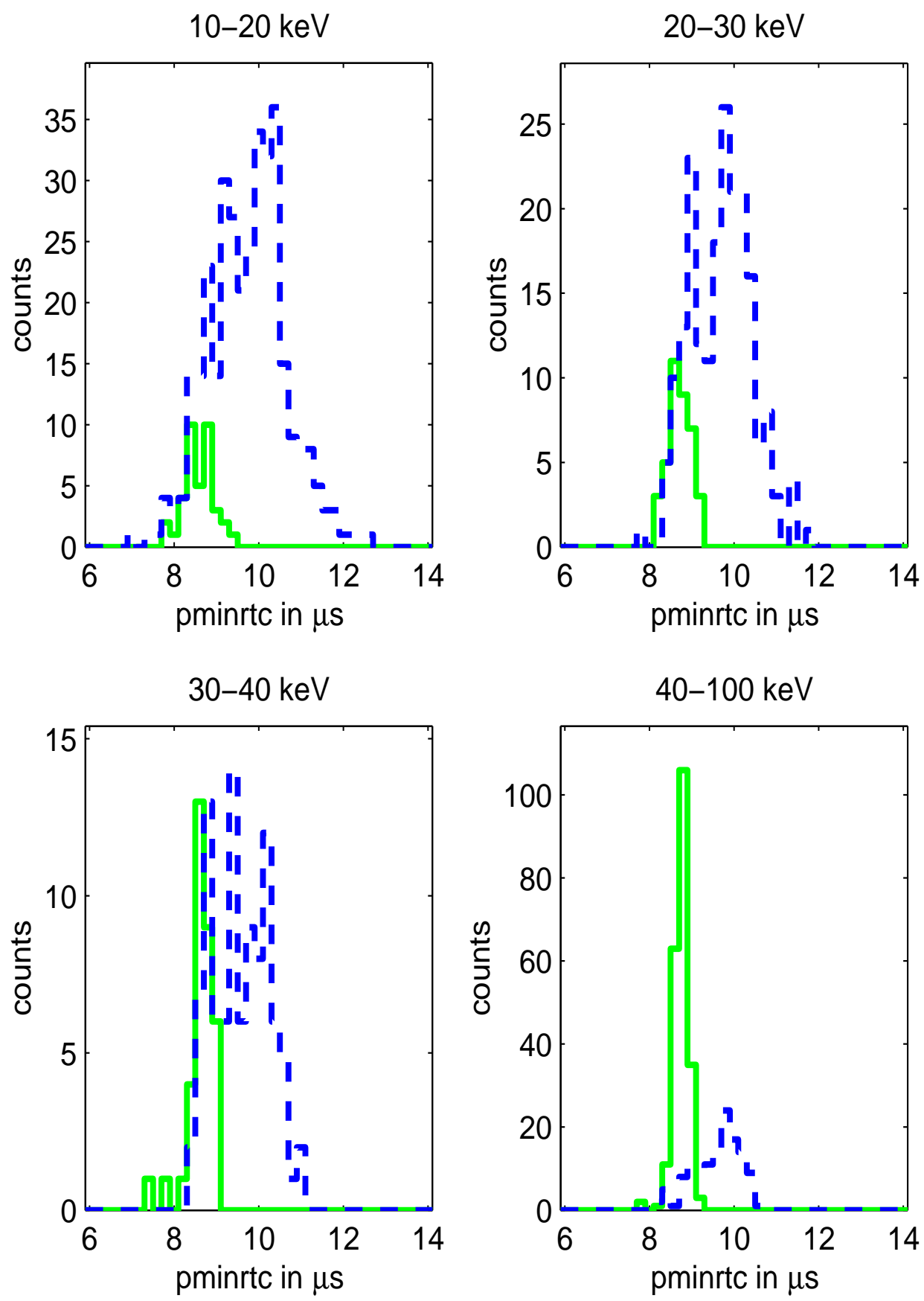

Figure 7.12: pminrtc distribution of neutrons (blue dashed lines) and ejectrons (green solid lines) of Z5 in different energy bins. 

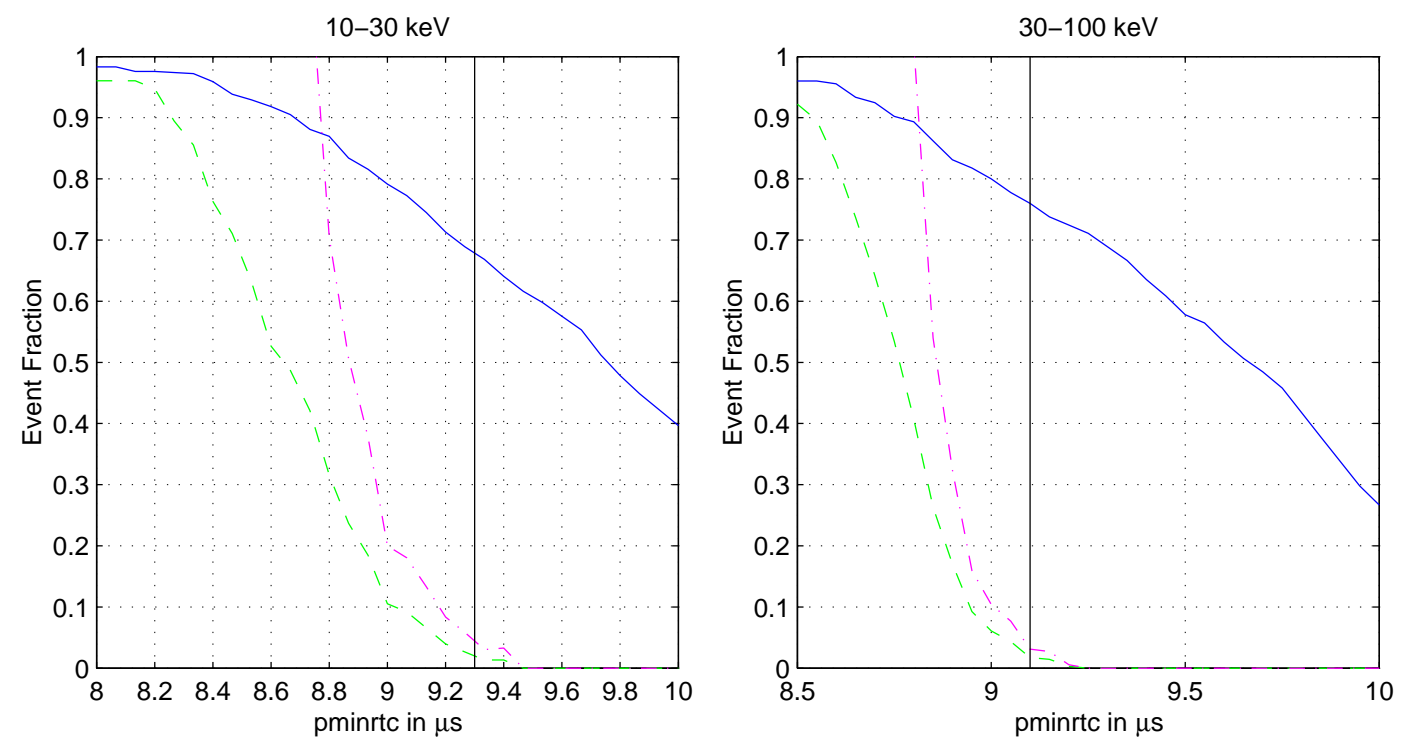

Figure 7.13: The optimized pminrtc cut value search for Z5 in energy bins 10-30 $\mathrm{keV}$ (the left graph) and 30-100 keV (the right graph). The blue solid line is selected neutron fraction, the green dashed line is the selected ejectron fraction, the dashed dot line is the quality factor $Q$.
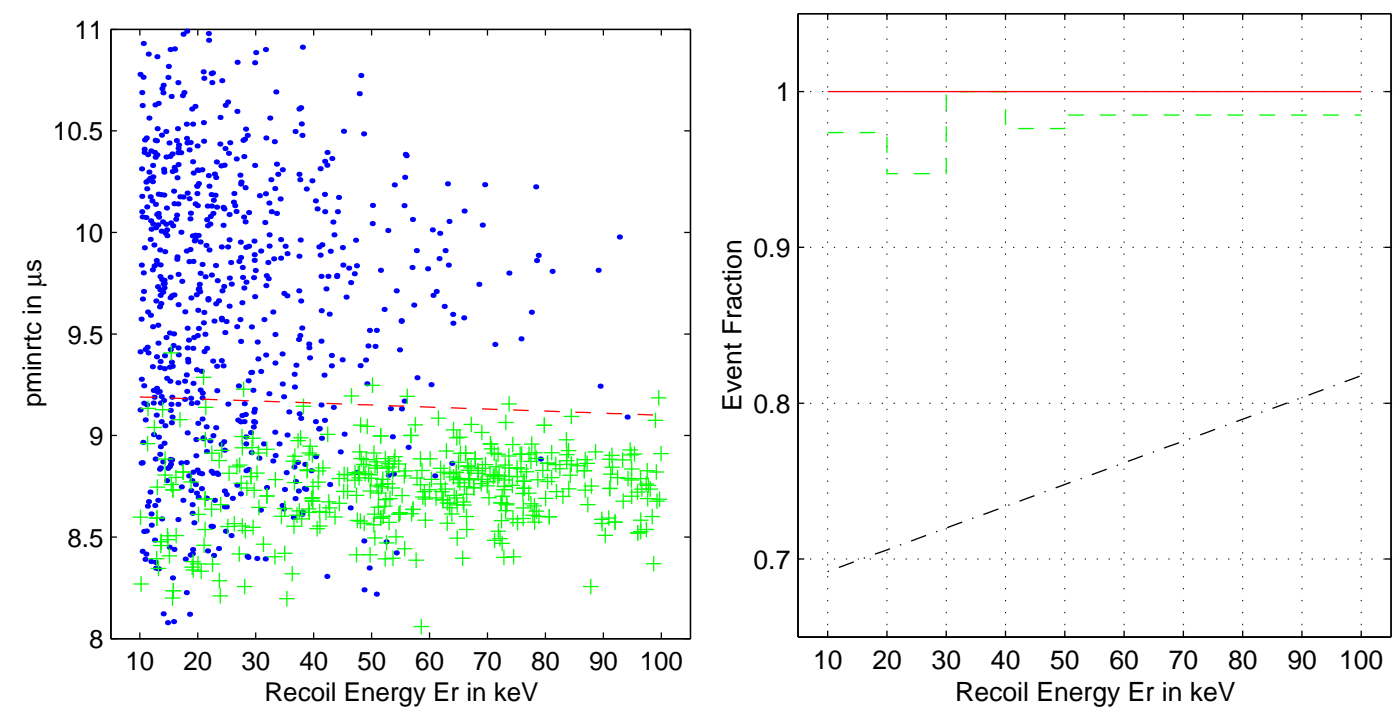

Figure 7.14: The pminrtc cut (the left graph) and efficiencies (the right graph) of Z5. The black dashed dot line is the neutron selection efficiency, the green dash line is ejectron rejection efficiency without ionization yield cut, the solid red line on the top is the ejectron rejection efficiency with the ionization yield cut ( $2 \sigma \mathrm{NR}$ band) and pminrtc cut. 

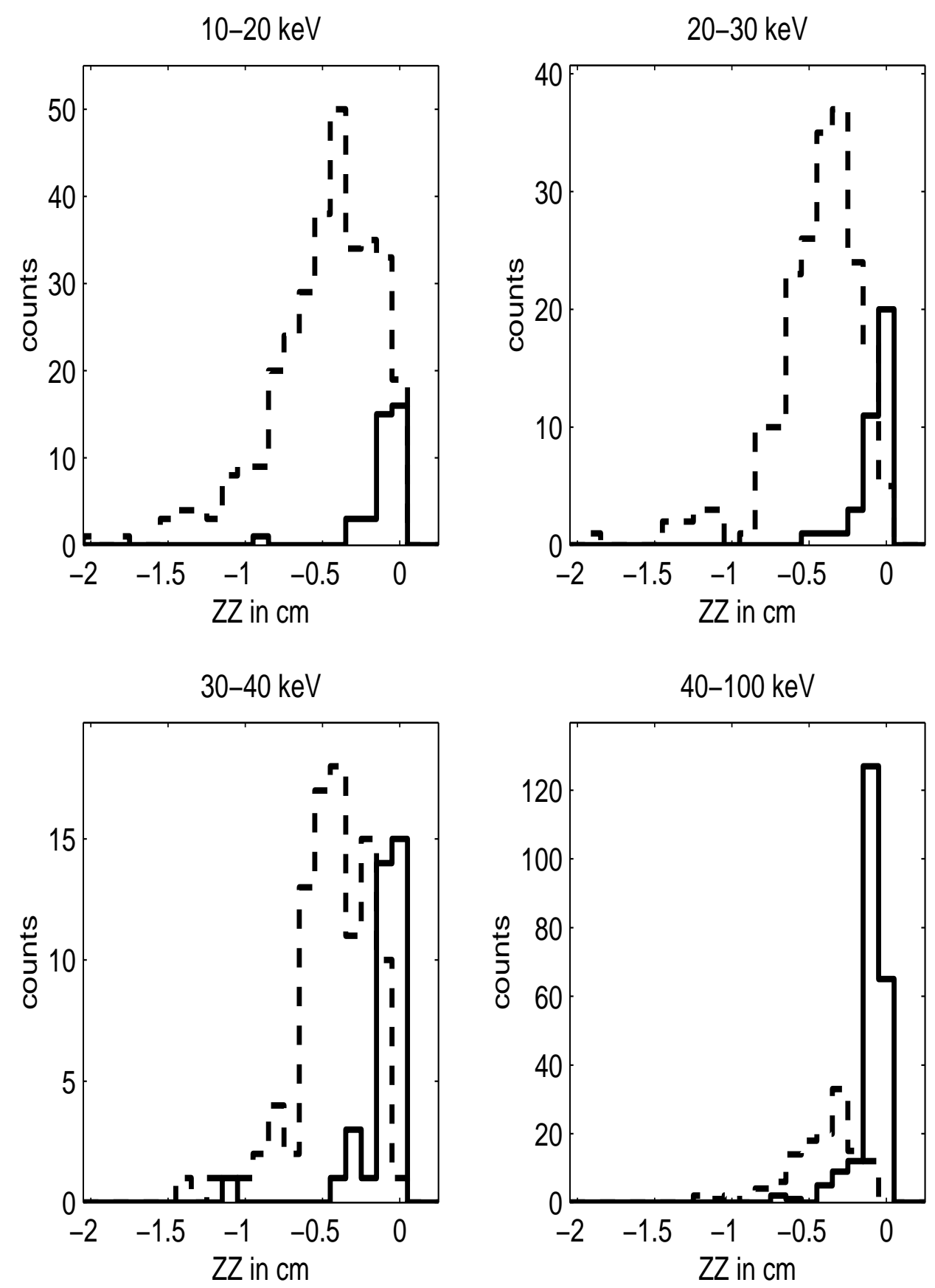

Figure 7.15: ZZ distribution of neutrons (blue dashed lines) and ejectrons (green solid lines) of Z5 in different energy bins. 

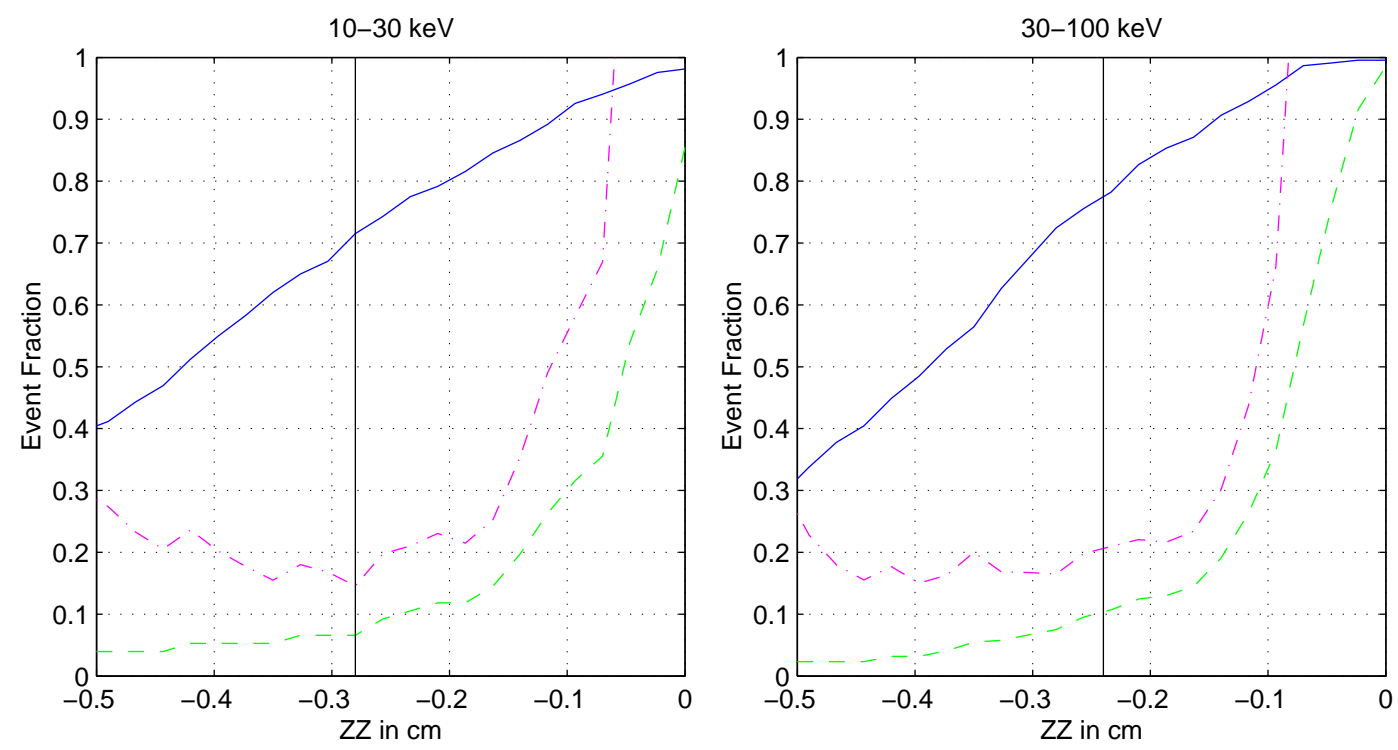

Figure 7.16: The optimized ZZ cut value search for Z5 in energy bins 10-30 keV (the left graph) and 30-100 keV (the right graph). Blue solid line is selected neutron fraction, green dashed line is selected ejectron fraction, dashed dot line is the quality factor $Q$.
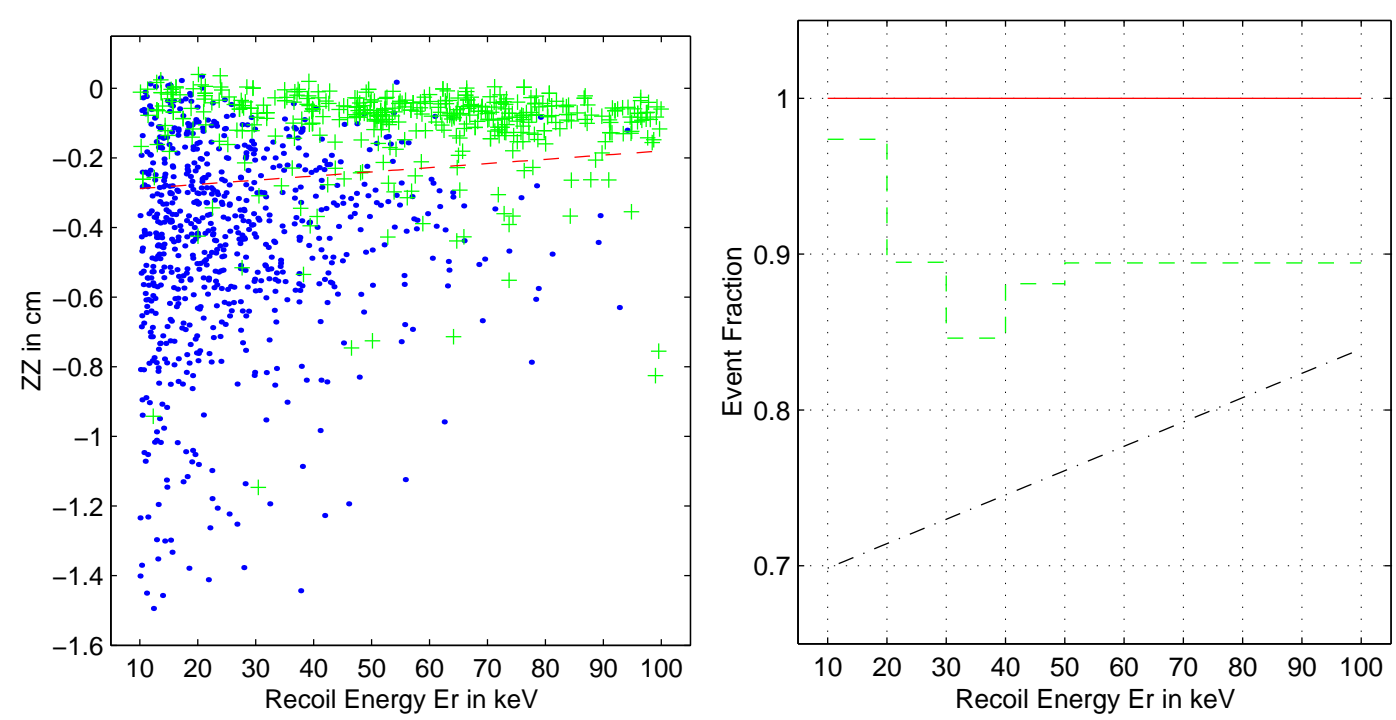

Figure 7.17: The ZZ cut (left plot) and efficiencies (right plot) of Z5. The black dash dot line is the neutron selection efficiency, the green dashed line is ejectron rejection efficiency without ionization yield cut, the solid red line on the top is the ejectron rejection efficiency with ionization yield cut ( $2 \sigma \mathrm{NR}$ band) and ZZ cut. 


\subsection{Phonon Partition for Events with Big Ra- dius}

The introduction of event location information helps us to identify the features of the surface events in the ZIP detector and to use them in backround rejection for WIMP searching. Figure 7.18 shows the neutrons and ejectrons in two categories: events with radius $R \leq 2.7 \mathrm{~cm}$ and events with radius $R>2.7 \mathrm{~cm}$ (the detector inner electrode radius is $3.5 \mathrm{~cm}$, the reconstructed radius parameter $R \leq 3.5 \mathrm{~cm}$ for electron recoils, but $R$ can be as big as $4.5 \mathrm{~cm}$ for nuclear recoils due to the

slow phonon times). Ejectrons that have a small radius can be rejected at high efficiency with timing parameters, as shown in the top graph. Ejectrons that have a big radius need harsh timing cuts, as shown in the bottom graph. Ejectrons between the two dashed lines in the bottom plot are in a controversial region. Figure 7.19 shows that most of the low yield ejectrons that need harsh timing cuts are at the edge of the phonon sensors. This is generally true for all timing parameters, as shown in Figures 7.20, 7.21, and 7.22. ptrtc, pminrtc, and pdelc are plotted as functions of radius $R$, respectively.

Because of the limitations in separating nuclear recoil and surafce events at a big radius, as illustrated by the above plots, we pursue a strategy that separates the events in the CDMS ZIP detector into the small radius category and the big radius category and allows us to select nuclear recoils at a higher confidence level. An independent surface rejection parameter, which should be used together with phonon timing parameters, is required for high nuclear recoil selection efficiency and an improved surface event rejection efficiency. This parameter is the phonon energy partition: the phonon energy in the local quadrant and the phonon energy in two neighbor quadrants minus the phonon energy in the opposite quadrant. 
For an event in the CDMS ZIP detector quadrant A, the phonon partition can be expressed as

$$
\text { partition }=\frac{p a+p b+p d-p c}{p t}
$$

where $p a$ is phonon energy in quadrant $\mathrm{A}, p b$ is phonon energy in quadrant $\mathrm{B}$, $p c$ is phonon energy in quadrant $\mathrm{C}, p d$ is phonon energy in quadrant $\mathrm{D}$, and $p t=p a+p b+p c+p d$ is total phonon energy.

The separation between neutrons and ejectrons with big radius parameters is good in the phonon partition versus pdelc two dimensional plot as shown in Figure 7.23 for neutrons and ejectrons (below the $3 \sigma$ gamma band and $y c<0.75$ ), and in Figure 7.24 (ejectrons are in the $3 \sigma$ nuclear recoil band).

The full understanding of the separation between neutrons and ejectrons in the phonon partition vs pdelc plot comes from several effects of phonon evolution and propagation in the ZIP detector. First, surface scattering leads to fast phonon timing parameters. Second, the phonon timing parameters are bigger for the same kind of scattering when the event approches the charge outer electrode, as seen in arguments at the beginning of this section. Finally, the diffusion reflection of the phonons gives a characteristic phonon partition distribution as a function of event position. I explain the first and the third of these arguments in detail:

First, the evolution of the nonequalibrium phonon distribution function $n_{\vec{q}}(\vec{r}, t)$ in space and time is governed by the Boltzmann-Peierls equation [106]

$$
\frac{\partial n_{\vec{q}}}{\partial t}=-\overrightarrow{v_{\vec{q}}} \cdot \nabla n_{\vec{q}}+S\left(n_{\vec{q}}\right)+J\left(n_{\vec{q}}\right)+G_{\vec{q}}
$$

where $v_{\vec{q}}$ is the group velocity of phonon $\vec{q}, S\left(n_{\vec{q}}\right)$ is a collision term for phononphonon scattering(usually it is called anharmonic decay, resulting in phonon split or coalescence), $J\left(n_{\vec{q}}\right)$ is another collision term for phonon-impurity (defect) scattering, and $G_{\vec{q}}$ is the phonon generation rate from external excitation. After an 
interaction occurs in the crystal of the ZIP detector and before the next event, $G_{\vec{q}}=0$.

There are three characteristic times of non-equilibrium phonon propagation. The first one is $\tau_{L}$, phonon propagation time from phonon source to the phonon sensor; the second one is $\tau_{R}$, phonon life time due to phonon impurity scattering; the third is $\tau_{N}$, phonon life time due to phonon phonon scattering. The values of $\tau_{L}, \tau_{R}$, and $\tau_{N}$ determine the real phonon propagation mode.

In the case of isotropic distribution, and the dispersion relation $\omega_{\vec{q}}=s q$ ( $\mathrm{s}$ is sound speed) under the long wave approximation, we have [106]

$$
\begin{aligned}
& S\left(n_{\omega}\right)=-\frac{n_{\omega}-n_{\omega 0}}{\tau_{N}} \\
& J\left(n_{\omega}\right)=-\frac{n_{\omega}-n_{\omega 0}}{\tau_{R}}
\end{aligned}
$$

where $n_{\omega 0}$ is the equilibrium phonon number with frequency $\omega$.

When the phonon impurity (or defects) scattering is important, $\tau_{L} \gg \tau_{R}$, the Boltzmann-peierls equation is simply the diffusion equation

$$
\frac{\partial n_{\vec{q}}}{\partial t}=D \nabla^{2} n_{\vec{q}}+S\left(n_{\vec{q}}\right)+G_{\vec{q}}
$$

where $D=v_{\vec{q}}^{2} \tau_{R}$, and $\tau_{R}$ is the phonon lifetime due to impurity scattering.

When phonon-phonon scattering is important, $\tau_{L} \ll \tau_{R}$, and $\tau_{L} \ll \tau_{N}$. At large scales $(\mathrm{cm})$, generally, phonon propagation distance $L \propto t$, where $t$ is the phonon propagation time.

Two kinds of impurities must be distinguished when phonon-impurity scattering is considered. The first kind of impurities are in the bulk of the ZIP detectors. They have a rigid electronic structure with excitation energy of the order of the 
atomic energy (a few eV). Such impurities are ionized donors and acceptors, and isotopes. The second kind of impurities have a soft electronic structure with low excitation energy levels. Such impurities are neutral donors and acceptors, and dangling chemical bonds.

The phonon scattering of the first kind of impurities, always the Rayleigh type, is usually referred to as isotope scattering. The phonon scattering of the second kind of impurities is generally considered in a simple model of a two-level impurity atom. The phonon scattering rate $\left(1 / \tau_{R 2}\right)$ of the second kind of impurities is much higher than the phonon scattering rate $\left(1 / \tau_{R 1}\right)$ of the first kind of impurities [106], i.e., $\tau_{R 1}>>\tau_{R 2}$.

CDMS ZIP detectors are high purity germanium or silicon detectors, so the first kind of impurities are mainly isotopes, and the second kind of impurities are the dangling chemical bonds on the surface of the detector. For surface events, the conversion time of primary optical phonons is determined by $\tau_{R 2}$, the second kind of phonon-impurity scattering time. After the conversion, lower frequency ballistic phonons travel through the ZIP detector at nearly uniformly high speed. For bulk events, the conversion time of primary optical phonons is determined by $\tau_{R 1}$, the first kind of phonon-impurity scattering time. $\tau_{R 1}>>\tau_{R 2}$ explains the phonon timing difference between ejectrons and neutrons.

The phonon diffusion reflection and limited phonon sensor coverage at the edge of the ZIP detector cause the phonon partition to be smaller for events near the charge outer electrode. This argument became clear to me after a discuusion with Richard Schnee in which he pointed out that the total number of phonons collected by a phonon sensor is proportional to the solid angle of the phonon sensor seeing the phonons. This solid angle of the opposite quadrant phonon sensor is enhanced for events near the charge outer electrode due to the phonon diffusion reflection. 
Figure 7.25 shows the phonon partion as a function of event radius parameter. Because neutrons generally have slow timing, the radius parameter is bigger for neutrons than for ejectrons. This means that the real event radius could be at $R_{0}=3 \mathrm{~cm}$ for a neutron having a recontructed radius parameter $R=3.5 \mathrm{~cm}$ or even slightly bigger. After this correction, there is no phonon partition difference between neutrons and ejectrons. But the feature is clear: events near the edge of the ZIP detector have a smaller phonon partition.

Figure 7.26 shows the phonon partition versus local quadrant phonon start time plot. Ejectrons with a big radius parameter may have a high phonon start time, but generally they have low phonon partition values, just like the ejectron distribution in the phonon partition versus pdelc plot of Figure 7.23.

Considering the phonon delay distribution as a function of radius parameter, the phonon partition as a function of radius parameter, and the fast phonon timing parameters for surface events, it is ideal to select nuclear recoils (and to reject surface events) in the phonon partition versus pdelc two dimensional plane for all events having big radius parameter $R$. This method can effectively cut off ejectrons that have big timing parameters, as illustrated in Figure 7.24. The WIMP search data analysis in the next section follows this idea. 

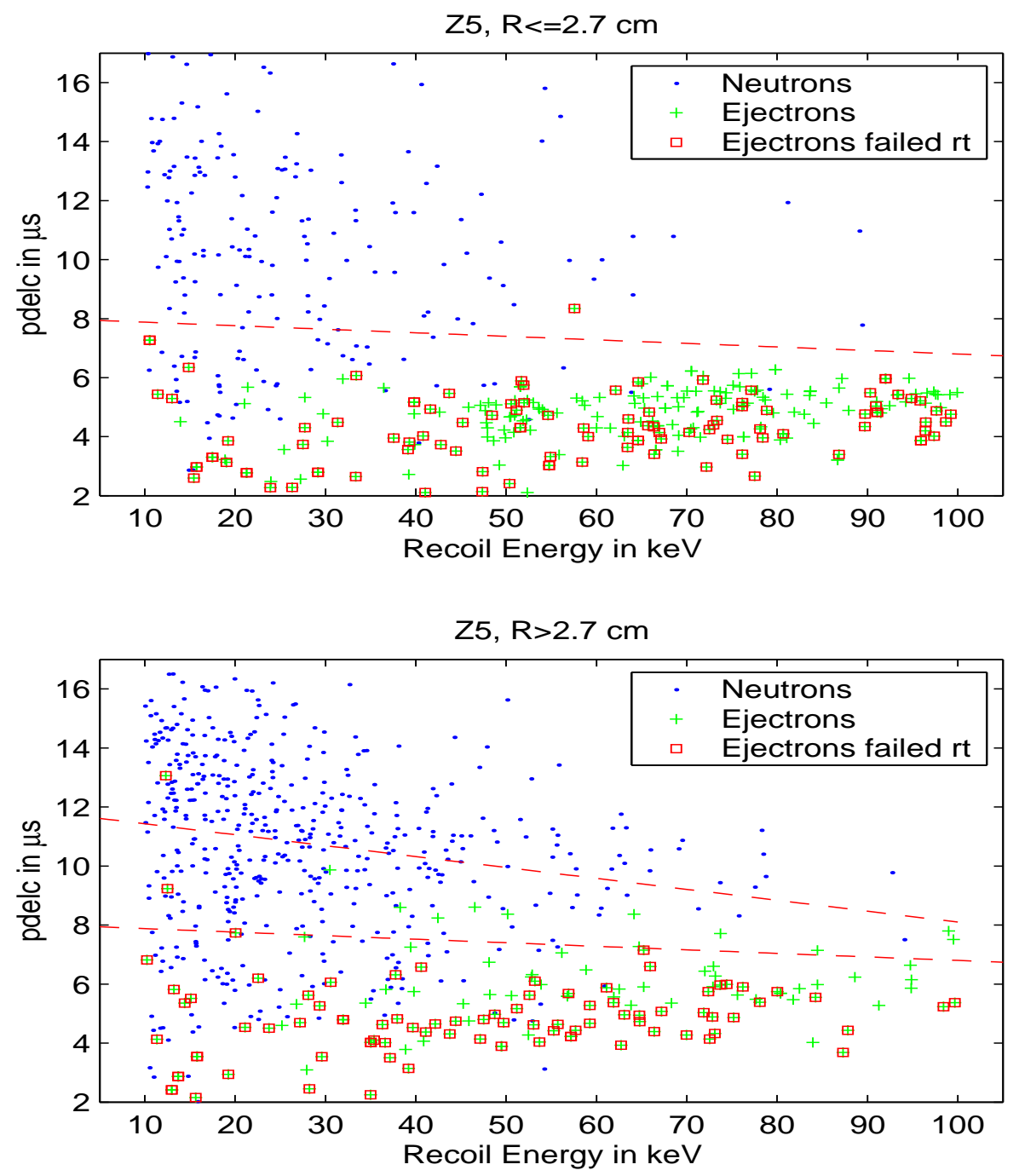

Figure 7.18: Separating events in Z5 into two categories. Events in the top plot have radius $R \leq 2.7 \mathrm{~cm}$. The events in the bottom plot have radius $R>2.7 \mathrm{~cm}$. The events falling into the area between the two dashed red lines in the bottom plot are events in the controversial region. The rt cut refers to: ptrtc $>11.5 \mu \mathrm{s}$ and pminrtc $>8.2 \mu s$. 


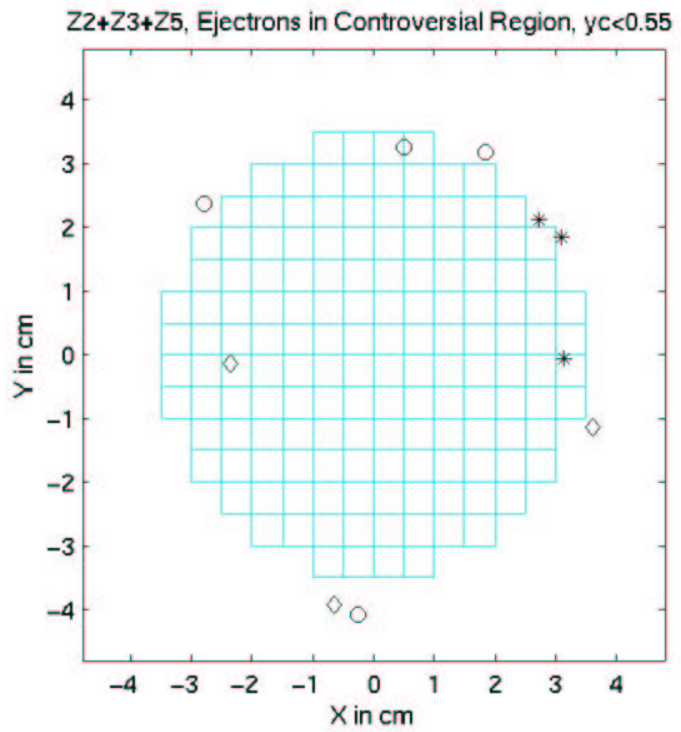

Figure 7.19: Big timing parameter ejectrons have a big radius. For the events shown in this graph, ptrtc $>11.5 \mu s$; pminrtc $>8.2 \mu s$; pdelc $>6.8 \mu s$ for Z2; pdelc $>$ $8.3-0.015 \cdot \operatorname{prc} \mu s$ for Z3; pdelc $>8.0-0.012 \cdot \operatorname{prc} \mu s$ for Z5. prc is recoil energy. The circles are for Z2, the diamonds are for Z3, and the stars are for Z5.
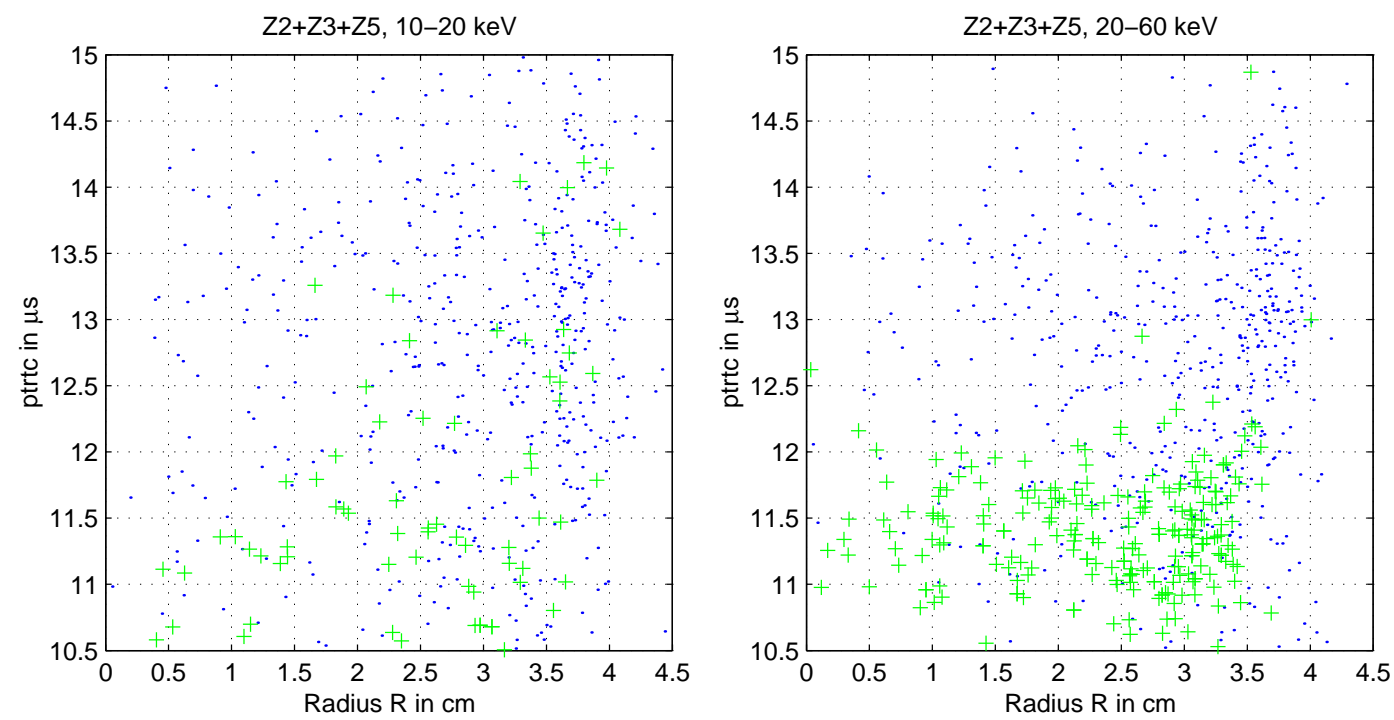

Figure 7.20: Z5, ptrtc distribution as a function of radius. Blue dots are neutrons, green crosses are ejectrons. Most big rise time ejectrons have a big radius. 

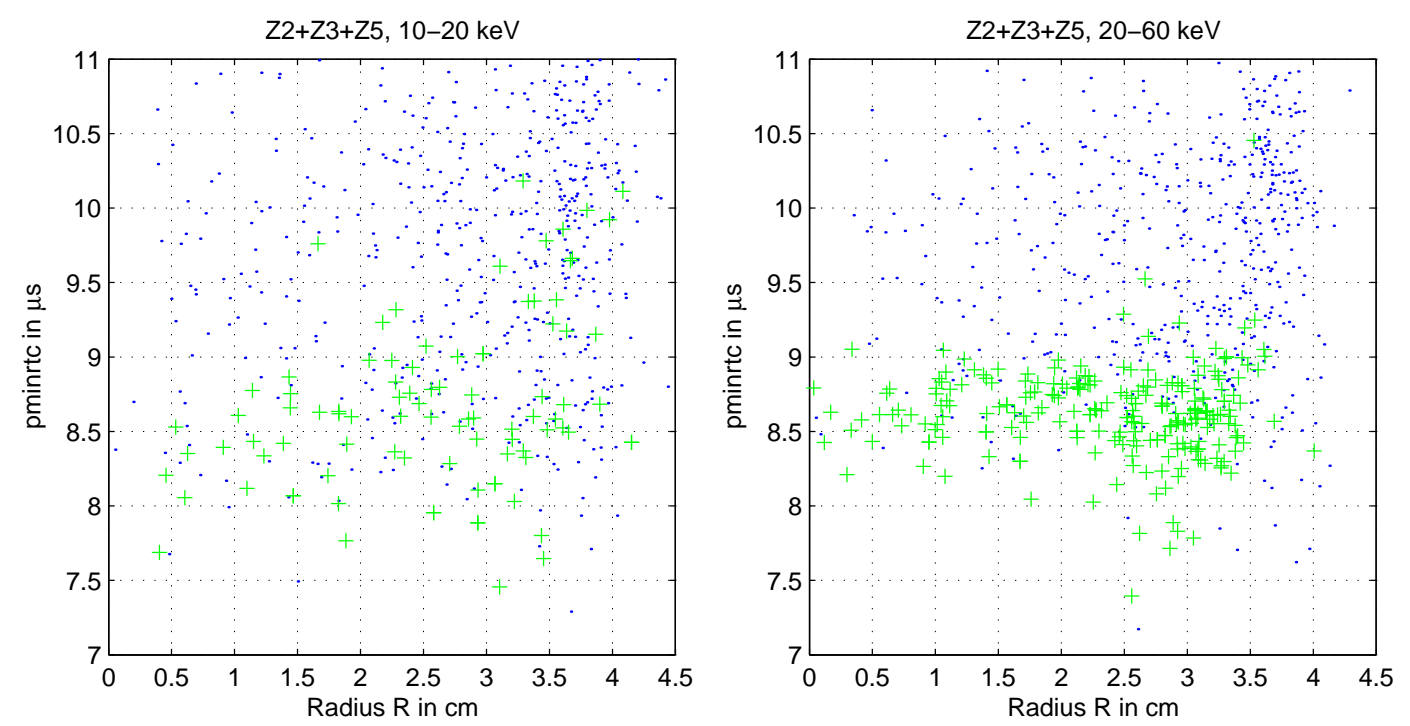

Figure 7.21: Z5, pminrtc distribution as a function of radius. Blue dots are neutrons, green crosses are ejectrons. Most big pminrtc ejectrons have big radius $R$.
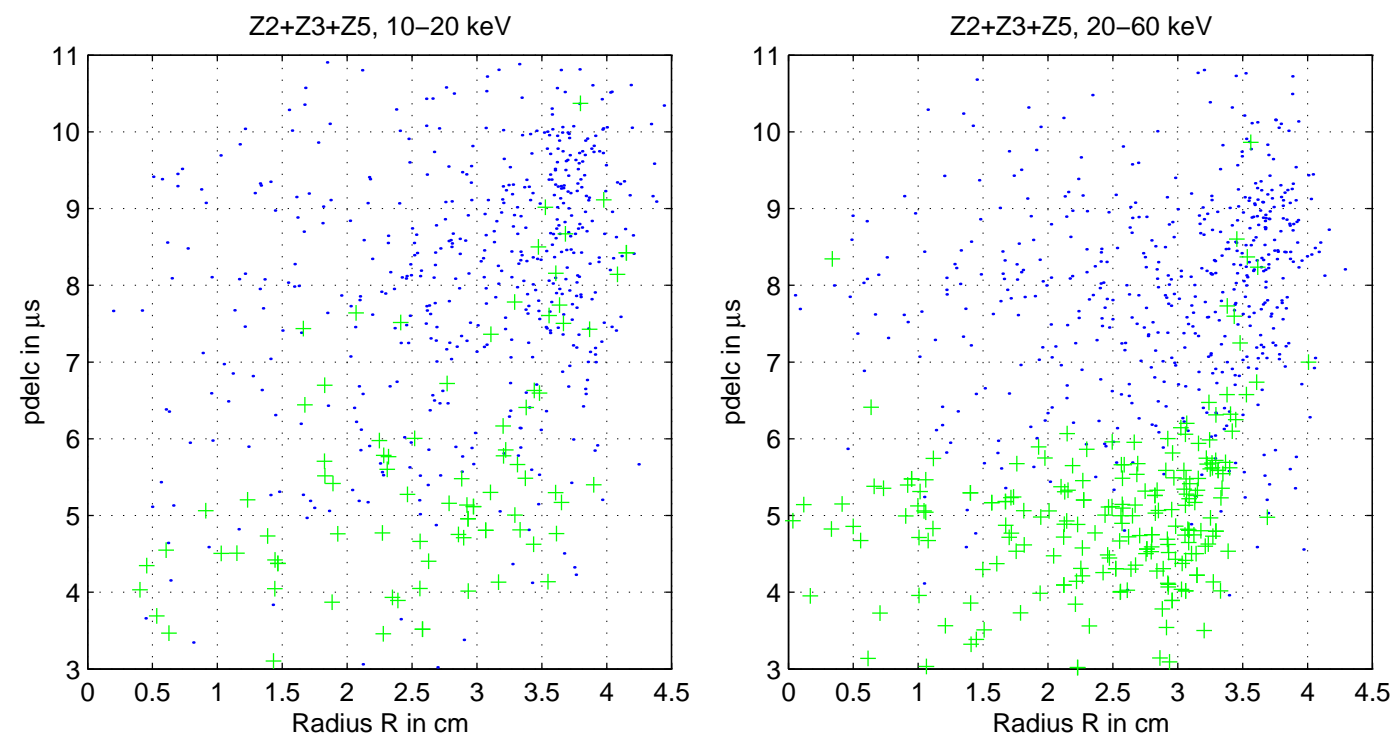

Figure 7.22: Z5, pdelc distribution as a function of radius. Blue dots are neutrons, green crosses are ejectrons. Most big phonon delay ejectrons have big radius $R$. 

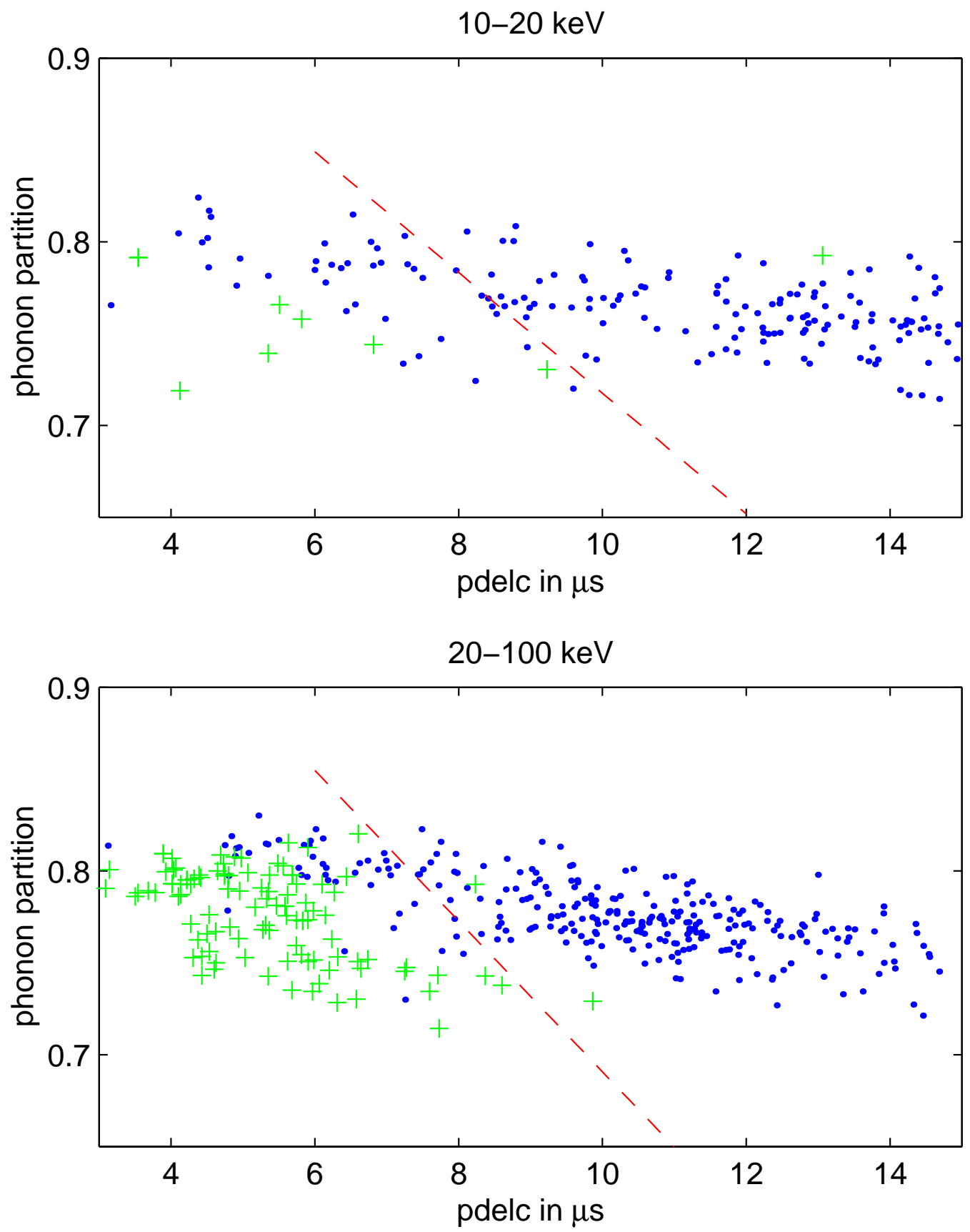

Figure 7.23: Z5, phonon partition distribution as a function of pdelc. The dashed red line is the surface event cut line, events below this line are supposed to be surface interactions. Blue dots are neutrons, green crosses are ejectrons. All events have radius $R>2.7 \mathrm{~cm}$. 

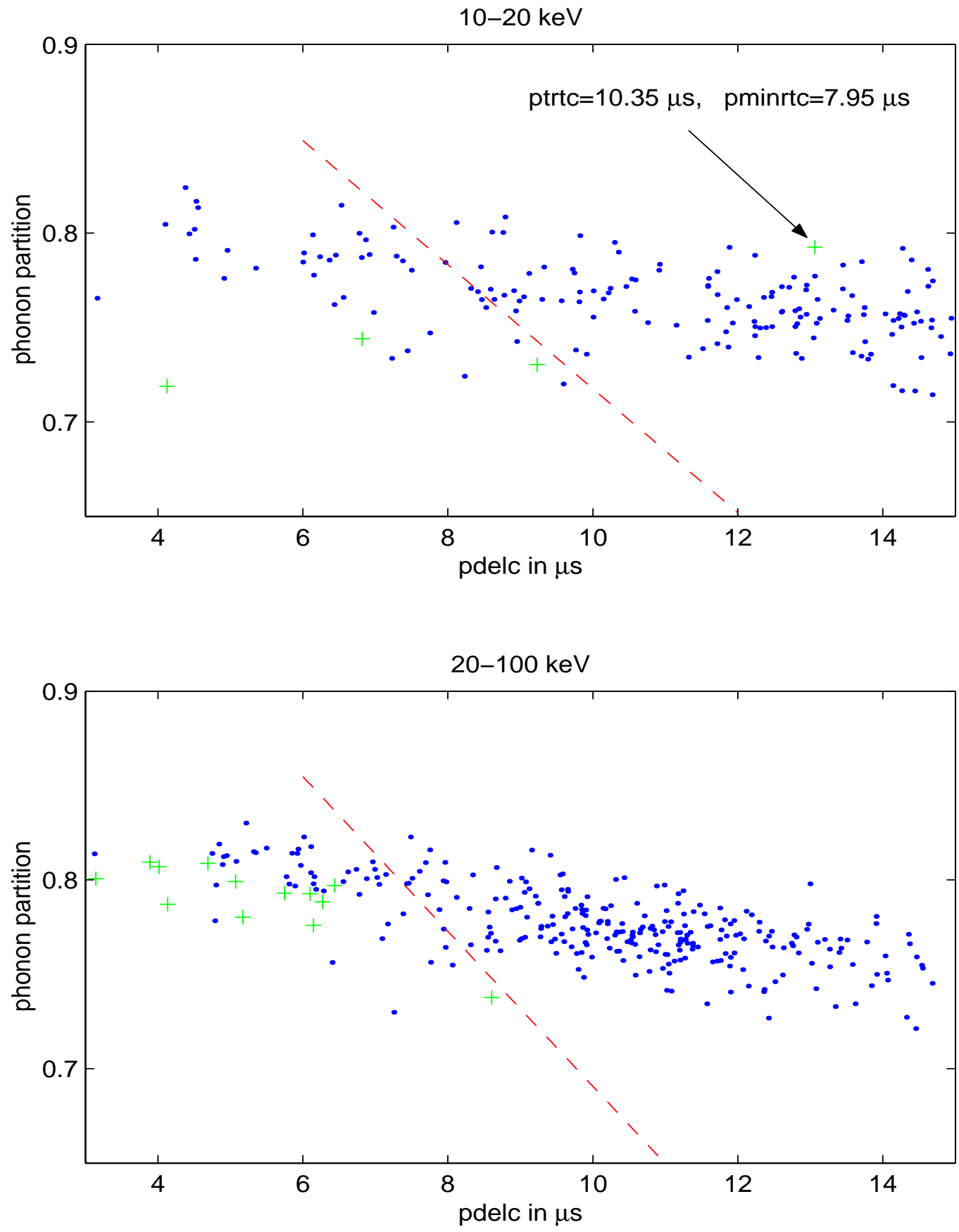

Figure 7.24: Z5, phonon partition distribution as a function of pdelc. The dashed red line is the surface event cut line; events below this line are supposed to be surface interactions. Blue dots are neutrons, green crosses are ejectrons. All events have radius $R>2.7 \mathrm{~cm}$. Only ejectrons within the $3 \sigma \mathrm{NR}$ band are shown. 


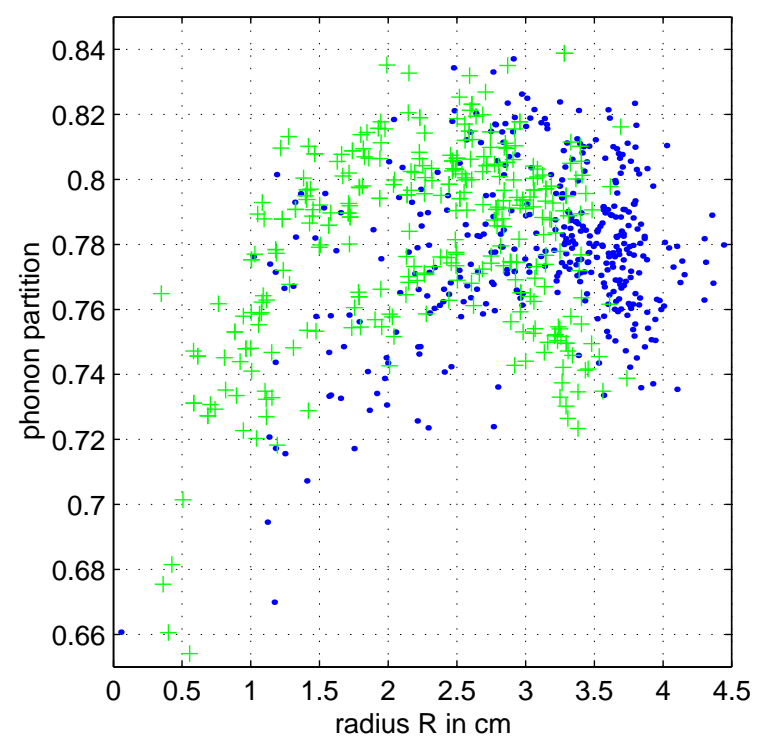

Figure 7.25: Phonon partition as a function of radius R. Blue dots are neutrons, green crosses are ejectrons. ptrtc $>11 \mu s$ for neutrons.

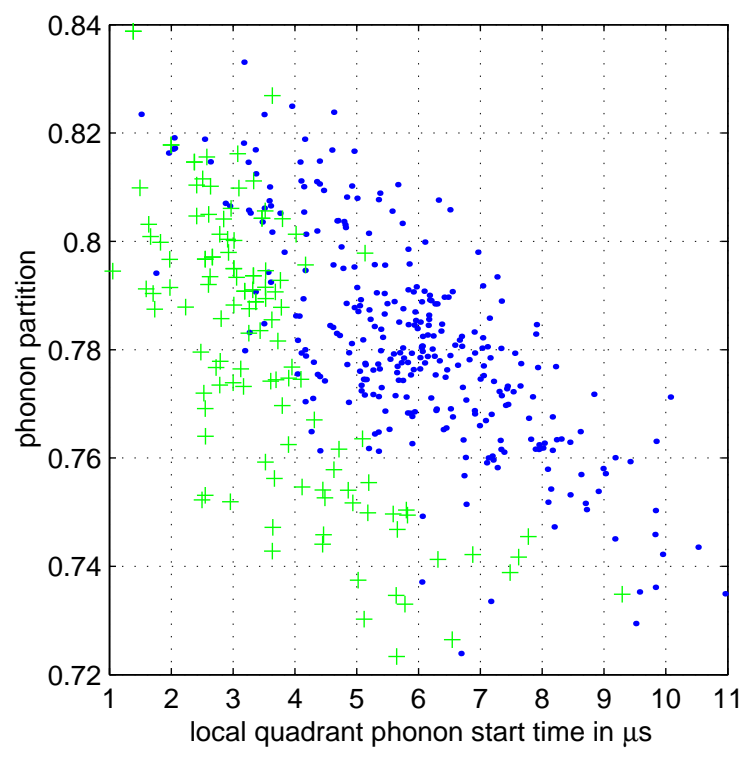

Figure 7.26: Phonon partition as a function of local quadrant phonon start time. Blue dots are neutrons, green crosses are ejectrons. All events have radius parameter $R>2.7 \mathrm{~cm}$. ptrtc $>11 \mu \mathrm{s}$ for neutrons. 


\subsection{Position Based WIMP Search Data Analysis}

As described in section 7.4, there is a separation between nuclear recoils and electron recoils in the phonon partition versus phonon delay two dimensional plot for events that have a big radius. So, in low background data analysis, it is ideal to separate all the events in the CDMS ZIP detector into two categories: events with radius $R \leq R_{0}$ and events with $R>R_{0}$. The following position information based on WIMP search data analysis uses this strategy. Events that have a small radius are subjected to phonon timing parameter cuts only, while events that have a big radius are subjected to phonon timing parameter cuts and phonon partition cuts.

Because the phonon partitions are not well separated between nuclear recoils and the electron recoils for events with a small radius, timing parameters are used for background reduction for the events in this category. The second reason for using timing parameters only in this category is that the timing parameters are well behaved for all events that have a small radius (see section 7.4 for details). Events that have phonon total rise time $p t r t c>R T 1$ (the average phonon rise time of ejectrons in the nuclear recoil band), the minimum rise time pminrtc $>R T 2$ in the maximum phonon pulse, and phonon delay pdelc $>R T 3$ are selected as nuclear recoils. $R T 3$ can be energy dependent. These cuts are set by looking at neutrons in the ${ }^{252} \mathrm{Cf}$ calibration data and ejectrons from the ${ }^{133} \mathrm{Ba}$ calibration data with two rules: $R T 3$ is optimized with quality factor $\mathrm{Q}$ minimum value search as described in section 7.3; ejectrons (in the ${ }^{133} \mathrm{Ba}$ calibration data from December 9, 2003 to December 16, 2003) that are in the $2 \sigma$ nuclear recoil band are not allowed to pass the timing cuts. Table 7.1 has the values of $R_{0}$ and details of the timing cut parameters for all six CDMS ZIP detectors of Tower I in Soudan 
run118.

\begin{tabular}{|c|c|c|c|c|c|c|}
\hline \hline parameter & Z1 & Z2 & Z3 & Z4 & Z5 & Z6 \\
\hline$R_{0}(\mathrm{~cm})$ & 2.5 & 2.7 & 2.7 & 2.5 & 2.7 & 2.5 \\
$R T 1(\mu s)$ & 11.0 & 11.4 & 11.0 & 5.5 & 11.2 & 5.8 \\
$R T 2(\mu s)$ & 8.2 & 8.2 & 8.2 & 9.0 & 8.2 & 9.0 \\
$a$ & 6.8 & 6.8 & 8.0 & 6.5 & 8.0 & 6.8 \\
$b$ & 0 & 0 & 0.01 & 0 & 0.012 & 0 \\
\hline$R T 3$ & \multicolumn{5}{|c|}{$a-b \cdot p r c$} \\
\hline
\end{tabular}

Table 7.1: Surface event rejection parameters for events having a small radius. $R_{0}$ is the small radius event selection parameter. a and $\mathrm{b}$ form a third timing parameter $R T 3=a-b \cdot p r c$, where prc is recoil energy in $\mathrm{keV}$. Assume that bulk nuclear interactions have ptrtc $>R T 1$, pminrtc $>R T 2$, and pdelc $>R T 3$.

The nuclear recoils and electron recoils are well separated in the phonon partition versus phonon delay pdelc in the big radius category. Both phonon partition and timing parameters are used for effective surface event rejection in this category. Because low recoil energy surface events could have an extremely long phonon delay at a big radius, events that have a big radius are divided into two classes: small energy class and big energy class. Events in the small energy class have recoil energy $E_{r} \leq E_{0}$. Events in the big energy class have recoil energy $E_{r}>E_{0} . E_{0}$ is in Table 7.2 for all six detectors. Events that have phonon total rise time ptrtc $>R T 1$, the minimum phonon pulse rise time pminrtc $>R T 2$, and phonon partition $>P A R T 1$ are selected as nuclear recoils in the big energy class. Equation 7.2 defines phonon partition. Events that have phonon total rise time ptrtc $>R T 1$, the minimum rise time pminrtc $>R T 2$, and phonon partition $>P A R T 2$ are selected as nuclear interactions in the small energy class.

These cuts have been selected in such a way that ejectrons in the $2 \sigma$ nuclear recoil band in ${ }^{133} \mathrm{Ba}$ calibration data (from December 9, 2003 to December 16, 2003) cannot pass the timing cuts and phonon partition cuts in all germanium 


\begin{tabular}{|c|c|c|c|c|c|c|}
\hline \hline parameter & $\mathrm{Z} 1$ & $\mathrm{Z} 2$ & $\mathrm{Z} 3$ & $\mathrm{Z} 4$ & $\mathrm{Z} 5$ & $\mathrm{Z} 6$ \\
\hline$R_{0}(\mathrm{~cm})$ & 2.5 & 2.7 & 2.7 & 2.5 & 2.7 & 2.5 \\
$E_{0}(\mathrm{keV})$ & 30 & 20 & 20 & 30 & 20 & 30 \\
$R T 1(\mu s)$ & 11.0 & 11.4 & 11.0 & 5.5 & 11.2 & 5.8 \\
$R T 2(\mu s)$ & 8.2 & 8.2 & 8.2 & 9.0 & 8.2 & 9.0 \\
$c$ & 1.1121 & 1.1050 & 1.1173 & 1.1664 & 1.1007 & 1.2750 \\
$d$ & 0.0536 & 0.5000 & 0.0467 & 0.0607 & 0.0410 & 0.0750 \\
$e$ & 0.9288 & 0.9482 & 0.9461 & 0.9884 & 1.0461 & 1.120 \\
$f$ & 0.0240 & 0.0255 & 0.0179 & 0.0320 & 0.0329 & 0.0500 \\
\hline PART1 & \multicolumn{7}{|c|}{$c-d \cdot$ pdelc } \\
PART2 & \multicolumn{7}{|c|}{ pdelc } \\
\hline
\end{tabular}

Table 7.2: Surface event rejection parameters for events having a big radius. $R_{0}$ is the big radius event selection parameter. Events that have recoil energy $E r>E_{0}$ are in the big energy class. c and d form phonon partition cut parameter $P A R T 1=c-d \cdot$ pdelc, where pdelc is phonon delay in $\mu s$. Nuclear interactions in the big energy class must have ptrtc $>$ RT1, pminrtc $>$ RT2, and partition $>$ $P A R T 1$. Events that have recoil energy $E r \leq E_{0}$ are in the small energy class. e and $\mathrm{f}$ form phonon partition cut parameter $P A R T 2=e-f \cdot$ pdelc. Nuclear interactions in the small energy class must have ptrtc $>R T 1$, pminrtc $>R T 2$, and partition $>P A R T 2$.

detectors. Ejectrons in the $3 \sigma$ NR band and the searched optimized value of pdelc with the method in section 7.3 have been also used for choosing the cut lines. Figures 7.23 and 7.24 show the details for Z5.

The two silicon detectors require pminrtc $>9 \mu \mathrm{s}$. The timing cuts are more important in silicon detectors. The phonon partition cut is not as good for silicon detectors as for germanium detectors. There are three possible reasons for this. First, the phonon energy collection efficiency in silicon detectors is not as good as in germanium detectors due to the old long aluminum fin structure for trapping phonons (significant quasi-particle loss). Second, phonons are generally fast in silicon. Finally the impurity density could be higher in the silicon detectors. The surface rejection cuts for silicon detectors have been selected by balancing the ejectron rejection efficiency and neutron selection efficiency in the $2 \sigma$ nuclear 
recoil band.

The purpose of requiring ptrtc $>R T 1$ and pminrtc $>R T 2$ is to remove the erratic surface events in one way or the other. It only has a limited effect on neutron selection efficiency. Also, RT1 and $R T 2$ cuts come from my first position based data analysis algorithm [131].

For all events in the big radius category, the surafce rejection cut parameter RT1, RT2, PART1, and PART2 are in Table 7.2 for all six detectors.

Only the lower half $(y<0$ or $y d e l<0)$ of the Z6 low background data is analyzed in this position information based data analysis. The reason is explained in reference [132]. Briefly, the ejectrons in the upper half $(y>0$ or ydel $>0)$ have a low yield and a big phonon rise time. These are the indications that there was a neutralization problem or position correction problem in the area.

For clarity, the surface event rejection method based on event location information can be reorganized as followings:

1. Events that have radius $R \leq R_{0}$ are in the small radius category. The surface rejection timing parameters are $R T 1, R T 2$, and $R T 3$, as listed in Table 7.1.

2. Events that have radius $R>R_{0}$ are in the big radius category.

- Events that have recoil energy $E r>E_{0}$ are in the big energy class. The surface rejection timing and phonon partition parameters are $R T 1$, $R T 2$, and $P A R T 1$, as listed in Table 7.2.

- Events that have recoil energy $E r \leq E_{0}$ are in the small energy class. The surface rejection timing and phonon partition parameters are $R T 1$, $R T 2$, and $P A R T 2$, as listed in Table 7.2. 
In this position based WIMP search data analysis, the applied data quality cuts are:

1. cGoodEvS. Cut off bad events based on pulse trace quality, baseline noise, charge bias issues, and noise issues.

2. cQinS. Select events in the ZIP detector fiducial volume.

3. cQThresS. Select events above the charge energy threshold.

4. Veto anti-coincident. Remove muon coincident events.

5. Recoil energy: $10 \mathrm{keV}$ to $100 \mathrm{keV}$.

Figure 7.23 shows the surface event rejection cut lines for events having radius parameter $R>2.7 \mathrm{~cm}$ in Z5. The left graph in Figure 7.28 shows the surface event rejection cut line for events having radius parameter $R \leq 2.7 \mathrm{~cm}$ in $\mathrm{Z} 5$.

\begin{tabular}{|c|c|c|c|c|c|c|}
\hline \hline $\begin{array}{c}\mathrm{Er} \\
(\mathrm{keV})\end{array}$ & $\begin{array}{c}\mathrm{R} \\
\mathrm{cm}\end{array}$ & yc & $\begin{array}{c}\text { pdelc } \\
\mu s\end{array}$ & $\begin{array}{c}\text { pminrtc } \\
\mu s\end{array}$ & $\begin{array}{c}\text { ptrtc } \\
\mu s\end{array}$ & phonon partition \\
\hline 16.3 & 4.69 & 0.146 & 1.4 & 8.3 & 10.4 & 0.836 \\
\hline 12.3 & 3.33 & 0.303 & 8.9 & 9.5 & 12.4 & 0.744 \\
\hline 34.6 & 3.30 & 0.269 & 5.2 & 8.7 & 11.2 & 0.813 \\
\hline 15.8 & 2.85 & 0.210 & 0.1 & 8.7 & 11.3 & 0.820 \\
\hline 35.9 & 2.15 & 0.264 & 5.2 & 8.8 & 11.7 & 0.813 \\
\hline 33.9 & 0.75 & 0.312 & 4.9 & 9.2 & 12.5 & 0.661 \\
\hline
\end{tabular}

Table 7.3: Anti-coincident single scattering events in Z5.

In the WIMP search data, we are looking for muon anti-coincident single scattering nuclear recoils as WIMP candidates. There are six anti-coincident single scattering events in the nuclear recoil band in Z5, as listed in Table 7.3. The 16.3 $\mathrm{keV}$ event has phonon delay pdelc $=1.4 \mu \mathrm{s}$, and the $15.8 \mathrm{keV}$ event has phonon delay $p d e l c=0.1 \mu s$. These two events are surface electron recoils because of their 
extremely fast phonon time (they are outer side of the top graph in Figure 7.27). The $12.3 \mathrm{keV}$ event (SeriesNumber $=1401061732$, Event Number $=100246$, pdelc $=8.9 \mu s$, pminrtc $=9.5 \mu s$, ptrtc $=12.4 \mu s)$ is the blue dot in the top graph in Figure 7.27. It is below the surface event cut line, which was set to reject all the ejectrons that fall in the $2 \sigma$ nuclear recoil band. The $34.6 \mathrm{keV}$ event is the blue dot in the bottom graph in Figure 7.27. It is far below the surface event cut line. Both the $35.9 \mathrm{keV}$ event and the $33.9 \mathrm{keV}$ event have radius parameter $R \leq 2.7 \mathrm{~cm}$; these two events are far below the surface event cut line, as shown in the right graph in Figure 7.28.

Figure 7.29 gives the neutron selection efficiency and surface event rejection efficiency with surface event rejection cuts for Z5.

The green circles in Figure 7.27 and in the right graph of Figure 7.28 are anticoincident multiple scattering events. The red square indicates ptrtc $<R T 1$ and pminrtc $<R T 2$.

Appendix B has the details of the surface event rejection cut settings for electron rejection, nuclear recoil selection efficiencies, the surface event rejection efficiencies, and anti-coincident single scattering events and multiple scattering events in the $2 \sigma$ nuclear recoil band for the six CDMS ZIP detectors in Soudan run118 WIMP search data.

The bottom line is that there are no muon-anticoincident single scattering nuclear recoils above $10 \mathrm{keV}$ energy threshold and below $100 \mathrm{keV}$ in CDMS II Soudan run118.

There are five double scattering events that passed the surface event rejection cuts. They appear to be nuclear recoils in one detector, but the double scattering partners of these five events failed the surface event rejection cuts in at least one way in the other detector. They are listed in Table 7.4. For example, event 1 


\begin{tabular}{|c|c|c|c|c|c|c|c|c|}
\hline \hline Event & SeriesNumber & EventNumber & detector & $\begin{array}{c}\text { Er } \\
(\mathrm{keV})\end{array}$ & yc & $\begin{array}{c}\text { pdelc } \\
\mu s\end{array}$ & $\begin{array}{c}\text { pminrtc } \\
\mu s\end{array}$ & $\begin{array}{c}\text { ptrtc } \\
\mu s\end{array}$ \\
\hline 1 & 1312211915 & 30209 & $\mathrm{Z} 4$ & 20.3 & 0.27 & 6.94 & 9.17 & 5.67 \\
& & & $\mathrm{Z} 5$ & 77.9 & 0.55 & 4.22 & 8.28 & 10.59 \\
\hline 2 & 1311160954 & 50026 & $\mathrm{Z} 4$ & 37.3 & 0.27 & 6.30 & 9.84 & 5.57 \\
& & & $\mathrm{Z} 3$ & 12.8 & 0.42 & 6.96 & 9.07 & 12.3 \\
\hline 3 & 1310241749 & 50266 & $\mathrm{Z} 5$ & 10.3 & 0.37 & 10.7 & 9.60 & 15.2 \\
& & & $\mathrm{Z} 6$ & 161 & 0.98 & 5.82 & 8.56 & 5.89 \\
\hline 4 & 1312041736 & 60317 & $\mathrm{Z} 6$ & 25.5 & 0.23 & 8.05 & 10.0 & 5.94 \\
& & & $\mathrm{Z} 5$ & 91.7 & 0.95 & 8.74 & 8.50 & 11.44 \\
\hline 5 & 1312202011 & \multirow{2}{*}{90254} & $\mathrm{Z} 6$ & 76.7 & 0.38 & 6.53 & 9.45 & 6.20 \\
& & & $\mathrm{Z} 5$ & 125 & 0.98 & 5.50 & 9.20 & 5.5 \\
\hline
\end{tabular}

Table 7.4: Multiple scattering events in position information based low background data analysis.

is selected as a nuclear recoil event in Z4. But this event failed the background reduction cut in ionization yield $y c$, surface event rejection cuts phonon delay pdelc, and phonon rise time ptrtc in Z5. These five events are not nuclear double scattering events. Most likely, they are gammas in one detector, and ejectrons in the other detector. Another possiblity is that they could be muon induced backgrounds; i.e., they are gammas in one detector, and neutrons in the other detector. But we expect to see 1 gamma and neutron combination in 30 neutrons from Monte Carlo simulations, and we see none of the latter.

Figures 7.30 to 7.35 show the anti-coincident single scattering events of low background data in Soudan run118 before and after position based surface event rejection cuts. After the cuts, the high ionization yield electron recoils are rejected uniformly in each detector, and there are no events in the $2 \sigma$ nuclear recoil bands. The event below the $2 \sigma$ NR band in the graph on the right in Figure 7.30 could be a leakage ejectron, because Z1 directly faces the DIB copper in the Tower. The same argument could be true for the event below the $2 \sigma$ NR band in the graph on the right in Figure 7.35. We also know that $\mathrm{Z} 6$ was contaminated with ${ }^{14} \mathrm{C}$, which is a beta source. 

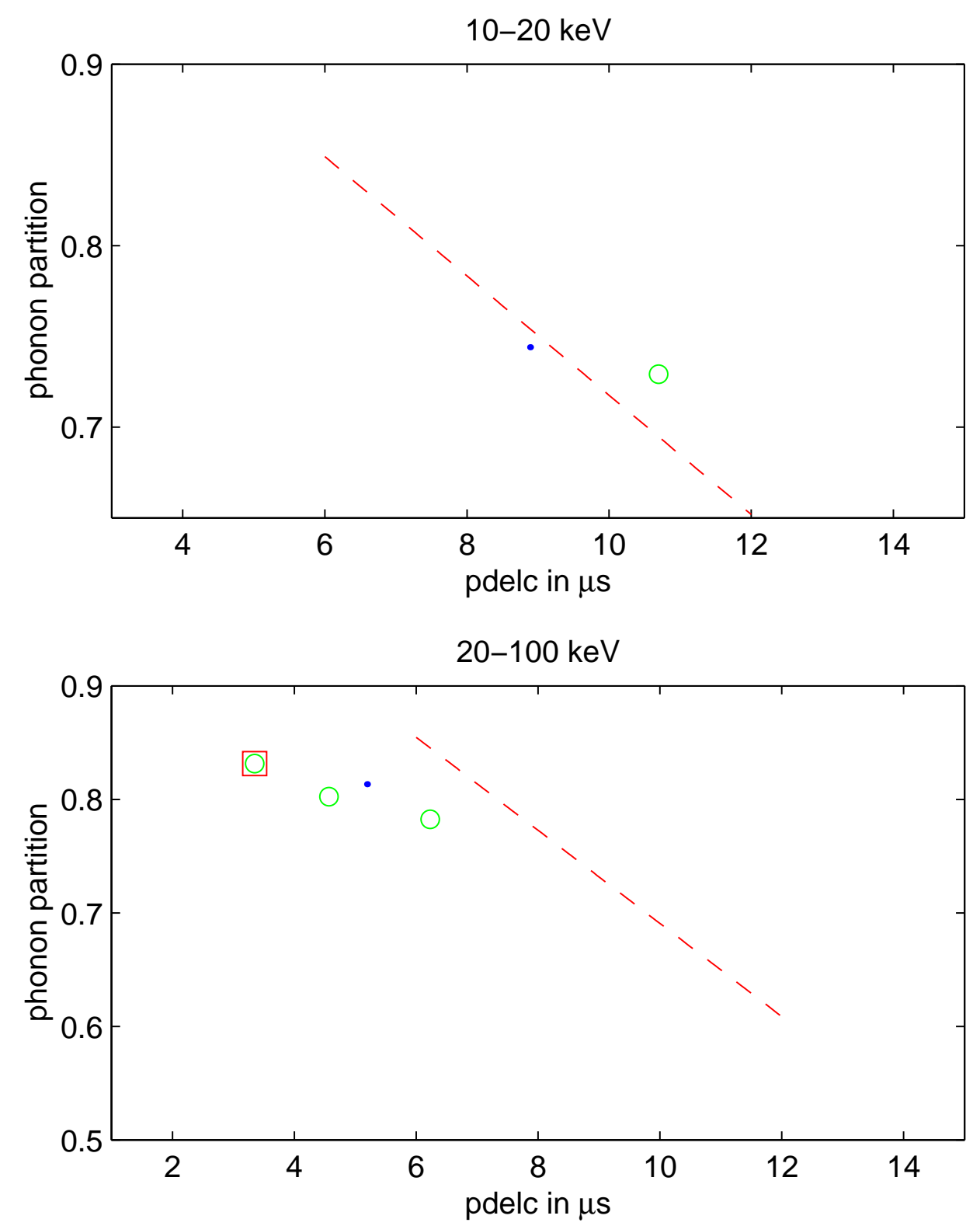

Figure 7.27: Z5. Soudan run118 anti-coincident low background data in the $2 \sigma$ nuclear recoil band with radius $R>2.7 \mathrm{~cm}$. Blue dots are single scatterings, green circles are multiple scatterings. The top plot is for events that have recoil energy $E r \leq 20 \mathrm{keV}$. The bottom plot is for events that have recoil energy $E r>20 \mathrm{keV}$. No single scattering nuclear recoils. 

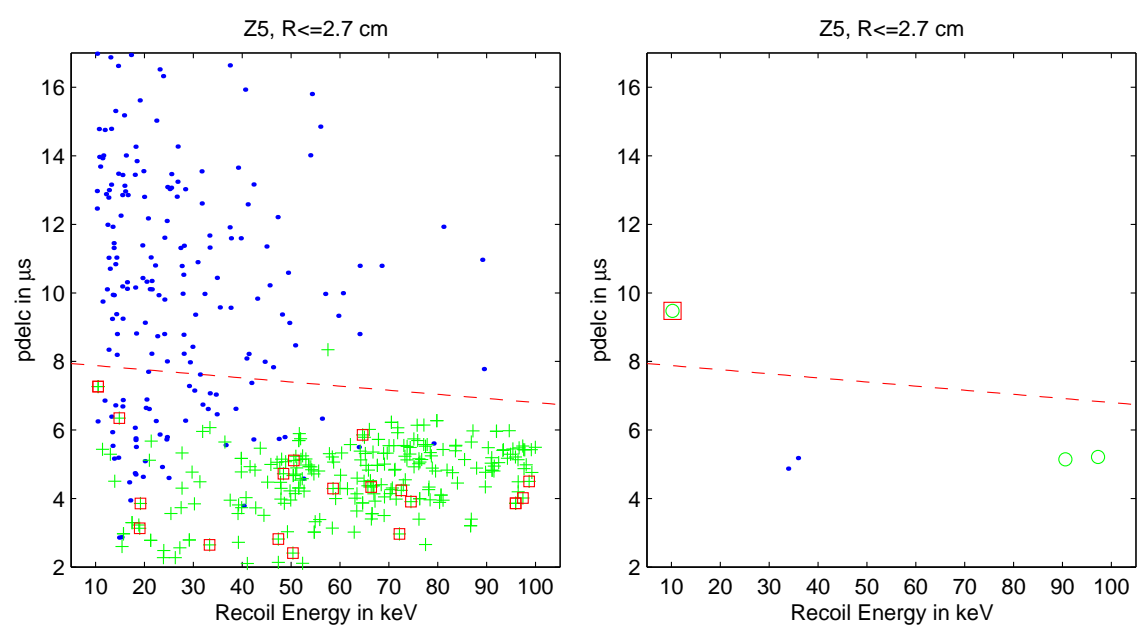

Figure 7.28: Left plot: Z5, phonon delay cut for events with radius $R \leq 2.7 \mathrm{~cm}$. Blue dots are neutrons, green crosses are ejectrons. Red squares are ejectrons that fail RT1 and RT2. The events above the dashed red line are assumed to be nuclear recoils if they pass RT1 and RT2 timing cuts. Right plot: Z5, Soudan run118 anti-coincident low background data in the $2 \sigma$ nuclear recoil band with radius $R \leq 2.7 \mathrm{~cm}$. Blue dots are single scatterings, green circles are multiple scatterings, the square is for events failing RT1 And RT2 cuts. No nuclear recoils.

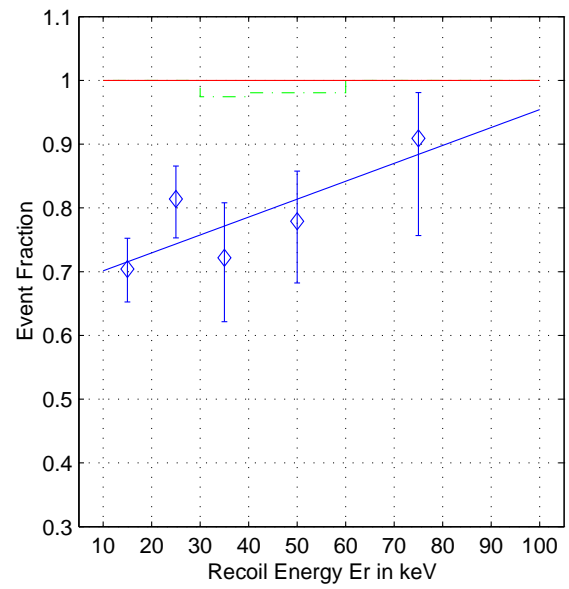

Figure 7.29: Z5, the blue line fitted to the data with error bars is neutron selection efficiency in the ${ }^{252} \mathrm{Cf}$ calibration data. The dashed green line is surface event rejection efficiency in the ${ }^{133} \mathrm{Ba}$ data without an ionization yield cut. The top red line is surface event rejection efficiency in the ${ }^{133} \mathrm{Ba}$ data within the $2 \sigma \mathrm{NR}$ band. 

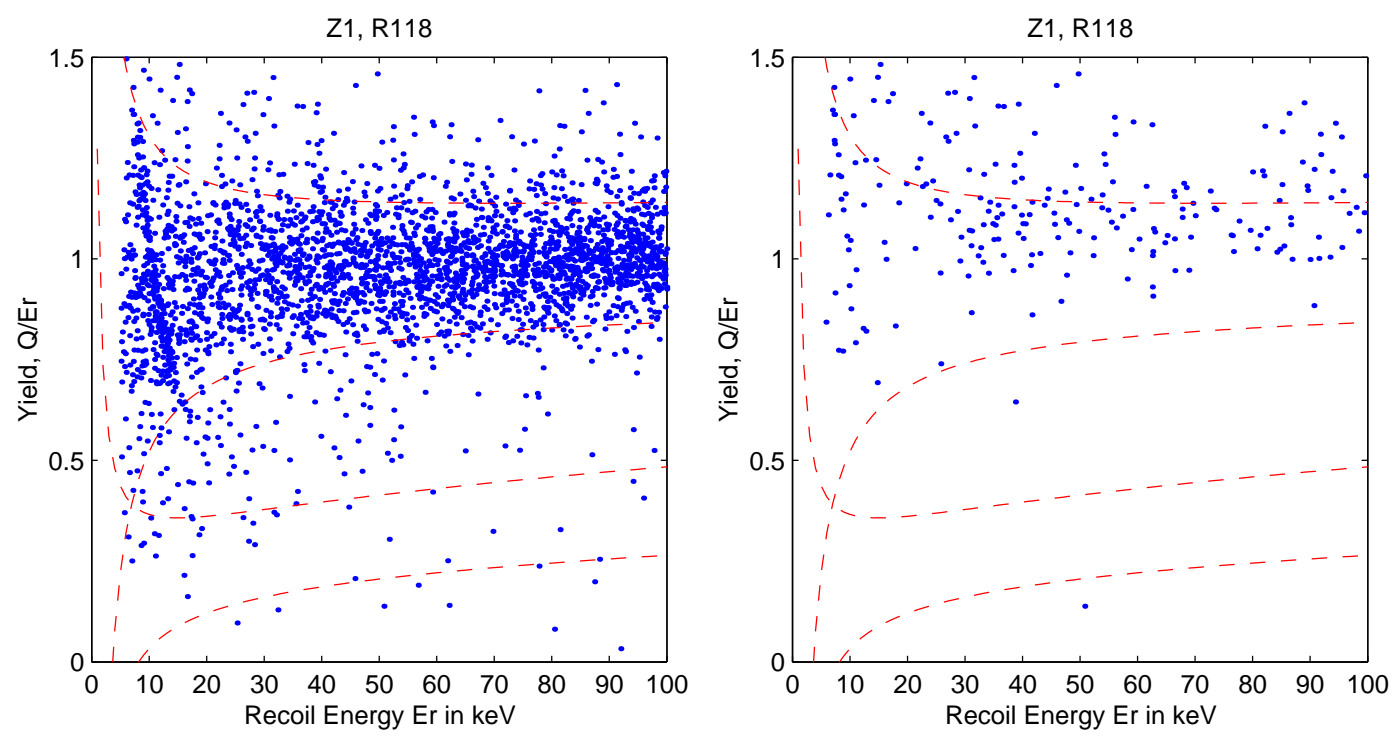

Figure 7.30: Left plot: Z1, Anti-coincident single scattering events before the surface event rejection cuts. Right plot: Z1, Anti-coincident single scattering events after the surface event rejection cuts. No single scattering nuclear recoils.
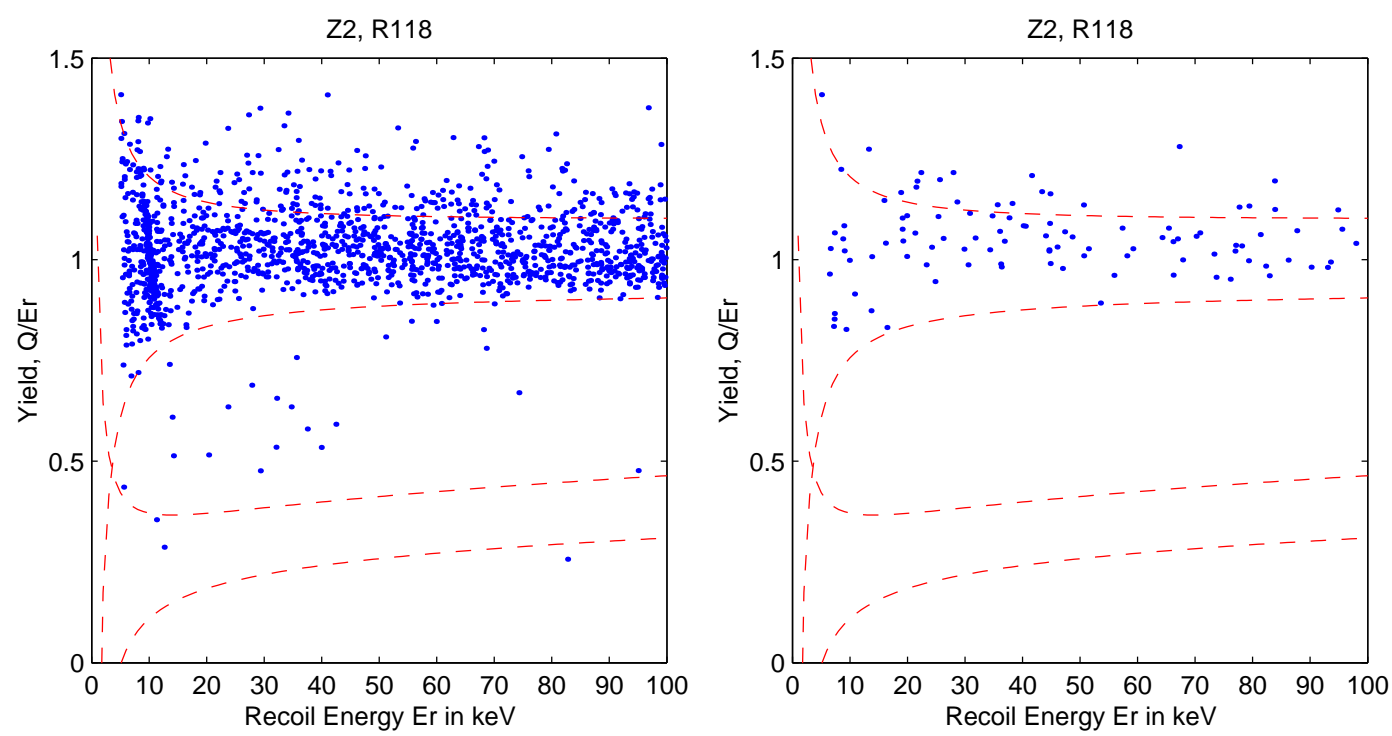

Figure 7.31: Left plot: Z2, Anti-coincident single scattering events before the surface event rejection cuts. Right plot: Z2, Anti-coincident single scattering events after the surface event rejection cuts. No single scattering nuclear recoils. 

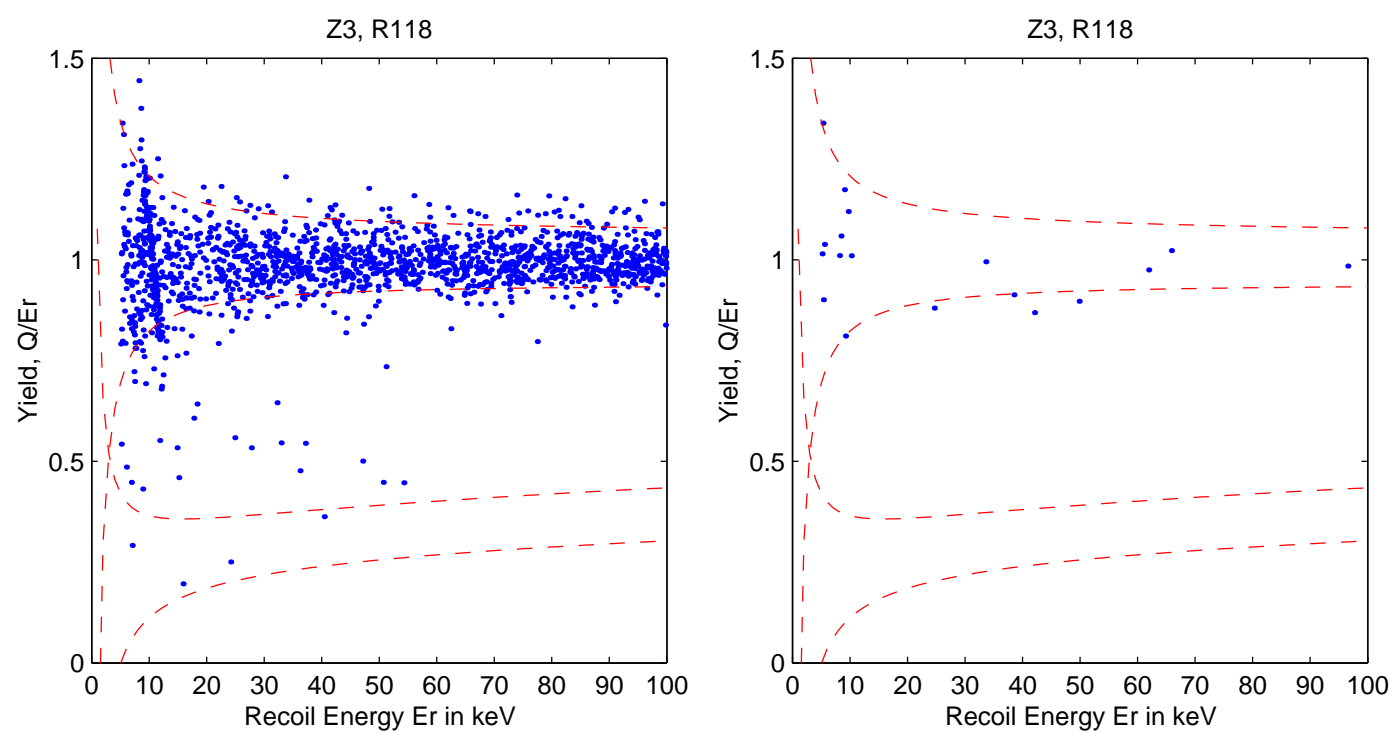

Figure 7.32: Left plot: Z3, Anti-coincident single scattering events before the surface event rejection cuts. Right plot: Z3, Anti-coincident single scattering events after the surface event rejection cuts. No single scattering nuclear recoils.
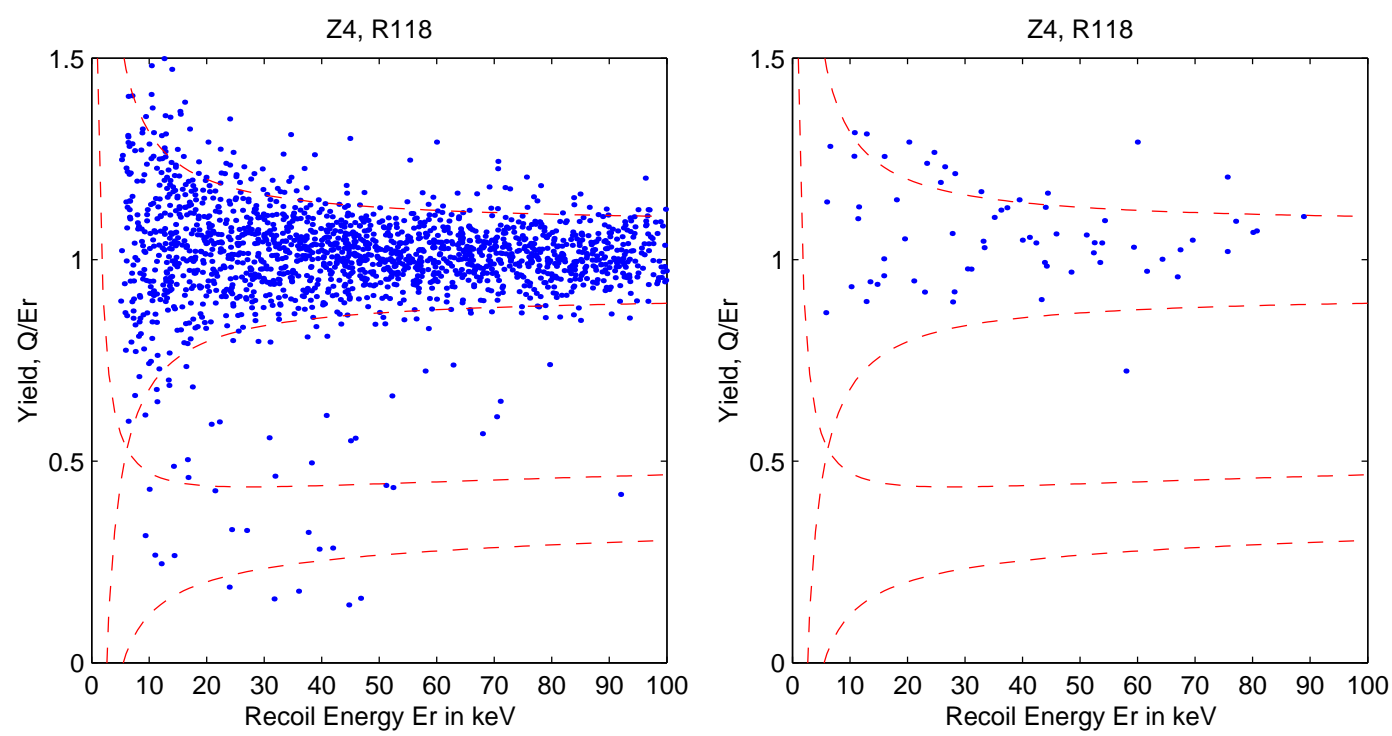

Figure 7.33: Left plot: Z4, Anti-coincident single scattering events before the surface event rejection cuts. Right plot: Z4, Anti-coincident single scattering events after the surface event rejection cuts. No single scattering nuclear recoils. 

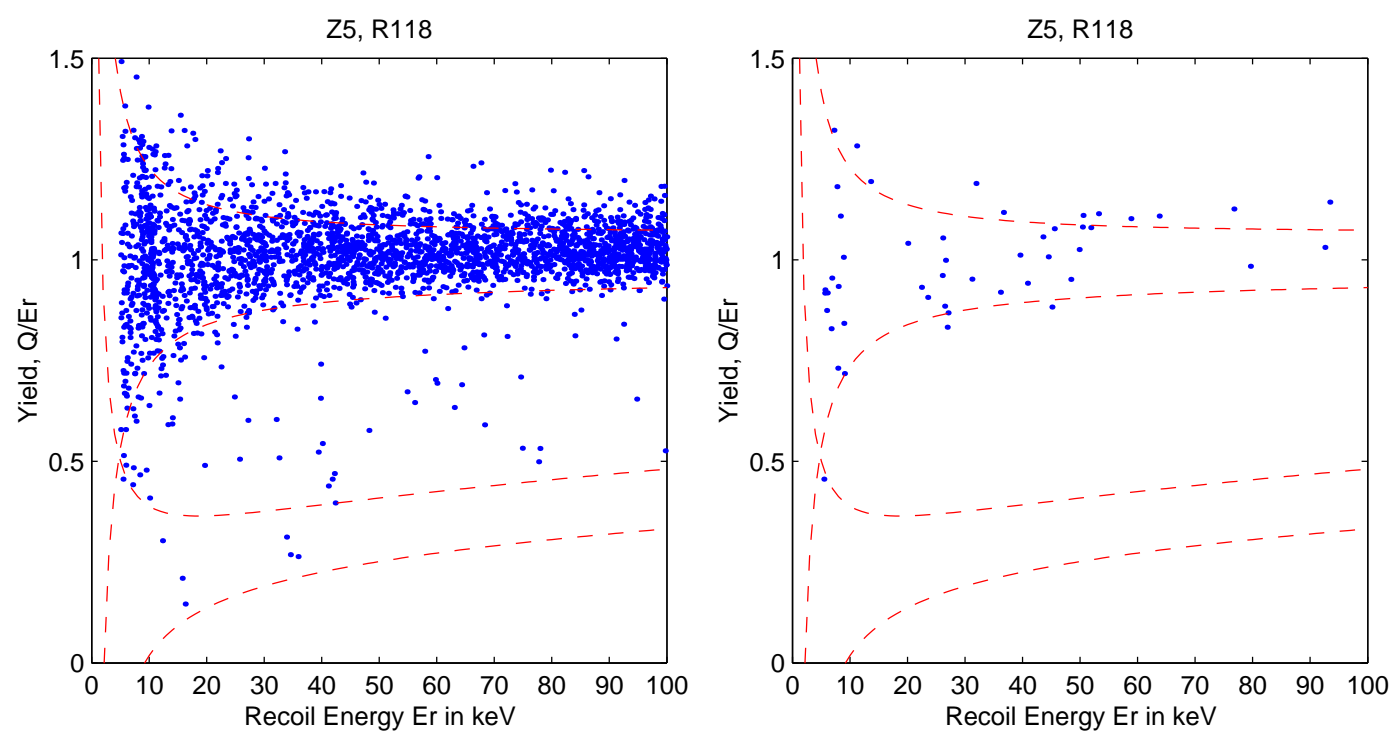

Figure 7.34: Left plot: Z5, Anti-coincident single scattering events before the surface event rejection cuts. Right plot: Z5, Anti-coincident single scattering events after the surface event rejection cuts. No single scattering nuclear recoils.
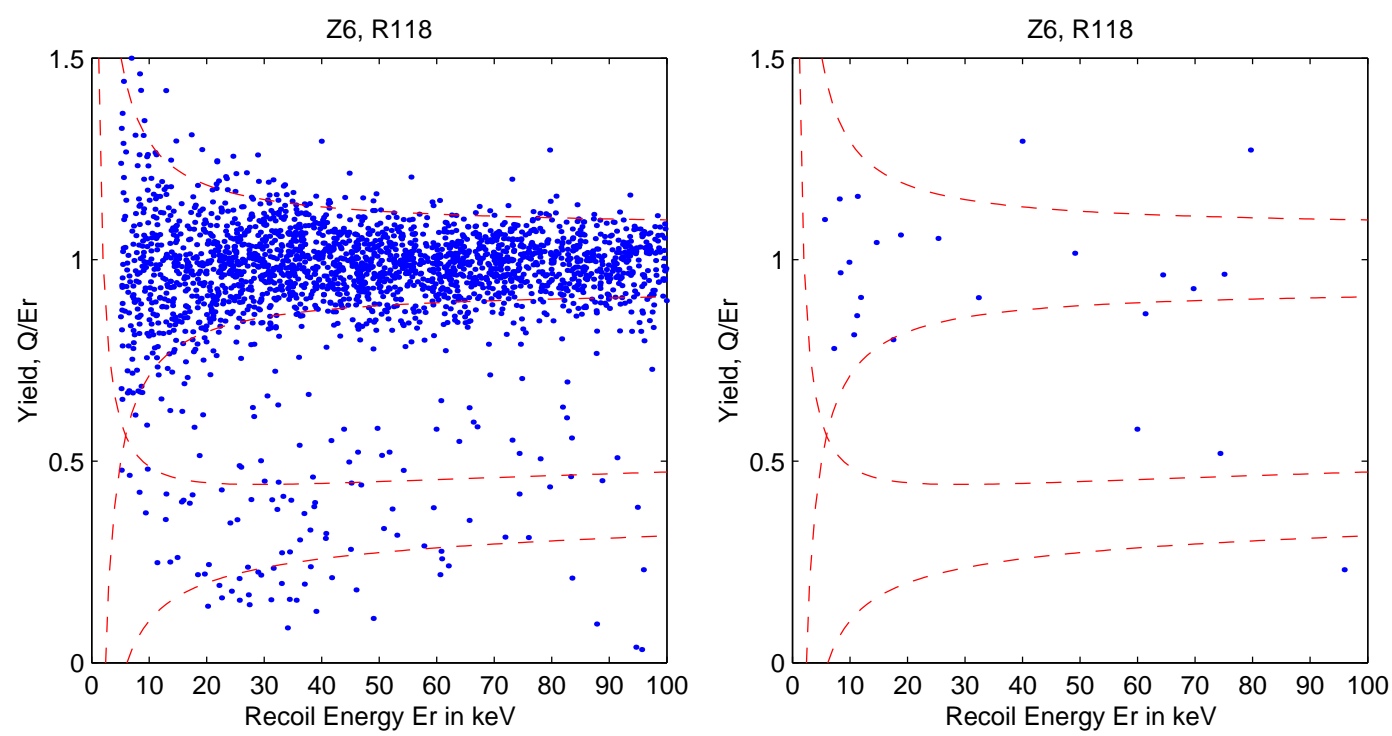

Figure 7.35: Left plot: Z6, Anti-coincident single scattering events before the surface event rejection cuts. Right plot: Z6, Anti-coincident single scattering events after the surface event rejection cuts. No single scattering nuclear recoils. 


\subsection{Blind WIMP Search Data Analysis}

A blind WIMP search data analysis was performed for CDMS run118. The search was blind in the sense that a widened nuclear recoil band was hidden, from view for unvetoed events. Before the low background data blinding was removed on March 20, 2004, the data quality cuts (cGoodEvS), inner elctrode event selection (cQinS), and charge energy threshold (cQThresS) were set with the ${ }^{133} \mathrm{Ba}$ calibration data and the ${ }^{252} \mathrm{Cf}$ calibration data, as described in Chapter 6 , and the surface event rejection cut (cRT2S) on phonon timing parameters was defined by rejecting the ejectrons in the nuclear recoil band.

The surface event rejection cut (cRT2S) was defined by Vuk Mandic [133]. Neutrons in the $2 \sigma$ nuclear recoil band of the ${ }^{252} \mathrm{Cf}$ are selected as nuclear recoils. Ejectrons (nearest neighbor double scatterings below the $3 \sigma$ gamma band and

$y c<0.75)$ in the ${ }^{133} \mathrm{Ba}$ calibration data are selected as surface events. The phonon timing parameter cuts were optimized by minimizing Po90/ $\alpha$ [133] as a reference, and by rejecting all ejectrons in the ${ }^{133} \mathrm{Ba}$ calibration data from December 9, 2003 to December 16, 2003. Figure 7.36 shows the phonon minimum rise time pminrtc cut search and the phonon delay pdelc cut search for Z5 in the 5-10 keV, 10-20 $\mathrm{keV}, 20-40 \mathrm{keV}$, and 40-100 keV energy bins, respectively. Po90 is the $90 \%$ upper limit for Poisson distribution for the given number of betas that survived the cut; $\alpha$ is the neutron selection efficiency on the timing parameter cut.

The phonon timing cut cRT2S for surface event rejection is cut2 in figure 7.36. This includes three parts in the interest energy range below $100 \mathrm{keV}$. These are:

1. Phonon minimum rise time cut

$$
R T 1=a 1 \cdot p r c+b 1
$$



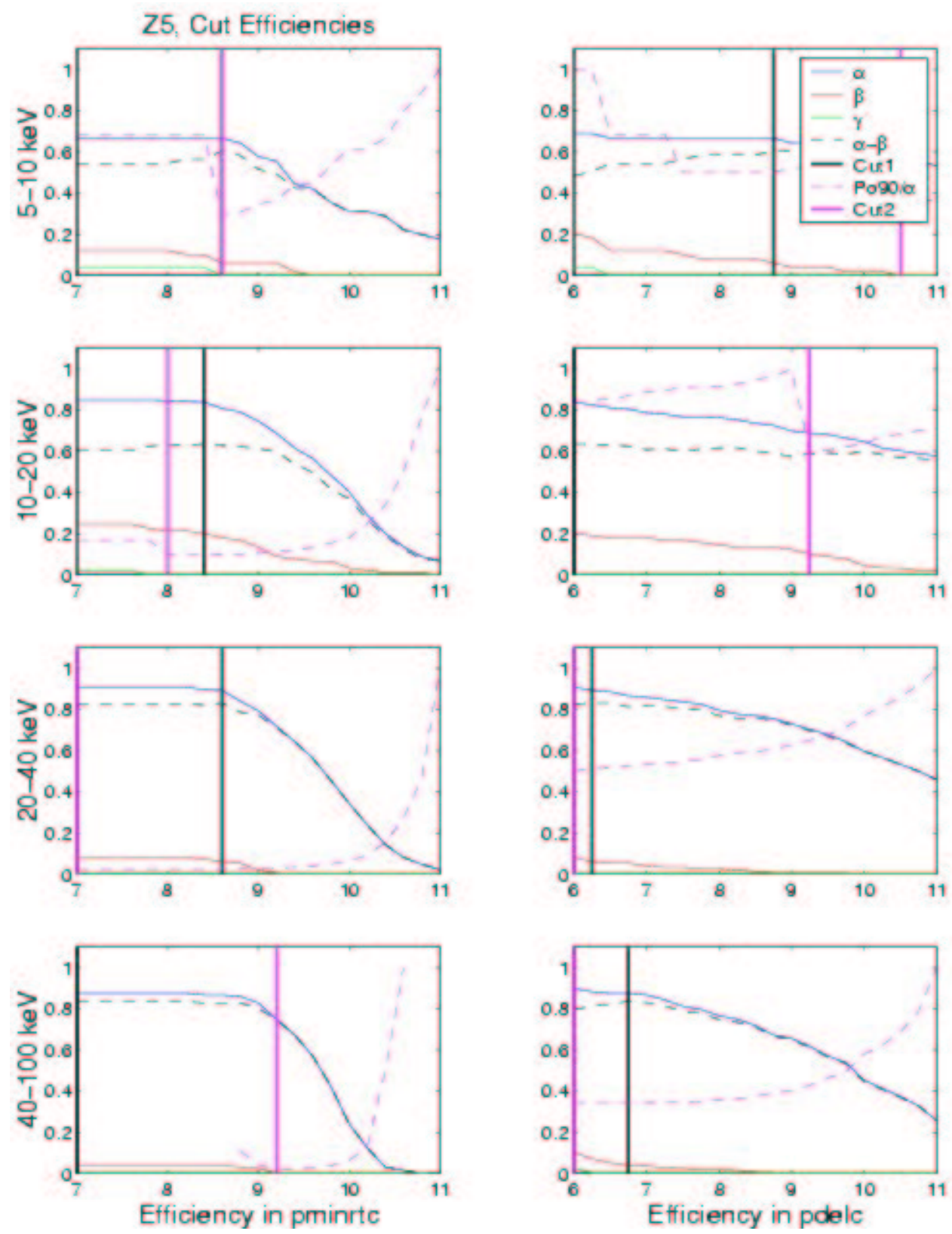

Figure 7.36: Searching phonon timing parameter cuts for surface event rejection. $\alpha$ is the neutron selection fraction, $\beta$ is the surface event selection fraction on timing parameter only, $\gamma$ is the surface event selection on timing parameter and in the nuclear recoil band. cut1 is set by maximizing $\alpha$ - $\beta$, cut2 is set by minimizing Po90/ $\alpha$. Figure from [133]. 


\begin{tabular}{|c|c|c|c|c|c|c|}
\hline \hline parameter & Z1 & Z2 & Z3 & Z4 & Z5 & Z6 \\
\hline$a_{1}$ & 0 & -0.17 & -0.18 & -0.01 & -0.07 & 0 \\
$b_{1}$ & 9.2 & 12.5 & 11.5 & 10.0 & 9.0 & 9.5 \\
$a_{21}$ & 0 & 0 & 0.013 & 0 & 0 & 0 \\
$b_{21}$ & 6.75 & 6.0 & 8.2 & 6.25 & 6.75 & 7 \\
$a_{22}$ & 0 & -0.17 & 0 & 0 & -0.17 & 0 \\
$b_{22}$ & 0 & 10 & 0 & 0 & 12 & 0 \\
\hline
\end{tabular}

Table 7.5: Timing cut parameters in low background data blinding analysis.

where prc is recoil energy in keV. $a_{1}$ and $b_{1}$ are listed in Table 7.5 for Z1 through Z6.

2. Phonon delay cut

$$
R T 21=a 21 \cdot p r c+b 21
$$

where prc is recoil energy in keV. $a_{21}$ and $b_{21}$ are listed in Table 7.5 for Z1 through Z6.

3. Phonon delay at low recoil energy

$$
R T 22=a 22 \cdot \operatorname{prc}+b 22
$$

where prc is recoil energy in keV. $a_{22}$ and $b_{22}$ are listed in Table 7.5 for Z1 through Z6.

The nuclear recoil selection efficiency and surface event rejection efficiency are calculated with ${ }^{252} \mathrm{Cf}$ calibration data 131125_1538, 131219_1447 and 140105_1556, and with ${ }^{133}$ Ba calibration data 131204_1416, 131205_0925, 131209_1153, 131210_1048, 131210_1342, 131211_0920, 131211_1159, 131211_1347, 131212_0904, 131212_1244, 131215_0937, 131215_1419, 131216_0925, 131216_1136, and 140106_1201. The efficiencies are shown in Figure 7.37 for all six ZIP detectors.

Extensive beta leakage research has been done with the surface event rejection timing cuts. Surface events in the background and calibration datasets (outside 
the NR band) are used to estimate the number of (single scatter) surface events that leak into the NR band in the background data. The estimated number of surface events that can leak into the nuclear recoil band for the 4 germanium detectors (Z2, Z3, and Z5 over the 10-100 keV energy range, and Z1 over the 20$100 \mathrm{keV}$ energy range) is 0.21 with uncertainty 0.58 . This leads to the $2 \sigma$ upper bound of the beta leakage of 1.37 events [134].

Figure 7.38 shows the WIMP search data from $5 \mathrm{keV}$ to $100 \mathrm{keV}$ after data quality cuts. After timing cuts, all single scattering events are in Figure 7.39. No single scattering events in the nuclear recoil band pass timing cuts in the four germanium detectors. Four events pass timing cuts in Z6, but these are consistent with the surface event leakage estimation, which is 3.94 events at $2 \sigma$ upper bound [134]. Z6 has problems in quadrant A and D; there is an unusually large number of low yield ejectrons in this area [132]. All four events passing the timing cuts are in the same area between quadrant A and quadrant D (see Figure 7.41). The conclusion is that there are no unvetoed single scatter nuclear recoils in Soudan run118 WIMP search data. The absence of such events will be interpreted as an upper limit on the WIMP-nucleon cross section in Chapter 8.

There are four double scattering events that pass the timing cuts in the nuclear recoil band in one detector (see Figure 7.40). Further study shows that these events are likely leakage electron recoils. The $37.3 \mathrm{keV}$ event in $\mathrm{Z} 4$ corresponds to a $12.8 \mathrm{keV}$ event in $\mathrm{Z} 3$, and fails timing cuts in Z3. Two double scattering events between Z6 and Z5 have a high ionization yield in Z5. They are most likely electron recoils. The high energy gammas knock off electrons from Z5, and the electrons interact with Z6. One double scattering between Z6 and Z3 has high ionization yield in Z3. The series number, event number, recoil energy, ionization yield and timing parameters of these four events are listed in Table 7.7. 


\begin{tabular}{|c|c|c|c|c|c|}
\hline SeriesNumber & EventNumber & $\begin{array}{c}\text { Er } \\
(\mathrm{keV})\end{array}$ & $\begin{array}{c}\text { yc } \\
\text { pdelc } \\
\mu s\end{array}$ & $\begin{array}{c}\text { pminrtc } \\
\mu s\end{array}$ \\
\hline 1311251831 & 90375 & 45.98 & 0.30 & 7.37 & 9.68 \\
1311271212 & 270187 & 13.04 & 0.27 & 7.76 & 10.41 \\
1312031901 & 60434 & 63.17 & 0.39 & 7.36 & 9.94 \\
1401042041 & 90416 & 70.98 & 0.42 & 7.46 & 9.62 \\
\hline
\end{tabular}

Table 7.6: Single scattering events in Z6.

\begin{tabular}{|c|c|c|c|c|c|c|}
\hline SeriesNumber & EventNumber & Detector & $\begin{array}{c}\text { Er } \\
(\mathrm{keV})\end{array}$ & yc & $\begin{array}{c}\text { pdelc } \\
\mu s\end{array}$ & $\begin{array}{c}\text { pminrtc } \\
\mu s\end{array}$ \\
\hline 1311160954 & 50026 & $\mathrm{Z} 4$ & 37.28 & 0.27 & 6.30 & 9.84 \\
1311160954 & 50026 & $\mathrm{Z} 3$ & 12.80 & 0.42 & 6.96 & 9.07 \\
\hline 1310170033 & 20067 & $\mathrm{Z} 6$ & 10.07 & 0.26 & 7.66 & 22.14 \\
1310170033 & 20067 & $\mathrm{Z} 5$ & 393.5 & 1.07 & 4.36 & 8.60 \\
\hline 1312041736 & 60317 & $\mathrm{Z} 6$ & 25.51 & 0.23 & 8.04 & 10.08 \\
1312041736 & 60317 & $\mathrm{Z} 5$ & 917.2 & 0.95 & 8.74 & 8.50 \\
\hline 1312111713 & 120083 & $\mathrm{Z} 6$ & 97.16 & 0.34 & 7.45 & 10.60 \\
1312111713 & 120083 & $\mathrm{Z} 3$ & 87.29 & 0.99 & 5.96 & 8.88 \\
\hline
\end{tabular}

Table 7.7: Multiple scattering events in blinding data analysis. One event in Z4 passes yield and timing cuts, but fails timing cuts in Z3; three events pass yield and timing cuts in Z6, but two of them fail the ionization yield cut in Z5, one of them fails the ionization yield cut in Z3. No multiple scattering nuclear recoil events are seen in WIMP search data. 

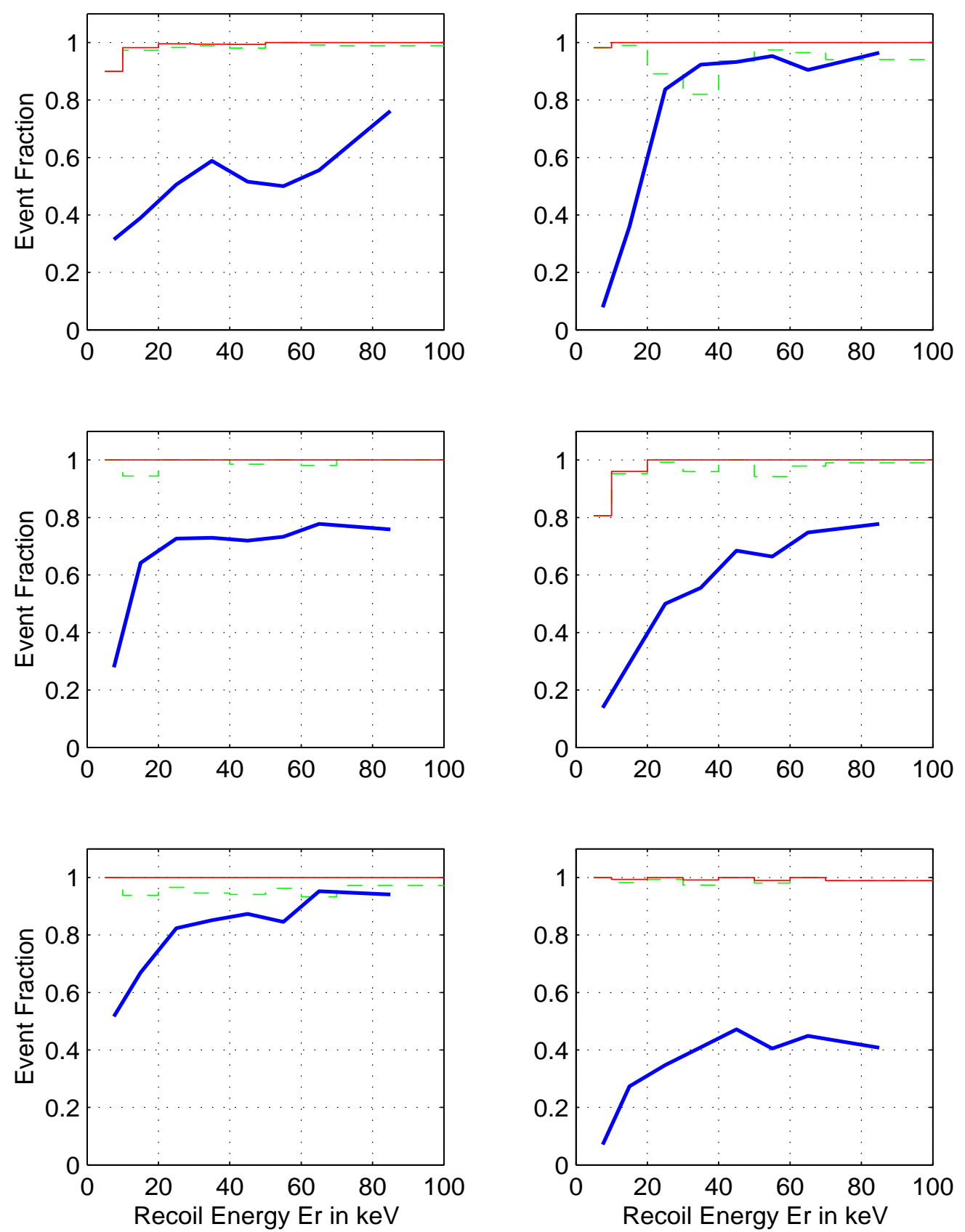

Figure 7.37: Timing cut efficiencies on neutron selection and surface event rejection in blinding low background data analysis. The heavy blue line is neutron selection efficiency, the dashed green line is surface event rejection efficiency with timing cut only, the top red line is surface rejection efficiency with both timing and ionization yield cuts. 

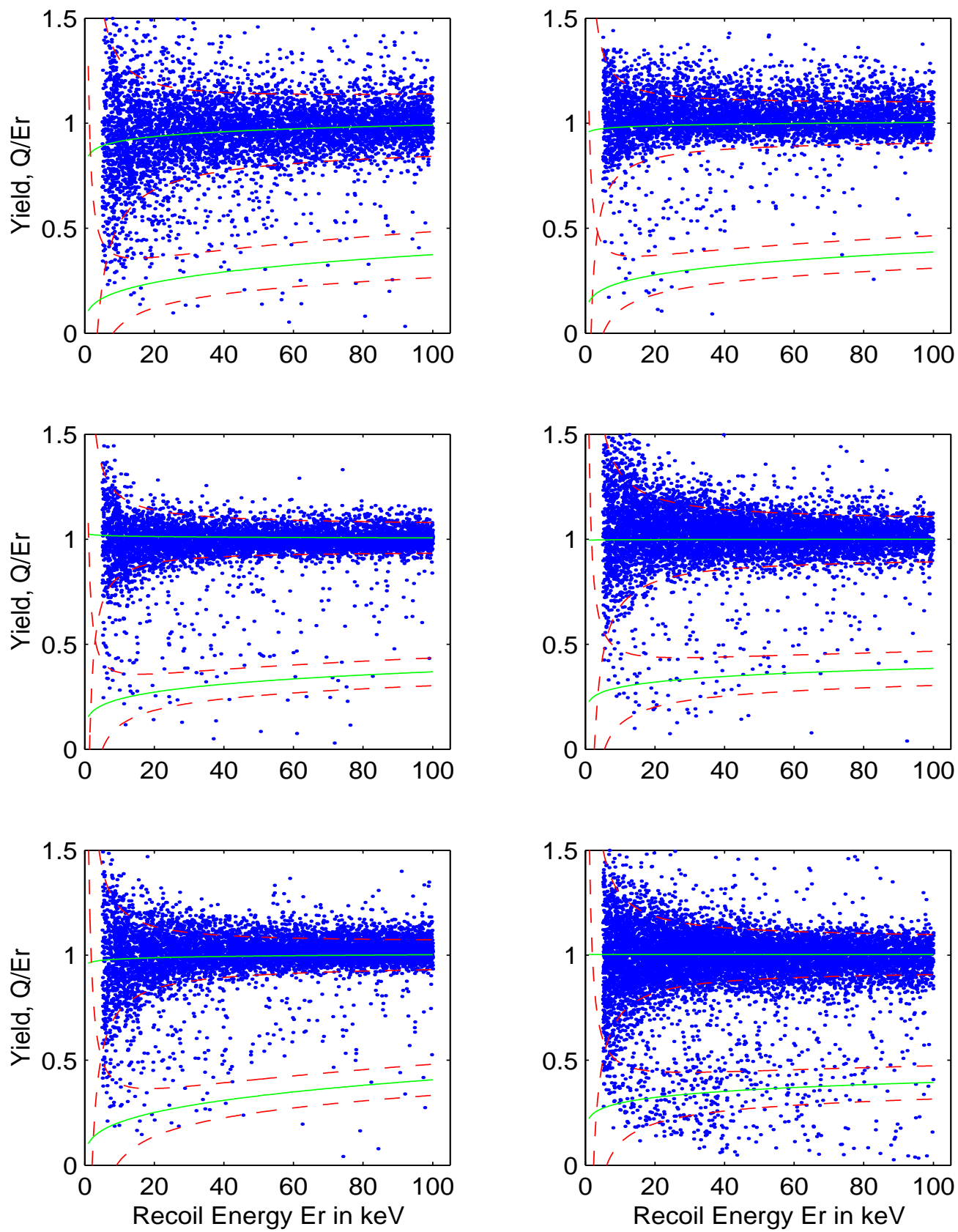

Figure 7.38: Muon anti-coincident WIMP search data from $5 \mathrm{keV}$ to $100 \mathrm{keV}$ before timing cuts. Data quality cuts, such as cGoodEvS, cQinS, and cQThresS, are applied. 

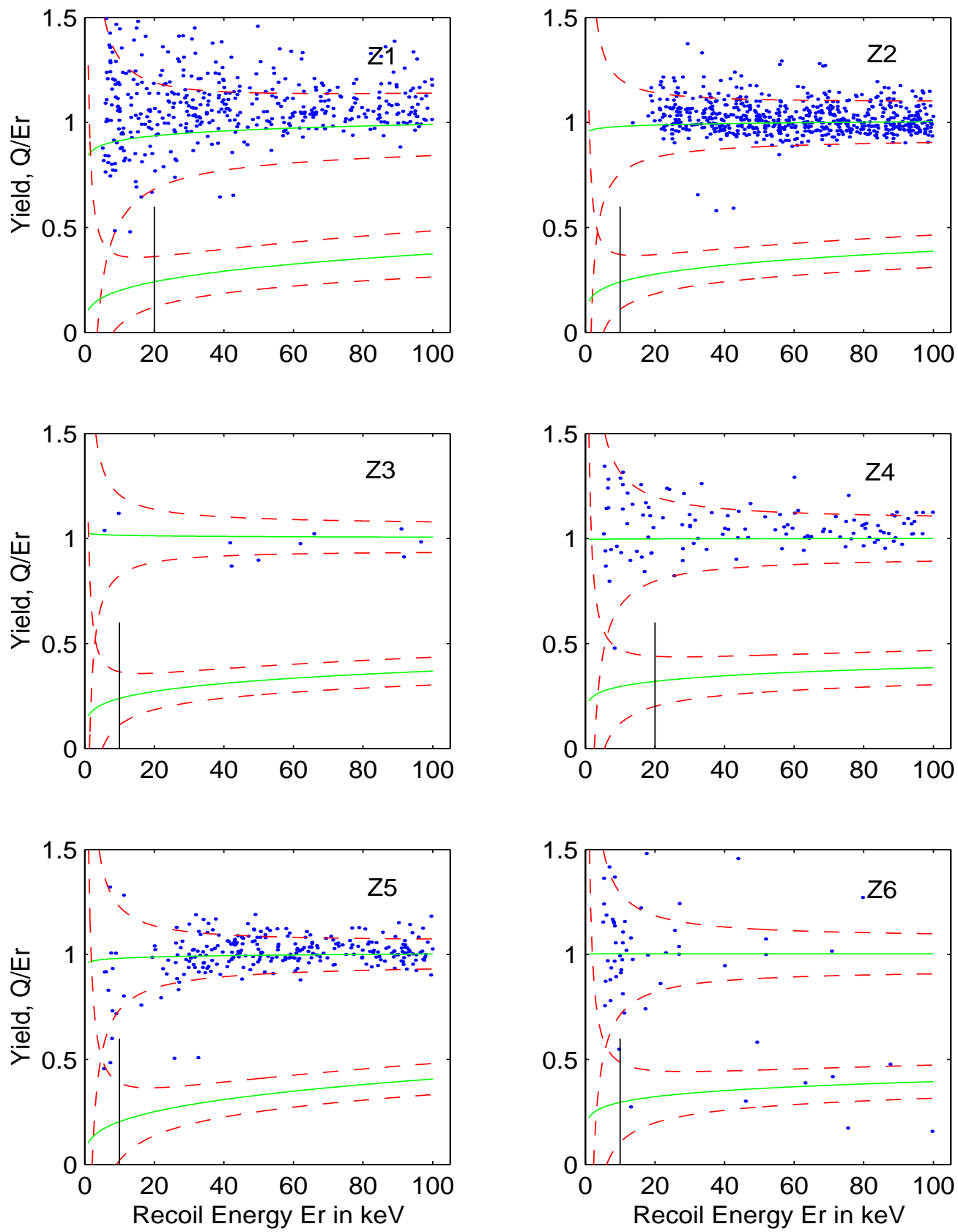

Figure 7.39: Muon anti-coincident single scattering events after timing cuts in blinding analysis. The black bar is the energy threshold. There are no single scattering nuclear recoils in Z1 through Z5; four leakage electron recoils exist in Z6. 

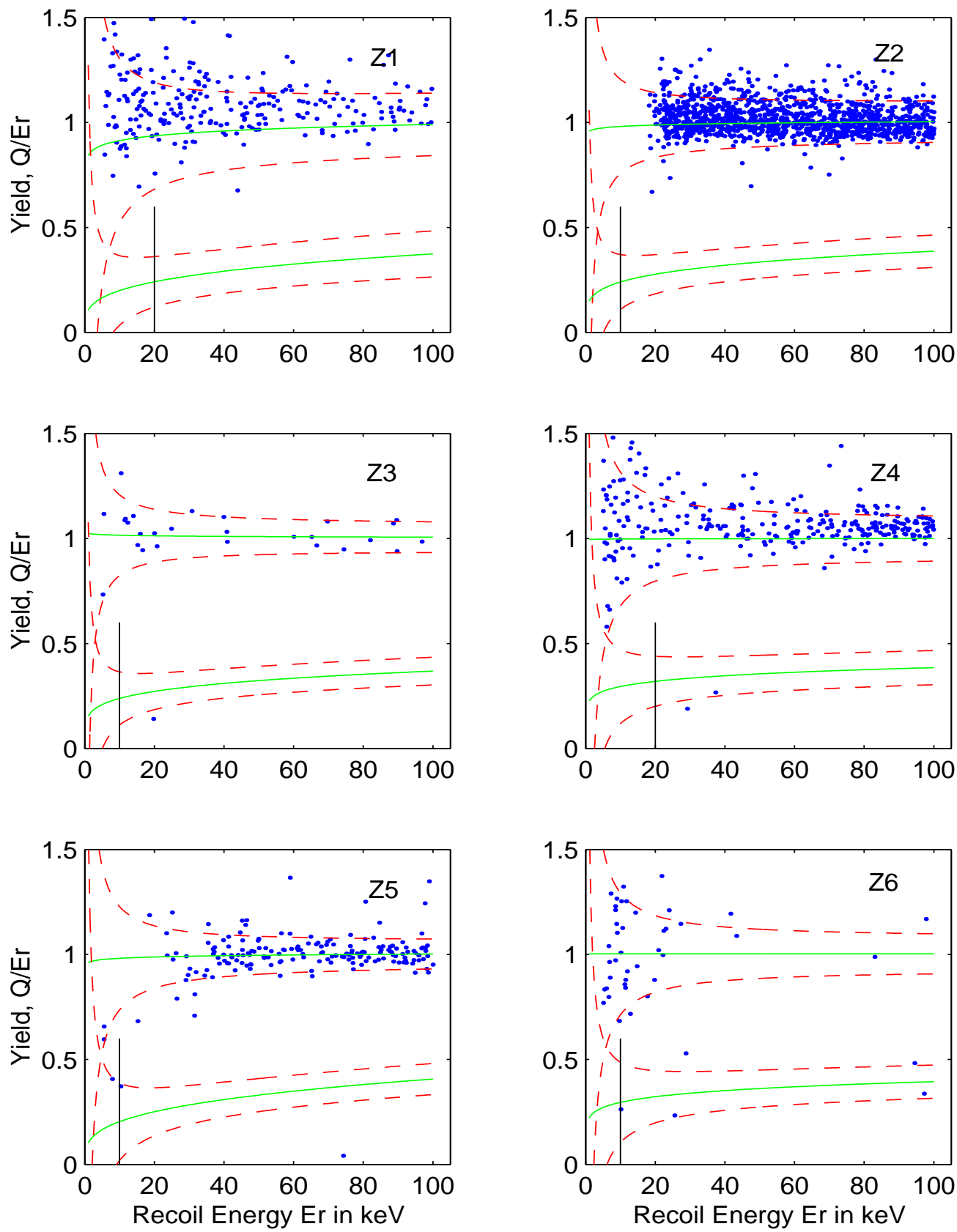

Figure 7.40: Multiple scattering events after timing cuts in blinding analysis. The black bar is the energy threshold. The multiple scattering events that pass the timing cuts in the nuclear recoil band in Z4 and Z6 are listed in Table 7.7; their partners are electron recoils in other detectors. 


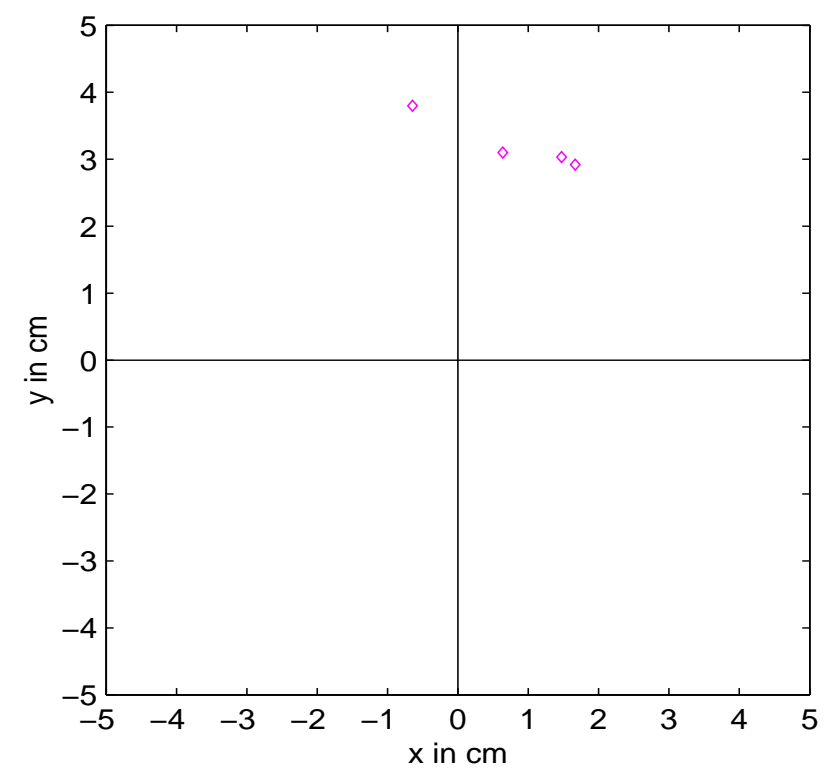

Figure 7.41: Single scattering events in Z6. All four single scattering events that pass timing cuts are in the same area.

\section{7 $\quad$ F5 bug}

In the first pass, the collected raw data in the CDMS experiment was analysed by running the CDMS analysis package, called DarkPipe. If charge traces were saturated for a particular event, the data should be analyzed by using a so-called F5 fit in the time domain for charges pulse. Otherwise, the data was analyzed by using a so-called OF (optimal filter) fit in the frequency domain, which has the background noise removed. But after the blinding data analysis of Soudan run118, it was found that some low background data were inadvertently analyzed with F5 fit, even though there were no saturation traces. This led to some events being cut off with cQinS, and caused the nuclear recoil band to shift. This case was called the "F5 bug".

The low background data was reanalyzed with the F5 bug removed. We found 
one event $($ SeriesNumber $=312231748$, EventNumber $=90179)$ that passed the timing cuts in the nuclear recoil band in Z5. (See the graph on the left in Figure 7.42). Although finding one event passing the timing cuts in the nuclear recoil band is consistent with the surface event leakage estimation, the direct evidence that this event is either nuclear recoil or electron recoil is needed.
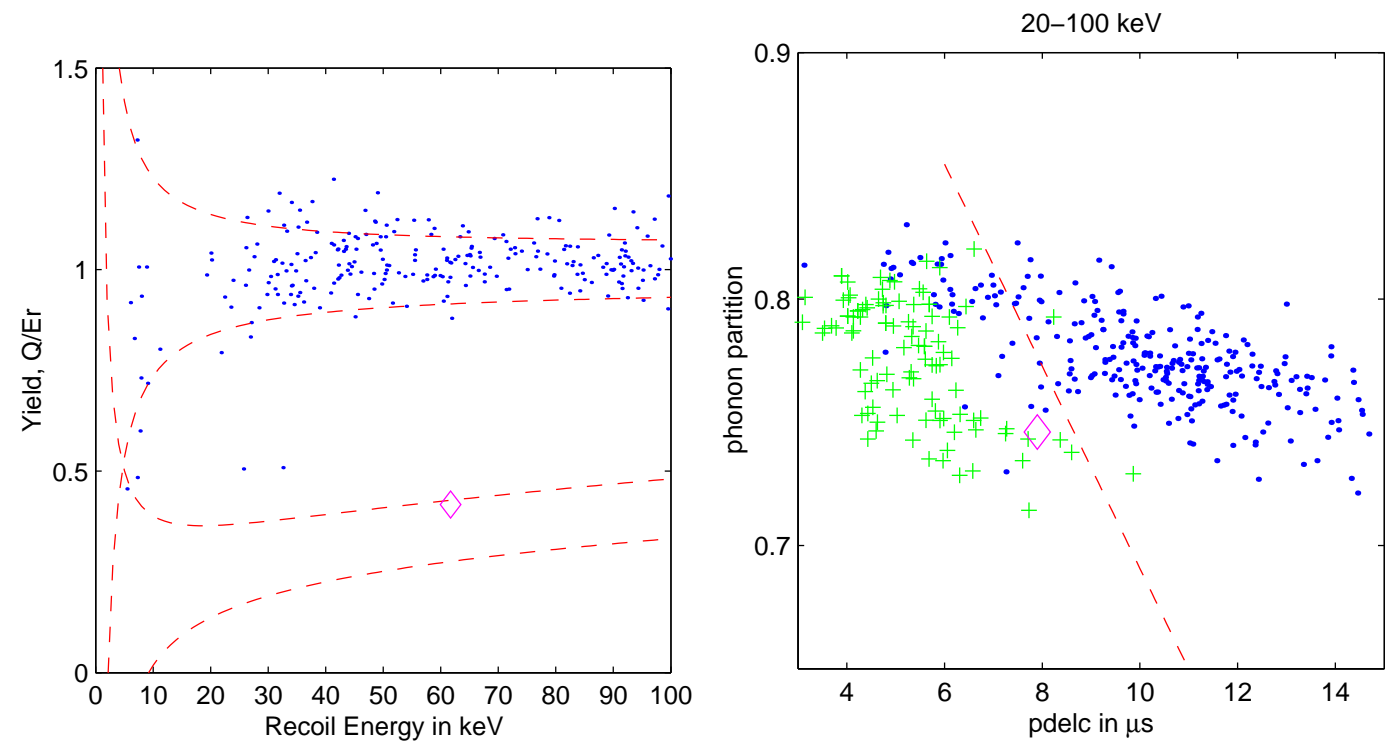

Figure 7.42: Left graph: Z5, Soudan run118 WIMP search data after surface event rejection timing cuts. The diamond is a anti-coincident single scattering event that is in the nuclear recoil band and passes timing cuts after F5 bug is removed in blinding data analysis. Right graph: In the phonon partition surface rejection method, the event (diamond) in the nuclear recoil band in the left graph is an electron recoil (diamond) in the right graph. The blue dots are neutrons in ${ }^{252} \mathrm{Cf}$ calibration. The green crosses are ejectrons in ${ }^{133} \mathrm{Ba}$ calibration.

In the phonon partition and pdelc surface event rejection method (in section 7.5), the event in the nuclear recoil band in Z5 is rejected; i.e., the event is a electron recoil. (See the graph on the right in Figure 7.42). The signature is very clear, the event falls in the ejectron cluster, away from nuclear recoils. This interpretation follows the unblinding of the analysis, but is based on the understanding of the fundamental difference between neutrons and ejectrons in the ZIP 
detector. In practice, seeing one electron recoil in the nuclear recoil band after the surface event rejection cuts is consistent with expected electron leakage, and only has a small effect on the interpretation of the data, which is further treated in Chapter 8. 


\section{Chapter 8}

\section{Conclusion}

\subsection{WIMP Exclusion Limit}

A WIMP only scatters off the nucleus in one detector because of its weak interaction with ordinary matter. In the CDMS WIMP search data, the WIMP signal can only be a muon anti-coincident single scattering nuclear recoil event. The possible background that has the same signature consists of external neutrons, which are generated in the mine rock from muon excitation or from radiaoactivity. These neutrons may penetrate the shield and scatter in one detector, but there is no record in the scintillator veto paddles.

Another possible single scattering event in the nuclear recoil band is an electron recoil due to its incomplete charge collection. The electron recoil is removed by using ionization yield, phonon energy distribution in the four phonon sensors of the ZIP detector, and the phonon timing parameters, as described in Chapter 7. There are no unvetoed single scatter nuclear recoils in the CDMS run118 52.6 kg-day data after the data quality and surface event cuts.

In the position information based WIMP search data analysis, the nuclear recoil event selection efficiency is calculated with data quality cuts (cChiSqS, cPstd), charge energy threshold (cThresS) on the events in the nuclear recoil 
band, the charge inner electrode cut (cQinS), the nuclear recoil band cut (cNRS), the surface event rejection cuts (the phonon partition vs pdelc in section 7.5), and the veto cut ( $\tilde{c} V T S)$. The veto cuts off $3 \%$ events in the low background operation mode in Soudan. The event selection efficiencies of all the cuts, and the total event selection efficiency after all the cuts are in Appendix C. These cuts result in an average nuclear recoil event selection efficiency for the four germanium detectors as shown in Figure 8.1. The peak of nuclear recoil selection efficiency is 0.4941 at $34 \mathrm{keV}$. This corresponds to a total exposure of $26 \mathrm{~kg}$-day.

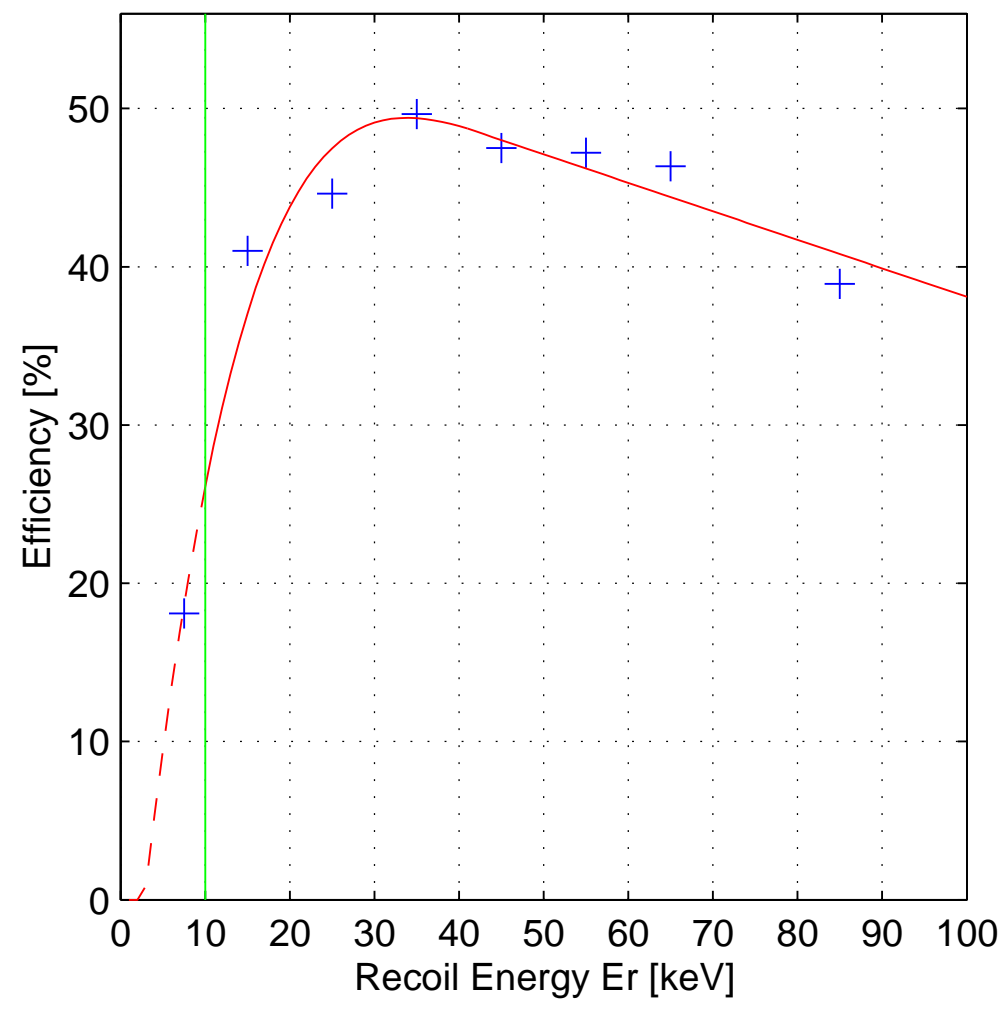

Figure 8.1: The average nuclear recoil event selection efficiency of four germanium detectors. The blue crosses are data, the red line is polynomial fit. Recoil energy threshold is $10 \mathrm{keV}$, as indicated by the vertical green line.

There are no nuclear recoil events in the CDMS II Soudan run118 WINP 


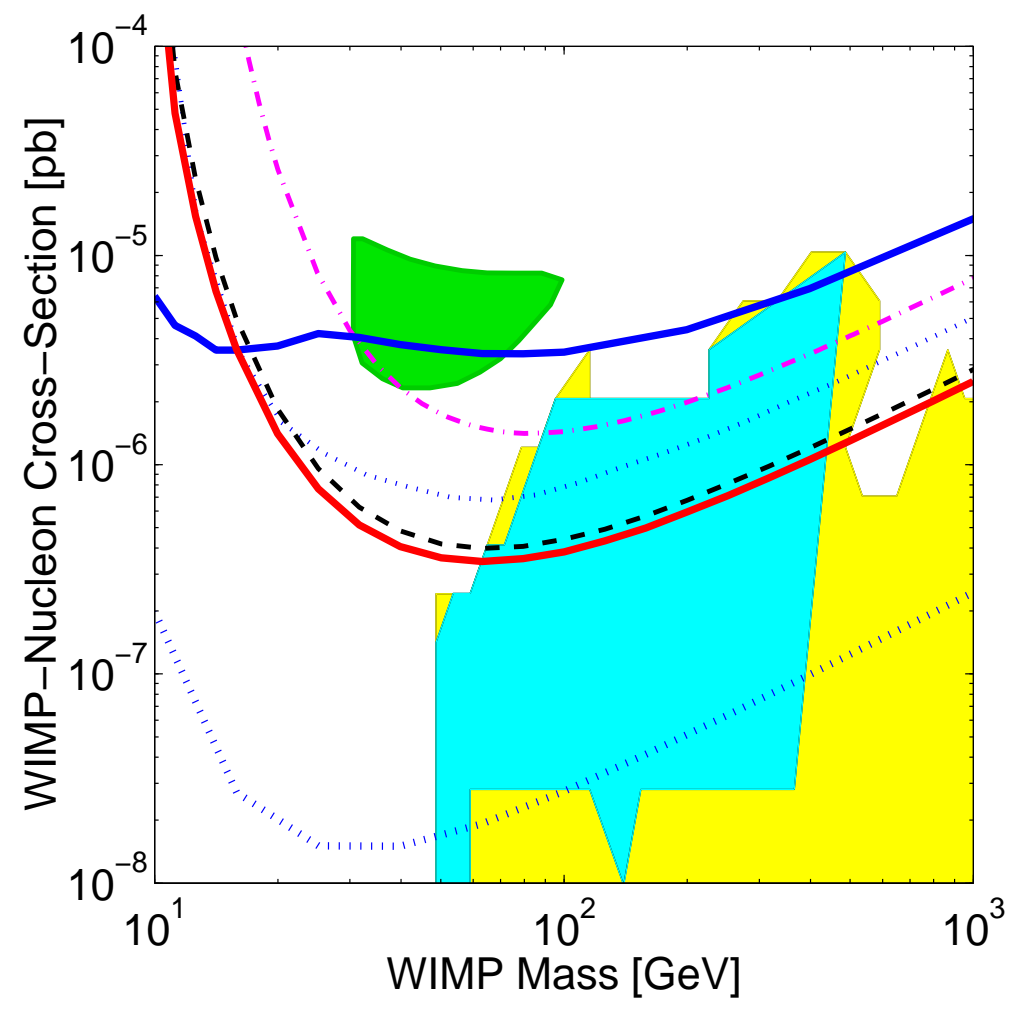

Figure 8.2: WIMP exclusion 90\% confidence level limits. The red line is the WIMP-nucleon cross-section exclusion limit from position information based WIMP search data analysis in the Soudan run118. The dashed black line just above the red line is from blind analysis. The blue line with a dip at $20 \mathrm{keV}$ is the CDMS II exclusion limit of run21 at Stanford Underground Facility [135]. The dotted line in the middle is the conservative CDMS estimation. The dotted line at the bottom is the expected limit the CDMS experiment will explore in the next few years. The green patch at the top is the $3 \sigma$ region for WIMPs from the DAMA 1-4 season data [136]. The magenta dashed dotted line is the Edelweiss experiment exclusion limit in 2002 [137]. The yellow patch that covers almost all of the area at the lower right corner is the WIMP parameter space in minimal supersymmetry [138]. The cyan area on the top of the yellow patch is the WIMP parameter space in minimal supersymmetry with the muon g-2 constraint [138].

search data after the background reduction cuts. Under the assumption of an isothermal dark matter (WIMP) distribution with a density $0.3 \mathrm{GeV} / \mathrm{c}^{2} \mathrm{~cm}^{-3}$ and a characteristic velocity $v_{0}=220 \mathrm{~km} / \mathrm{s}$ assumption, and mean Earth velocity $v_{E}=$ 
$232 \mathrm{kms}^{-1}$, the calculated WIMP exclusion limit for spin-independent WIMPnucleus interaction is shown in Figure 8.2. This result is slightly better than the conservative CDMS estimated sensitivity, and about four times lower than the Eldweiss result in 2002. It excludes the DAMA 1-4 season $3 \sigma$ region.

\subsection{Outlook}

The CDMS II experiment at Soudan is expected to explore the WIMP-nucleon cross section down to $2 \times 10^{-8} \mathrm{pb}$. This will incude a big part of the parameter space in minimal supersymmetry theory, and most of the parameter space in minimal supersymmetry theory with the muon g-2 constraint [138]. To achieve this goal, a large amount of low background data will be collected with tower I and tower II in 2004. Three more towers will be installed by the end of 2004 .

With an increase of the total exposure of the CDMS experiment in the near future, the effective background rejection will become more important. This requires precise neutron background simulation and a high efficiency of surface event rejection. The developed event-by-event background rejection methods in the CDMS collaboration with ionization yield, phonon timing parameters, and event location information will help to select nuclear recoils at the high confidence levels.

Before this thesis, only phonon timing parameters had been used for rejection of surface events in the nuclear recoil band in the CDMS collaboration. But the surface events near the charge outer electrode in the ZIP detector have higher values of phonon timing parameters due to the limited phonon sensor coverage at the edge of the ZIP detector.

The reconstructed $\mathrm{x}$ and $\mathrm{y}$ parameters in the ZIP detector allow us to use both phonon energy distribution and phonon timing parameters for surface event 
rejection. The phonon partition is an independent parameter that effectively rejects the surface events near the charge outer electrode in the ZIP detector.

Event location information based data analysis helps us to find out the location of the surface electron recoils that are most likely to fail the phonon timing parameter cuts. The complementary phonon partition parameter and phonon timing parameters together are used to achieve a nearly perfect event-by-event surface electron recoil rejection. This method and event position reconstruction offer a new window for surface event rejection. I hope that the position based data analysis technique will be tested with a large amount of ejectrons from ${ }^{133} \mathrm{Ba}$ calibration in the CDMS Soudan run119, and will be used as an independent WIMP search data analysis algorithm in the CDMS collaboration.

The reconstructed $\mathrm{z}$ parameter carries the phonon timing information in the local quadrant phonon sensor and two neighboring quadrant phonon sensors. It has its own surface rejection capability, as described in Chapte 5. The application of the $\mathrm{z}$ parameter combined with ionization yield and phonon timing parameters is a new mehtod to have an improved surface event rejection in the CDMS experiment.

\subsection{On My Contributions to A Broad Collabo- rative Experiment}

The CDMS experiment is accomplished through the work of many individual and group contributions. In this section, I highlight some of the contributions I've made or been involved, in addition to the event position reconstruction in chapter 5 and the position based analysis work on surface event rejection in chapter 7 .

Half of my graduate study time was on the testing and optimizing the use 
of CDMS detectors, using the Oxford Kelvinox dilution refrigerator, which has a base temperature of $12 \mathrm{mK}$.

Working with the CDMS test facility, most of the fun and learning came from CDMS detector testing. This testing is an important part of CDMS detector fabrication, as described in chapter 4 . The installation of the detector wiring \& electronics package, the Tower, from the bottom of the dilution refrigerator was a challenging job. Lying on the floor for hours, we made the thermal connections layer by layer. The difficulties came from the "ears" on the Tower being in a line. The screws must be held with a long Allen wrench at the proper angle to connect the detector stage of the Tower to the dilution refrigerator mixing chamber stage with extreme care. After working under difficult conditions in a Tyvek suit and a long struggle with the Tower, making the indium seal for the IVC vacuum was one of my favorite rewards.

Individual tungsten-sample superconducting transition temperature measurements served as an independent diagnostic for the transition temperature profile mapping on the surface of the CDMS ZIP detectors. To carry out these tests, we put twenty tungsten samples on a copper holder, using a piece of cigarette paper to electrically separate the samples from the copper. For good thermal contact, no air bubbles were allowed between the copper and the cigarette paper, or between the sample and the cigarette paper. After this, I did enjoy the aluminum wire bonding with the K\&S model 4123 Wedge Bonder to make electrical connections. I would like to say this supersonic wire bounding machine is the finest machine a human has ever invented. It was neccesary for me to adjust the aluminum wire tail, and set the bonding power and time interval very carefully to get reliable results.

Our test facility at CWRU was completely instrumented for operating with 
CDMS power supply, front end board (FEB), Receiver/Trigger/Filter (RTF) board, the detector holder Tower, the SQUET cards, FET cards, and three stripelines connecting the FET card at the cold stage inside the dilution refrigerator to warm electronics FEB. The most exciting moment was when we first got the SQUID modulation curve under current bias in 2000. After this, we have continuously contributed to CDMS ZIP detector tests. These tests in the detector charge channels, TES transition temperature and critical current are the crucial tools for understanding the individual ZIP detector and for making the phonon sensor transition temperature adjustment with iron implantation. We tested four of the six ZIP detectors in Tower I at CWRU before they were used for CDMS II at the Stanford Underground Facility starting in 2001. We oparated these same detectors at Soudan for my thesis data.

These detector tests were also a good opportunity to understand ZIP detector physics. One avenue we pursued was operating a CDMS silicon ZIP detector at alevated bias voltage to have an altra-low energy threshold for a neutrino magnetic moment measurement. We demonstrated stable operation at 200 volts bias across the silicon detector, and measured the expected level of thermal amplification. Our conclusion is that a CDMS silicon ZIP detector with a $20 \mathrm{eV}$ energy threshold for neutrino-electron scattering is feasible.

Another example of work with the CWRU test facility has been the TES thermal parameter measurements (part of Chapter 5 in this thesis). In the routine diagnostic tests, the superconducting transition temperature range has been assumed to be narrow (but the actual value could be from 5 to $15 \mathrm{mK}$ depending on the uniformity of individual phonon sensors). The dynamics of the TES in the transition range had not yet been fully studied. But all the physics in the TES phonon sensor is in this transition range. Knowledge of TES dynamics can thus 
be used for detector improvements. The ultimate goal is having a lower threshold energy and better phonon energy resolution detector, as well as optimizing the TES bias. I initiated a TES dynamics study with slow-mode TES bias current versus resistance and TES temperature versus resistance relation measurements. From these measurements, I extracted useful TES thermal parameters, such as the TES heat capacity and the thermal coupling constant between the TES and the detector substrate. I found useful insights into TES dynamics and hope someone else will do further studies of TES dynamics in the CDMS collaboration.

I very much enjoyed applying Rydberg model in semiconductor crystals to the CDMS-detector charge-trapping physics. The details are given in chapter 5. I 'd like to point out that the study of charge trapping with Rydberg series is not for increasing the external bias voltage of the CDMS detectors, but for finding the optimized external bias voltage, because too may Nagenov-Luke phonons lower the sensitivity of the recoil energy measurement.

My contributions to the CDMS II experiment work at Soudan have been in the experiment construction, experiment operation, and WIMP-search data analysis.

From the beginning of 2002 to the end of 2003, I usually traveled to Soudan twice in three months, each trip lasting from 10 days to two weeks, depending on the schedule.

There was a lot of experience in dilution refrigerator installation and ice box building in the CDMS collaboration before CDMS II started at Soudan. Building a large cryogenic experiment, such as the CDMS experiment, is not simple. To cool down the inner volume of the CDMS experiment, leaks in the vacuum thermal insulation layers (OVC and IVC) and in the mixture circulation loop is not allowed. But the leaking tends happen in these systems. Unfortunately, the CDMS experiment had a collapsed liquid-helium bath wall in the summer of 2001. 
After that, we failed in several attempts at cooling down the dilution refrigerator, because the leaking at the bottom flange from the liquid helium bath to the OVC caused a high rate of liquid helium consumption, and other leaks in the IVC gave the dilution unit a big thermal load. The CDMS cryogenic crew led by Professor Blas Cabrera put most of their time in the dilution refrigerator debugging in 2002. I was one of the cryogenic crew members. Several practical steps were taken in the dilution refrigerator debugging. The indium seal was redone to make sure there was no leaking from the mixture circulation loop to the IVC; the dilution unit was tested separately in liquid helium dewar, reaching a base temperature of $20 \mathrm{mK}$; the collapsed liquid helium bath wall was eventually extracted, straightened, and leak checked; and a "T" bellow in the dilution refrigerator Still line was installed for releasing the strain on the liquid helium bath wall. In the fall of 2002, the dilution refrigerator was finally put back together again, and was cooled to 20 $\mathrm{mK}$. The dilution refrigerator and the ice box together were tested at the end of 2002. The base temperature went down to $30 \mathrm{mk}$, and was a great milestone in the CDMS collaboration.

I was also involved in the commissioning of the detector installation in the first half of 2003, and in the experiment operation in the second half of 2003. Balancing TES biases for Tower I detectors was one example of my specific contributions. Another example I would like to mention here was the situation that Clarence Chang and I encountered in the Soudan mine. A whole bath of liquid helium evaporated in just a few minutes; we saw the liquid helium level drop down a few percent at each step. It was the leaking helium gas in the OVC that released from the liquid helium bath wall. The big thermal load was from $77 \mathrm{~K}$ stage due to the released helium gas. The turbo pump eventually pumps away the helium gas and restore the insulating vacuum. In this situation, there are three important 
steps to follow: make sure there is enough OVC vacuum pumping speed; do not allow the liquid helium bath to run dry; and watch the mixture circulation in case the dilution unit warms up too quick. Ultimately we were able to obtain enough stable operation for extensive data taking.

I regard my most important work in the CDMS collaboration to be data analysis, which includes the cross-talk analysis of Stanford Underground Facility run21 data, the exploration of ptrtcQ (phonon rise time plus the time difference between phonon trig time and the charge channel time) as a potential improvement to surface event rejection, the phonon and charge start times for event-position reconstruction, etc. In particular I emphasized the event-position reconstruction in the ZIP detector and its application in surface event rejection, which have been addressed in chapters 5 and 7 .

To summarize, my graduate study at Case Western Reserve University, I will say that the knowledge of cosmology and particle physics, and the scientific and technical training in Cryogenic Dark Matter Search collaboration have been rich and enjoyable. 


\section{Appendix A}

\section{LED Study}

\section{A.1 Introduction}

At the beginning of run21 at the Stanford Underground Facility (SUF), there were charge collection deficiency problems in the two silicon detectors Z4 and Z6. Dan Akerib suggested that I do LED tests in the CWRU test facility, and investigate the neutralization physics of the detectors. It led to the work that is summarized here.

The purpose of LED flashing in the DIB is to neutralize the trapped charges in silicon detectors and in germanium detectors. So we performed two procedures: we checked the LED's function, and tested how the detectors respond to LED flashing.

The LEDs in the DIB are PDI-E940SM high power GaAs infrared emitters from Photonic Detectors Inc., having a peak wavelength of $940 \mathrm{~nm}$ at room temperature.

As for the neutralization physics in the detector, there are four important physics processes to be considered. The first is the photon energy spectrum from the GaAs LED. The second is the electron hole excitation threshold energy in silicon or germanium. The third is the photon penetration depth (optical ab- 
sorption coefficient) in the detector, and the last is the electron diffusion length in the detector. Investigating these detector parameters will improve our understanding of detector neutralization physics, and allow us to optimize the detector neutralization procedure.

Section A.2 is devoted to LED I-V curve measurement, section A.3 to the charging time measurement of the LED, and section A.4 to energy power estimations of the LED. In section A.5, detector neutralization physics is discussed with detector photon conductance measurements and the fundamental semiconductor physics analysis in the detector.

\section{A.2 LED I-V Measurement}

The LED I-V measurement is quite straightforward. A $1 \mathrm{~K}$ resistor is in series with the LED, and external voltage is applied across them. (See Figure A.1.) The applied voltage $V_{0}$ and the current flow through the LED were measured. The actual voltage across the LED is $V=V_{0}-I \cdot R$.

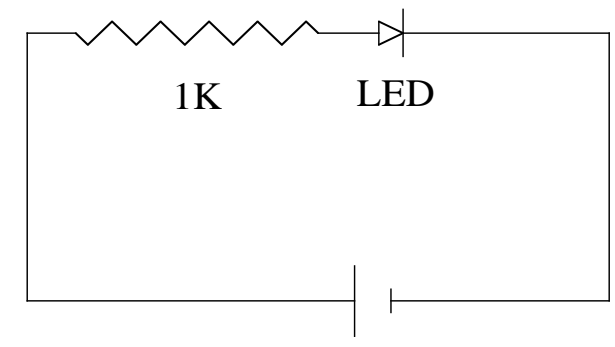

Figure A.1: LED I-V measurement circuit.

There are two characteristics in LED I-V curves, as shown in Figure A.2. First, there is a clear turn-on voltage; below this voltage, the current through the LED is 


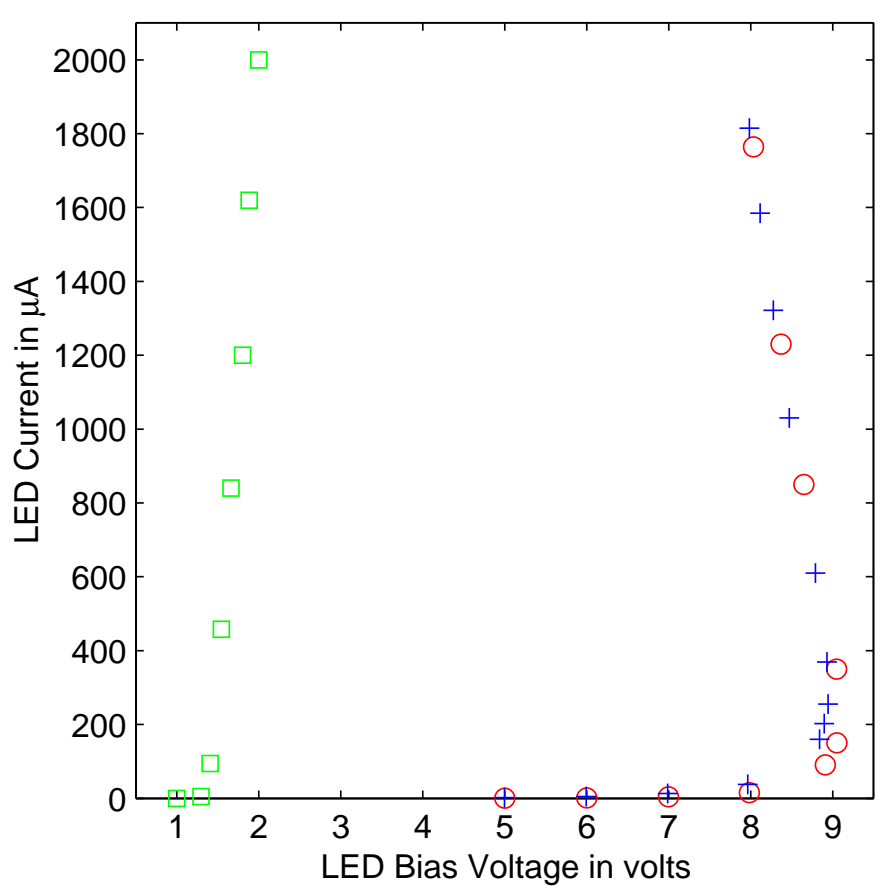

Figure A.2: LED I-V relation at different temperatures. Squares are LED at 77K, crosses are LED at $4.2 \mathrm{~K}$, circles are LED at $300 \mathrm{mK}$.

small. After the turn-on voltage, the current through the LED increases sharply. Second, there is I-V curve foldback at high LED bias at low temperatures. The LED is a current driving bipolar device. Its flashing light power is proportional to the bias current. The injected electron and hole annihilation gives off photons, but there are injected electron and hole scattering before they are annihilated, and the kinetic energy of the scattering electron and hole go to the lattice system, so there is internal self-heating after the LED is turned on. Because it is used at extremely low temperatures, the self-heating effect has a huge effect, and it gives a special I-V relation. Paul Brink at Stanford University has a matlab program that shows that there is an LED I-V curve foldback when the LED internal self-heating effect is considered.

Semiconductor device physics (for example,the book by Sze [139]) of diodes 


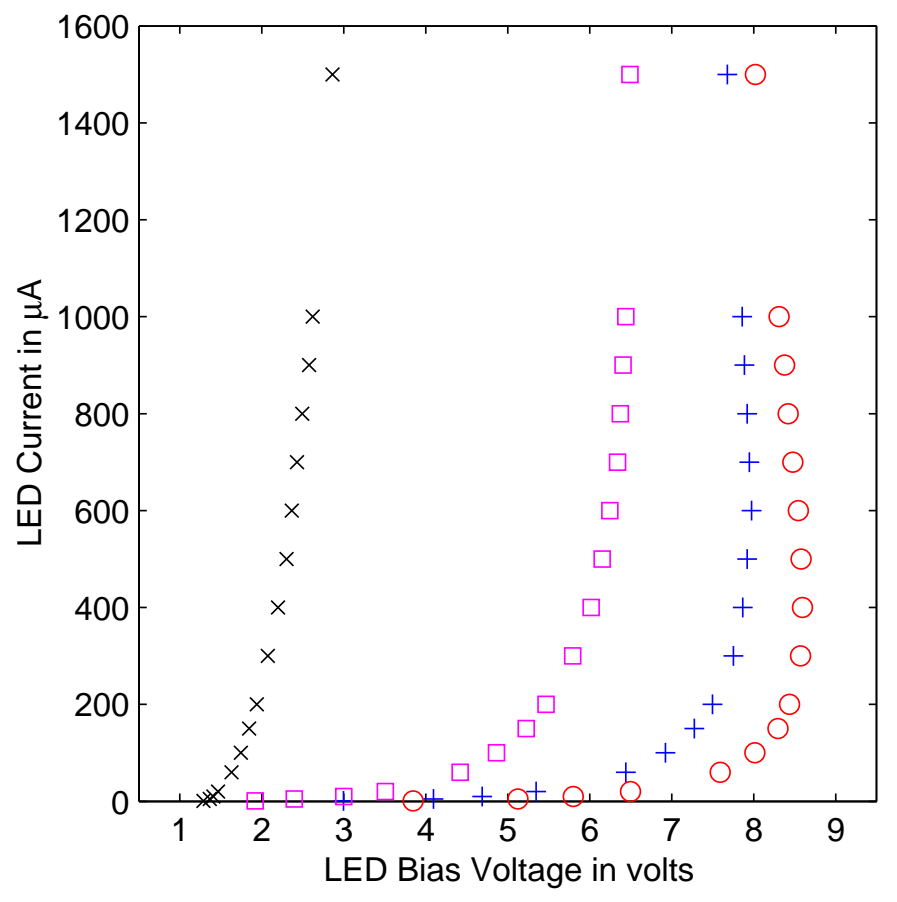

Figure A.3: LED I-V relation at different temperatures. The Xes are for LED at $48.1 \mathrm{~K}$, squares are for LED at $26.1 \mathrm{~K}$, crosses are for LED at $13.5 \mathrm{~K}$, and circles are for LED at 7.4K.

gives the explicit I-V relation of a diode

$$
\begin{gathered}
I=I_{s}\left(\exp \left(\frac{q V}{k T}\right)-1\right) \\
I_{s} \propto T^{3+\frac{\gamma}{2}} \exp \left(-\frac{E_{g}}{k T}\right)
\end{gathered}
$$

where $q$ is electron charge, $V$ is bias volatge, $k$ is the Boltzmann constant, and $\gamma$ describes how the ratio of the diffusion coefficient and life time of electron changes with temperature, $\frac{D}{\tau} \propto T^{\gamma}$.

Besides the emission of $\mathrm{eV}$ energy photons, there is IR radiation from the LED. So we need to know the LED temperature while flashing. We did this measurement at the end of CWRU run17 for S4 LED2, and the results are plotted in Figure A.3. We estimated that LED I-V curves stop folding back at about 
$30 \mathrm{~K}$. The corresponding thermal radiation energy spectrum peaks at $12.5 \mathrm{meV}$, according to Wien's displacement law. It should not be a problem for silicon, because its shallow impurity ionization energy is about $50 \mathrm{meV}$.

\section{A.3 Charging Time Constant}

Because a long stripline is used, the LED charging time is long for small bias currents. When current pulses are applied to the LED, the actual current through the LED is really tiny before the stripline is fully charged. We need to know how big the pulse width should be in order to keep the fridge at the appropriate temperature, in case the self-heating effect of the LED drives its temperature too high.

The diode junction capacity is about $25 \mathrm{pF}$ from the LED specification. But the stripline capacitance is about $1 \mathrm{nF}$ in parallel with the LED.

With $9.5 \mathrm{~V}-p p$ external voltage pulses applied in the LED I-V measurement circuit, the charging time constant is measured with FLUKE PM3394B oscilloscope by monitoring the voltage across the LED. The charging time is $2.5 \mu s$ for S4 LED1, and 3.0 $\mu$ s for S4 LED2, with a 1K resistor in series with the LED.

In the CDMS experiments, the LED is biased with current, and the LED charging time depends strongly on bias current. The frontend board was used as the current source. The current pulse width of the LED flashing can be adjusted in a $256 \mathrm{~ms}$ time window, i.e., from $10 \mu \mathrm{s}$ to $256 \mathrm{~ms}$.

The characteristic foldback I-V relation of the LED can tell us when the LED is turned on. We did this study by using $200 \mu A$ current, but the current pulse width is tunable. Figure A.4 shows the cases of $160 \mu s$ width current pulse and of $320 \mu s$ width current pulse. In the first case, no sign of the LED is fully turned 

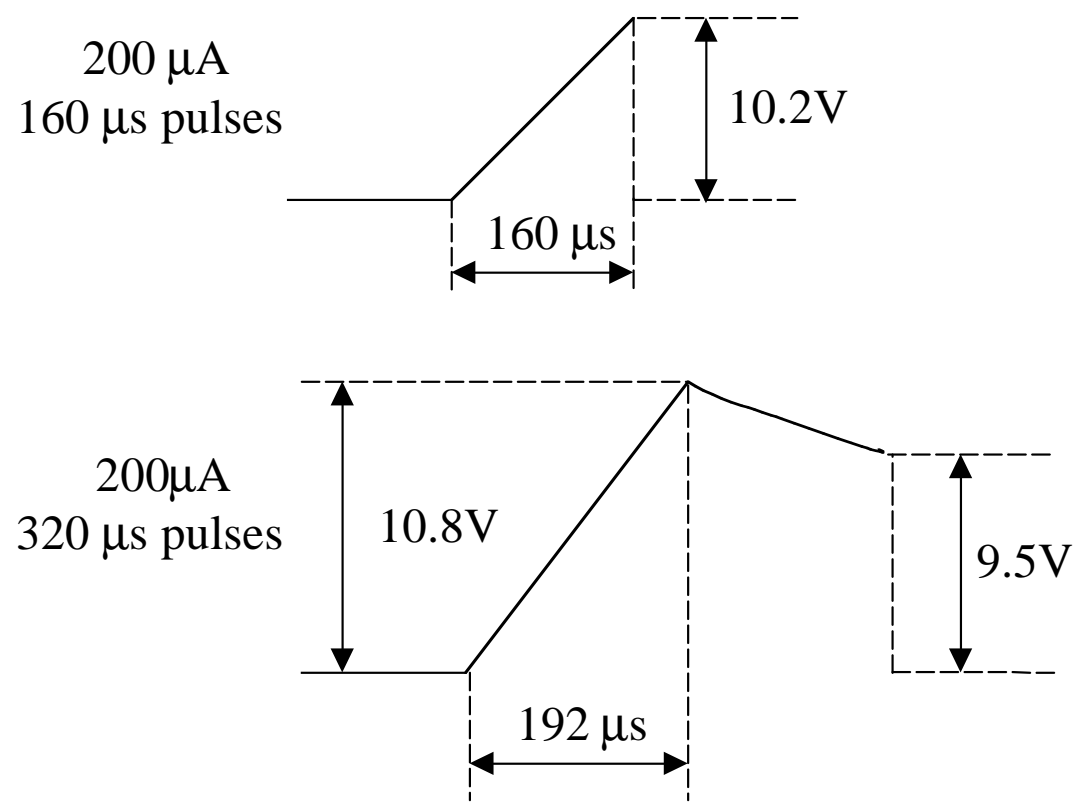

Figure A.4: LED turn-on time.

on. But in the second case, we can clearly see the signature of the characteristic foldback of the I-V relation, as the voltage across the LED starts to drop down at $192 \mu s$.

We investigated five LEDs in this way. Because of the LED long turn-on time, we realized that current times current pulse width should be greater than 40000 $\mu A \cdot \mu s$ to guarantee that the LED is on.

There are advantages to bias LED with current; for example, the LED's power is stable once the LED is turned on, and accidental LED burning can be avoided. But the LED turn on time is too long. Shorter LED pulse time means longer cooling down time, and longer cooling time guarantees smaller fridge temperature perturbation and quick data collection recovery from short time detector neutralization in the middle of data taking.

Our experience has been that the LED is easily burned off when it is voltage 
biased. The probable reason for this is that we lacked the knowledge of LED I-V relations at base temperature. If the LED I-V curve is measured before it is voltage biased, the bias voltage can be chosen exactly. Short LED flashing pulses(for example $10 \mu s$ ) can be used safely under voltage bias.

\section{A.4 Heating Power Estimation}

The typical bias voltage across S4 LED2 under current bias with FEB.vi in time domain has the shape shown in Figure A.5.

Before LED gets its full bias current $\left(I_{0}\right)$, the current through the LED increases exponentially with the actual bias voltage across the LED. This is the LED I-V relation. Before V1, the current through the LED is really small, so we can take a current cut at V1. The current is $\mathrm{I}=0$ if voltage across the LED is lower than V1. Approximately, the current I increases linearly with V-V1. The average power is approximately $\frac{V 2}{2} I_{0}(t 2-t 1)$ from the LED having significant current at time t1 to the LED, reaching its maximum voltage at $\mathrm{t} 2$.

With a $320 \mu s$ current pulse width, and peak to peak $200 \mu \mathrm{A}$ current bias for S4 LED2, V1=8.5V; just before LED turn-on, $t 1=160 \mu s ; \mathrm{V} 2=10.8 \mathrm{~V}$ at $t 2=192 \mu S$.

The relaxation is from the self-heating effect of the LED. Empirically, $\tau=$ $200 \mu s$. Then

$$
V 3=V 1+(V 2-V 1) \cdot \exp \left(-\frac{t 3-t 2}{\tau}\right)
$$

t3=LED bias on time ; T=LED bias off time

After the LED gets its full bias current, the voltage across the LED drops exponentially to V3, due to its self-heating effect. The average power is a rectangular area plus a triangular area. This is because the current through the LED is constant once it is turned on. With the LED flashing current $I_{0}$, LED total 


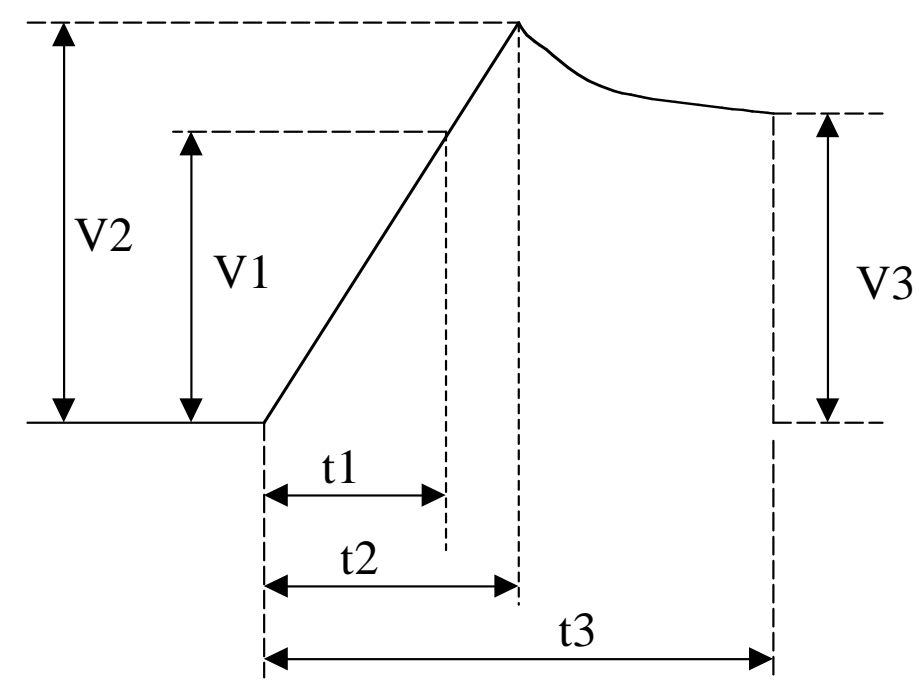

Figure A.5: LED turn-on time and heating power estimation.

dissipating power is

$$
P=\left(\frac{1}{T+t 3}\right)\left[I_{0}\left(\frac{V 2}{2}\right)(t 2-t 1)+I_{0}\left(\frac{V 2+V 3}{2}\right)(t 3-t 2)\right]
$$

The excited electron will eventually come back to its ground state in the detector, so, except for the escaped light, almost all the LED dissipation power should be transformed to heat.

The LED flashing bias current and duty time can be determined with the references of the fridge's performance and the temperature at which it should be operated.

Actually, the precision of calculation strongly depends on the choice of V1 when short current pulses are applied. If V1 can be chosen appropriately, that formula gives a good power dissipation estimate. 


\section{A.5 CDMS Detector Neutralization}

We will try to understand detector neutralization with the following measurement and analysis.

\section{A.5.1 LED Photon Energy Spectrum}

The characteristic energy of infrared photons from the LED ( GaAs, at room temperature, lamda $=940 \mathrm{~nm}$ ) is about $1.32 \mathrm{eV}$. The GaAs's energy gap changes with temperature [140]:

$$
E_{g}=1.519-5.405 \times 10^{-4} \frac{T^{2}}{T+204} \mathrm{eV}
$$

The energy gap change is $\delta E_{g}=0.0965 \mathrm{eV}$ from room temperature down to base temperature. If other factors are ignored with the temperature change, the photon energy spectrum from the LED shifts up $0.0965 \mathrm{eV}$, peaking around 1.417 $\mathrm{eV}$. The reason we can make this inference is that GaAs is a direct semiconductor, and electron hole pairs can be excited or annihilated with little interaction with the lattice. This is consistent with the data from literature, for example, Sze [139]. Aaron Miller and Sae Woo Nam at Stanford tested the photon energy spectrum of UC-Berkeley IR LED (I understand that it was the same LED we are using). They found that the spectrum peaks at $1.3 \mathrm{eV}(950 \mathrm{~nm})$. A possible explanation for this is that the energy spectrum of this kind of LED does not peak at $1.32 \mathrm{eV}$ (940nm) at room temperature. Here we use the measured energy spectrum.

\section{A.5.2 Intrinsic Excitation Threshold of Silicon}

The energy gap of silicon changes with temperature [140]. The energy gap of silicon at base temperature is about $1.17 \mathrm{eV}$.

$$
E_{g}=1.17-4.73 \times 10^{-4} \frac{T^{2}}{T+636} \mathrm{eV}
$$




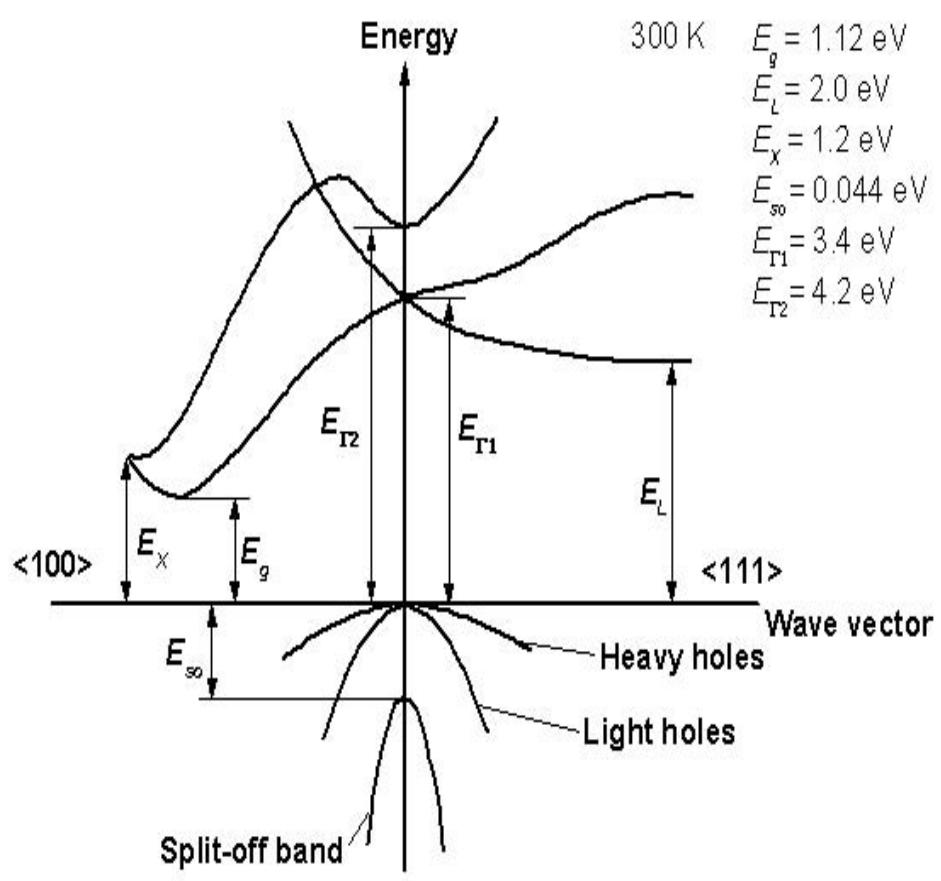

Figure A.6: Energy gap structure of silicon. Figure from [140].

Silicon is an indirect semiconductor, as shown in Figure A.6. The excitation energy for intrinsic electron hole pair production should be above the energy gap $E_{g}$. When an electron is excited from the valence band to the conduction band, there is a wave vector change, $\delta k=0.85 \cdot \frac{\pi}{a}, a=0.543 \mathrm{~nm}$, (see the silicon band diagram in figure A.6). Therefore, some excitation energy must go to the lattice system, and some excitation energy must go to the electron system. Paul Brink at Stanford University suggested that there are two optical phonon excitations and some low energy acoustic phonons when there is an electron hole pair excitation in silicon. If we only count two typical optical phonons $(65 \mathrm{meV}$ each), the lower limit of intrinsic electron hole excitation energy is about $1.296 \mathrm{eV}$. The LED we 
used has a continuous photon energy spectrum that peaks at $1.32 \mathrm{eV}$. There are enough energetic photons for electron-hole pair creation in silicon.

\section{A.5.3 Detector's Conductance with LED Flashing}

The photons from the LED cause excitations at the crystal's surface and in the bulk of the detector, depending on the photon's wavelength. There is a measurable photo-conductance between the outer charge collection electrode and the ground, a photo-conductance between the inner charge collection electrode and the ground, and a photon-conductance between the inner charge collection electrode and the outer charge collection electrode.

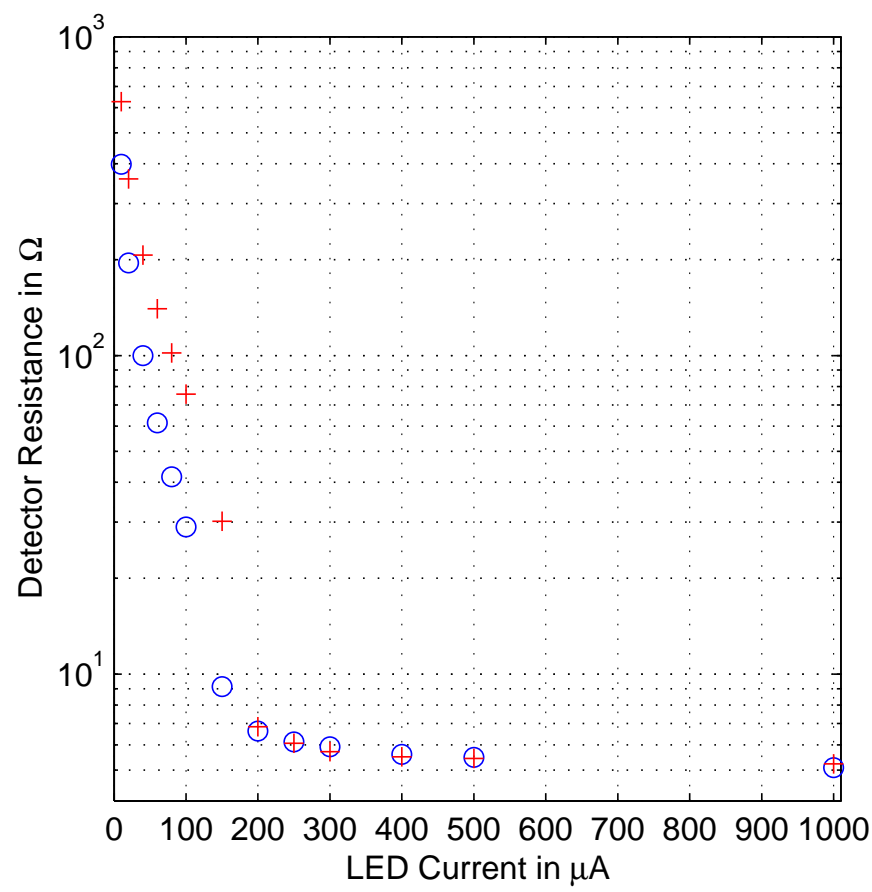

Figure A.7: Detector resistance with LED flashing current.

The detectors' conductance helps us to understand the charge neutralization physics processes in the detector by providing a direct measurement with the light from the LED. In the photoresistance-LED flashing current plots, as shown 
in Figure A.7, photoresistance decreases exponentially until it is saturated with a bias current around $200 \mu \mathrm{A}$.

In principle, the LED luminescent intensity increases monotonously (linear is a good approximation under modest bias currents) with the LED bias current. There are both intrinsic electron hole pair excitation and impurity ionization in the detector with the LED flashing. Electron hole pair excitation is what we expect for trapped charge neutralization. Impurity ionization is bad for the detector neutralization. The net neutralization effect depends on which process is dominant.

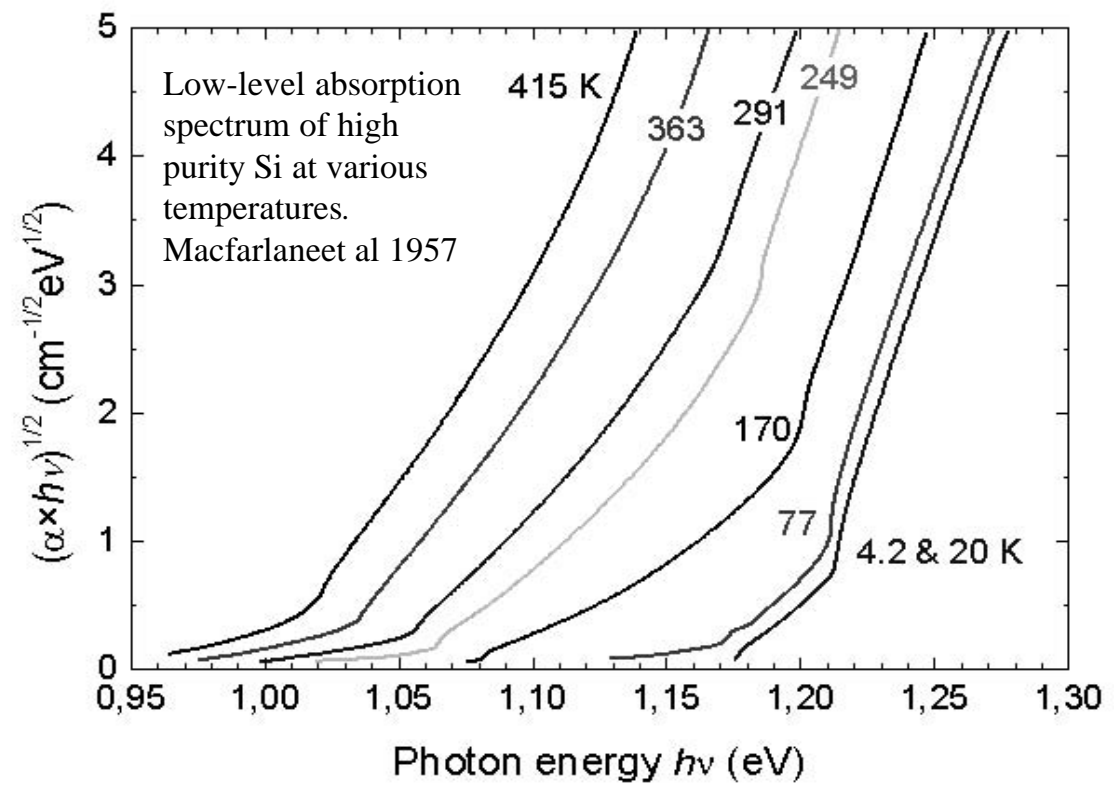

Figure A.8: Photon absorption coefficient with photon energy. Figure from [140].

To understand the conductance measurement, we need to look at the intrinsic absorption coefficient of silicon. The intrinsic absorption coefficient increases with photon energy. (See Figure A.8.) This means that the lower photon has a bigger 
penetration depth in silicon. The penetration depth of $1.25 \mathrm{eV}$ photons is about 3 $\mathrm{mm}$. So, the photons that have energy bigger than $1.25 \mathrm{eV}$ from the LED create electron-hole pairs in the surface region as well as in the bulk of the detector.

When the LED flashing current is high enough, and the excited electron density is getting big, the number of high energy photons that can reach the bulk of the detector increase. This helps to increase the detector neutralization efficiency. But we can not have the LED bias current too high, because high LED bias current warms up the dilution refrigerator.

The number of photons that can reach the bulk of the detector decrease expo-

nentially [141]. There are much more electron hole pairs created near the surface of the detector, we should use these electron hole pairs for detector neutralization.

\section{A.5.4 Electron Diffusion in the Crystal}

In the ideal case, most intrinsic excitation occurs at the surface, because the number of energetic photons decreases exponentially [141] in the bulk of the detector. The electrons and holes can diffuse into the bulk of the detector to neutralize the trapped charges.

To understand the physics better, we start with Einstein's relation in semiconductor physics

$$
D_{n}=\mu_{n} \frac{k T}{q}
$$

where $q$ is electron charge, $k$ is the Boltzmann constant, and $T$ is the detector temperature.

At $50 \mathrm{mK}$, the mobility of electron $\mu_{n} \sim 10^{6} \mathrm{~cm}^{2} / V \cdot s$, the diffusivity $D_{n} \sim$ $4.3 \mathrm{~cm}^{2} / \mathrm{s}$, and the life time of electron $\tau_{n} \sim 2 \mathrm{~ms}$, which has only a very weak dependence at temperatures below $4 \mathrm{~K}$ in high purity silicon [141]. So the diffusion 
length of the electron is

$$
L_{n}=\sqrt{D_{n} \tau_{n}} \sim 0.94 \mathrm{~mm}
$$

At $1 \mathrm{~K}, \mu_{n} \sim 5 \times 10^{5} \mathrm{~cm}^{2} / V \cdot s$, the diffussvity $D_{n} \sim 43 \mathrm{~cm}^{2} / \mathrm{s}$, and the life time of the electron $\tau_{n} \sim 1 \mathrm{~ms}$ [141]. So the diffusion length of the electron is

$$
L_{n}=\sqrt{D_{n} \tau_{n}} \sim 2.9 \mathrm{~mm}
$$

It is clear that free electrons can diffuse into the bulk of the detector crystal more easily at higher temperatures, say at $1 \mathrm{~K}$, but the situation is different at the base temperature of the dilution refrigerator. We strongly suggest that a two step neutralization strategy should be used. The initial detector neutralization starts at $1 \mathrm{~K}$ (just pumping the $1 \mathrm{~K}$ pot; keep the LED bias on overnight). The final detector neutralization is when the detectors are at the base temperature.

\section{A.6 Summary}

- It is better to choose an LED flashing current above $200 m u A$. This conclusion is based on the detector's surface conductance measurement.

- Under current bias conditions, LEDs have a long turn on time; for example, at $200 \mathrm{uA}$ bias for S4 LED2, the LED turn on time is about $200 \mu s$. Generally, we can guarantee that current times current pulse width is greater than $40000 \mu A \cdot \mu s$. 


\section{Appendix B}

\section{Plots of Position Based Data Analysis}

This appendix has all the plots for the settings of surface event rejection cuts, the plots of surface event discrimination for the anti-coincident single scattering events and anti-coincident multiple scattering events in the nuclear recoil band, and the plots of neutron selection efficiency and surface event rejection efficiency.

The exact values of surface event rejection parameters can be found in Tables 7.1 and 7.1 in section 7.5 . 

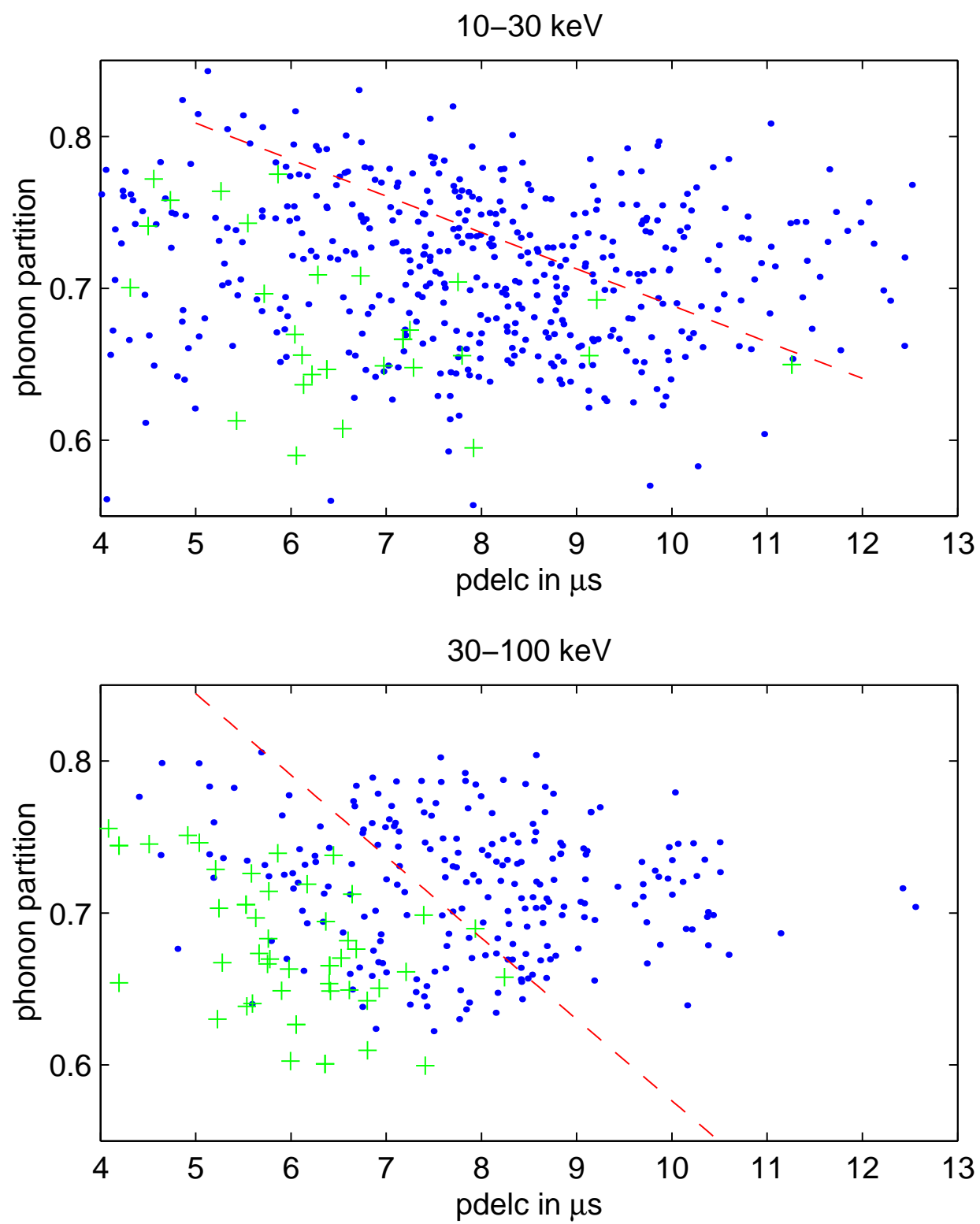

Figure B.1: Z1, phonon partition cut for events with radius $R>2.5 \mathrm{~cm}$. Blue dots are neutrons, green crosses are ejectrons. The top plot is for events that have recoil energy $E r \leq 30 \mathrm{keV}$. The bottom plot is for events that have recoil energy $E r>30 \mathrm{keV}$. The events above the dashed red line are assumed to be nuclear recoils if they pass RT1 and RT2 timing cuts. 

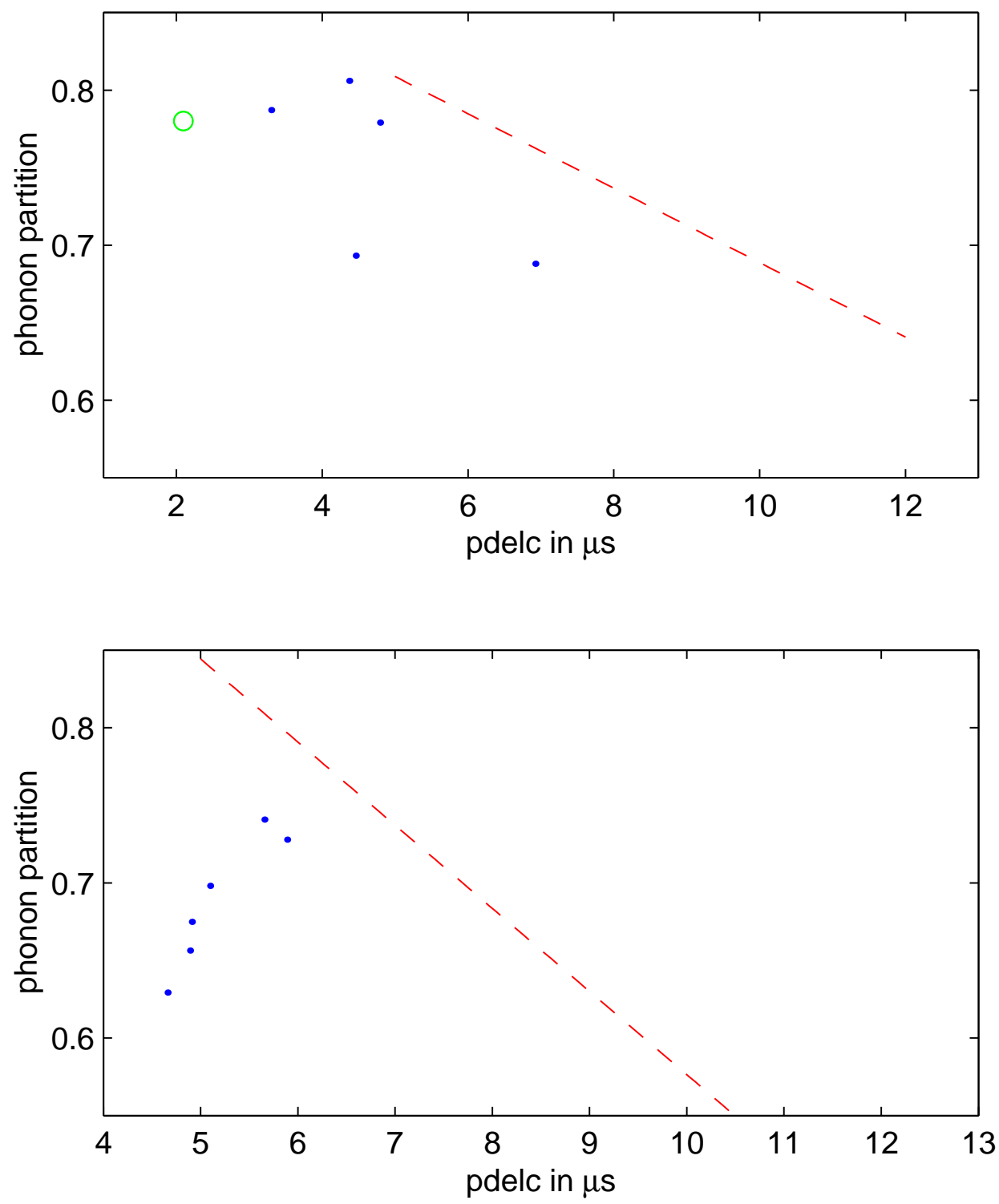

Figure B.2: Z1, Soudan run118 anti-coincident low background data in the $2 \sigma$ nuclear recoil band with radius $R>2.5 \mathrm{~cm}$. Blue dots are single scatterings, green circles are multiple scatterings. The top plot is for events that have recoil energy $E r \leq 30 \mathrm{keV}$. The bottom plot is for events that have recoil energy $E r>30$ $\mathrm{keV}$. No nuclear recoils. 

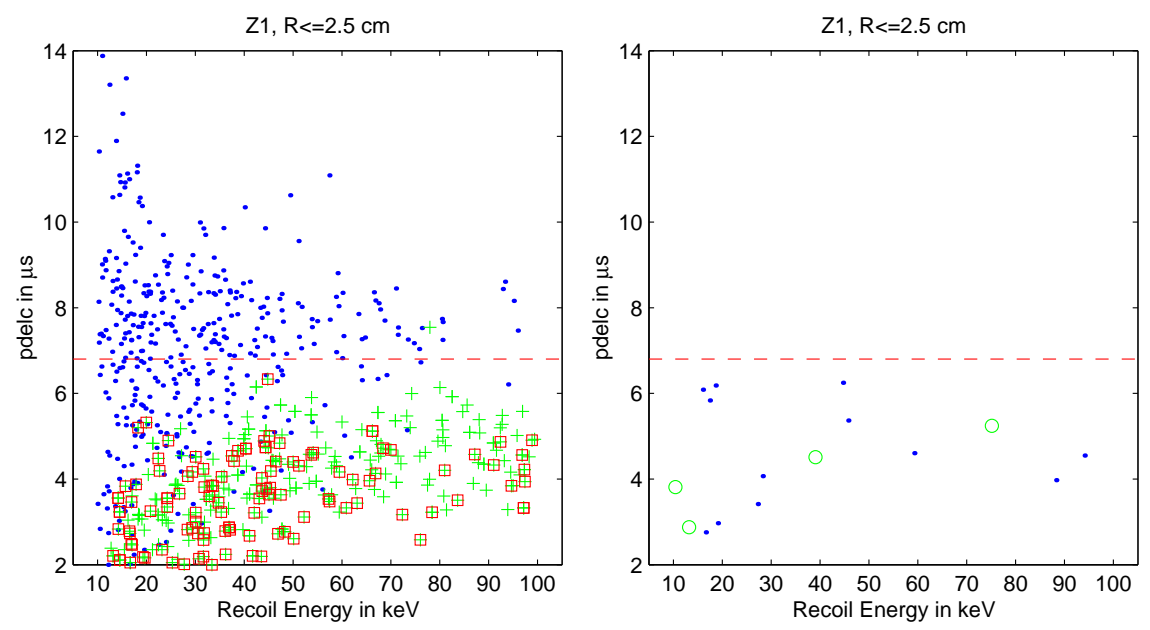

Figure B.3: Left plot: Z1, phonon delay cut for events with radius $R \leq 2.5 \mathrm{~cm}$. Blue dots are neutrons, green crosses are ejectrons. Red squares are ejectrons that fail RT1 and RT2. The events above the dashed red line are assumed to be nuclear recoils if they pass RT1 and RT2 timing cuts. Right plot: Z1, Soudan run118 anti-coincident low background data in the $2 \sigma$ nuclear recoil band with radius $R \leq 2.5 \mathrm{~cm}$. Blue dots are single scatterings, green circles are multiple scatterings. No nuclear recoils.

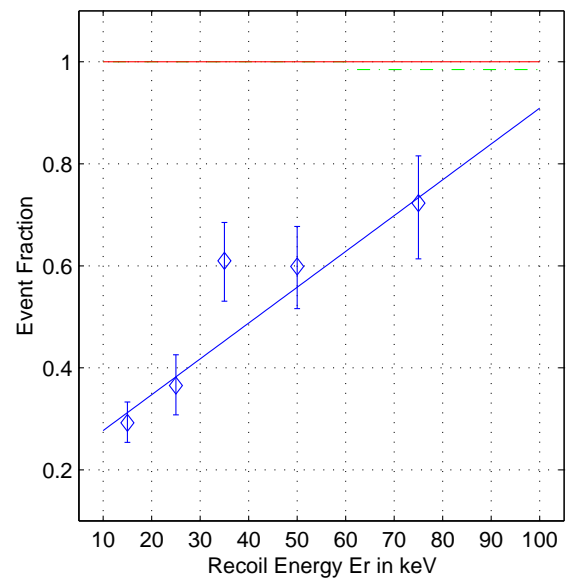

Figure B.4: Z1. The blue line fitted to the data with error bars is neutron selection efficiency in the ${ }^{252} \mathrm{Cf}$ calibration data. The dashed green line is surface event rejection efficiency in the ${ }^{133} \mathrm{Ba}$ data without an ionization yield cut. The top red line is surface event rejection efficiency in the ${ }^{133} \mathrm{Ba}$ data within the $2 \sigma \mathrm{NR}$ band. 

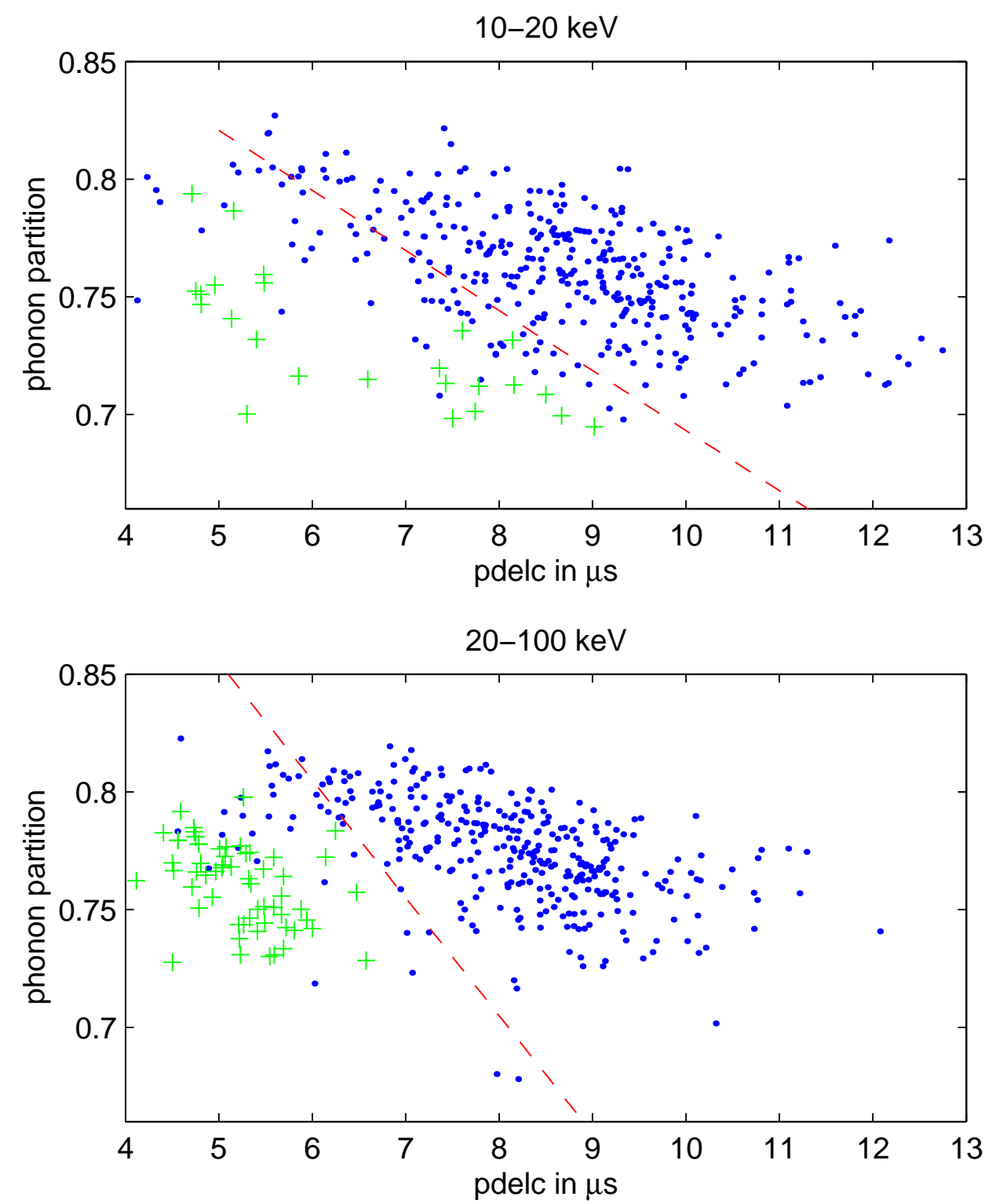

Figure B.5: Z2, phonon partition cut for events with radius $R>2.7 \mathrm{~cm}$. Blue dots are neutrons, green crosses are ejectrons. The top plot is for events that have recoil energy $E r \leq 20 \mathrm{keV}$. The bottom plot is for events that have recoil energy $E r>20 \mathrm{keV}$. The events above the dashed red line are assumed to be nuclear recoils if they pass RT1 and RT2 timing cuts. 

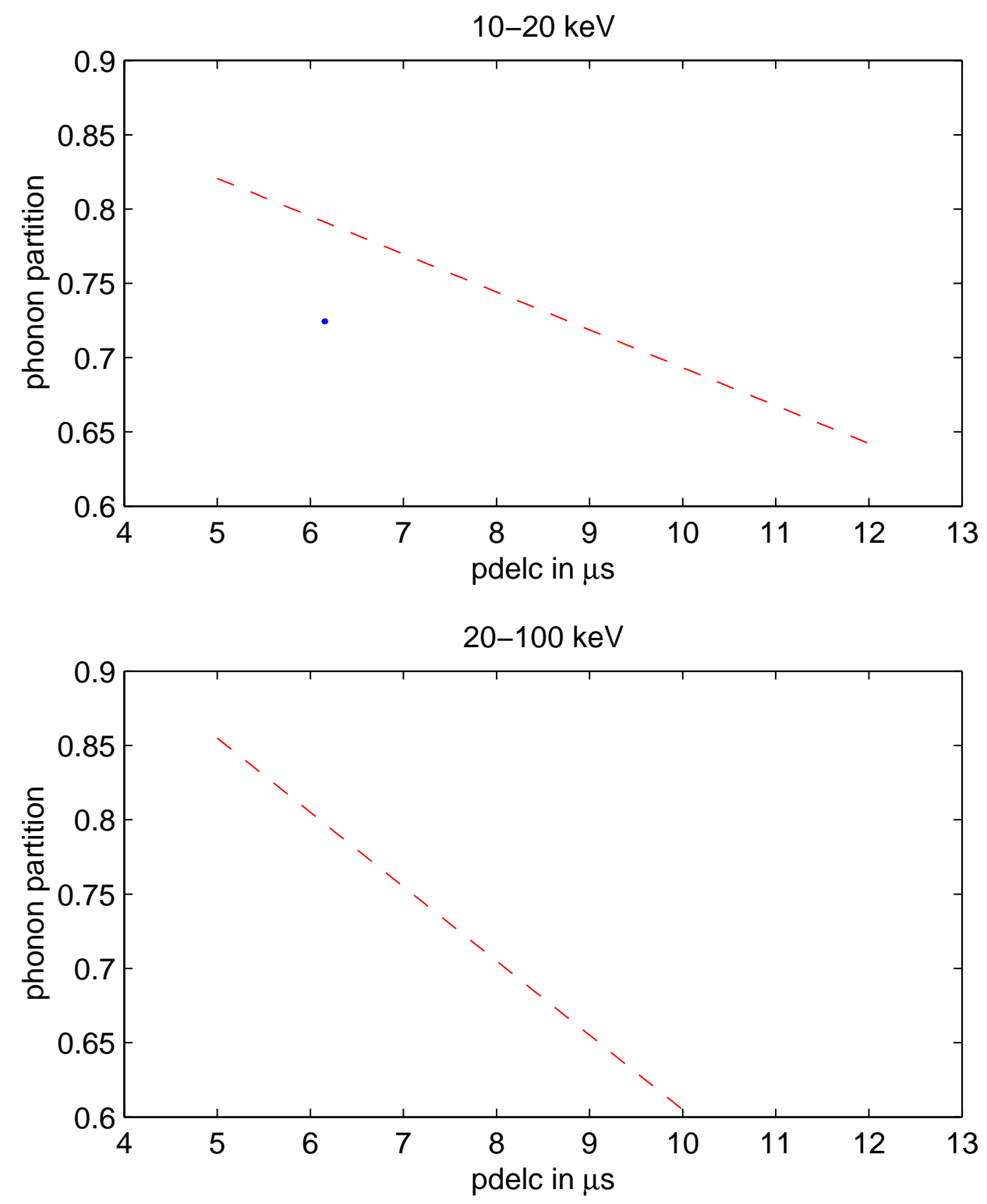

Figure B.6: Z2, Soudan run118 anti-coincident low background data in the $2 \sigma$ nuclear recoil band with radius $R>2.7 \mathrm{~cm}$. Blue dots are single scatterings, green circles are multiple scatterings. The top plot is for events that have recoil energy $E r \leq 20 \mathrm{keV}$. The bottom plot is for events that have recoil energy $E r>20$ $\mathrm{keV}$, there are no events. No nuclear recoils. 

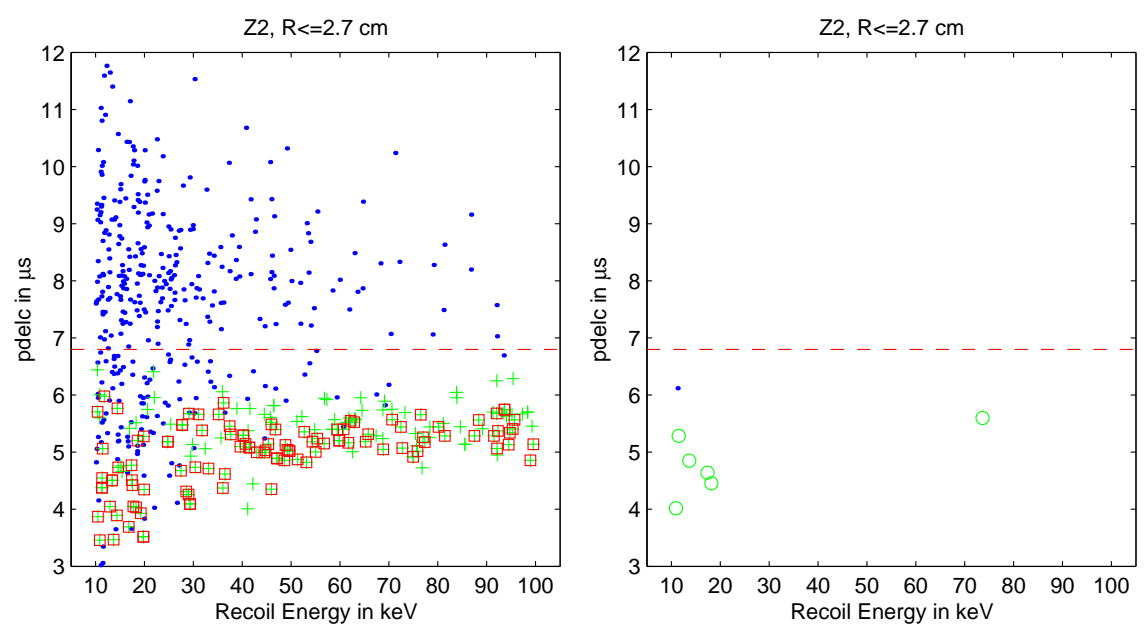

Figure B.7: Left plot: Z2, phonon delay cut for events with radius $R \leq 2.7 \mathrm{~cm}$. Blue dots are neutrons, green crosses are ejectrons. Red squares are ejectrons that fail RT1 and RT2. The events above the dashed red line are assumed to be nuclear recoils if they pass RT1 and RT2 timing cuts. Right plot: Z2, Soudan run118 anti-coincident low background data in the $2 \sigma$ nuclear recoil band with radius $R \leq 2.7 \mathrm{~cm}$. Blue dots are single scatterings, green circles are multiple scatterings. No nuclear recoils.

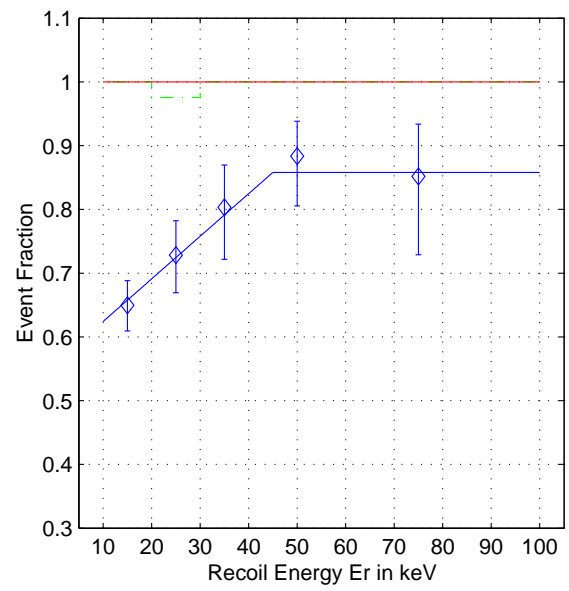

Figure B.8: Z2. The blue line fitted to the data with error bars is neutron selection efficiency in the ${ }^{252} \mathrm{Cf}$ calibration data. The dashed green line is surface event rejection efficiency in the ${ }^{133} \mathrm{Ba}$ data without an ionization yield cut. The top red line is surface event rejection efficiency in the ${ }^{133} \mathrm{Ba}$ data within the $2 \sigma \mathrm{NR}$ band. 

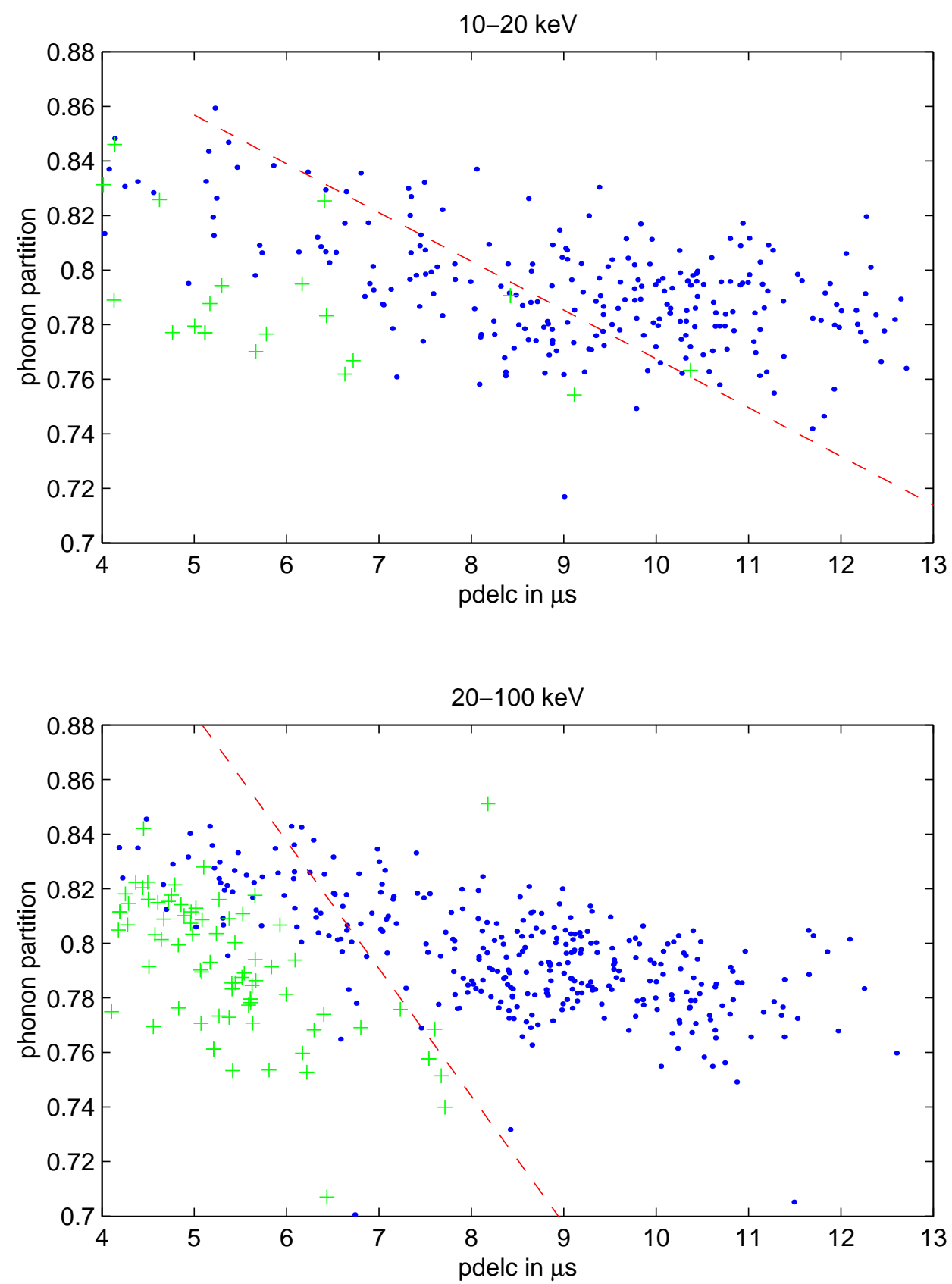

Figure B.9: Z3, phonon partition cut for events with radius $R>2.7 \mathrm{~cm}$. Blue dots are neutrons, green crosses are ejectrons. The top plot is for events that have recoil energy $E r \leq 20 \mathrm{keV}$. The bottom plot is for events that have recoil energy $E r>20 \mathrm{keV}$. The events above the dashed red line are assumed to be nuclear recoil if they pass RT1 and RT2 timing cuts. 

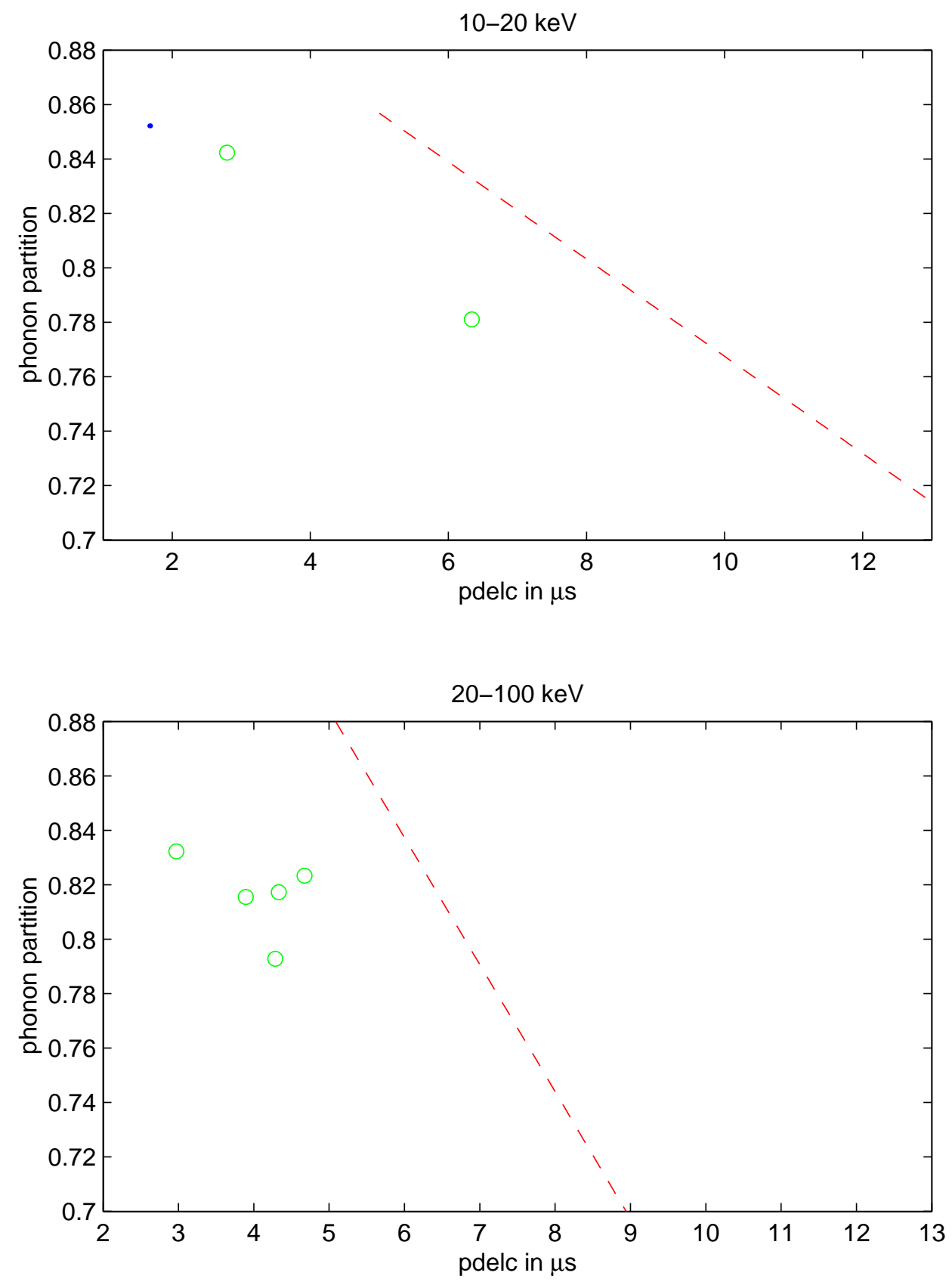

Figure B.10: Z3, Soudan run118 anti-coincident low background data in the $2 \sigma$ nuclear recoil band with radius $R>2.7 \mathrm{~cm}$. Blue dots are single scatterings, green circles are multiple scatterings. The top plot is for events that have recoil energy $E r \leq 20 \mathrm{keV}$. The bottom plot is for events that have recoil energy $E r>20 \mathrm{keV}$. No nuclear recoils. 

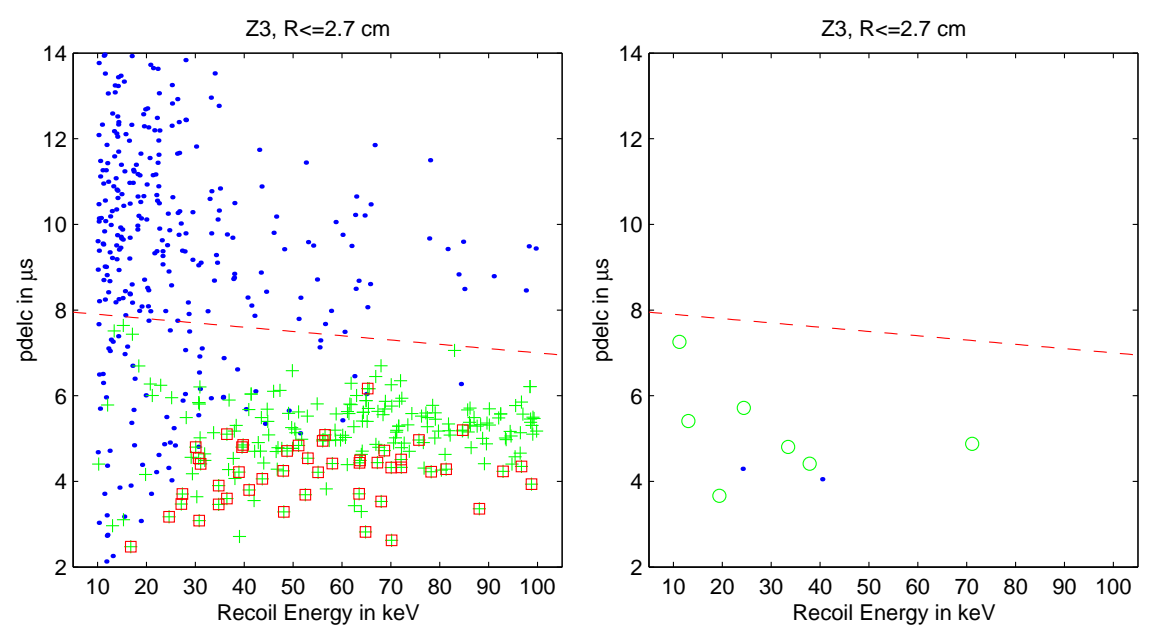

Figure B.11: Left plot: Z3, phonon delay cut for events with radius $R \leq 2.7 \mathrm{~cm}$. Blue dots are neutrons, green crosses are ejectrons. Red squares are ejectrons that fail RT1 and RT2. The events above the dashed red line are assumed to be nuclear recoils if they pass RT1 and RT2 timing cuts. Right plot: Z3, Soudan run118 anti-coincident low background data in the $2 \sigma$ nuclear recoil band with radius $R \leq 2.7 \mathrm{~cm}$. Blue dots are single scatterings, green circles are multiple scatterings. No nuclear recoils.

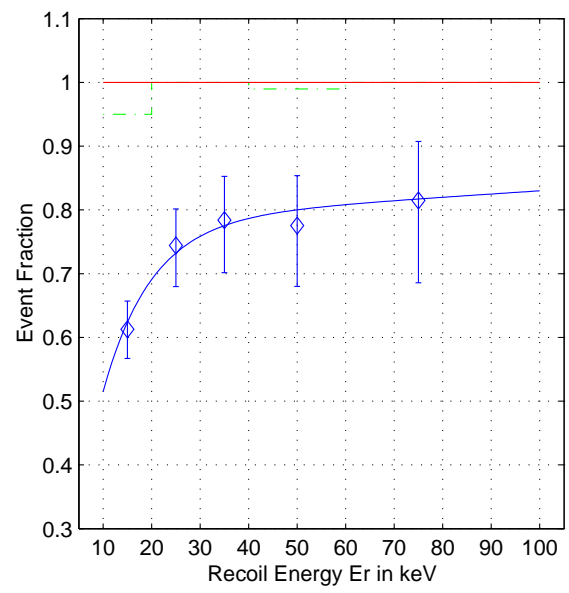

Figure B.12: Z3. The blue line fitted to the data with error bars is neutron selection efficiency in the ${ }^{252} \mathrm{Cf}$ calibration data. The dashed green line is surface event rejection efficiency in the ${ }^{133} \mathrm{Ba}$ data without an ionization yield cut. The top red line is surface event rejection efficiency in the ${ }^{133} \mathrm{Ba}$ data within the $2 \sigma$ NR band. 

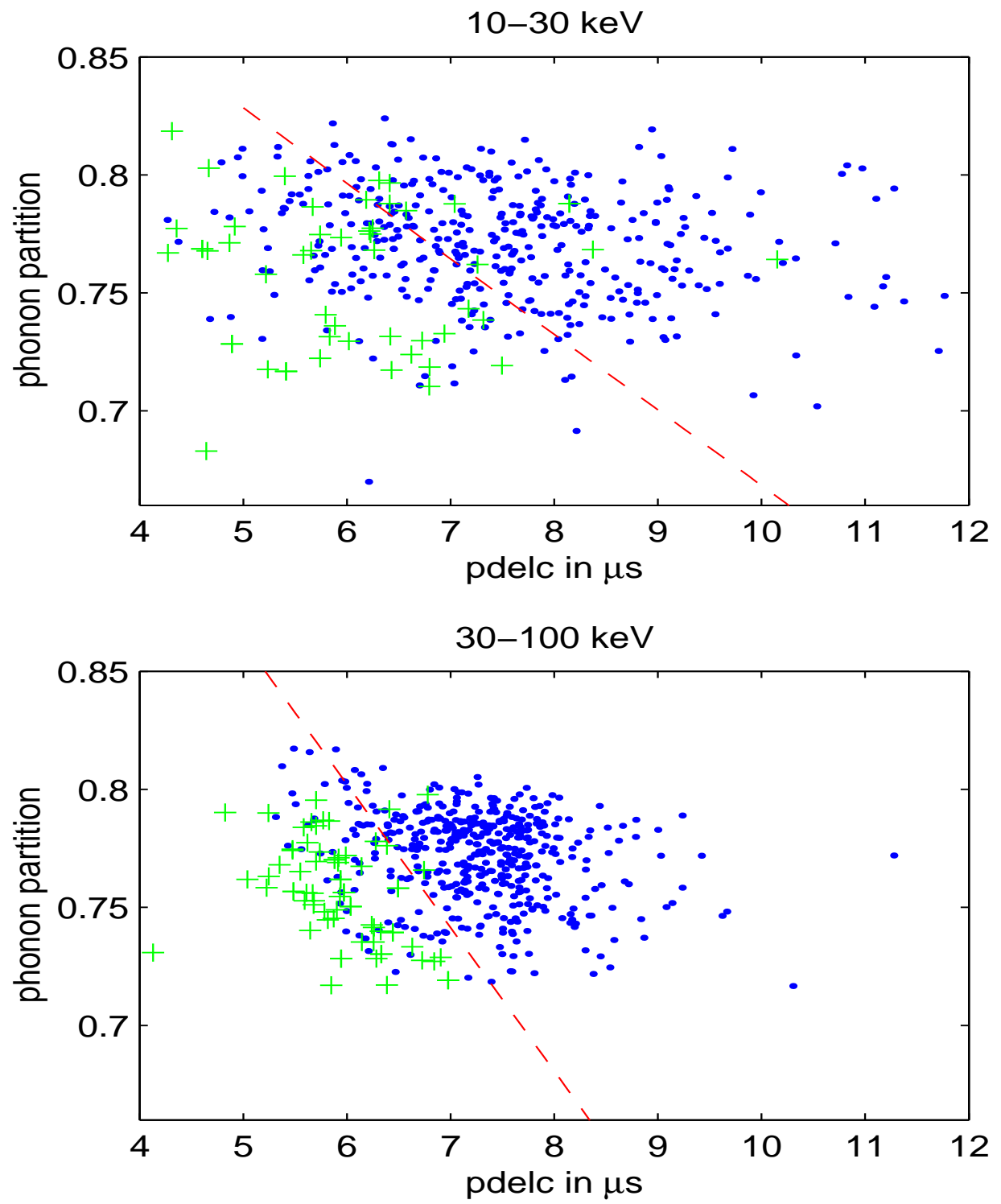

Figure B.13: Z4, phonon partition cut for events with radius $R>2.5 \mathrm{~cm}$. Blue dots are neutrons, green crosses are ejectrons. The top plot is for events that have recoil energy $E r \leq 30 \mathrm{keV}$. The bottom plot is for events that have recoil energy $E r>30 \mathrm{keV}$. The events above the dashed red line are assumed to be nuclear recoil if they pass RT1 and RT2 timing cuts. 

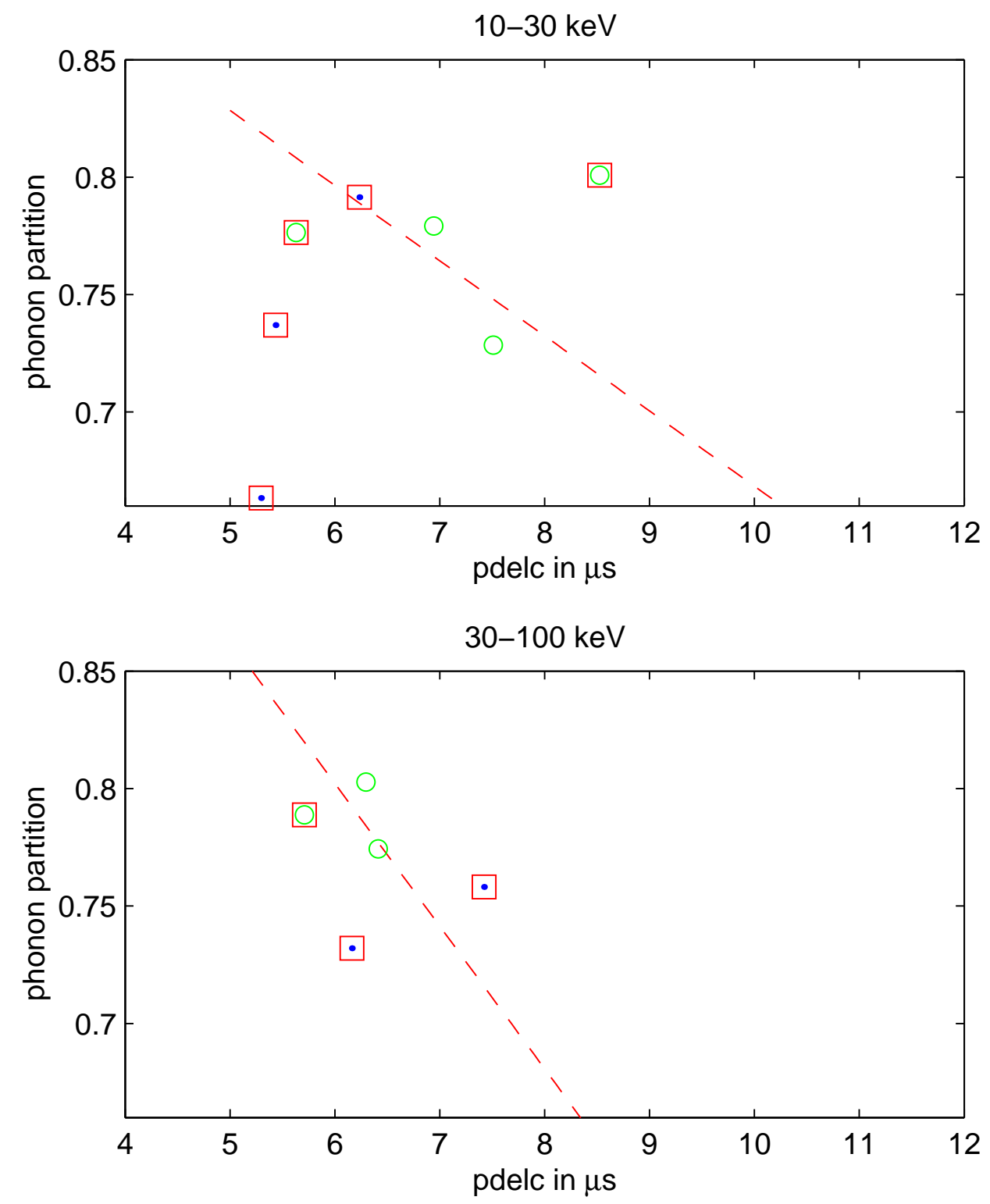

Figure B.14: Z4, Soudan run118 anti-coincident low background data in the $2 \sigma$ nuclear recoil band with radius $R>2.5 \mathrm{~cm}$. Blue dots are single scatterings, green circles are multiple scatterings, red squares are events that fail RT1 and RT2 cuts. The top plot is for events that have recoil energy $E r \leq 30 \mathrm{keV}$. The bottom plot is for events that have recoil energy $E r>30 \mathrm{keV}$. No single scattering nuclear recoils. 

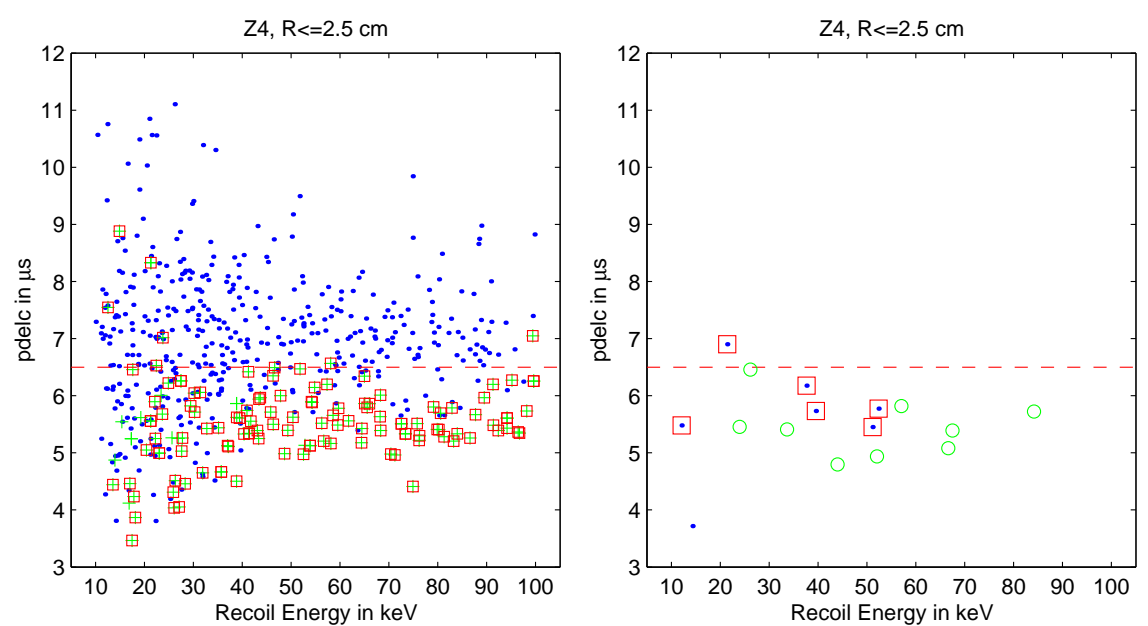

Figure B.15: Left plot: Z4, phonon delay cut for events with radius $R \leq 2.5 \mathrm{~cm}$. Blue dots are neutrons, green crosses are ejectrons. Red squares are ejectrons that fail RT1 and RT2. The events above the dashed red line are assumed to be nuclear recoils if they pass RT1 and RT2 timing cuts. Right plot: Z4, Soudan run118 anti-coincident low background data in the $2 \sigma$ nuclear recoil band with radius $R \leq 2.5 \mathrm{~cm}$. Blue dots are single scatterings, green circles are multiple scatterings, red squares are events that fail RT1 and RT2. No nuclear recoils.

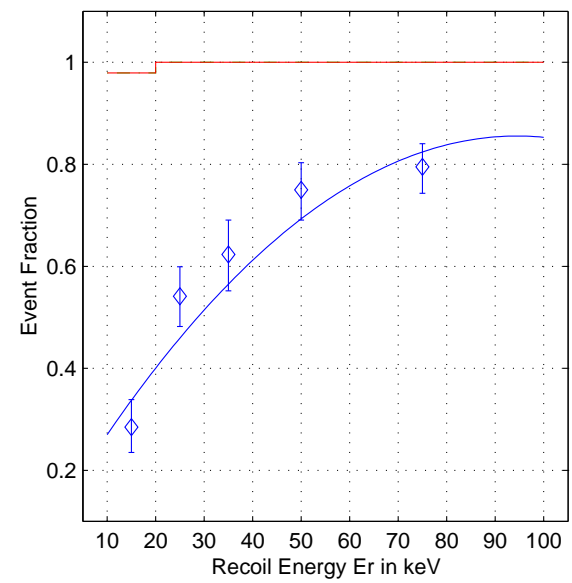

Figure B.16: Z4. The blue line fitted to the data with error bars is neutron selection efficiency in the ${ }^{252} \mathrm{Cf}$ calibration data. The dashed green line is surface event rejection efficiency in the ${ }^{133} \mathrm{Ba}$ data without an ionization yield cut. The top red line is surface event rejection efficiency in the ${ }^{133} \mathrm{Ba}$ data within the $2 \sigma$ NR band. 

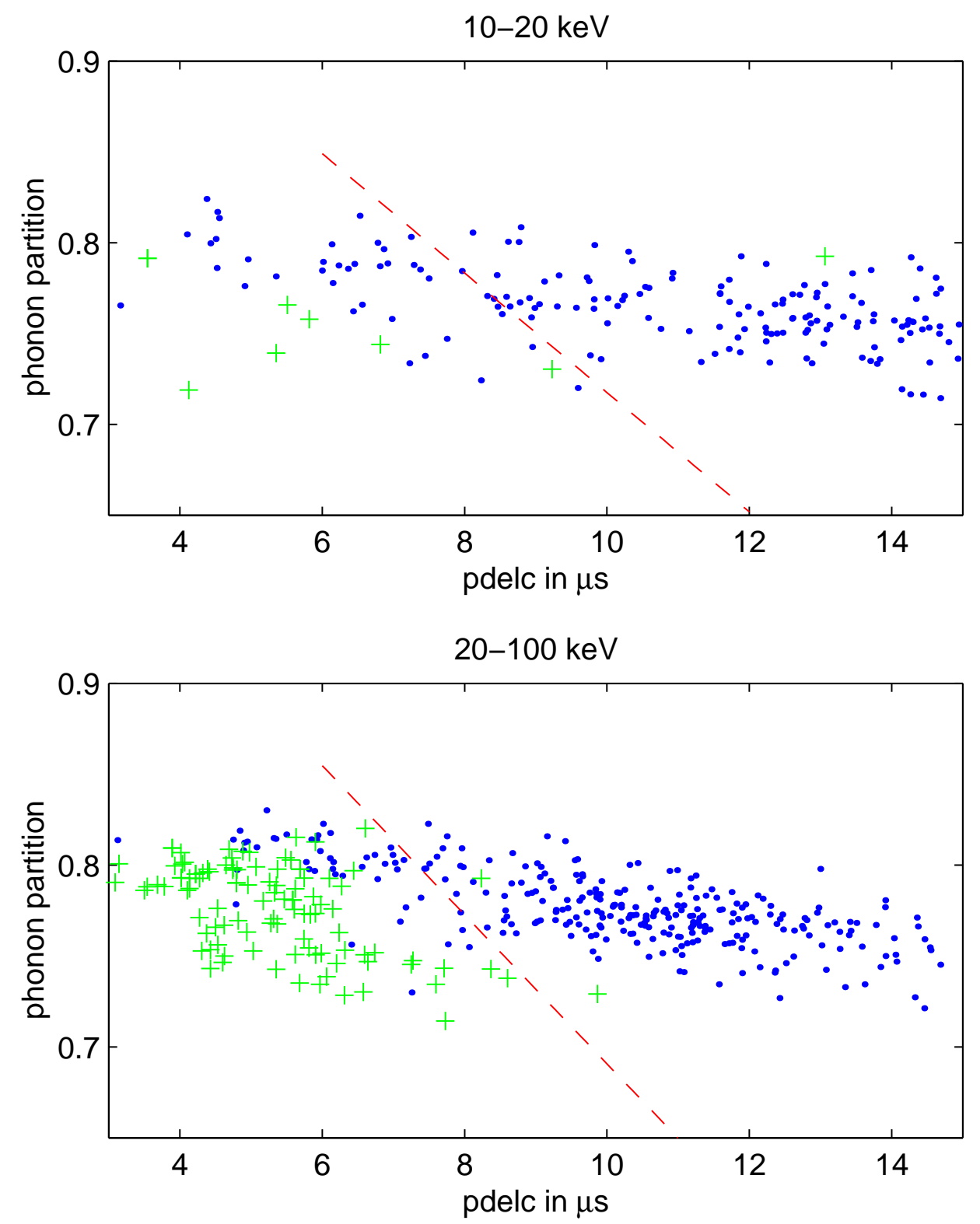

Figure B.17: Z5, phonon partition cut for events with radius $R>2.7 \mathrm{~cm}$. Blue dots are neutrons, green crosses are ejectrons. The top plot is for events that have recoil energy $E r \leq 20 \mathrm{keV}$. The bottom plot is for events that have recoil energy $E r>20 \mathrm{keV}$. The events above the dashed red line are assumed to be nuclear recoils if they pass RT1 and RT2 timing cuts. 

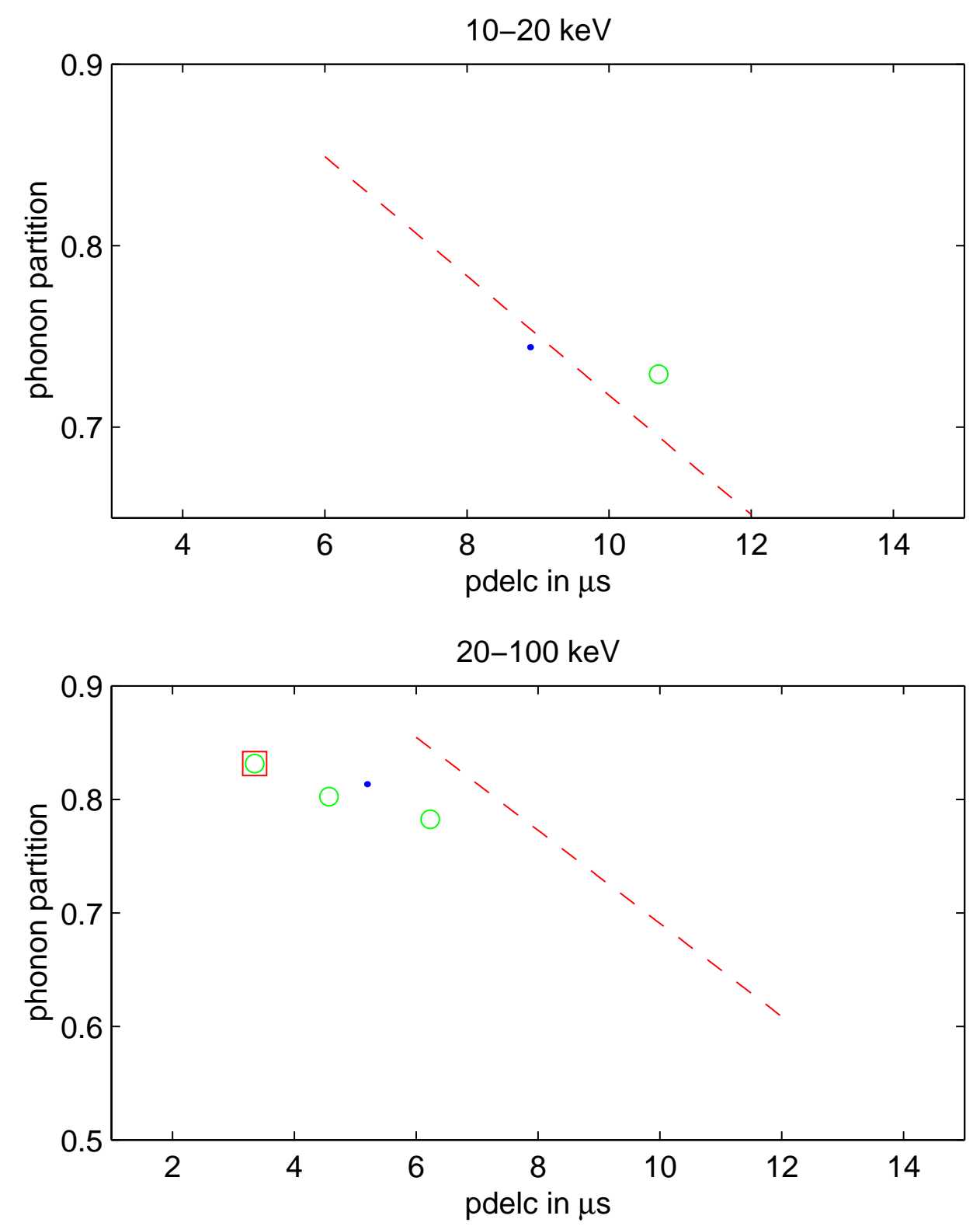

Figure B.18: Z5, Soudan run118 anti-coincident low background data in the $2 \sigma$ nuclear recoil band with radius $R>2.7 \mathrm{~cm}$. Blue dots are single scatterings, green circles are multiple scatterings. The top plot is for events that have recoil energy $E r \leq 20 \mathrm{keV}$. One multiple event is above the surface event cut line. The bottom plot is for events that have recoil energy $E r>20 \mathrm{keV}$. No single scattering nuclear recoils. 

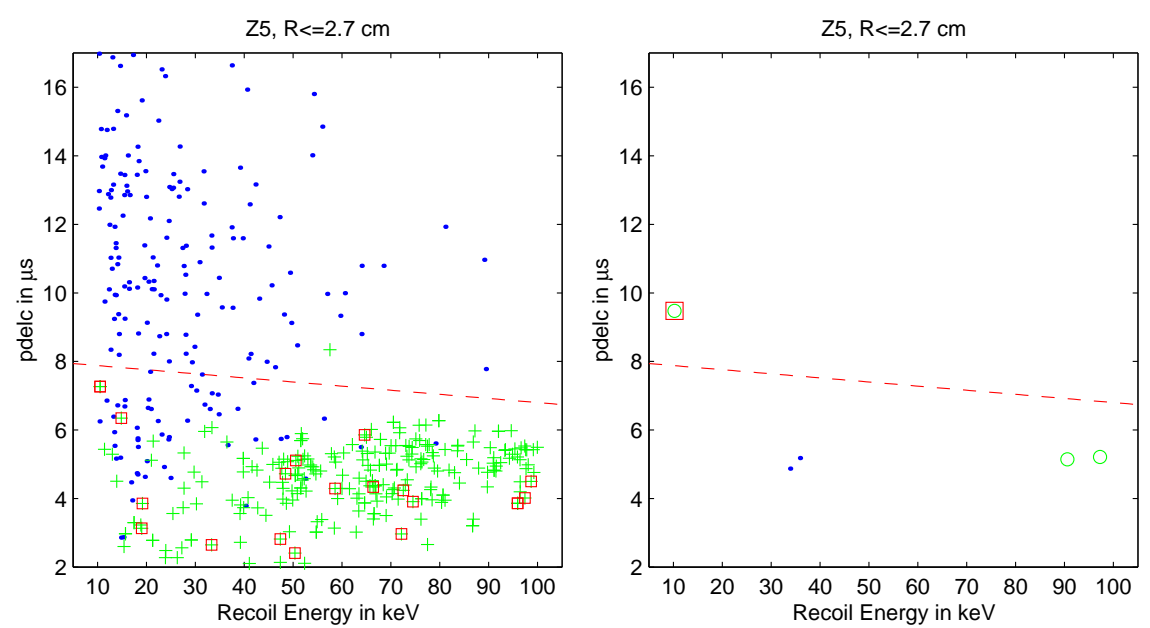

Figure B.19: Left plot: Z5, phonon delay cut for events with radius $R \leq 2.7 \mathrm{~cm}$. Blue dots are neutrons, green crosses are ejectrons. Red squares are ejectrons that fail RT1 and RT2. The events above the dashed red line are assumed to be nuclear recoil if they pass RT1 and RT2 timing cuts. Right plot: Z5, Soudan run118 anti-coincident low background data in the $2 \sigma$ nuclear recoil band with radius $R \leq 2.7 \mathrm{~cm}$. Blue dots are single scatterings, green circles are multiple scatterings. The red square indicates that the nultiple scattering event fails RT1 and RT2 cuts. No nuclear recoils.

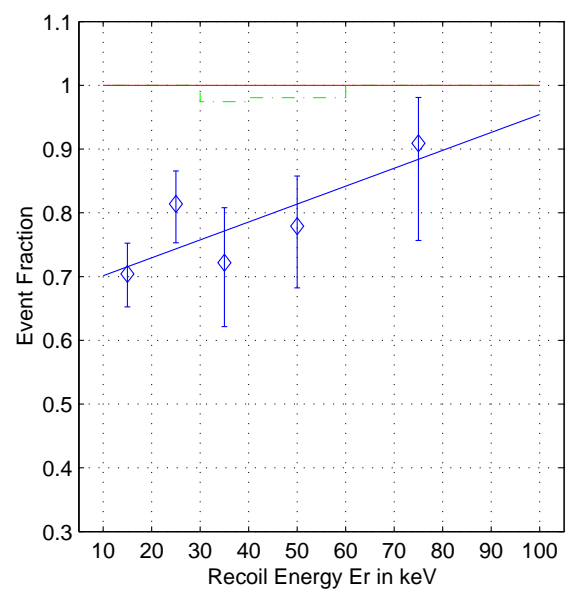

Figure B.20: Z5. The blue line fitted to the data with error bars is neutron selection efficiency in the ${ }^{252} \mathrm{Cf}$ calibration data. The dashed green line is surface event rejection efficiency in the ${ }^{133} \mathrm{Ba}$ data without an ionization yield cut. The top red line is surface event rejection efficiency in the ${ }^{133} \mathrm{Ba}$ data within the $2 \sigma$ NR band. 

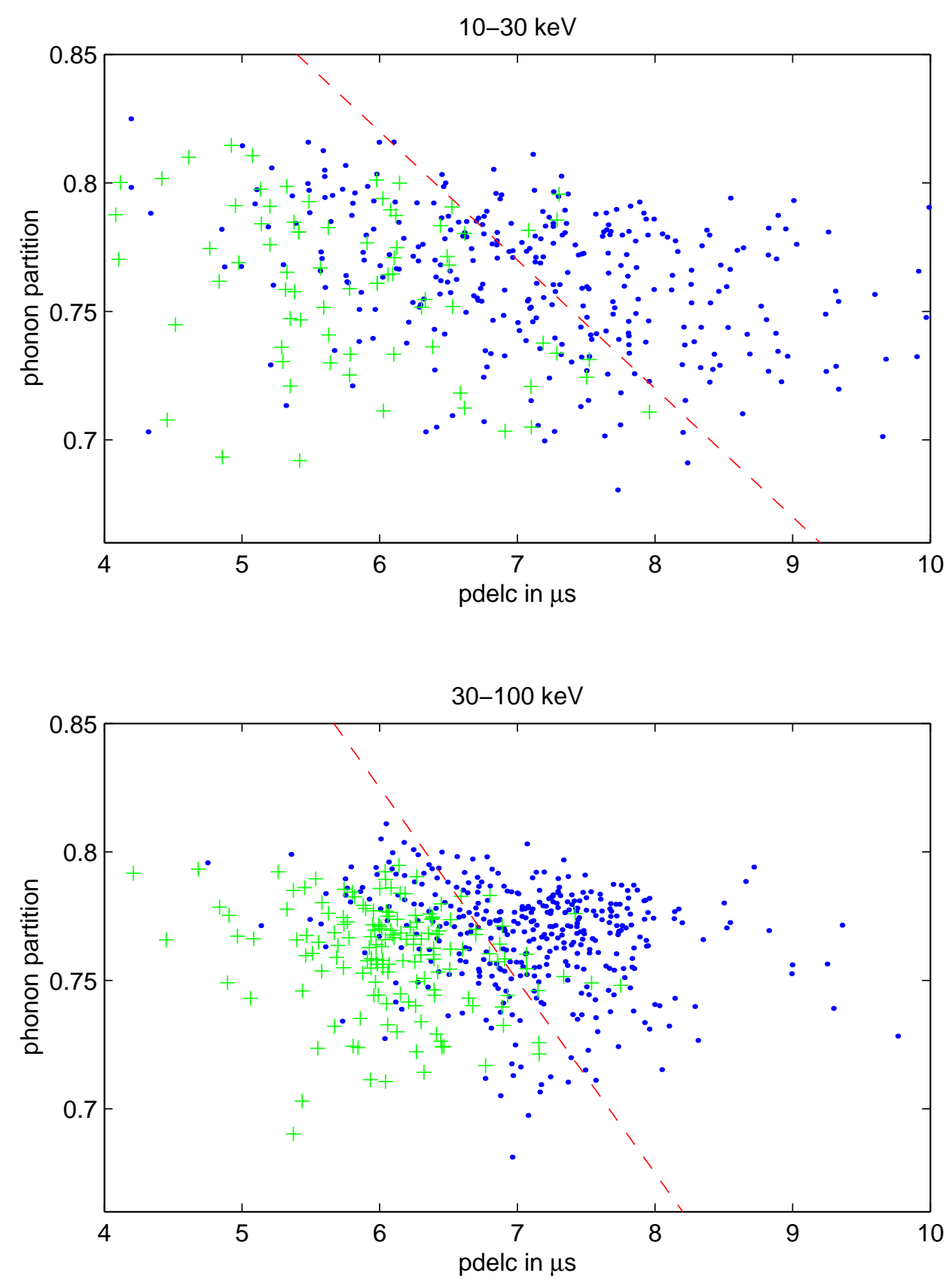

Figure B.21: Z6, phonon partition cut for events with radius $R>2.5 \mathrm{~cm}$. Blue dots are neutrons, green crosses are ejectrons. The top plot is for events that have recoil energy $E r \leq 30 \mathrm{keV}$. The bottom plot is for events that have recoil energy $E r>30 \mathrm{keV}$. The events above the dashed red line are assumed to be nuclear recoil if they pass $\mathrm{RT} 1$ and $\mathrm{RT} 2$ timing cuts. 

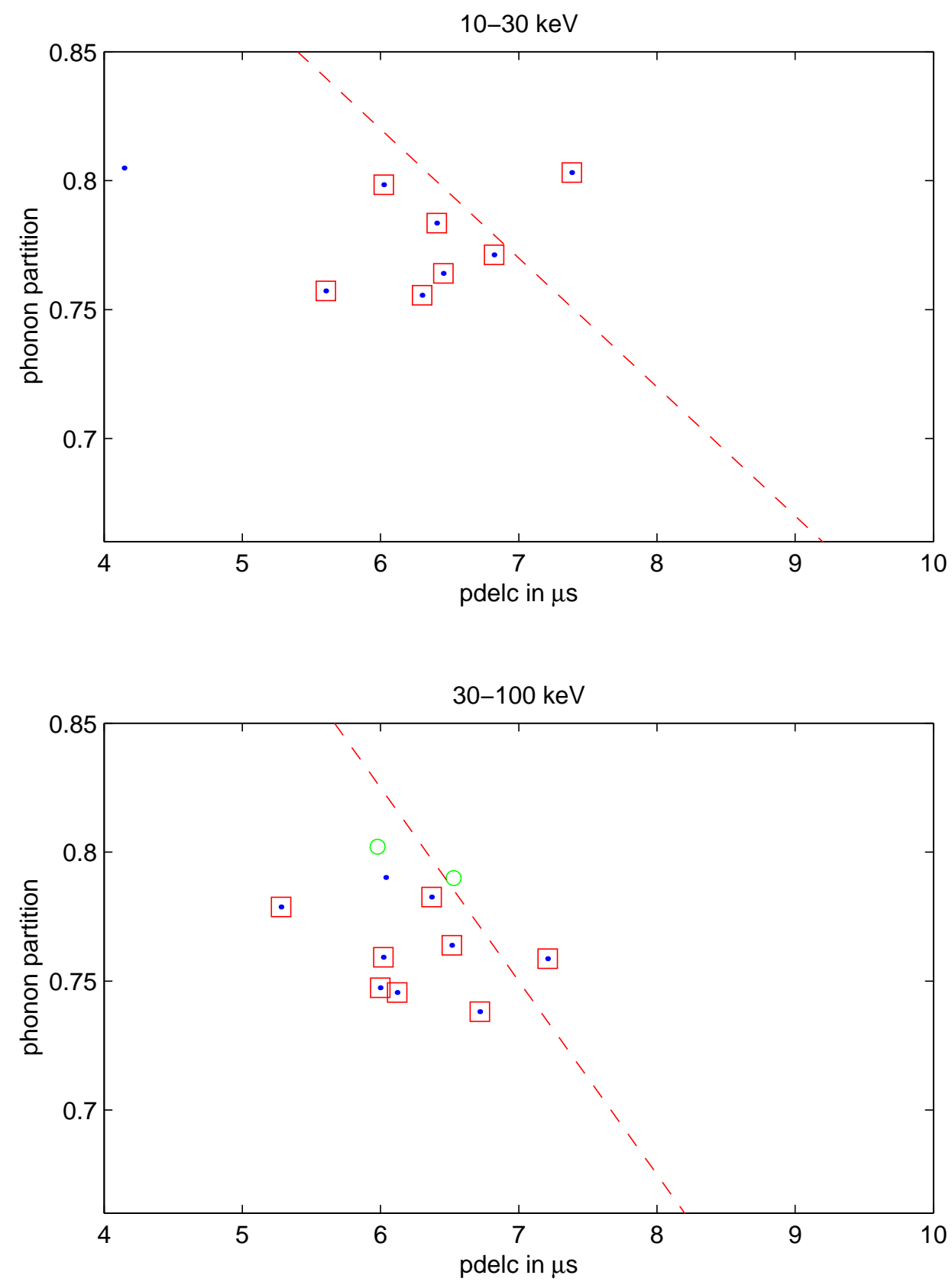

Figure B.22: Z6, Soudan run118 anti-coincident low background data in the $2 \sigma$ nuclear recoil band with radius $R>2.5 \mathrm{~cm}$. Blue dots are single scatterings, green circles are multiple scatterings, red squares are events that fail RT1 and RT2 cuts. The top plot is for events that have recoil energy $E r \leq 30 \mathrm{keV}$. The bottom plot is for events that have recoil energy $E r>30 \mathrm{keV}$. No single scattering nuclear recoils. 

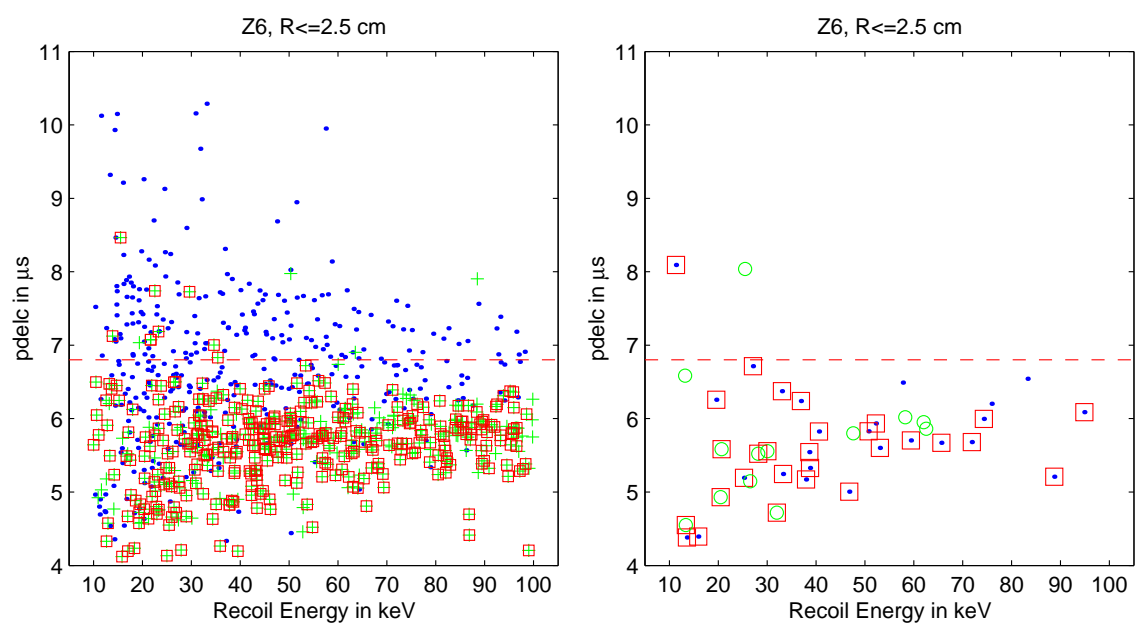

Figure B.23: Left plot: Z6, phonon delay cut for events with radius $R \leq 2.5 \mathrm{~cm}$. Blue dots are neutrons, green crosses are ejectrons. Red squares are ejectrons that fail RT1 and RT2. The events above the dashed red line are assumed to be nuclear recoil if they pass RT1 and RT2 timing cuts. Right plot: Z6, Soudan run118 anticoincident low background data in the $2 \sigma$ nuclear recoil band with radius $R \leq 2.5$ $\mathrm{cm}$. Blue dots are single scatterings, green circles are multiple scatterings, red squares are events that fail RT1 and RT2 cuts. No single scattering nuclear recoils.

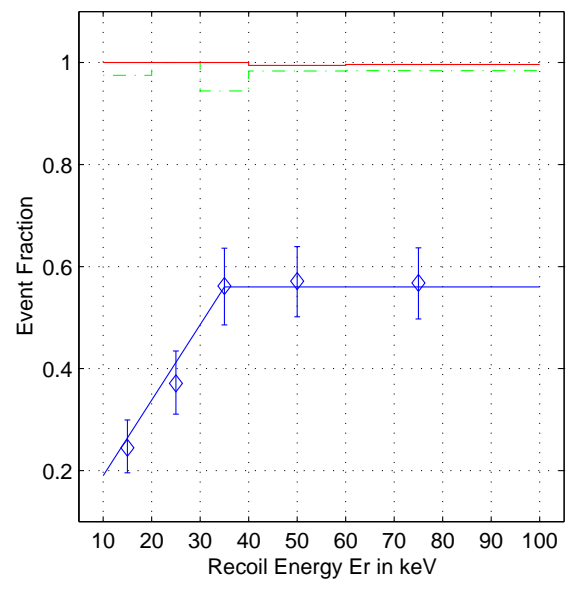

Figure B.24: Z6. The blue line fitted to the data with error bars is neutron selection efficiency in the ${ }^{252} \mathrm{Cf}$ calibration data. The dashed green line is surface event rejection efficiency in the ${ }^{133} \mathrm{Ba}$ data without an ionization yield cut. The top red line is surface event rejection efficiency in the ${ }^{133} \mathrm{Ba}$ data within the $2 \sigma$ NR band. 


\section{Appendix C}

\section{The Efficiency}

This appendix lists the nuclear recoil selection efficiencies for the following cuts:

1. Data quality (cChiSqS and cPstd) cuts

2. Charge inner electrode(cQThesS) cut

3. Charge energy threshold (cQThesS) cut

4. Surface event rejection cuts

5. Nuclear recoil band cut

The event preselection conditions are described in the captions with the plots. The event selection efficiency after veto cut is counted as 0.97 .

The cut efficiencies are calculated in the following energy bins: $5-10 \mathrm{keV}, 10-20$ $\mathrm{keV}, 20-30 \mathrm{keV}, 30-40 \mathrm{keV}, 40-50 \mathrm{keV}, 50-60 \mathrm{keV}, 60-70 \mathrm{keV}$, and 70-100 keV. The average nuclear recoil selection efficiency of the four germanium detectors (Z1, Z2, Z3, and Z5) is summarized in chapter 8.1. 

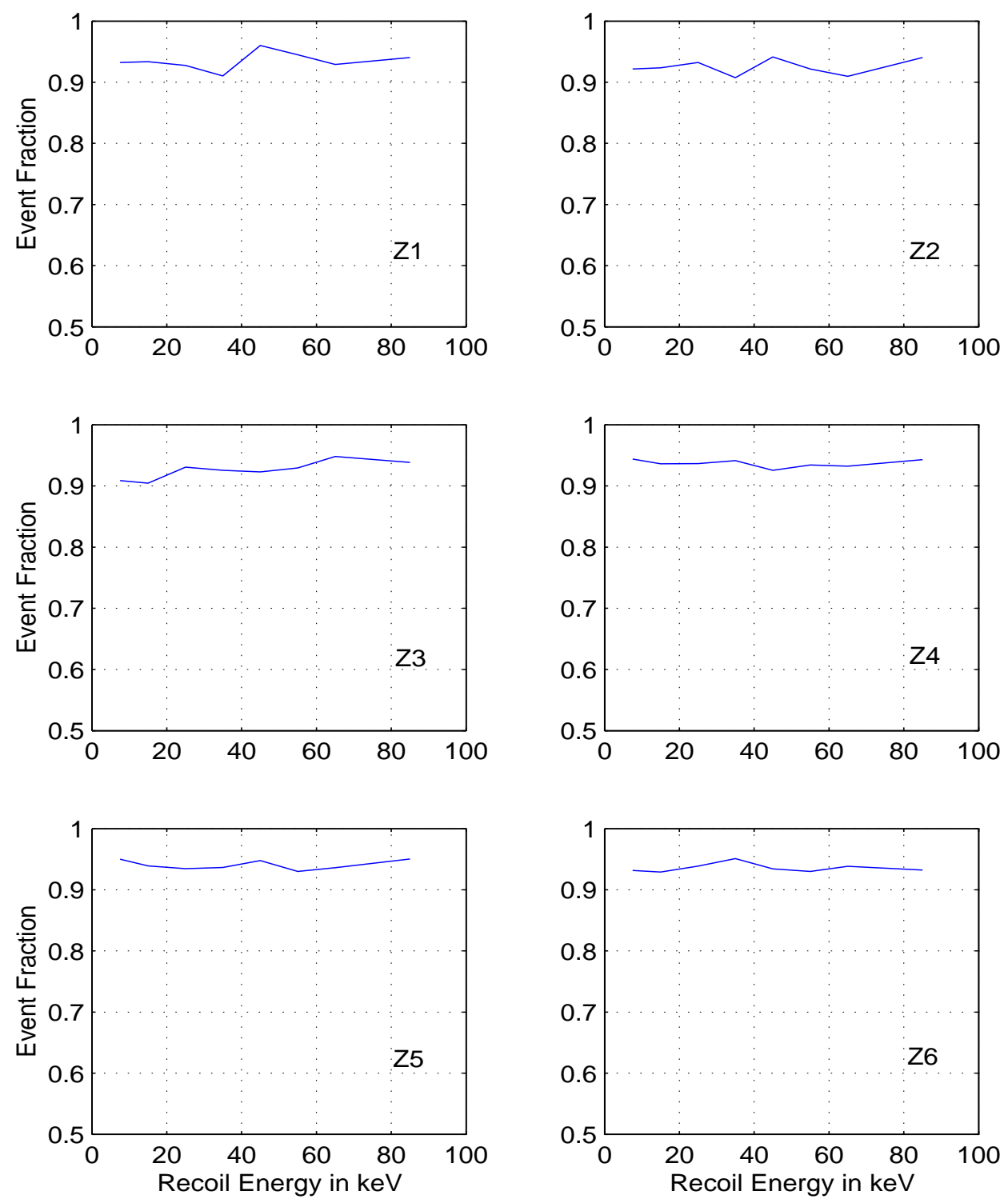

Figure C.1: Efficiency of data quality cuts. Low background events in the gamma band of the 52.6 live days are used for the efficiency calculation. Before the data quality cuts, the events are selected by applying the cuts: cBad \& cQThresS \& cQinS \& cERS. 

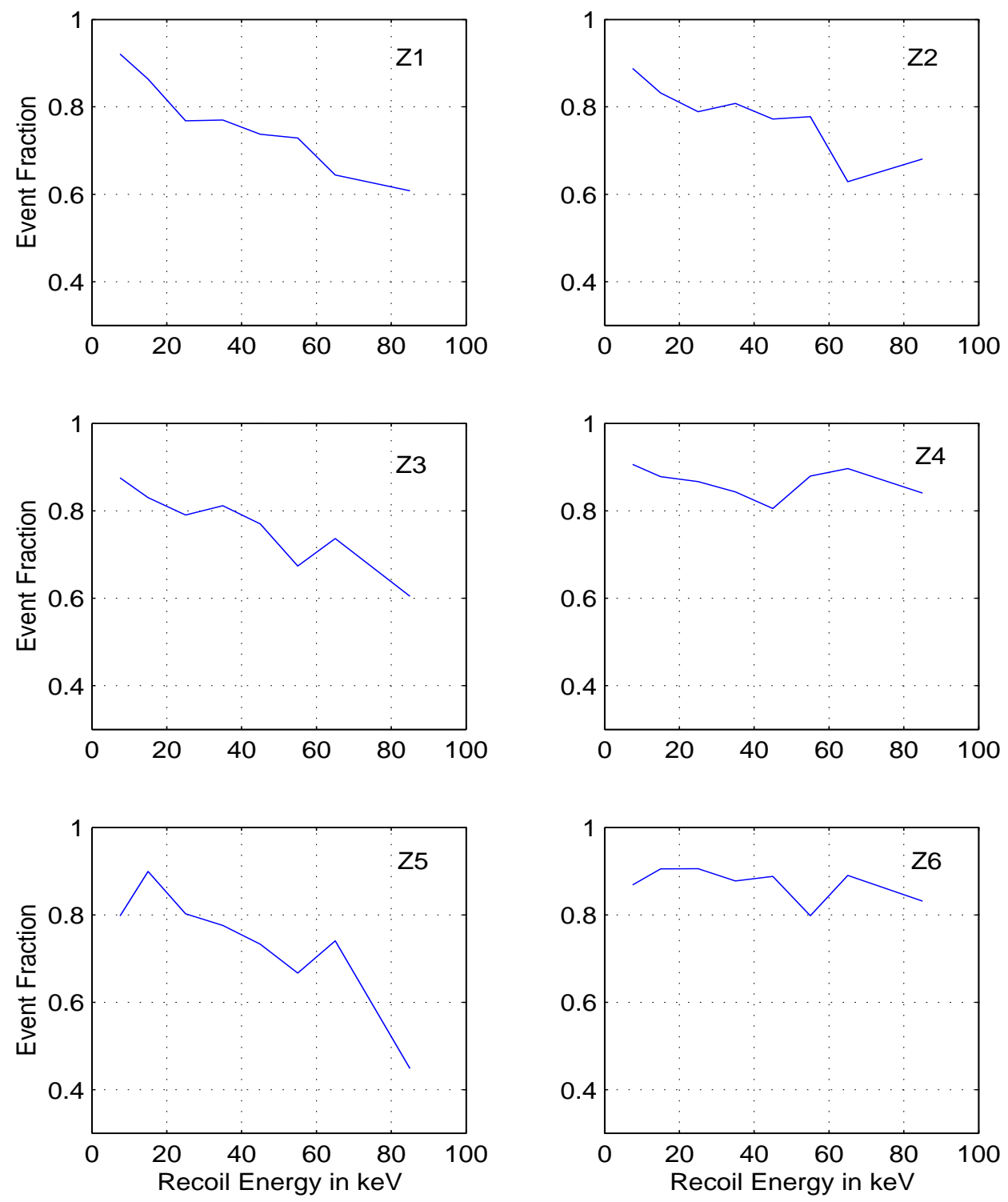

Figure C.2: Efficiency of charge inner electrode cut. Neutrons in the $2 \sigma$ nuclear recoil band in the ${ }^{252} \mathrm{Cf}$ calibration are used for the efficiency calculation. Before the charge inner electrode cut, the events are selected by applying the cuts: cGoodEvS1004 \& cNRS \& cQThresS. 

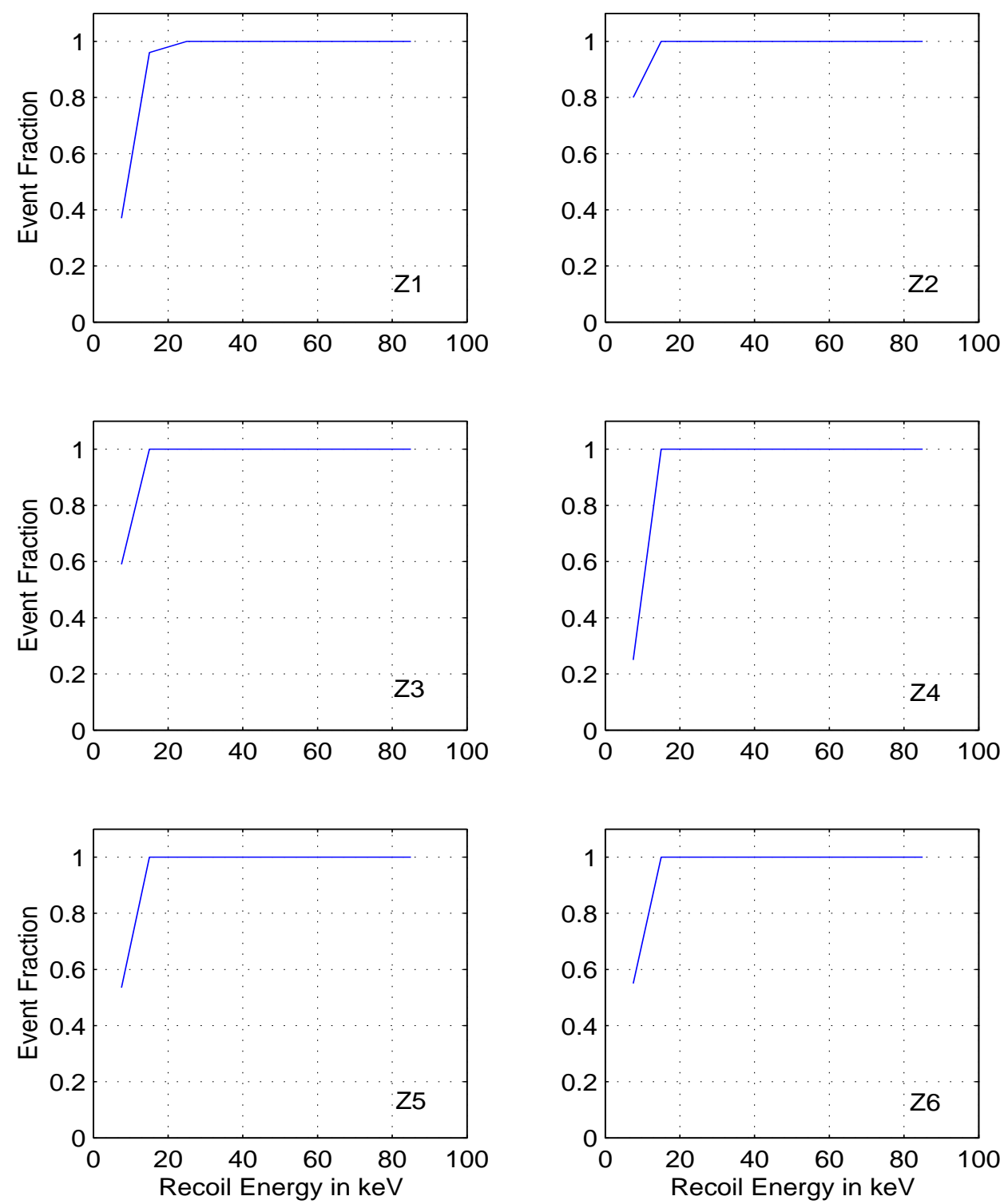

Figure C.3: Efficiency of charge energy threshold cut. Neutrons in the $2 \sigma$ nuclear recoil band in the ${ }^{252} \mathrm{Cf}$ calibration data are used for the efficiency calculation. Before the charge energy threshold cut, the events are selected by applying the cuts: cChiSqS \& cPstd \& cQinS \& cNRS. 

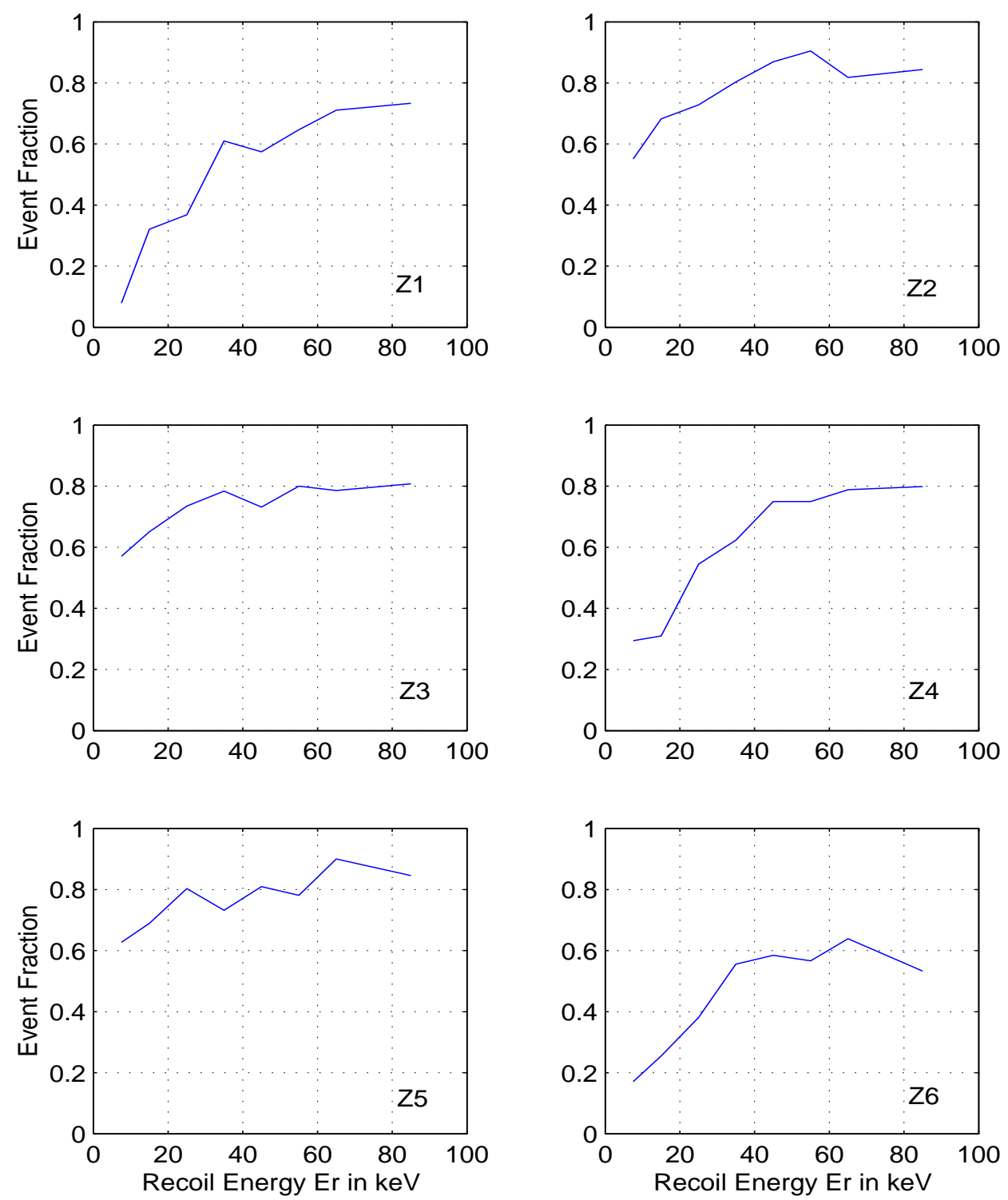

Figure C.4: Efficiency of surface event rejection cuts. The cuts are described in section 7.5. Neutrons in the $2 \sigma$ nuclear recoil band in the ${ }^{252} \mathrm{Cf}$ calibration data are used for the efficiency calculation. Before the surface event rejection cuts, the events are selected by applying the cuts: cGoodEvS1004 \& cNRS \& cQThresS \& cQinS. 

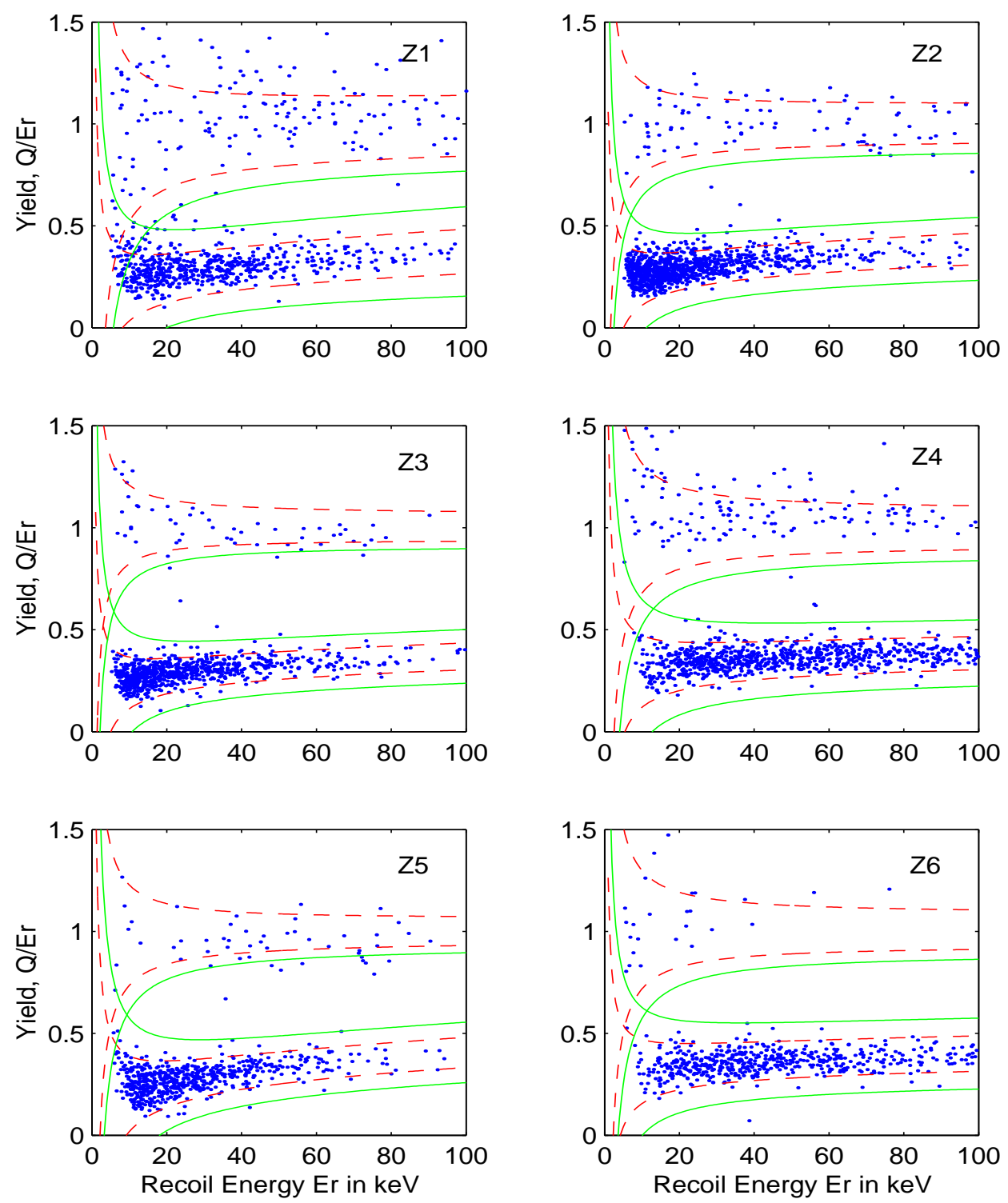

Figure C.5: Before the nuclear recoil band cut, the events are selected by applying the cuts: cGoodEvS1004 \& cQThresS \& cQinS \& surface event rejection cuts as described in section 7.5. The events that pass the preselection cuts in the $4 \sigma$ nuclear recoil band and below the $3 \sigma$ electron recoil band are assumed to be neutrons. 

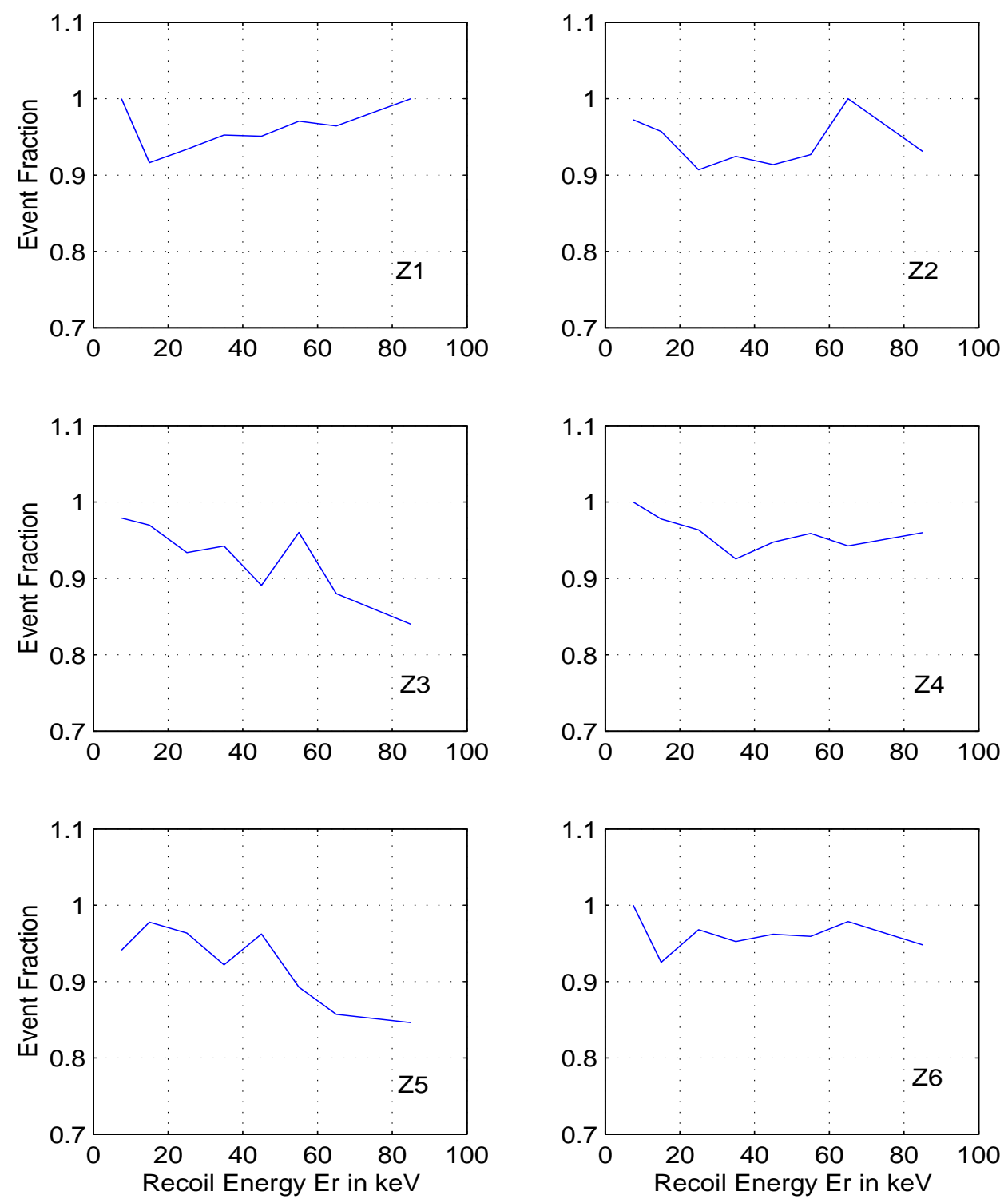

Figure C.6: Efficiency of nuclear recoil band cut with ${ }^{252} \mathrm{Cf}$ calibration data. For a given energy bin, the number of neutrons in the $2 \sigma$ nuclear recoil band divided by the number of neutrons in the $4 \sigma$ nuclear recoil band and below the $3 \sigma$ gamma band (see Figure C.5) is the nuclear band cut efficiency. 

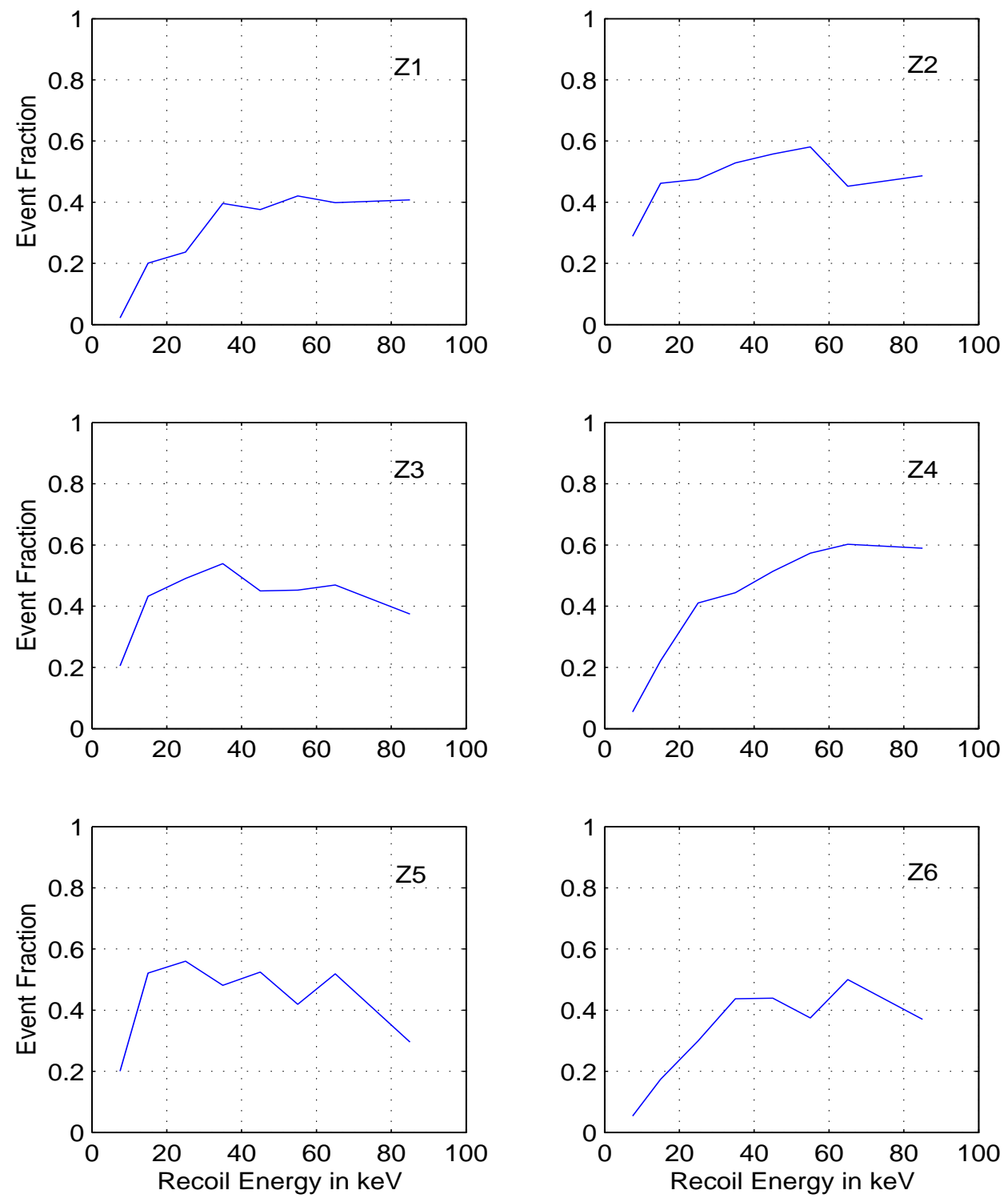

Figure C.7: Efficiency of nuclear recoil selection. It includes data quality cuts, charge inner electrode cut, surface event rejection cuts, nuclear recoil band, and veto cut. The event selection efficiency after veto cut is 0.97 . 


\section{Bibliography}

[1] Barbara Ryden. Introduction to Cosmology. Addison Wesley, 2003.

[2] J. A. Peacock. Cosmological Physics. Cambridge University Press, 1999.

[3] W. L. Freedman and M. S. Turner. Measuring and understanding the universe. Reviews of Modern Physics, 75:1443, 2003.

[4] W. L. Freedman et al. Final results from the hubble space telescope key project to measure the hubble constant. Astrophysical Journal, 553:47, 2001.

[5] A. G. Riess et al. Type ia supernova discoveries at $z_{i} 1$ from the hubble space telescope: Evidence for past deceleration and constraints on dark energy evolution. astro-ph/0402512, 2004.

[6] S. Perlmutter et al. Measurements of omega and lambda from 42 highredshift supernovae. Astrophysical Journal, 517:565, 1999.

[7] A. G. Riess et al. Observational evidence from supernovae for an accelerating universe and a cosmological constant. Astronomical Journal, 116:1009, 1998.

[8] C. L. Bennettand others. First year wilkinson microwave anisotropy probe (wmap) observations: Preliminary maps and basic results. Astrophysical Journal Supplement, 148:1, 2003.

[9] E. M. Leitch et al. Measurement of polarization with the degree angular scale interferometer. Nature, 420:763, December 2002.

[10] D. N. Spergel et al. First year wilkinson microwave anisotropy probe (wmap) observations: Determination of cosmological parameters. Astrophyical Journal Supplement, 148:175, 2003. astro-ph/0302209.

[11] K. A. Olive. Tasi lectures on dark matter. astro-ph/0301505, 2003.

[12] M. Tegmark et al. Cosmological parameters from sdss and wmap. Physical Review D, 69:10350, 2004.

[13] D. Tytler et al. Review of big bang nucleosynthesis and primordial abundance. Physica Script, T85:12, 2000. 
[14] D. Tytler et al. The deuterium abundance at $\mathrm{z}=0.701$ toward qso 1718+4807. Astronomical Journal, 117:63, 1999.

[15] J. A. Peacock et al. A measurement of the cosmological density from clustering in the 2 df galaxy redshift survey. Nature, 410:167, 2001.

[16] J. A. Peacock et al. Studying large-scale structure with the 2df galaxy redshift survey. astro-ph/0204239, 2002.

[17] M. Tegmark and M. Zaldarriaga. Separating the early universe from the late universe: cosmological parameter estimation beyond the black box. Physical Review D, 66:103508, 2002.

[18] S. W Allen et al. Cosmological constraints from the x-ray gas mass fraction in relaxed lensing clusters observed with chandra. Monthly Notice to Royal Astronomy Society, 334:L11, 2002.

[19] S. D. M. White et al. The baryon content of galaxy clusters: a challenge to cosmological orthodoxy. Nature, 366:429, 1993.

[20] D. Clowe et al. Weak lensing mass reconstruction of the interacting cluster 1e0657-558: Direct evidence for the existence of dark matter. Astrophyscal Journal, 604:569, 2004.

[21] L. Grego et al. Galaxy cluster gas mass fractions from sunyaev-zel'dovich effect measurements: Constraints on $\omega_{m}$. Astrophysical Journal, 552:2, 1002.

[22] K. G. Begeman, A. H. Broeils, and R. H. Sanders. Extended rotation curves of spiral galaxies: dark haloes and modified dynamics. Monthly Notices of the Royal Astronomical Society, 249:523, 1991.

[23] F. F. Navarro. The inner density cusp of cold dark matter halos. astro$\mathrm{ph} / 0110680,2001$.

[24] R. P. Olling and M. R. Merrifield. Two measures of the shape of the milky way's dark halo. astro-ph/9907353, 1999.

[25] L. Bergstrom et al. Observability of gamma rays from dark matter neutralino annihilations in the milky way halo. Astroparticle Physics, 9:137, 1998. hep$\mathrm{ph} / 9704384$.

[26] J. N. Bahcall et al. The galactic spheroid. Astrophysical Journal, 265:730, 1983.

[27] R. R. Caldwell and J. P. Ostriker. The mass distribution within our galaxy: a three component model. Astrophysical Journal, 251:61, 1981.

[28] M. S. Turner. Cosmic and local mass density of 'invisible' axions. Physical Rview D, 33:889, 1986. 
[29] B. Sadoulet. Deciphering the nature of dark matter. Reviews of Modern Physics, 71:97, 1999.

[30] Y. Fukuda et al. Evidence for oscillation of atmospheric neutrinos. Physical Review Letters, 81:1562, 1998.

[31] Q. R. Ahmad et al. Direct evidence for neutrino flavor transformation from neutral-current interactions in the sudbury neutrino. Physical Review Letters, 89:11301, 2002.

[32] K. Hagiwara et al. Review of particle physics. Physical Review D, 66:10001, 2002 .

[33] P. Gondolo. Introduction to non-baryonic dark matter. astro-ph/0403064, March 2004.

[34] D. N. Spergel et al. First-year wilkinson microwave anisotropy probe (wmap) observations: Determination of cosmological parameters. Astrophysical Journal Supplement, 148:175, 2003.

[35] J. R. Bond et al. Massive neutrinos and the large-scale structure of the universe. Physical Review Letters, 45(24):1980-3, December 1980.

[36] H. Goldberg. Constraint on the photino mass from cosmology. Physical Review Letters, 50:1419, 1983.

[37] L. M. Krauss. New constraints on 'ino' masses from cosmology. i. supersymmetric 'inos'. Nuclear Physics B, Particle Physics, B227:556, 1983.

[38] J. R. Ellis et al. Supersymmetric relics from the big bang. Nuclear Physics $B, 238: 453,1984$.

[39] G. Jungman M. Kamionkowski and K. Griest. Supersymmetric dark matter. Physics Reports, 267:195, 1996.

[40] B. W. Lee and S. Weinberg. Cosmological lower bound on heavy-neutrino masses. Physical Review Letters, 39:165, 1977.

[41] J. Edsjo and P. Gondolo. Neutralino relic density including coannihilations. Physical Review D, 56:1879, 1997.

[42] J. Edsjo. Aspects of Neutrino Detection of Neutralino Dark Matter. PhD thesis, Uppsala University, Fall 1997. hep-ph/9704384.

[43] R. D. Peccei and H. R. Quinn. Cp conservation in the presence of instantons. Physical Review Letters, 38:1440, 1977.

[44] J. E. Kim. Weak interaction singlet and strong cp invariance. Physical Review Letters, 43:103, 1979.

[45] M. A. Shifman et al. Can confinement ensure natural cp invariance of strong interactions? Nuclear Physics B, 166:493, 1980. 
[46] A. R. Zhitnitsky. On possible suppression of the axion hadron interactions. Soviet Journal of Nuclear Physics, 31:260, 1980.

[47] S. J. Asztalos et al. An improved rf cavity search for halo axions. Physical Review D, 69:011101, 2004.

[48] L. E. Ibanez. The scalar neutrinos as the lightest supersymmetric particles and cosmology. Physics Letter B, 137:160, 1984.

[49] T. Falk et al. Heavy sneutrinos as dark matter. Physics Letter B, 339:248, 1994.

[50] S. P. Ahlen et al. Limits on cold dark matter candidates from an ultralow background germanium spectrometer. Physics Letter B, 195:603, 1987.

[51] H. Pagels and J. R. Primack. Supersymmetry, cosmology and new tev physics. Physical Review Letters, 48:223, 1982.

[52] E. W. Kolb et al. Wimpzillas. astro-ph/9810361, 1998.

[53] G. Servant and T. M. P. Tait. Is the lightest kaluza-klein particle a viable dark matter candidate? Nuclear Physics B, 650:391, 2003.

[54] S. Raby and G. B. P. West. Detection of galactic halo magninos via their coherent interaction with heavy nuclei. Physics Letters B, 202:47, 1988.

[55] G. Gelmini et al. Neutralino dark matter searches. Nuclear Physics B, 313:623, 1991.

[56] M. Srednicki and R. Watkins. Coherent couplings of neutralinos to nuclei from squark mixing. Physics Letters B, 225:149, 1989.

[57] M. Drees and M. M. Nojiri. Neutralino relic density in minimal $n=1$ supergravity. Physical Review D, 47:379, 1993.

[58] C. Munos. Dark matter detection in the light of recent experimental results. International Journal of Modern Physics A, 2003. astro-ph/0309346.

[59] H. Baer and M. Brhlik. Neutralino dark matter in minimal supergravity: Direct detection vs. collider searches. Physical Review D, 58:015007, 1998.

[60] V. A. Bednyakov and H. V. Klapdor-Kleingrothaus. Update of the direct detection of dark matter and the role of the nuclear spin. Physical Review $D, 63: 095005,2001$.

[61] P. Nath and R. Arnowitt. Supersymmetric dark matter. hep-ph/9610460, 1996.

[62] U. Chattopadhyay et al. Wmap constraints, susy dark matter and implications for the direct detection of susy. Physical Review D, 68:035005, 2003. 
[63] J. D. Lewin and P. F. Smith. Review of mathematics, numerical factors, and corrections for dark matter experiments based on elastic nuclear recoil. Astroparticle Physics, 6:87-112, 1996.

[64] C. J. Copi and L. M. Krauss. Angular signatures for galactic halo wimp scattering in direct detectors: Prospects and challenges. Physical Review D, 63:043507, 2001.

[65] D. P. Snowden-Ifft et al. Low pressure negative ion drift chamber for dark matter search. Physical Review D, 61:101301, 2000.

[66] R. Bernabei. Dark matter search. astro-ph/0307403, 2003.

[67] F. Hasenbslg et al. Cold dark matter identification: Diurnal modulation reexamined. Physical Review D, 55:7350, 1997.

[68] J. Ahrens et al. Search for extraterrestrial point sources of neutrinos with amanda-ii. Physical Review Letters, 92(7):071102, February 2004.

[69] V. I. Lyashuk. Antares-deep undersea neutrino observatory: status report. Physics of Atomic Nuclei, 66(3):509, March 2003.

[70] S. Yoshida et al. Propagation of extremely high energy leptons in earth: implications for their detection by the icecube neutrino telescope. Physical Review D, 69(15):103004, May 2004.

[71] S. Desai et al. Search for dark matter wimps using upward through-going muons in super-kamiokande. hep-ex/0404025, 2004.

[72] A. Cesarini et al. The galactic centeras a dark matter gamma-ray source. Astroparticle Physics, 21:267, 2004. astro-ph/0305075.

[73] D. Hooper and B. Dingus. Improving the angular resolution of egret and new limits on supersymmetric dark matter near the galactic center. astroph/0212509, 2002.

[74] L. Baudis et al. New limits on dark-matter weakly interacting particles from the heidelberg-moscow experiment. Physical Review D, 59:022001, 2000.

[75] A. Morales et al. New constraints on wimps from the canfranc igex dark matter search. Physics Letter B, 489:268, 2000.

[76] CDMS Collaboration. Cdms status and roadmap. April 2005.

[77] Eric J. Weinberg and D.L. Nordstrom, editors. Particles and Fields. American Physical Society, 1993.

[78] A.J. da Silva. Development of a Low Background Environment for the Cryogenic Dark Matter Search. PhD thesis, The University of British Columbia, April 1997. 
[79] Sunil R. Golwala. Exclusion Limits on the WIMP-Nucleon ElasticScattering Cross Section from the Cryogenic Dark Matter Search. PhD thesis, The University of California at Berkeley, Fall 2000.

[80] T. A. Perera. The Limiting Background in a Dark Matter Search at Shallow Depth. PhD thesis, Case Western Reserve University, January 2002.

[81] T. Saab. Search for Weakly Interacting Massive Particles with the Cryogenic Dark Matter Search Experiment. PhD thesis, Stanford University, August 2002 .

[82] D.S. Akerib et al. New results from the cryogenic dark matter search experiment. Physical Review D, 68, October 2002.

[83] S. Kamat. Neutrons at soudan. CDMS run118 ebook \#64, November 2003.

[84] R. Alig et al. Scattering by ionization and phonon emission in semiconductors. Physical Review B, 22:5565, 1980.

[85] W. E. Drummond and J. L. Moll. Hot carriers in si and ge radiation detectors. Journal of Applied Physics, 42:5556, 1971.

[86] D. Abrams et al. Exclusion limits on the wimp-nucleon cross section from the cryogenic dark matter search. Physical Review D, 66, 2002.

[87] B. Neganov and V. Trofimov. Otkrytia, Izobreteniya, 146:215, 1985.

[88] P. N. Luke. Journal of Applied Physics, 64:6858, 1988.

[89] D. Yvon et al. Low noise voltage and charge amplifiers for phonon and ionization detectors at very low temperature. Nuclear Instrumentation and Methods A, 368:778, 1996.

[90] Charge collection and electrode structures in ionization and phonon based dark matter detectors, volume 605. American Institute of Physics, July 2001.

[91] G. Wang. To understand charge trapping. CDMS run118 ebook, \#14, November 2003.

[92] M. Lax. Cascade capture of electrons in solids. Physical Review, 119:1502, 1960.

[93] V. N. Abakumov et al. Capture of carriers by attractive centers in semiconductors. Soviet Physics of Semiconductors, 12:1, 1978.

[94] B. Cabrera. Electron phonon scattering, note93. CDMS note, March 2003.

[95] M. J. Penn et al. Charge collection and trapping effect in cryogenic silicon detectors. Nuclear Instrumentation and Methods A, 370:215, 1996.

[96] E. Pop. Pop researchh page. http://www.stanford.edu/ epop/research.html, May 2002. 
[97] S. Tamura et al. Lattice dynamics and elastic phonon scattering in silicon. Physical Review B, 44:3001, 1991.

[98] P. Meunier. Quasi-particles. CDMS internal notes 0202002, July 2002.

[99] T. Saab et al. Design of qet phonon sensors for the cdms detetcors. Nuclear Instrumentation and Methods A, 444:300, 2000.

[100] K. D. Irwin. Phonon-Mediated Particle Detection Using Superconducting Tungsten Transition-Edge Sensors. PhD thesis, Stanford University, February 1995.

[101] Sae Woo Nam. Development of Phonon-Mediated Cryogenic Particle Detectors with Electron and Nuclear Recoil Discrimination. PhD thesis, Stanford University, December 1998.

[102] D. D. Driscoll. Development and performance of detectors for the cryogenic dark matter search experiment with an increased sensitivity based on a maximum likelihood analysis of beta contamination. PhD thesis, Case Western Reserve University, May 2004.

[103] D. S. Akerib others. Demonstration of feasibioity of operating silicon zip detector with $20 \mathrm{ev}$ threshold. Nuclear Instrumentation and Methods A, $520: 163,2004$.

[104] G. Wang. Superconducting transition edge sensor dynamics. CWRU run20 ebook, Summary of S11, March 2003.

[105] M. E. Msall and J. P. Wolfe. Phonon production in weakly photoexcited semiconductors:quasidiffusion in Ge, GaAs, and Si. Physical Review B, 56:9557, 1997.

[106] Y. B. Levinson. Phonon propagation with frequency down-conversion, in Nonequaillibrium Phonons in Nonmetallic Crystals. Elsevier Science Publisher, 1986.

[107] C. Kittel. Introduction To Solid State Physics. John Wiley \& sons, 1976.

[108] B. K. Ridley. Quantum processes in semiconductors. Oxford University Press, 1993.

[109] T. A. Shutt. A dark matter detector based on the simultaneous measurement of phonons and ionization at $20 \mathrm{mK}$. PhD thesis, University of California, Berkeley, May 1993.

[110] J. A. Shields et al. Propagation of optically generated acoustic phonons in si. Physical Review B, 47:12510, 1993.

[111] R. J. Gaitskell et al. The statistics of background rejection in direct detection experiements for dark matetr. Nuclear Physics B, Proceedings Supplements, 51:279, 1996. 
[112] G. Wang. Tes bias. CDMS run118 ebook \#26, October 2003.

[113] W. Ogburn. Correcting and calibrating qi, qo. CDMS run118 ebook \#114, February 2004.

[114] L. Baudis. Ba: tower 1 data vs mc. CDMS run118 ebook \#149, March 2004.

[115] V. Mandic. Charge threshold cut. CDMS run118 ebook \#131, March 2004.

[116] G. Wang and S. Kamat. Update on qinner cut. CDMS run118 ebook \#125, March 2004.

[117] S. Kamat. Efficiency (data and mc)- of quantities. CDMS run118 ebook \#193, April 2004.

[118] V. Mandic. Stability issues. CDMS run118 ebook \#71, November 2003.

[119] J. Sander. Defining a new cpstd cut. CDMS run118 ebook \#101, March 2004.

[120] R. Mahapatra. A quick look at charge chisq efficiency. CDMS run118 ebook \#110, March 2004.

[121] A. H. Sonnenschein. A search for weakly interacting dark matter particles with low temperature detectors capable of simultaneously measuring ionization and heat. PhD thesis, University of California, Santa Barbara, July 1999.

[122] V. Mandic. Calculating bands. CDMS run118 ebook \#132, March 2004.

[123] T. Shutt et al. Measurement of ionization and phonon production by nuclear recoils in a $60 \mathrm{~g}$ crystal of germanium at $25 \mathrm{mk}$. Physical Review Letters, 69:3425, 1992.

[124] D. S. Akerib et al. Preliminary limits on the wimp-nucleon cross section from the cryogenic dark matter search. Nuclear Physics B, Proceedings Supplements, 70:64, 1999.

[125] R. Clarke et al. Enhanced ballistic phonon production for surface events in cryogenic silicon detector. Applied Physics Letters, 76:2958, 2000.

[126] D. S. Akerib and G. Wang. Define new time parameter with neutron calibration data. CDMS SUF run21 ebook \#300, March 2003.

[127] G. Wang. charge collection time. CDMS SUF run21 ebook \#302, March 2003.

[128] G. Wang. Position information of zip detector. CDMS run118 ebook \#92, January 2004.

[129] V. Mandic. Z-position information in phonon delay. CDMS G31 ebook \#40, August 2003. 
[130] V. Mandic. Calculating risetime cuts - case of z3. CDMS run118 ebook \#133, March 2004.

[131] G. Wang. Surafce event rejection with timing and position information. CDMS run118 ebook \#203, April 2004.

[132] G. Wang. Z6 problem. CDMS run118 ebook \#195, April 2004.

[133] V. Mandic. Z1-6 risetime cut using two parameters. CDMS run118 ebook \#139, March 2004.

[134] V. Mandic. Surface events rejection and leakage. CDMS run118 ebook \#144, March 2004.

[135] D. S. Akerib et al. New results from the cryogenic dark matter search experiment. Physical Review D, 68:082002, 2003.

[136] R. Bernabei et al. Dark matter search with the dama experiments. Nuclear Physics B, Proceedings Supplements, 87:67, 2000.

[137] B. Benoit et al. Improved exclusion limits from the edelweiss wimp search. Physics Letters B, 545:43, 2002.

[138] E. A. Baltz and P. Gondolo. Improved constraint on supersymmetric dark matter from muon g-2. Physical Review D, 67:063503, 2003.

[139] S. M. Sze. Semiconductor devices, physics and technology. John Wiley \& sons, 1985.

[140] M. Balkanski. Optical properties of semiconductors. North-Holland Pub. Co., 1994.

[141] K. Seeger. Semiconductor physics : an introduction. Springer-Verlag, 1991. 